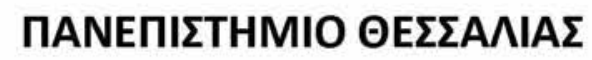

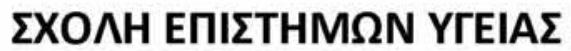

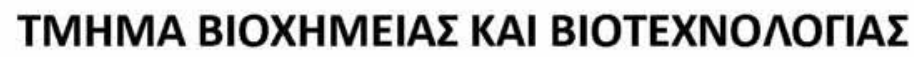

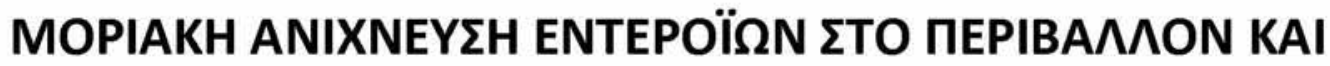

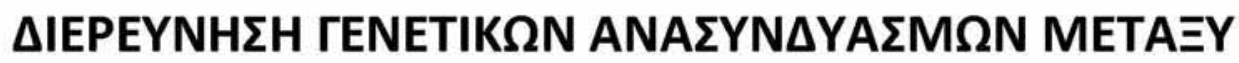

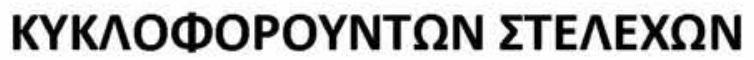

$\Delta$ I $\triangle$ AKTOPIKH $\triangle$ IATPIBH

ZAXAPOYAA Г. KYPIAKOПOYАOY

вІологог

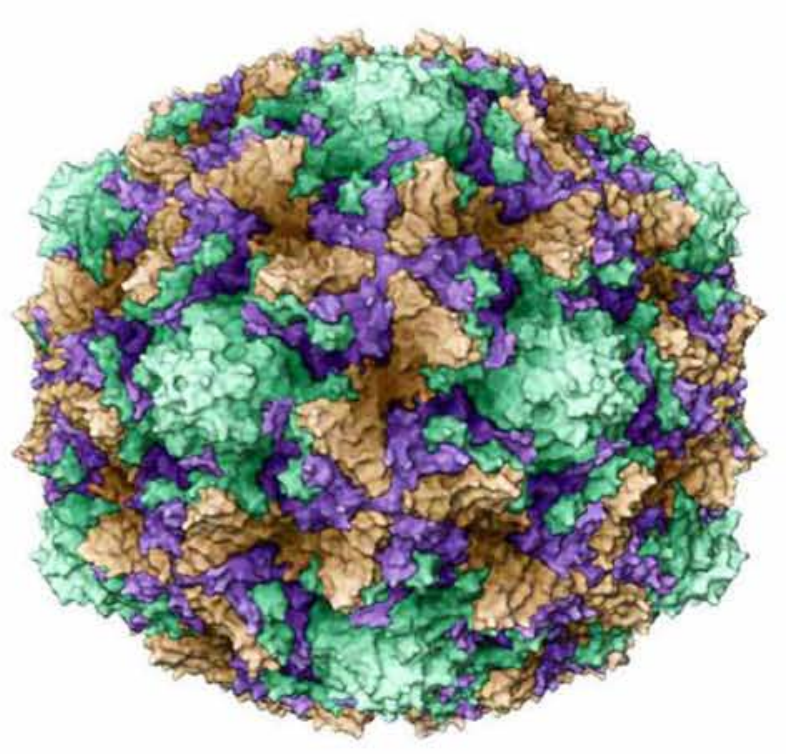

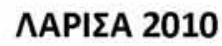




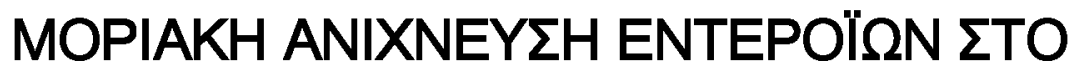

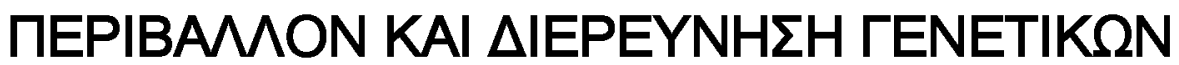

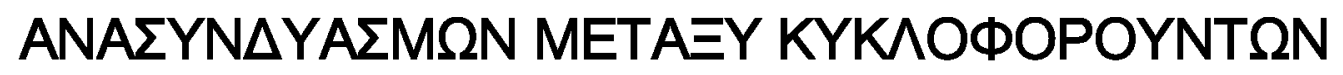 \\ $\Sigma T E \wedge E X \Omega N$}




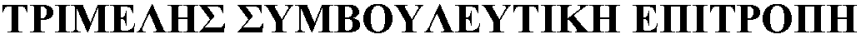

\section{П. МАРКОYААТО ( ЕПIB}

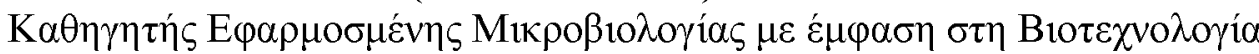

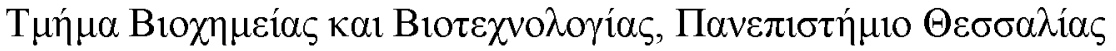

\section{Z. MAMOYPH $\boldsymbol{2}$}

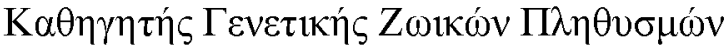

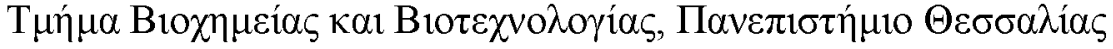

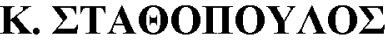

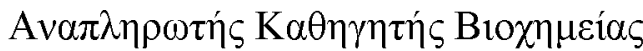

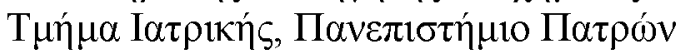

\section{ЕПТАМЕАН $\Sigma$ ЕЕЕТА}

\section{N. KATH 2}

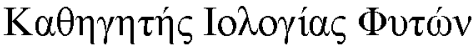

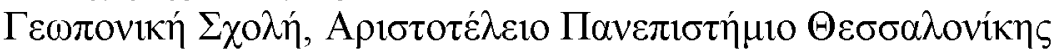

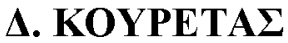

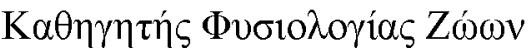

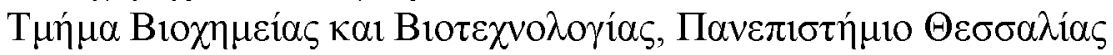

\section{Z. MAMOYPH 2}

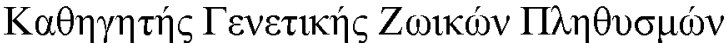

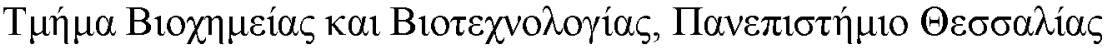

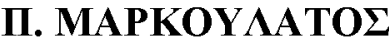

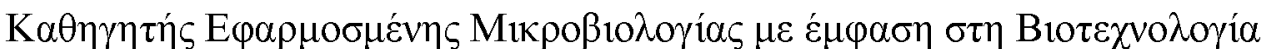

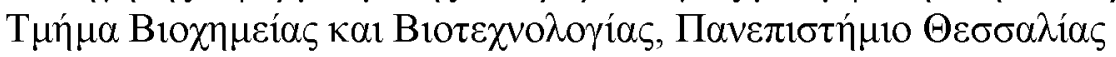

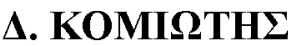

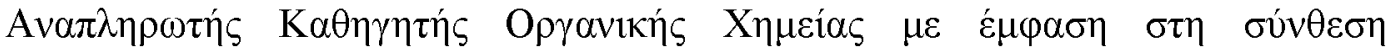

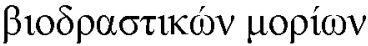

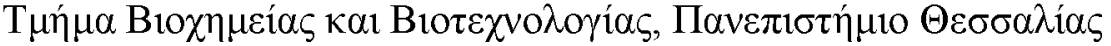

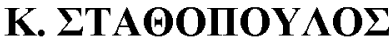

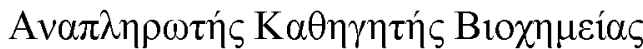

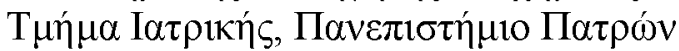

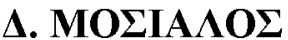

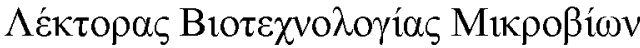

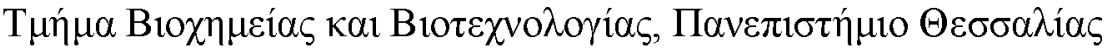




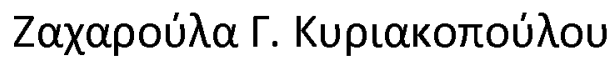

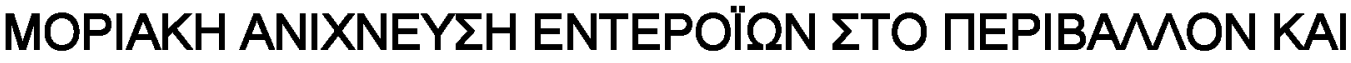 $\triangle I E P E Y N H \Sigma H \Gamma E N E T I K \Omega N$ ANA $\Sigma Y N \Delta Y A \Sigma M \Omega N$ METAEY

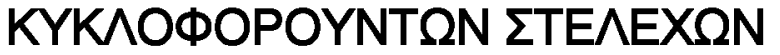

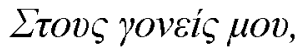

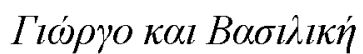
$\kappa \alpha \imath \tau \alpha \alpha \delta \varepsilon \dot{\rho} \varphi \imath \alpha \mu o v$,

$O \lambda v \mu \pi i \alpha \kappa \alpha l H \lambda i \alpha$ 


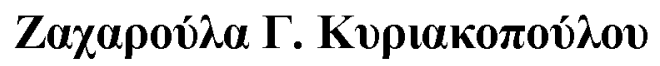

2010

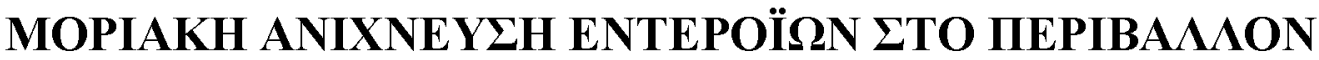 KAI $\triangle I E P E Y N H \Sigma H ~ \Gamma E N E T I K \Omega N ~ A N A \Sigma Y N \Delta Y A \Sigma M \Omega N$

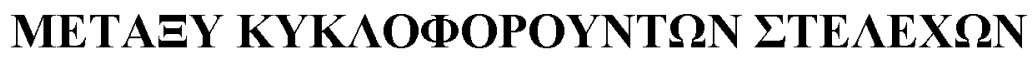

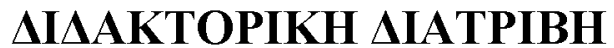

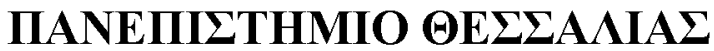

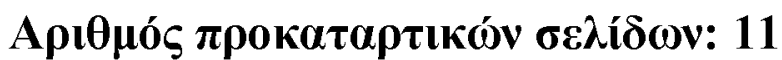

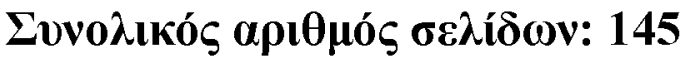

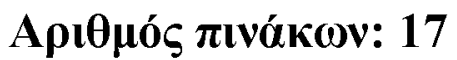

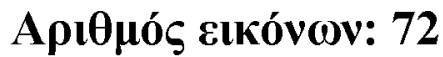

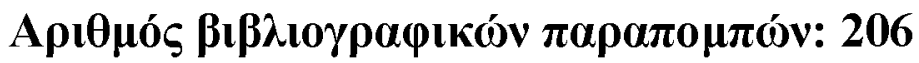




\section{ПЕРІАНЧН}

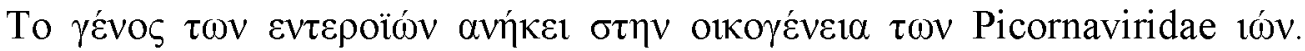

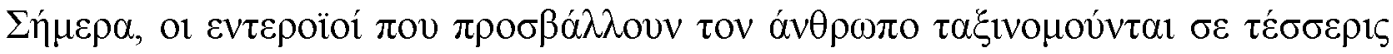

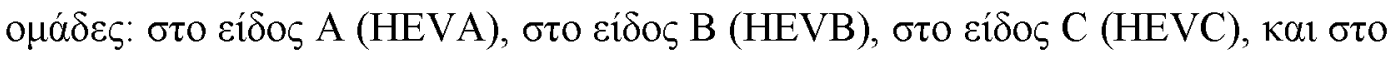

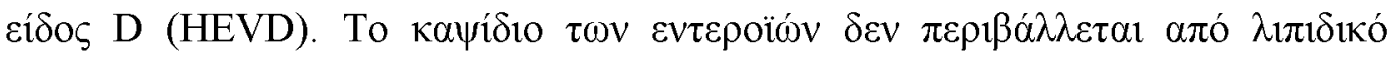

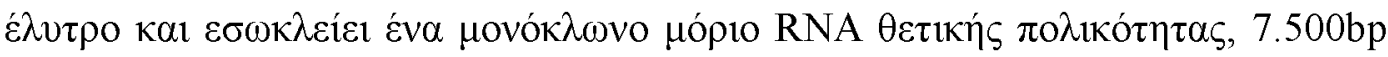

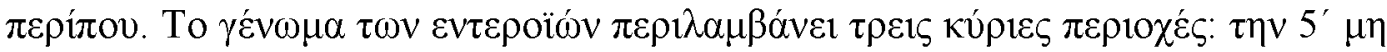

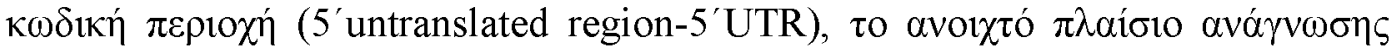

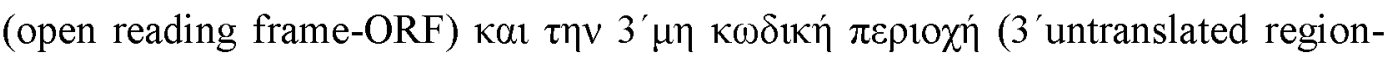

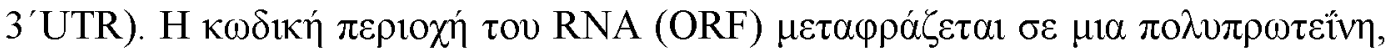

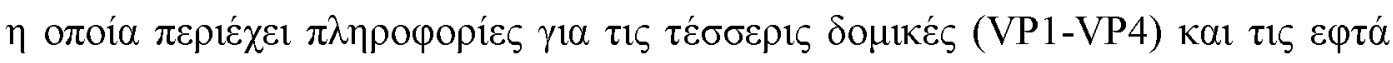

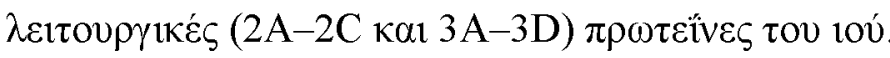

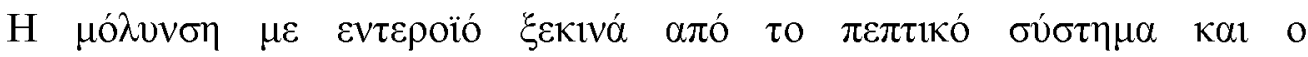

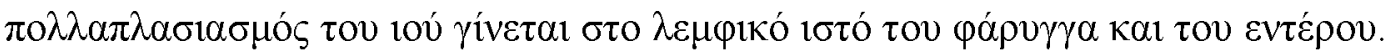

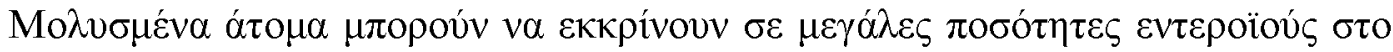

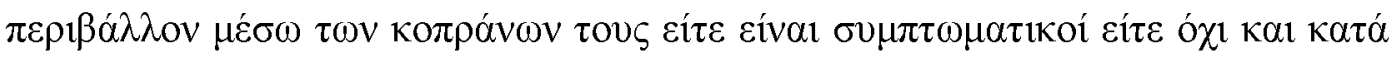

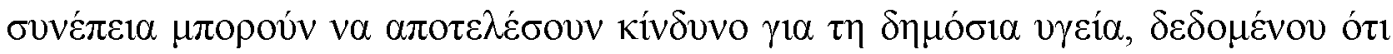

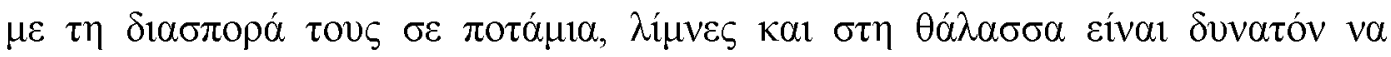

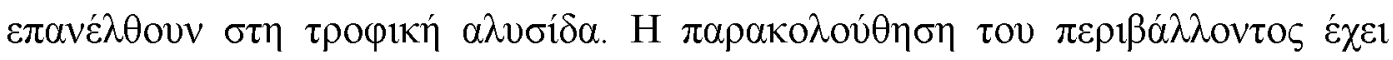

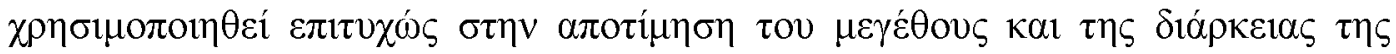

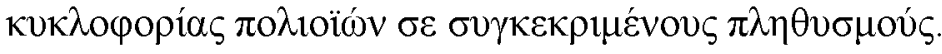

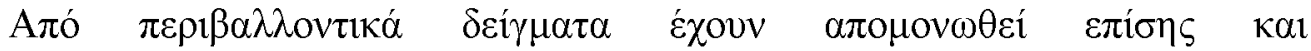

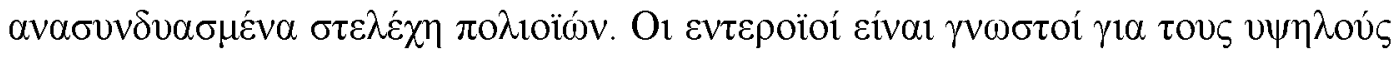

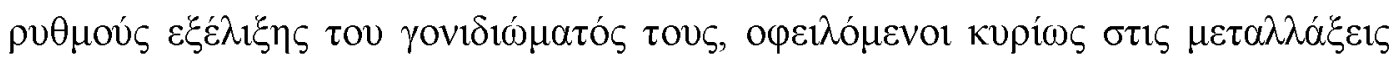

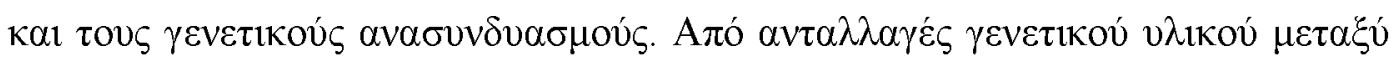
$\tau \omega \vee$ 1

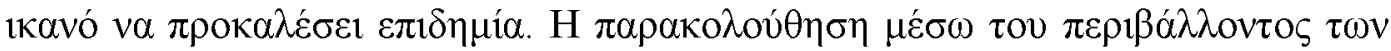

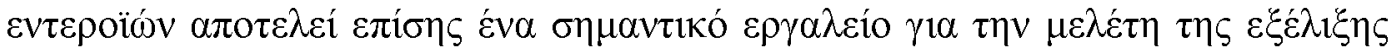

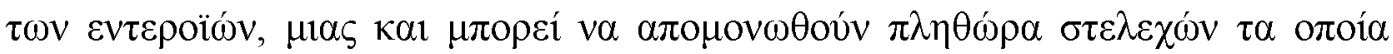

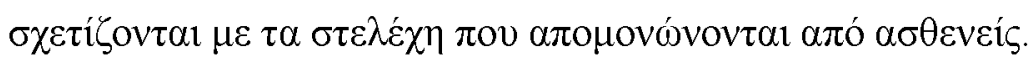




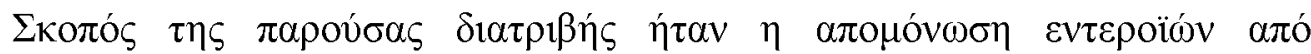

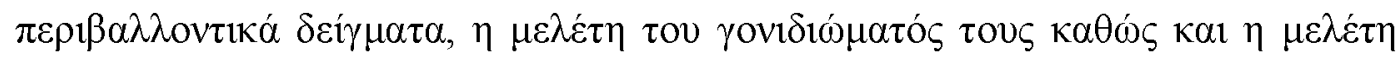

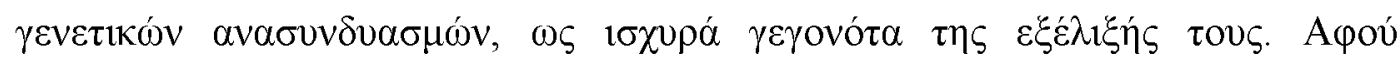

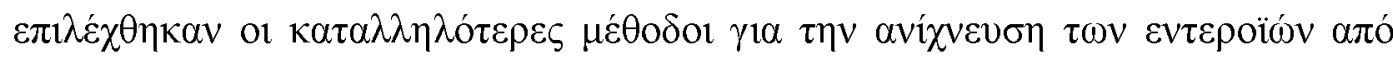

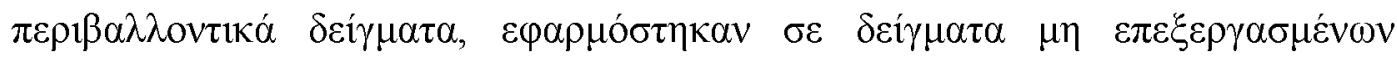

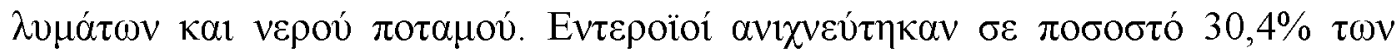

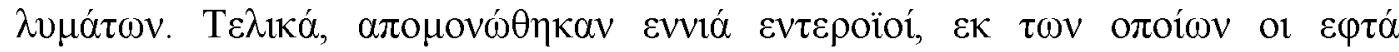

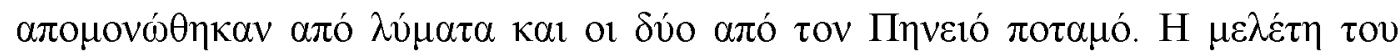

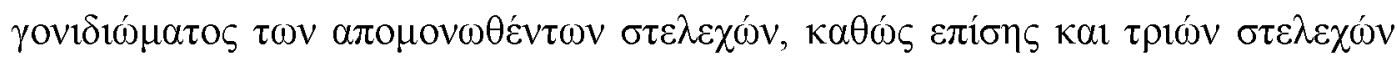

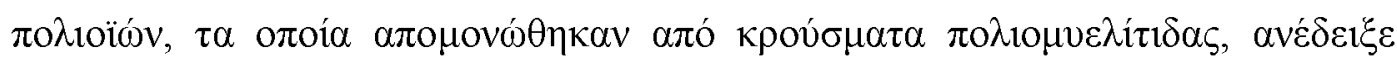

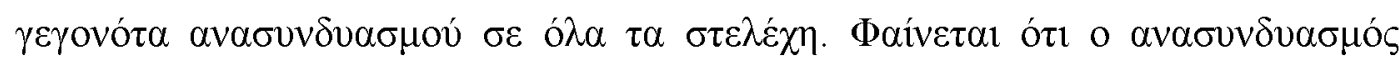

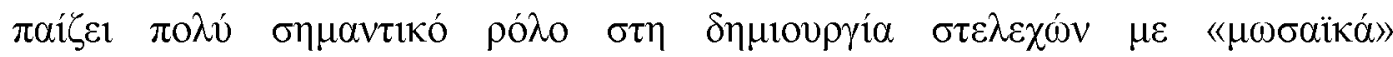

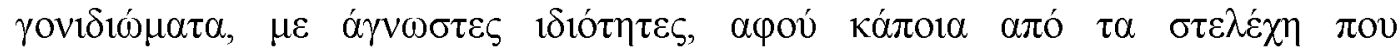

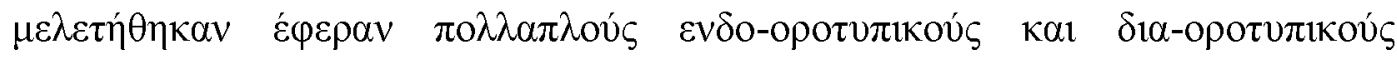
$\alpha v \alpha \sigma v v \delta v a \sigma \mu o u ́ s$. 


\section{ПРОАОГОГ}

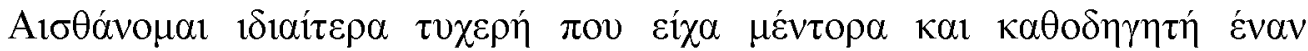

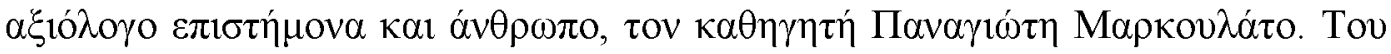

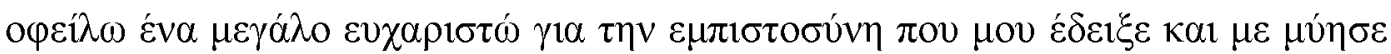

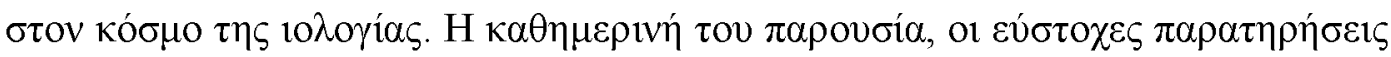

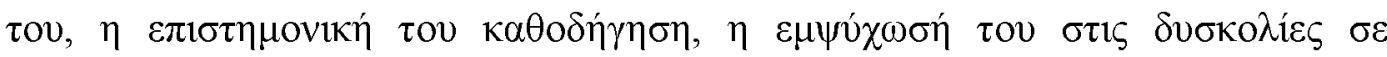

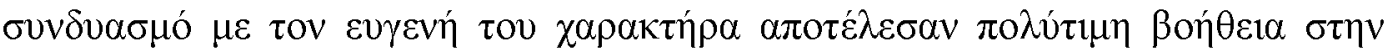

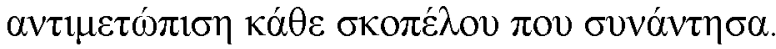

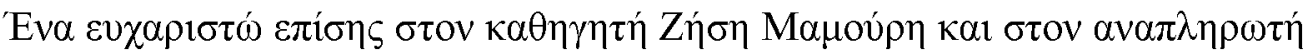

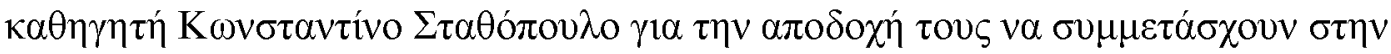

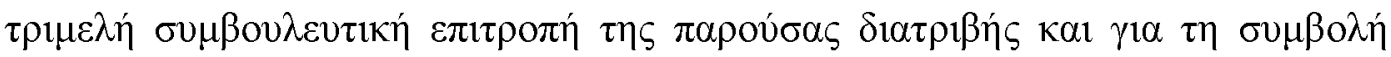

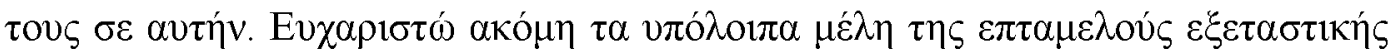

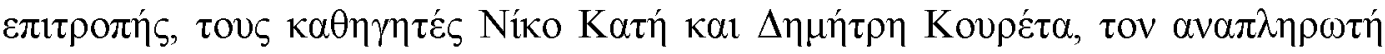

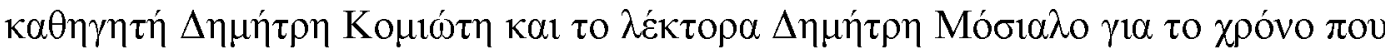

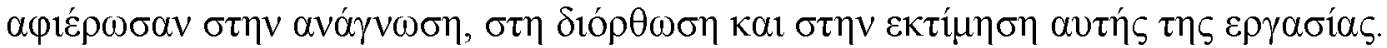

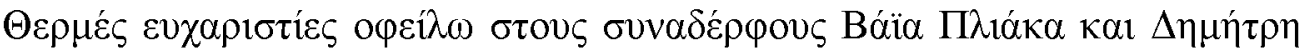

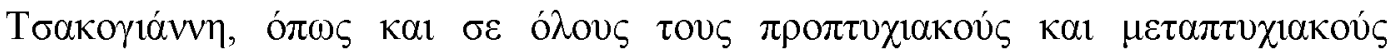


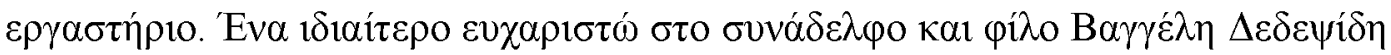

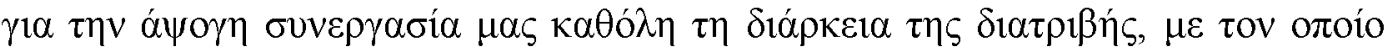

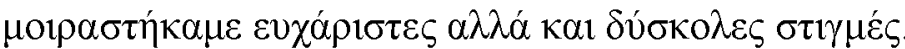

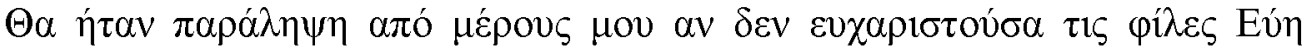

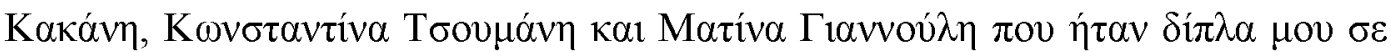

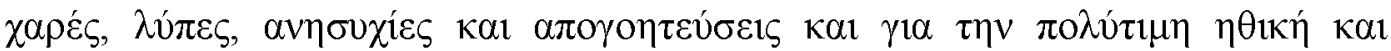

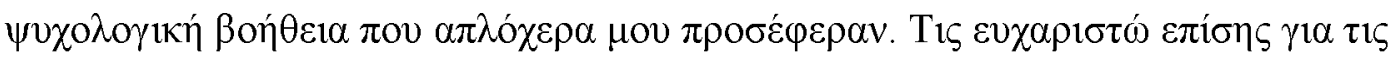

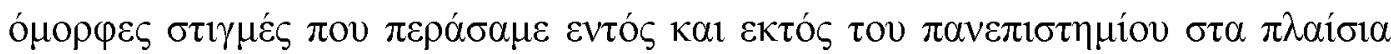

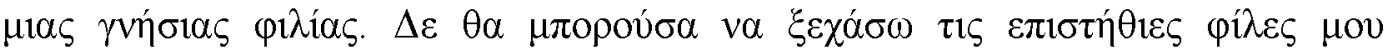

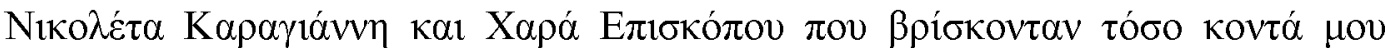

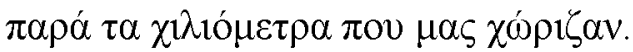

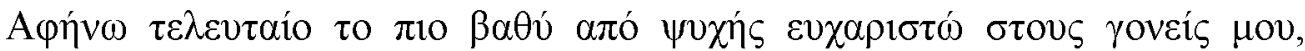

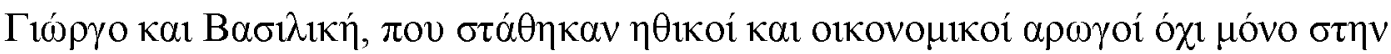




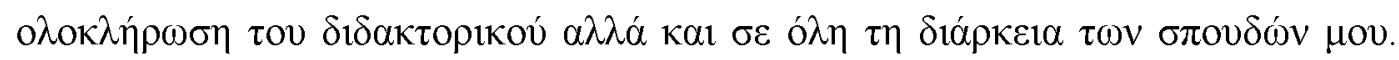

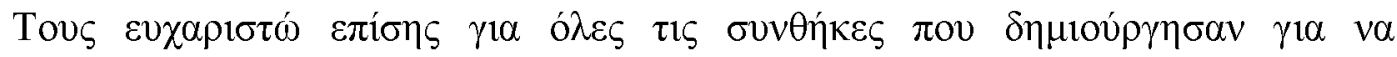

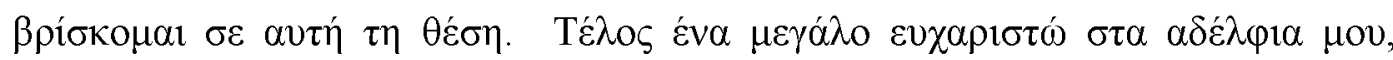

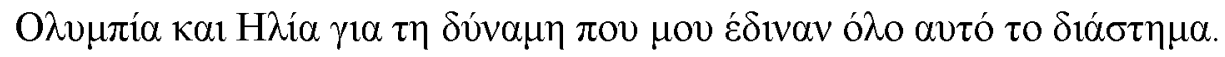




\section{IEPIEXOMENA}

\section{КЕФАМАIO 1}

ЕГГАГ ЛГН

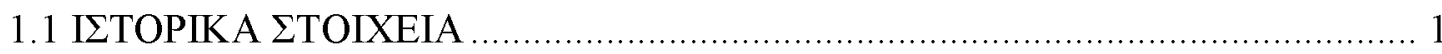

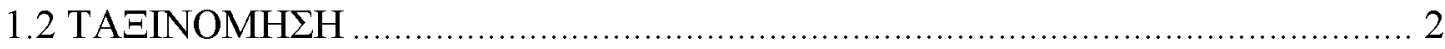

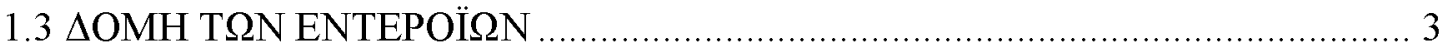

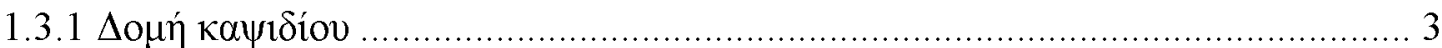

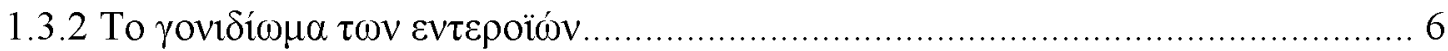

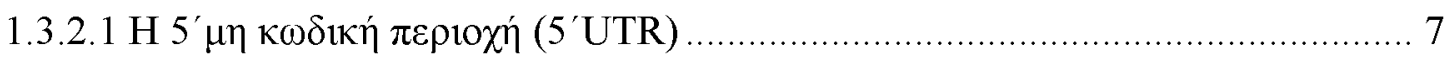

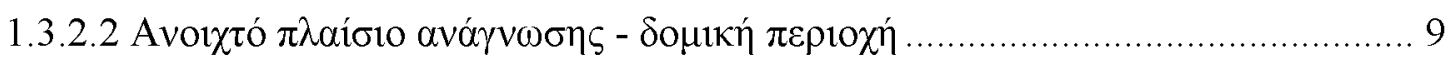

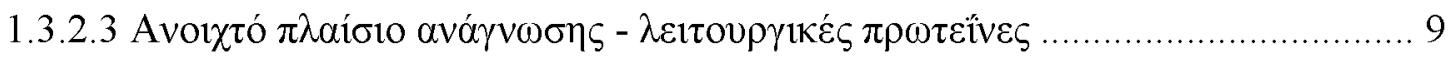

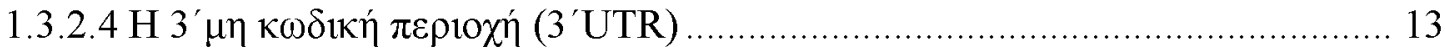

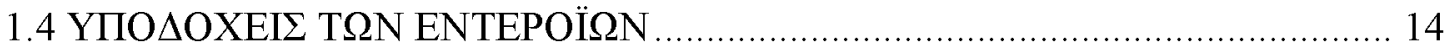

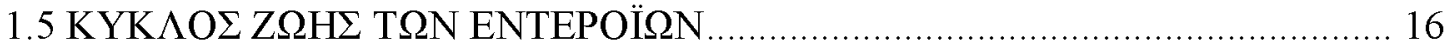

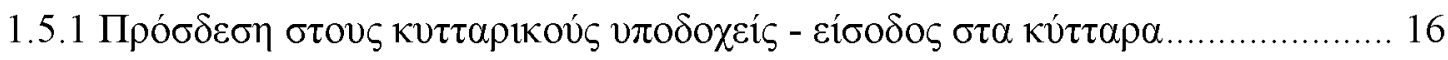

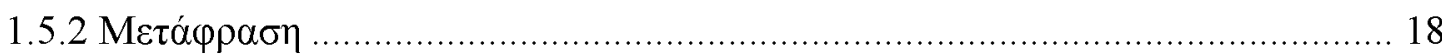

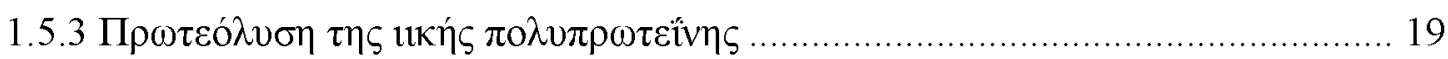

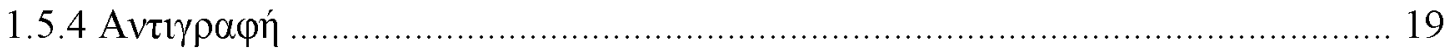

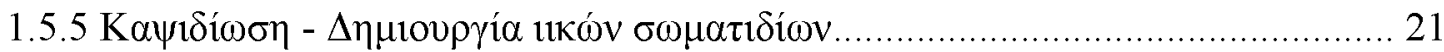

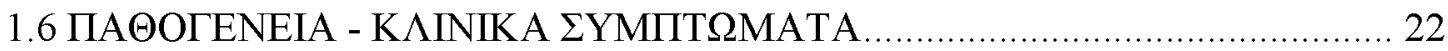

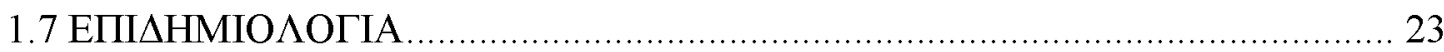

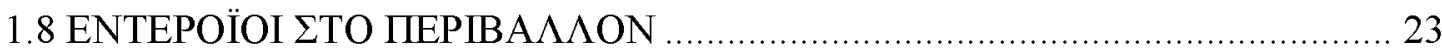

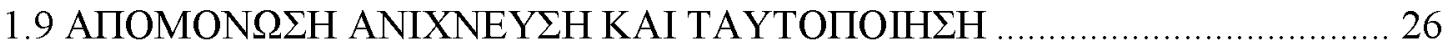

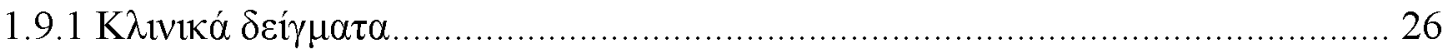

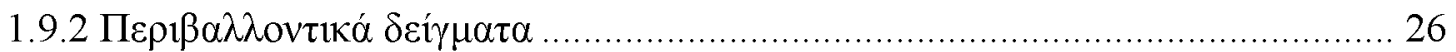

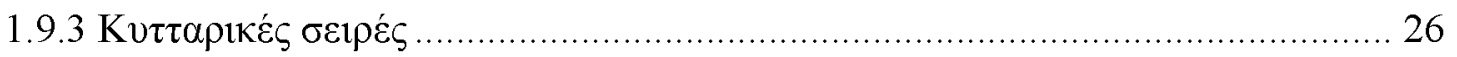

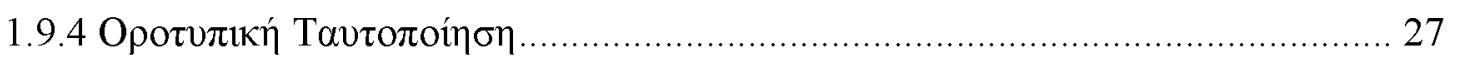

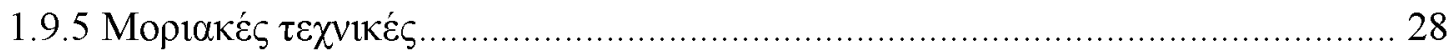

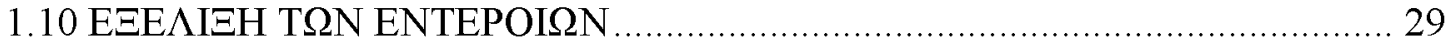

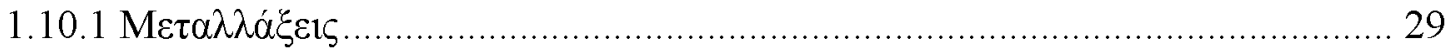

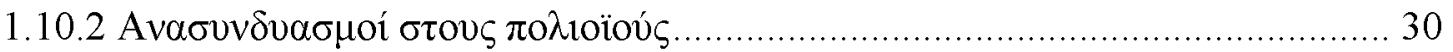




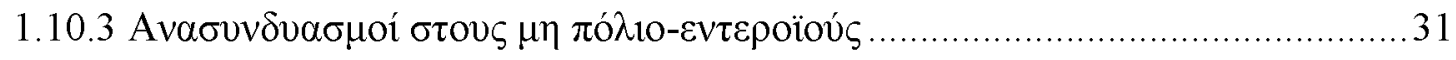

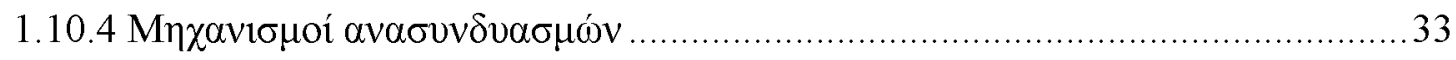

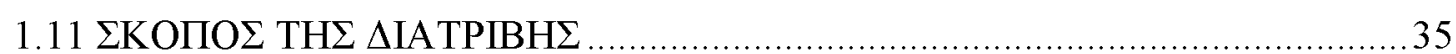

\section{КЕФА АAIO 2}

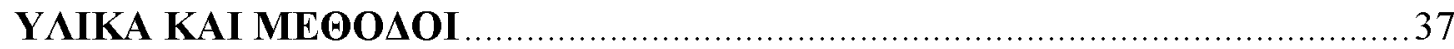

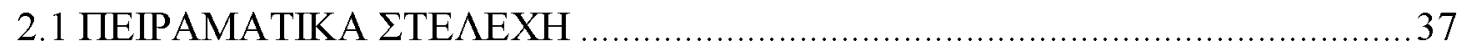

2.1.1 Про́ $\tau$

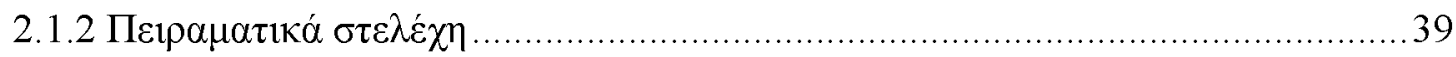

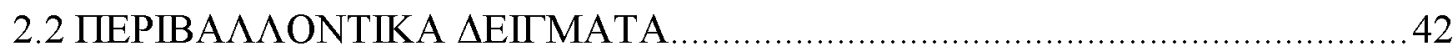

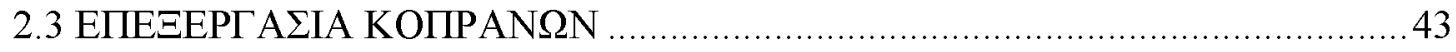

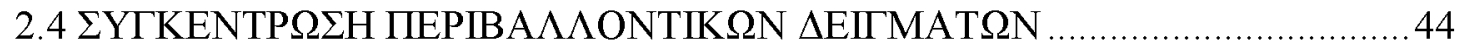

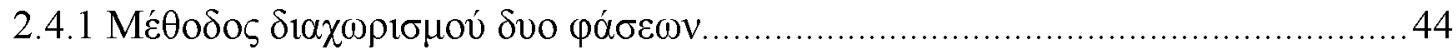

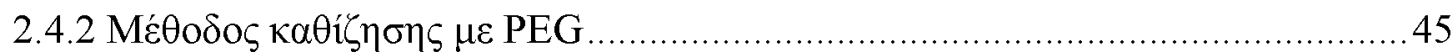

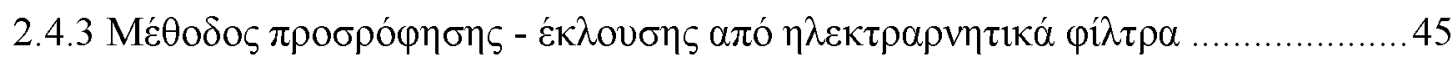

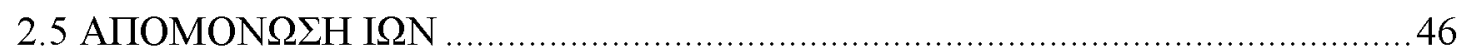

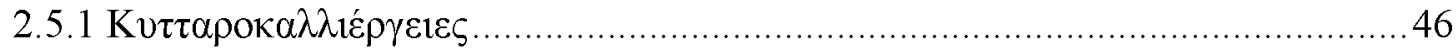

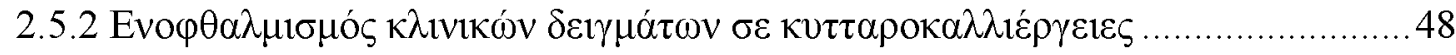

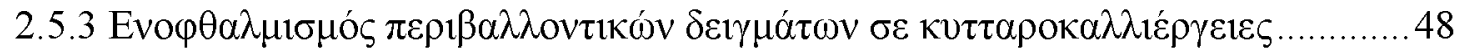

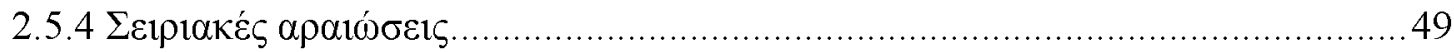

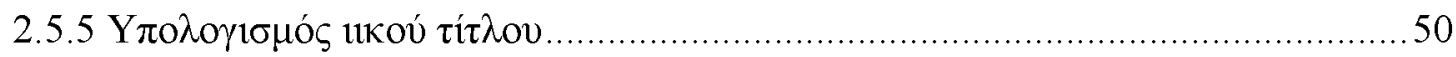

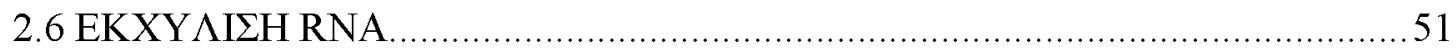

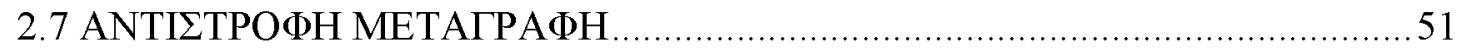

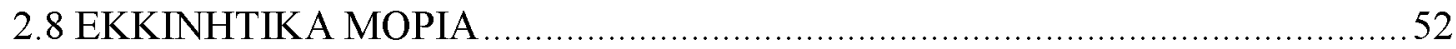

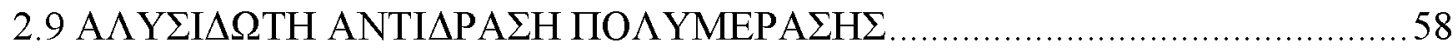

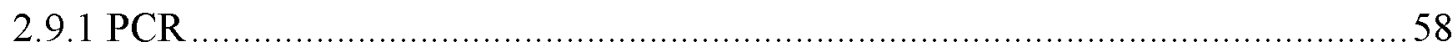

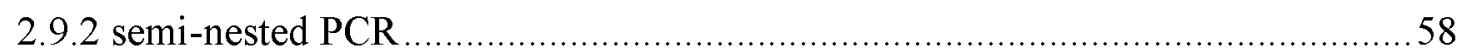

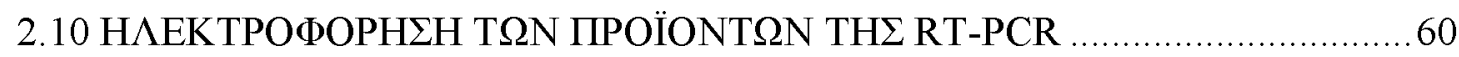

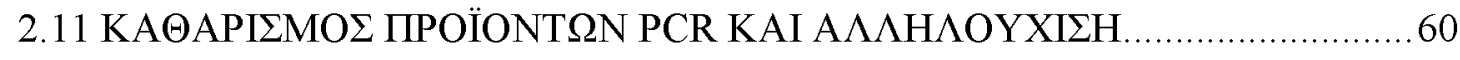

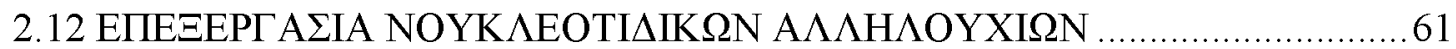

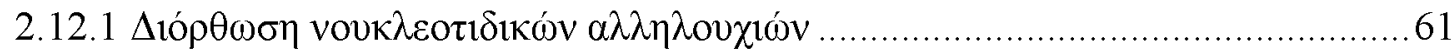

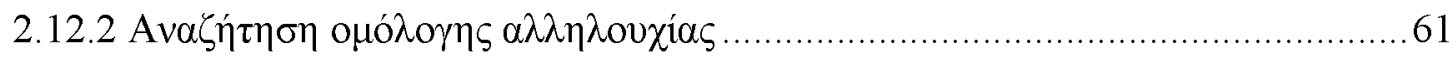

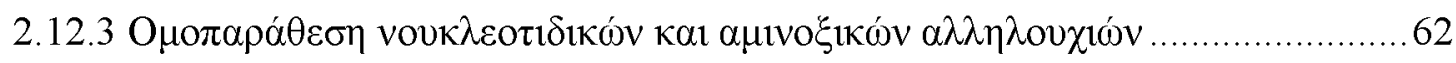

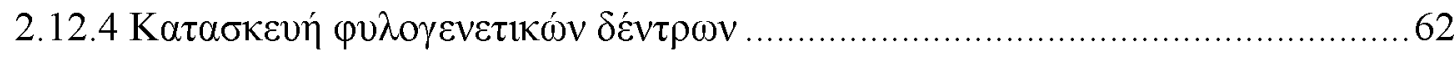




\section{КЕФАМАIO 3}

AIIOTEAEহMATA 65

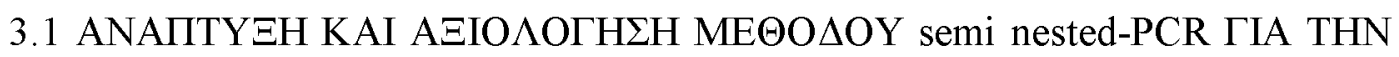
ANIXNEY $\Sigma H$ ENTEPOÏ $\Omega N$

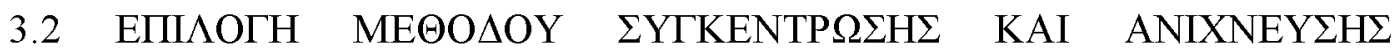
ENTEPOÏ $\Omega N \Sigma E$ ПЕPIBA $\Lambda \Lambda$ ONTIKA $\triangle$ EIГMATA 65

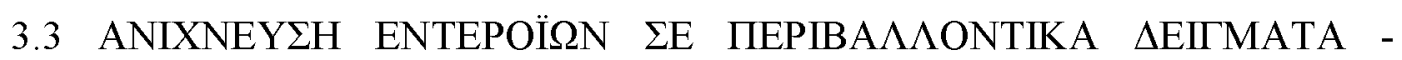
$\Sigma$ YГKPI $\Sigma H \mathrm{ME} \Theta \mathrm{O} \Delta \Omega \mathrm{N}$ ANIXNEY $\Sigma \mathrm{H} \Sigma$.. 66

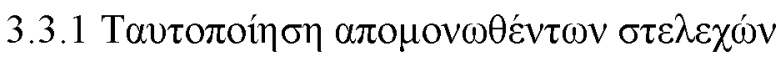
67

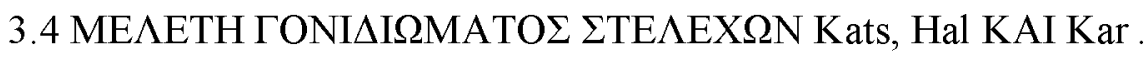
69

3.5 ME $\Lambda$ ЕTH ГONI $\triangle \mathrm{I} \Omega$ MATO $\Sigma \Sigma$ TE $\Lambda$ EXOY $\Sigma$ LR11F7 ..................................... 77

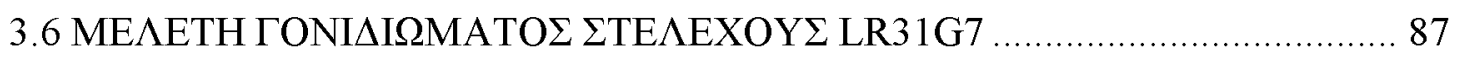

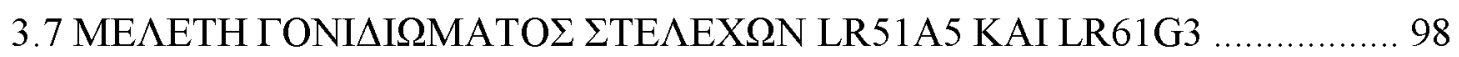

КЕФАААIO 4

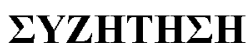

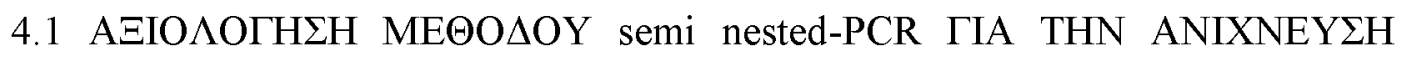
ENTEPOÏ $\Omega N$

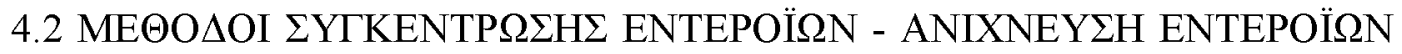

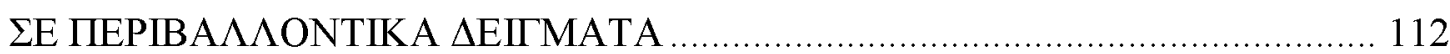

4.3 ME $\Lambda$ ETH ГONI $\triangle \mathrm{I} \Omega$ MATO $\Sigma \Sigma T E \Lambda E X \Omega N$ Kats, Hal KAI Kar ....................... 118

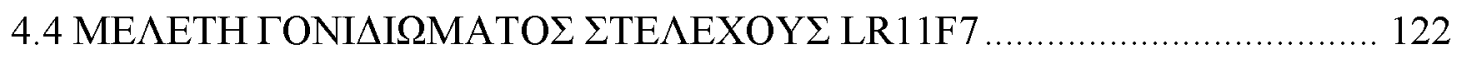

4.5 ME $\Lambda$ ETH ГONI $\triangle \mathrm{I} \Omega M A T O \Sigma \Sigma T E \Lambda E X O Y \Sigma$ LR31G7 ................................. 125

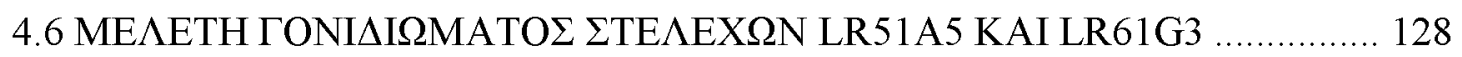

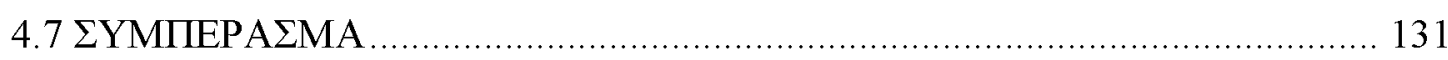

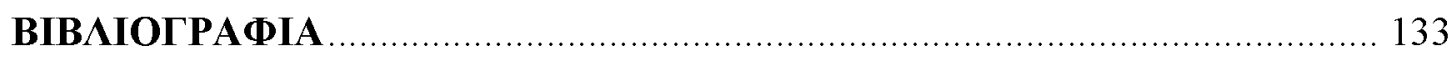

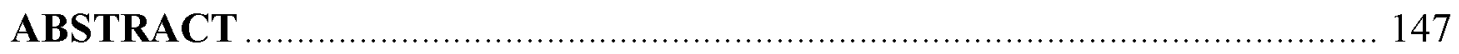

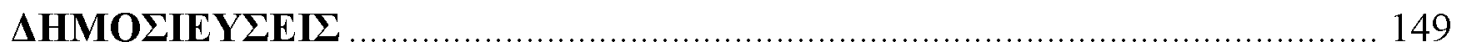




\section{KЕФААAIO 1}

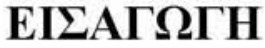

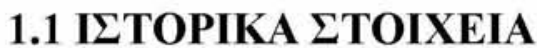

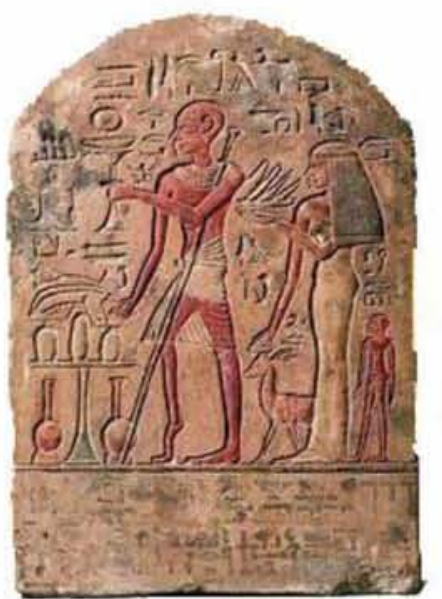

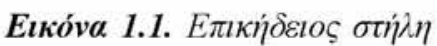

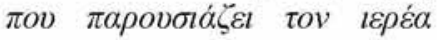

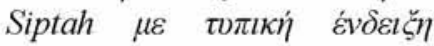
$\pi \alpha \rho \alpha \lambda \nu \tau \imath \kappa \eta ் \varsigma ~ \pi о \lambda \imath o \mu v \varepsilon \lambda i t \imath \delta \alpha \varsigma$.

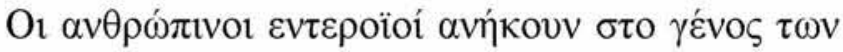

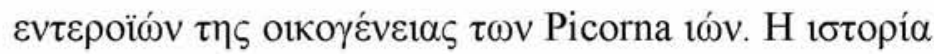

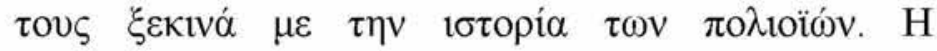

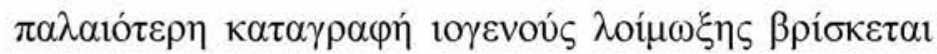

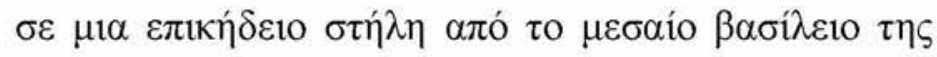

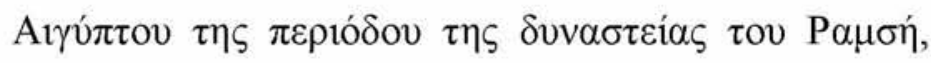

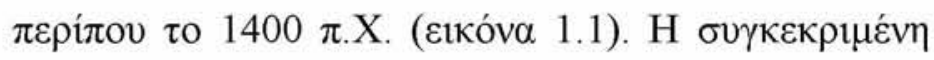

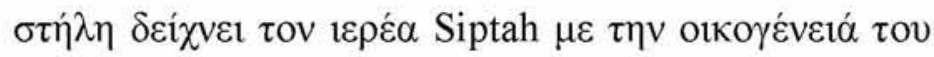

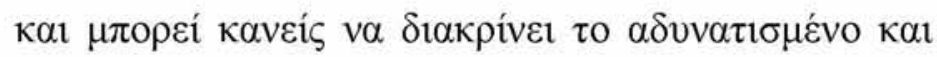

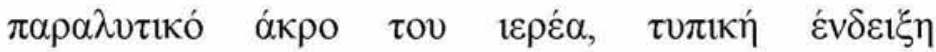

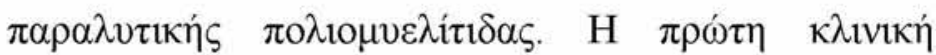

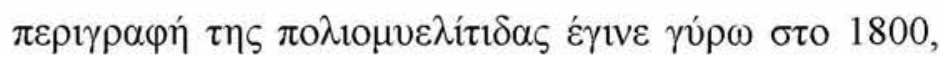

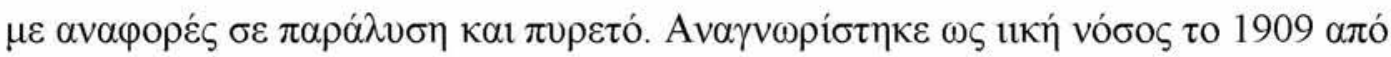

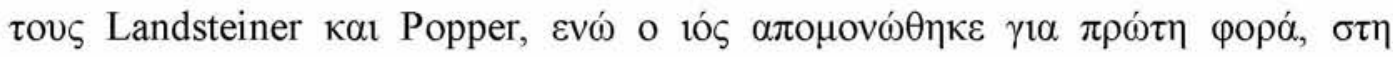

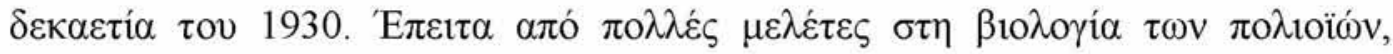

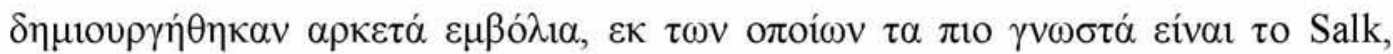

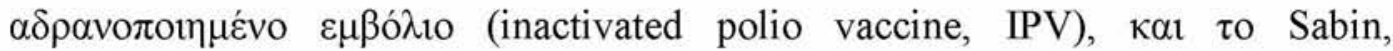

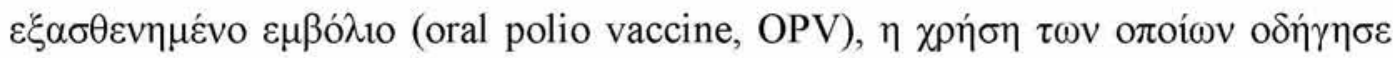

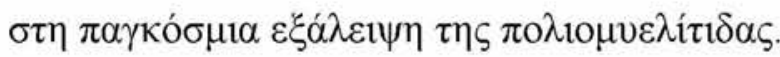

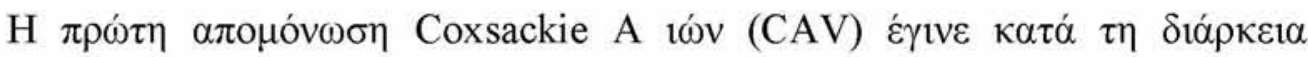

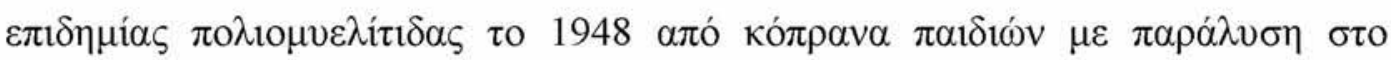

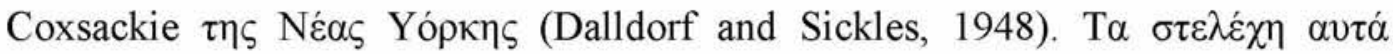

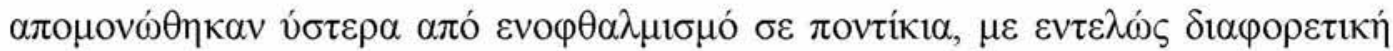

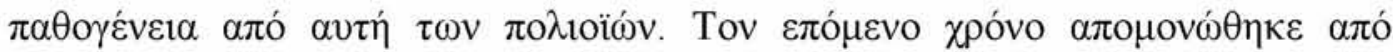

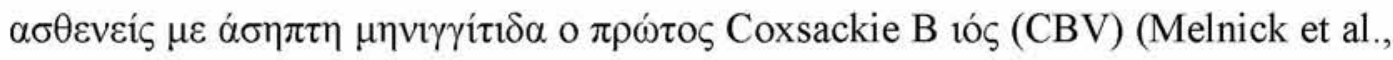

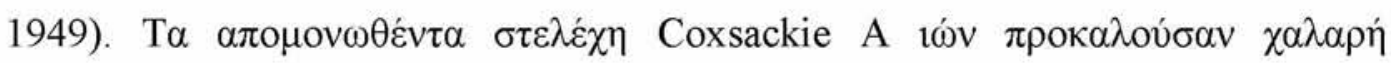




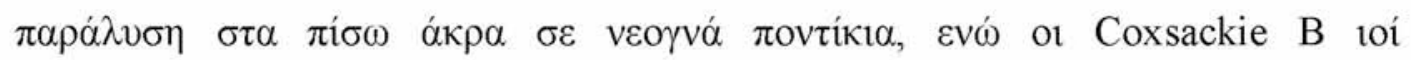

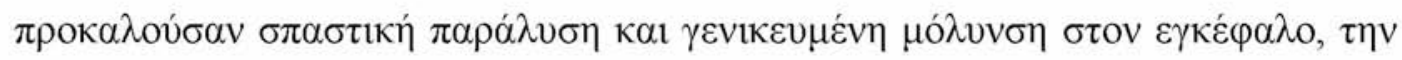

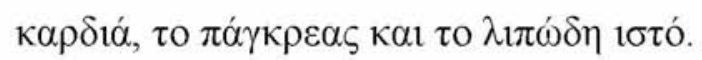

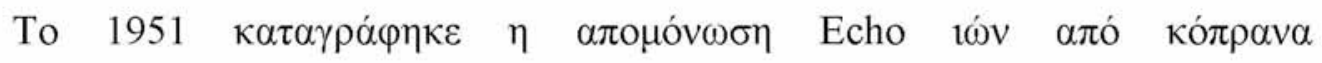

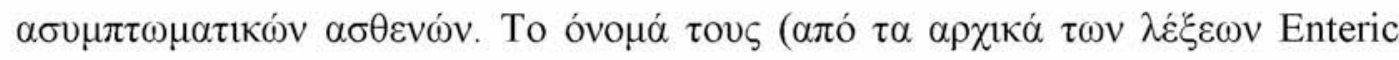

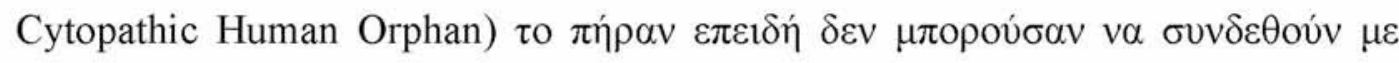

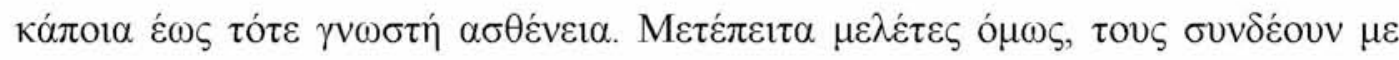

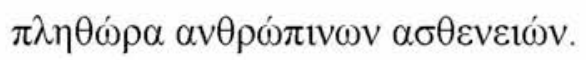

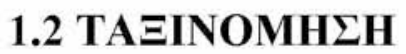

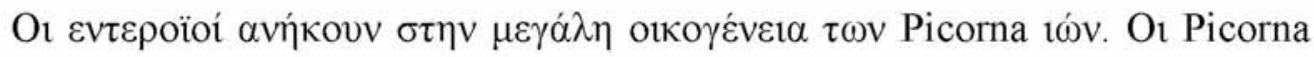

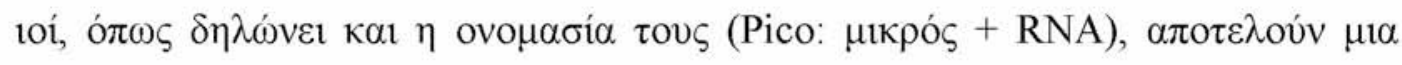

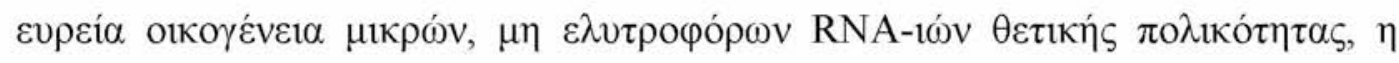

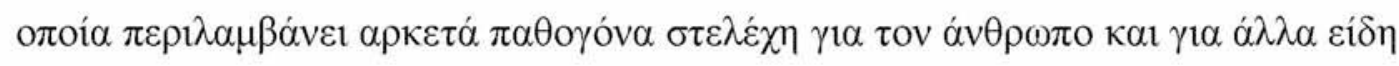

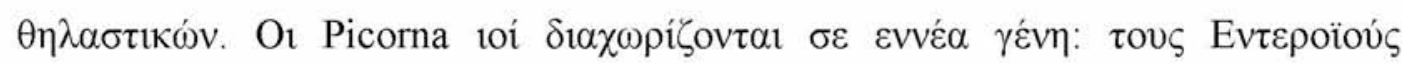

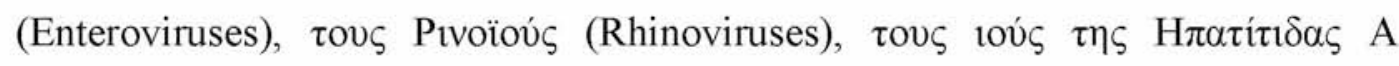

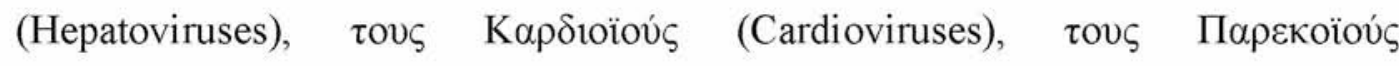

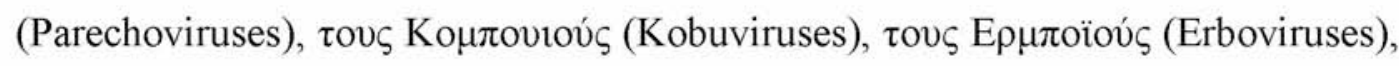

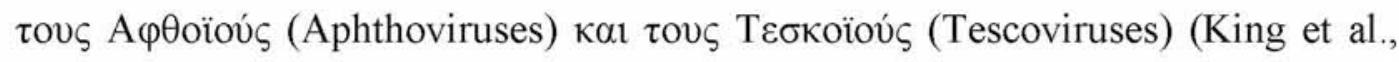

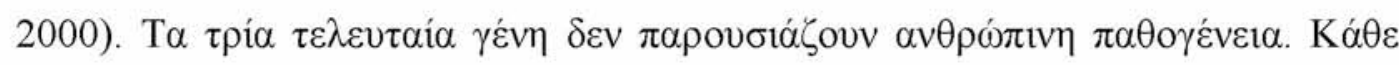

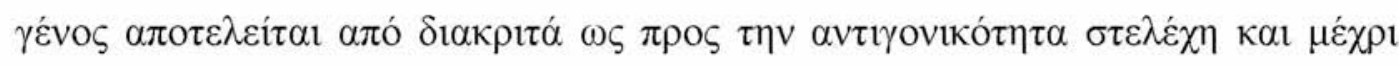

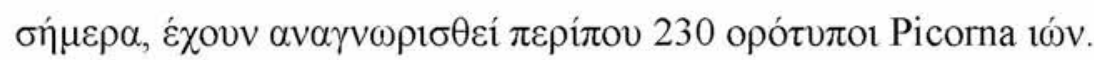

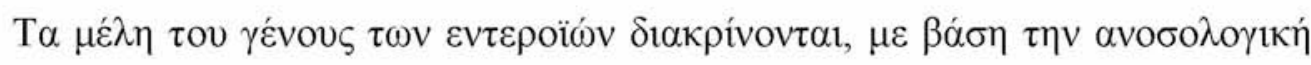

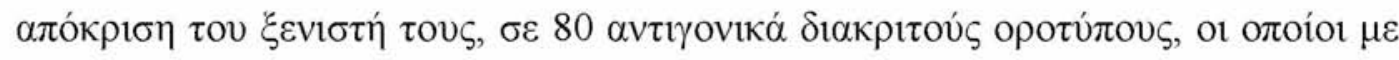

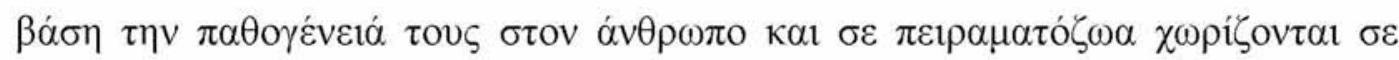

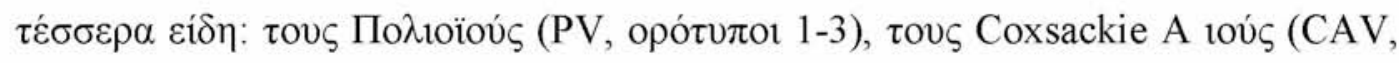

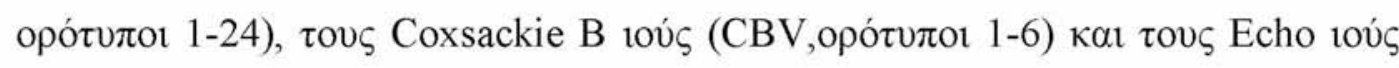

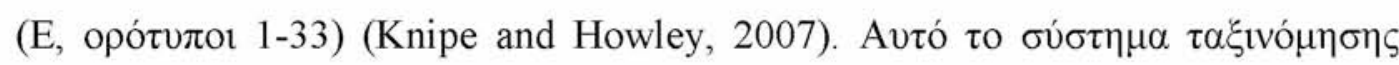

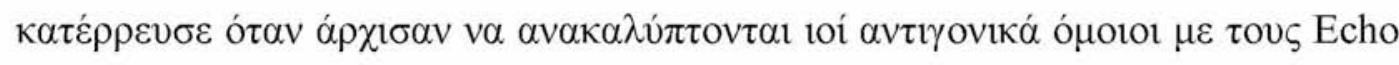

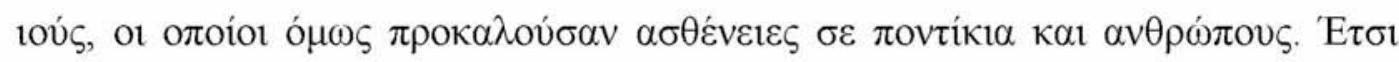

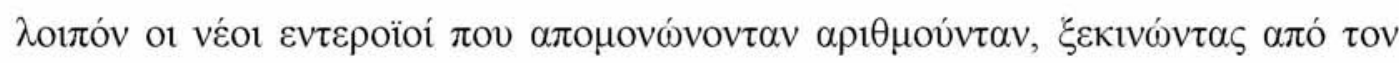
Evזєроїo 68 (EV68). 


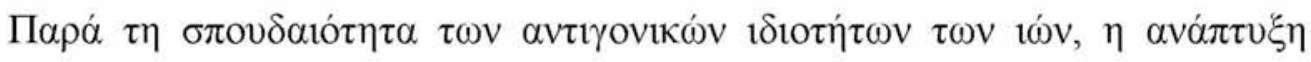

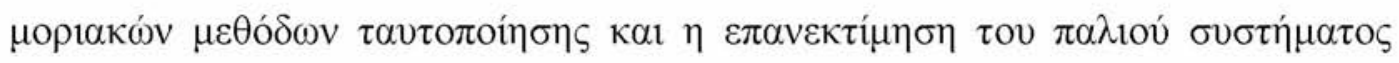

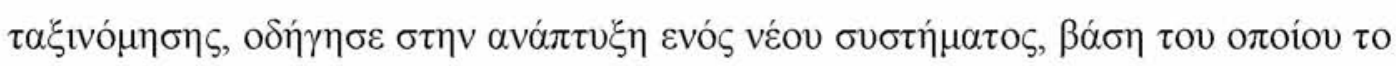

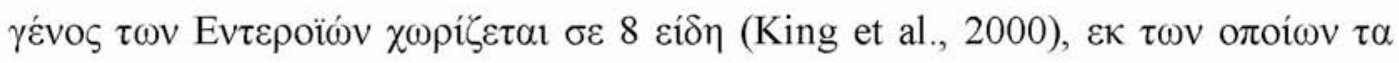
$\pi \varepsilon \dot{\varepsilon} \tau \varepsilon$ (Poliovirus $\kappa \alpha$ Human enterovirus A-D) $\pi \alpha \rho o v \sigma i \alpha ́ \zeta o v v a v \theta \rho \omega ́ \pi ı v \eta$

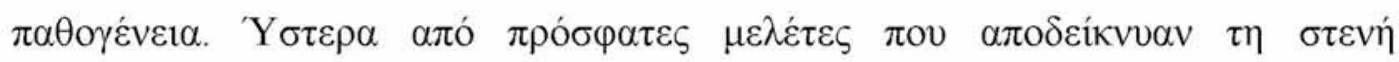

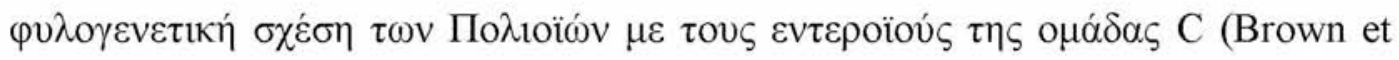

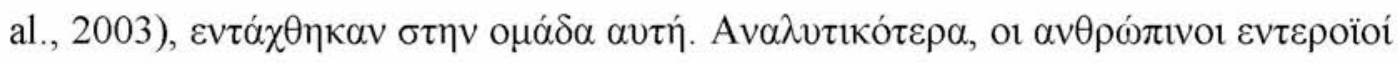

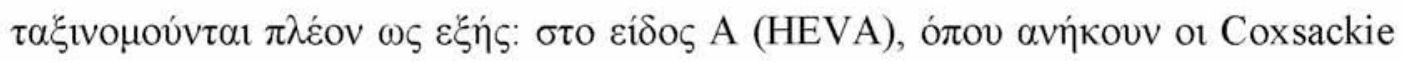

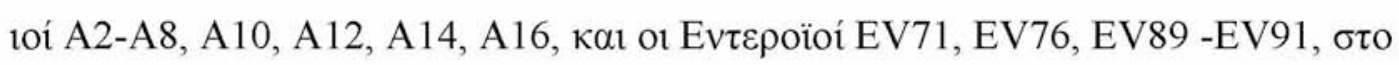

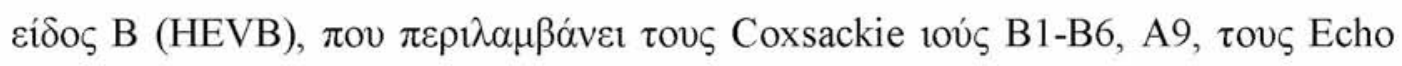

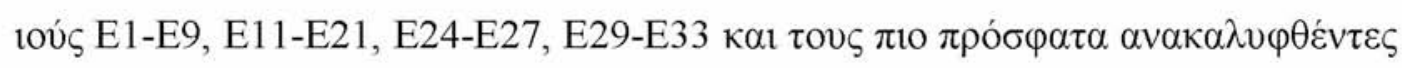

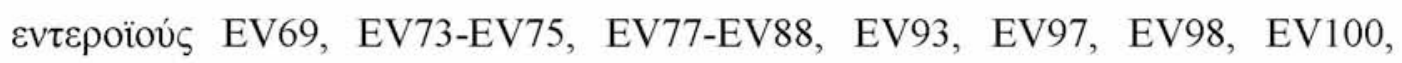

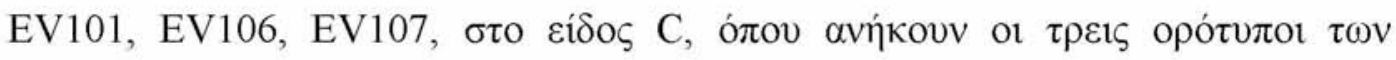

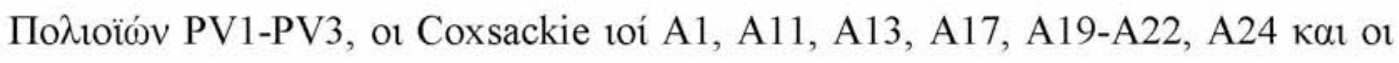

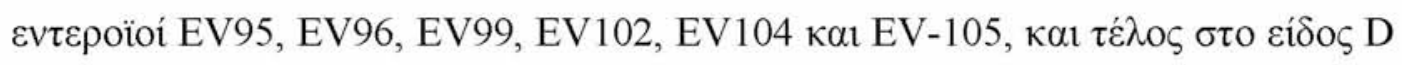

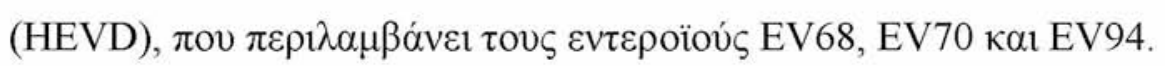

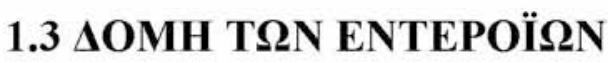

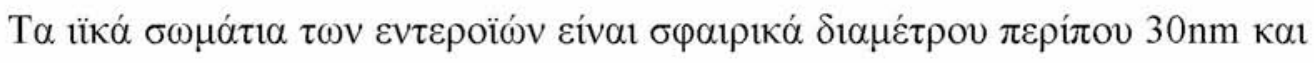

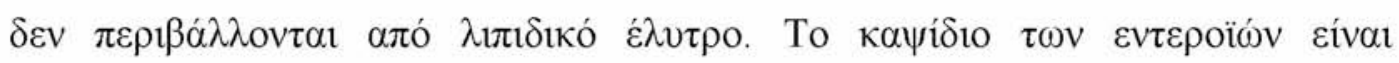

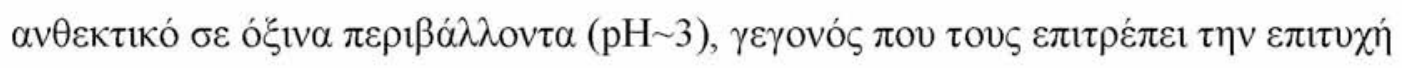

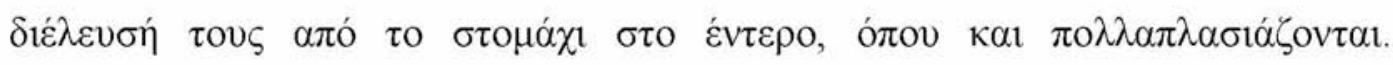

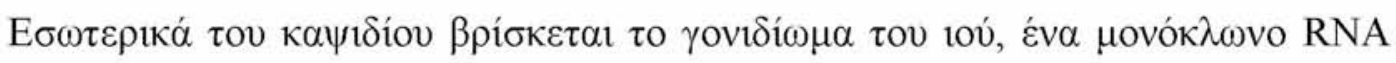

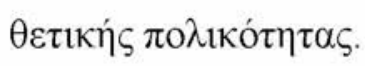

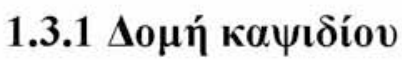

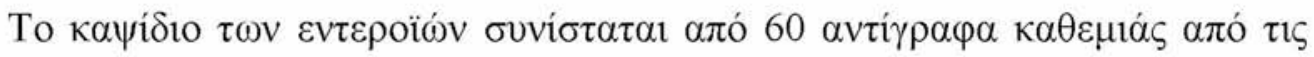

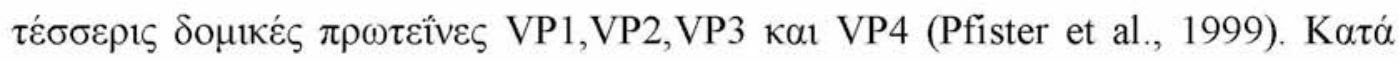

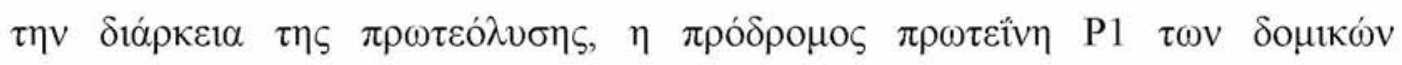

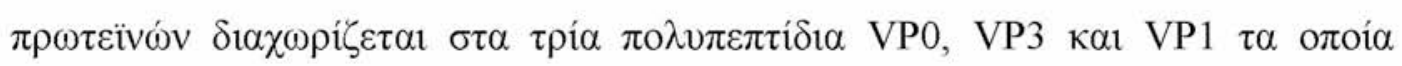

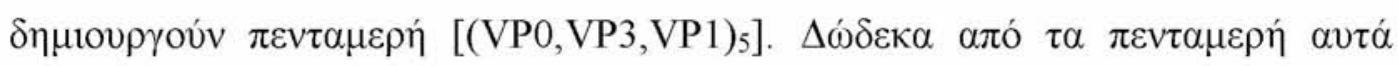

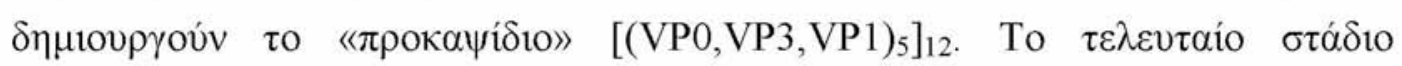




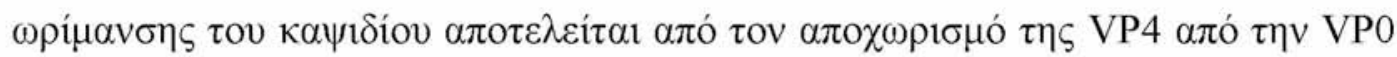

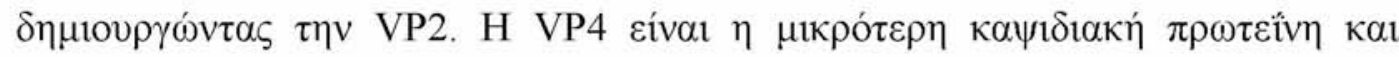

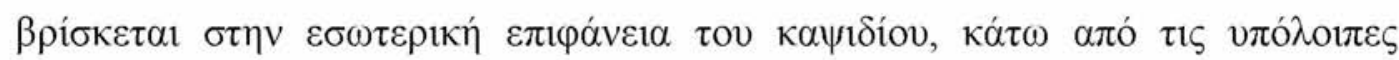

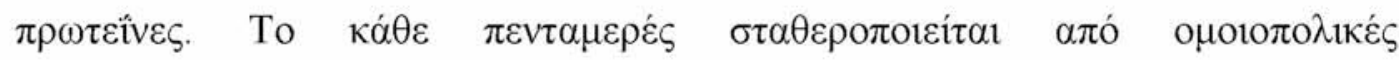

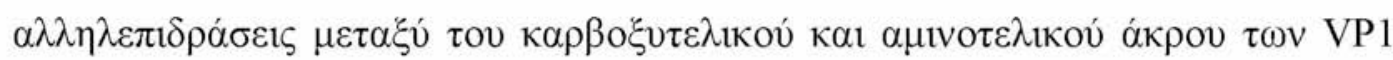

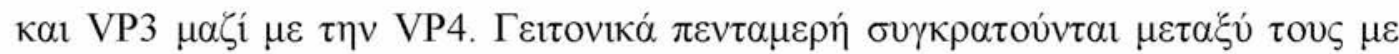

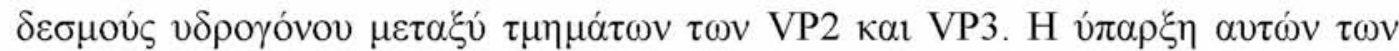

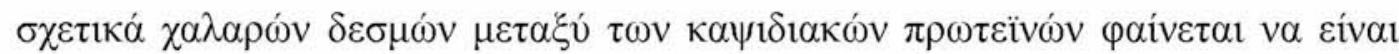

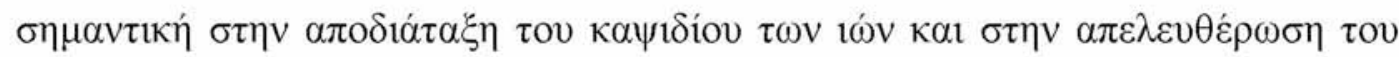

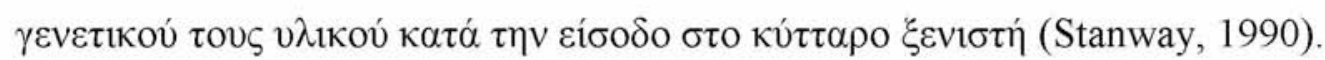

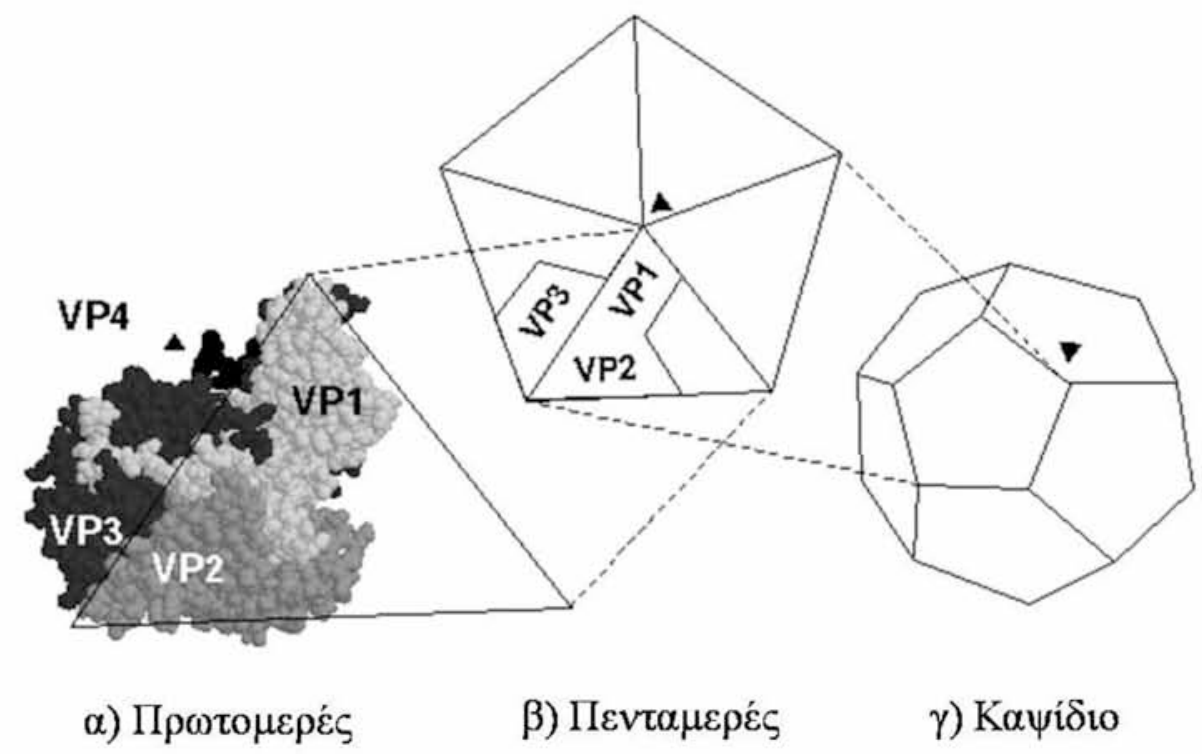

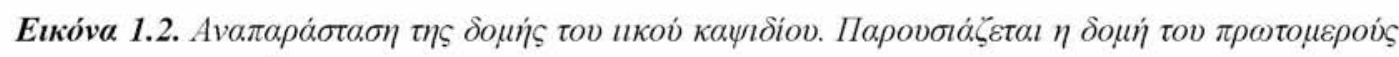

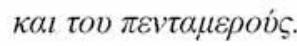

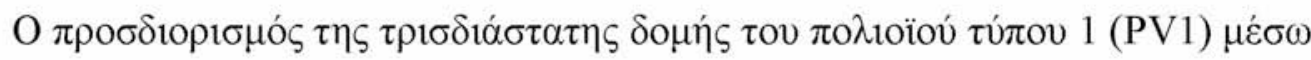

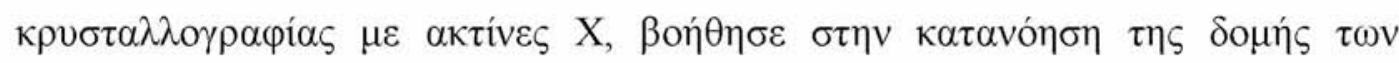

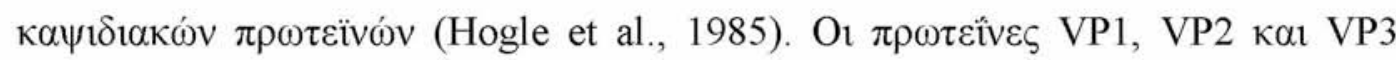

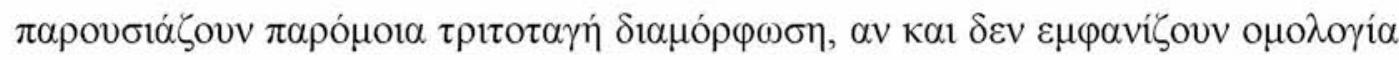

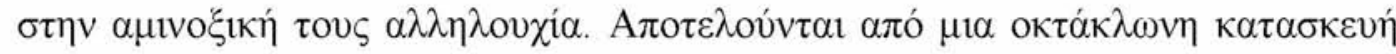

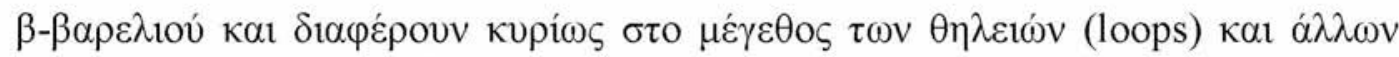

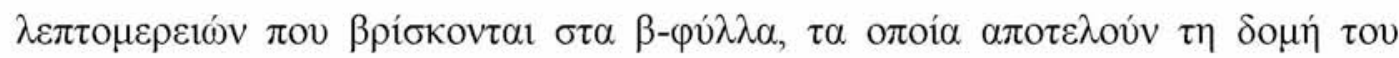

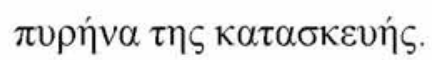




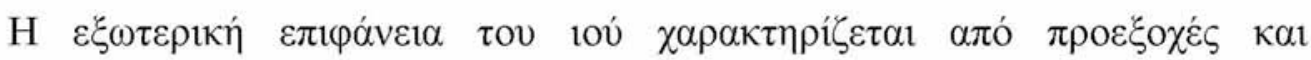

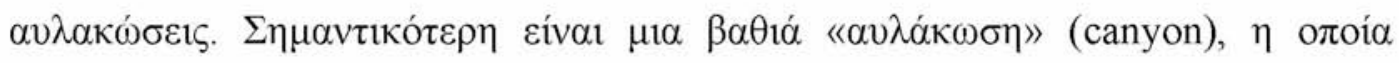

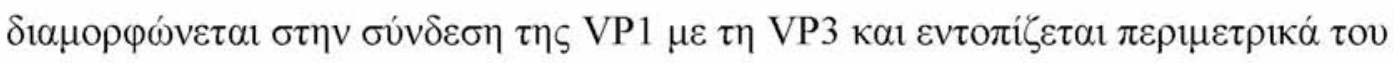

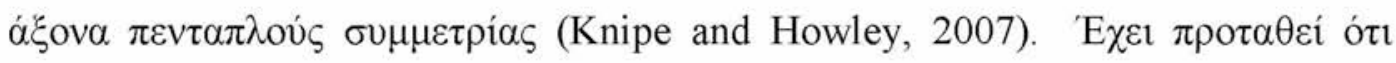

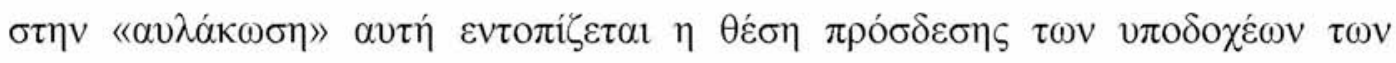

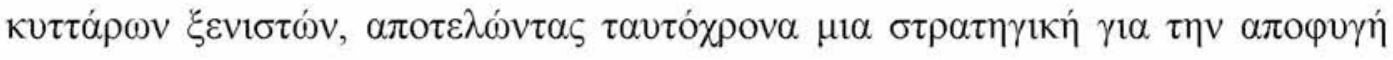

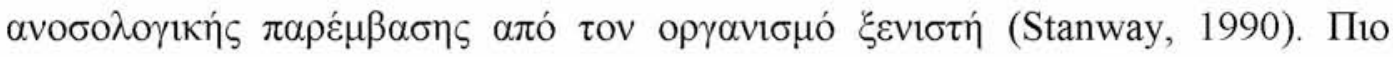

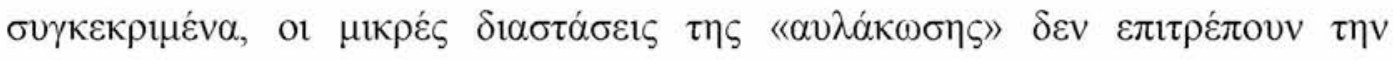

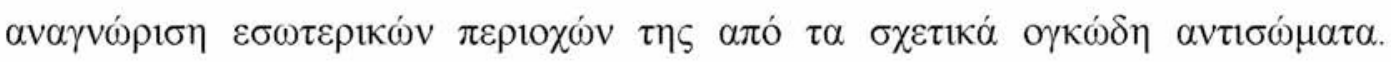

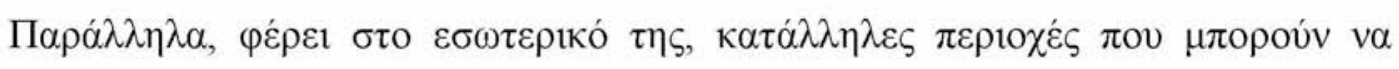

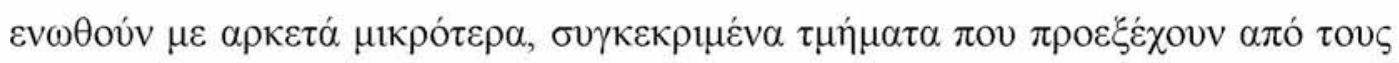

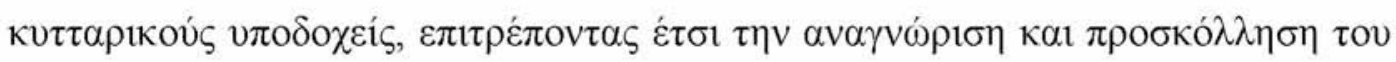

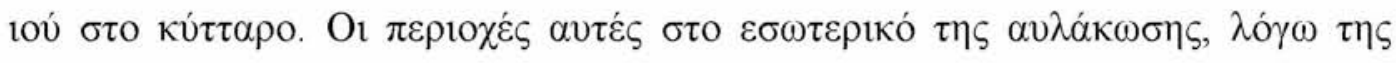

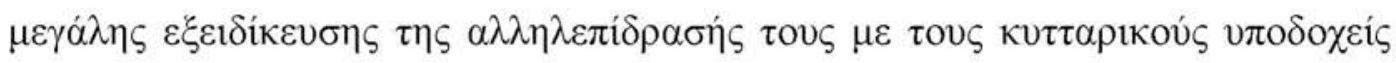

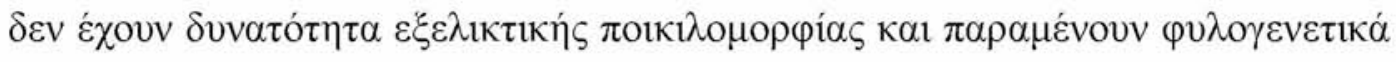

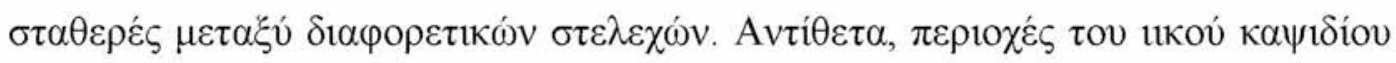

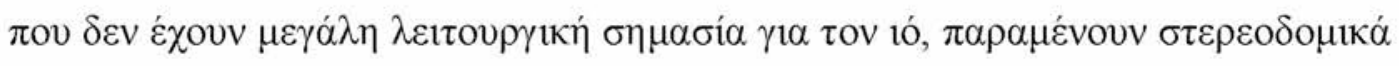

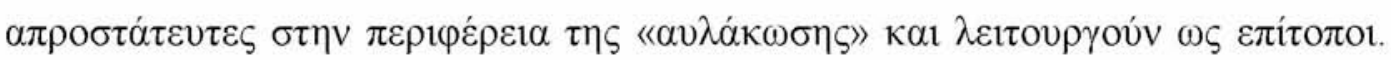

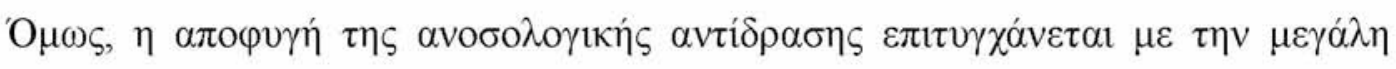

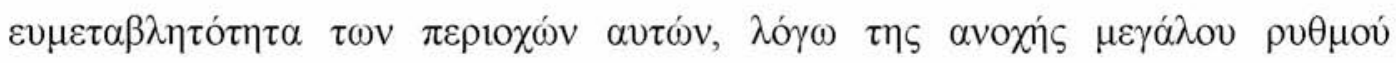

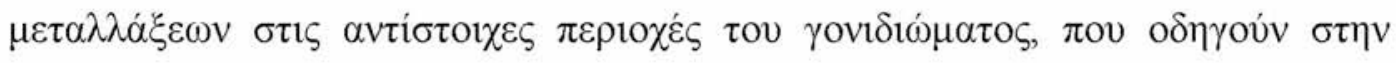

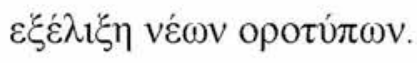

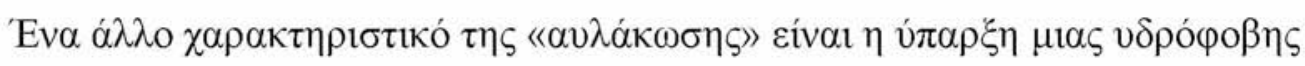

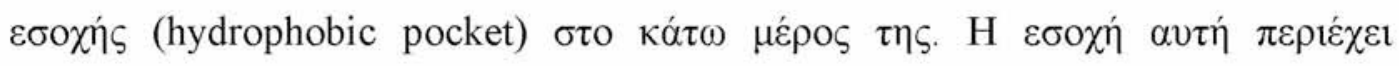

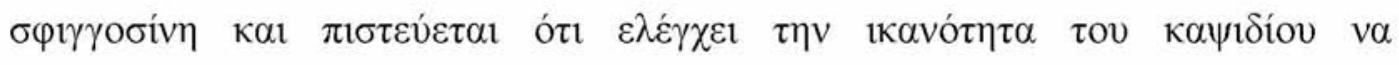

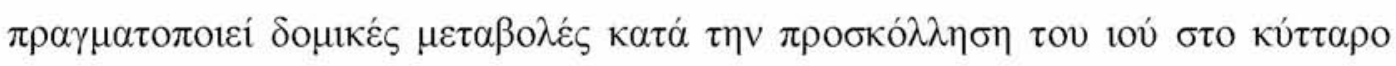

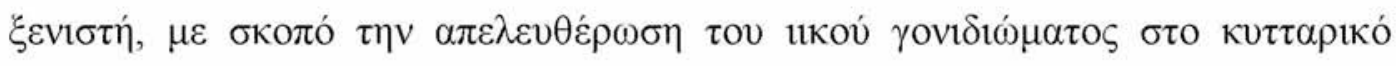

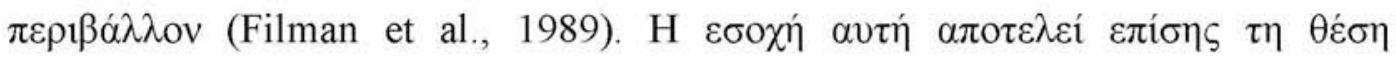

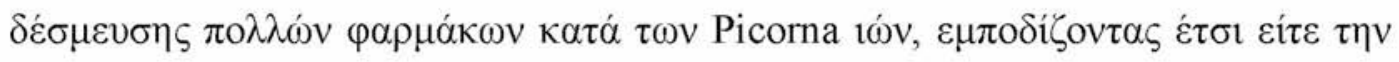

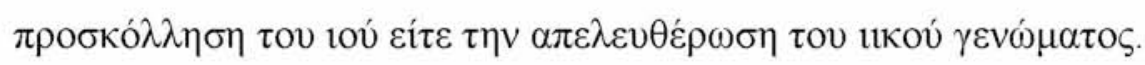

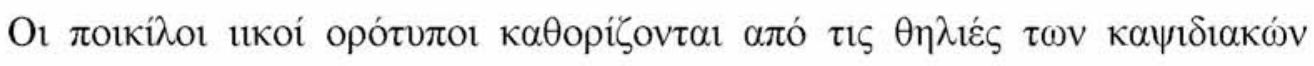

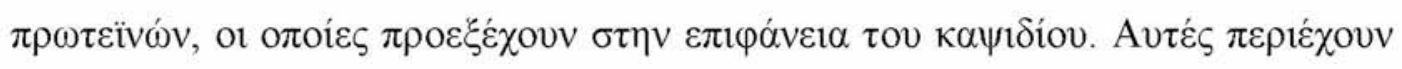

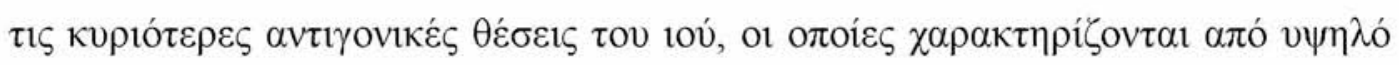




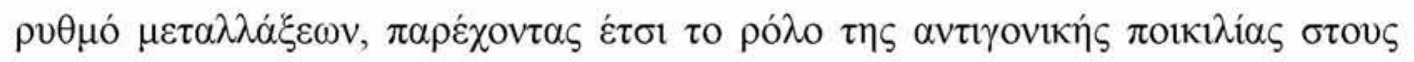

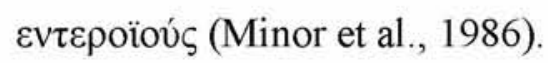

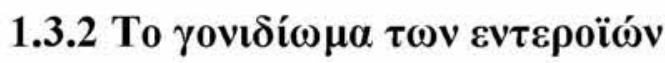

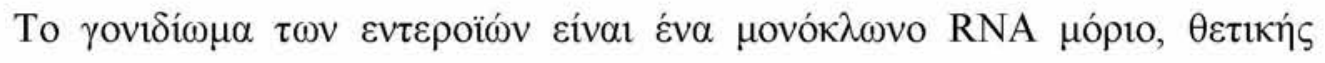

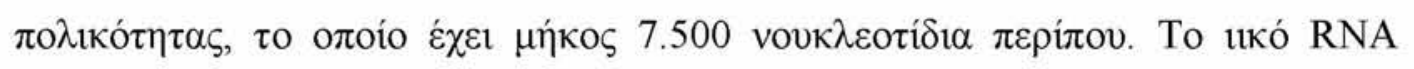

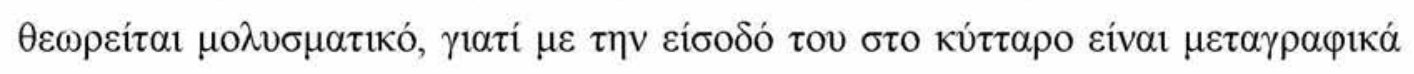

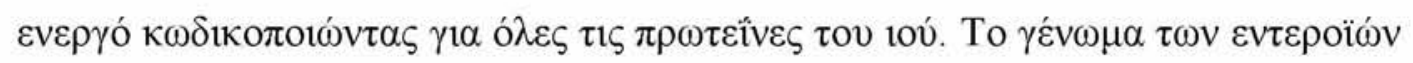

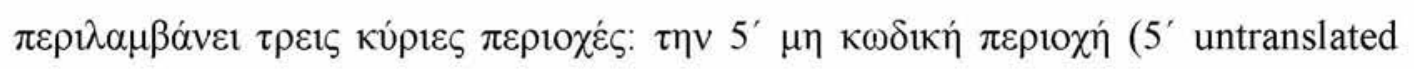

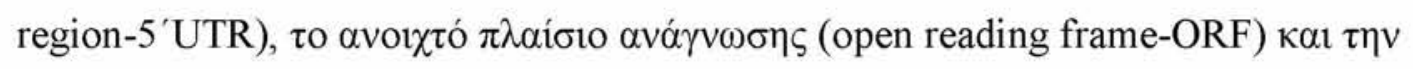

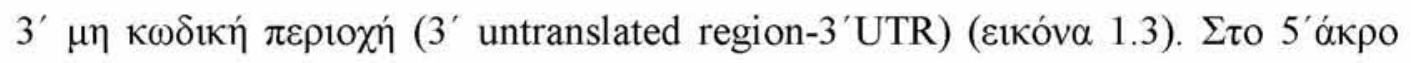

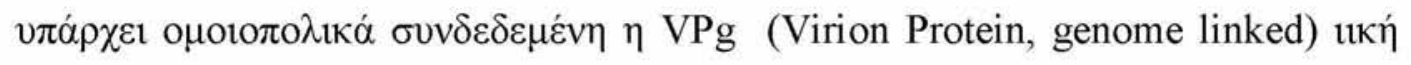

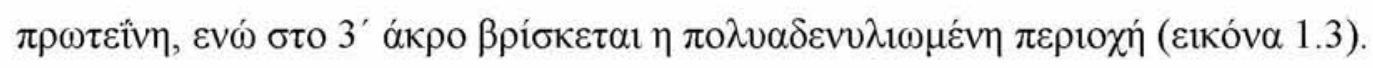

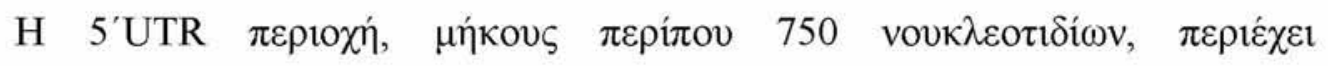

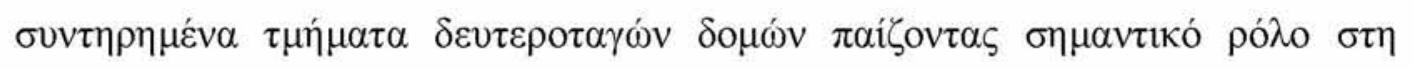

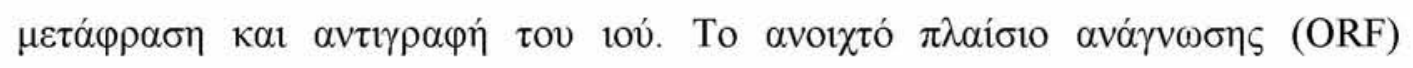

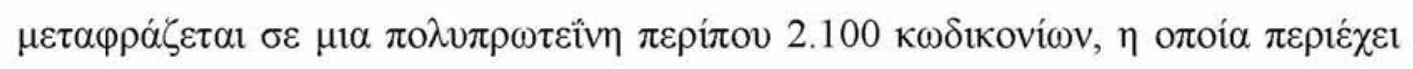

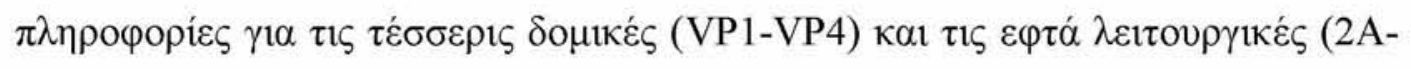

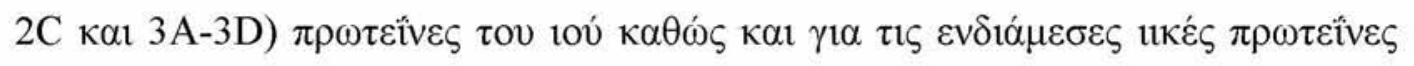
ó $\pi \omega \varsigma$ or VP0, 2BC, 3AB каı 3CD (Knipe and Howley, 2007). H 3' UTR

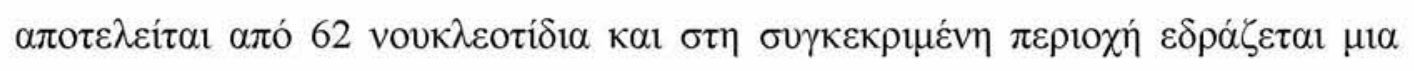

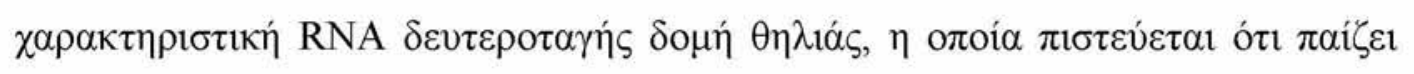

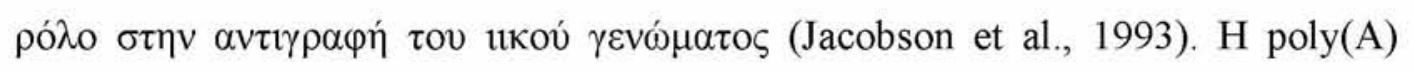

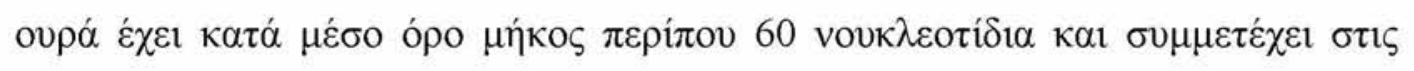

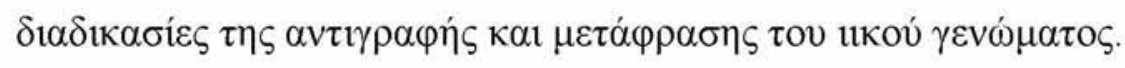

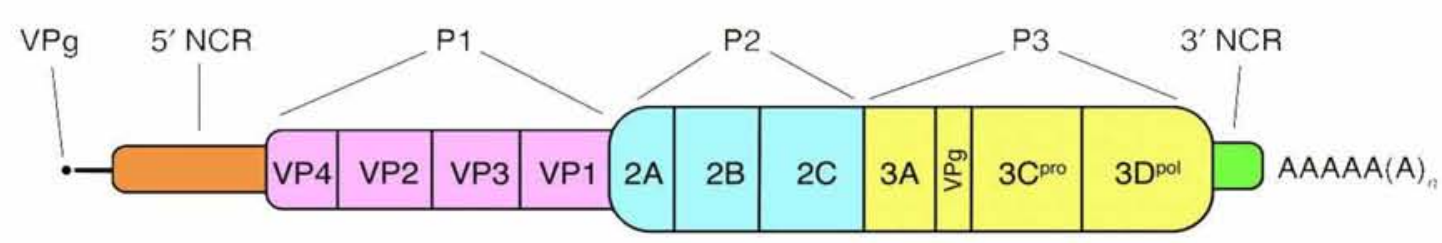

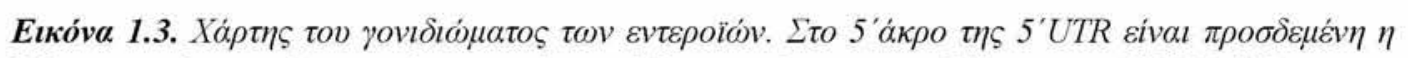

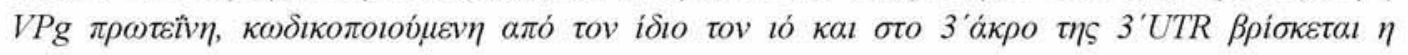

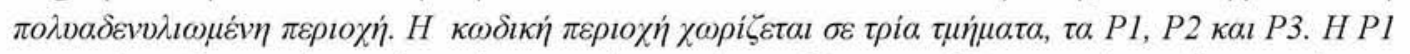

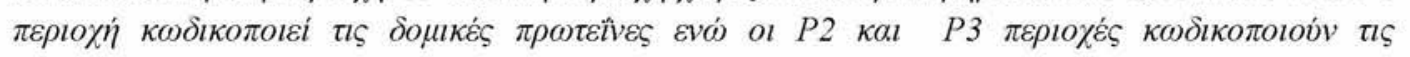

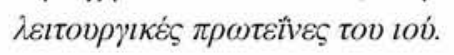




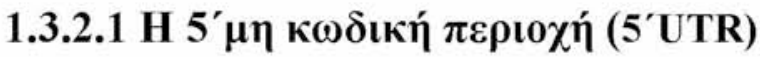

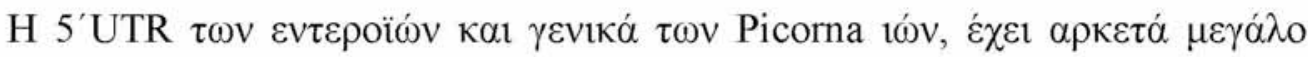

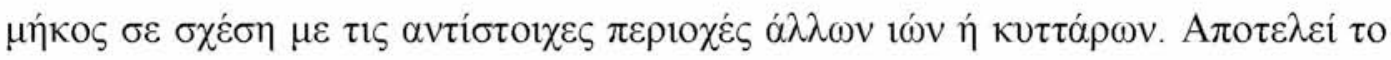

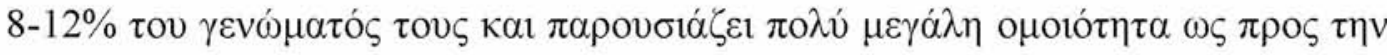

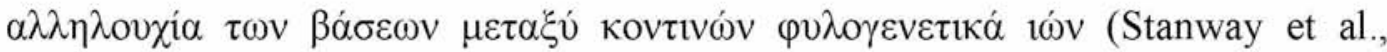

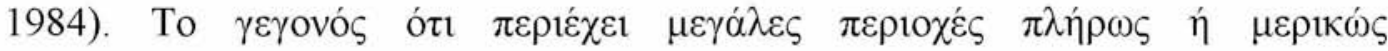

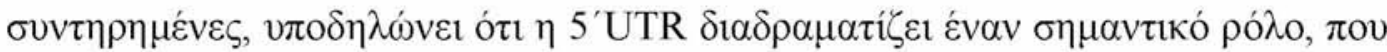

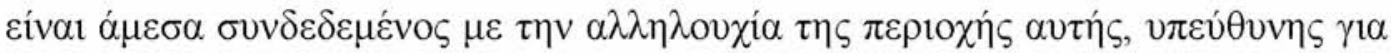

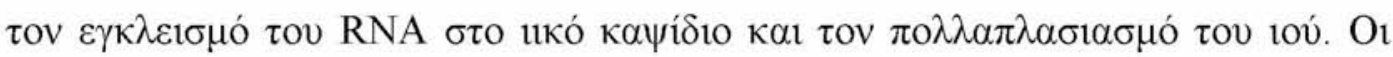

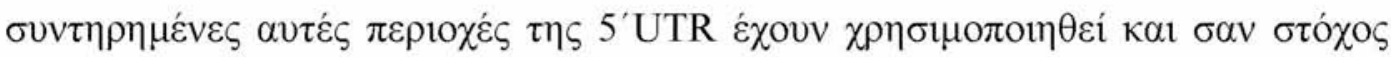

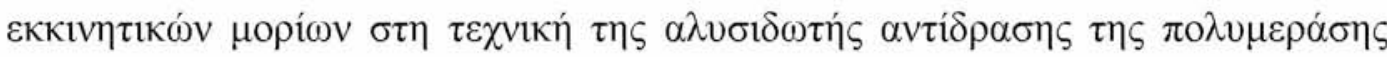

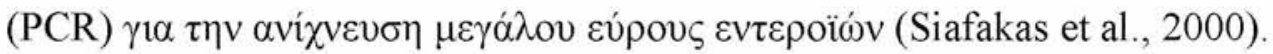

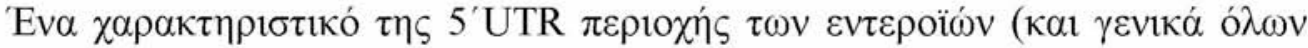

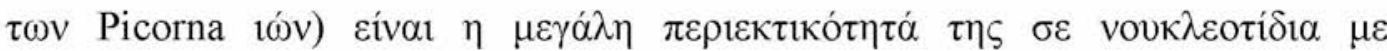

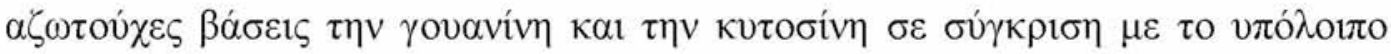

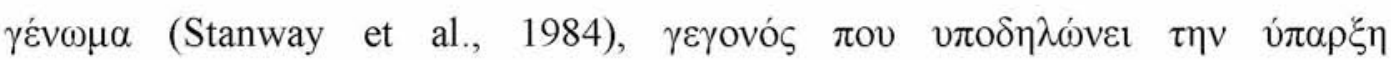

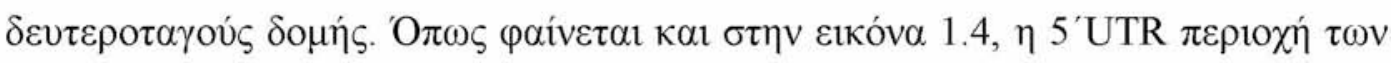

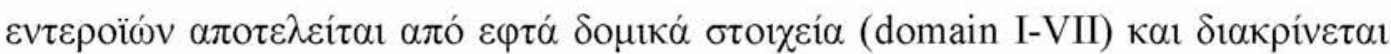

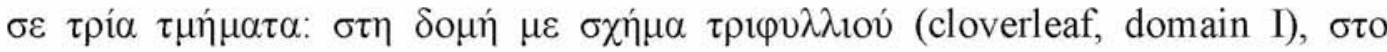

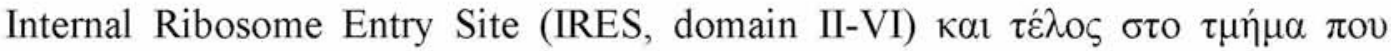

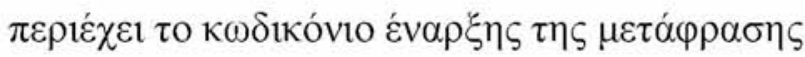

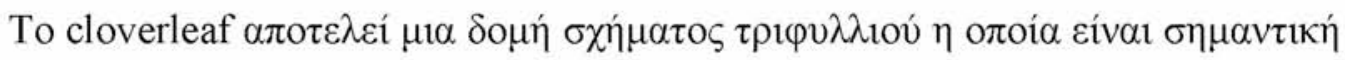

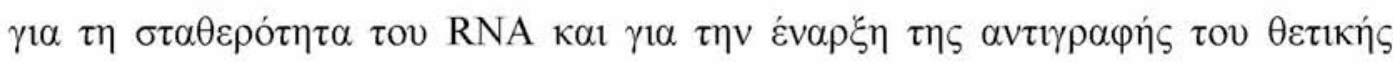

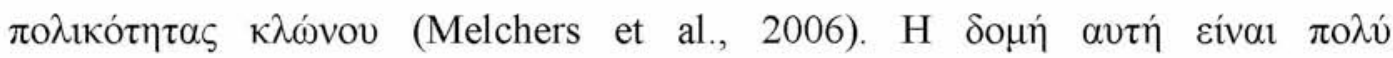

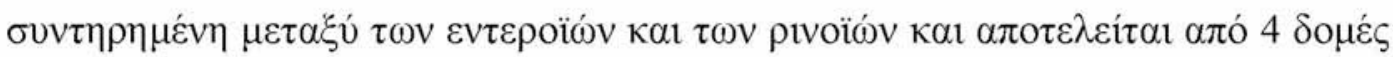

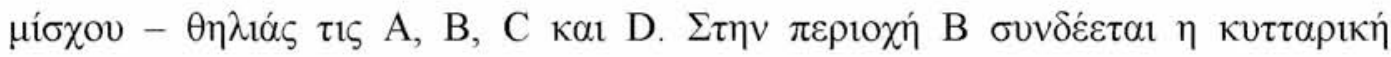

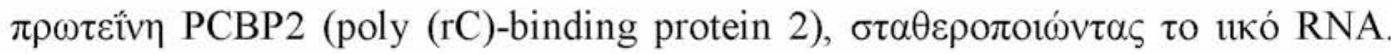

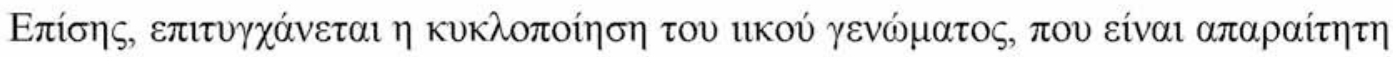

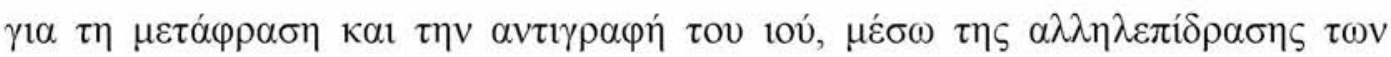
PCBP2 ( $\pi \rho \circ \sigma \delta \varepsilon \delta \varepsilon \mu \varepsilon \dot{v} \eta \sigma \varepsilon$ cloverleaf) $\kappa \alpha \iota \tau\rceil \varsigma$ Poly (A) binding protein (PABP) $\eta$

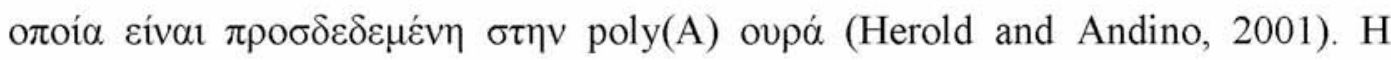

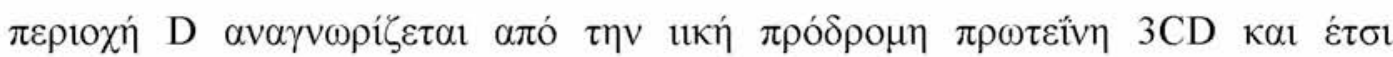

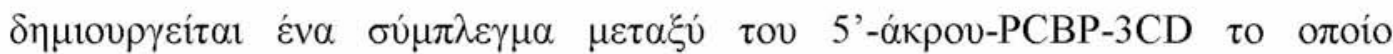




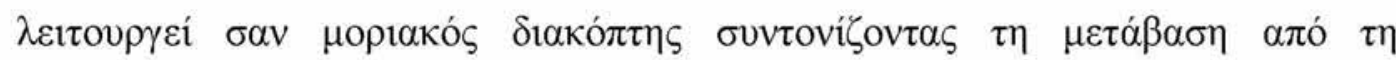

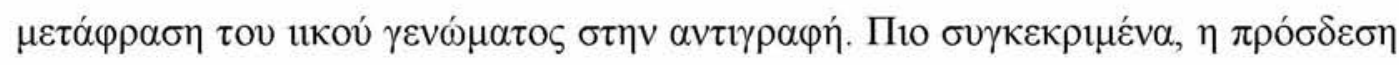

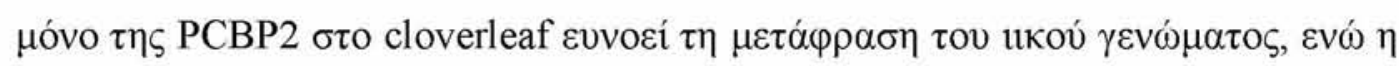

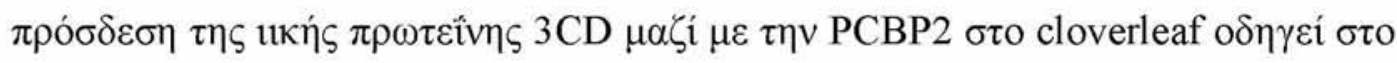

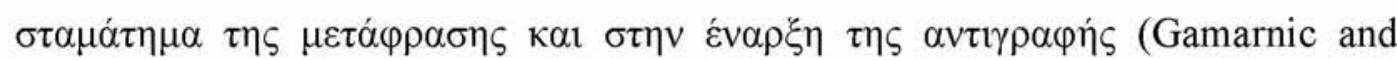
Andino, 1998).

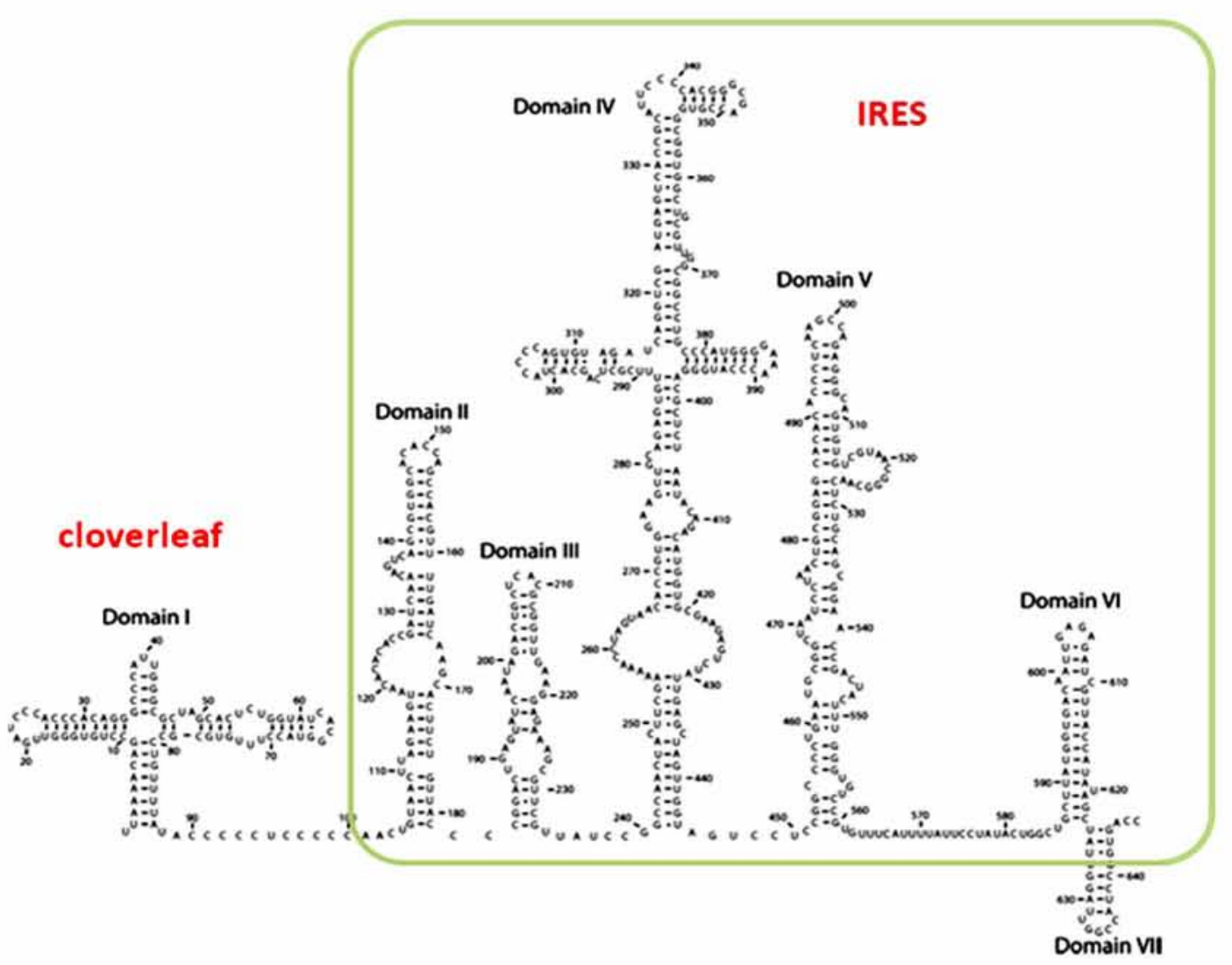

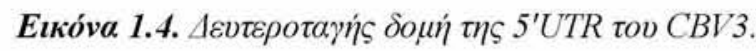

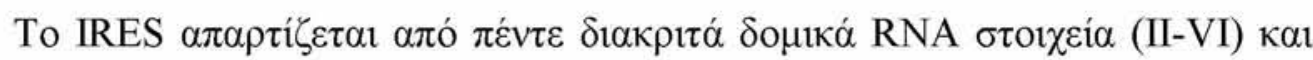

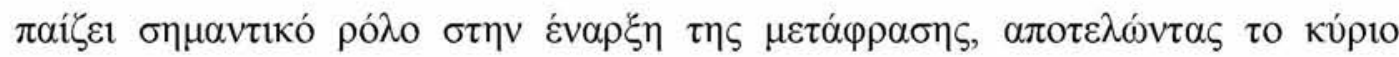

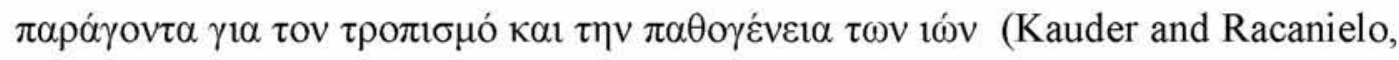

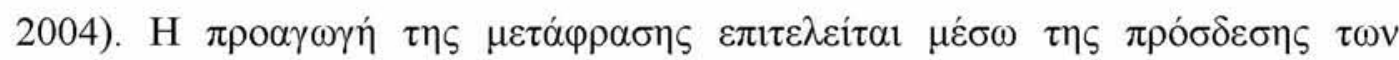

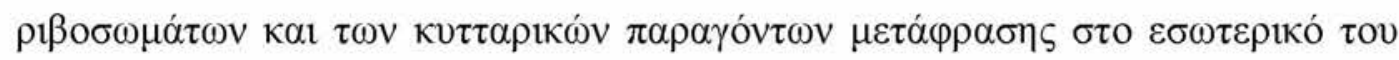

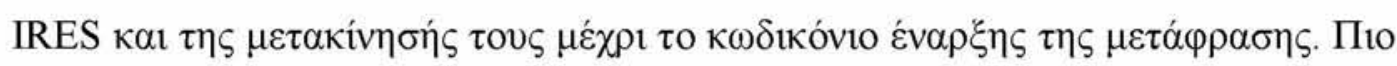

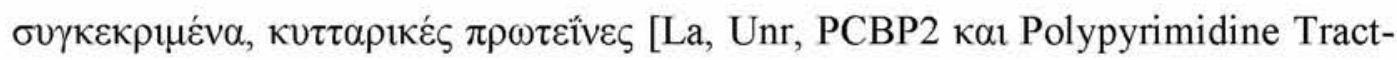

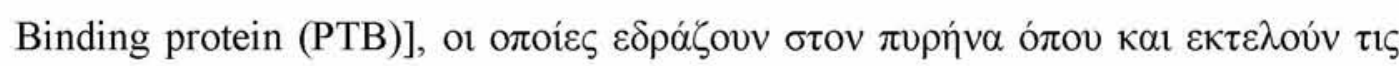

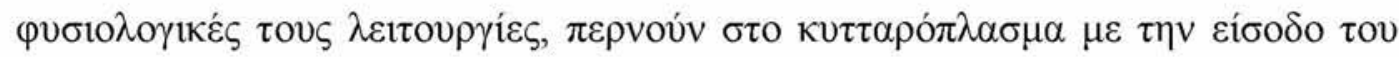

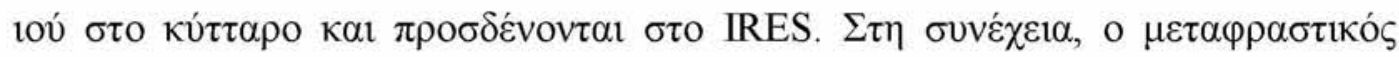




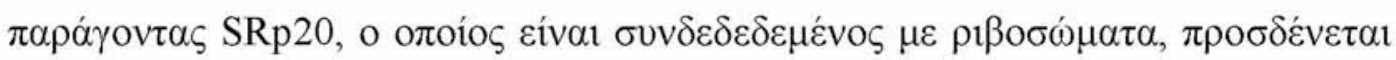

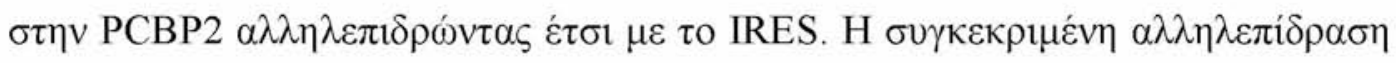

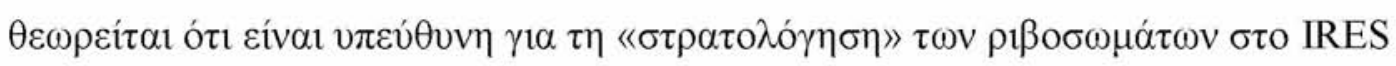
(Sharma et al., 2004).

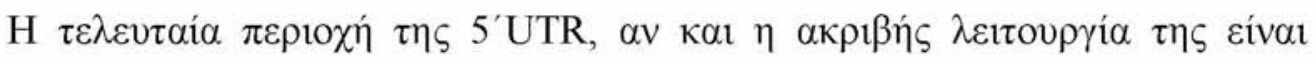

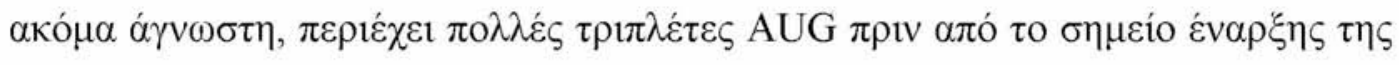

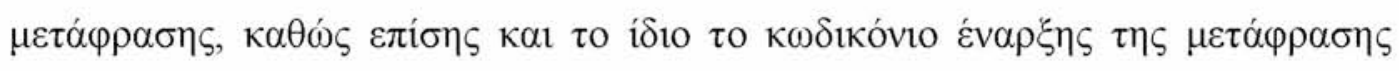
(Witwer et al., 2001).

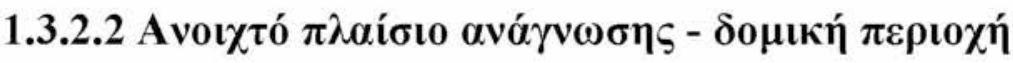

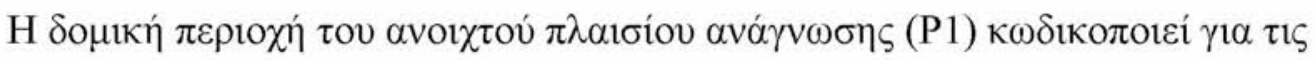

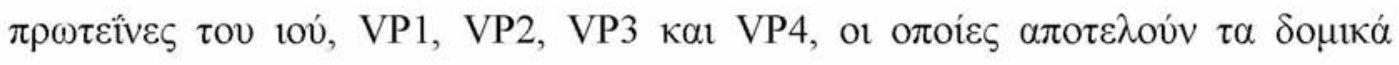

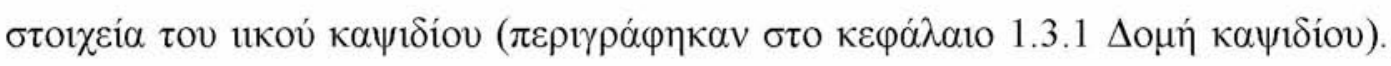
H P1

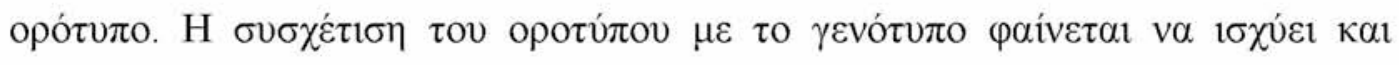

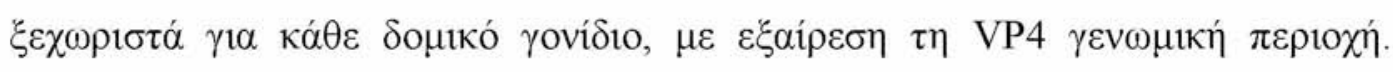

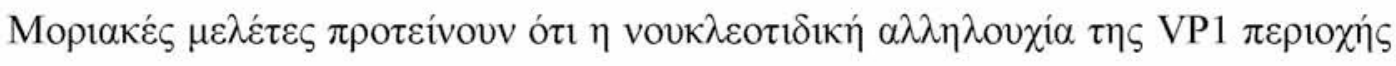

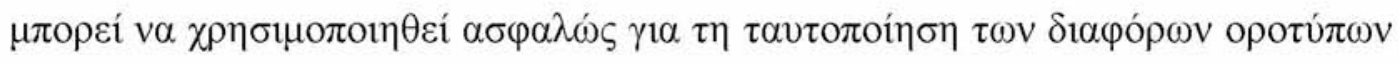

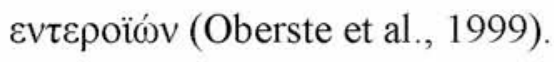

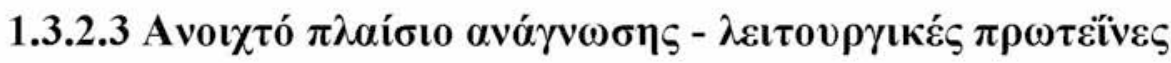

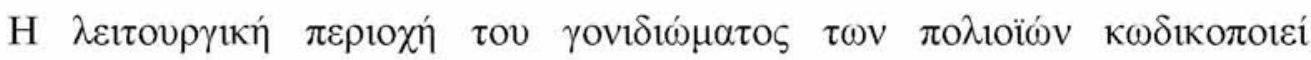

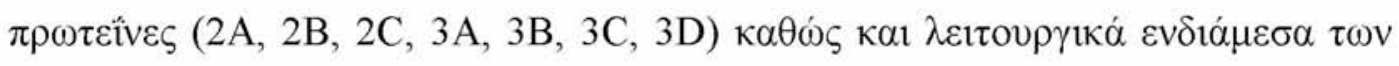

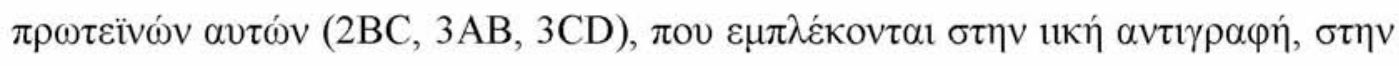

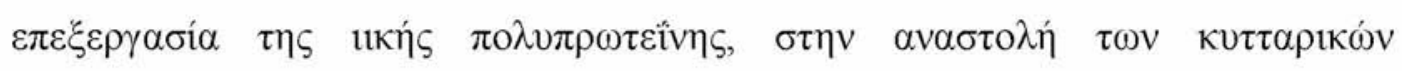

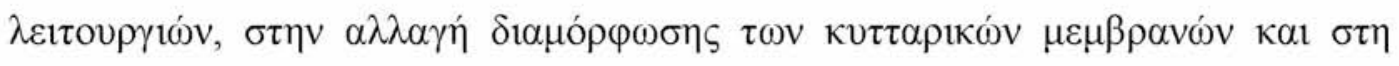

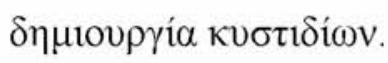

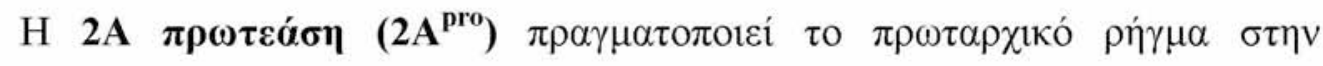

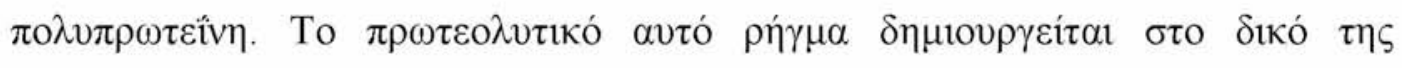

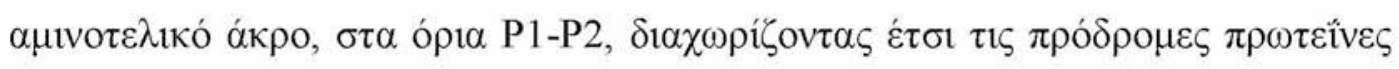

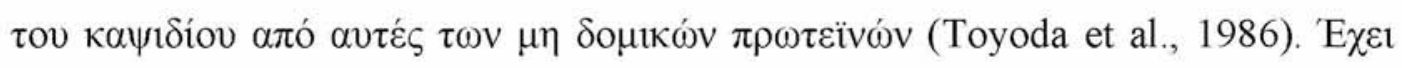

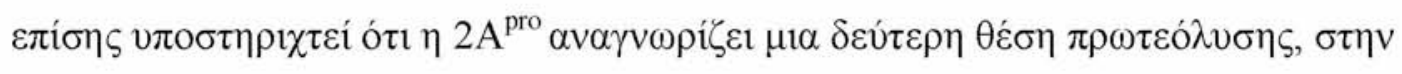

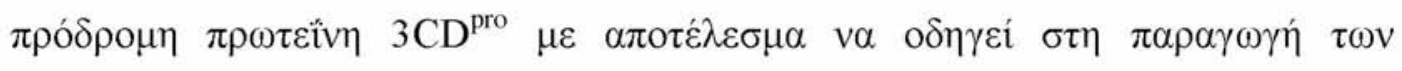




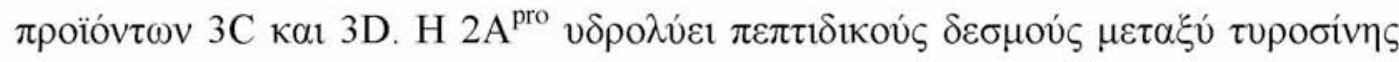

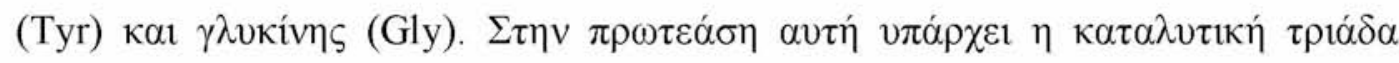

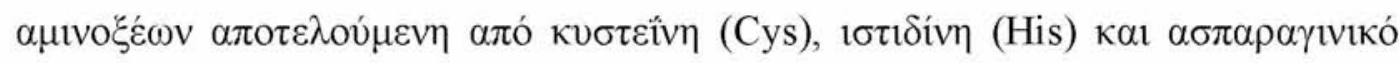

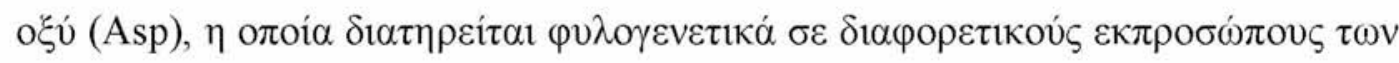

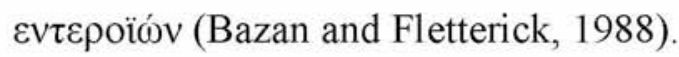

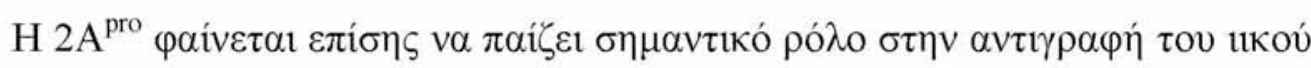

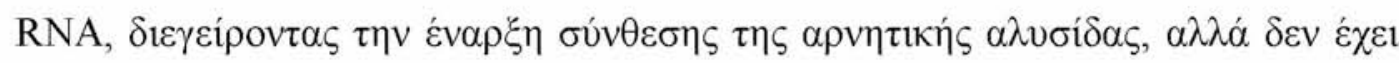

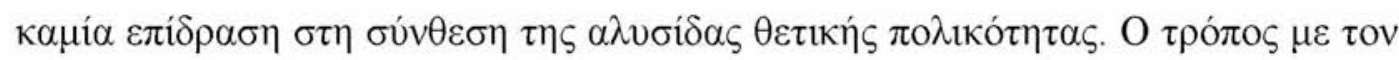

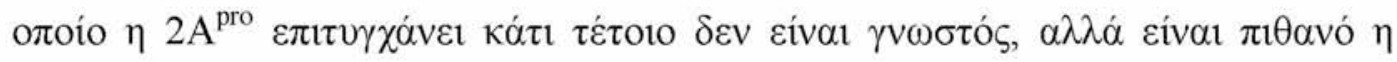

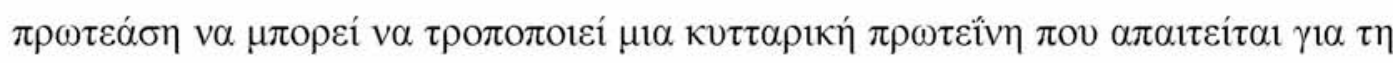

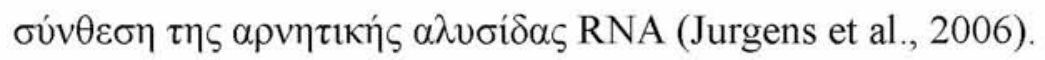

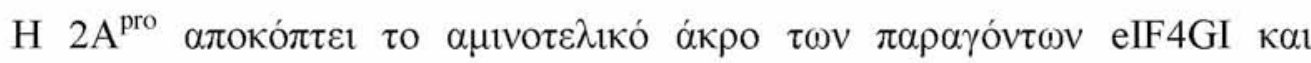

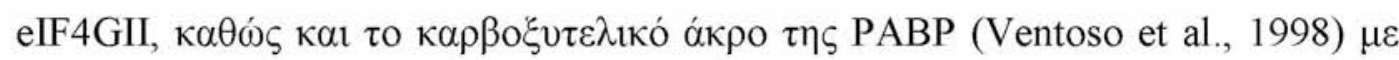

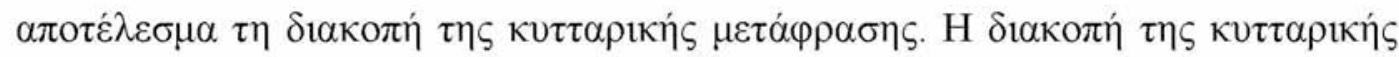

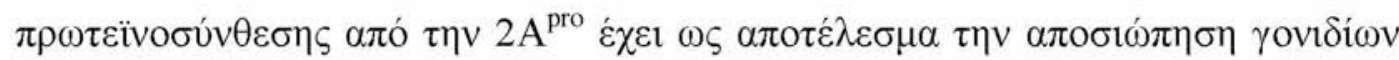

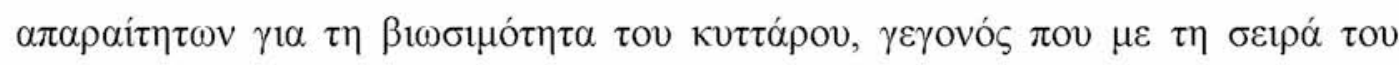

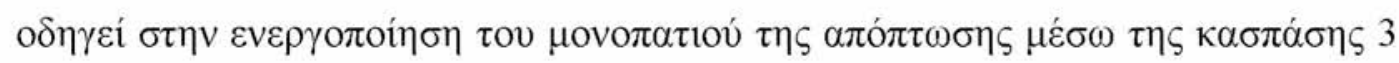
(Calandria et al., 2004).

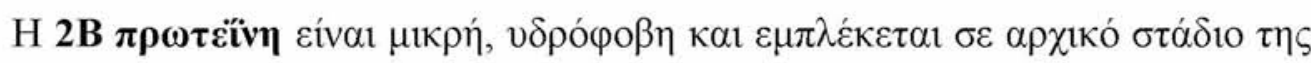

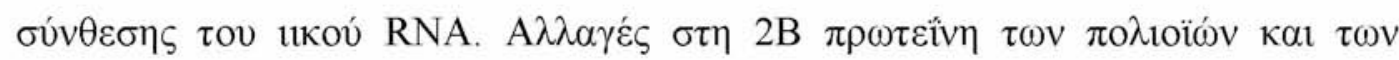

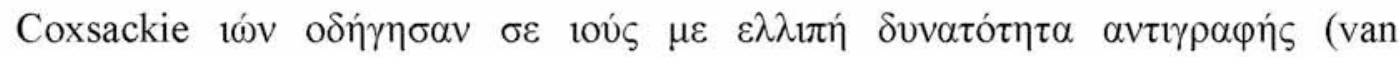

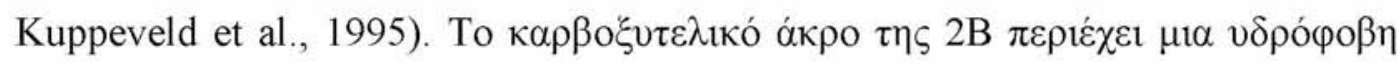

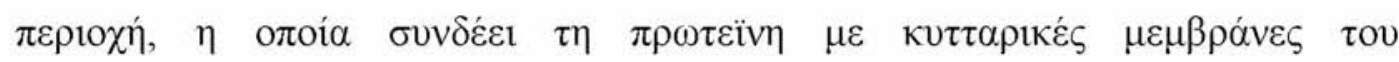

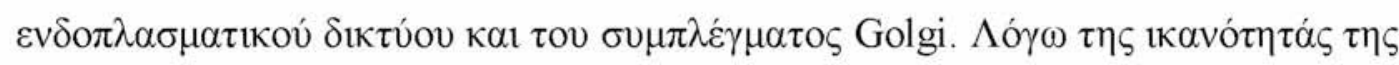

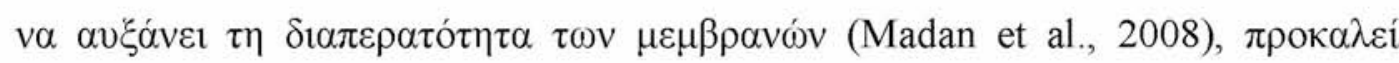

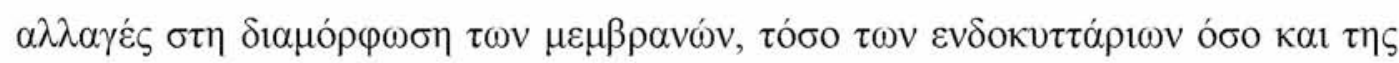

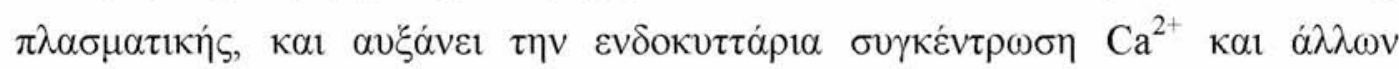

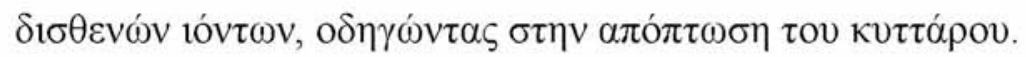

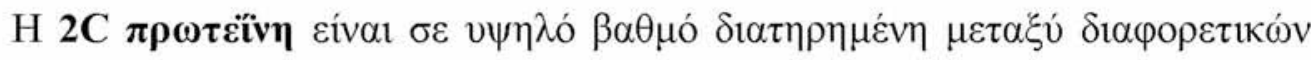

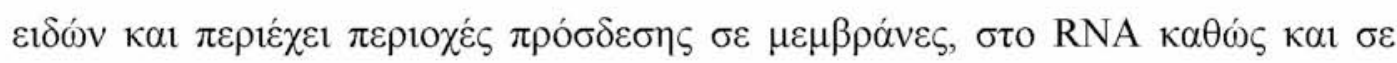

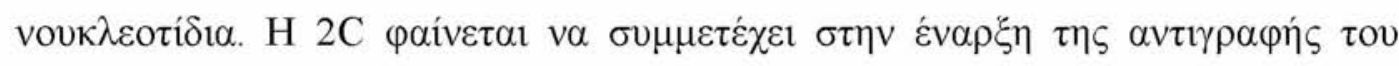

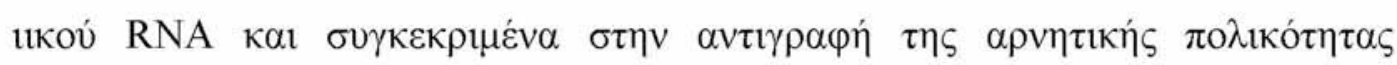

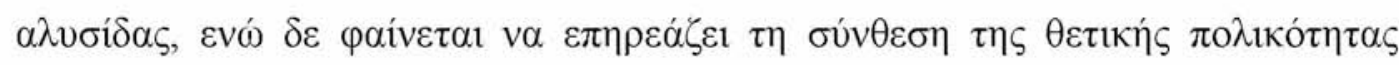

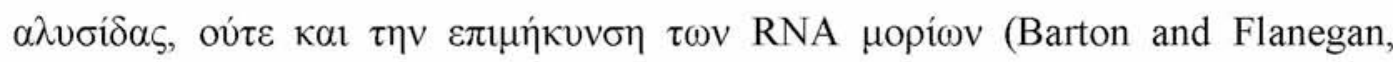




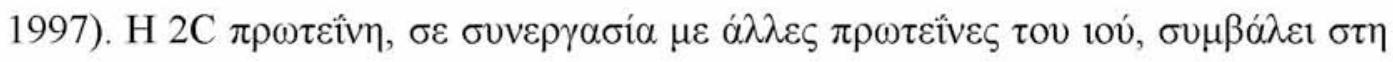

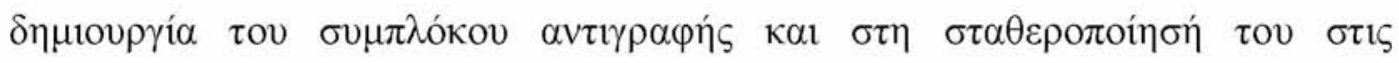

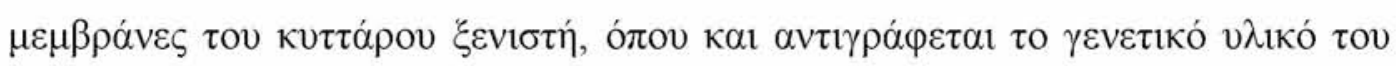

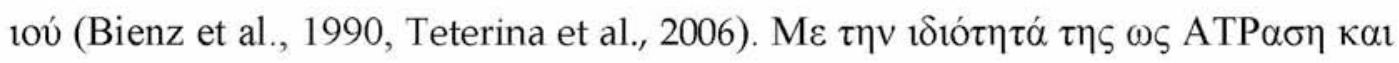

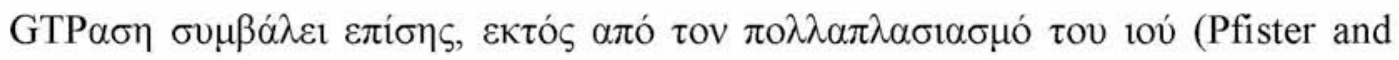

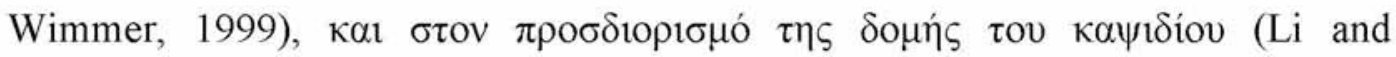
Baltimore, 1990).

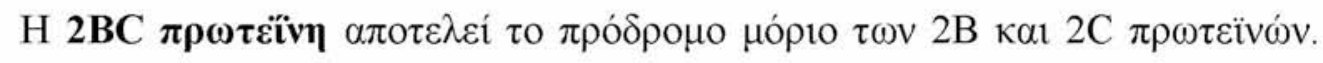

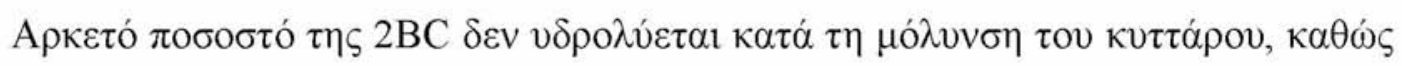

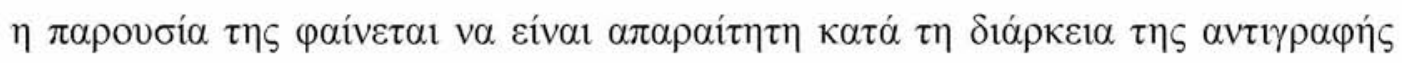

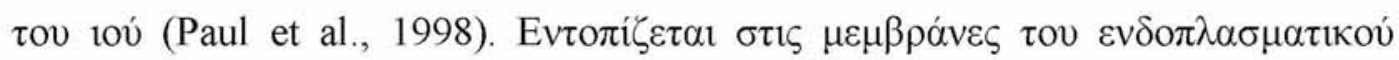

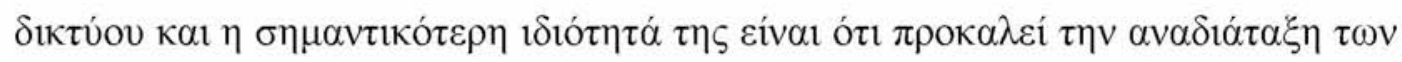

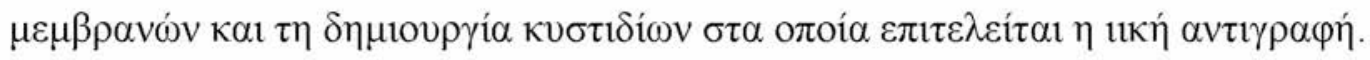

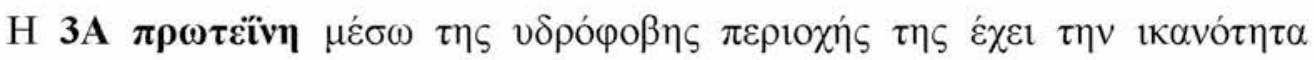

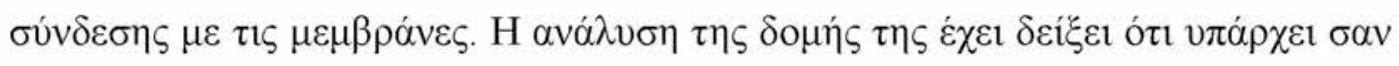

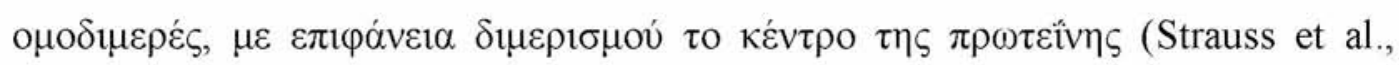

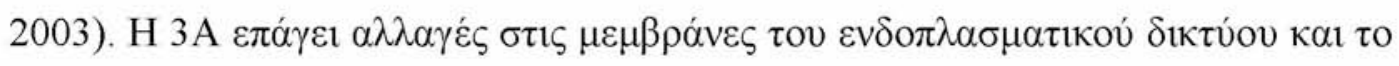

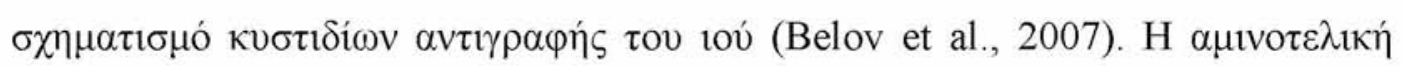

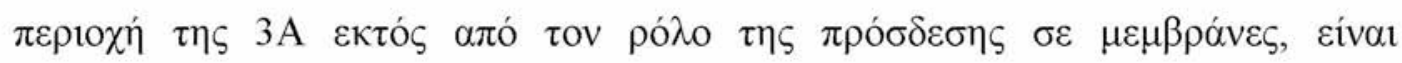

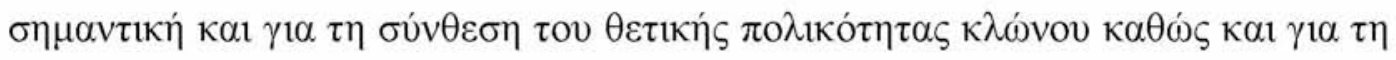

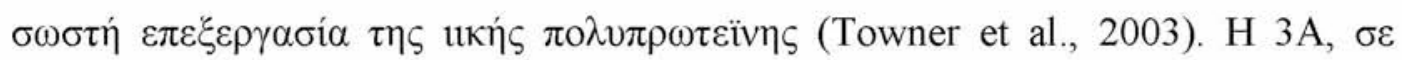

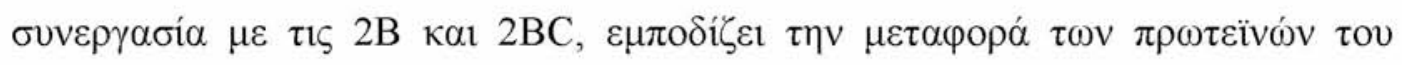

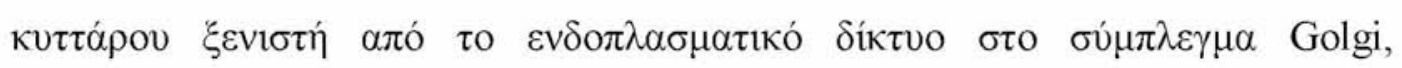

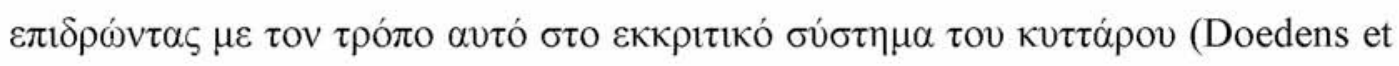
al., 1997).

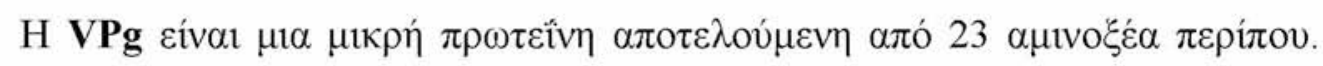

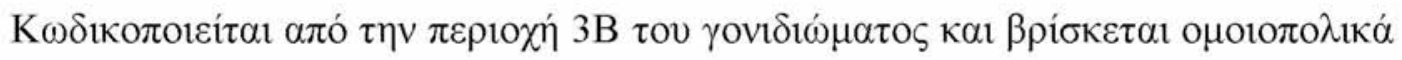

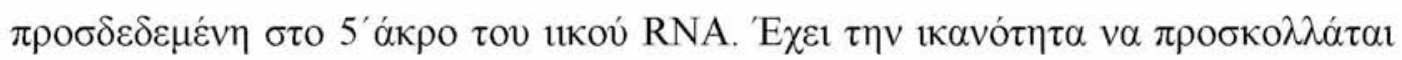

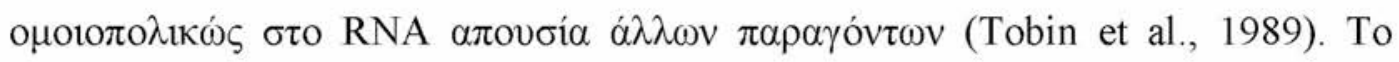

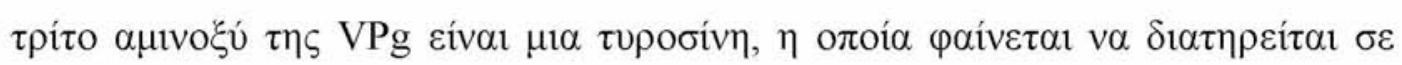

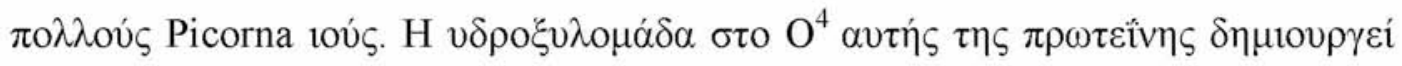

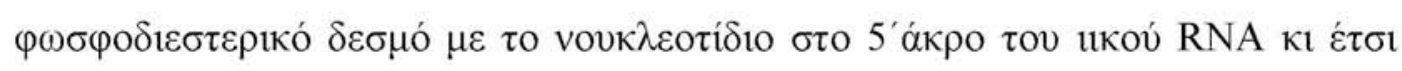

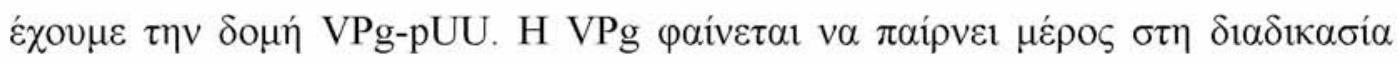

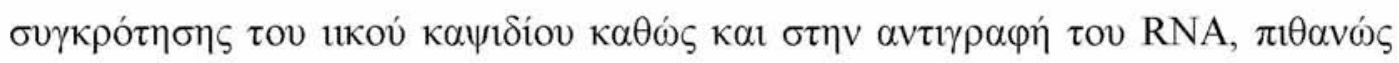




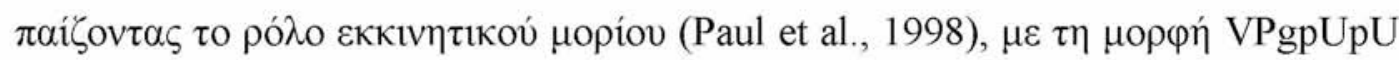

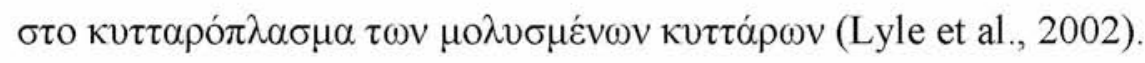

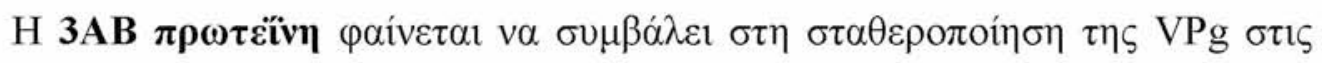

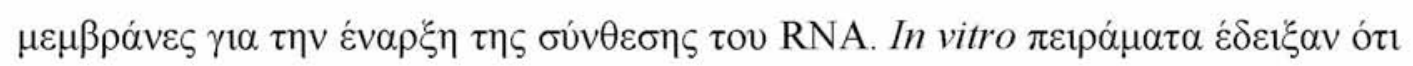

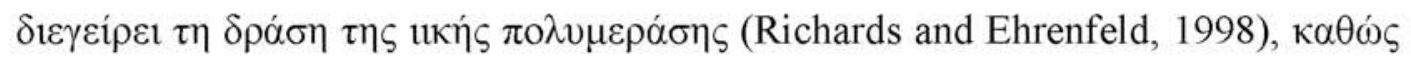

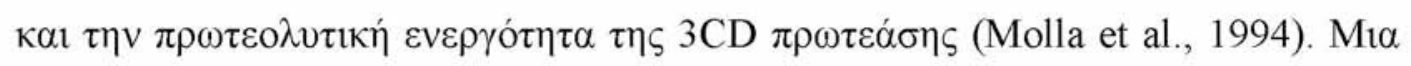

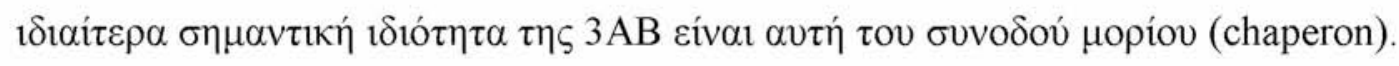

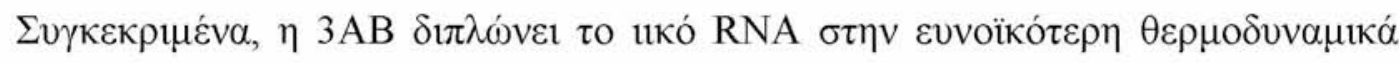

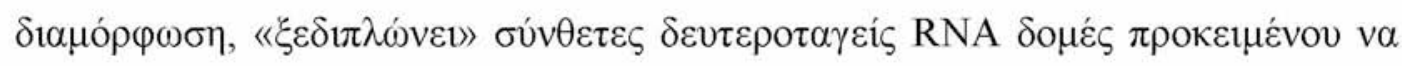

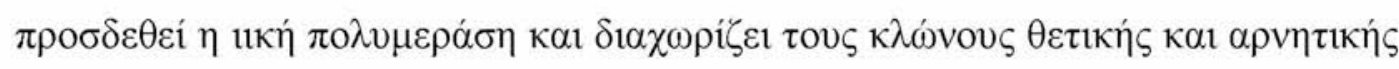

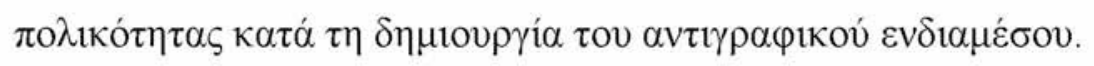

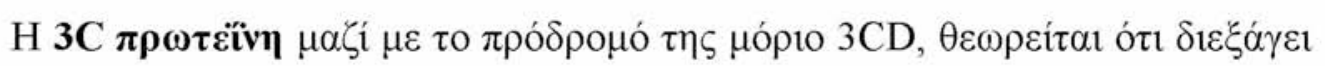

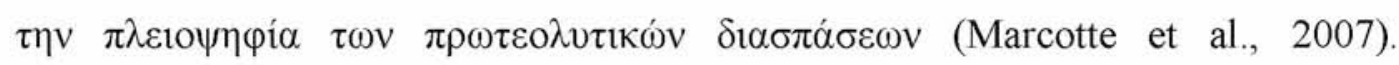

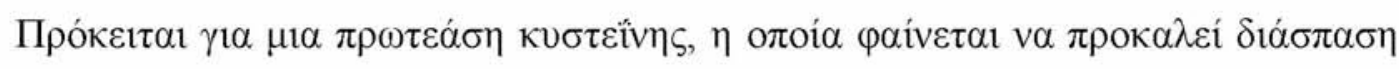

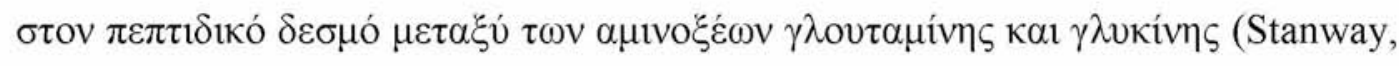

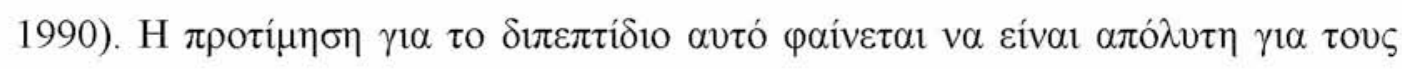

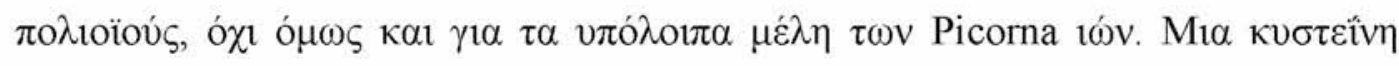

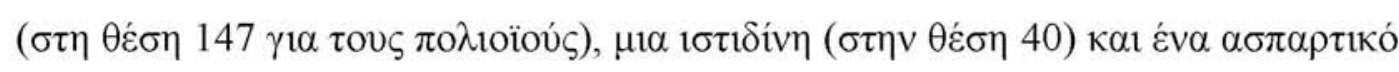

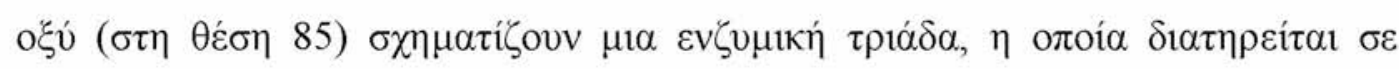

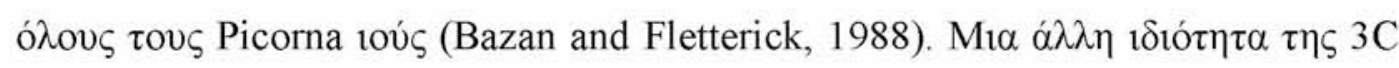

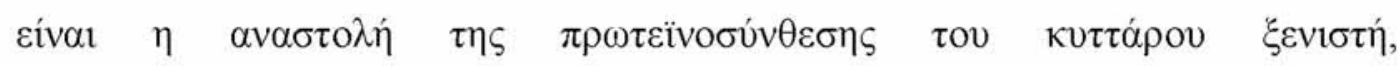

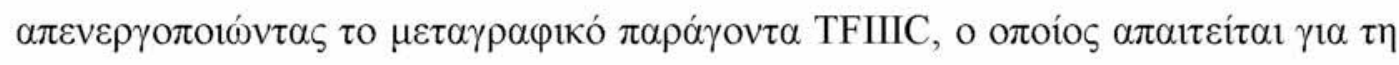

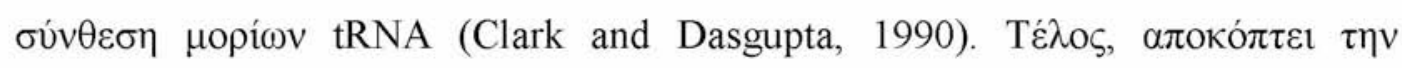

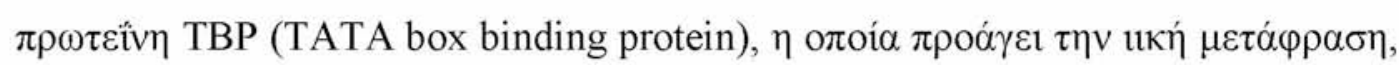

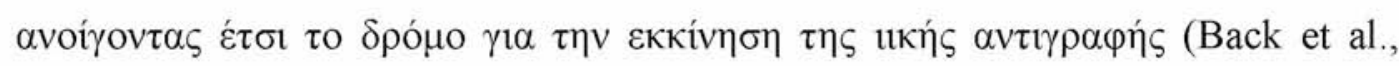
2002).

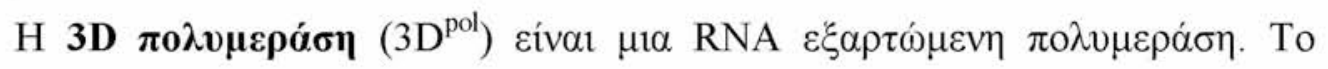

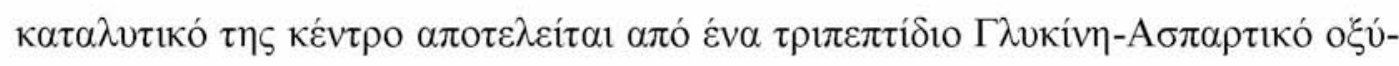

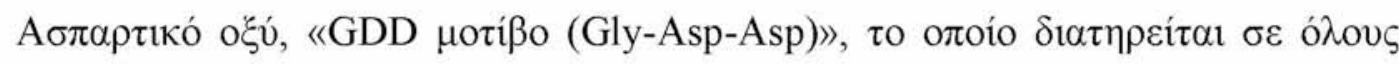

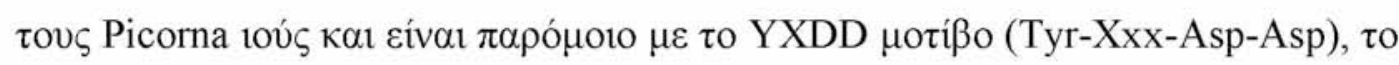

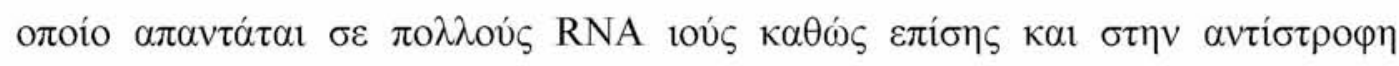

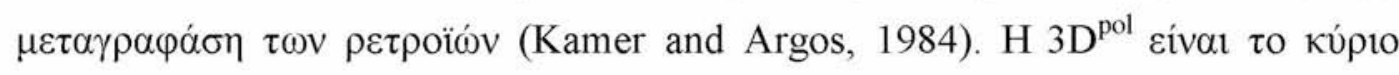

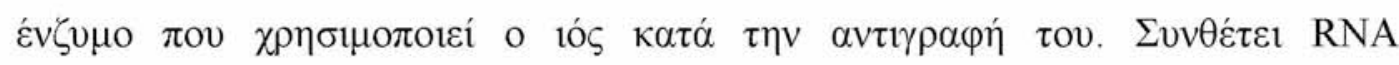

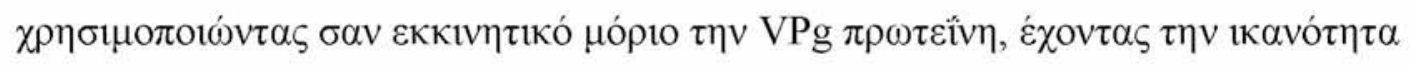




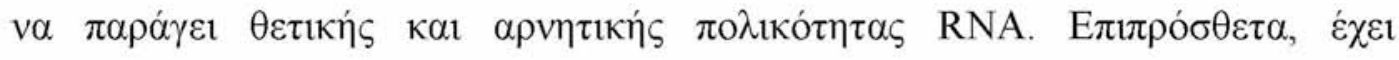

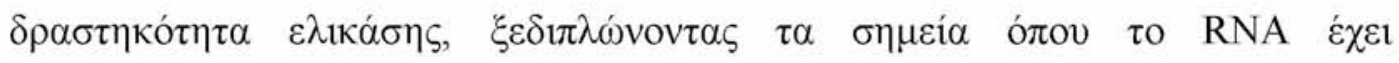

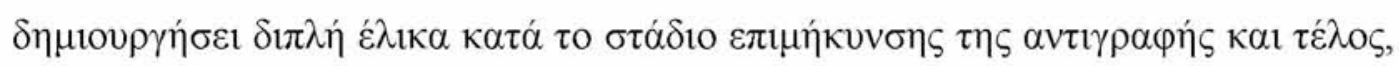

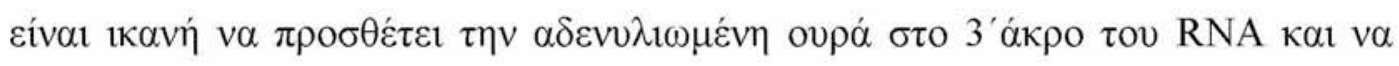

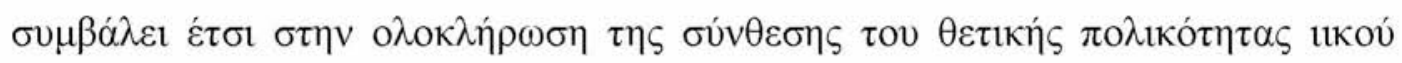
RNA (Pfister et al., 1999).

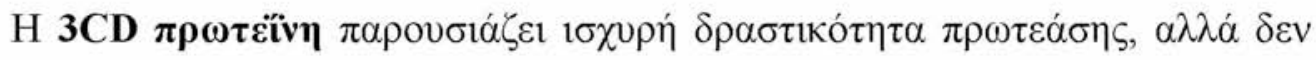

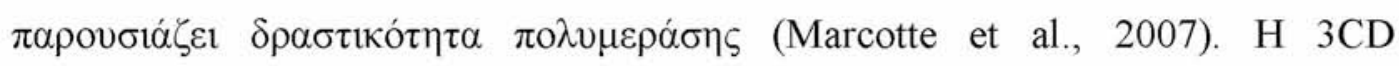

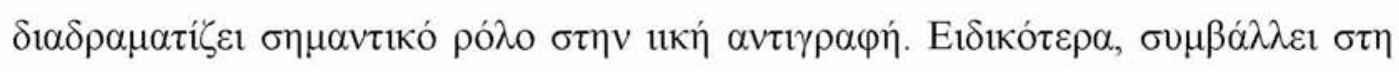

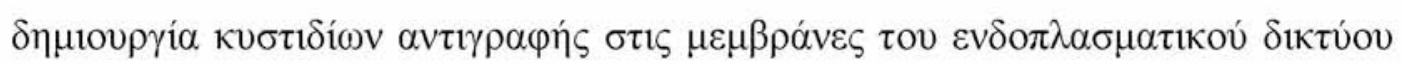

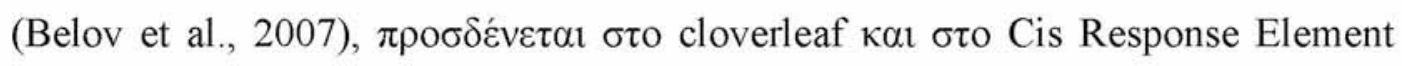

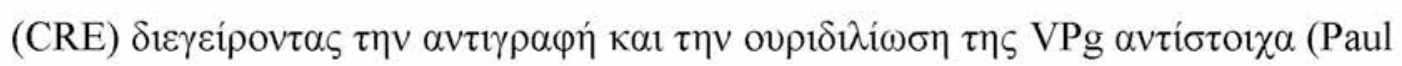

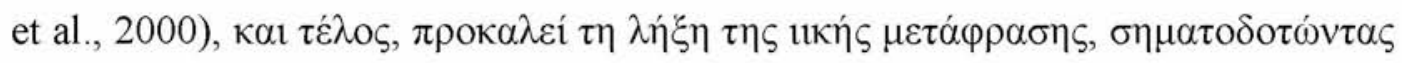

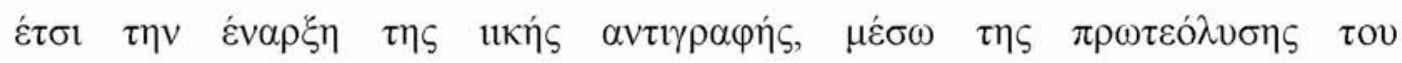

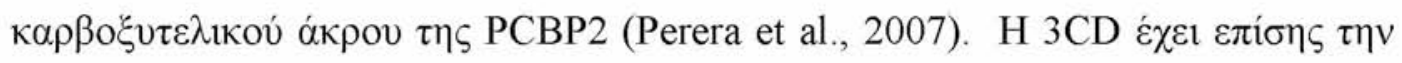

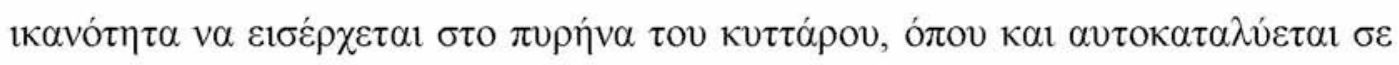

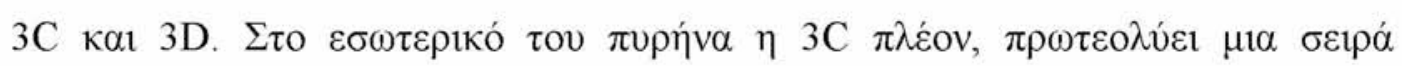

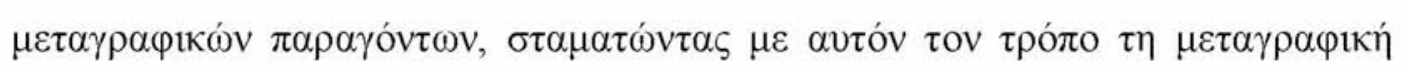

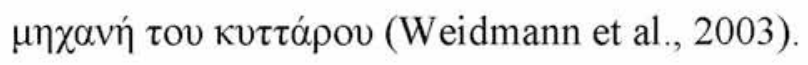

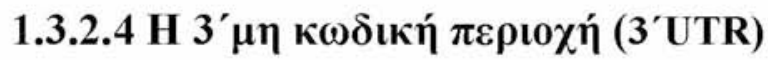

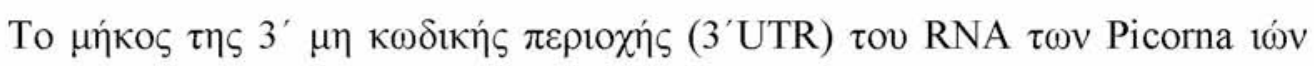

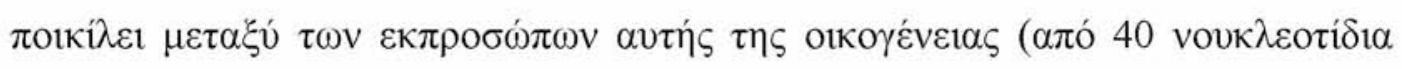

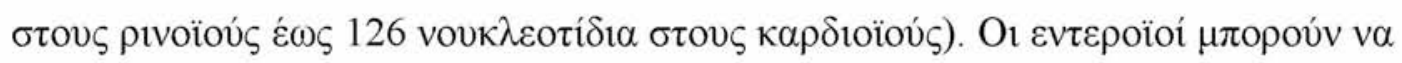

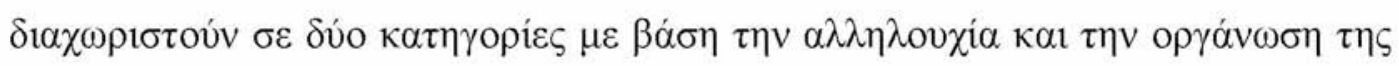

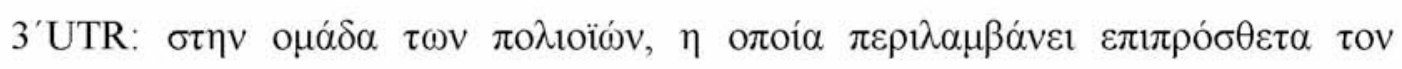

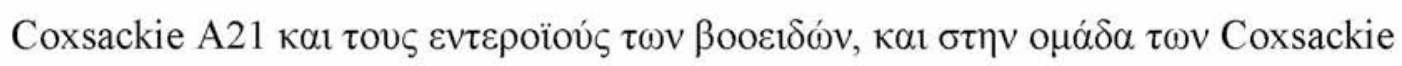

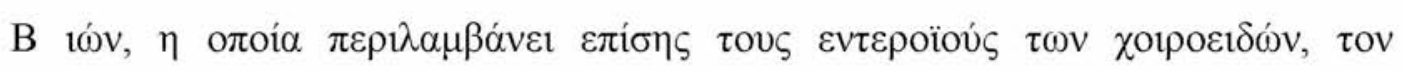

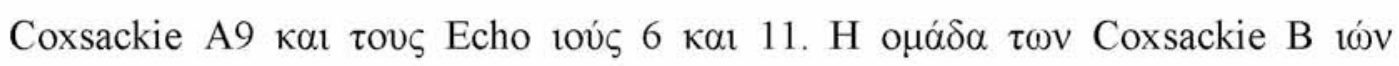

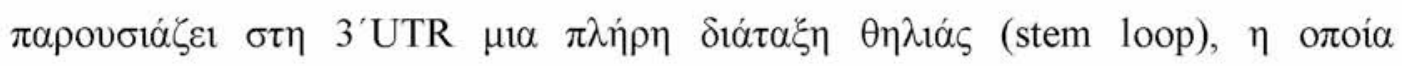

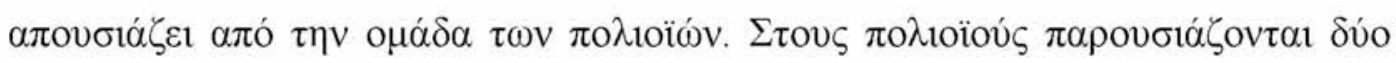

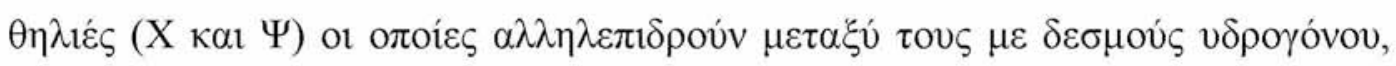

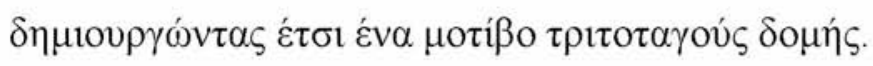




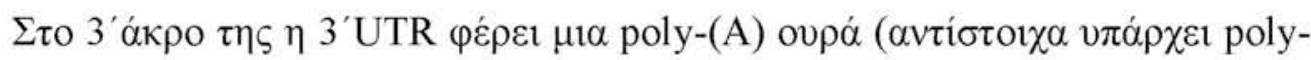

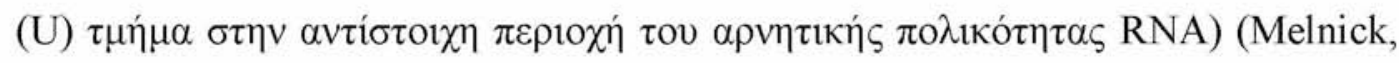

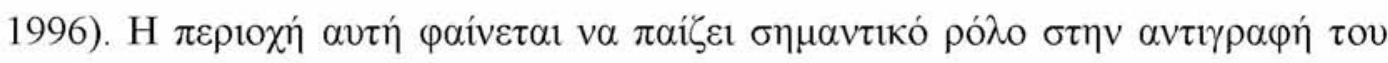

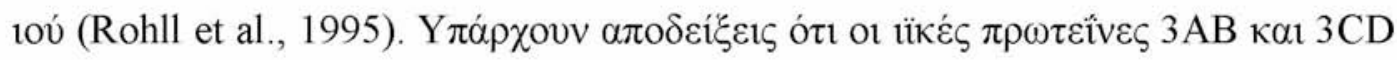

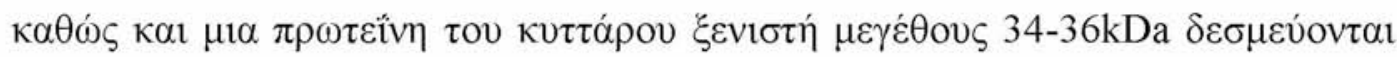

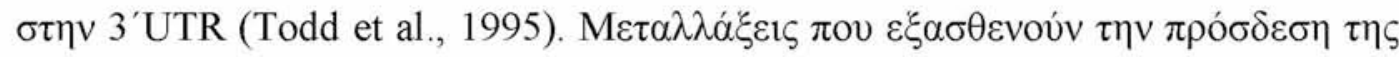

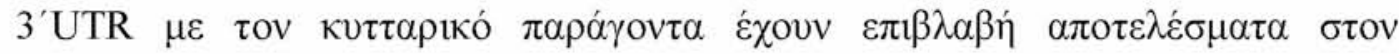

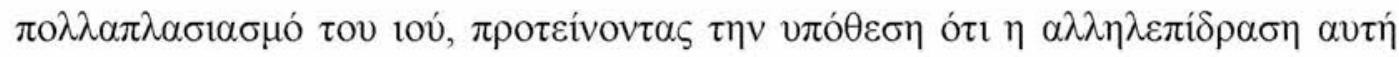

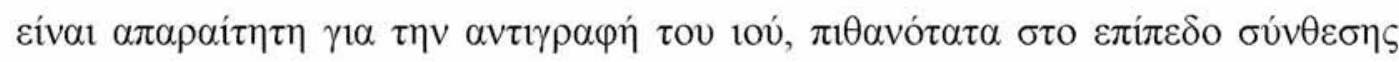

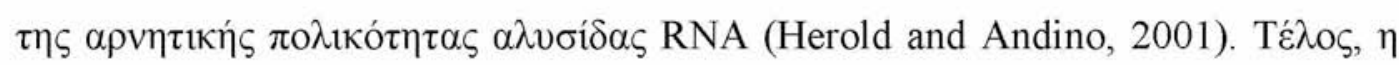

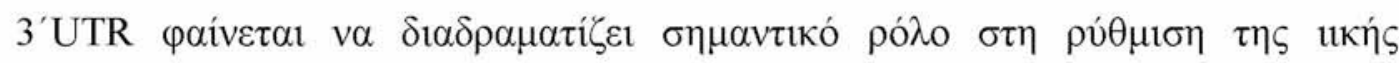

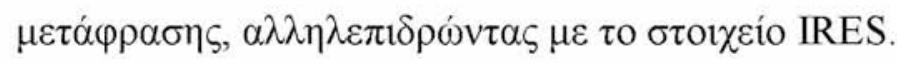

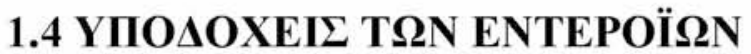

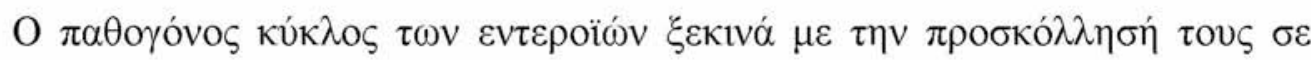

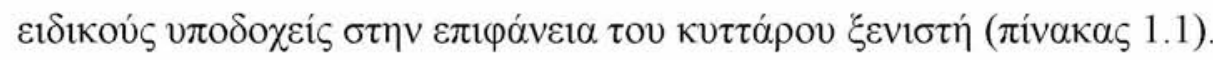

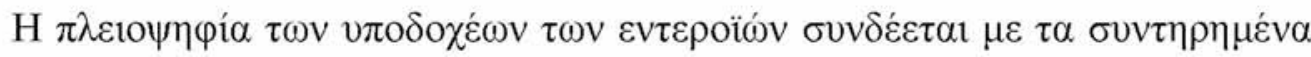

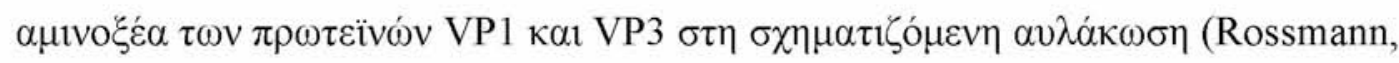

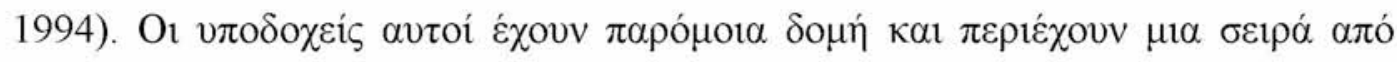
$\pi \varepsilon \rho 10 \chi \varepsilon \dot{\varepsilon} \varsigma$ ó

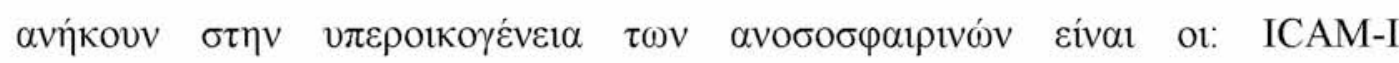

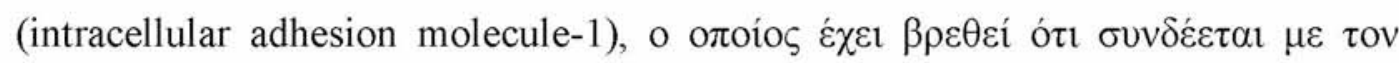
Coxsackie A21 (Xiao et al., 2001), CAR (Coxsackie-Adenovirus Receptor), o

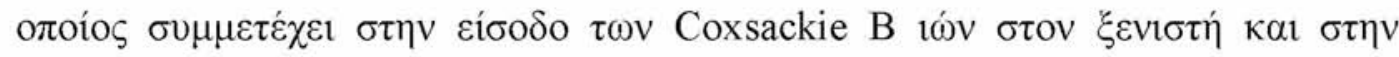

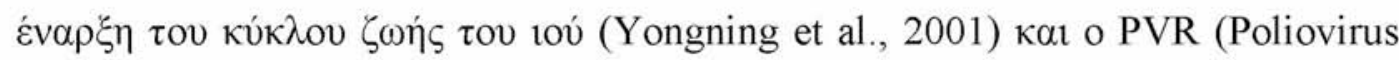

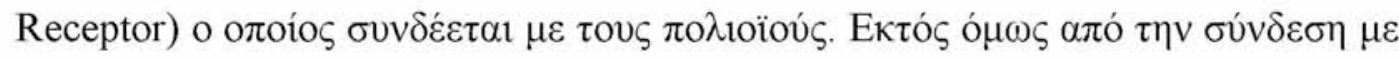

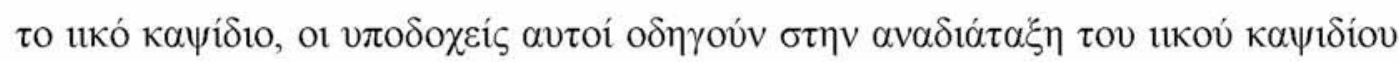

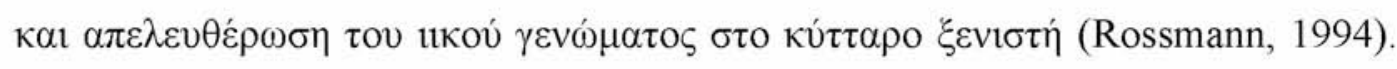

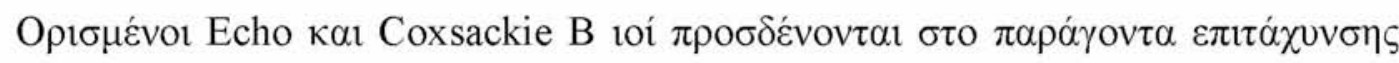

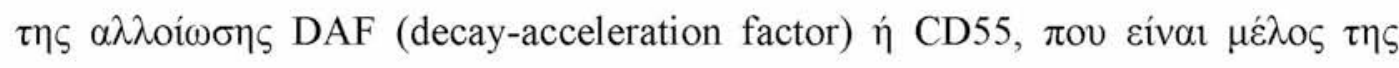

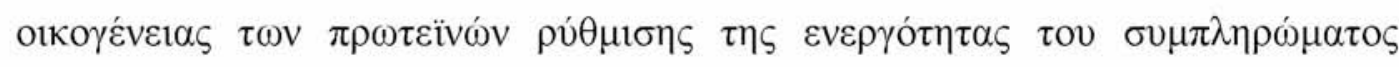

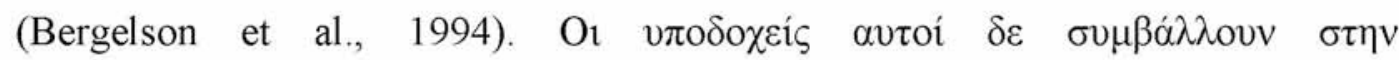

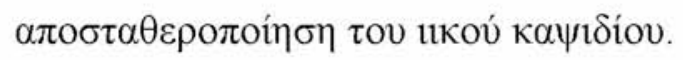




\begin{tabular}{|c|c|c|c|}
\hline Iós & 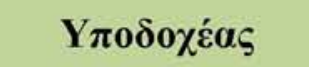 & 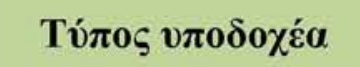 & 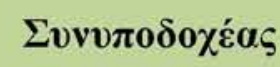 \\
\hline Под $\lambda$ เö̀ó 1-3 & PVR & lg-like & \\
\hline $\begin{array}{c}\text { Coxsackie A13, } \\
\text { A18, A21 }\end{array}$ & Icam-1 & lg-like & \\
\hline Enterovirus 70 & CD55 & $\begin{array}{c}\text { SCR-like } \\
\text { (complement cascade) }\end{array}$ & \\
\hline Coxsackie A21 & CD55 & $\begin{array}{c}\text { SCR-like } \\
\text { (complement cascade) }\end{array}$ & Icam-1 \\
\hline $\begin{array}{c}\text { Coxsackie B1, B3, } \\
\text { B5 }\end{array}$ & CD55 & $\begin{array}{c}\text { SCR-like } \\
\text { (complement cascade) }\end{array}$ & $\alpha_{4} \beta_{6}$-Integrin \\
\hline $\begin{array}{c}\text { Echo } 3,6,7,11-13, \\
20,21,24,29,33\end{array}$ & CD55 & $\begin{array}{c}\text { SCR-like } \\
\text { (complement cascade) }\end{array}$ & $\beta_{2}$-Microglobulin \\
\hline Echo 1,8 & $\begin{array}{c}\alpha_{2} \beta_{1} \text {-Integrin (VIa- } \\
2 \text { ) }\end{array}$ & Integrin & $\beta_{2}$-Microglobulin \\
\hline Coxsackie B1-B6 & CAR & lg-like & \\
\hline
\end{tabular}

lg, Immunoglobulin; SCR, short consensus repeat

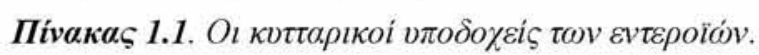

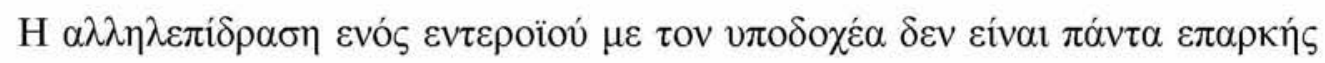

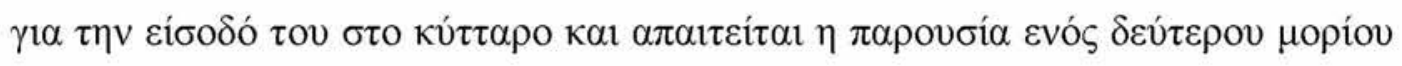

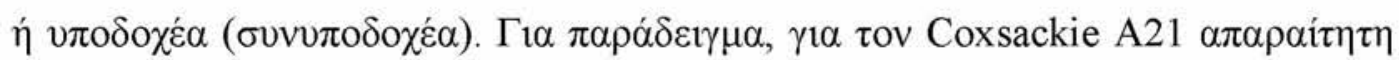

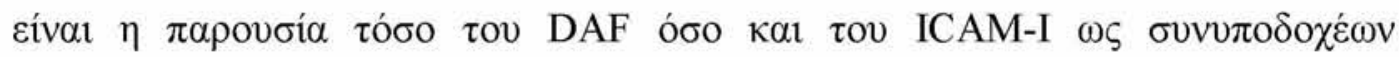

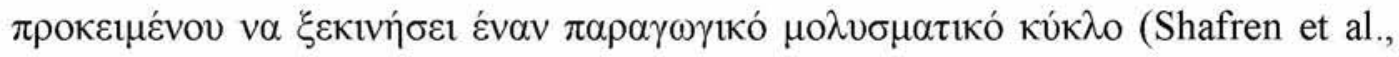

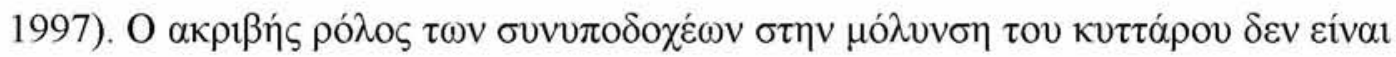

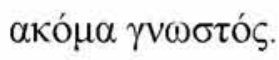




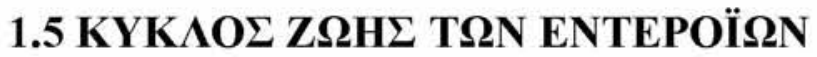

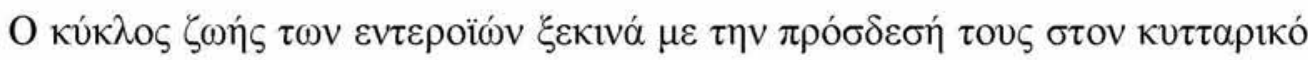

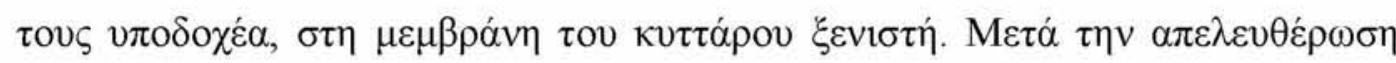

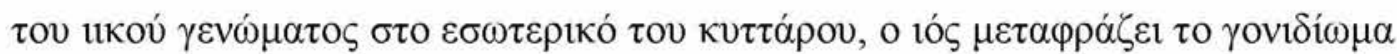

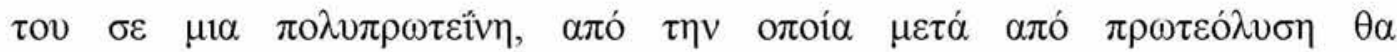

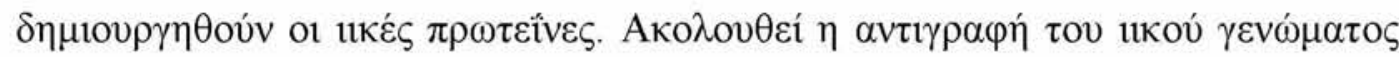

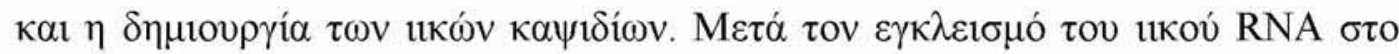

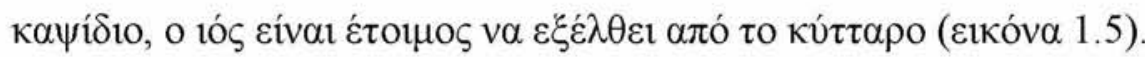

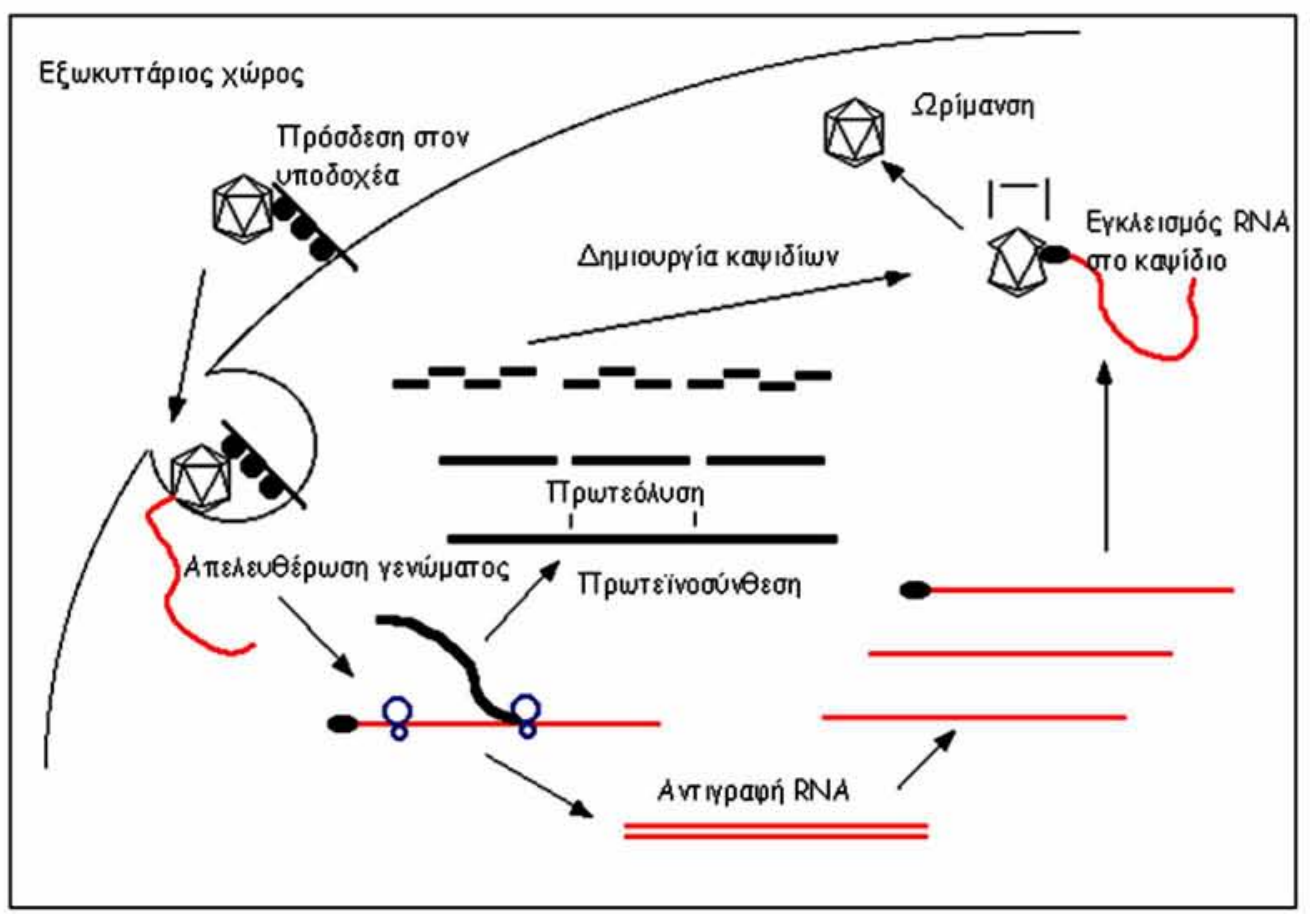

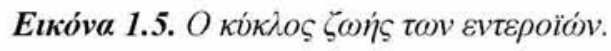

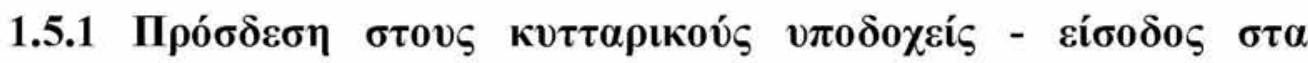

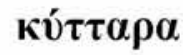

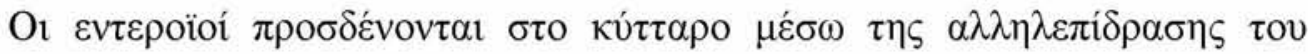

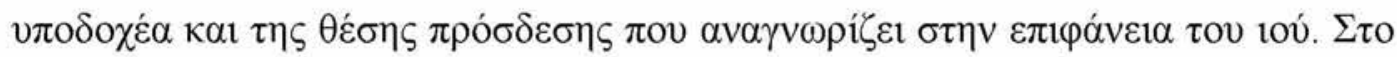

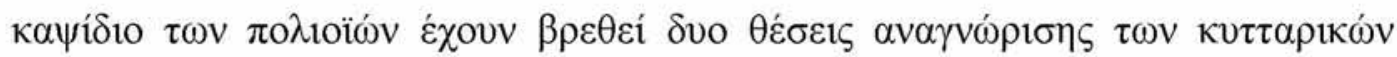

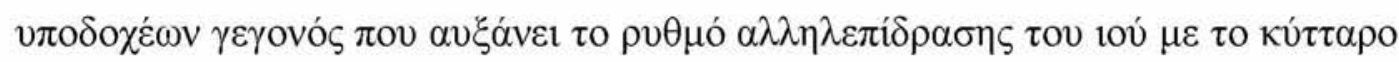

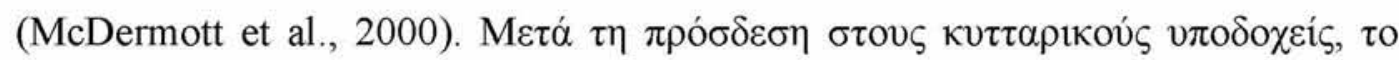

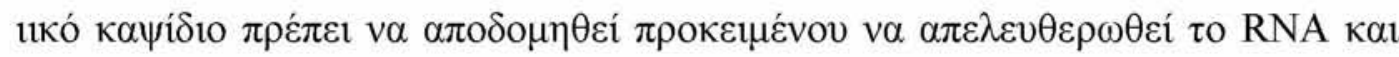

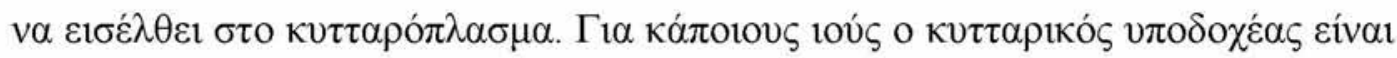




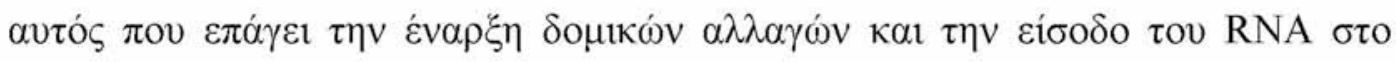

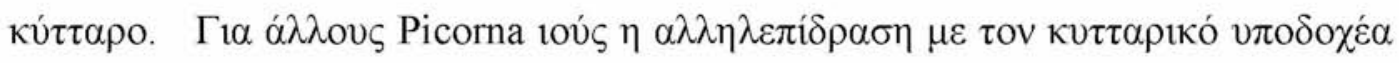

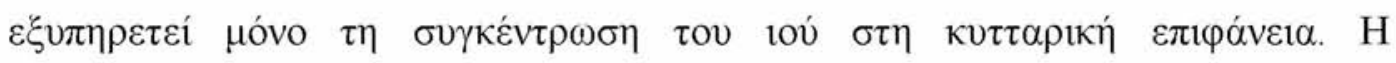

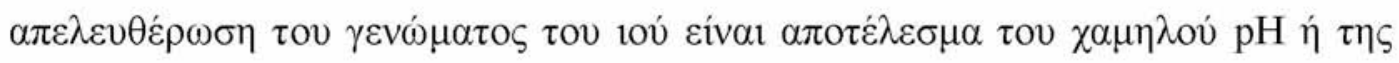

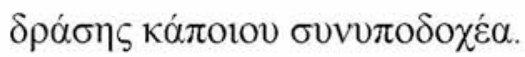

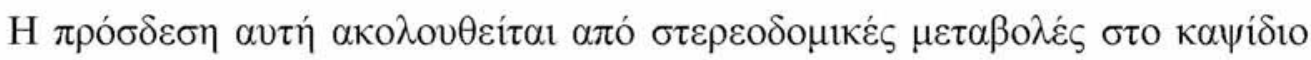

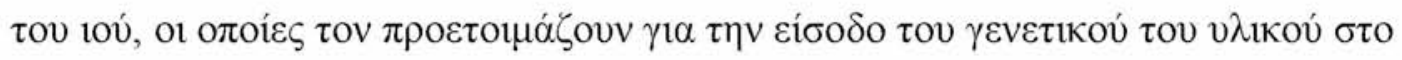

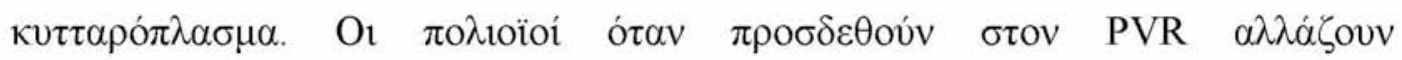

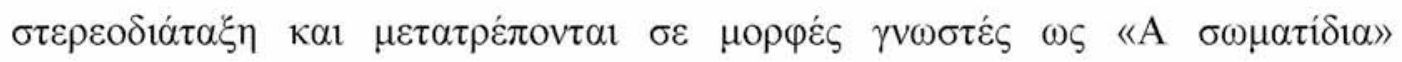

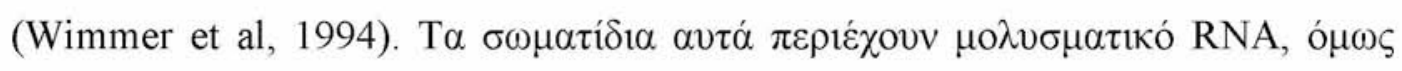

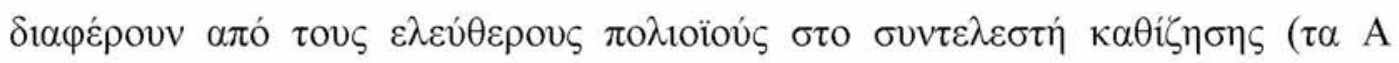

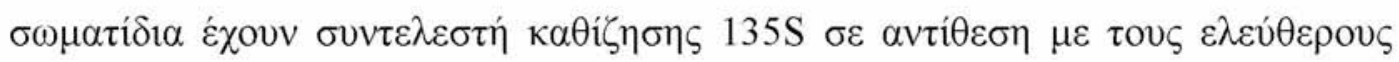

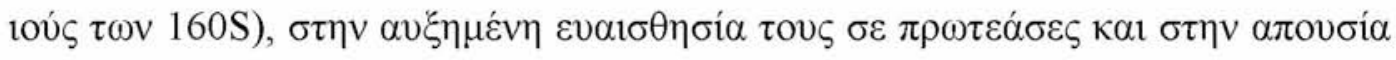

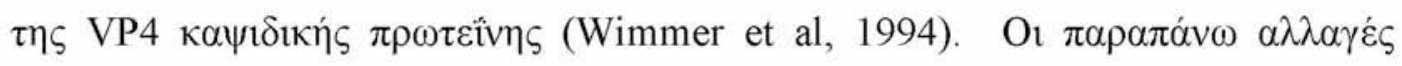

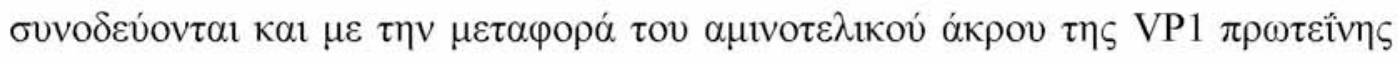

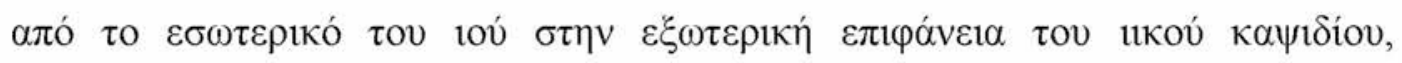

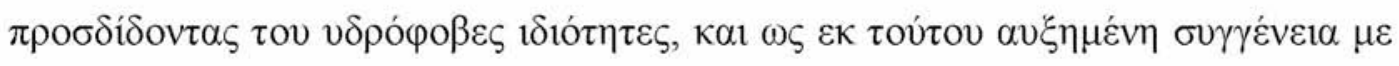

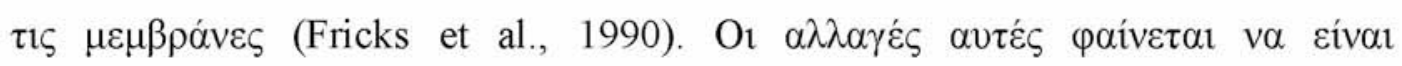

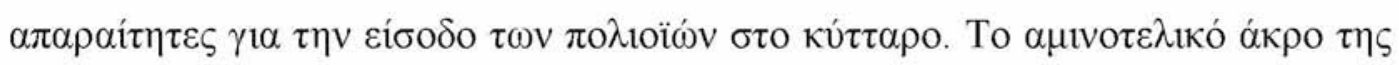

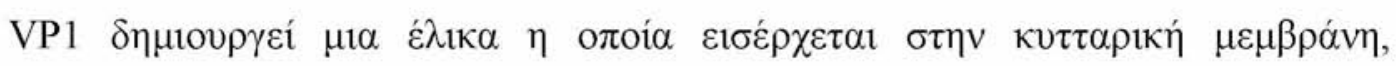

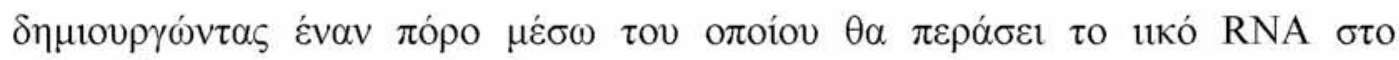

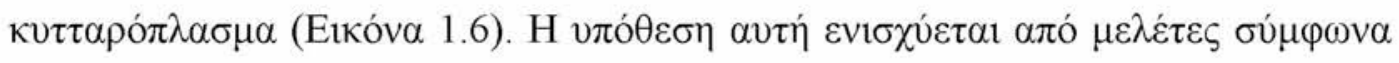

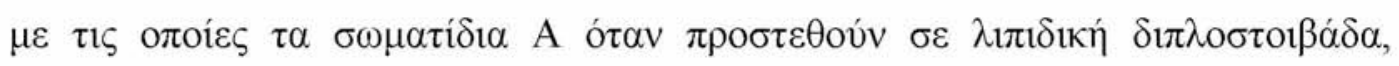

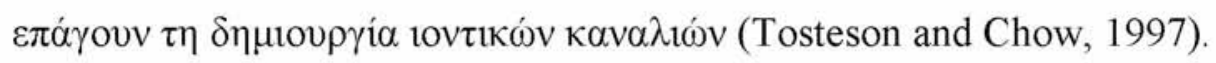




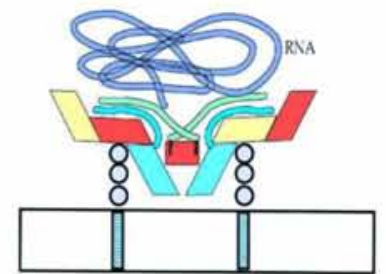

$160 \mathrm{~S}$

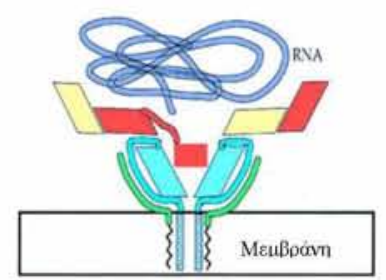

$135 S$
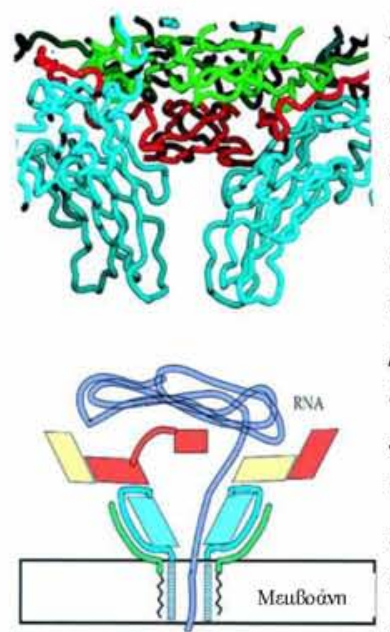

PORE

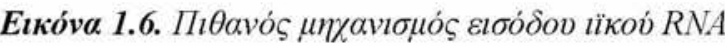

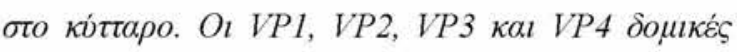

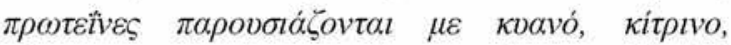

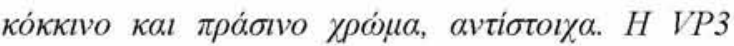

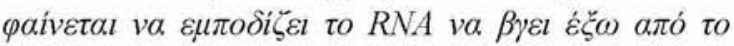

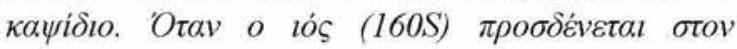

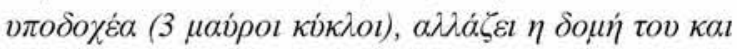

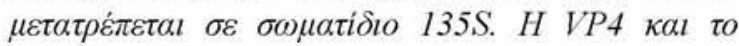

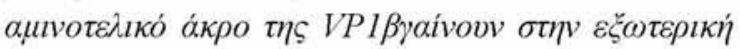

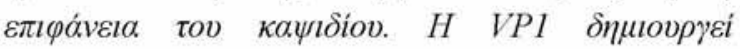

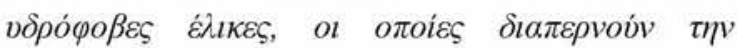

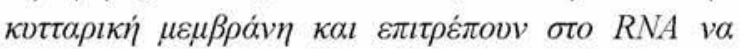

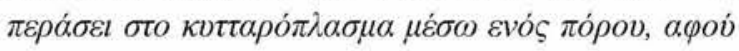

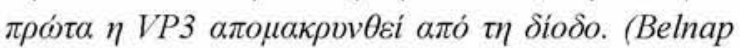
et al., 2000)

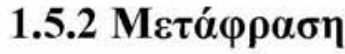

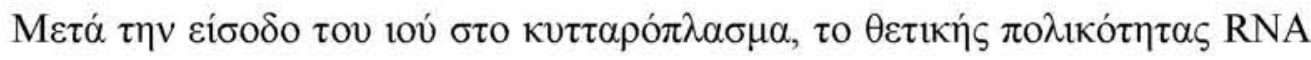

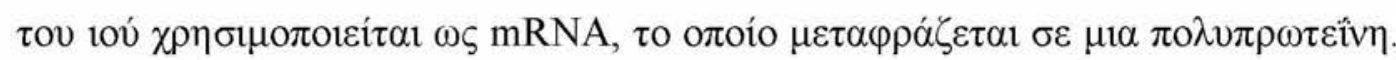

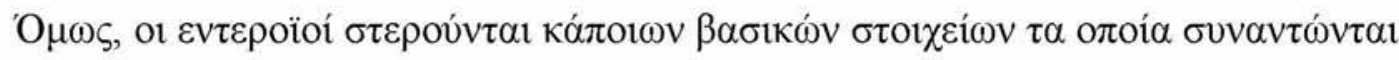

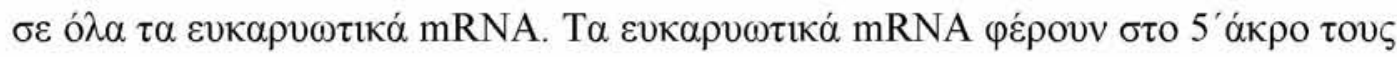

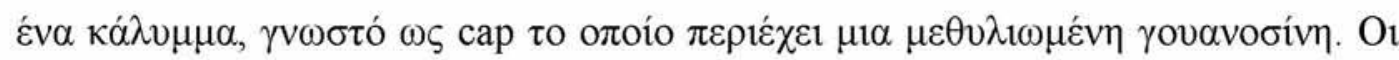

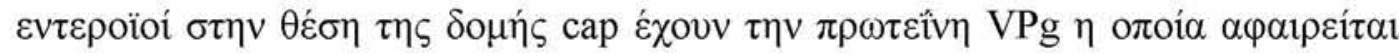

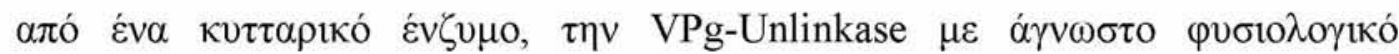

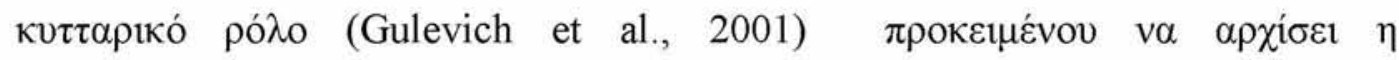
$\pi \rho \omega \tau \varepsilon \ddot{v} \circ \sigma u ́ v \theta \varepsilon \sigma \eta$.

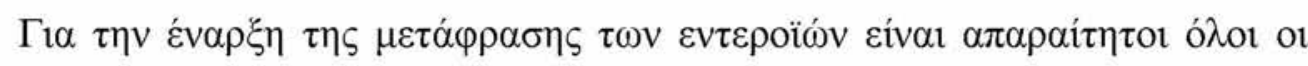

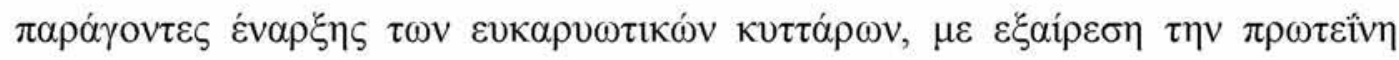

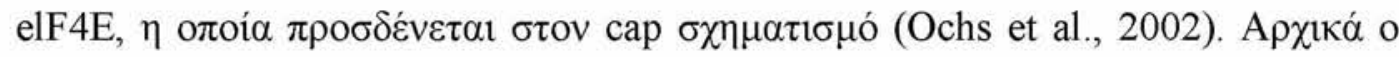

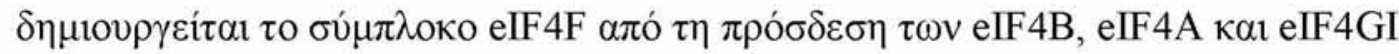
$\mu \varepsilon \tau \alpha \gamma \rho \alpha \varphi$ «

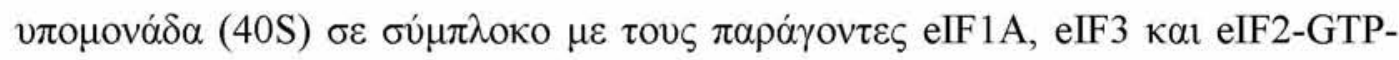

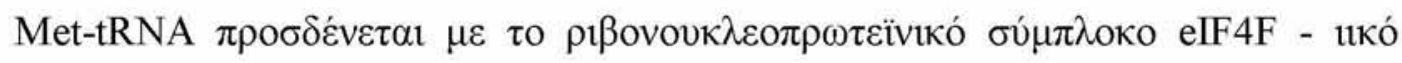

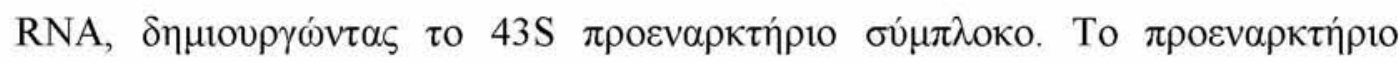

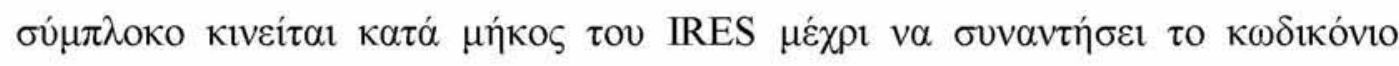

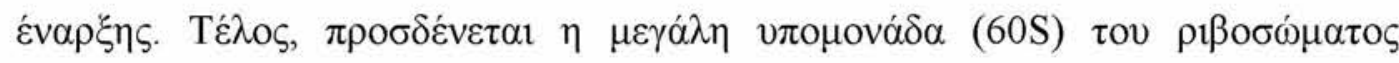

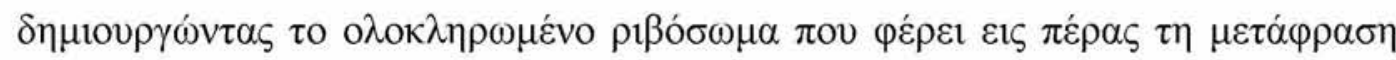
(Prevot et al., 2003). 


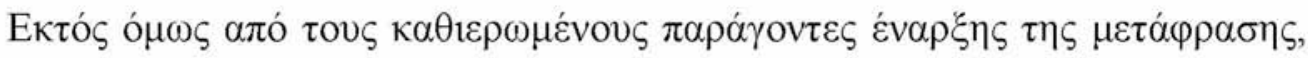

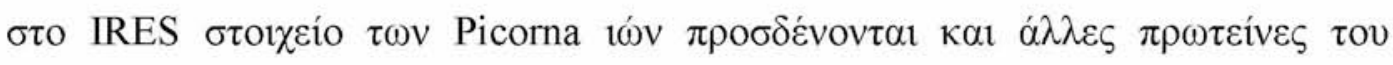

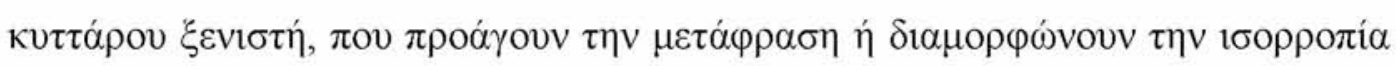

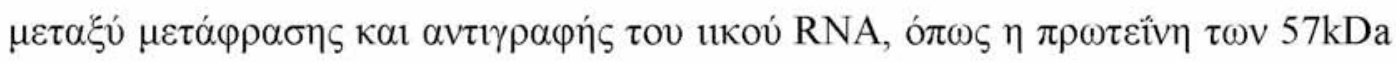

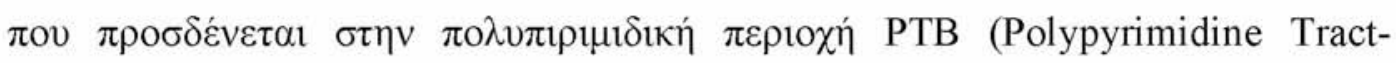
Binding protein), $\eta \pi \rho \omega \tau \varepsilon i v \eta \mathrm{La} \tau \omega \nu 52 \mathrm{kDa}$ (La auto antigen), $\eta$ poly(rC) $\pi \rho \omega \tau \varepsilon i v \eta$ PCBP(Poly(rC) Binding Protein) $\tau \omega \nu 39 \mathrm{kDa} \kappa \alpha \iota \eta$ Unr (Upstream of NRas) (Murray et al, 2001; Costa-Mattioli et al, 2004; Boussadia et al., 2003). Ot

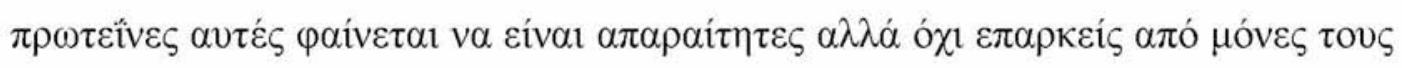

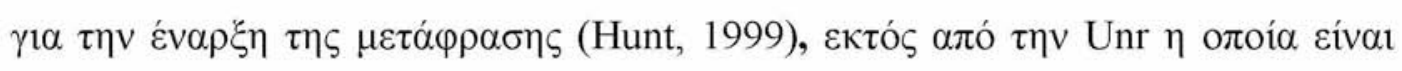
$\pi \rho \alpha ́ \gamma \mu \alpha \tau \iota ~ \alpha v \alpha \gamma \kappa \alpha i \alpha \sigma \varepsilon \alpha v \tau \eta \dot{~ \tau \eta ~ \delta ı \alpha \delta ı к \alpha \sigma i ́ \alpha ~(B o u s s a d i a ~ e t ~ a l ., ~ 2003) . ~}$

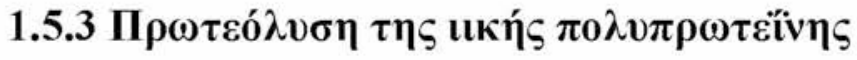

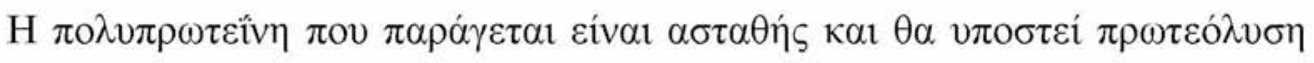

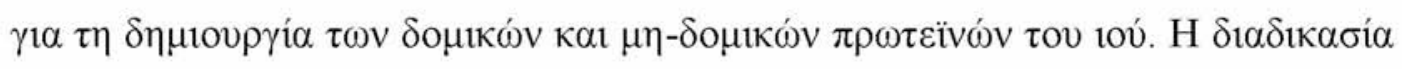

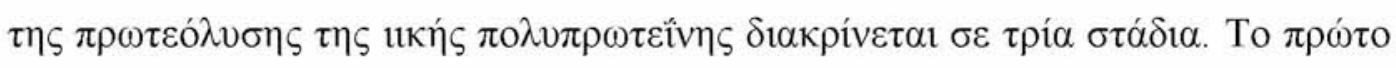

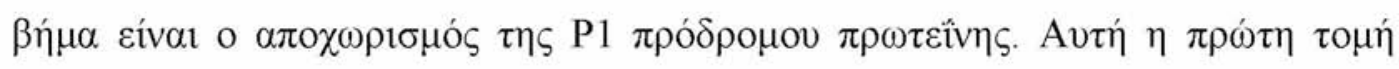

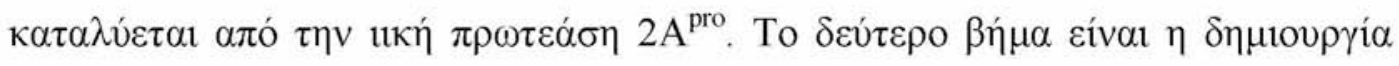

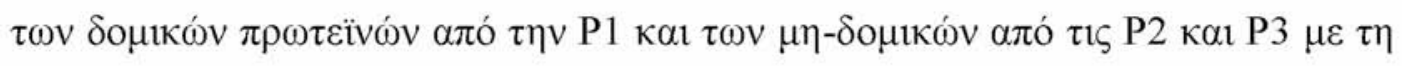

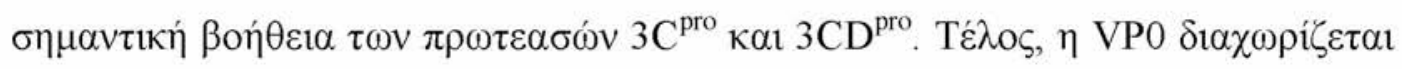

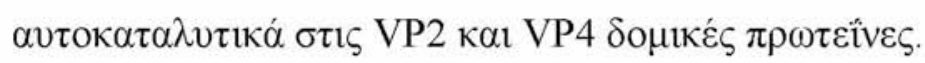

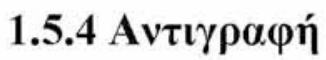

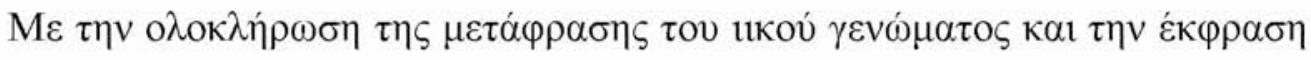

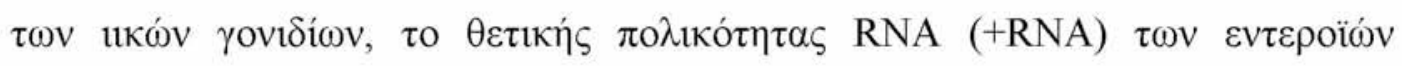

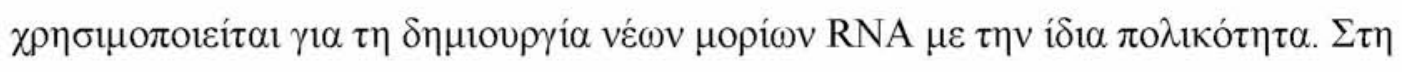

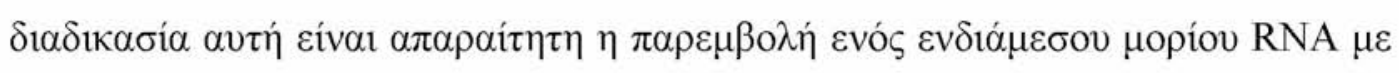

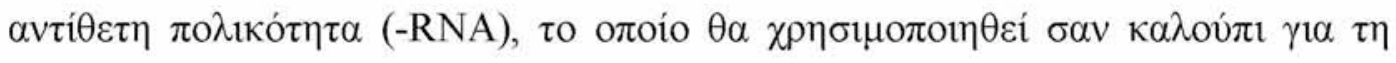

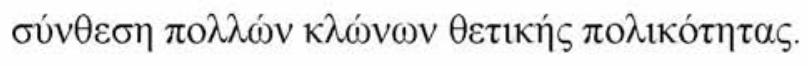

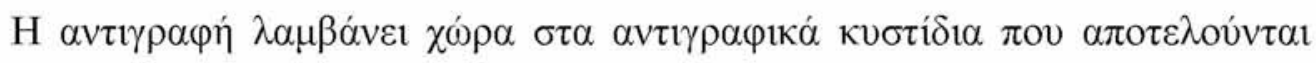

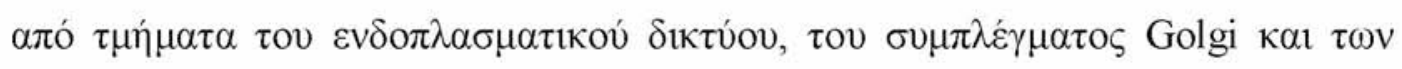

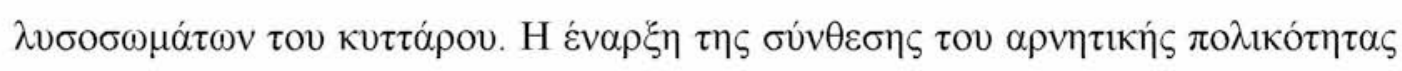

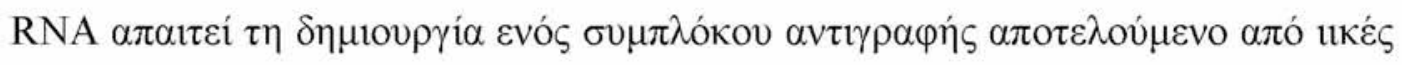




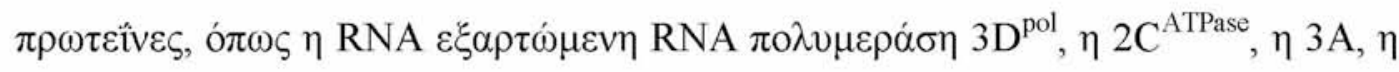

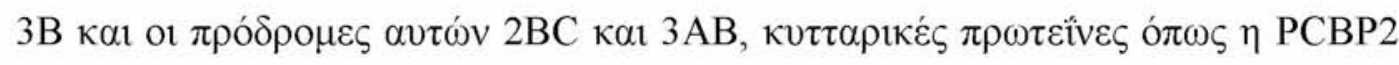

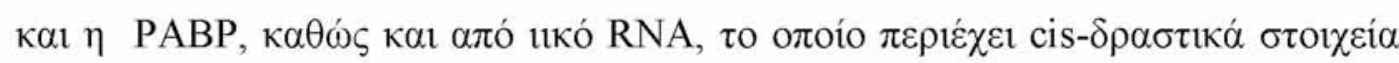

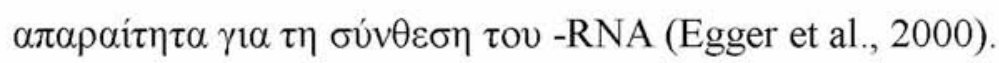

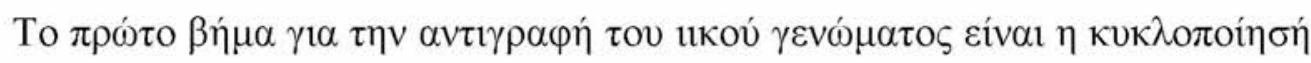

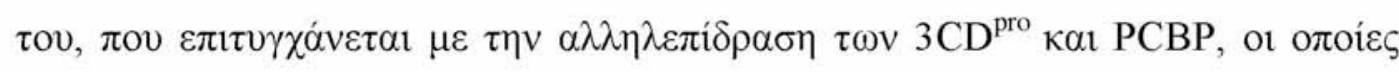

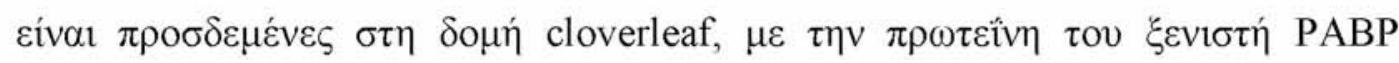

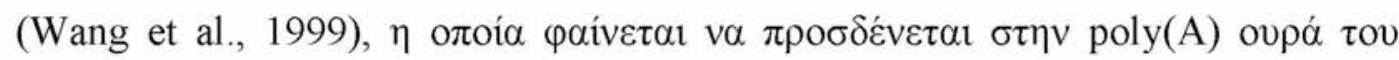

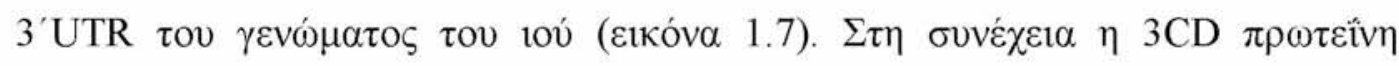

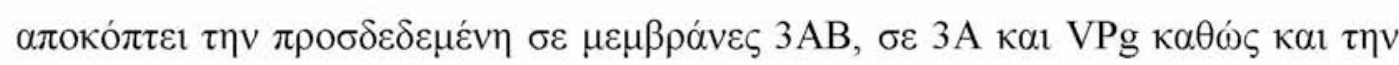
3CD $\sigma \varepsilon 3 \mathrm{C} \kappa \alpha 1$ 3D (Murray and Barton, 2003). $\Sigma \tau \eta v \varepsilon \pi 1 \varphi \alpha ́ v \varepsilon 1 \alpha \tau\rceil \varsigma 3 \mathrm{D}, \eta \mathrm{VPg}, \eta$

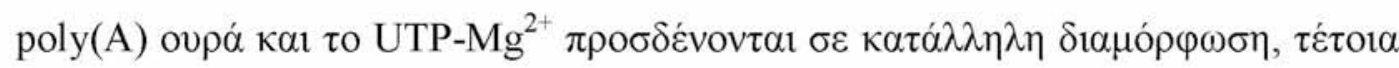

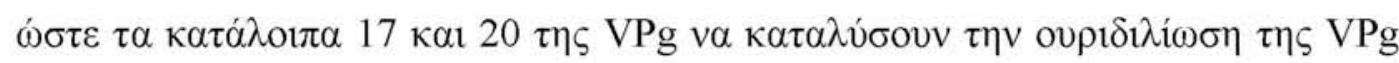

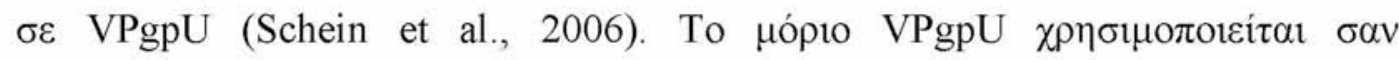

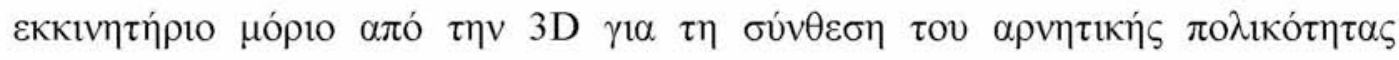

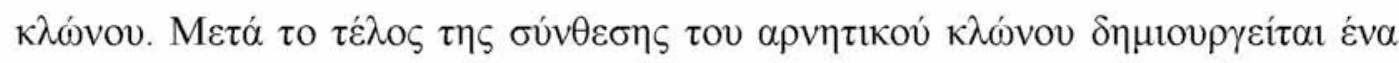

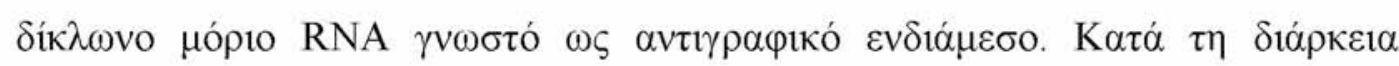

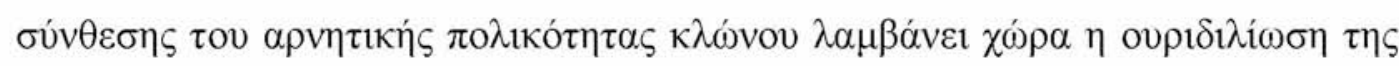

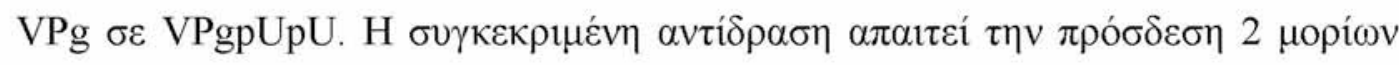

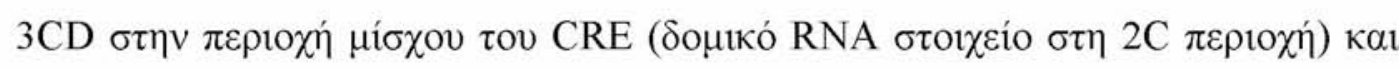

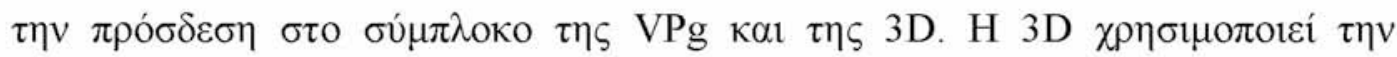

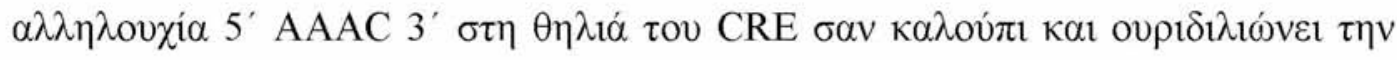

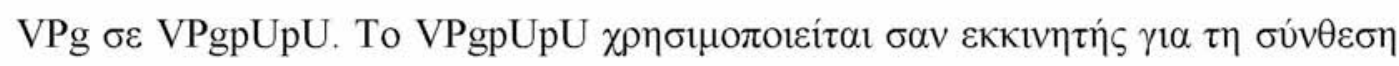

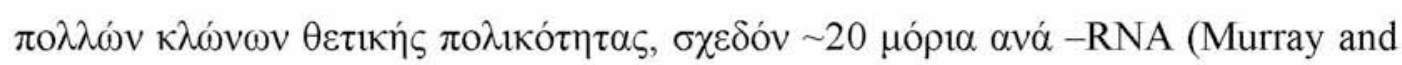
Barton, 2003). 


\section{Kukגoroínon RNA}
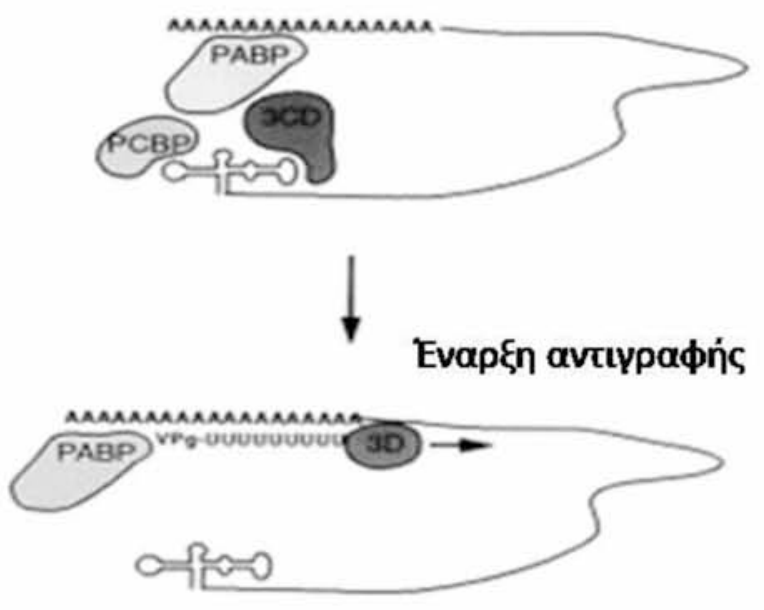

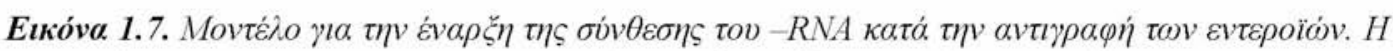

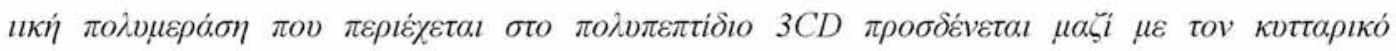

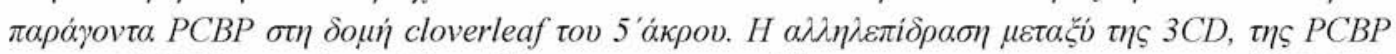

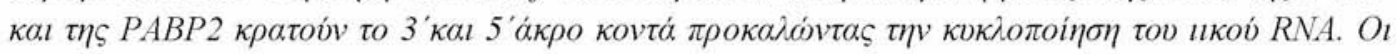

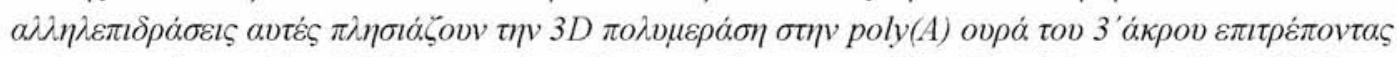

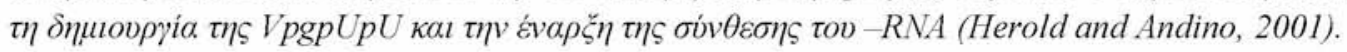

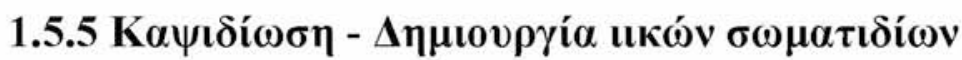

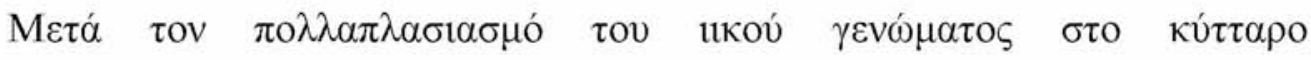

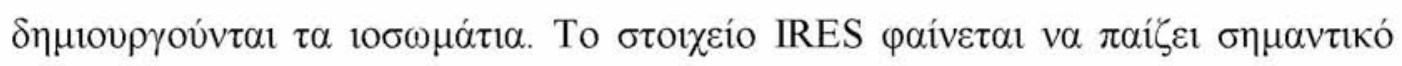

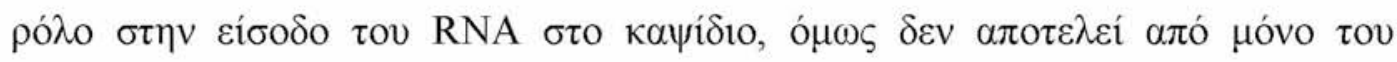

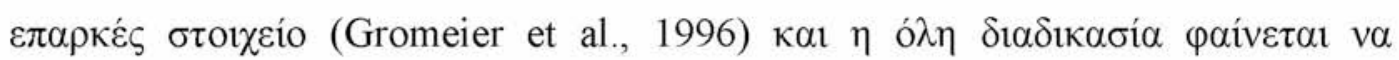

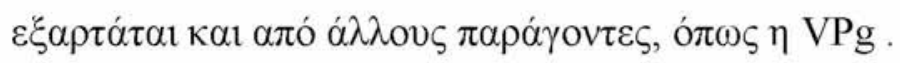

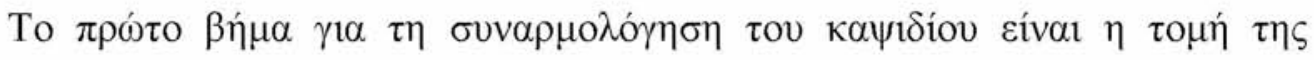

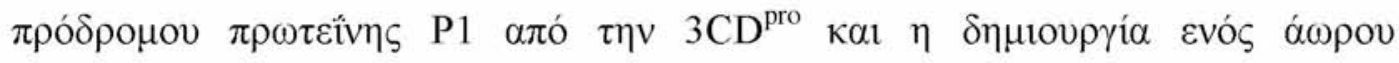

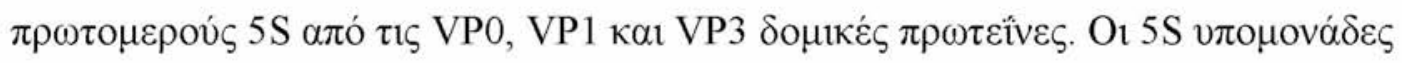

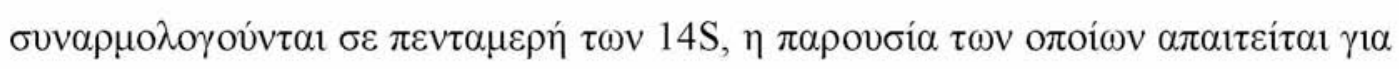

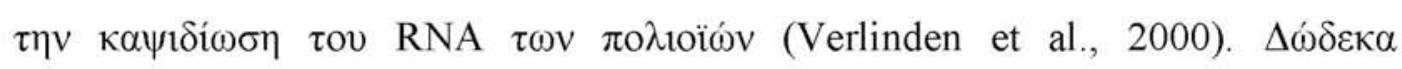

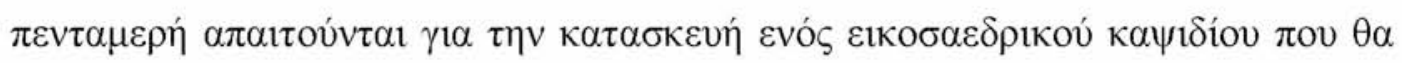

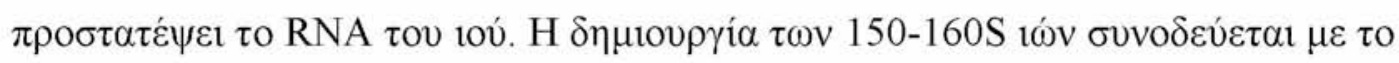

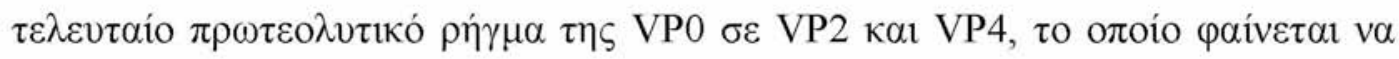

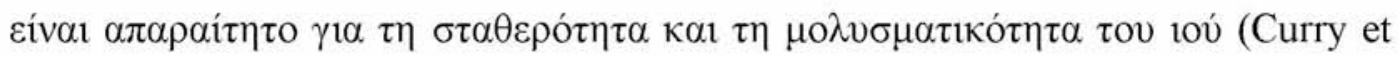




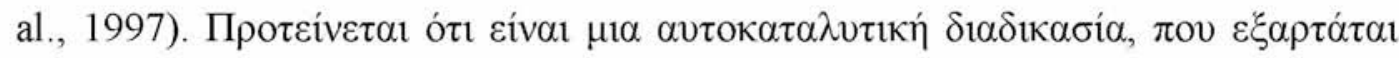

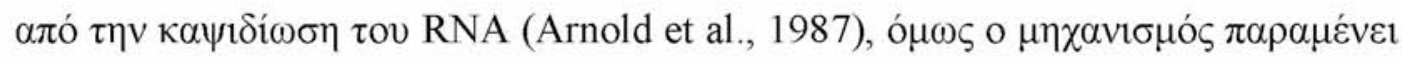
$\alpha \gamma v \omega \sigma \tau o s$.

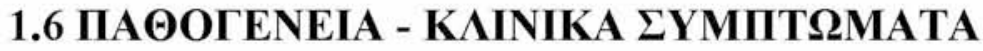

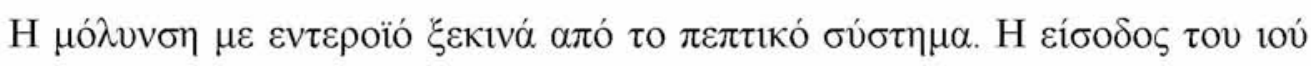

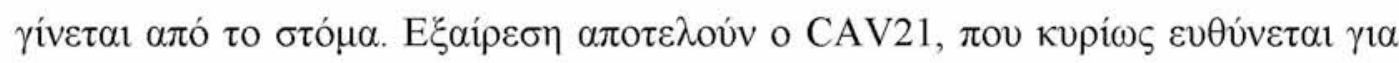

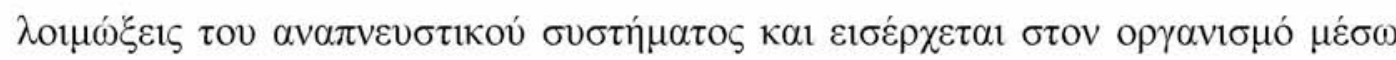

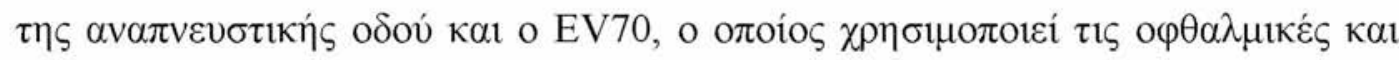

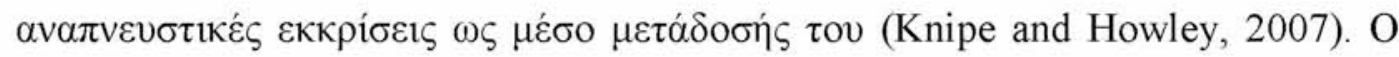

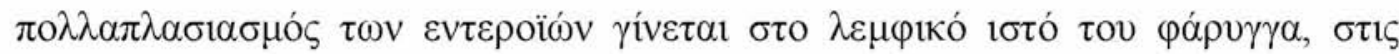

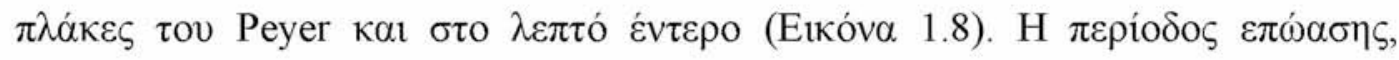

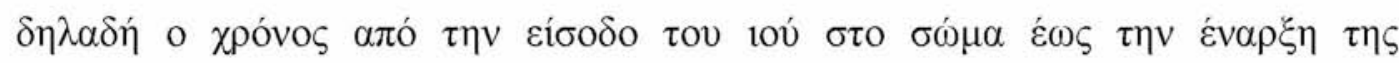

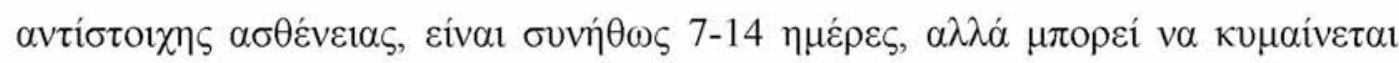

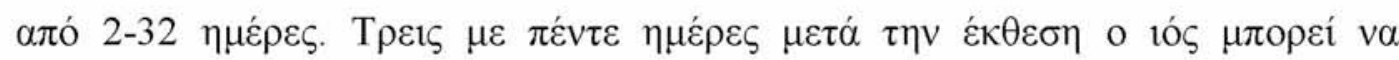

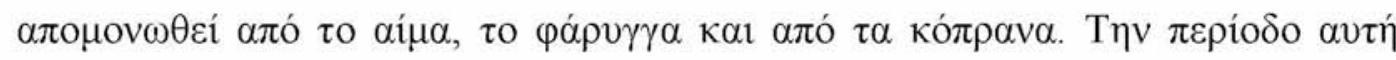

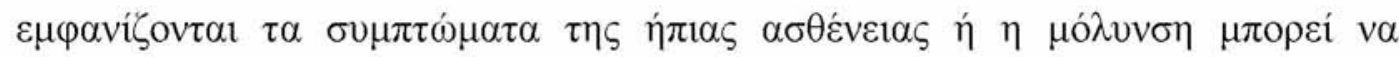

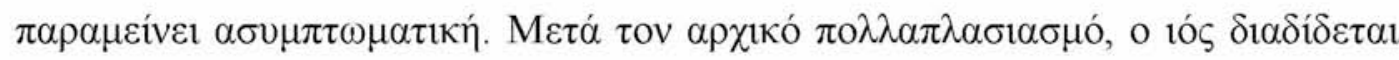

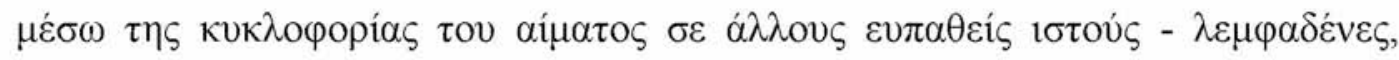

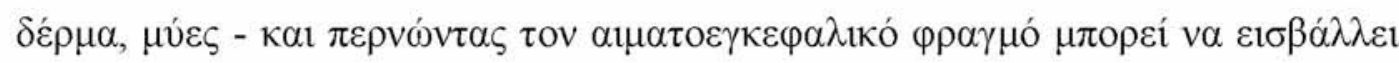

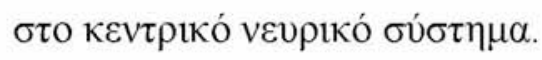

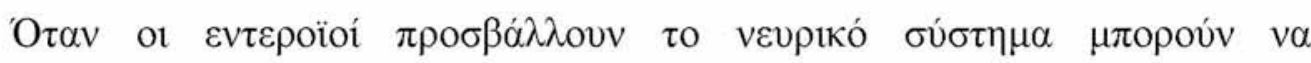

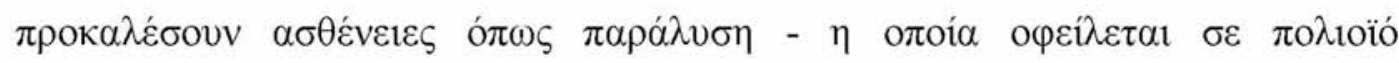

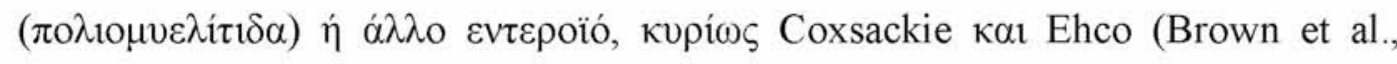

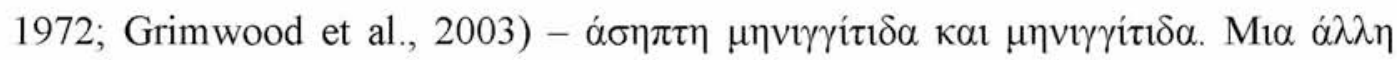

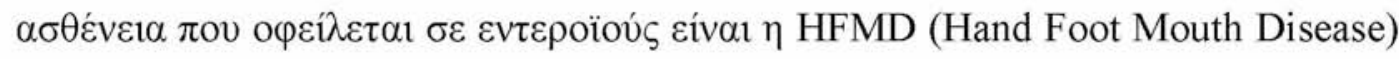

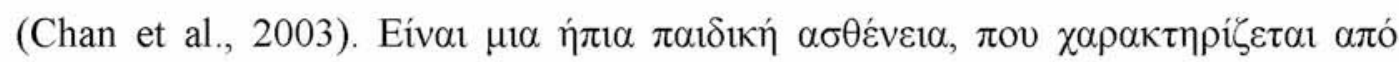
$\alpha \pi$ ó

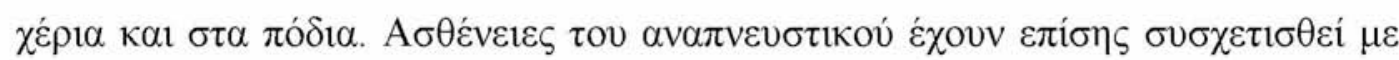

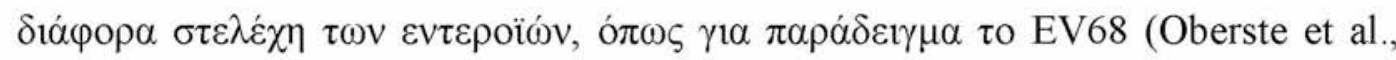

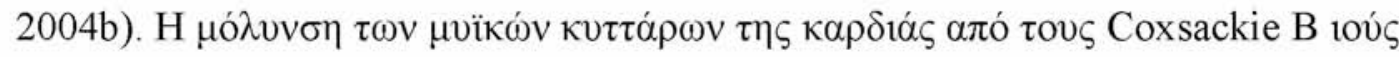

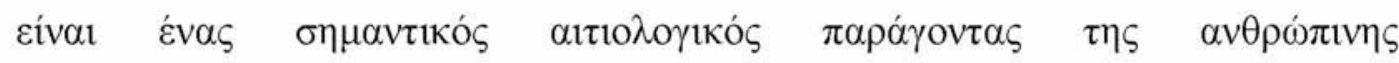

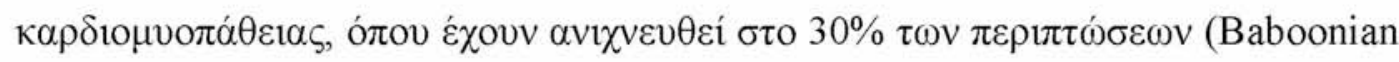




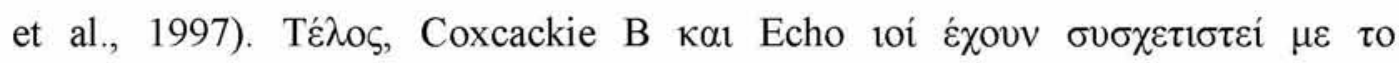

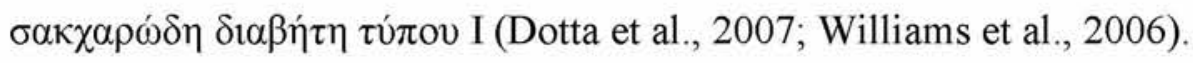

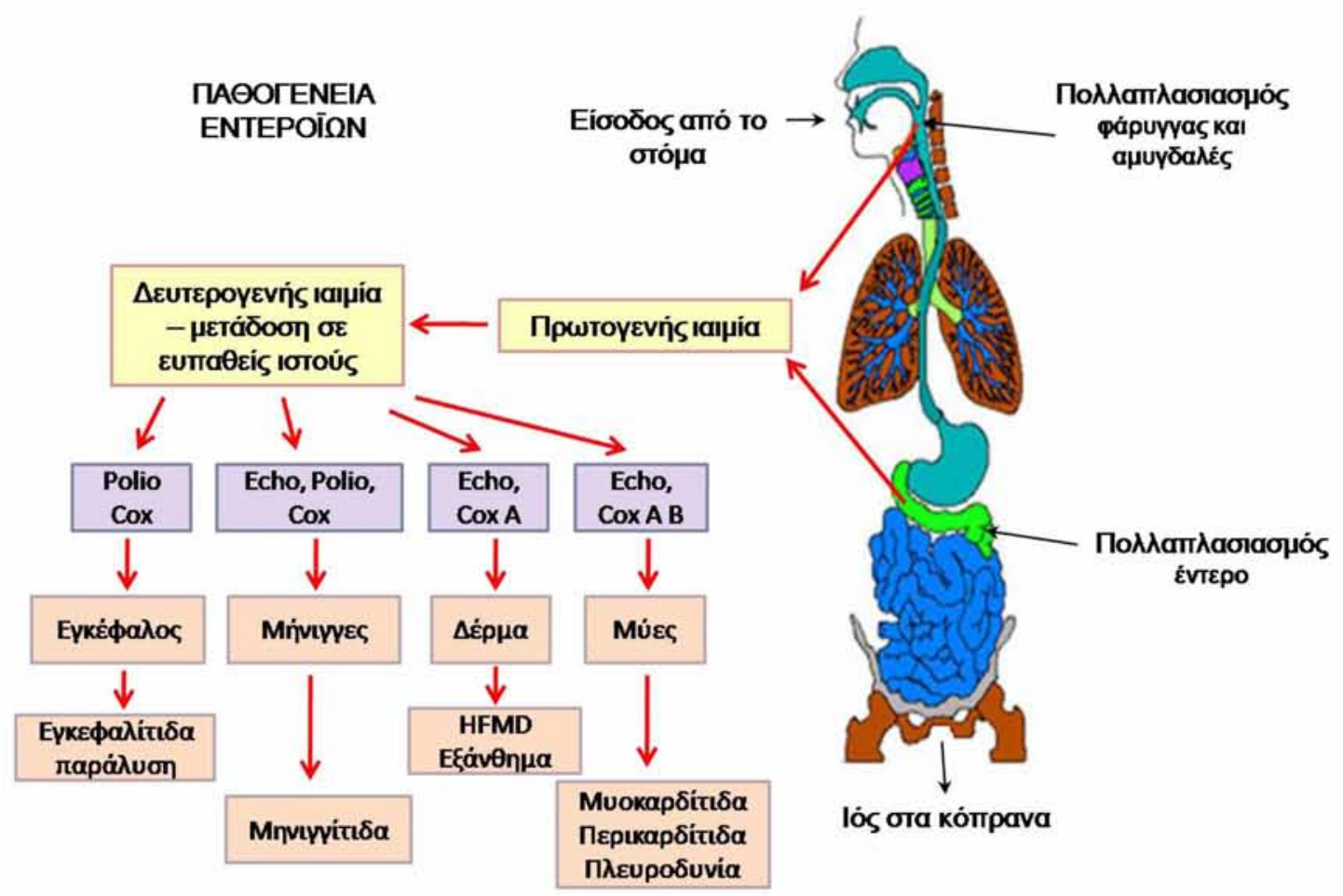

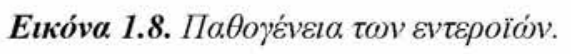

\section{7 ЕПІАНМІОАОГІА}

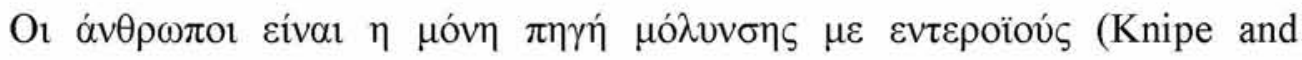

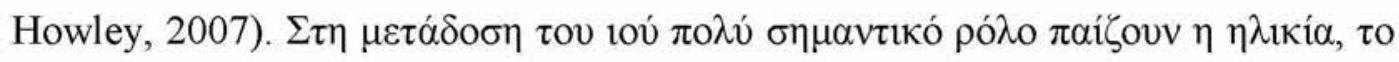

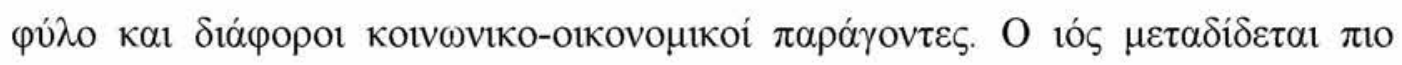

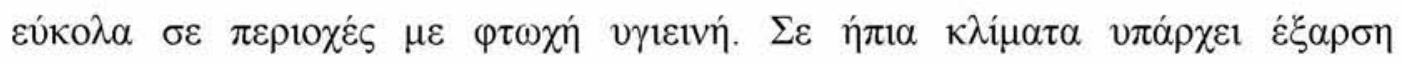

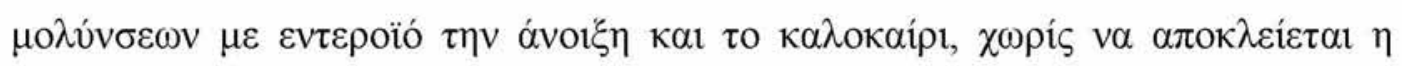

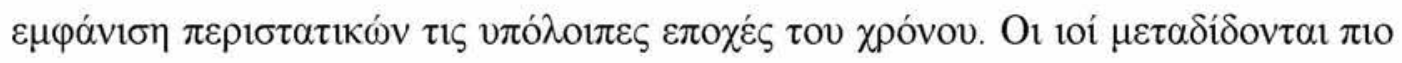

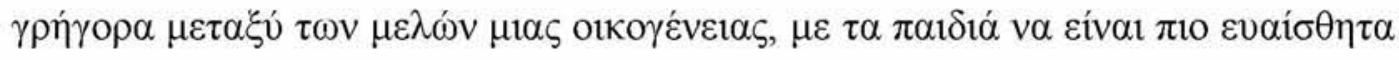

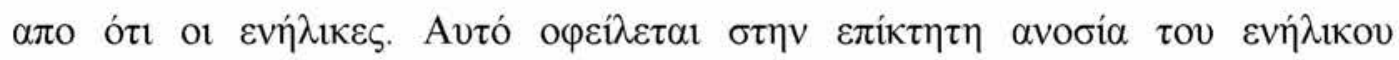

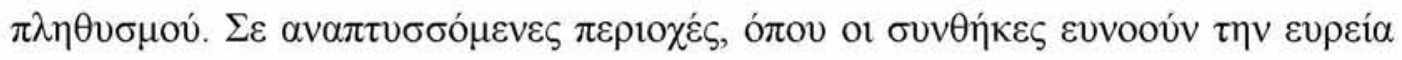

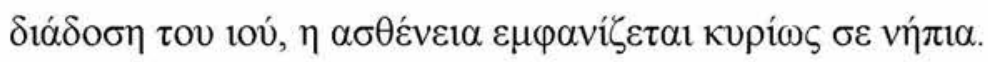

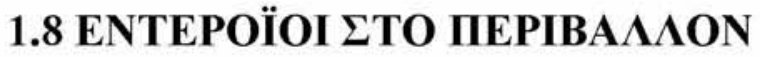

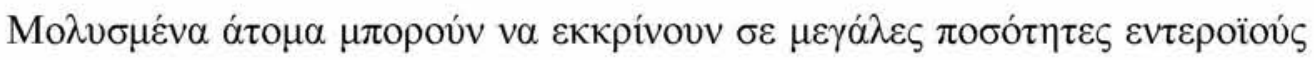

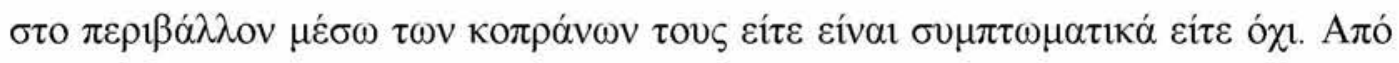




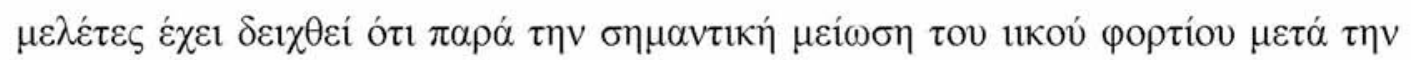

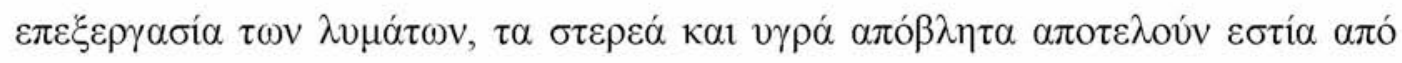

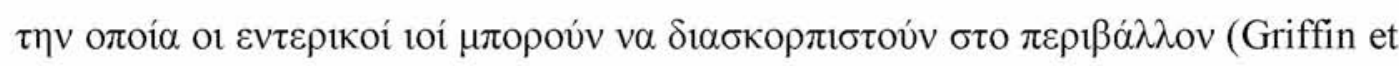

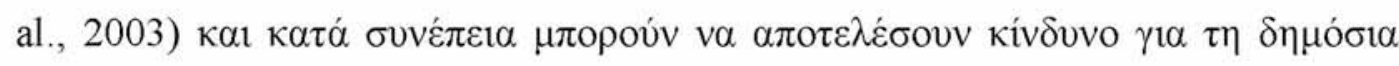

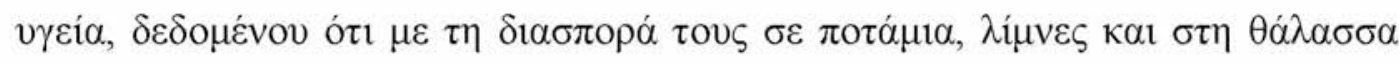

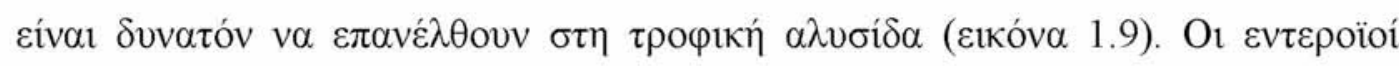

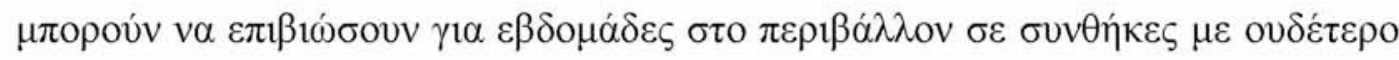

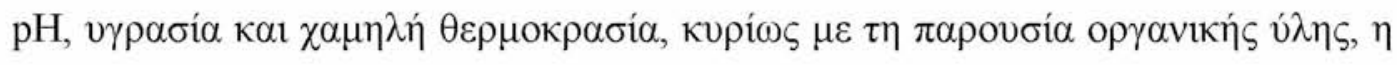

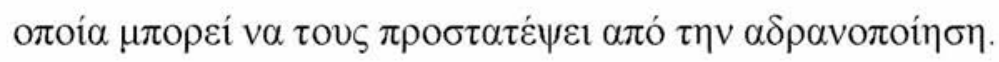

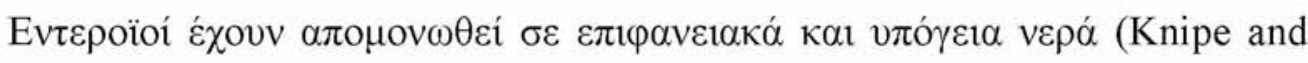

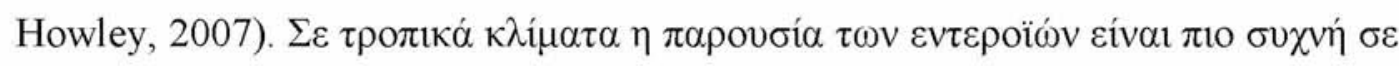

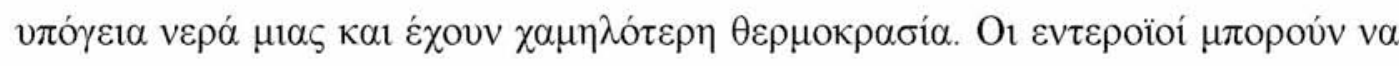

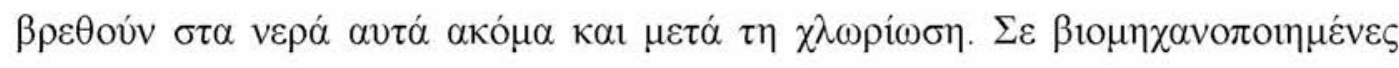

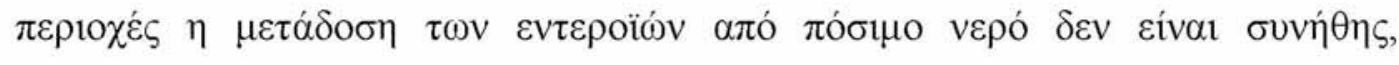

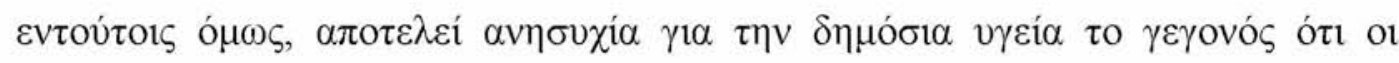

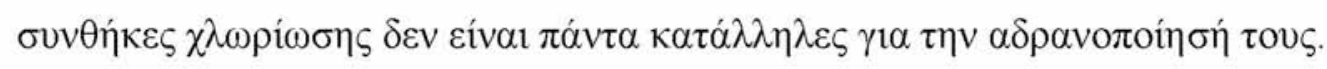

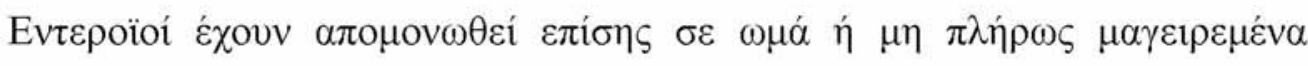

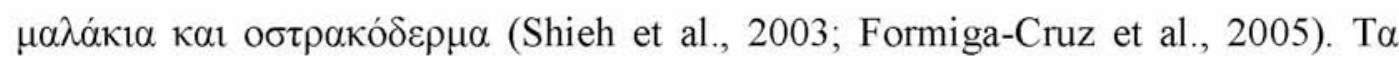

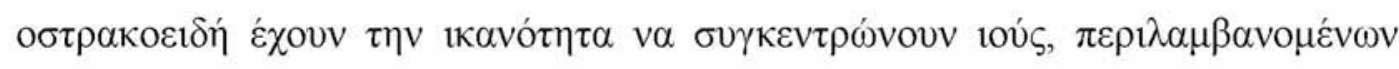

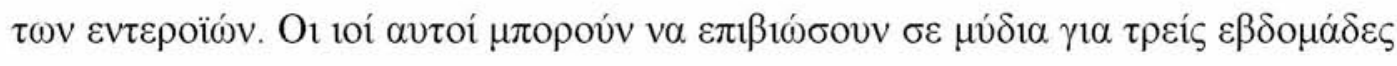

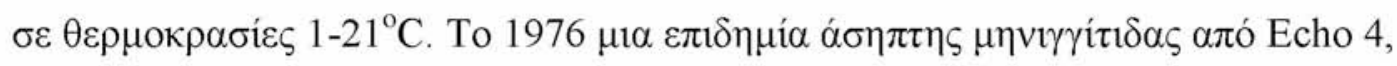

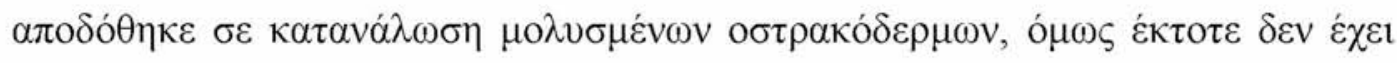

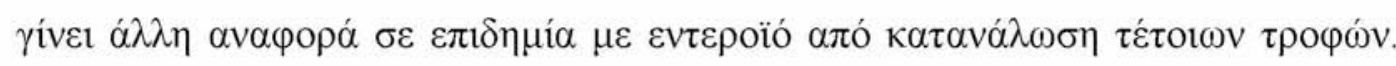

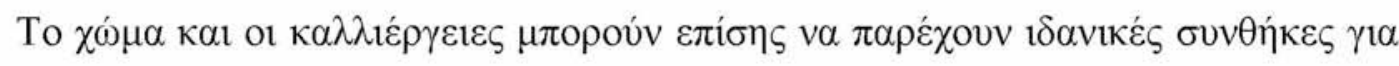

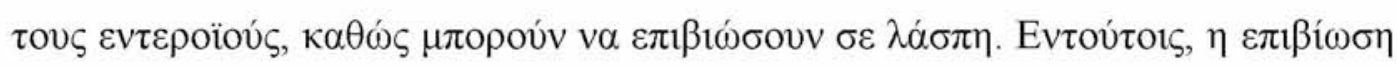

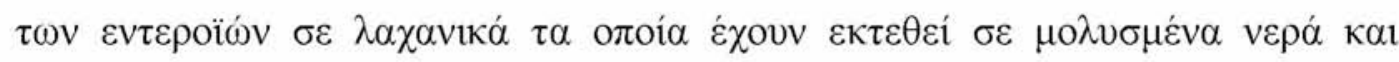

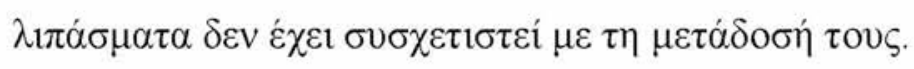




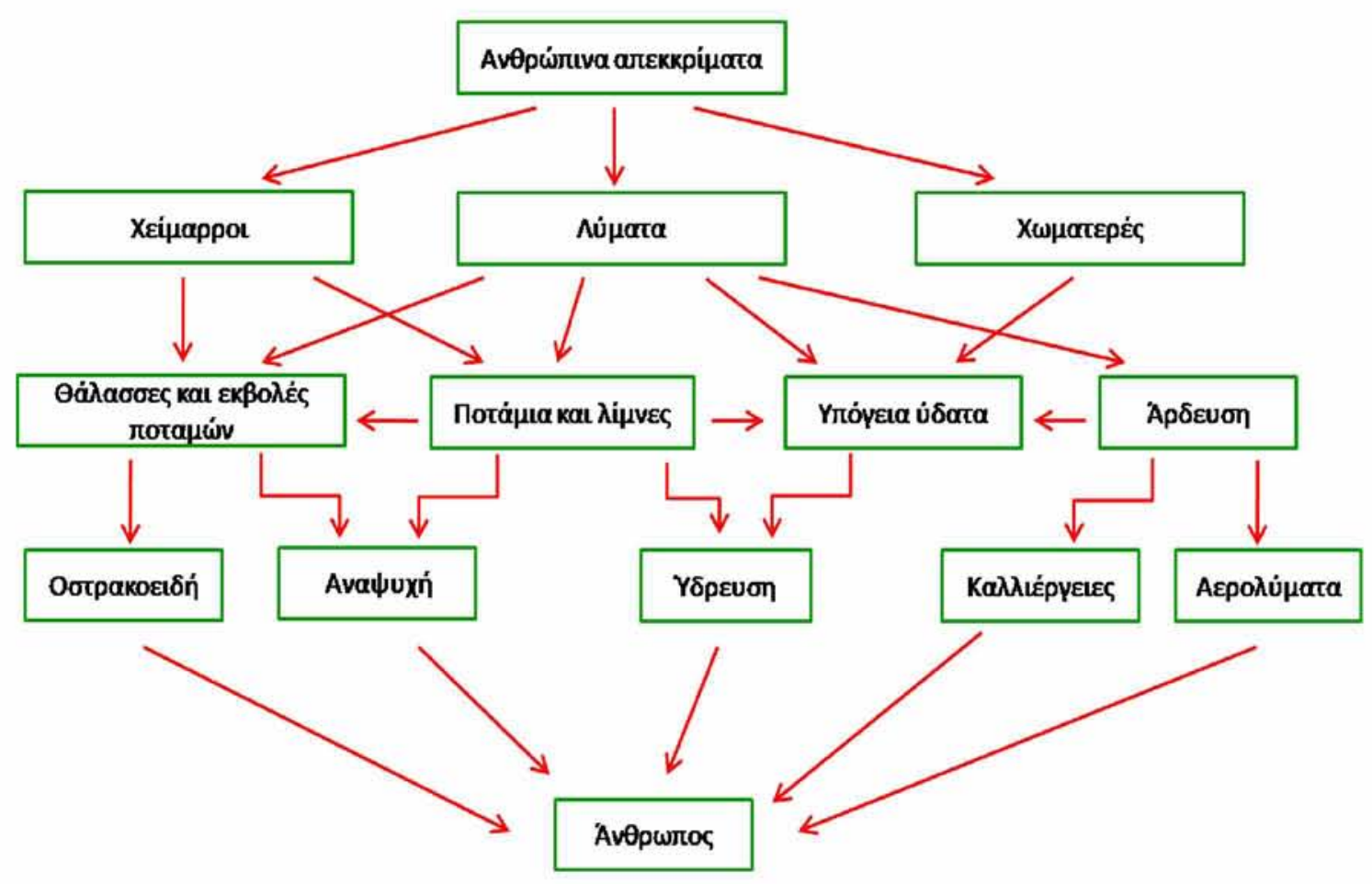

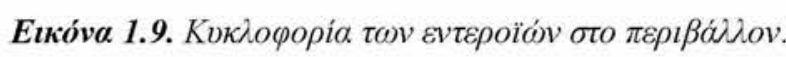

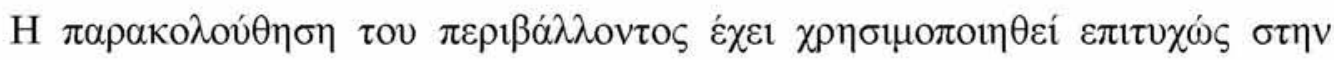

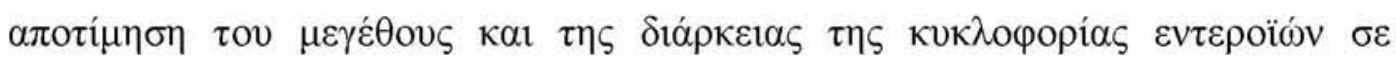

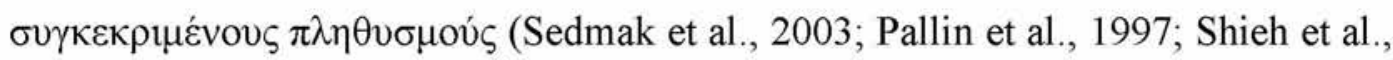

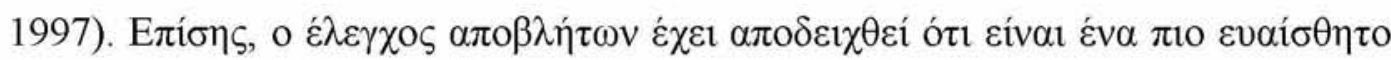

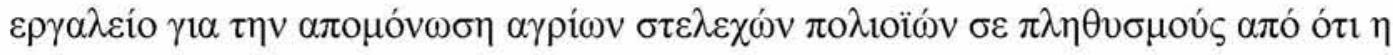

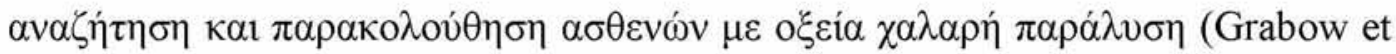

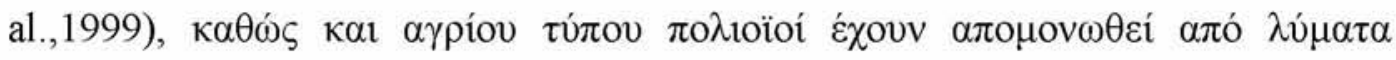

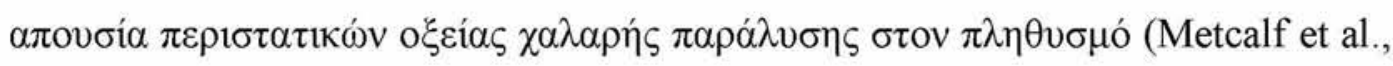

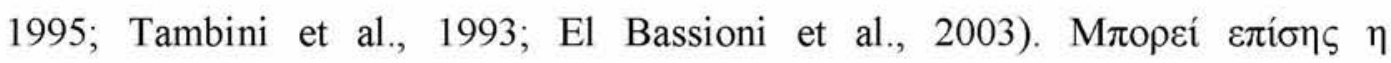

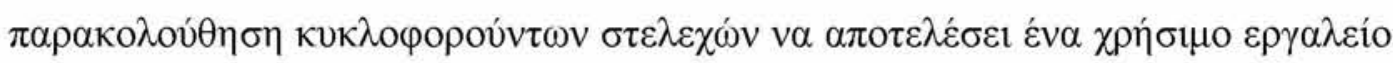

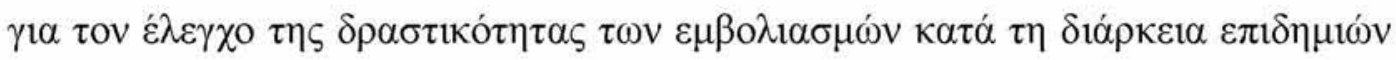

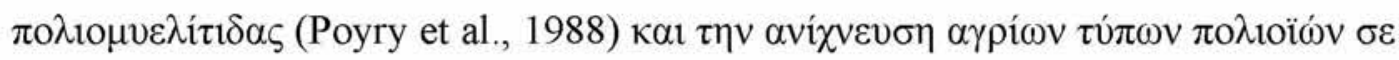

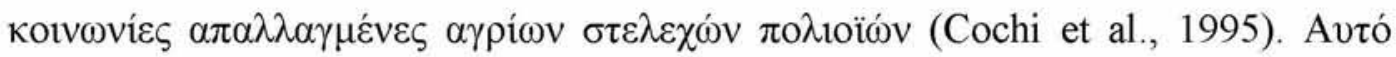

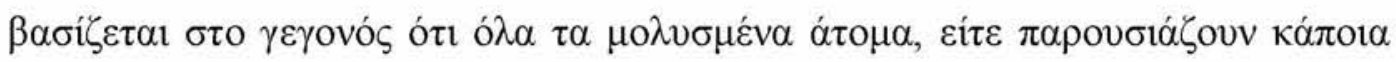

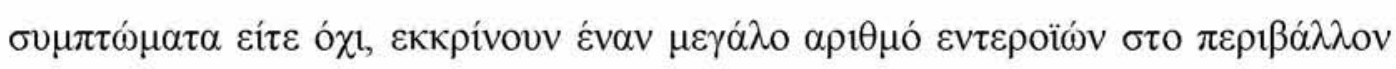

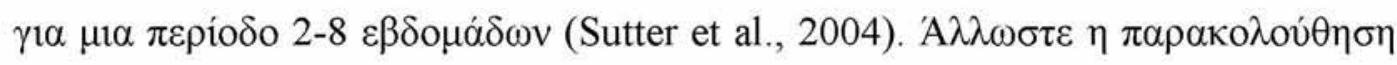

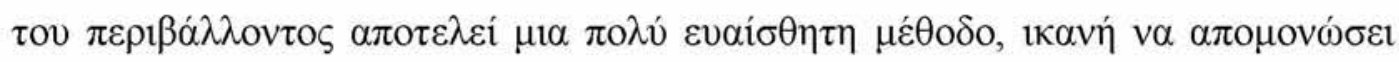

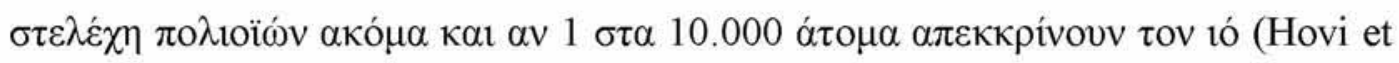




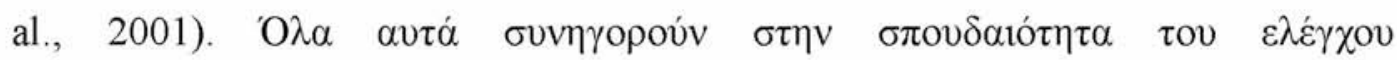

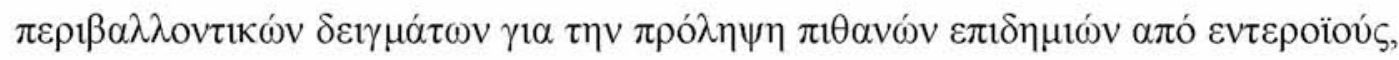

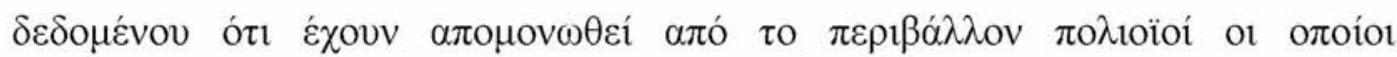

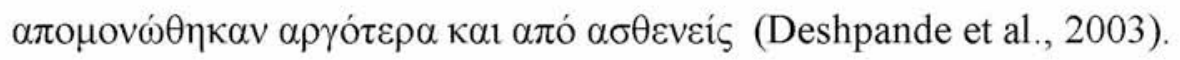

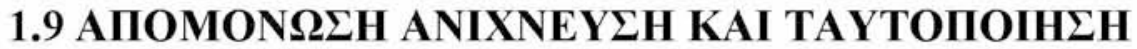

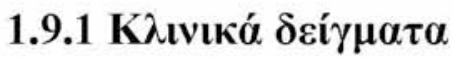

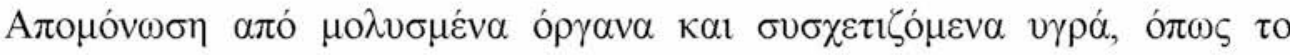

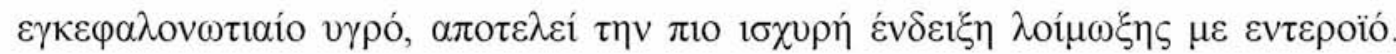

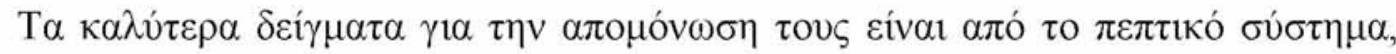

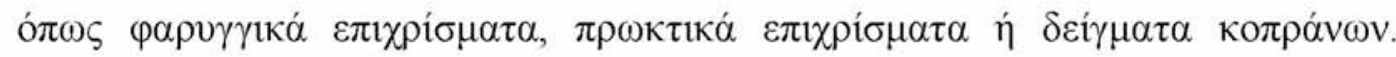

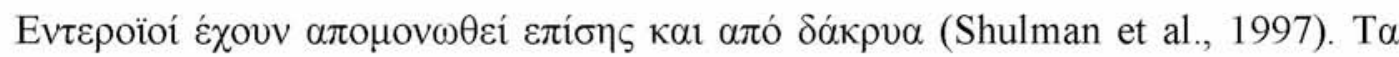

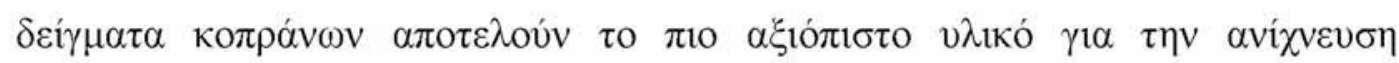

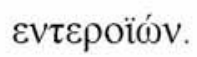

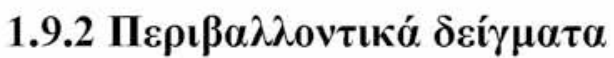

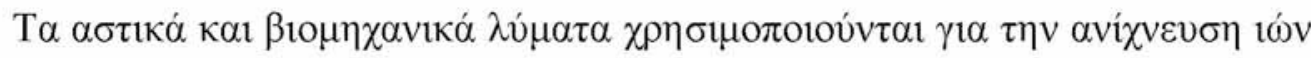

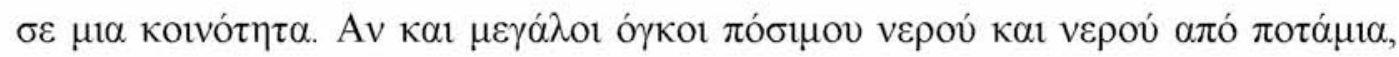

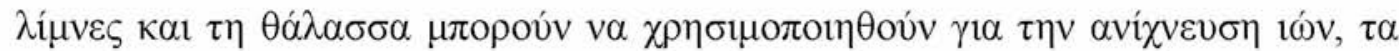

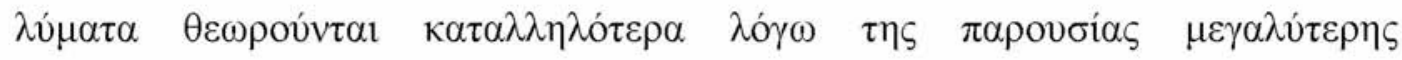

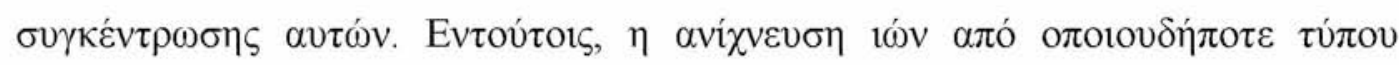

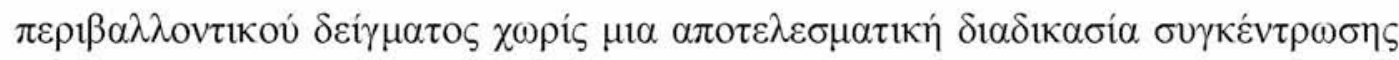

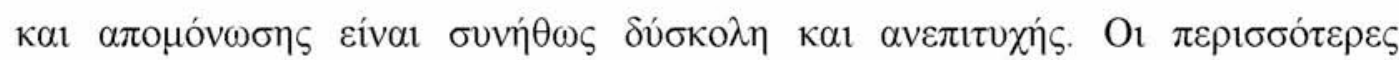

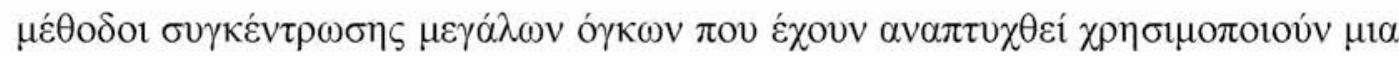

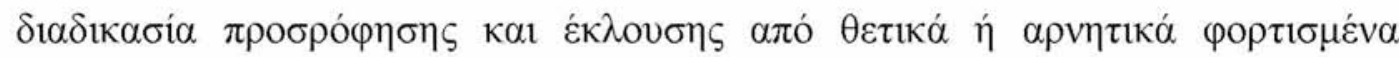
$\varphi \hat{i} \lambda \tau \rho \alpha$ (Crabtree et al., 1997; Katayama et al., 2002; Mocé-Llivina et al., 2002),

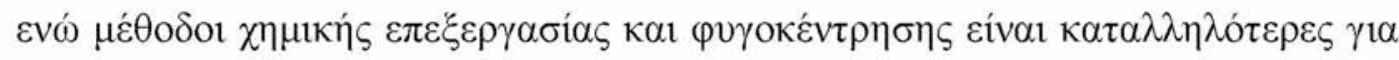

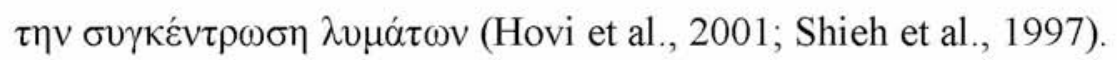

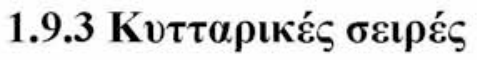

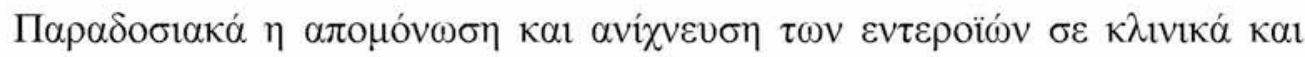

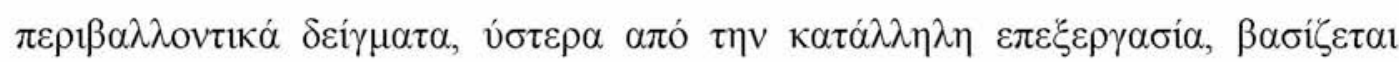




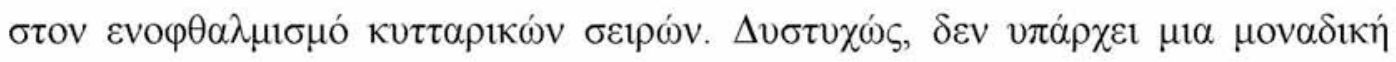

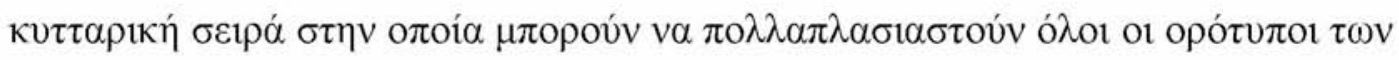

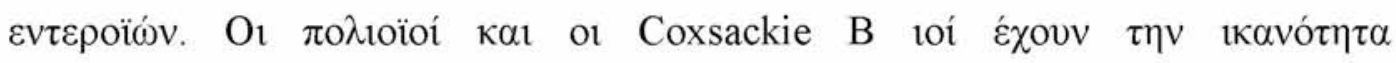

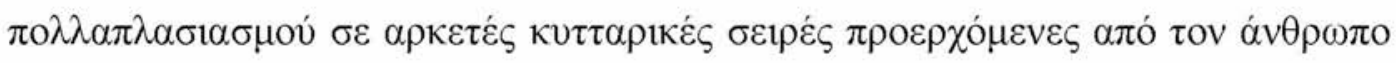

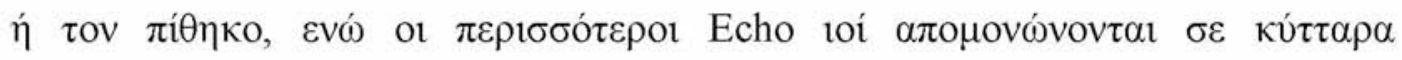

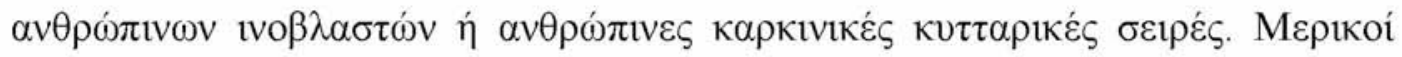

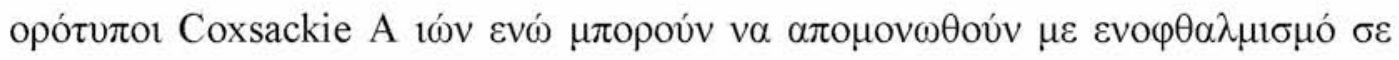

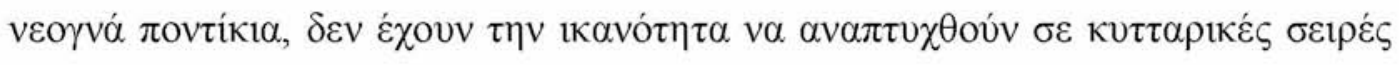

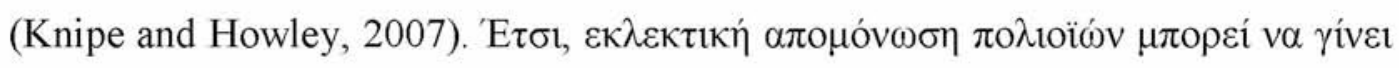

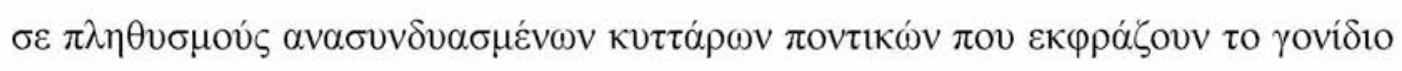

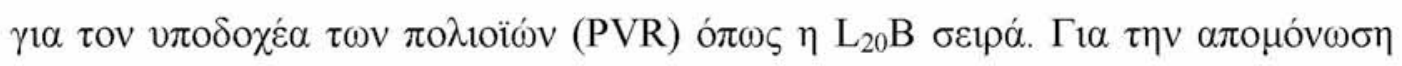

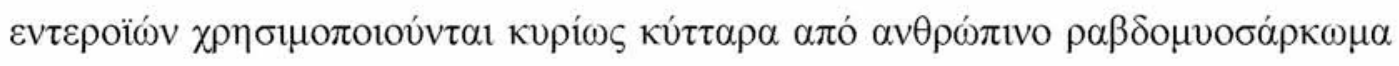

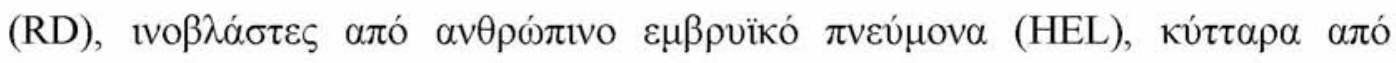

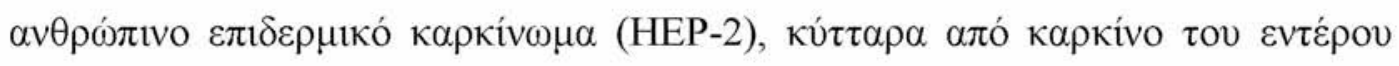

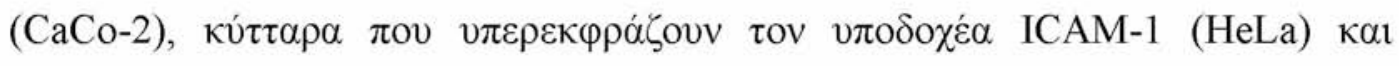

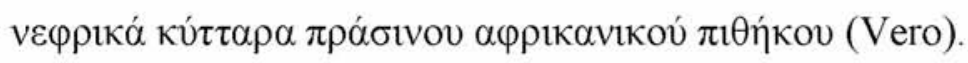

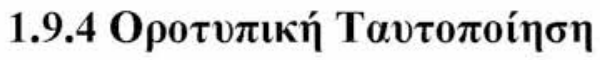

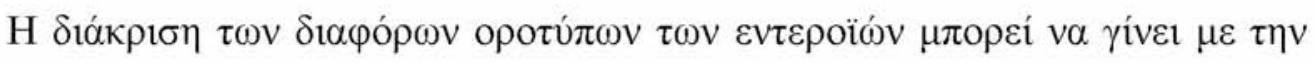

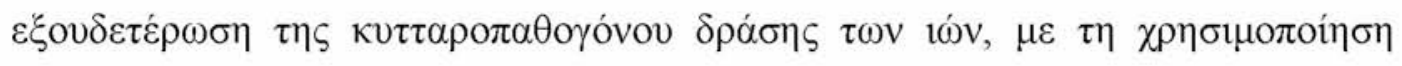

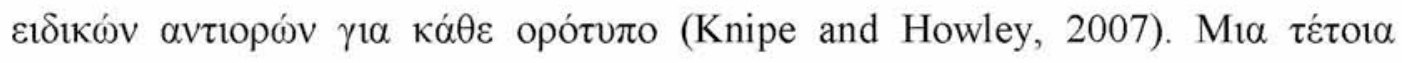

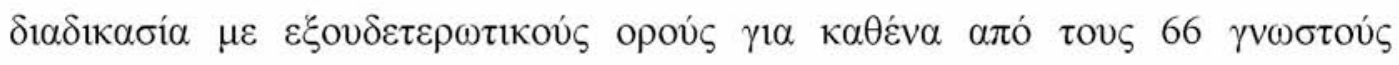

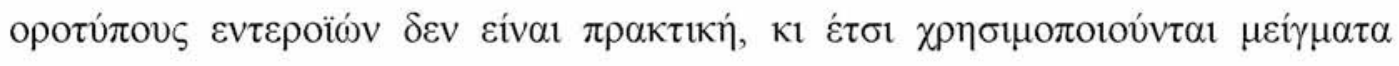

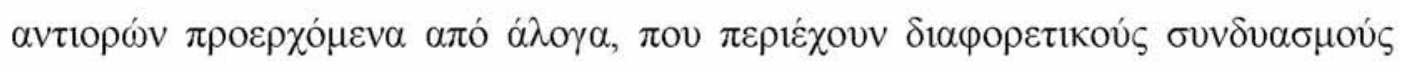

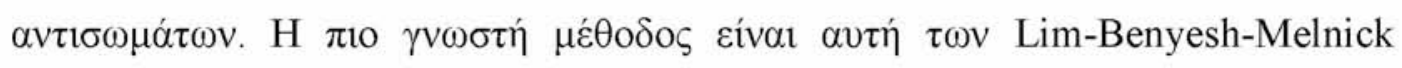

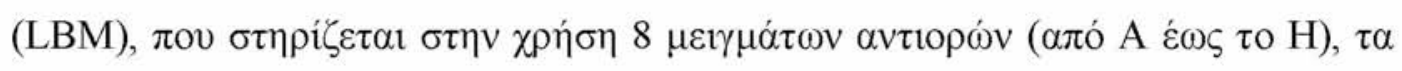

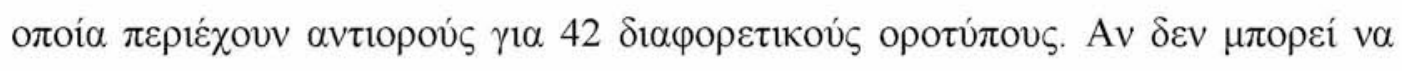

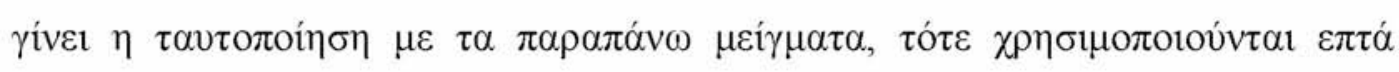

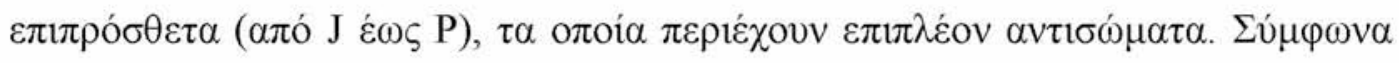

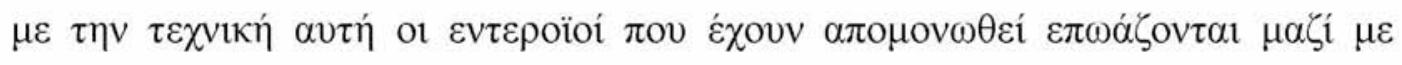

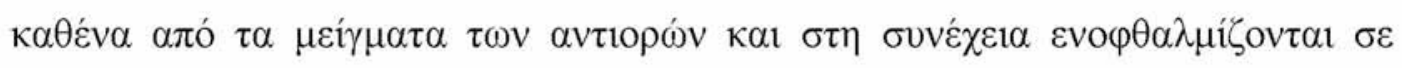

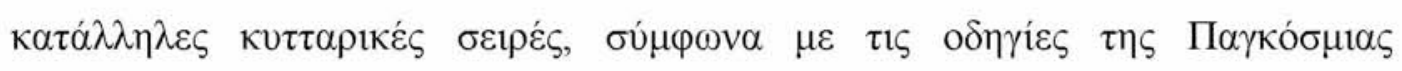

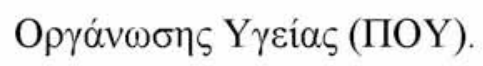




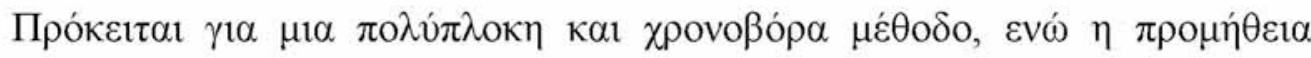

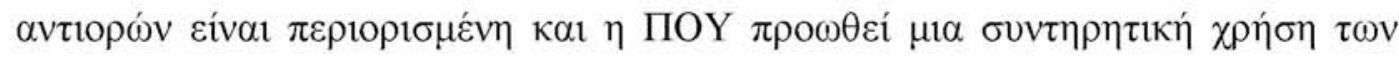

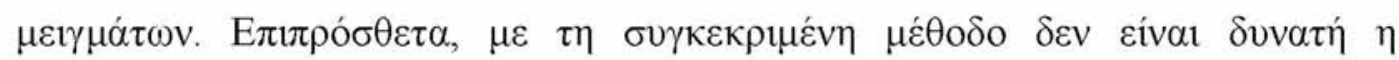

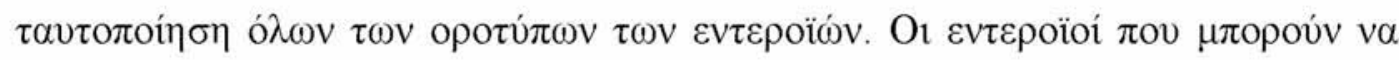

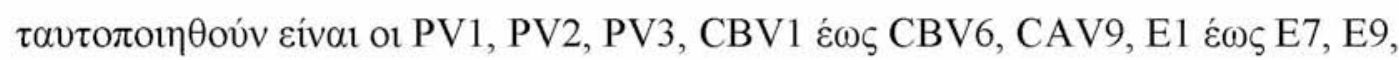

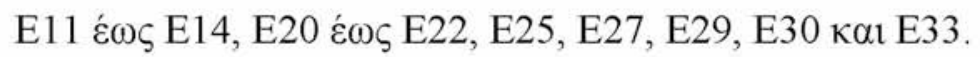

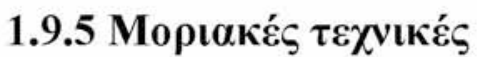

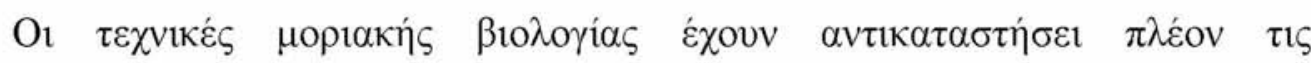

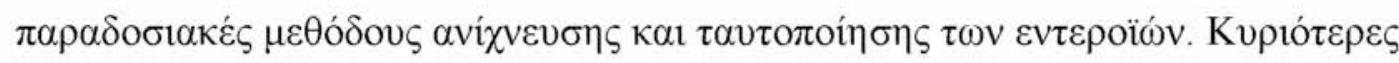

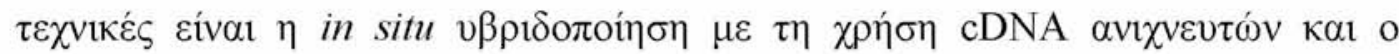

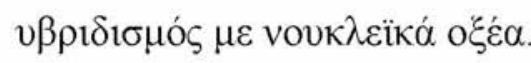

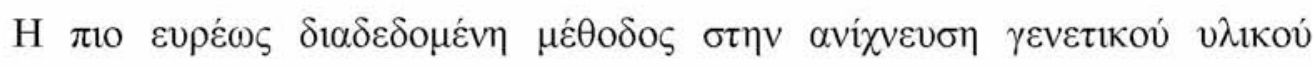

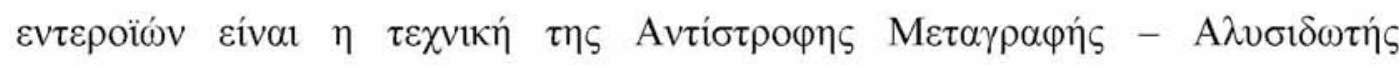

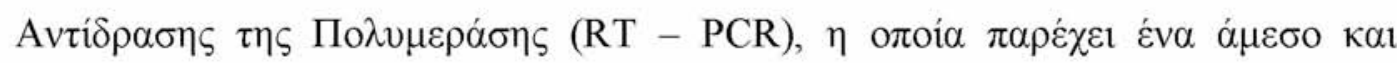

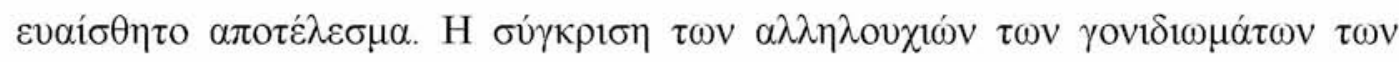

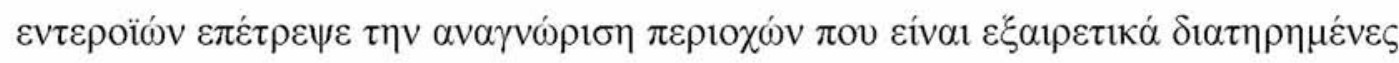

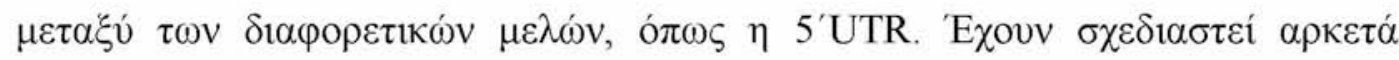

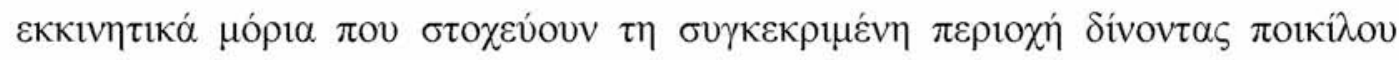

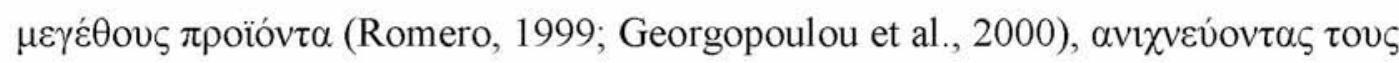

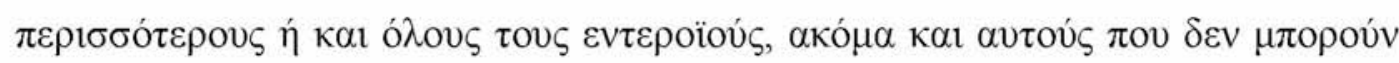

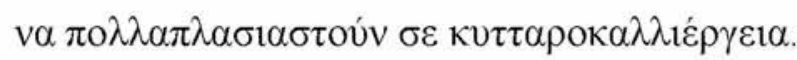

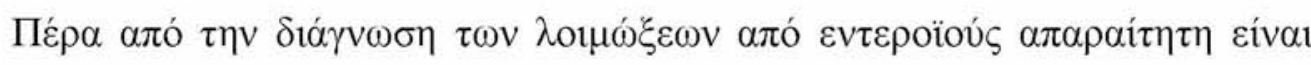

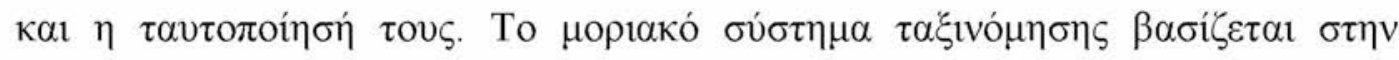

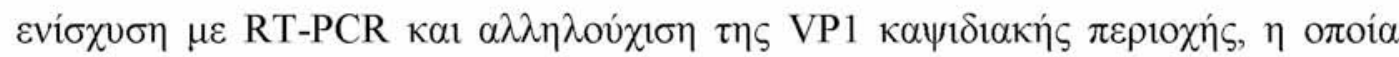

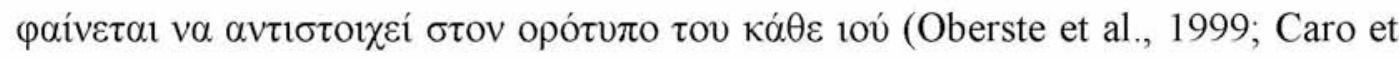

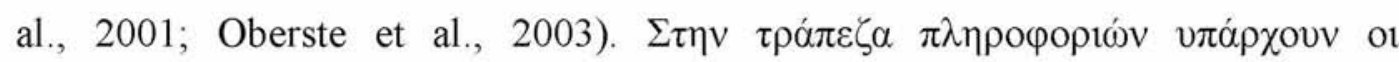

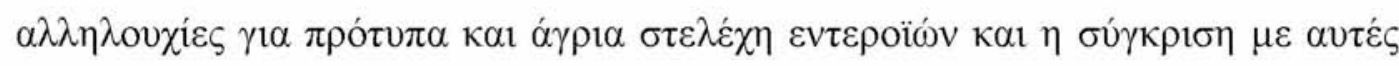

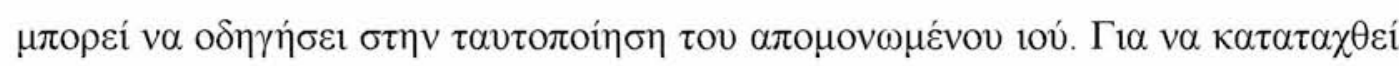

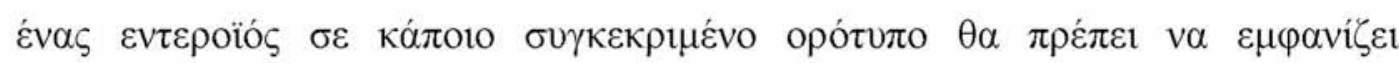

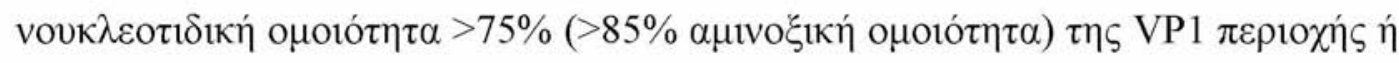

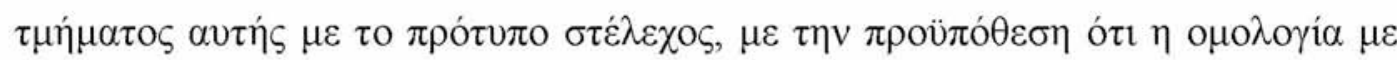




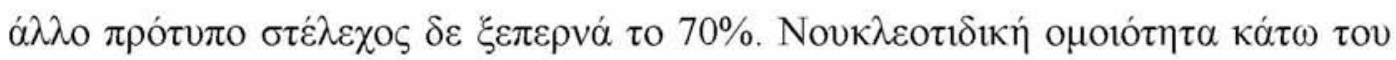

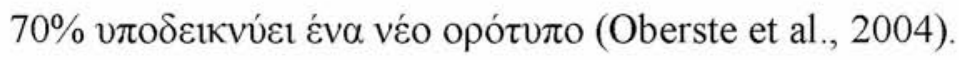

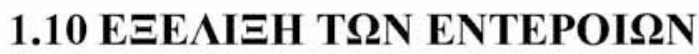

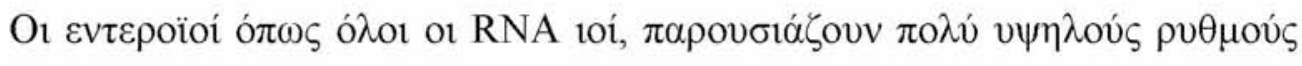

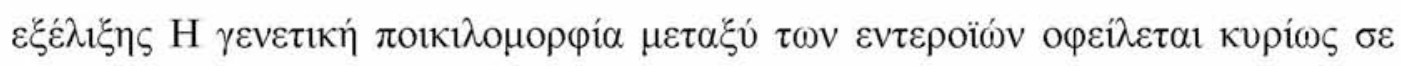

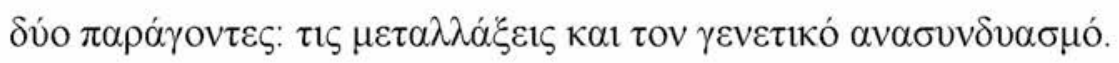

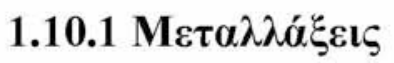

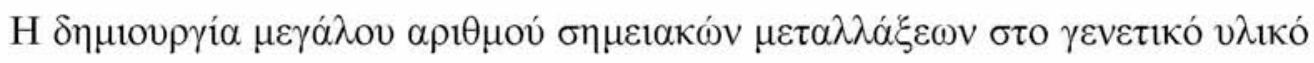

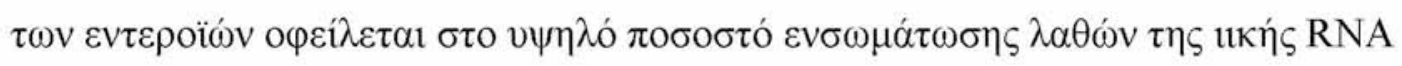

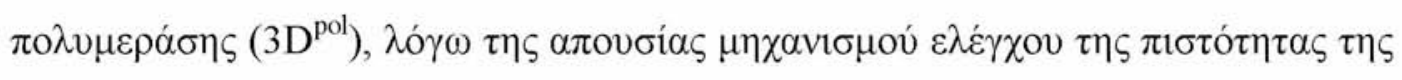

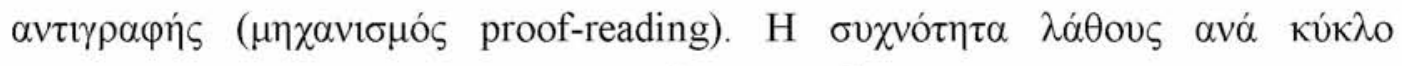

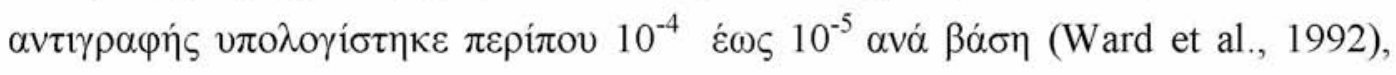

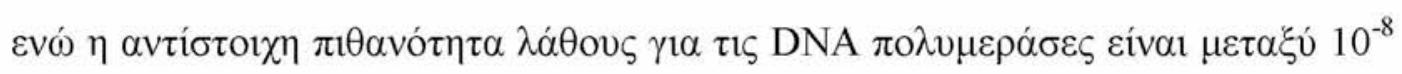

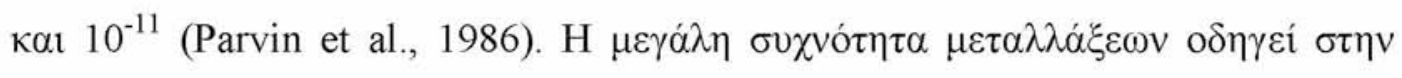

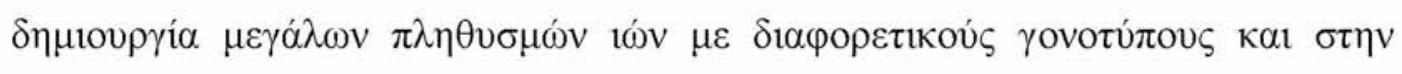

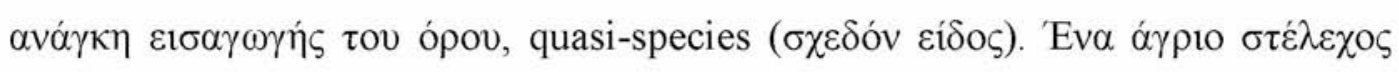

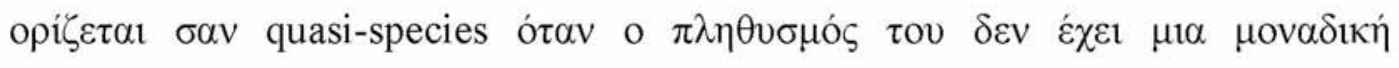

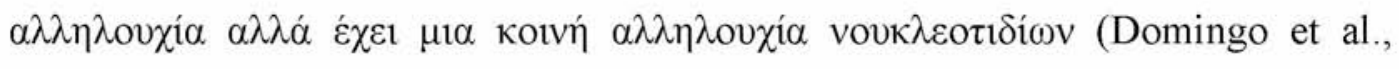

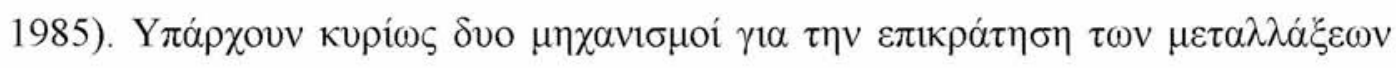

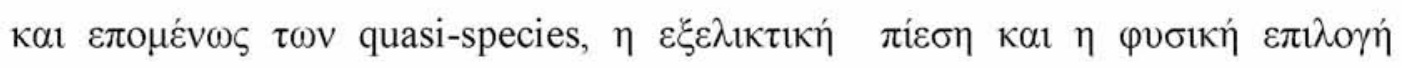

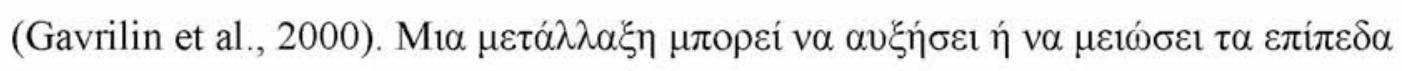

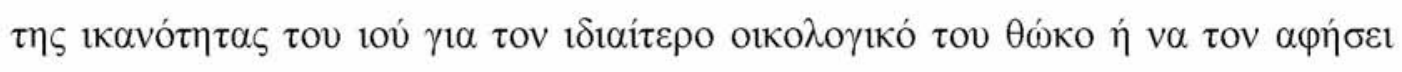

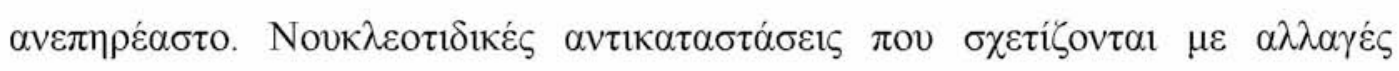

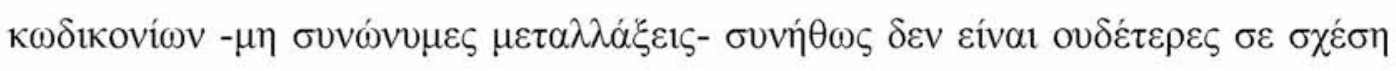

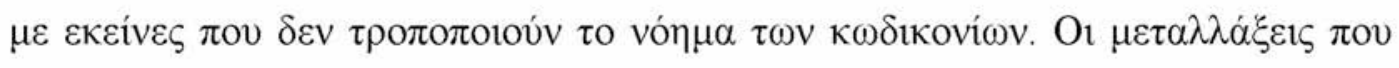

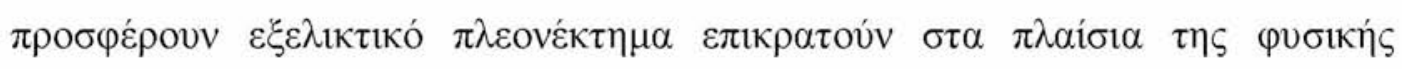
$\varepsilon \pi i \lambda o \gamma \dot{s}$.

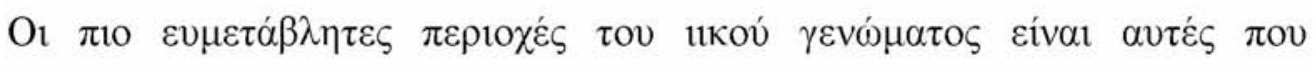

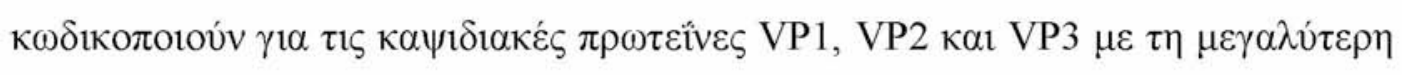

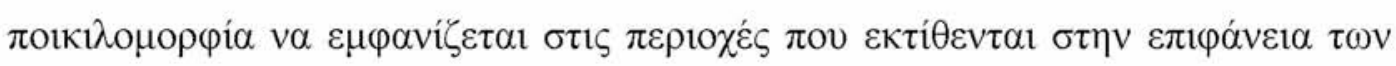

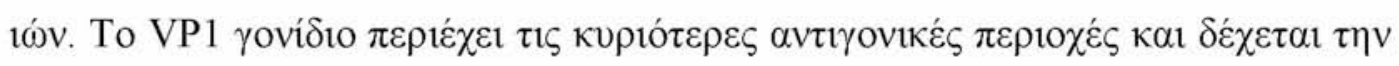




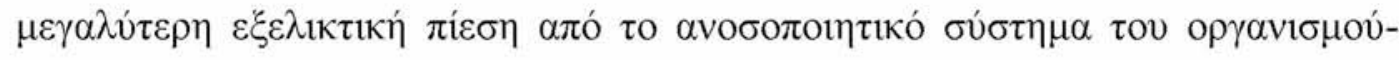

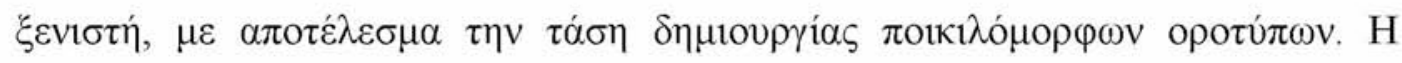

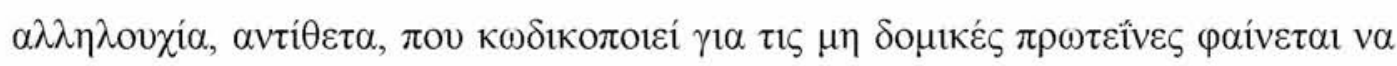

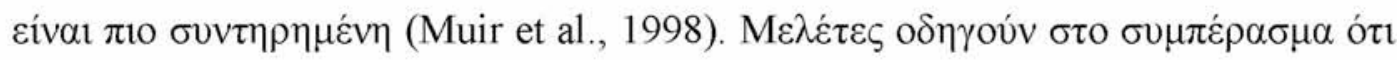

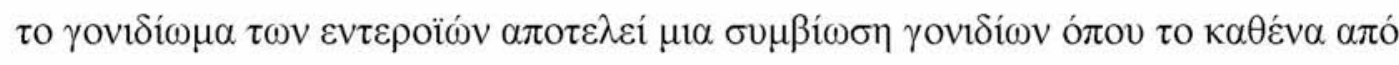

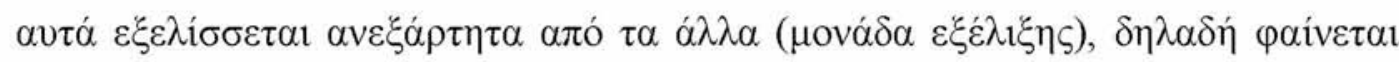

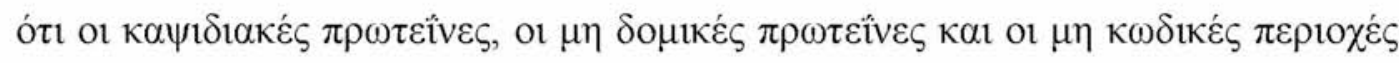

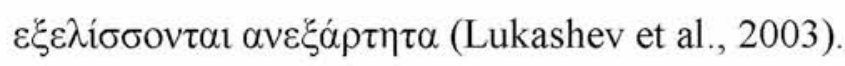

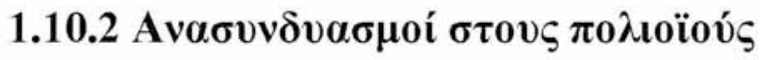

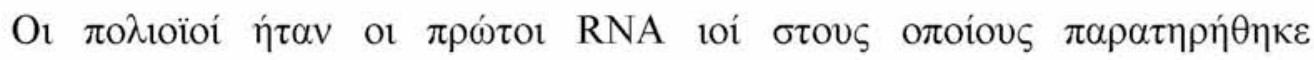

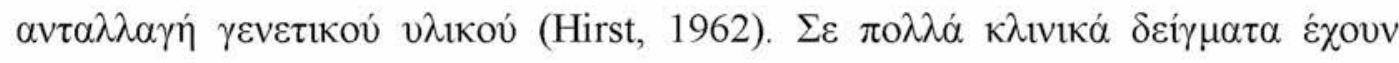

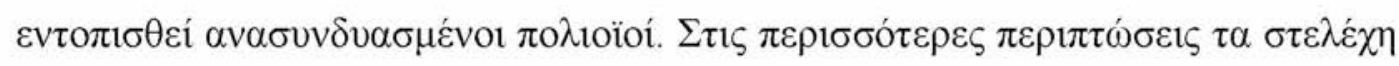

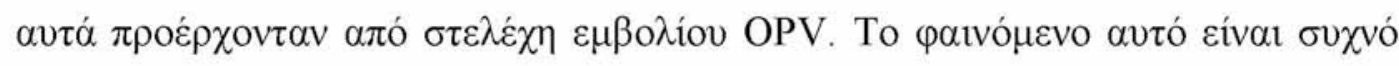

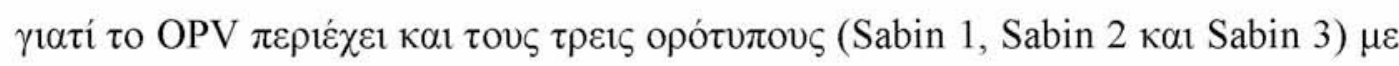

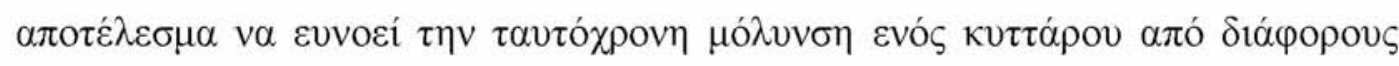

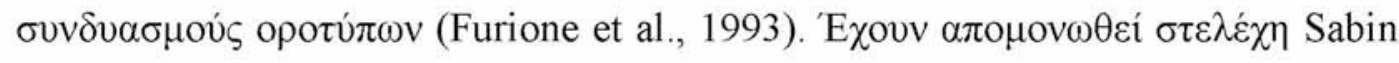

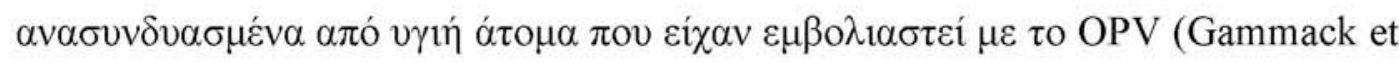

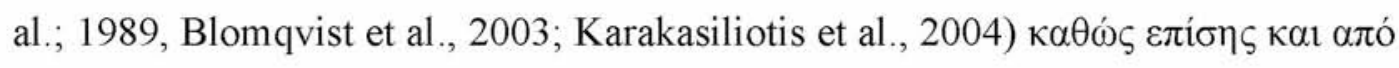

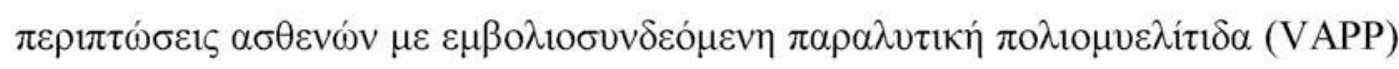

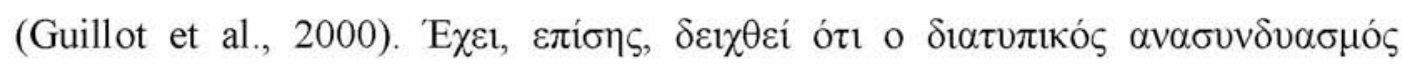

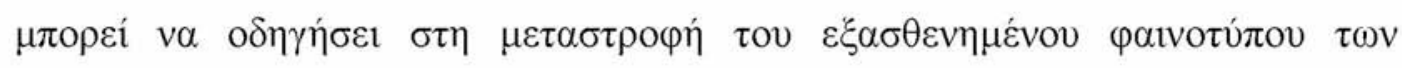

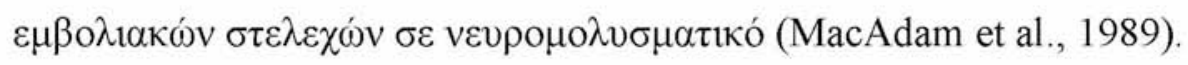

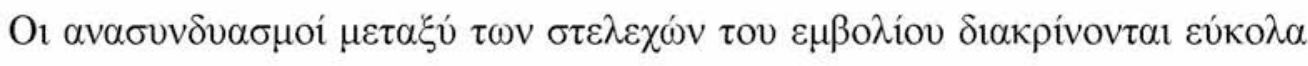

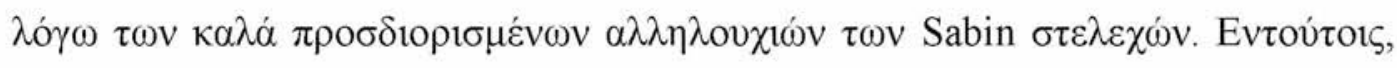

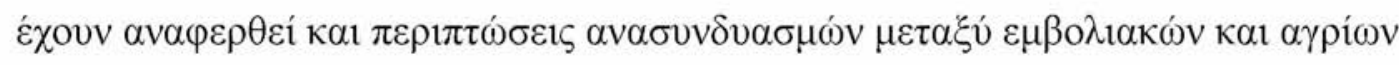

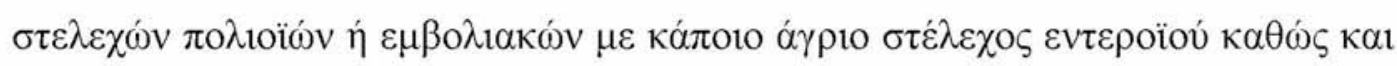

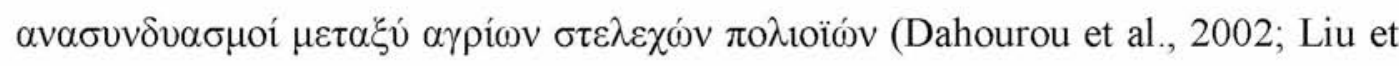

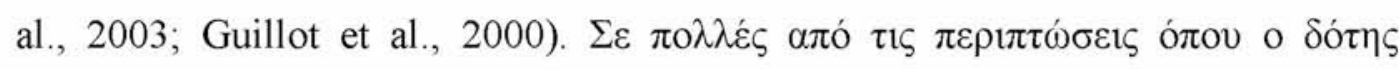

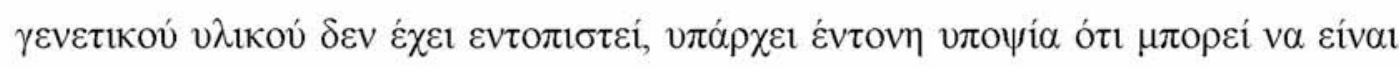

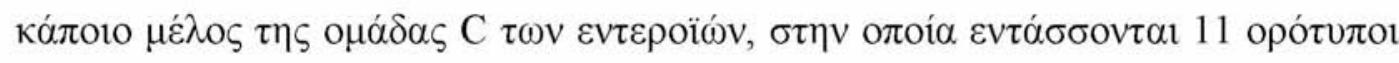
Coxsackie A (Liu et al., 2003; Guillot et al., 2000). 


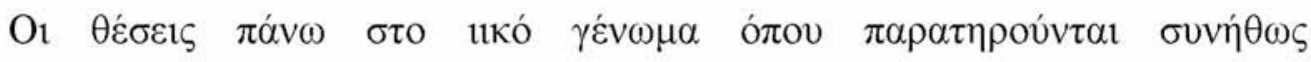

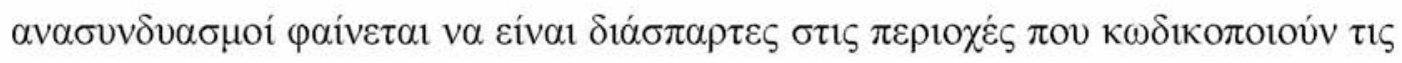

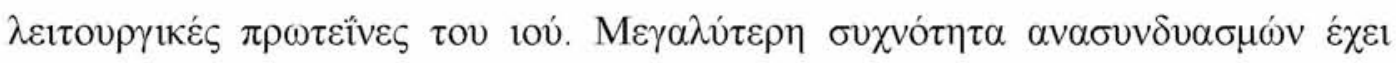

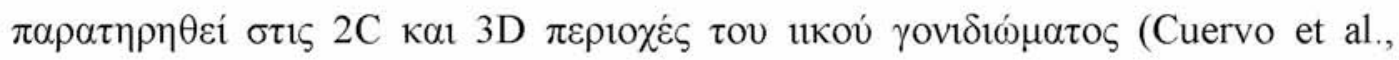
2001; Georgopoulou and Markoulatos, 2001; Paximadi et al., 2006; Paximadi et

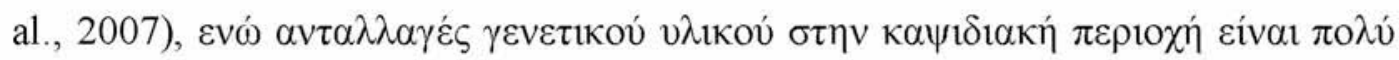

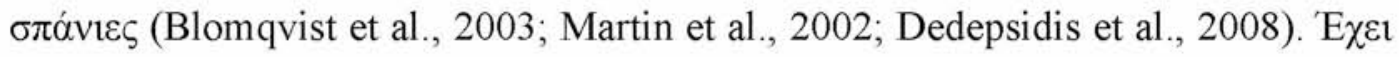

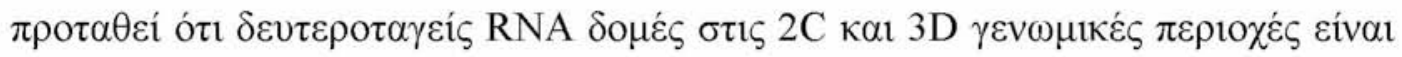

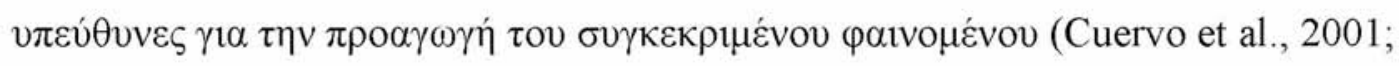

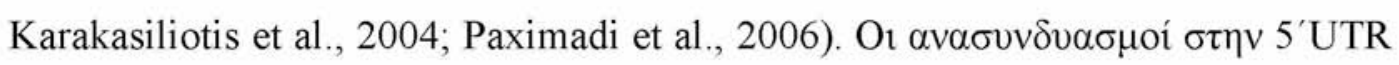

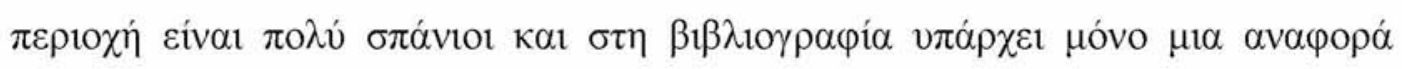

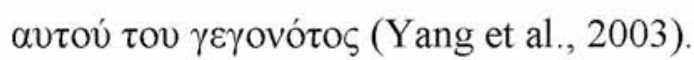

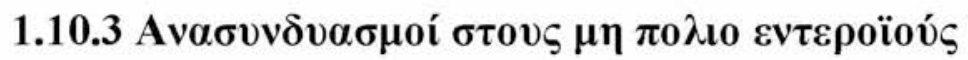

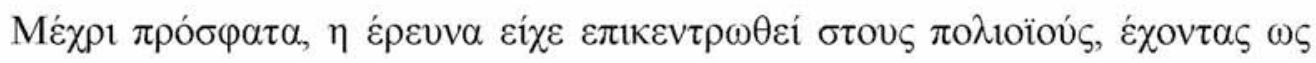

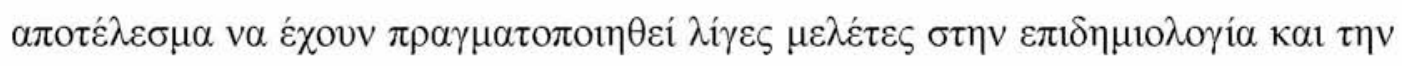

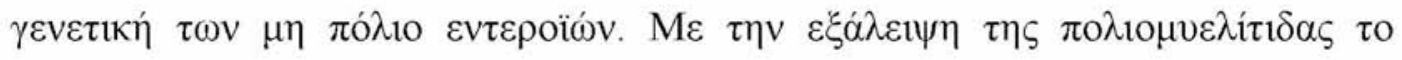

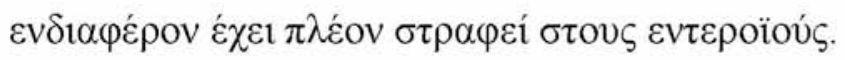

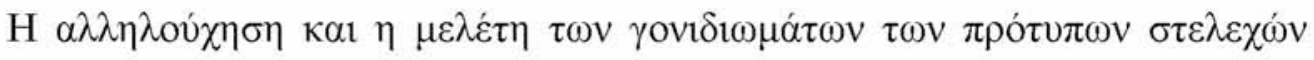

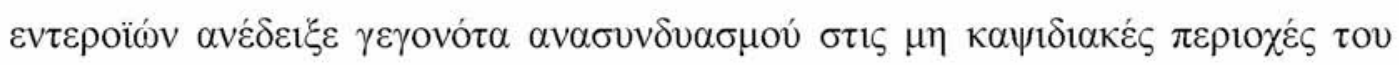

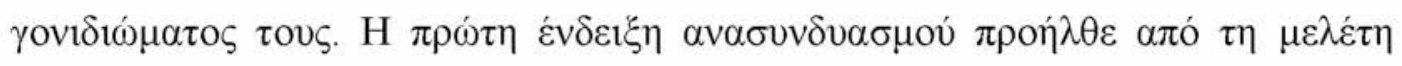

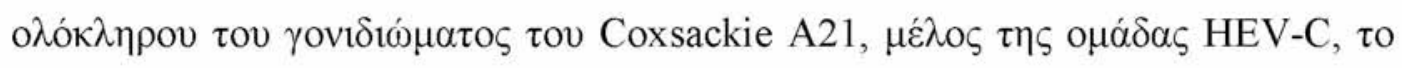

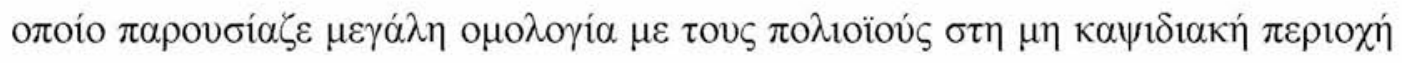

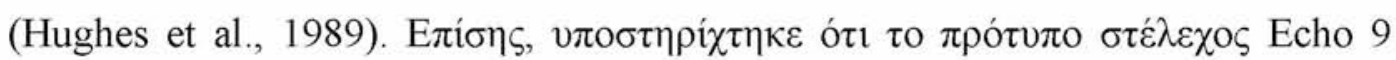

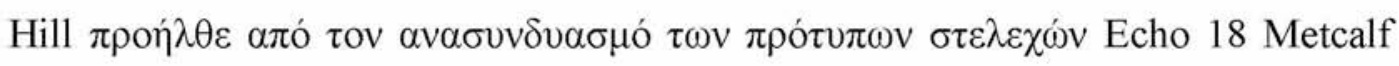

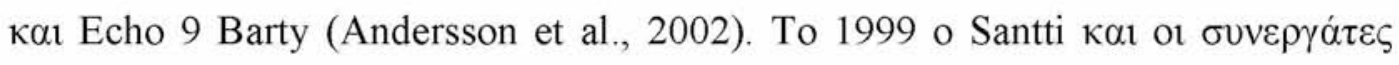

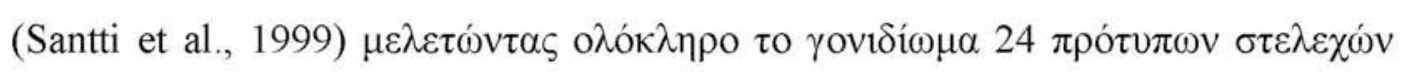

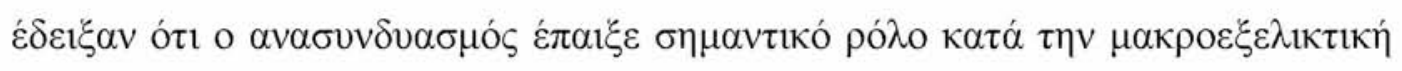

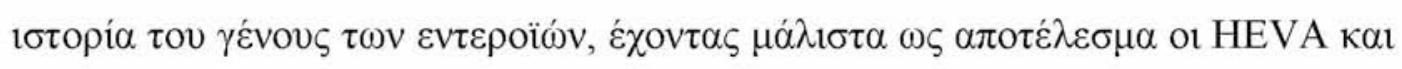

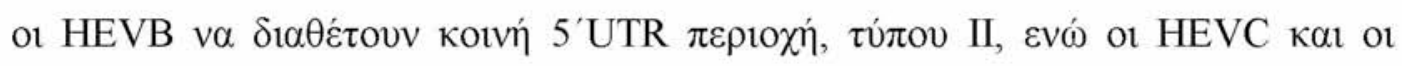

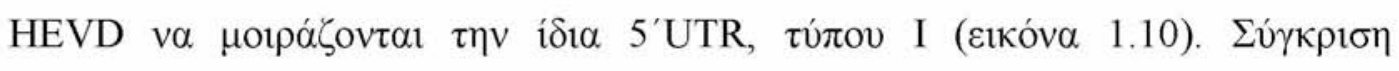

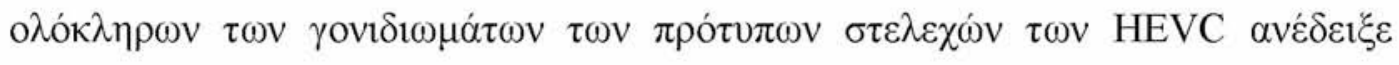

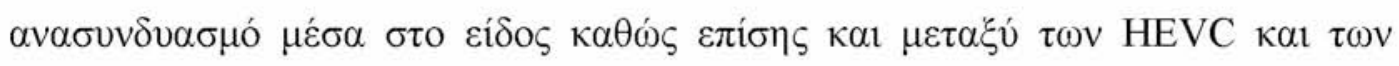




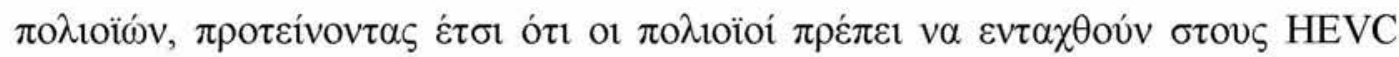

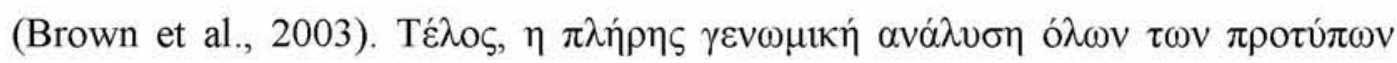

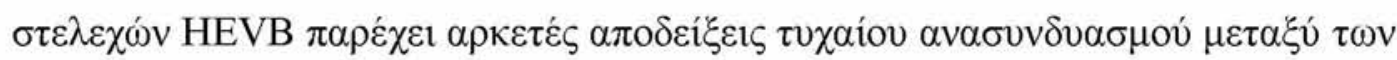

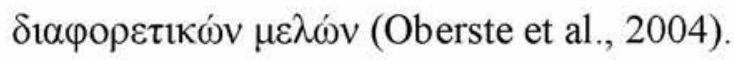

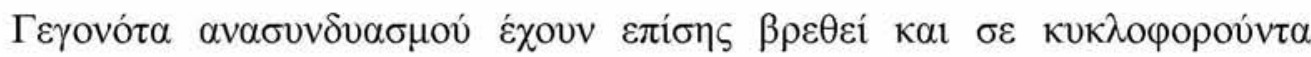

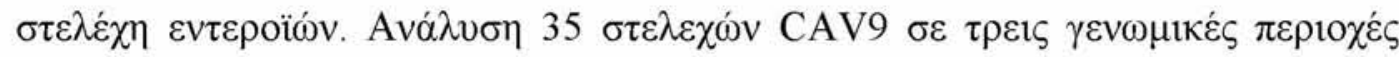

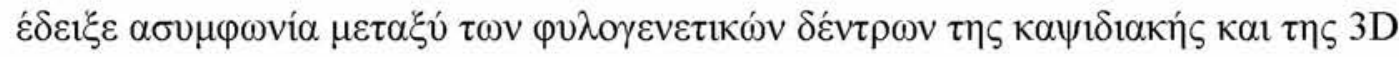

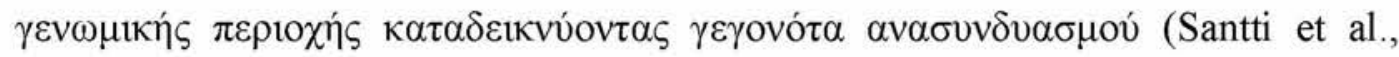

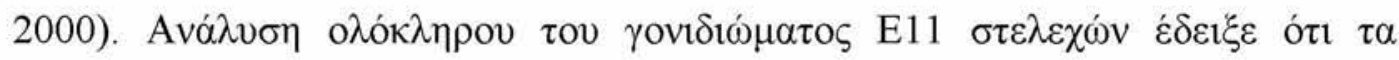

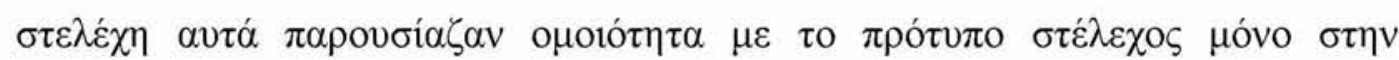

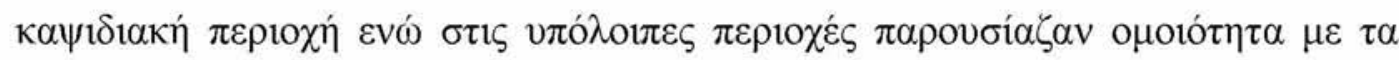

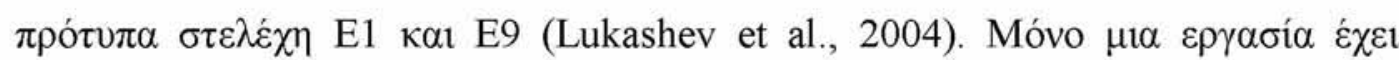

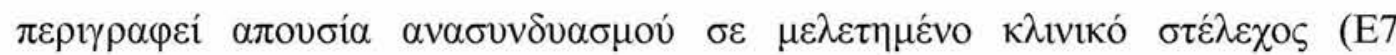

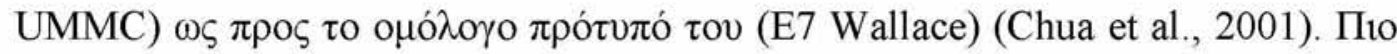

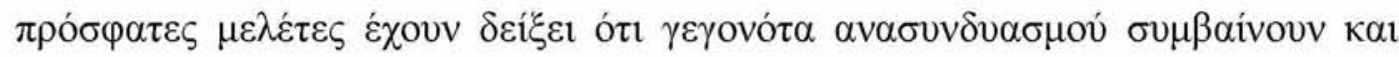

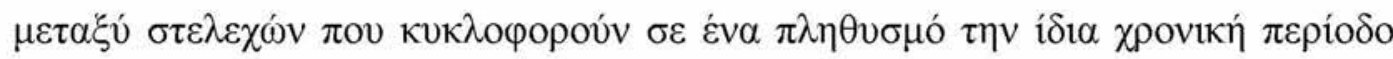
(Bolanaki et al., 2007; Kottaridi et al., 2007; Mirand et al., 2007).

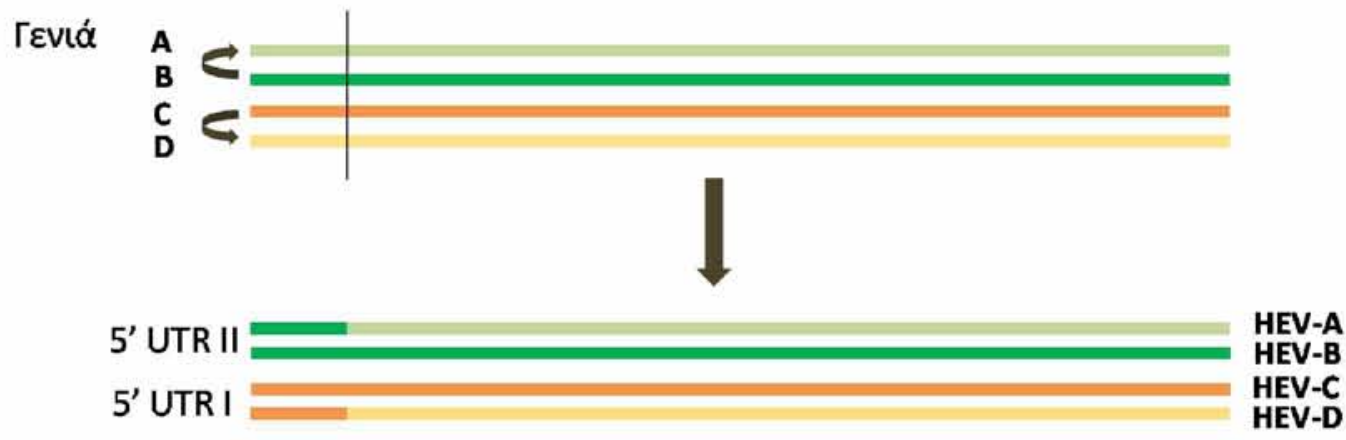

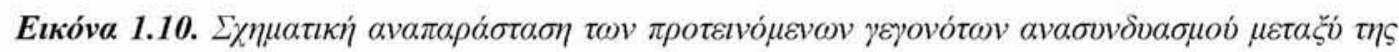

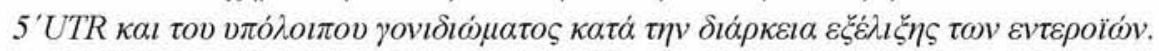

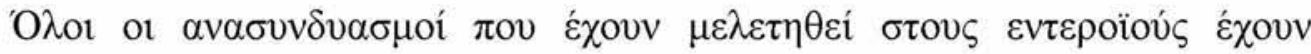

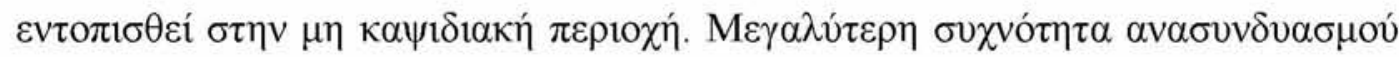

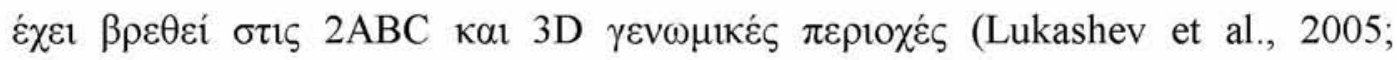

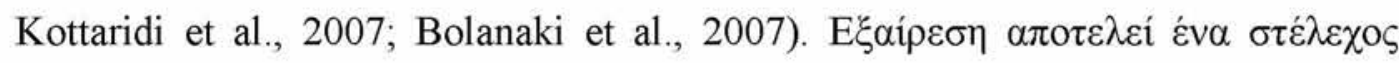

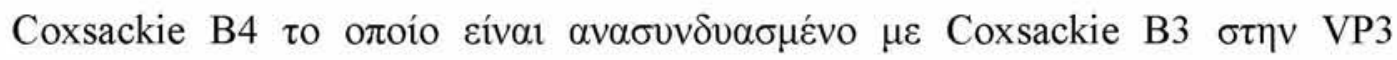

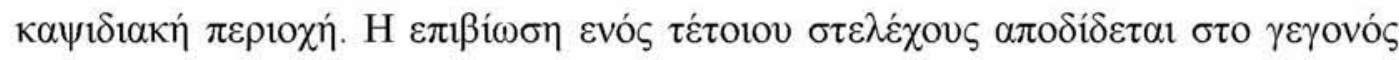




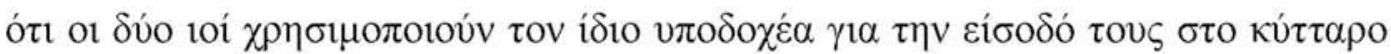
(Buslama et al., 2007).

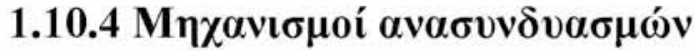

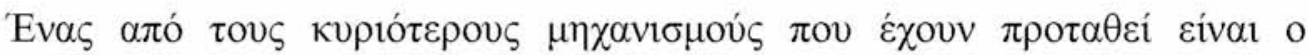
$\mu \eta \chi \alpha v i \sigma \mu o ́ \varsigma \alpha \lambda \lambda \alpha \gamma \eta \dot{\zeta} \mu \dot{\tau} \tau \rho \alpha \varsigma$ (template switch mechanism) (Kirkegaard and

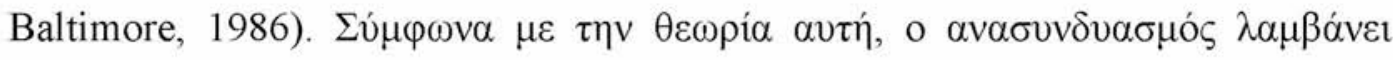

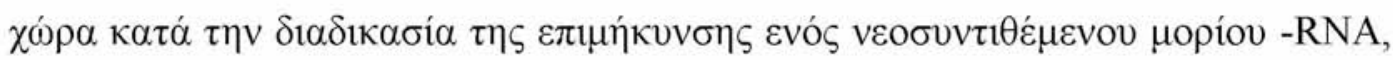

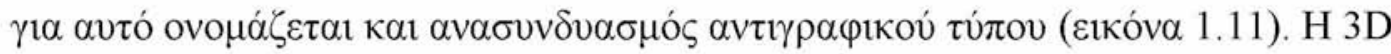

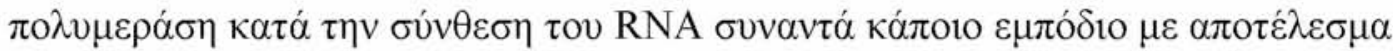

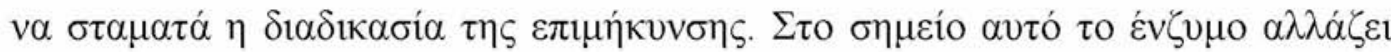

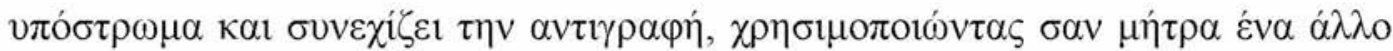

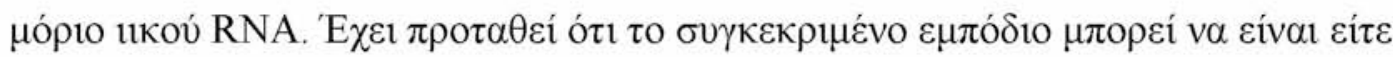

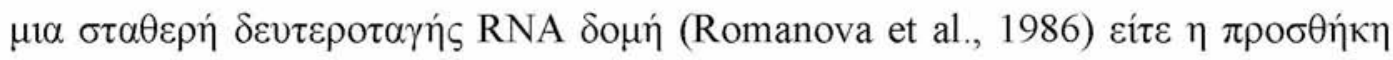

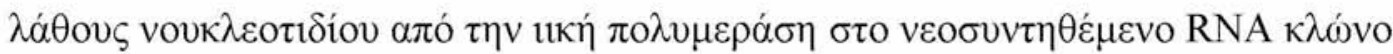
(Pilipenko et al., 1995, Paximadi et al., 2007, Paximadi et al., 2008).
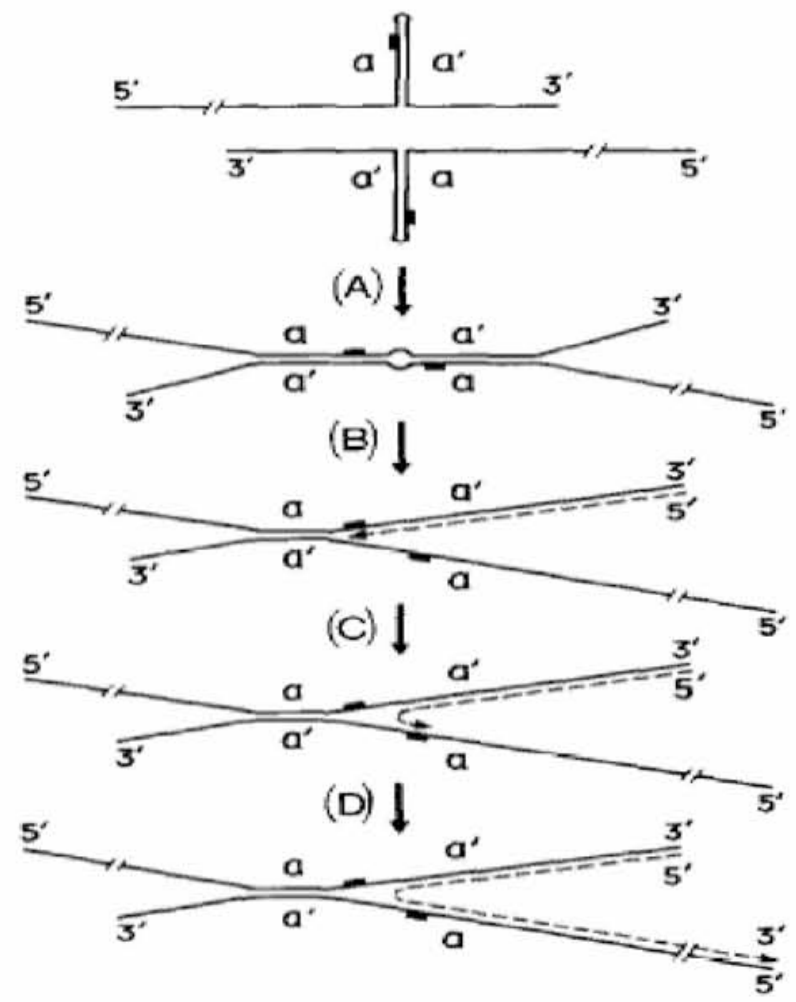

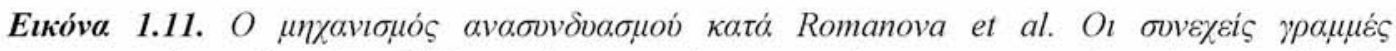

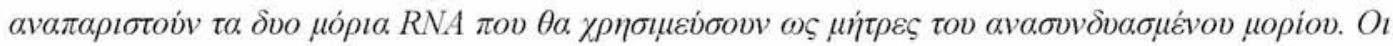

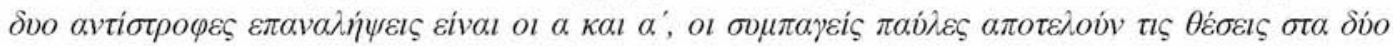

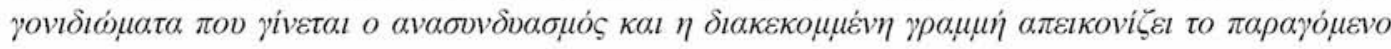

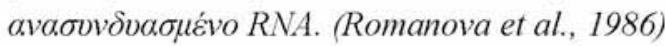




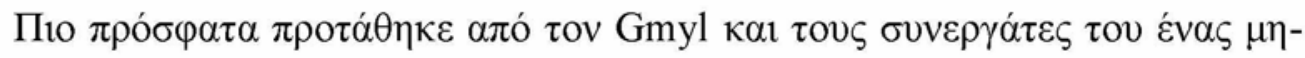

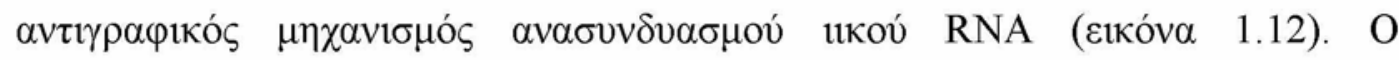

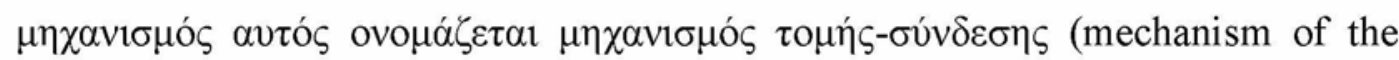

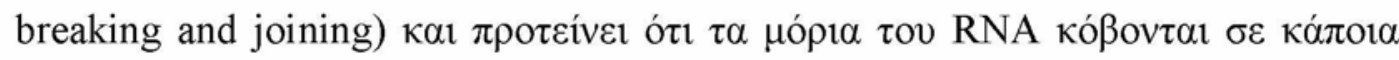

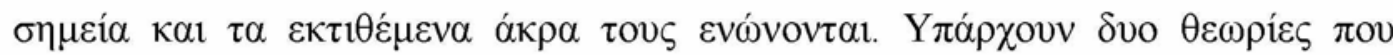

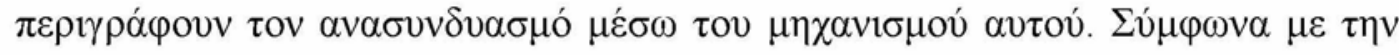

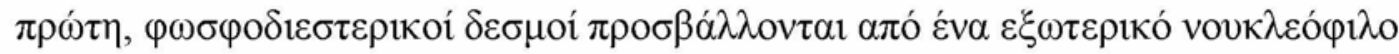

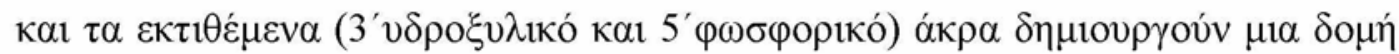

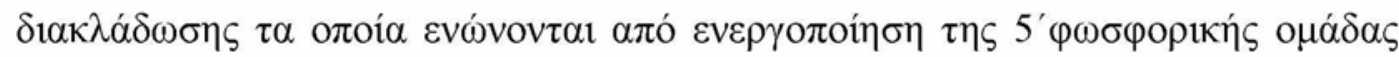
(Chetverin, 1999). H

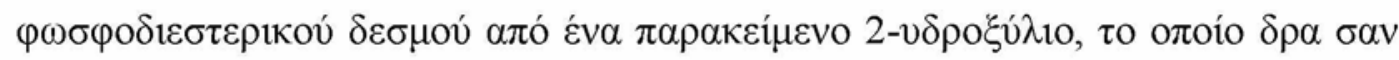

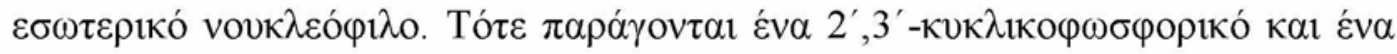

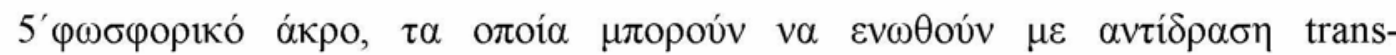

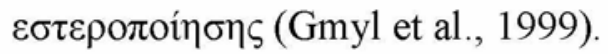

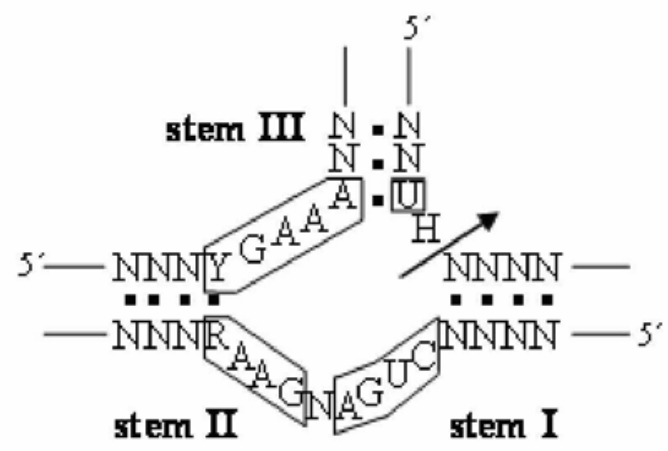

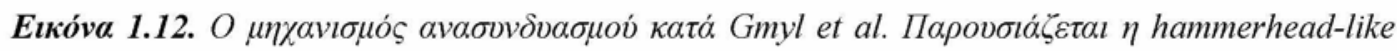

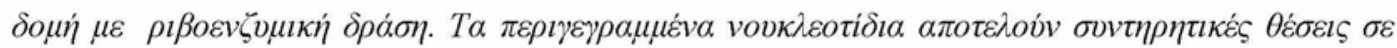

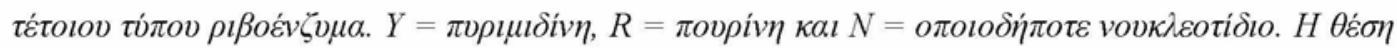

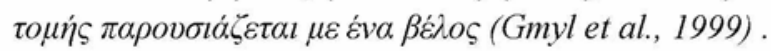




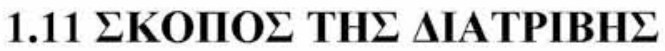

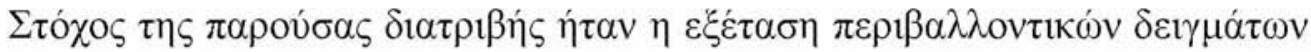

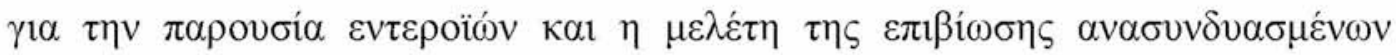

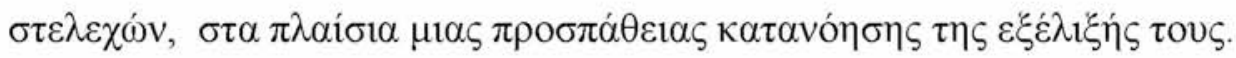

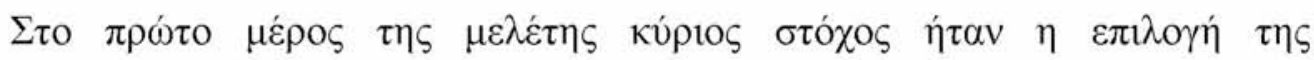

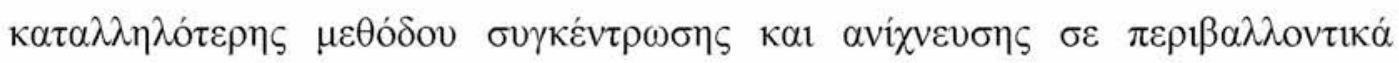

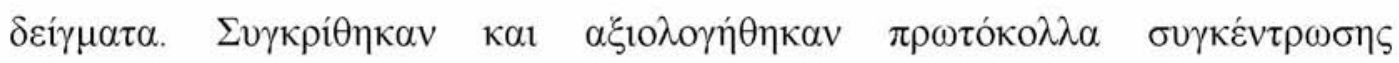

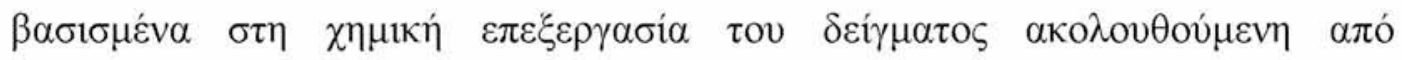

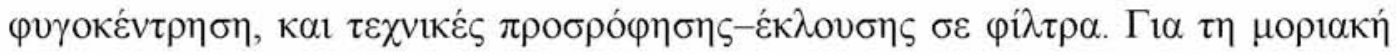

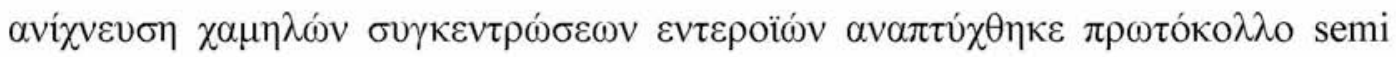

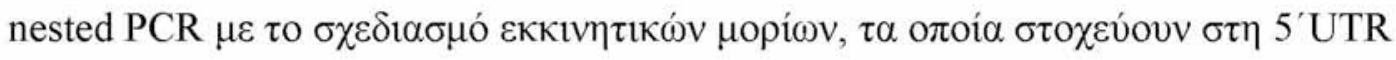

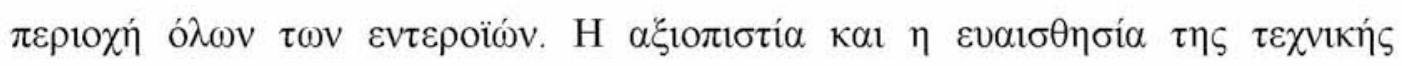

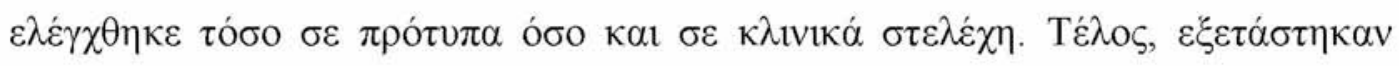

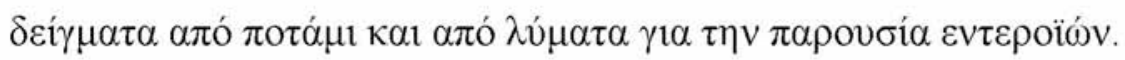

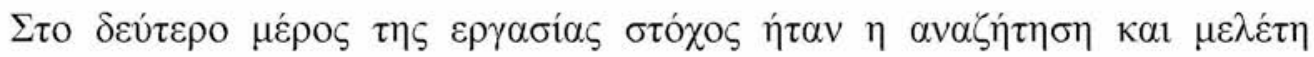

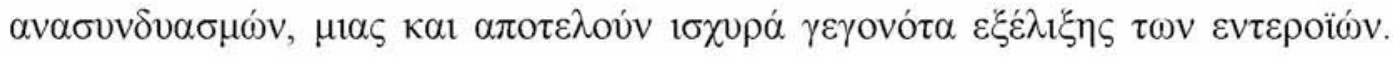

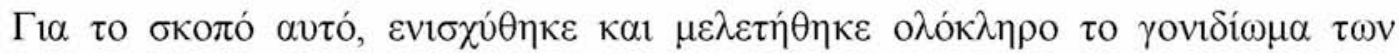

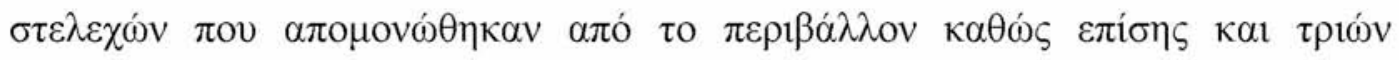

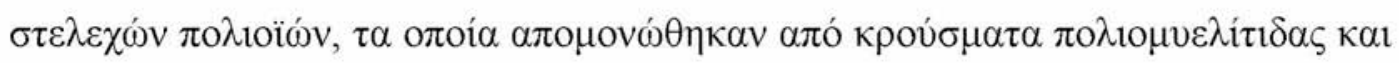

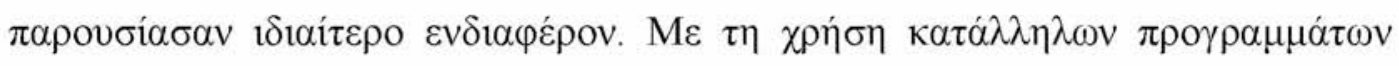

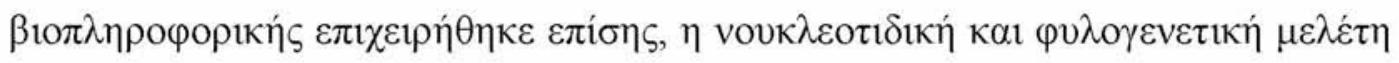

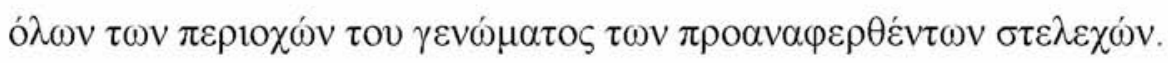




\section{КЕФАААIO 2}

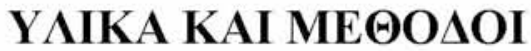

\section{1 ПIEIPAMATIKA $\Sigma$ TEAEXH}

\subsection{1 Прó $\tau v \pi \alpha \sigma \tau \varepsilon \lambda \dot{\chi} \chi \eta$}

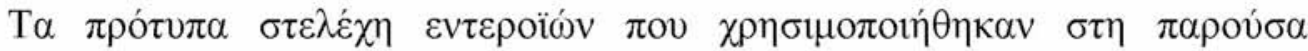

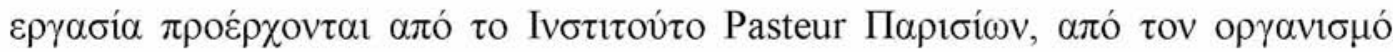

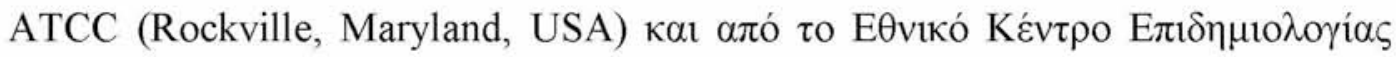

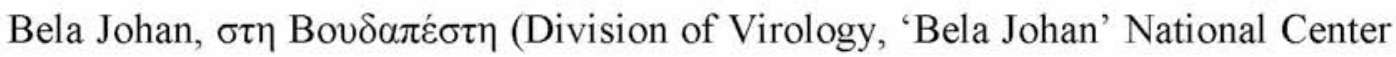

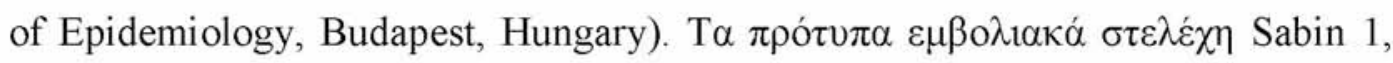

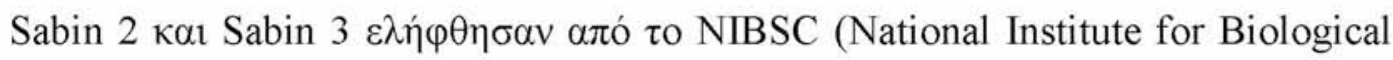

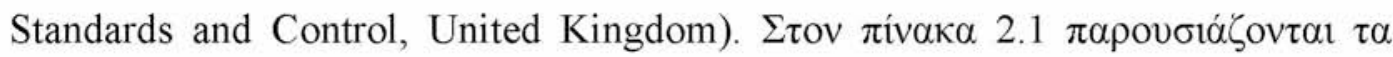

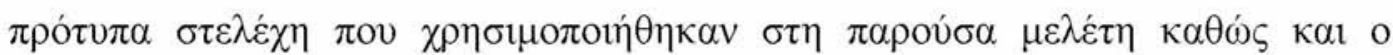

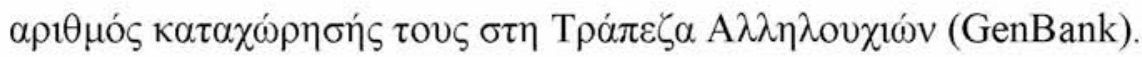

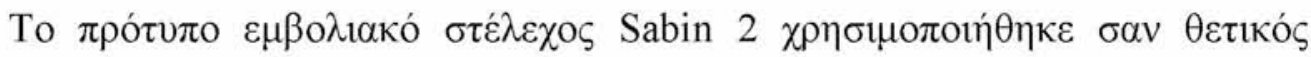

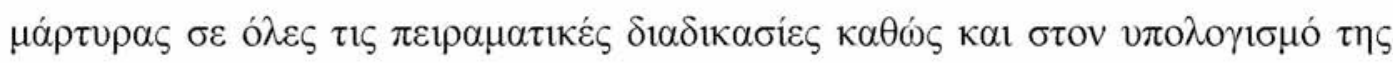

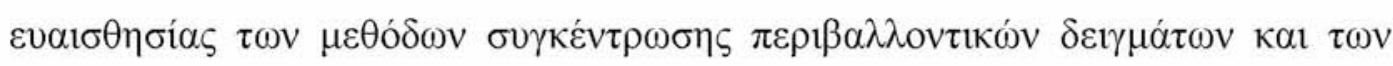

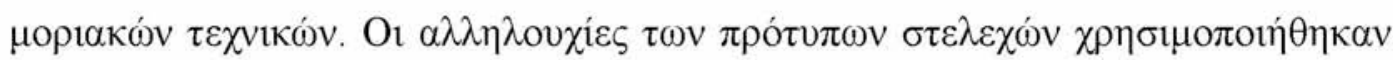

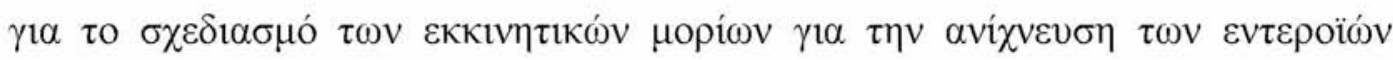

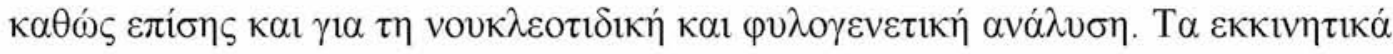

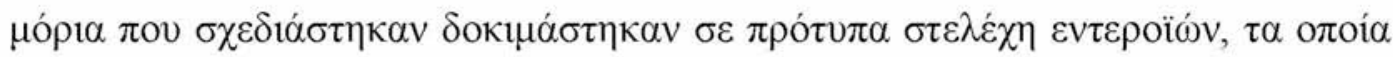

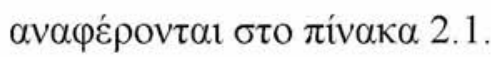




\begin{tabular}{|c|c|c|c|c|c|}
\hline 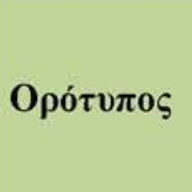 & $\Sigma \tau \varepsilon \dot{\lambda} \varepsilon \chi 0 \varsigma$ & 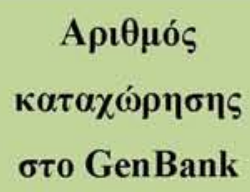 & 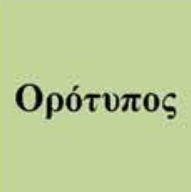 & $\Sigma \tau \varepsilon \dot{\lambda} \varepsilon \chi 0 \varsigma$ & 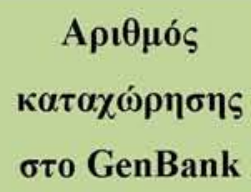 \\
\hline CAV1* & Tompkins & AF499635 & Echo12* & Travis & X79047 \\
\hline CAV2* & Fleetwood & AY421760 & Echo13* & Del Carmen & AY302539 \\
\hline CAV3* & Olson & AY421761 & Echo14* & Tow & AY302540 \\
\hline CAV4* & High Point & AY421762 & Echo15* & Ch 96-51 & AY302541 \\
\hline CAV5 & Swartz & AY421763 & Echo16* & Harrington & AY302542 \\
\hline CAV6* & Gdula & AY421764 & Echo17** & CHHE-29 & AY302543 \\
\hline CAV7* & Parker & AY421765 & Echo18* & Metcalf & AF317694 \\
\hline CAV8* & Donovan & AY421766 & Echo19 & Burke & AY302544 \\
\hline CAV9 & Griggs & D00627 & Echo20* & JV-1 & AY302546 \\
\hline CAV10* & Kowalik & AY421767 & Echo21* & Farina & AY302547 \\
\hline CAV11* & Belgium 1 & AF499636 & Echo24* & DeCamp & AY302548 \\
\hline CAV12* & Texas-12 & AY421768 & Echo25 & $\mathrm{JV}-4$ & AY302549 \\
\hline CAV13* & Flores & AF303040 & Echo26* & Coronel & AY302550 \\
\hline CAV14* & G-14 & AF329687 & Echo27* & Bacon & AY302551 \\
\hline CAV15* & G-9 & AY028217 & Echo29* & JV-10 & AY302552 \\
\hline CAV16 & G-10 & U05876 & Echo30 & Bastianni & AF162711 \\
\hline CAV17 & G-12 & AF499639 & Echo31* & Caldwell & AY302554 \\
\hline CAV18* & G-13 & AF499640 & Echo32* & PR-10 & AY302555 \\
\hline CAV19 & Dohi & AF499641 & Echo33* & Toluca-3 & AY302556 \\
\hline CAV20* & IH-35 & AF499642 & EV68 & Fermon & AY426531 \\
\hline CAV21* & Coe & D00538 & EV69 & Toluca-1 & AY302560 \\
\hline CAV22 & Chulman & AF499643 & EV70* & $\mathrm{J} 670 / 71$ & D00820 \\
\hline CAV24 & EH 24/70 & D90457 & EV71 & Tainan/5746/98 & AF304457 \\
\hline CBV1* & Japan & M16560 & EV73 & CA55-1988 & AF241359 \\
\hline CBV2* & Ohio-1 & AF081485 & EV74 & CA75-10213 & AY556057 \\
\hline CBV3 & Nancy & M88483 & EV75 & OK85-10362 & AY556070 \\
\hline CBV4 & JVB & X05690 & EV77 & TX97-10394 & AY843302 \\
\hline CBV5* & Faulkner & AF114383 & EV79 & CA82-10385 & AY843309 \\
\hline CBV6* & Schmitt & AF039205 & EV79 & CA79-10384 & AY843297 \\
\hline Polio 1 & Mahoney & V01148 & EV80 & CA67-10387 & AY843298 \\
\hline Polio 1* & Sabin & V01150 & EV81 & CA68-10389 & AY843299 \\
\hline Polio 2 & Lansing & M12197 & EV82 & CA64-10390 & AY843300 \\
\hline Polio 2* & Sabin & X00595 & EV83 & CA76-10392 & AY843301 \\
\hline Polio 3 & Leon & K01392 & EV84 & CIV2003-10603 & DQ902712 \\
\hline Polio 3* & Sabin & K00043 & EV85 & BAN00-10353 & AY843303 \\
\hline Echo1* & Farouk & AF029859 & EV86 & BAN00-10354 & AY843304 \\
\hline Echo2* & Cornelis & AY302545 & EV87 & BAN01-10396 & AY843305 \\
\hline Echo3* & Morrisey & AY302553 & EV88 & BAN01-10398 & AY843306 \\
\hline Echo4* & Pesacek & AY302557 & EV89 & BAN00-10359 & AY697459 \\
\hline Echo5 & Noyce & AF083069 & EV90 & BAN99-10399 & AY697460 \\
\hline Echo6 & D'Amori & AY302558 & EV91 & BAN00-10406 & AY697461 \\
\hline Echo7 & Wallace & AY036579 & EV97 & BAN99-10355 & AY843307 \\
\hline Echo9* & Barty & X92886 & EV100 & BAN2000-10500 & NC009887 \\
\hline Echo11* & Gregory & X80059 & EV101 & CIV03-10361 & AY843308 \\
\hline
\end{tabular}

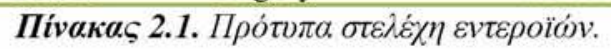

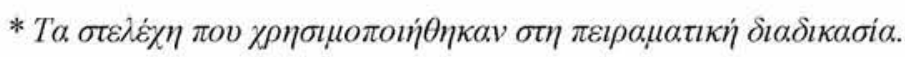




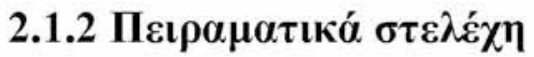

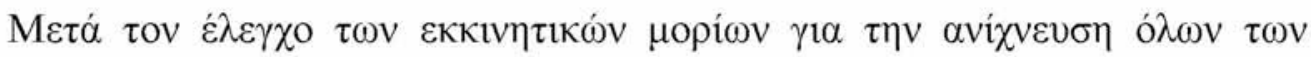

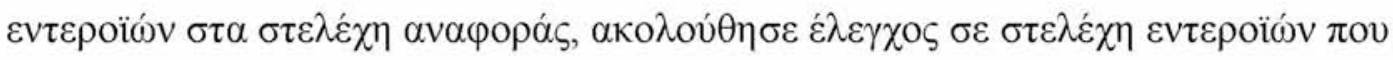
$\alpha \pi$ o

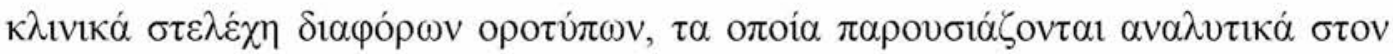

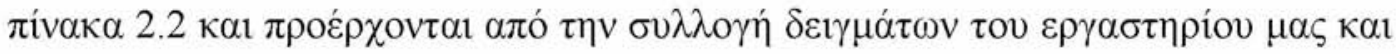
to National Reference Center for Enteroviruses Cantacuzino Institute, Bucharest,

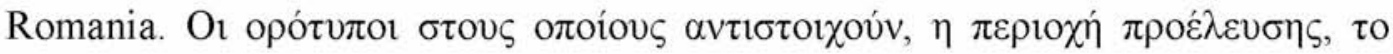

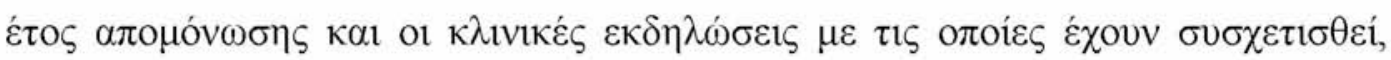

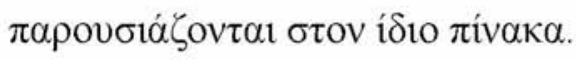

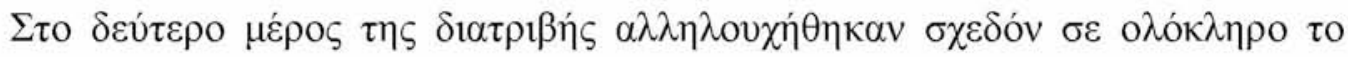

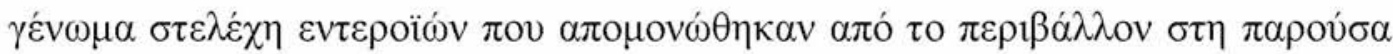

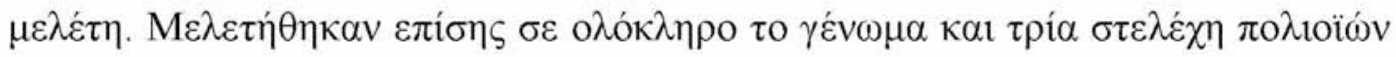

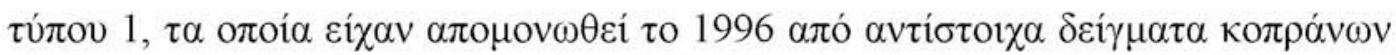

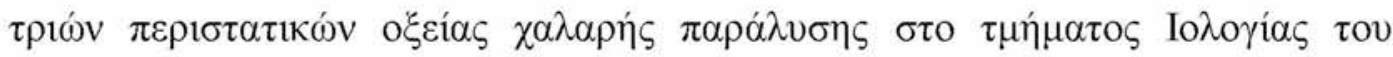

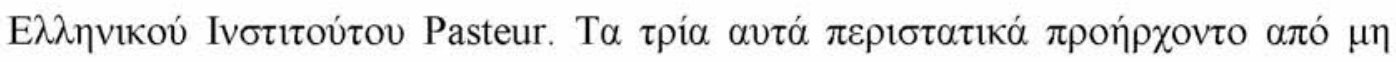

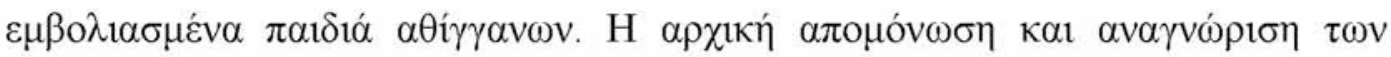

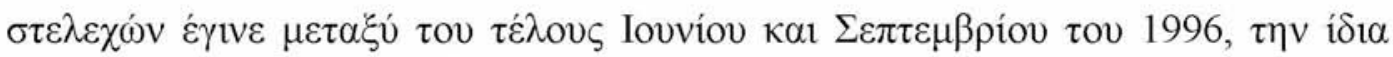

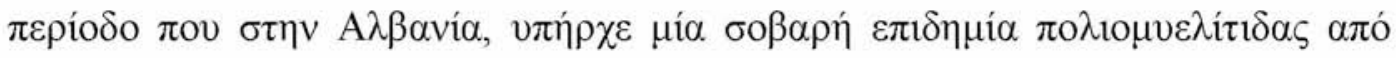
$\pi \circ \lambda$ ıӧо $\tau$

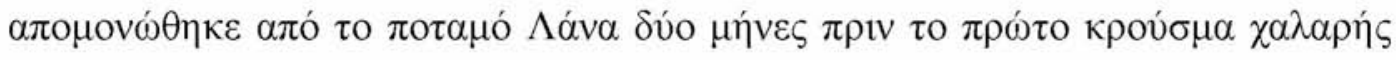

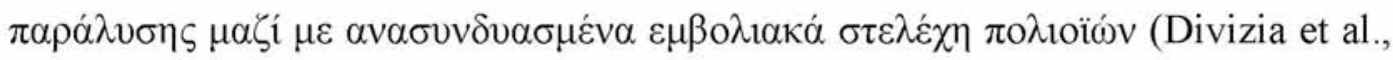

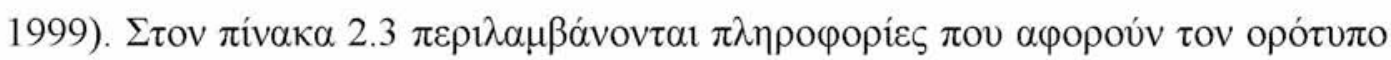

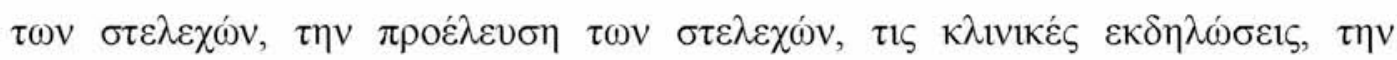

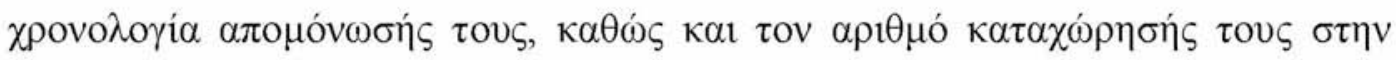

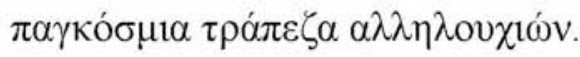




\begin{tabular}{|c|c|c|c|c|c|}
\hline 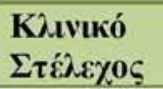 & 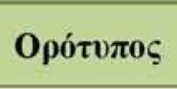 & $\begin{array}{c}\text { Гвюүрафчки́ } \\
\pi \rho 0 \hat{\lambda \varepsilon v \sigma \eta ~}\end{array}$ & 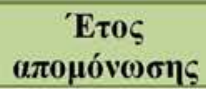 & 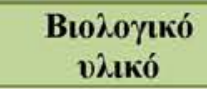 & 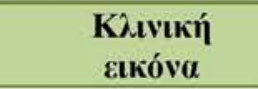 \\
\hline $\mathrm{Sm} 2 / 71 / 1$ & CAV4 & Povpavía & 1971 & А $\gamma \nu \omega \sigma \tau o$ & 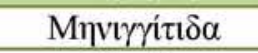 \\
\hline $243 / 7 / 99$ & CAV8 & Povpucvía & 1971 & Kó $\pi \rho \alpha v \alpha$ & 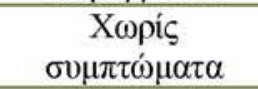 \\
\hline $611 / 80 / 1$ & CAV9 & Povenovía & 1980 & A $\gamma \nu \omega \sigma \tau o$ & 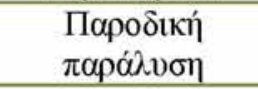 \\
\hline $65 / 89 / 1$ & CAV9 & Povpovía & 1989 & A $\gamma v \omega \sigma \tau o$ & 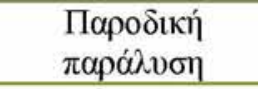 \\
\hline $113 / 73 / 2$ & CAV9 & Povpavía & 1973 & A $\gamma v \omega \sigma \tau o$ & 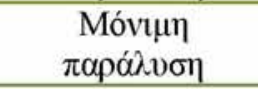 \\
\hline $\mathrm{A} 9 / \mathrm{Hel}$ & CAV9 & Kúmpos & 2002 & 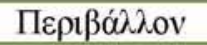 & - \\
\hline $\mathrm{A} 9 / \mathrm{He} 2$ & CAV9 & 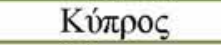 & 2002 & 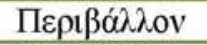 & - \\
\hline $\mathrm{A} 9 / \mathrm{He} 3$ & CAV9 & Kó $\pi \rho \circ \varsigma$ & 2002 & 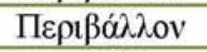 & - \\
\hline A9/He5 & CAV9 & 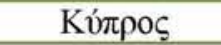 & 2002 & 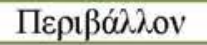 & - \\
\hline 5441 & CAV16 & E $\lambda \lambda \alpha ́ \delta \alpha$ & 1994 & A $\gamma v \omega \sigma \tau o$ & HFMD \\
\hline $99 / 74 / 1$ & CBV1 & Povpavia & 1974 & A $\gamma v \omega \sigma \tau o$ & $\begin{array}{l}\text { Паробики́ } \\
\pi \alpha \rho \alpha ́ \lambda v \sigma \eta\end{array}$ \\
\hline $98 / 74 / 2$ & CBV1 & Povpovía & 1974 & А $\gamma \nu \omega \sigma \tau o$ & 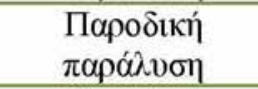 \\
\hline $88 / 74 / 9$ & CBV1 & Povpovía & 1974 & A $\gamma \nu \omega \sigma \tau о$ & 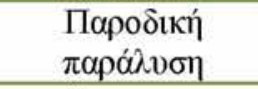 \\
\hline $116 / 95 / 2$ & CBV2 & Povenovía & 1974 & А $\gamma v \omega \sigma \tau 0$ & A $\gamma \nu \omega \sigma \tau o$ \\
\hline $118 / 95 / 1$ & CBV2 & Povenovía & 1974 & А $\gamma v \omega \sigma \tau 0$ & A $\gamma v \omega \sigma \tau 0$ \\
\hline 5207 B & CBV2 & $E \lambda \lambda \alpha \dot{\alpha} \alpha \alpha$ & 1984 & A $\gamma v \omega \sigma \tau 0$ & A $\gamma v \omega \sigma \tau 0$ \\
\hline $5211 \mathrm{~B}$ & CBV2 & E $\lambda \lambda \alpha ́ \delta \alpha$ & 1984 & A & A $\gamma v \omega \sigma \tau o$ \\
\hline 109/85/1 & CBV3 & Povpovía & 1985 & Кó $\rho \rho \alpha v \alpha$ & 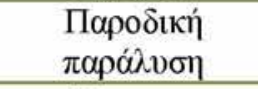 \\
\hline $123 / 95 / 1$ & CBV3 & Povenvía & 1995 & А $\gamma \nu \omega \sigma \tau o$ & 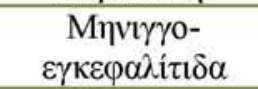 \\
\hline $77 / 78 / 3$ & CBV3 & Povpavía & 1978 & A $\gamma v \omega \sigma \tau o$ & 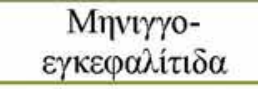 \\
\hline $169 / 75 / 1$ & CBV4 & Povpavía & 1986 & 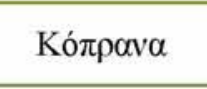 & 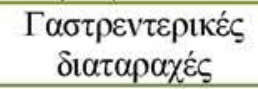 \\
\hline 69/86/1 & CBV4 & Povenovía & 1986 & Ко́ $\rho \rho \alpha \alpha$ & 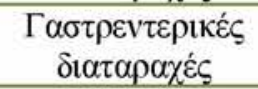 \\
\hline $14 / 70 / 1$ & CBV5 & Povpovía & 1970 & ENY & 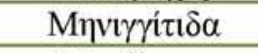 \\
\hline $254 / 77 / 1$ & CBV5 & Povpovía & 1977 & 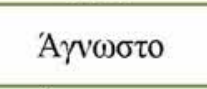 & 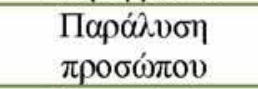 \\
\hline 81020 & CBV5 & E $\lambda \lambda \alpha ́ \delta \alpha$ & 2001 & A $\gamma v \omega \sigma \tau o$ & 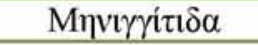 \\
\hline $6451 \mathrm{G}$ & CBV5 & E $\lambda \lambda \alpha \dot{\alpha} \delta \alpha$ & 1981 & A $\gamma \nu \omega \sigma \tau о$ & A $\gamma v \omega \sigma \tau o$ \\
\hline $86 / 73 / 1$ & CBV6 & Povpavia & 1973 & А $\gamma \nu \omega \sigma \tau о$ & 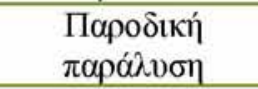 \\
\hline $7 / 73 / 19$ & CBV6 & Povpovía & 1973 & A $\gamma v \omega \sigma \tau 0$ & 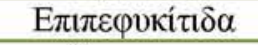 \\
\hline AB-19 & CBV6 & Povpovía & 1973 & A $\gamma v \omega \sigma \tau o$ & A $\gamma v \omega \sigma \tau 0$ \\
\hline 138/79/1 & Echo3 & Povpovía & 1979 & Ко́ $\tau \rho \alpha v \alpha$ & 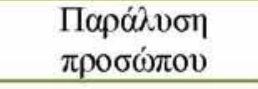 \\
\hline $243 / 97 / 1$ & Echo3 & Povpovía & 1997 & Ко́ $\pi \rho \alpha v \alpha$ & 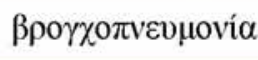 \\
\hline $156 / 98 / 1$ & Echo5 & Povpavía & 1998 & Ко́ $\rho \rho \alpha \alpha$ & 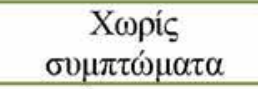 \\
\hline Foun & Echo6 & $E \lambda \lambda \alpha \dot{\delta} \delta \alpha$ & 2001 & Кó $\pi \rho \alpha v \alpha$ & 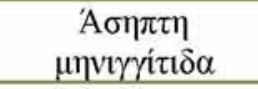 \\
\hline Bled & Echo6 & $E \lambda \lambda \alpha ́ \delta \alpha$ & 2001 & Kó $\pi \rho \alpha v \alpha$ & 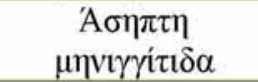 \\
\hline Tsikan & Echo6 & E $\lambda \lambda \alpha \dot{\alpha} \delta \alpha$ & 2001 & Ко́ $\tau \rho \alpha v \alpha$ & 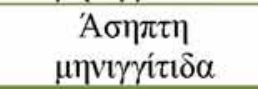 \\
\hline
\end{tabular}




\begin{tabular}{|c|c|c|c|c|c|}
\hline Freg & Echo6 & $\mathrm{E} \lambda \lambda \alpha \dot{\alpha} \delta \alpha$ & 2001 & Ко́ $\pi \rho \alpha v \alpha$ & $\begin{array}{c}\text { А } \sigma \eta \pi \tau \eta \\
\mu \eta v \imath \gamma \gamma i \tau t \delta \alpha\end{array}$ \\
\hline $44 / 73 / 1$ & Echo7 & 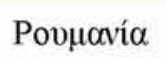 & A $\gamma \nu \omega \sigma \tau o$ & A $\gamma \nu \omega \sigma \tau o$ & A $\gamma v \omega \sigma \tau 0$ \\
\hline $307 / 77 / 1$ & Echo9 & Povpavía & 1977 & Ко́ $\pi \rho \alpha v \alpha$ & $\begin{array}{c}\text { A } \sigma \eta \pi \tau \eta \\
\mu \eta v \imath \gamma \gamma i \tau \imath \delta \alpha\end{array}$ \\
\hline $121 / 82 / 1$ & Echo9 & Povpovía & 1982 & Ко́ $\pi \rho \alpha v \alpha$ & $\begin{array}{c}\text { Абๆ } \pi \tau \eta \\
\mu \eta v \imath \gamma \gamma i \tau t \delta \alpha\end{array}$ \\
\hline $257 / 98 / 1$ & Echol1 & Povpuvía & 1998 & Ко́ $\pi \rho \alpha v \alpha$ & 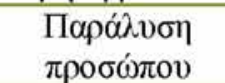 \\
\hline Papad & Echo13 & E $\lambda \lambda \alpha ́ \delta \alpha$ & 2001 & Ко́ $\pi \rho \alpha v \alpha$ & 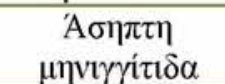 \\
\hline Pleur & Echo 13 & $E \lambda \lambda \alpha ́ \delta \alpha$ & 2001 & Ко́ $\pi \rho \alpha v \alpha$ & 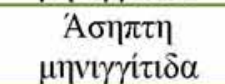 \\
\hline Pap & Echo13 & $\mathrm{E} \lambda \lambda \alpha \dot{\alpha} \alpha \alpha$ & 2001 & Kó $\pi \rho \alpha v \alpha$ & 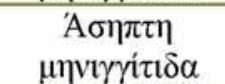 \\
\hline 1817 & Echol4 & $\mathrm{E} \lambda \lambda \alpha \dot{\alpha} \alpha \alpha$ & 1980 & ENY & $\begin{array}{c}\Psi \varepsilon v \delta 0 \gamma \rho \iota \pi \pi \iota \kappa \alpha ́ \\
\sigma \nu \mu \pi \tau \omega \dot{\alpha} \alpha \tau \alpha\end{array}$ \\
\hline 1729 & Echol4 & $\mathrm{E} \lambda \lambda \alpha \dot{\alpha} \delta \alpha$ & 1980 & ENY & $\begin{array}{l}\Psi \varepsilon v \delta о \gamma \rho \iota \pi \iota \kappa \alpha ́ \\
\sigma \nu \mu \pi \tau \omega ́ \mu \alpha \tau \alpha\end{array}$ \\
\hline 1374 & Echol4 & $\mathrm{E} \lambda \lambda \alpha \dot{\alpha} \alpha \alpha$ & 1980 & ENY & $\begin{array}{l}\Psi \varepsilon v \delta о \gamma \rho л \pi t \kappa \alpha ́ \\
\sigma \nu \mu \pi \tau \omega \dot{\mu} \alpha \tau \alpha\end{array}$ \\
\hline 85671 & Echo30 & $\mathrm{E} \lambda \lambda \alpha \dot{\alpha} \delta \alpha$ & 2001 & Ко́ $\pi \rho \alpha v \alpha$ & 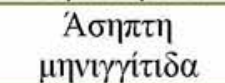 \\
\hline 86027 & Echo30 & $\mathrm{E} \lambda \lambda \alpha \alpha \delta \alpha$ & 2001 & Ко́ $\pi \rho \alpha v \alpha$ & $\begin{array}{c}\text { A } \sigma \eta \pi \tau \eta \\
\mu \eta v \imath \gamma \gamma i \tau 1 \delta \alpha\end{array}$ \\
\hline 85929 & Echo30 & $E \lambda \lambda \alpha \dot{\alpha} \delta \alpha$ & 2001 & Kó $\pi \rho \alpha v \alpha$ & 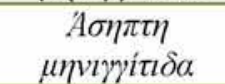 \\
\hline
\end{tabular}

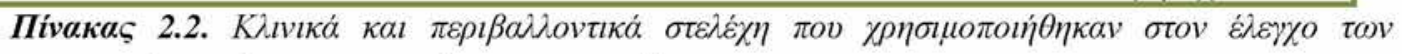

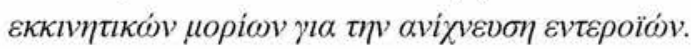

\begin{tabular}{|c|c|c|c|c|c|c|}
\hline$\Sigma \tau \varepsilon \dot{\lambda} \varepsilon \chi \chi 0 \varsigma$ & 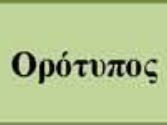 & 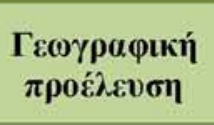 & 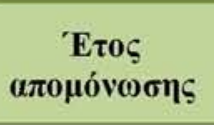 & 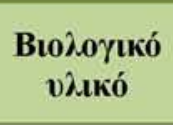 & $\begin{array}{l}\text { Кдıvıкฑ́ } \\
\text { \&เкóva }\end{array}$ & 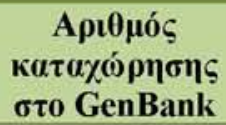 \\
\hline Kats & Polio1 & $E \lambda \lambda \alpha \dot{\alpha} \delta \alpha$ & 1996 & Ко́ $\pi \rho \alpha v \alpha$ & 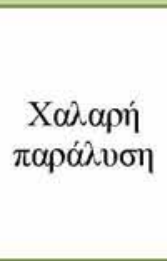 & $\begin{array}{l}\text { AY956405 } \\
\text { AY960848 } \\
\text { AY } 956408 \\
\text { AY962580 } \\
\text { AY962579 } \\
\text { AY962578 } \\
\text { AY962577 }\end{array}$ \\
\hline Hal & Polio1 & $\mathrm{E} \lambda \lambda \alpha \dot{\delta} \alpha \alpha$ & 1996 & Кó $\pi \rho \alpha v \alpha$ & 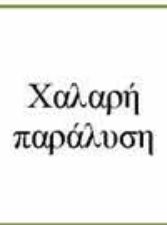 & $\begin{array}{l}\text { AY956406 } \\
\text { AY960849 } \\
\text { AY962582 } \\
\text { AY962583 } \\
\text { AY956409 } \\
\text { AY962584 }\end{array}$ \\
\hline Kar & Polio1 & $E \lambda \lambda \alpha \delta^{\delta} \alpha$ & 1996 & Ко́ $\pi \rho \alpha v \alpha$ & 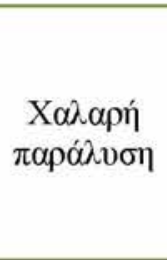 & $\begin{array}{l}\text { AY962586 } \\
\text { AY962585 } \\
\text { AY962587 } \\
\text { AY962588 } \\
\text { AY956407 } \\
\text { AY960850 } \\
\text { AY956410 }\end{array}$ \\
\hline LR11F7 & Echo7 & $\mathrm{E} \lambda \lambda \alpha \dot{\alpha} \delta \alpha$ & 2005 & $\Lambda v ́ \mu \alpha \tau \alpha$ & - & FJ460595 \\
\hline LR31G7 & Echo3 & $\mathrm{E} \lambda \lambda \alpha \alpha \delta \alpha$ & 2005 & $\Lambda v ́ \mu \alpha \tau \alpha$ & - & FJ766334 \\
\hline LR51A5 & Echo6 & $\mathrm{E} \lambda \lambda \alpha \dot{\delta} \delta \alpha$ & 2006 & $\Lambda v ́ \mu \alpha \tau \alpha$ & - & - \\
\hline LR61G3 & Echo6 & $\mathrm{E} \lambda \lambda \alpha \dot{\alpha} \alpha \alpha$ & 2006 & $\Lambda v ́ \mu \alpha \tau \alpha$ & - & - \\
\hline
\end{tabular}

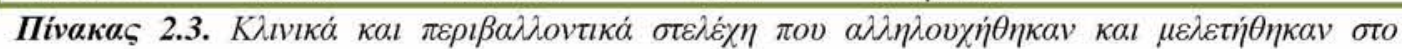

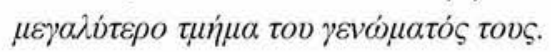




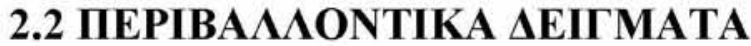

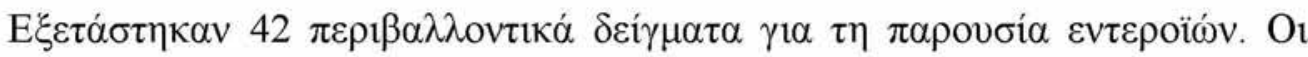

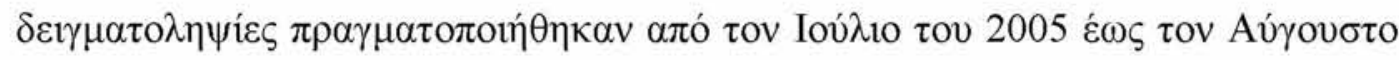

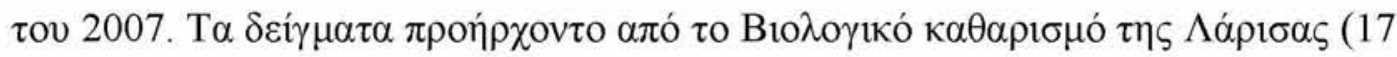

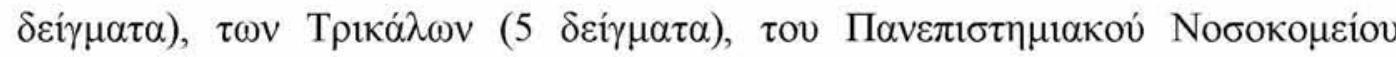

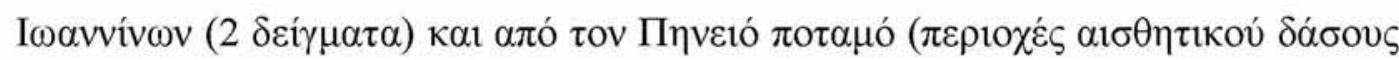

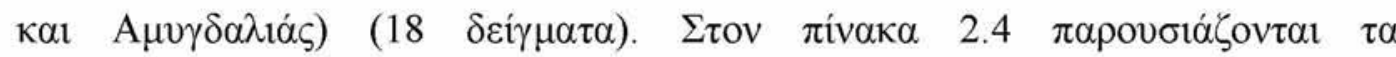

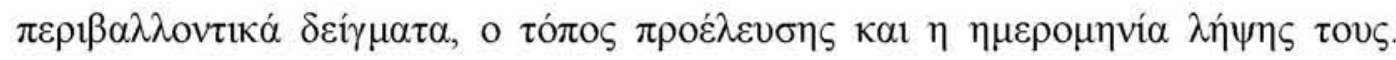

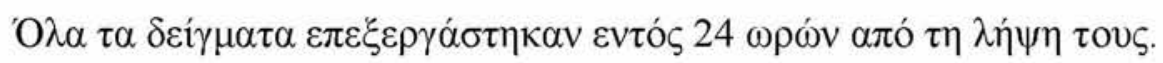

\begin{tabular}{|c|c|c|c|}
\hline$\Delta \varepsilon \dot{\gamma} \gamma \mu \alpha$ & 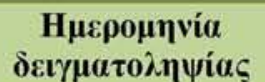 & 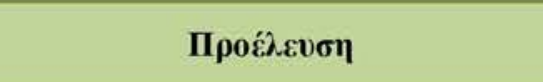 & 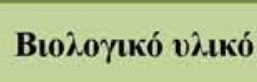 \\
\hline B1 & $13 / 07 / 2005$ & 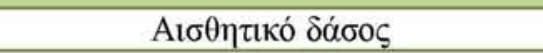 & 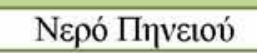 \\
\hline$\Gamma 1$ & $13 / 07 / 2005$ & A & 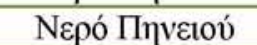 \\
\hline LR1 & $14 / 07 / 2005$ & 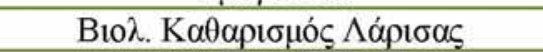 & 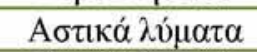 \\
\hline B2 & $7 / 09 / 2005$ & 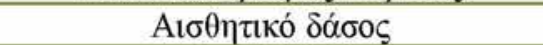 & 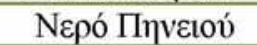 \\
\hline$\Gamma 2$ & $7 / 09 / 2005$ & 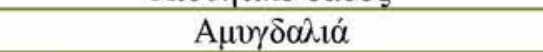 & 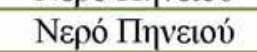 \\
\hline LR2 & $8 / 09 / 2005$ & 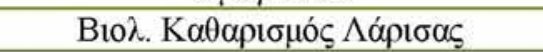 & 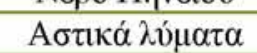 \\
\hline B3 & $5 / 10 / 2005$ & 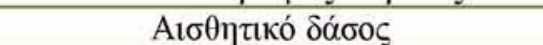 & 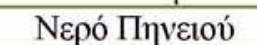 \\
\hline$\Gamma 3$ & $5 / 10 / 2005$ & 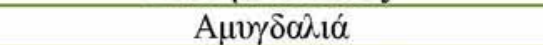 & 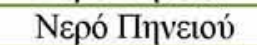 \\
\hline LR3 & $6 / 10 / 2005$ & 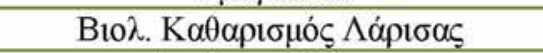 & 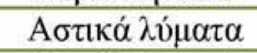 \\
\hline B4 & $16 / 11 / 2005$ & 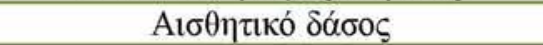 & 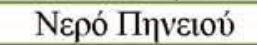 \\
\hline$\Gamma 4$ & $16 / 11 / 2005$ & 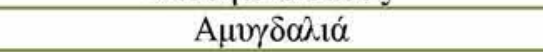 & 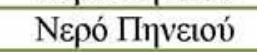 \\
\hline B5 & $24 / 05 / 2005$ & 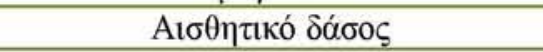 & 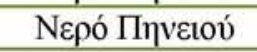 \\
\hline$\Gamma 5$ & $24 / 05 / 2005$ & 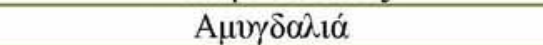 & 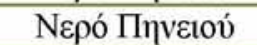 \\
\hline LR5 & $25 / 05 / 2006$ & 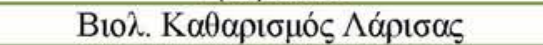 & 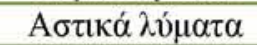 \\
\hline B6 & $15 / 06 / 2006$ & 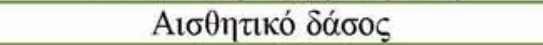 & 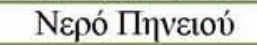 \\
\hline Г6 & $15 / 06 / 2006$ & 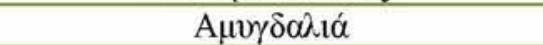 & 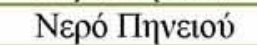 \\
\hline LR6 & $16 / 06 / 2006$ & 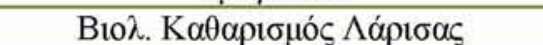 & 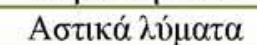 \\
\hline B7 & $29 / 06 / 2006$ & 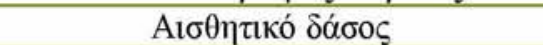 & 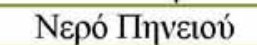 \\
\hline$\Gamma 7$ & $29 / 06 / 2006$ & A $\mu \gamma \gamma \delta \alpha \lambda i \alpha$ & 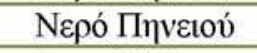 \\
\hline LR7 & $4 / 07 / 2006$ & 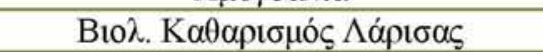 & 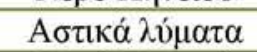 \\
\hline B8 & $12 / 07 / 2006$ & 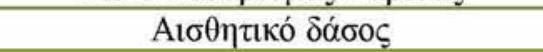 & 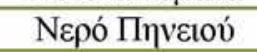 \\
\hline$\Gamma 8$ & $12 / 07 / 2006$ & A $\mu \gamma \gamma \delta \alpha \lambda \lambda_{1} \alpha$ & 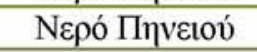 \\
\hline LR8 & $13 / 07 / 2006$ & 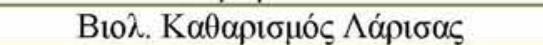 & 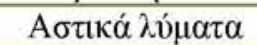 \\
\hline LR9 & $19 / 07 / 2006$ & 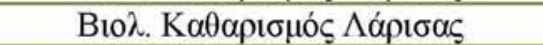 & 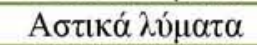 \\
\hline LR10 & $2 / 08 / 2006$ & 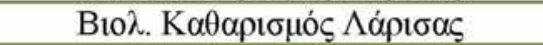 & 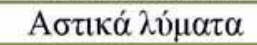 \\
\hline B11 & $14 / 09 / 2006$ & 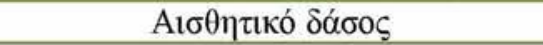 & 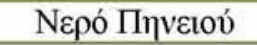 \\
\hline$\Gamma 11$ & $14 / 09 / 2006$ & A & 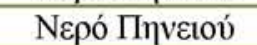 \\
\hline TR1 & $15 / 09 / 2006$ & 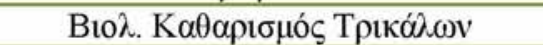 & 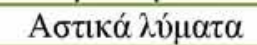 \\
\hline LR11 & $18 / 09 / 2006$ & 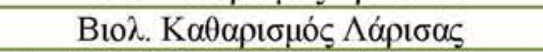 & 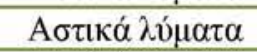 \\
\hline GN2 & $14 / 09 / 2006$ & 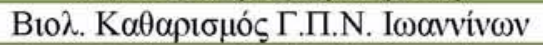 & $\Lambda v ́ \mu \alpha \tau \alpha$ \\
\hline GNl & $21 / 09 / 2006$ & 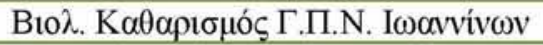 & $\Lambda v ́ \mu \alpha \tau \alpha$ \\
\hline TR2 & $28 / 11 / 2006$ & 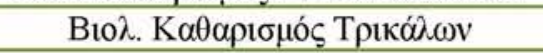 & 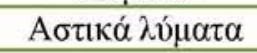 \\
\hline TR3 & $18 / 01 / 2007$ & 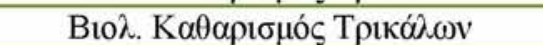 & 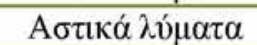 \\
\hline LR12 & $5 / 02 / 2007$ & 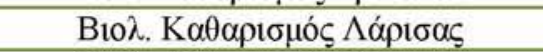 & 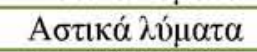 \\
\hline LR13 & $14 / 02 / 2007$ & 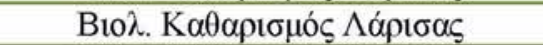 & 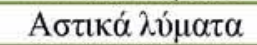 \\
\hline TR4 & $18 / 04 / 2007$ & 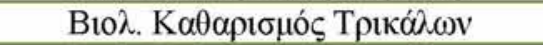 & 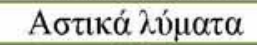 \\
\hline TR5 & $3 / 06 / 2007$ & 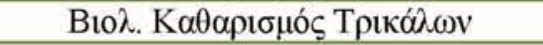 & 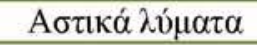 \\
\hline LR14 & $24 / 07 / 2007$ & 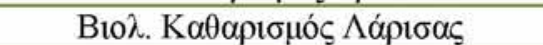 & 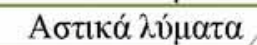 \\
\hline
\end{tabular}




\begin{tabular}{|c|c|c|c|}
\hline LR15 & $26 / 07 / 2007$ & 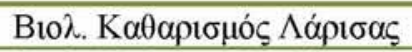 & 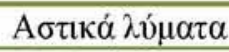 \\
\hline LRB1 & $26 / 07 / 2007$ & 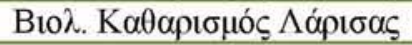 & 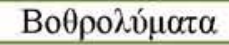 \\
\hline LR16 & $1 / 08 / 2007$ & 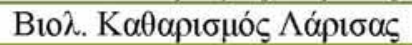 & 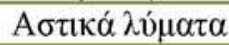 \\
\hline LRB2 & $1 / 08 / 2007$ & 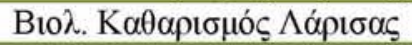 & 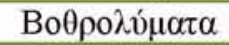 \\
\hline
\end{tabular}

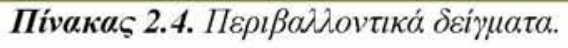

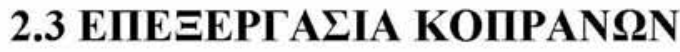

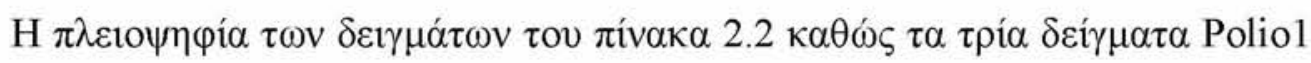

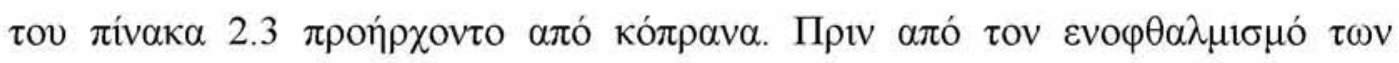

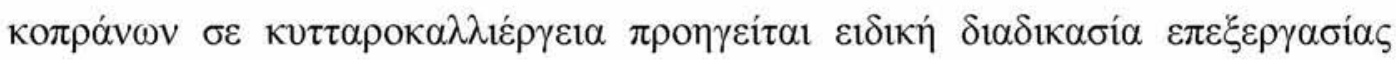

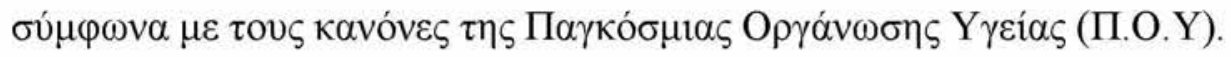

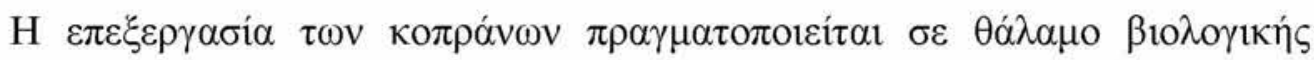
$\pi \rho \circ \sigma \tau \alpha \sigma i \alpha \varsigma$ (Biological Safety Cabinet, BSC) $\varepsilon \pi \imath \varepsilon \dot{\varepsilon} \delta o v 2$ (Biological Safety

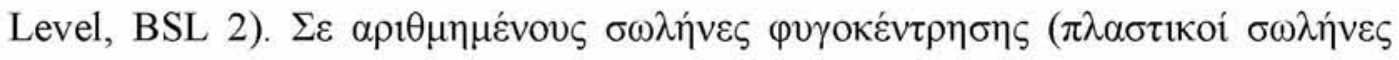

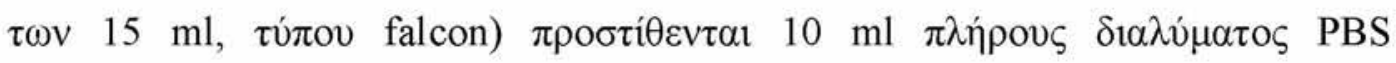

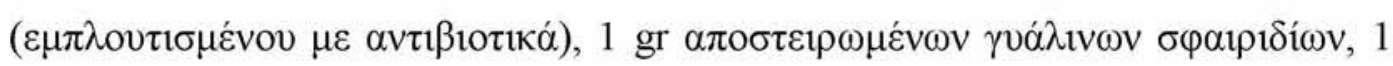

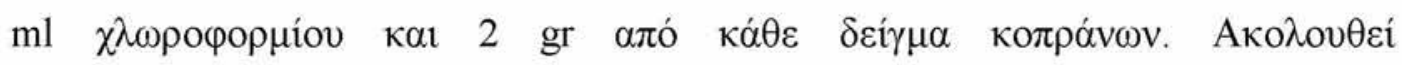

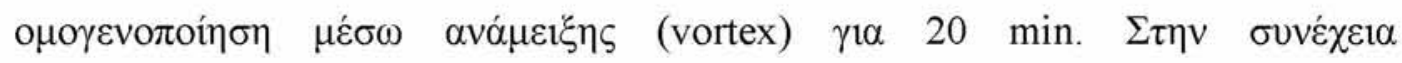

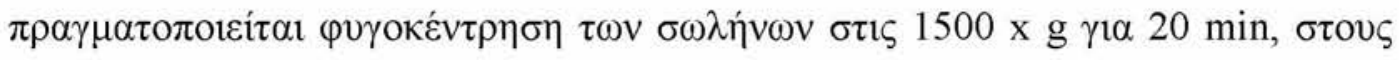

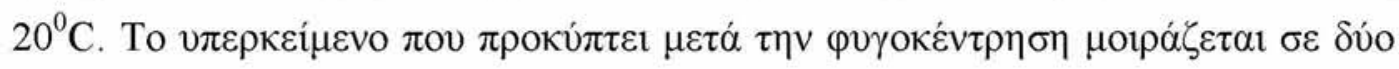

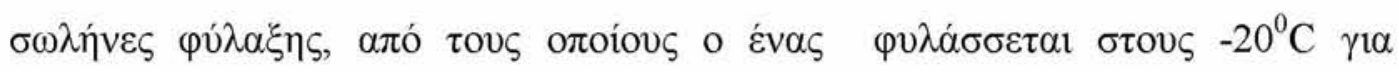

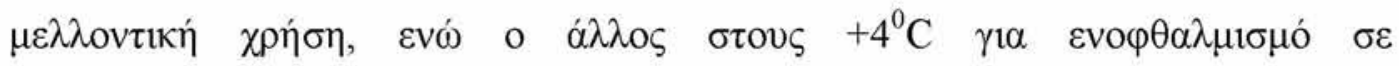

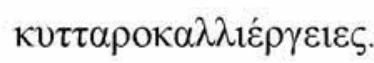

\section{$\Delta \iota \alpha \lambda \hat{\mu \alpha \alpha \alpha:}$}

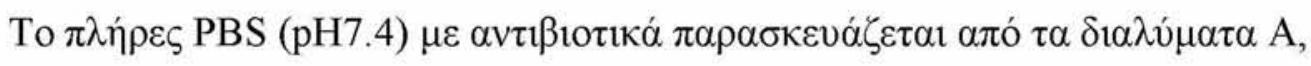

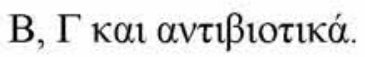

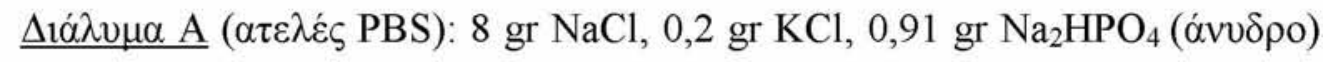

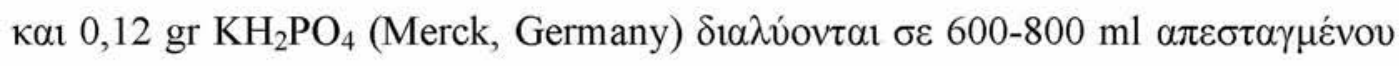

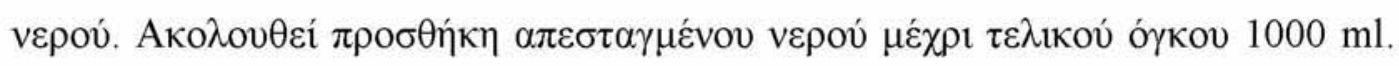

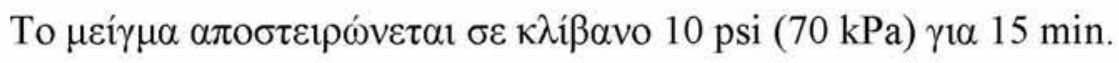

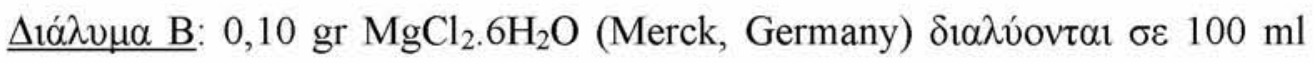

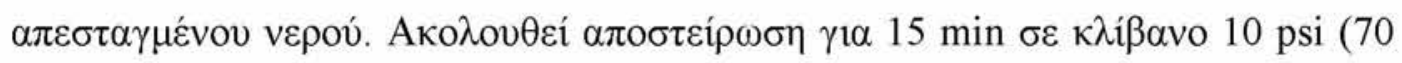
$\mathrm{kPa})$. 


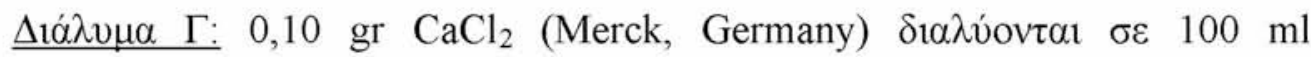

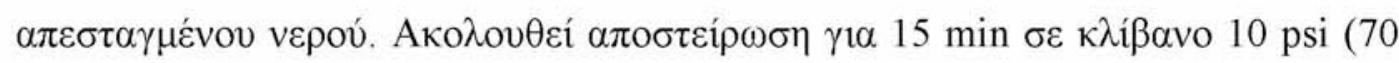
$\mathrm{kPa})$.

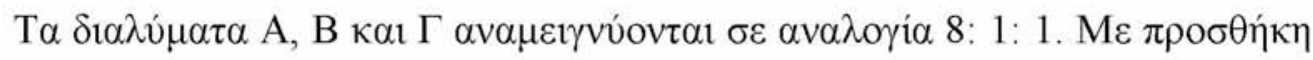

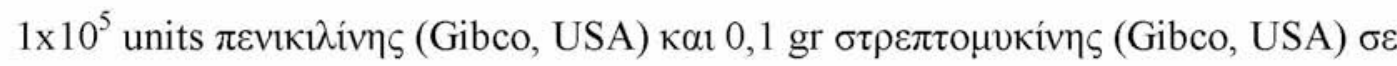

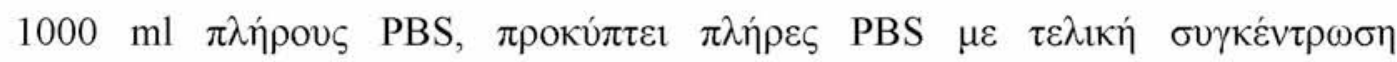

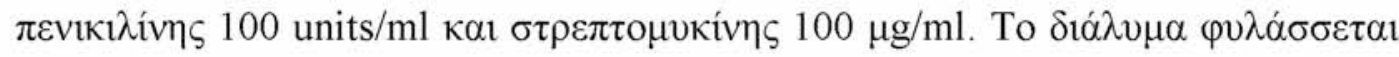

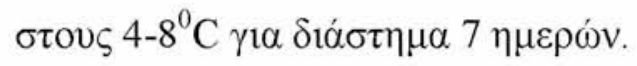

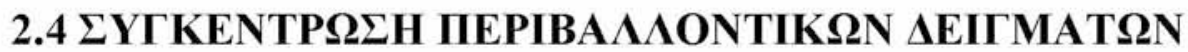

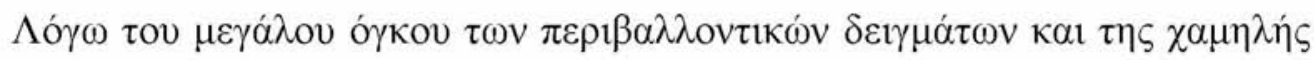

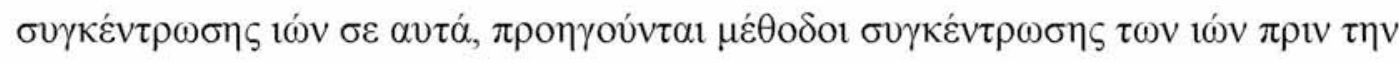

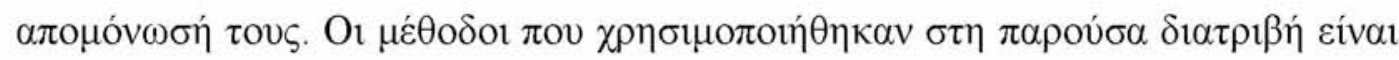

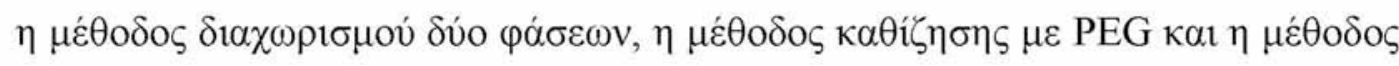

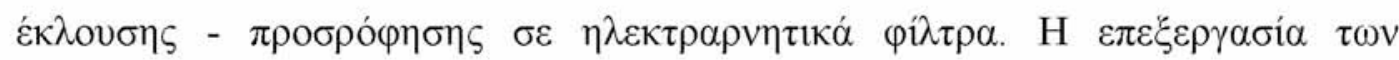

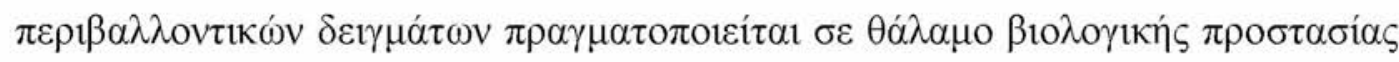

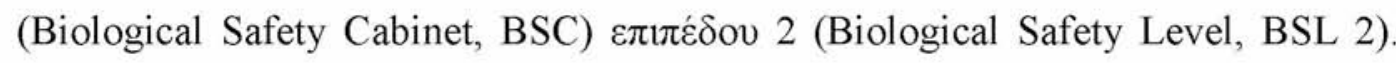

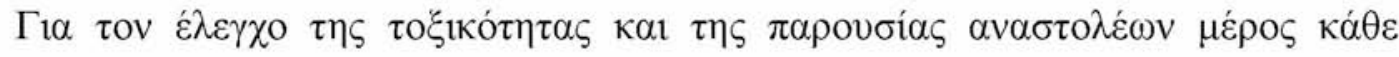

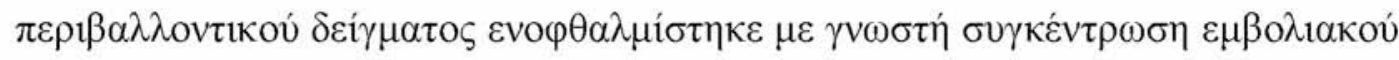

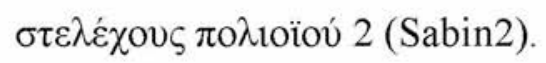

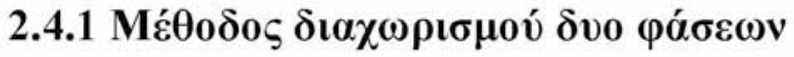

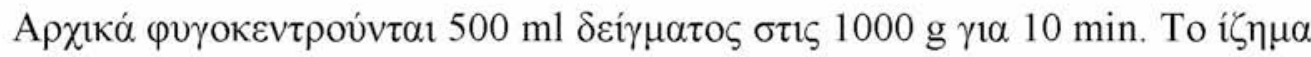

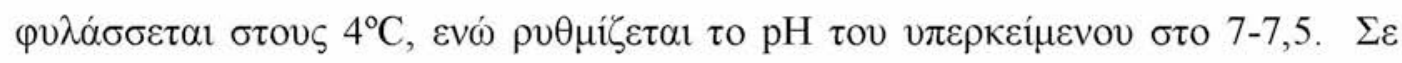

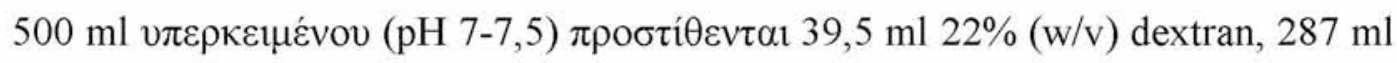

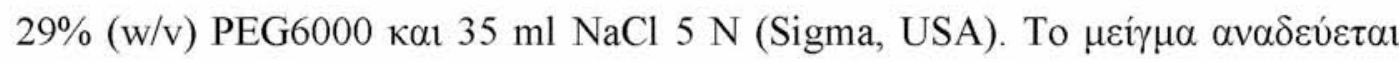

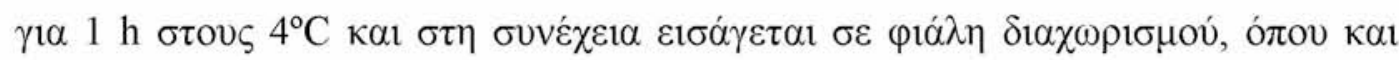

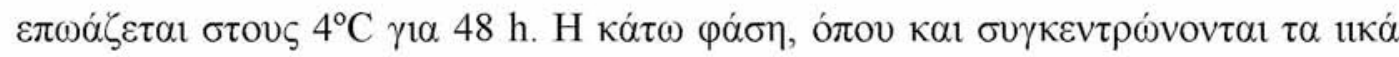

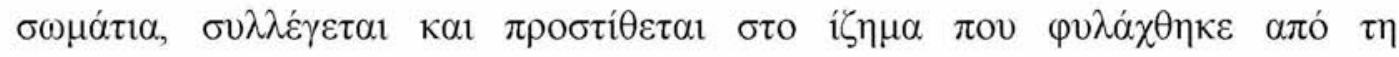

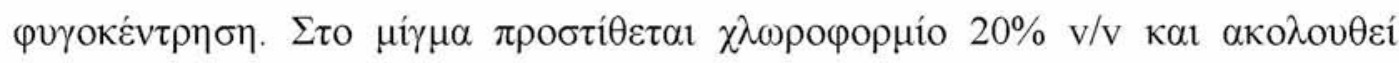

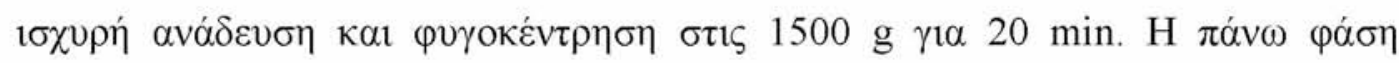

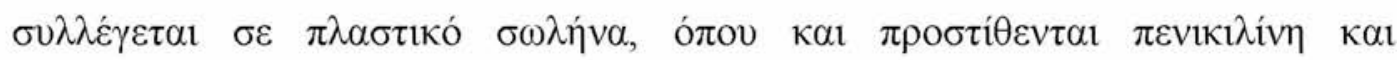




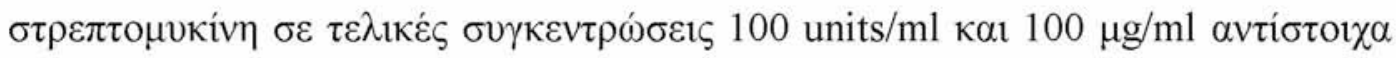
(WHO 2003).

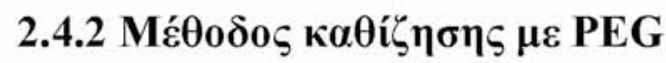

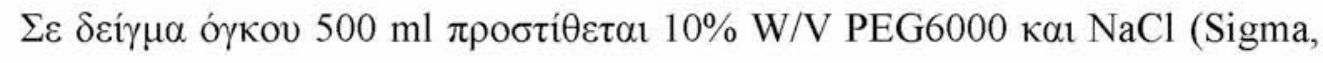

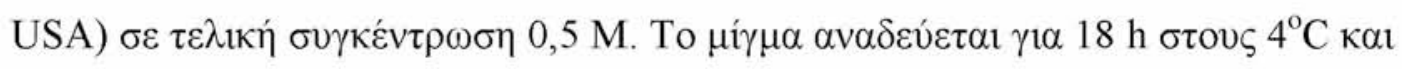

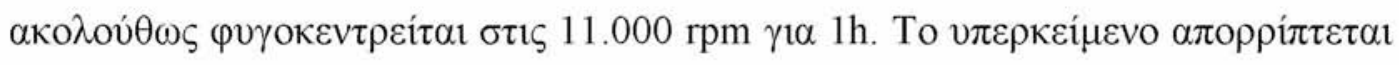

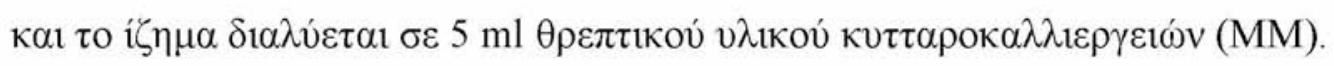

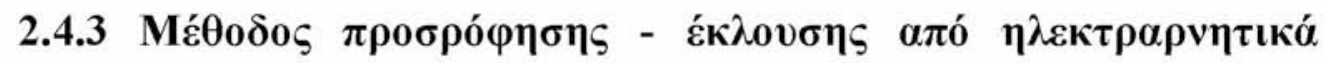
$\varphi \hat{i} \lambda \tau \rho \alpha$

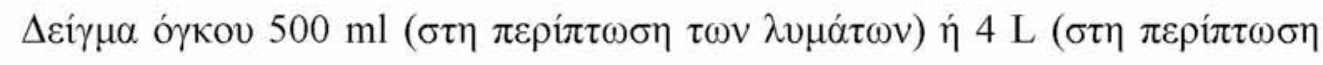

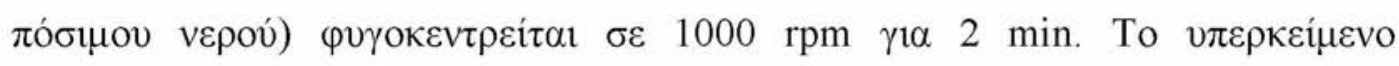

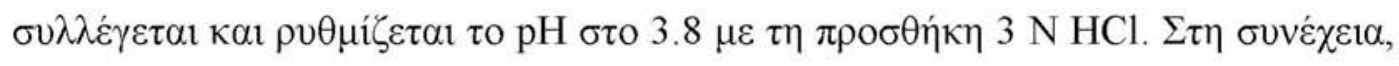

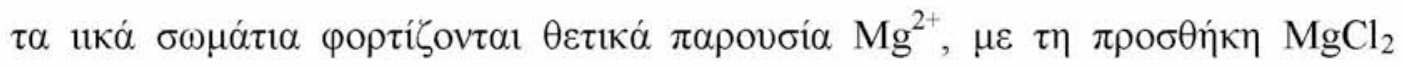

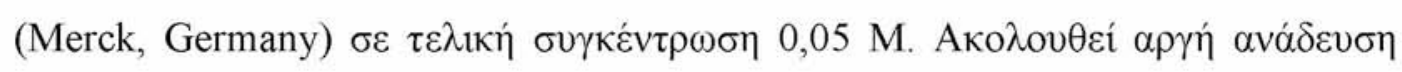

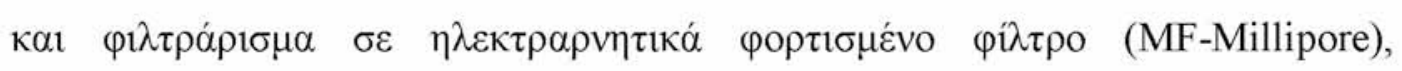

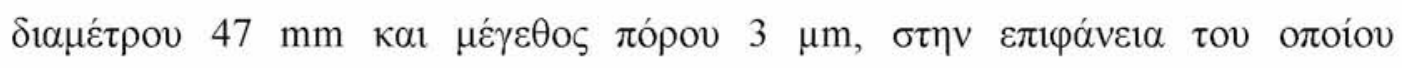

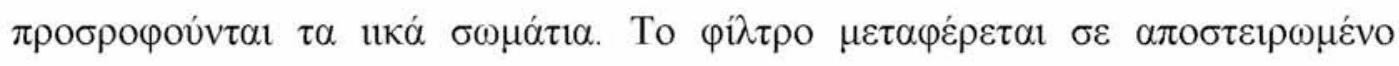

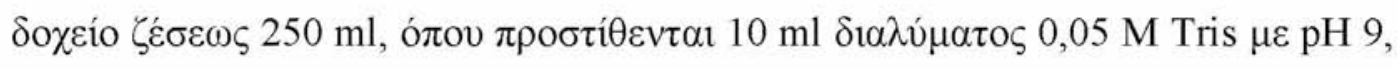

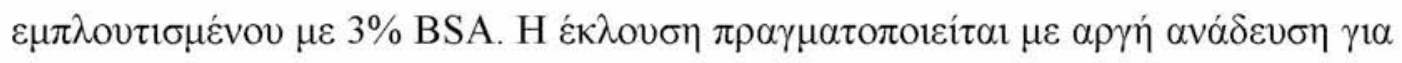

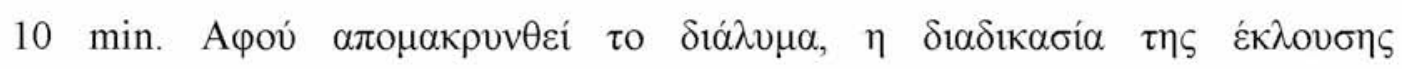

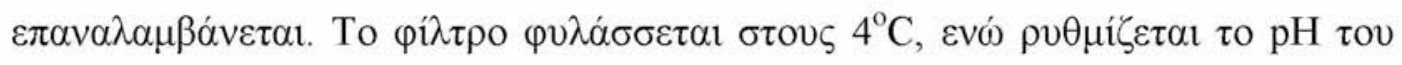

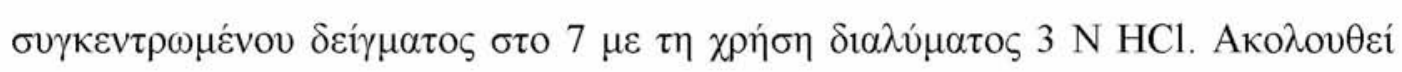

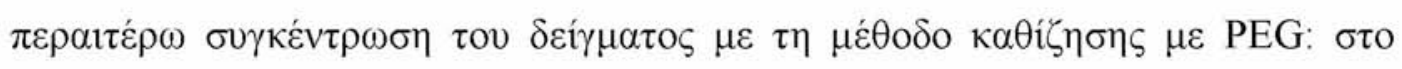

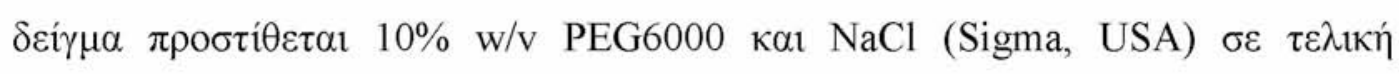

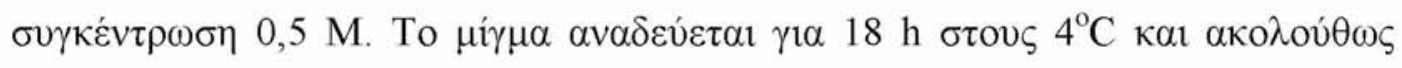

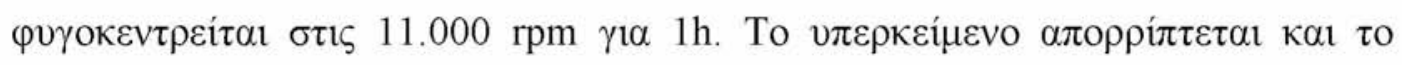

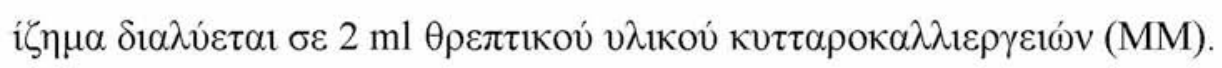




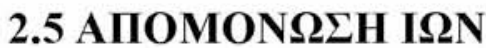

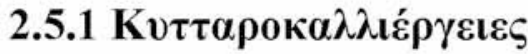

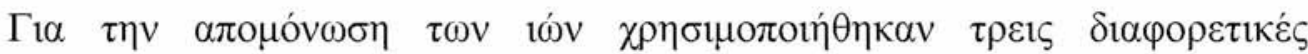

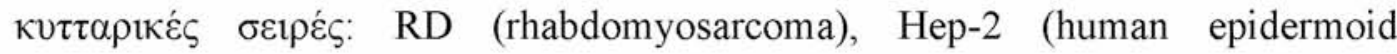

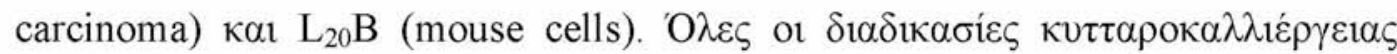

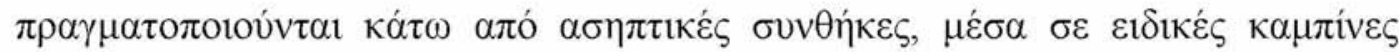

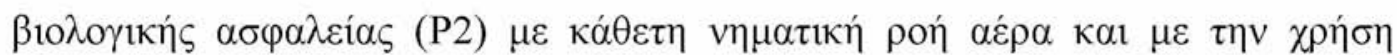

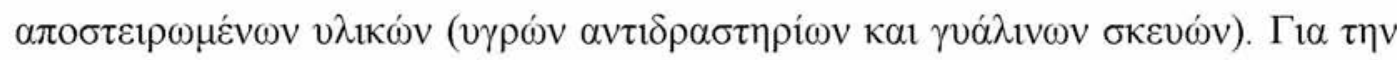

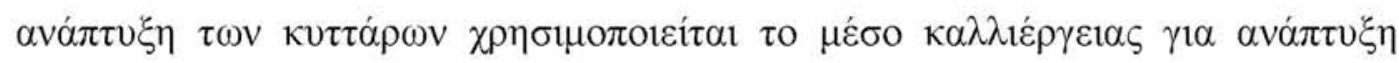

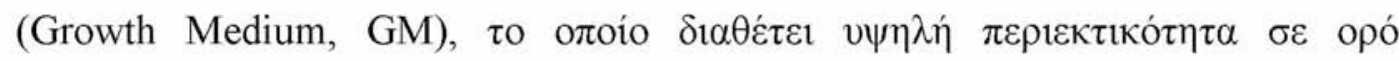

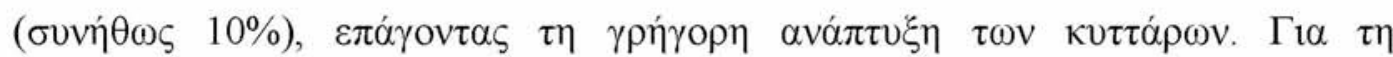

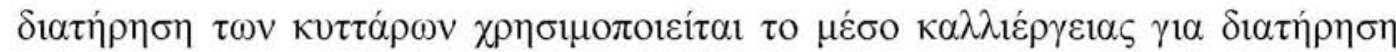

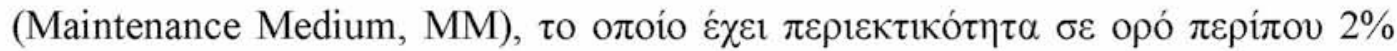

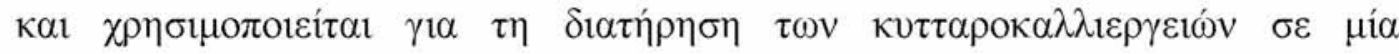

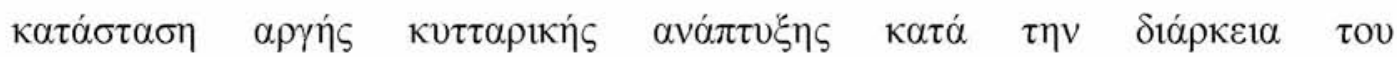
$\pi \mathrm{\partial} \lambda \alpha \pi \lambda \alpha \sigma i \alpha \sigma \mu \sigma \dot{\tau} \tau \omega \mathrm{v}$ เóv.

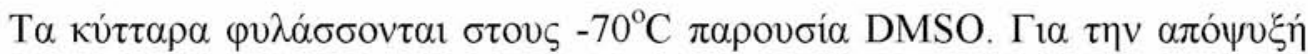

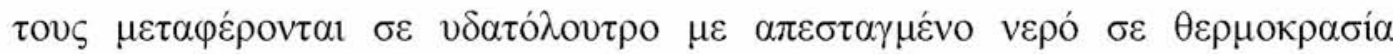

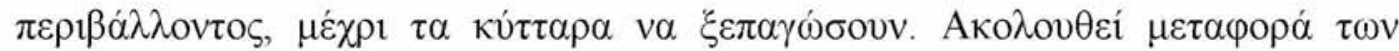

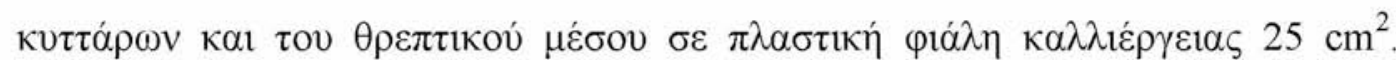

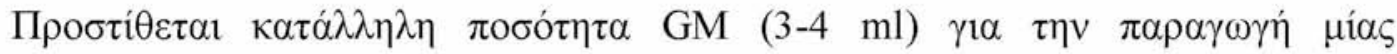

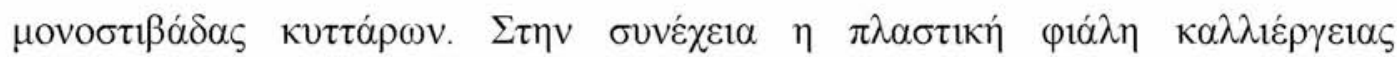

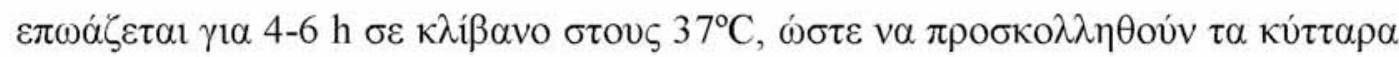

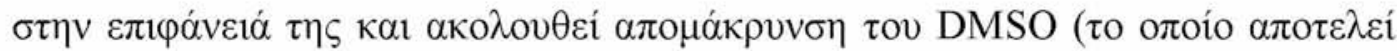

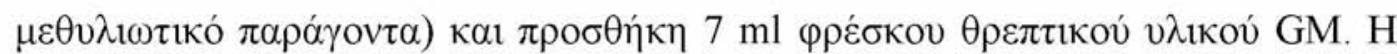

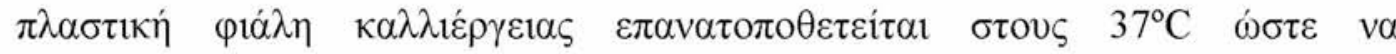

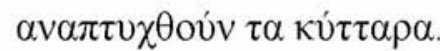

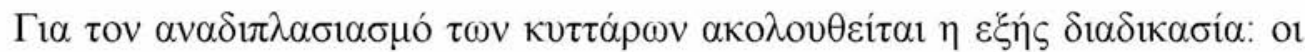

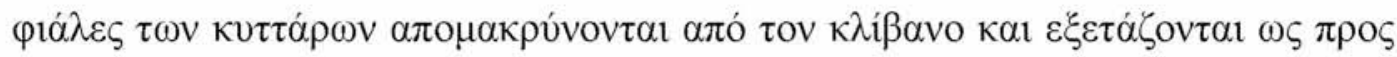

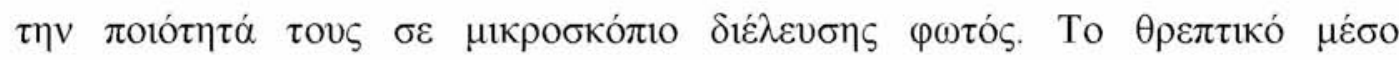

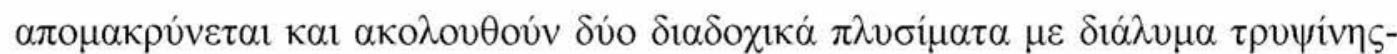

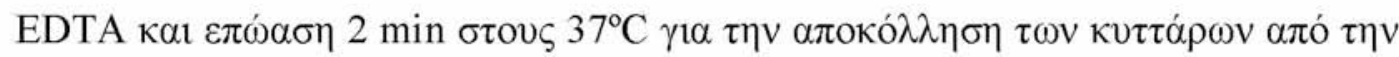




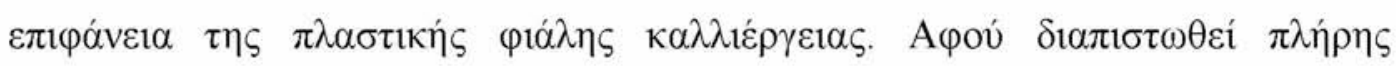

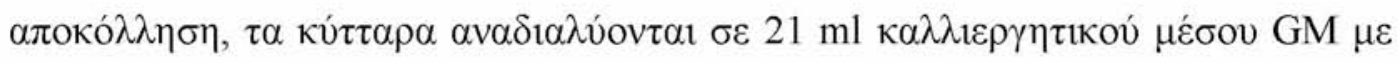

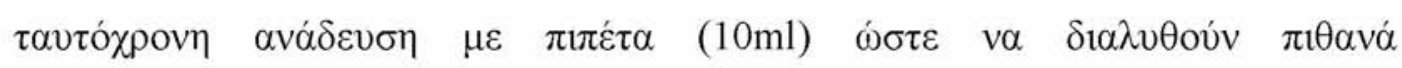

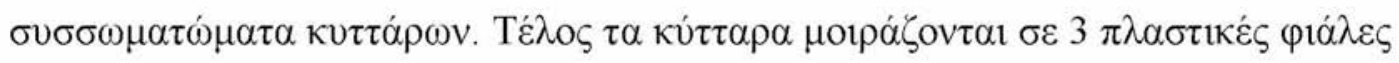

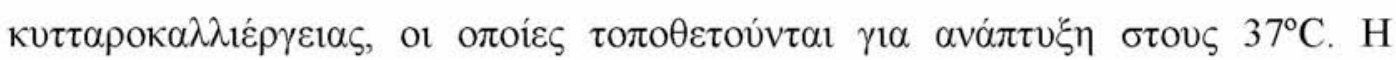

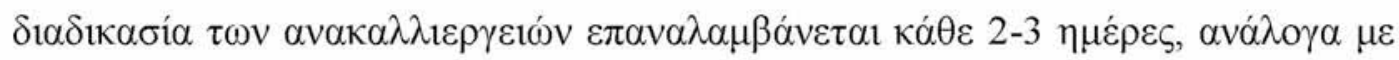

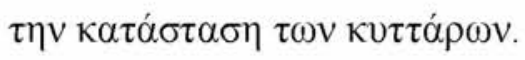

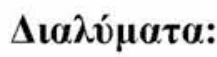

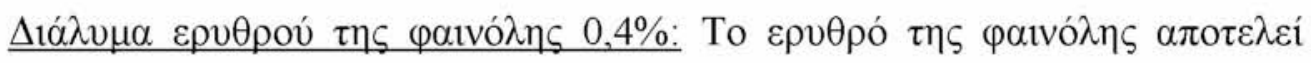

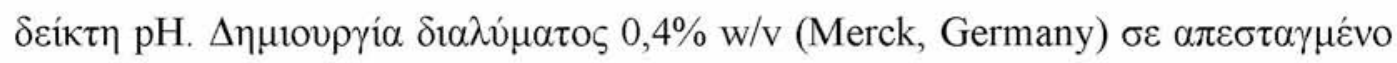

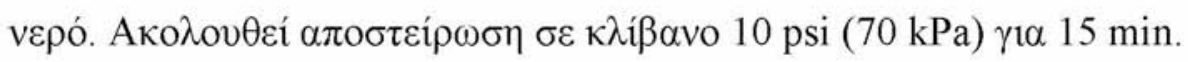

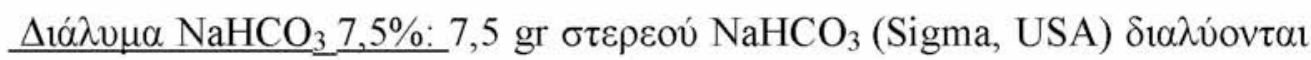

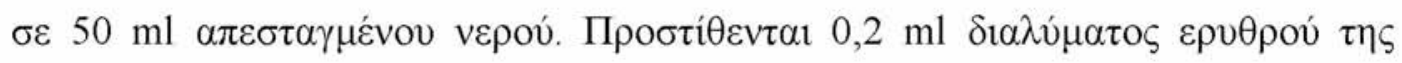

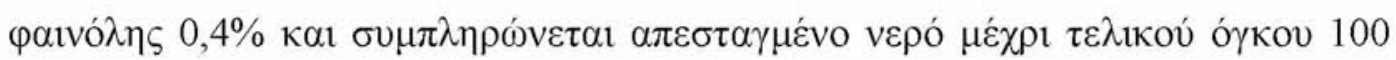

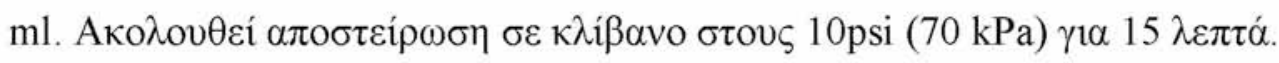

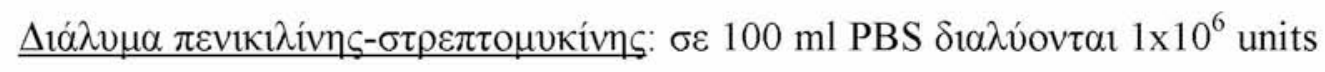

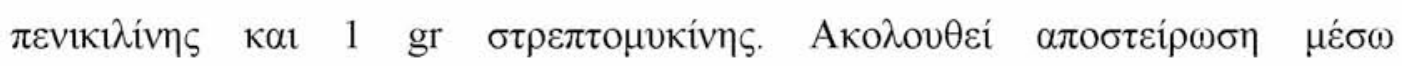
$\varphi \imath \lambda \tau \rho \alpha \rho \dot{\sigma} \sigma \alpha \alpha$ os.

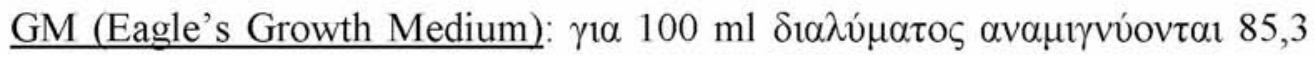
ml Eagle's minimum essential medium (Earl's salts base, no bicarbonate) (Sigma,

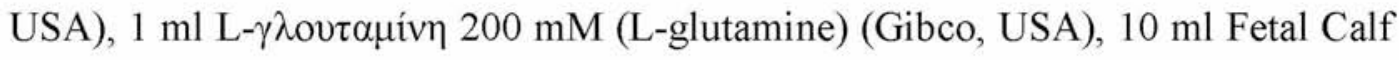
Serum (Sigma, USA), 1,5 ml $\delta i \alpha ́ \lambda v \mu \alpha \mathrm{NaHCO}_{3}$ 7,5\%, $1 \mathrm{ml}$ HEPES $1 \mathrm{M}$ (Merck,

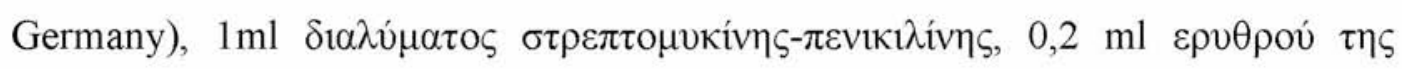

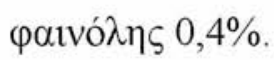

MM (Eagle's Meintenance medium): $\gamma 1 \alpha 100 \mathrm{ml} \delta 1 \alpha \lambda \dot{\mu} \mu \alpha \tau \sigma \varsigma \alpha v \alpha \mu \gamma v v \dot{o v \tau \alpha l}$ 92,3 ml Eagle's minimum essential medium (Earl's salts base, no bicarbonate), 1

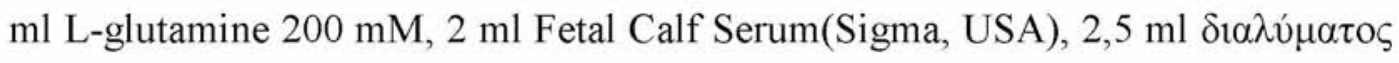
$\mathrm{NaHCO}_{3} 7,5 \%, 1 \mathrm{ml}$ HEPES 1M, $1 \mathrm{ml} \delta 1 \alpha \lambda \hat{\mu} \mu \alpha \tau$

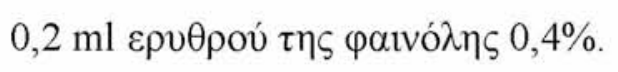

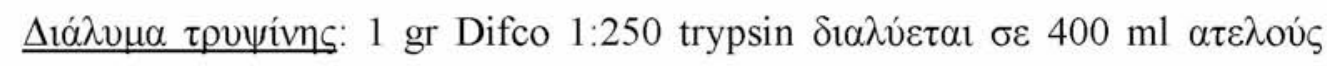

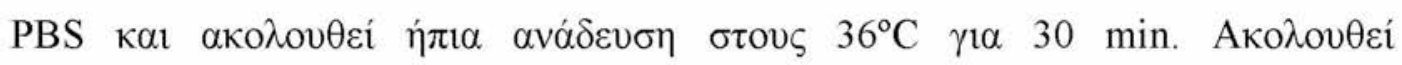

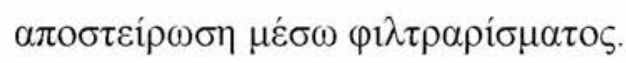




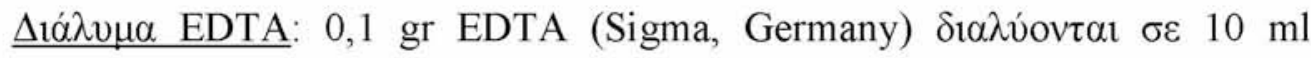

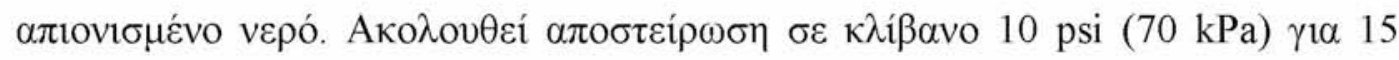

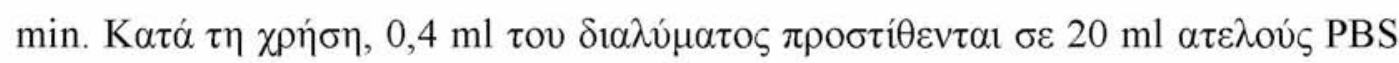

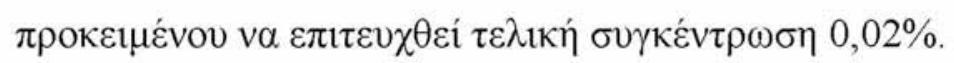

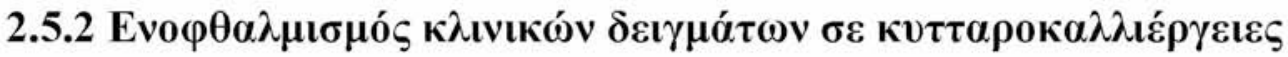

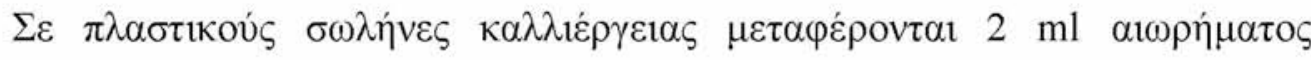

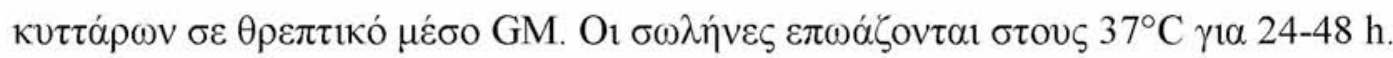
A

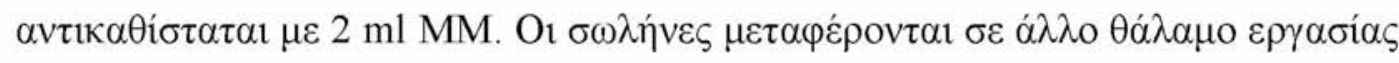

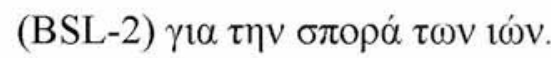

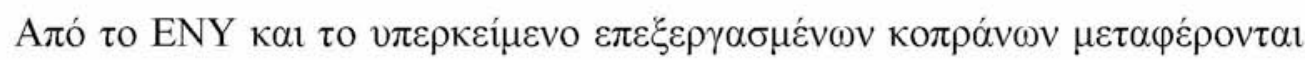

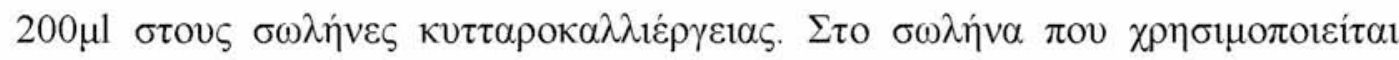

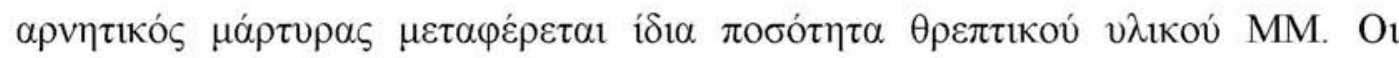

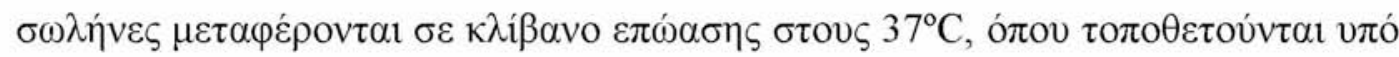

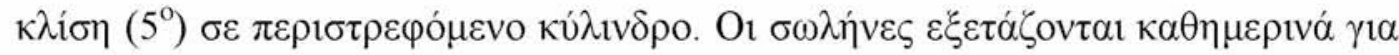

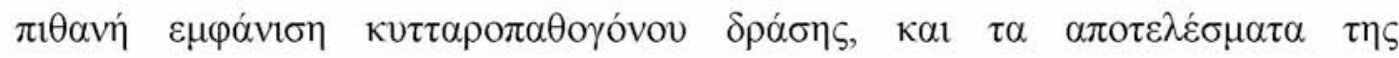

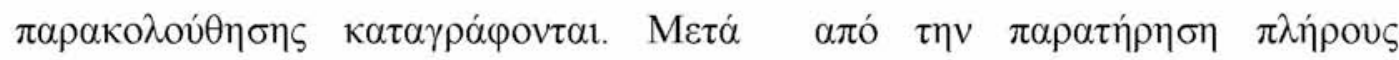

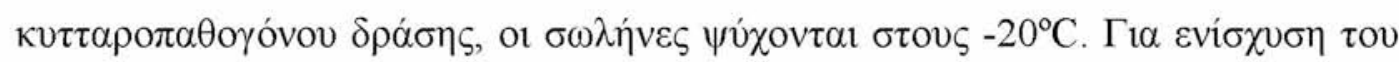

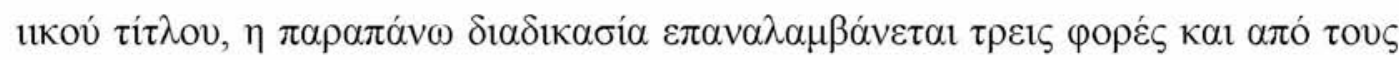

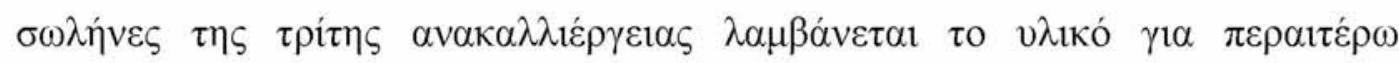
$\varepsilon \pi \varepsilon \xi \varepsilon \rho \gamma \alpha \sigma i \alpha$.

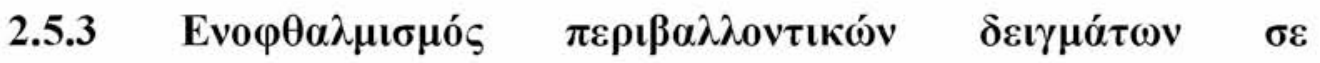

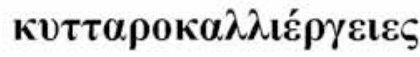

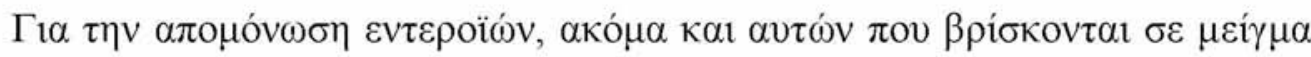

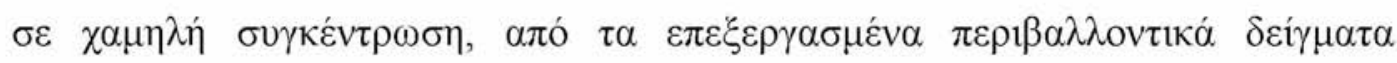

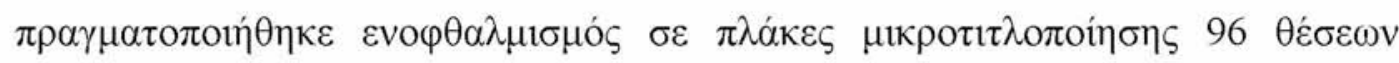

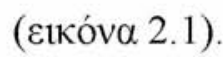

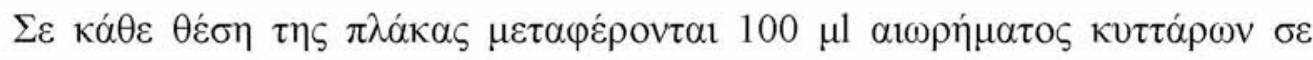

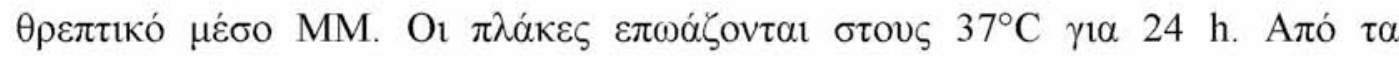

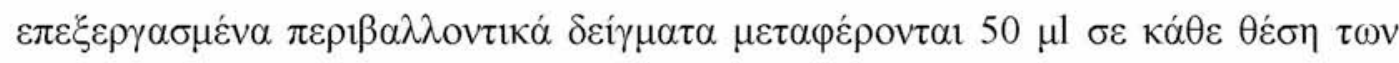

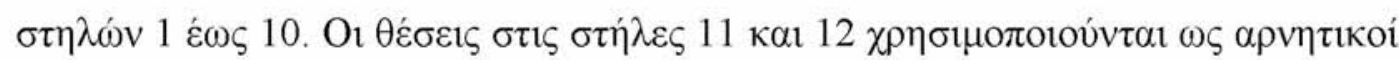




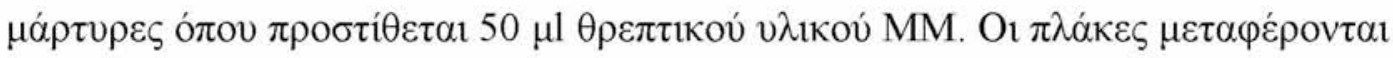

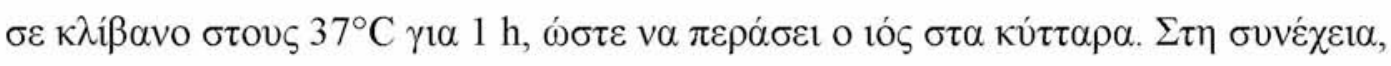

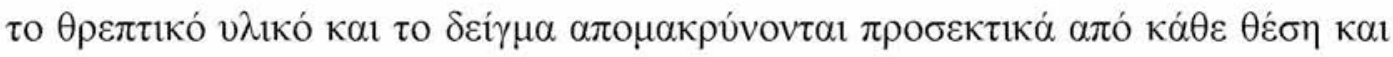

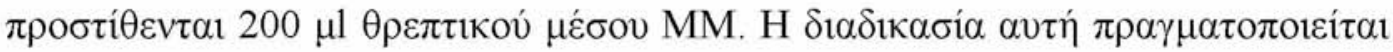

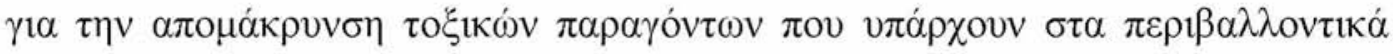

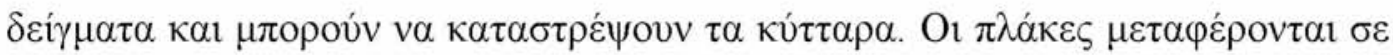

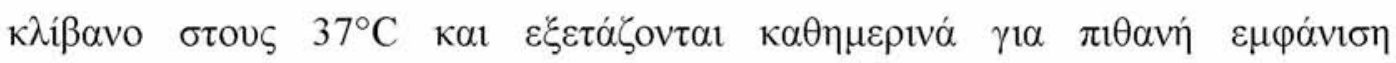

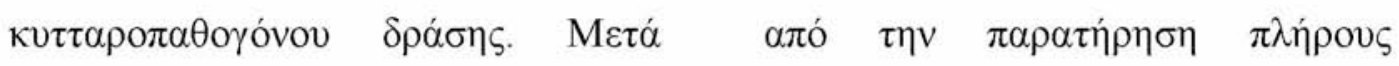

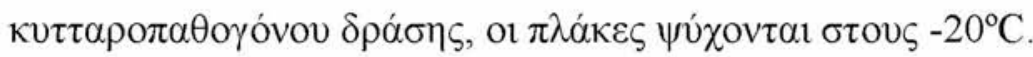

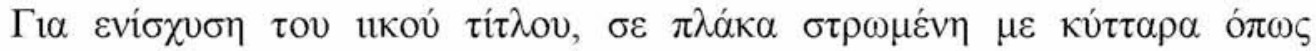

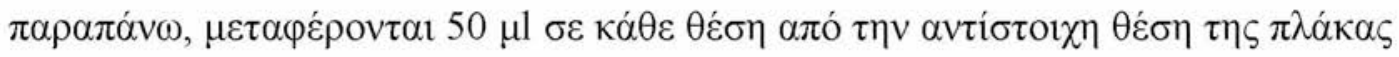

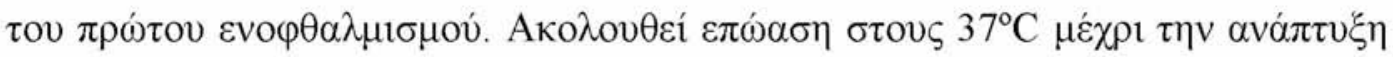

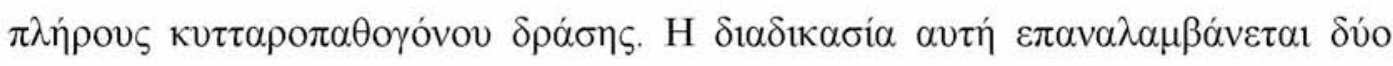

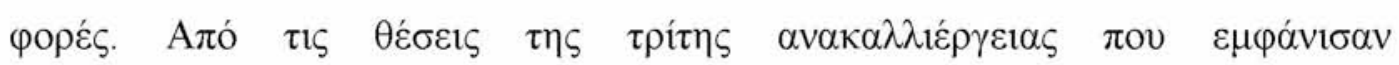

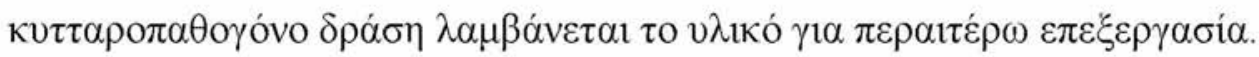

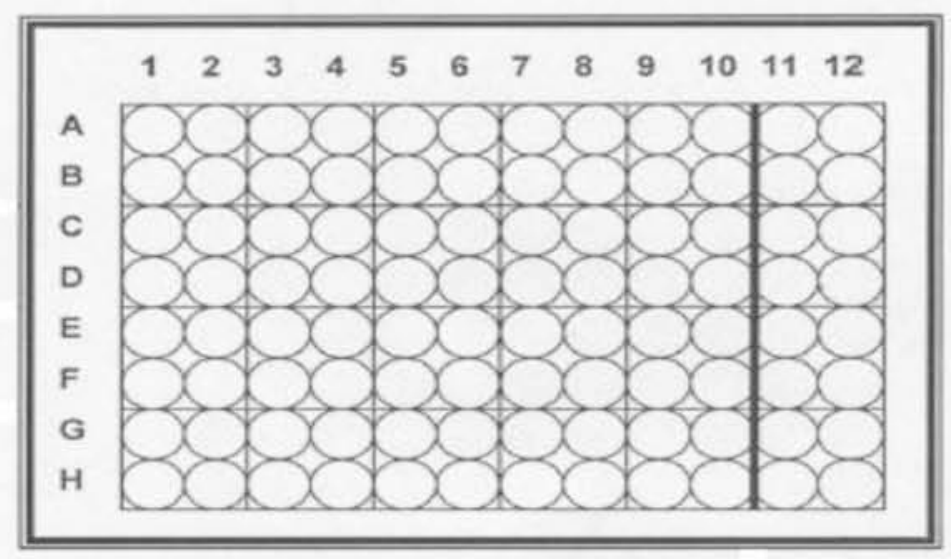

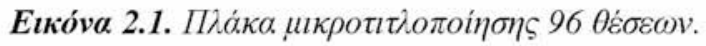

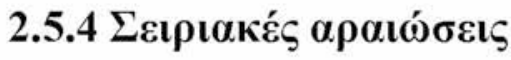

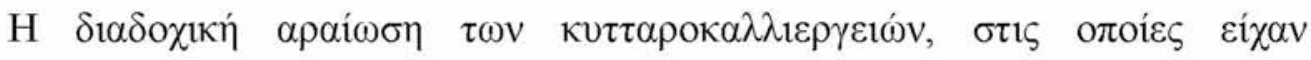

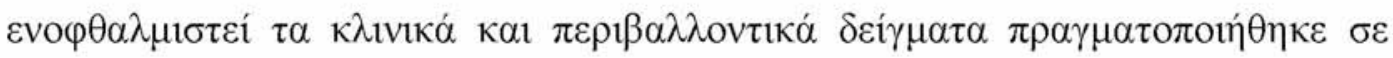

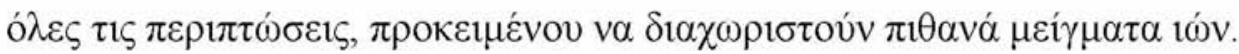

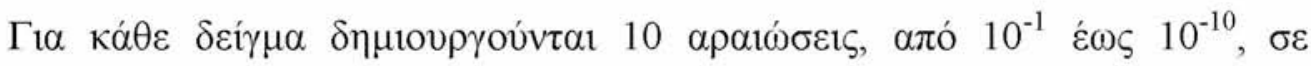

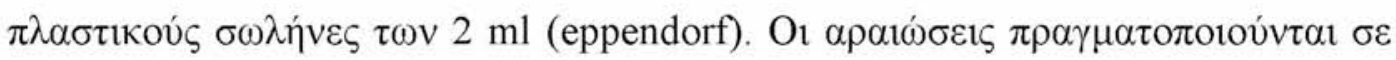

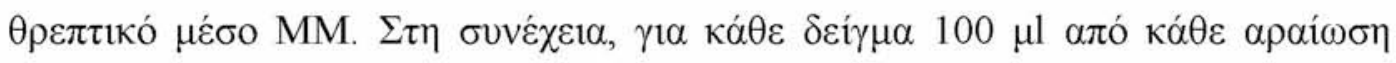

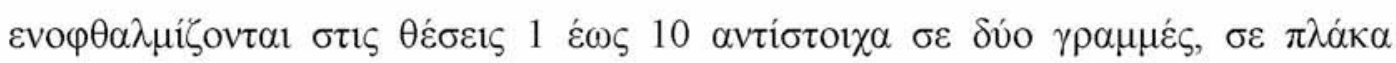




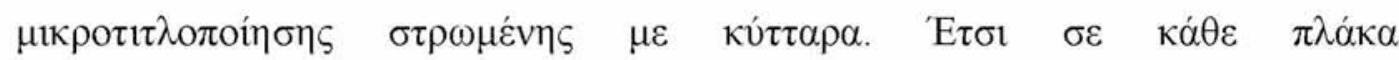

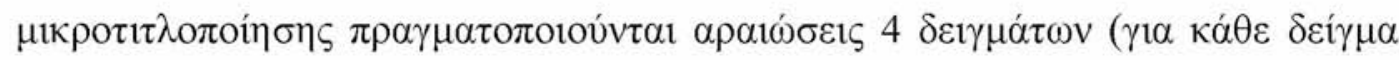

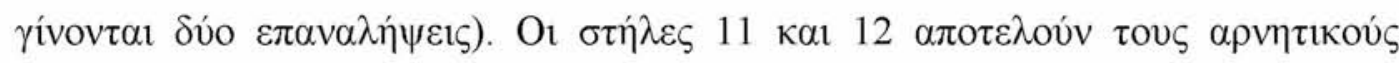

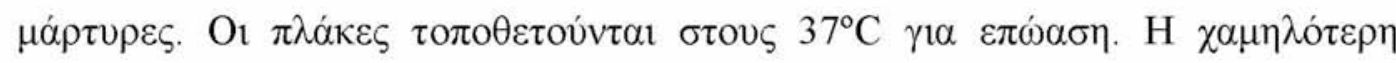

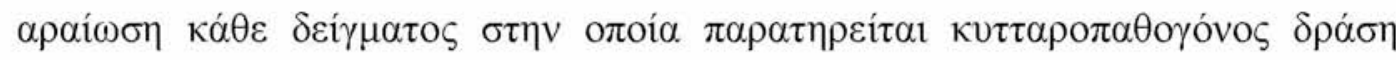

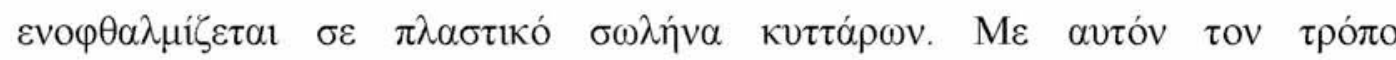

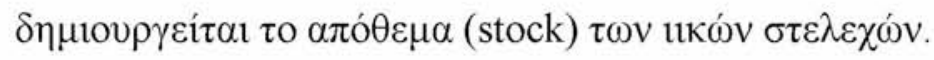

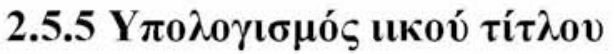

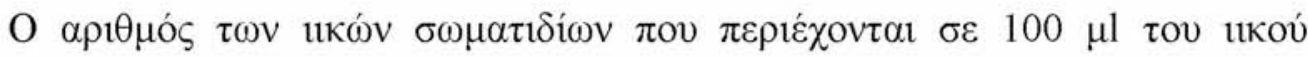

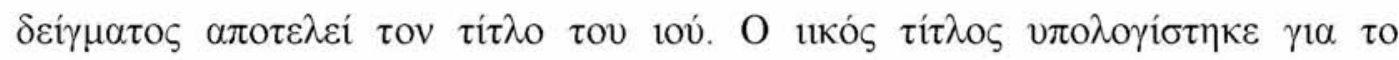

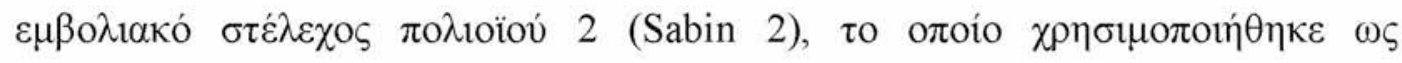

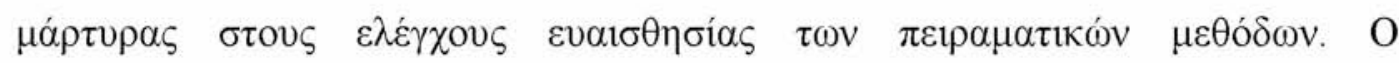

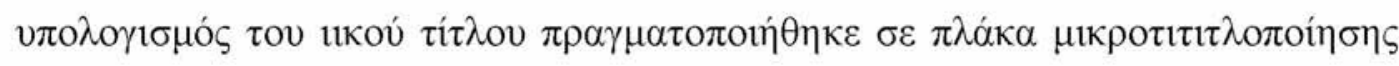

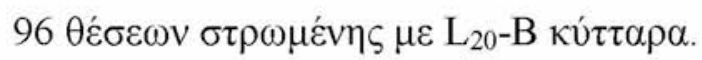

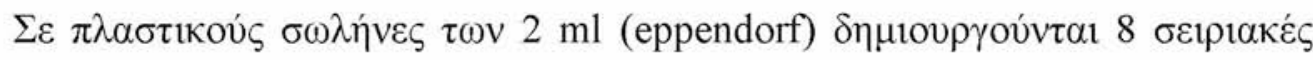

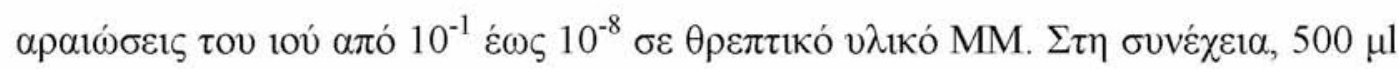

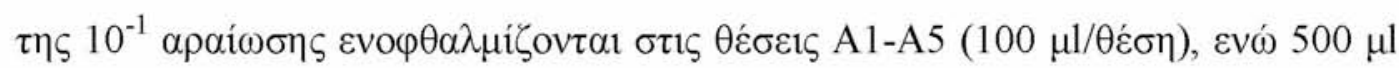

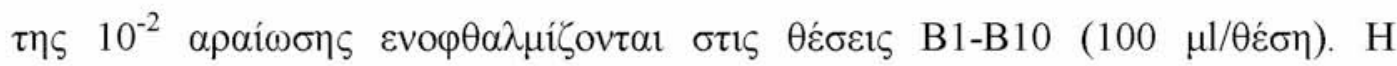

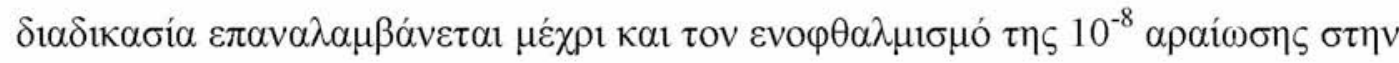

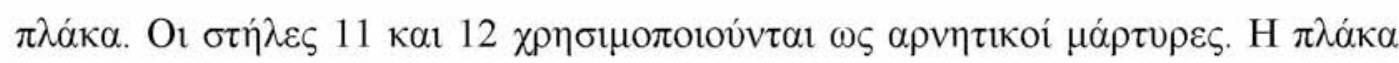

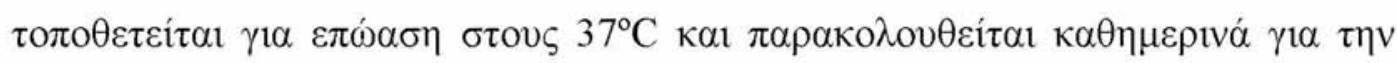

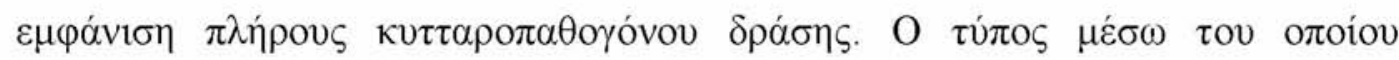

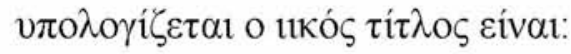

$\log \mathrm{TCID}_{50}=\mathrm{L}-\mathrm{d}(\mathrm{S}-0,5)$

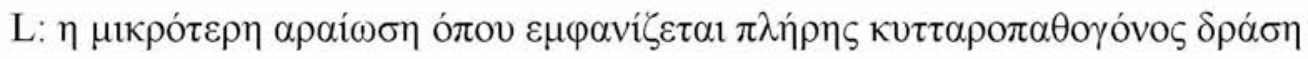

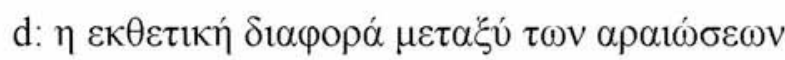

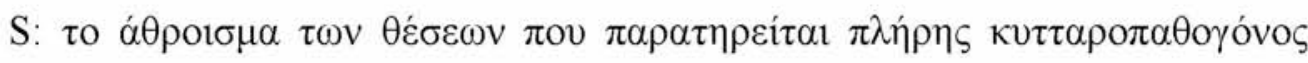

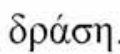

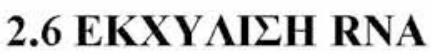

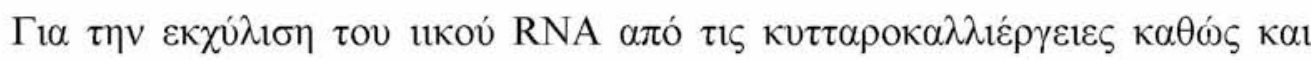

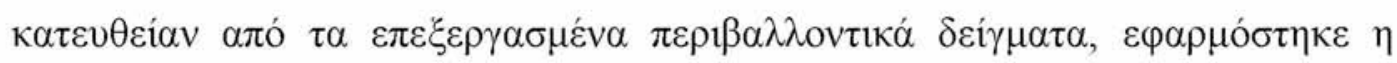




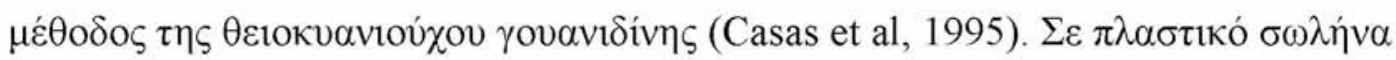

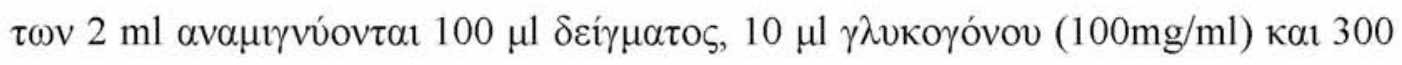

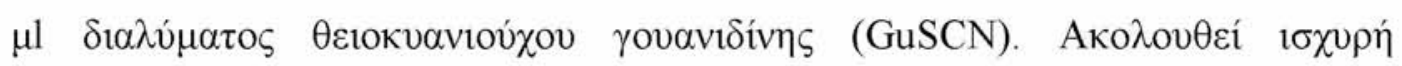

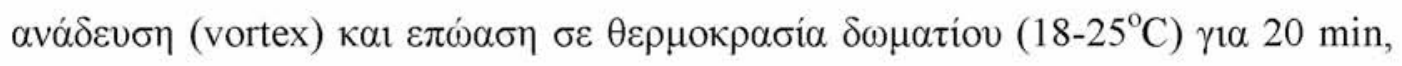

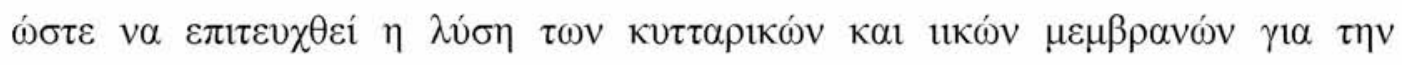

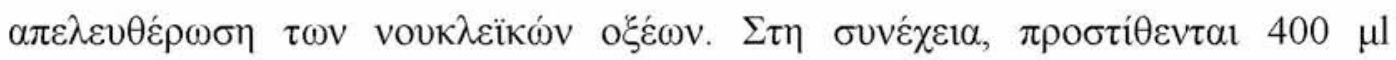

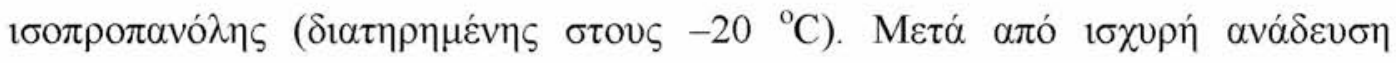

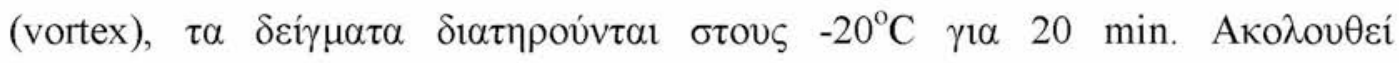

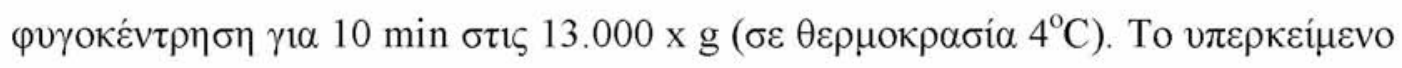

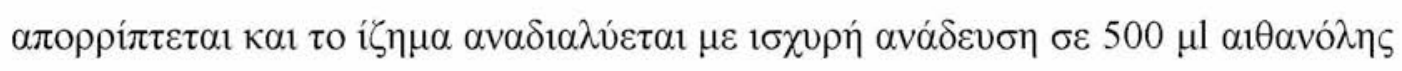

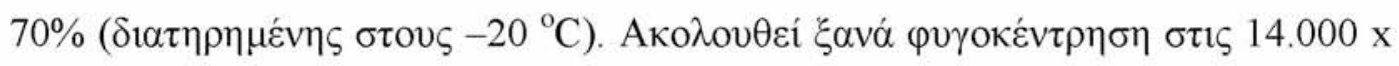

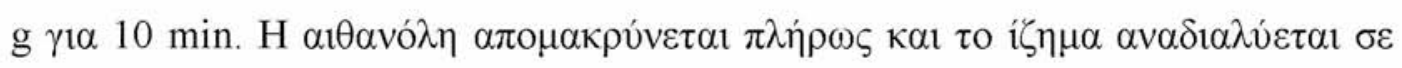

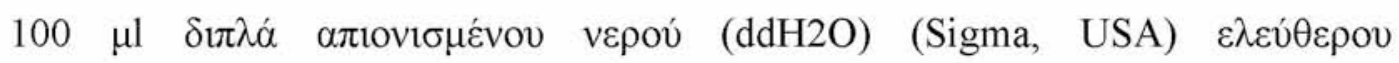

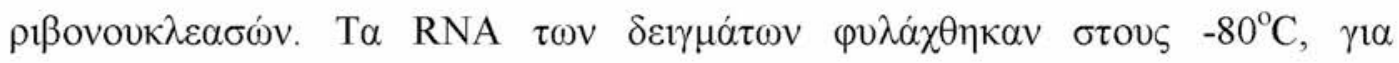
$\mu \alpha \kappa \rho о \pi \rho \dot{\theta \varepsilon \sigma \mu \eta ~ \chi \rho \eta ́ \sigma \eta . ~}$

\section{$\Delta \iota \alpha \hat{\jmath} \mu \alpha \tau \alpha:$}

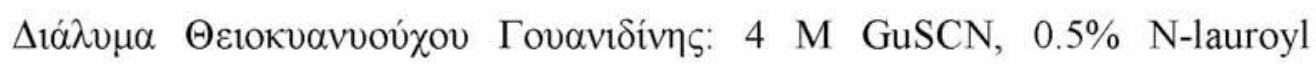
sarcosine, $1 \mathrm{mM}$ dithiotreitol, $25 \mathrm{mM}$ sodium citrate (Merck, Germany).

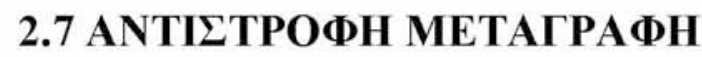

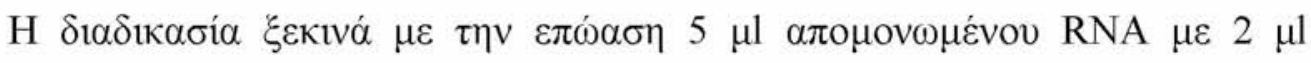

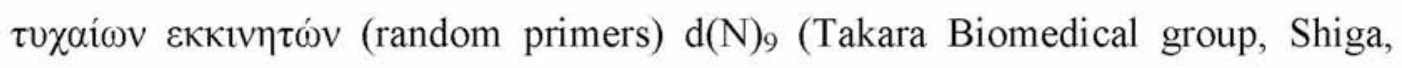

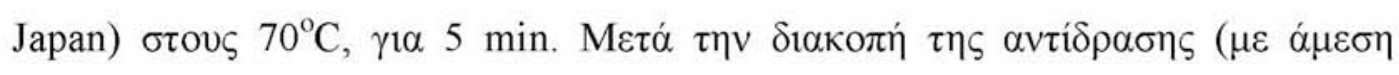

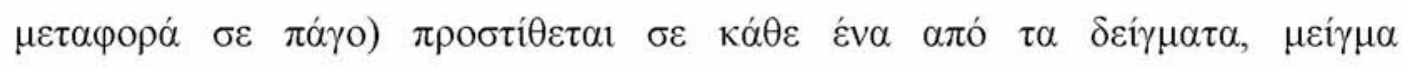

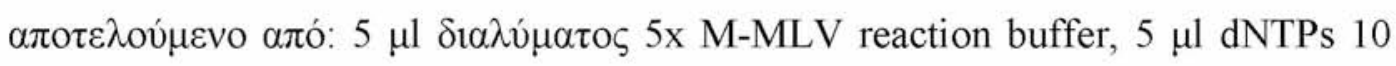

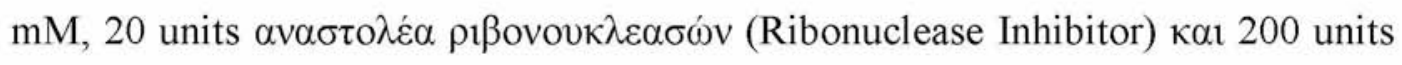
$\alpha v \tau i ́ \sigma \tau \rho \circ \eta \varsigma \mu \varepsilon \tau \alpha \gamma \rho \alpha \varphi \alpha ́ \sigma \eta \varsigma$ M-MLV Reverse Transcriptase (Promega, USA).

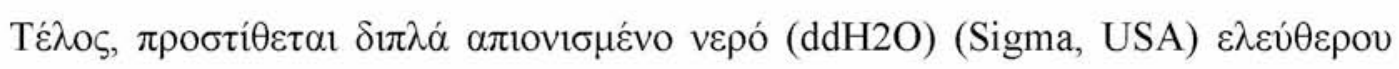

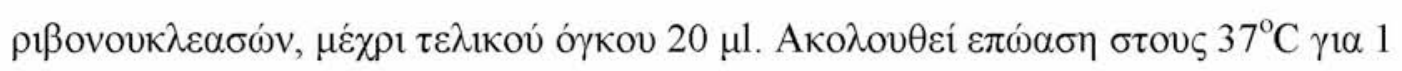

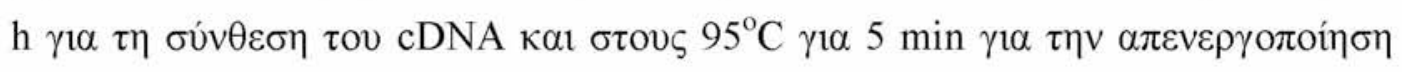
$\tau \eta \varsigma \alpha v \tau i \sigma \tau \rho \circ \varphi \eta \varsigma \mu \varepsilon \tau \alpha \gamma \rho \alpha \varphi \alpha ́ \sigma \eta \zeta$.

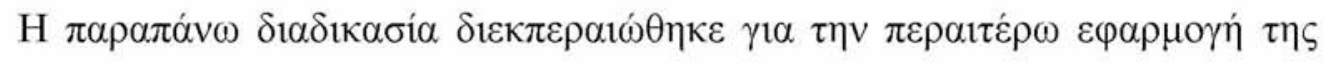

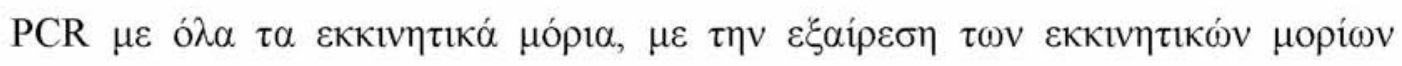




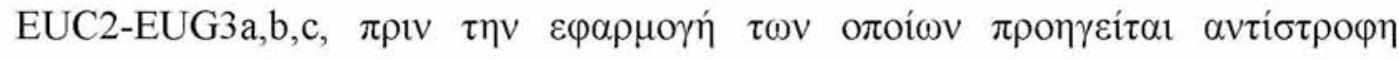

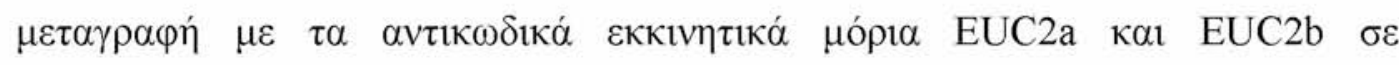

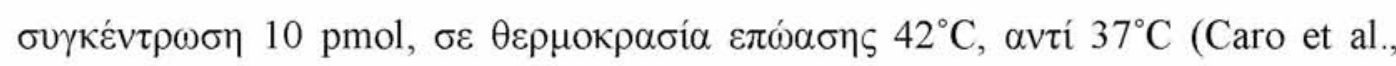
2001).

\subsection{EKKINHTIKA MOPIA}

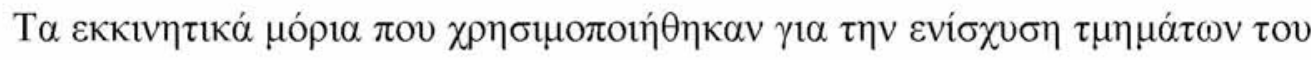

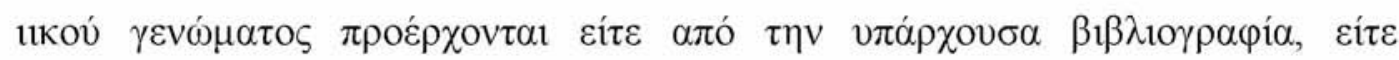

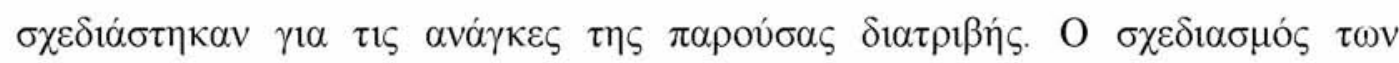

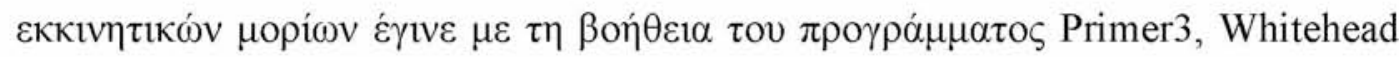
Institute (http://www.genome.wi.mit.edu/genomesoftware/other/).

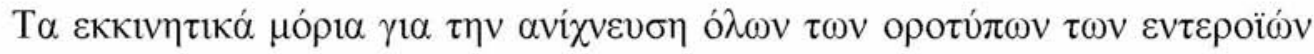

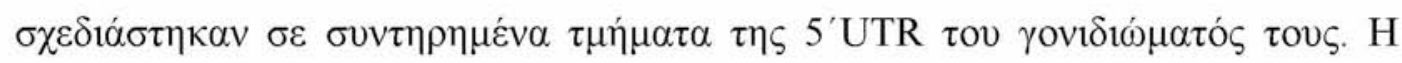

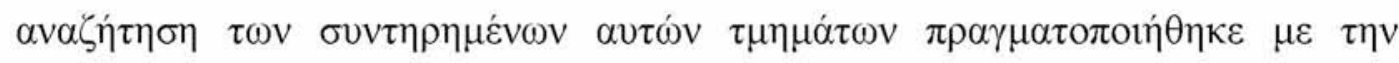

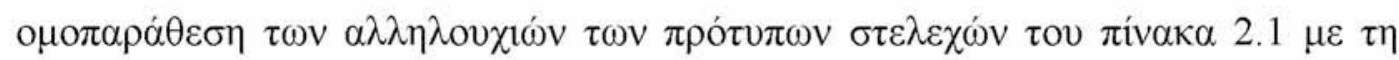

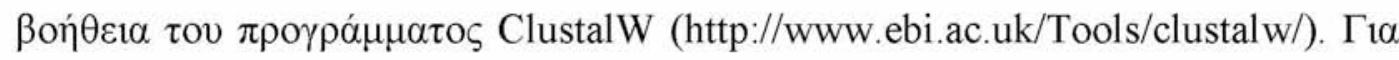

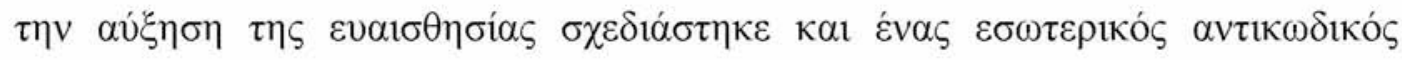

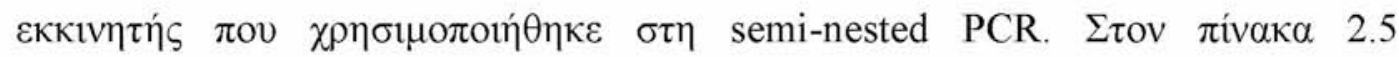

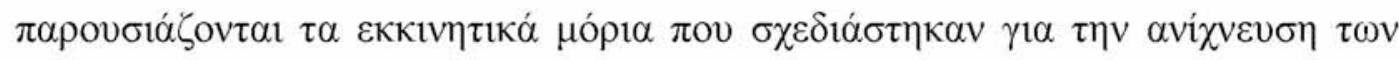

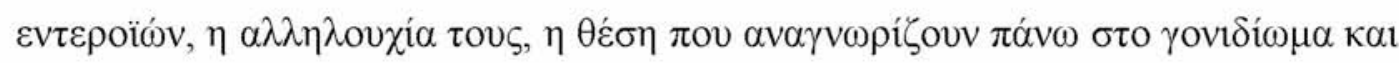

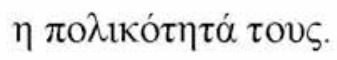

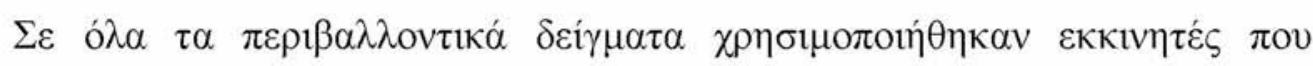

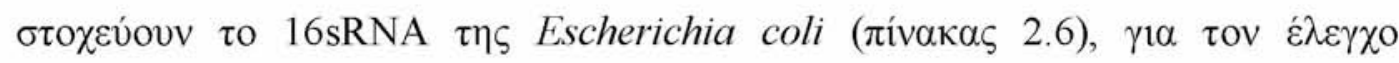

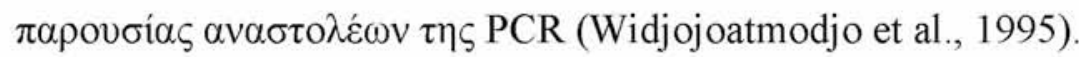

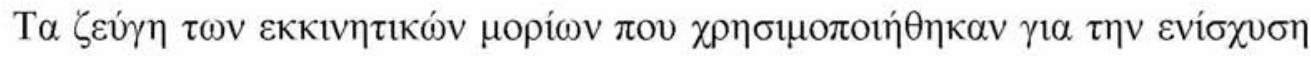

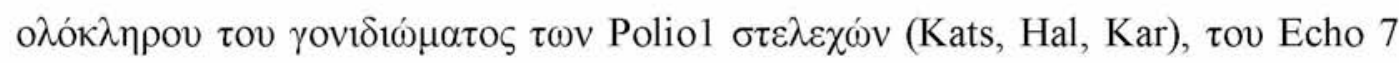
(LR11F7), $\tau$ ov Echo 3 (LR31G7) $\kappa$ al $\tau \omega v$ Echo 6 (LR51A5, LR61G3)

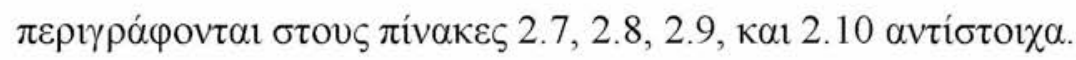




\begin{tabular}{|c|c|c|c|}
\hline Оуона & 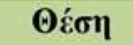 & 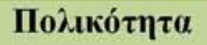 & 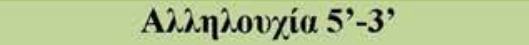 \\
\hline TS1 & $66-85$ & Sense & TACC(CT)TTGTACGCCTGTTTT \\
\hline TS3 & $582-599$ & Antisense & TTCTCACCATAAGCAGCC \\
\hline HEV-C-9 & $537-558$ & Antisense & GGACACCCAAAGTAGTCGGTTC \\
\hline
\end{tabular}

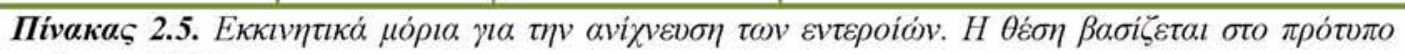

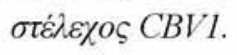

\begin{tabular}{|c|c|c|c|}
\hline Ovона & 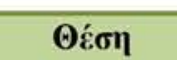 & 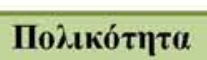 & 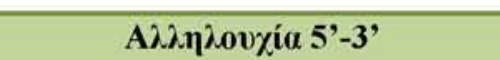 \\
\hline P11P & $1173-1192$ & Sense & GAGGAAGGTGGGGATGACGT \\
\hline P13P & 1370-1389 & Antisense & AGGCCCGGGAACGTATTCAC \\
\hline
\end{tabular}

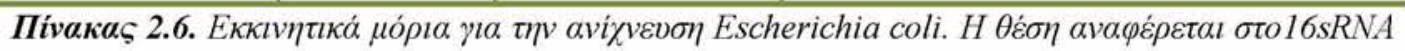
$\tau \eta \varsigma$ Escherichia coli . 


\begin{tabular}{|c|c|c|c|c|}
\hline Оvона & $\Theta \varepsilon \dot{\sigma} \eta$ & 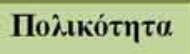 & 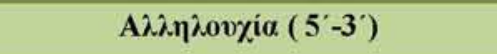 & Вıріноүрафік \\
\hline 72437 & $1-20$ & Sense & TTAAAACAGCTCTGGGGTTG & Mulders et al. 1999 \\
\hline 216616 & $545-565$ & Antisense & GGAACACGGACACCCAAAGTA & Blomqvist et al. 1999 \\
\hline $\mathrm{Z752}$ & $752-774$ & Sense & CAGGTCTCATCCCAGAAAGTTG & 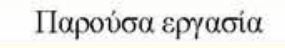 \\
\hline Z1461 & $1461-1483$ & Antisense & GGGTGTCGTTTGGTTATTGTCT & 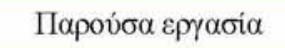 \\
\hline Z1196 & $1196-1216$ & Sense & ACCGAACACACTAAGGGACA & 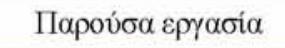 \\
\hline Z1941 & $1941-1963$ & Antisense & GGTAGCACTCAAATCAAAAGGA & 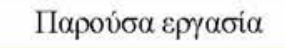 \\
\hline $\mathrm{Z} 1814$ & $1814-1836$ & Sense & GACAACTTTCAGTCTCCGTGTG & 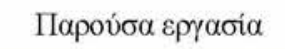 \\
\hline $\mathrm{Z} 2478$ & $2478-2500$ & Antisense & GCCTTGTGCTATTGCTTTTTGT & 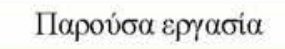 \\
\hline $\mathrm{Z} 2378$ & $2378-2400$ & Sense & GAAATGGATATTCTCGGGTTTG & П $\alpha \rho \circ v \sigma \alpha \alpha \varepsilon \rho \gamma \alpha \sigma i \alpha$ \\
\hline Z3021 & $3021-3043$ & Antisense & TTGATGGGTTTGATGAAGTCTG & 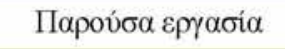 \\
\hline EUC2 & $4413-4433$ & Antisense & TTTGCACTTGAACTGTATGTA & Caro et al. 2001 \\
\hline EUC2a & $4428-4448$ & Antisense & GGTTCAATACGGCATTTG & Caro et al. 2001 \\
\hline EUC2b & $4428-4448$ & Antisense & GGTTCAATACGGTGTTTGCT & Caro et al. 2001 \\
\hline EUG3a & $2946-2965$ & Sense & TGGCAAACTTCCWCCAACCC & Caro et al. 2001 \\
\hline EUG3b & $2946-2965$ & Sense & TGGCAAACATCTTCMAATCC & Caro et al. 2001 \\
\hline EUG3c & $2946-2965$ & Sense & TGGCAGACTTCAACHAACCC & Caro et al. 2001 \\
\hline ZA202 & $4202-4223$ & Sense & CATTGACTGGCTGAAGGAGAG & 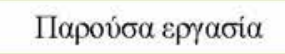 \\
\hline Z4809 & $4809-4831$ & Antisense & GGCTACAGTGGGTGGAGTTATT & 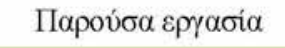 \\
\hline Z4677 & $4677-4697$ & Sense & AGAACCCAGAAGGTGCTGAC & 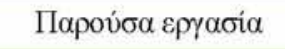 \\
\hline Z5309 & $5309-5331$ & Antisense & TGTCATTGCTCTGTTGATGTTG & 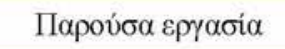 \\
\hline $\mathrm{Z} 5228$ & $5228-5250$ & Sense & GAACATTTCAAGTCAGGTCCAA & 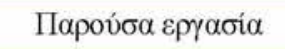 \\
\hline Z5890 & $5890-5912$ & Antisense & TGCCAGTACAAGTGATGATTCC & 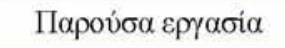 \\
\hline UG16 & $5921-5940$ & Sense & GTTGGTGGGAACGGTTCACA & Guillot et al, 2000 \\
\hline $\mathrm{UC} 12$ & $6516-6494$ & Antisense & TCAATTAGTCTGGATTTTCCCTG & Guillot et al, 2000 \\
\hline Z6206 & $6206-6228$ & Sense & GACCACTATGCTGGACAACTCA & 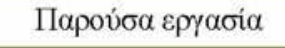 \\
\hline Z6643 & $6643-6665$ & Antisense & AGCACTGGGATCTTACTCCAAA & 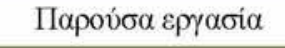 \\
\hline $\mathrm{S}_{2} 107$ & $6541-6562$ & Sense & TCCCAGTGCTAATGGAAGAGA & Paximadi et al., 2006 \\
\hline $\mathrm{S}_{2} 872$ & $7421-7441$ & Antisense & ACAACAGCATGACCCAATCC & Paximadi et al., 2006 \\
\hline
\end{tabular}

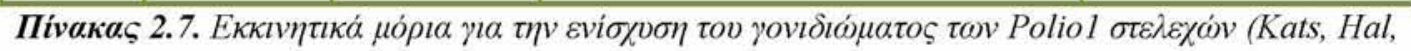

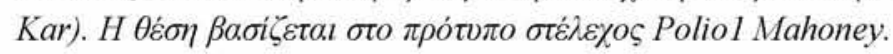




\begin{tabular}{|c|c|c|c|c|}
\hline Ovo $\mu \alpha$ & 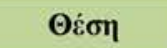 & 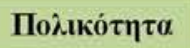 & 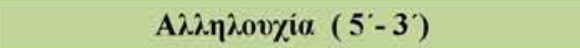 & Вıрілюүрафік \\
\hline 72437 & $001-020$ & Sense & TTAAAACAGCTCTGGGGTTG & Mulders et al., 1999 \\
\hline 216616 & $545-565$ & Antisense & GAAACACGGACACCCAAAGTA & Blomqvist et al. 1999 \\
\hline 0340F & $310-333$ & Sense & TAGATCAGGCYGATGAGTCACCGC & Lukashev et al., 2005 \\
\hline $1200 \mathrm{R}$ & $1177-1196$ & Antisense & GGGAATTTCCACCACCACCC & Lukashev et al., 2005 \\
\hline AL897 & $897-1007$ & Sense & GGAAGTTTACCGAGCCAGTT & 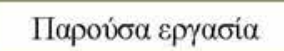 \\
\hline AR3091 & 3071-3091 & Antisense & TTGTTCAGAGCGTTGTAGGC & 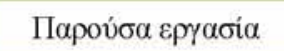 \\
\hline AL939 & $939-959$ & Sense & TGCCTGCTCTAAACTCACCA & 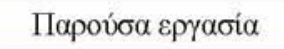 \\
\hline AR2751 & $2731-2751$ & Antisense & GCACCATCCTTCTTGCATTT & 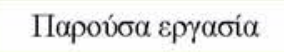 \\
\hline AL1632 & $1632-1652$ & Sense & GCACCCTTGGATTACACTGC & 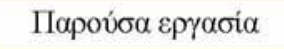 \\
\hline AR2180 & $2160-2180$ & Antisense & CATTGCCTGTTTCCTGTTCG & 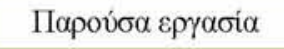 \\
\hline 292 & $2612-2627$ & Sense & MIGCIGYIGARACNGG & Oberste et al., 2003 \\
\hline 222 & $2969-2951$ & Antisense & CICCIGGIGGIAYRWACAT & Oberste et al., 2003 \\
\hline ZL2550 & $2550-2570$ & Sense & AGACGGGGCATACATCACAG & 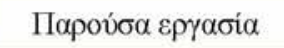 \\
\hline ZR3040 & $3040-3060$ & Antisense & TACCCCATTTTGCGAGAAGT & 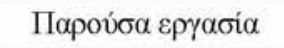 \\
\hline EUG3a & $2946-2965$ & Sense & TGGCAAACTTCCWCCAACCC & Caro et al., 2001 \\
\hline EUG3b & $2946-2965$ & Sense & TGGCAAACATCTTCMAATCC & Caro et al., 2001 \\
\hline EUG3c & $2946-2965$ & Sense & TGGCAGACTTCAACHAACCC & Caro et al., 2001 \\
\hline EUC2 & $4413-4433$ & Antisense & TTTGCACTTGAACTGTATGTA & Caro et al., 2001 \\
\hline EUC2a & $4428-4448$ & Antisense & GGTTCAATACGGCATTTG & Caro et al., 2001 \\
\hline EUC2b & $4428-4448$ & Antisense & GGTTCAATACGGTGTTTGCT & Caro et al., 2001 \\
\hline CHR1 & $4284-4308$ & Sense & CNTCHCARAGTGAYCARGARCARYT & Kottaridi et al., 2007 \\
\hline $\mathrm{CHR} 2$ & $5084-5081$ & Antisense & GTAYACYGGTGGWCCYTGRAAKA & Kottaridi et a.,12007 \\
\hline AL4872 & $4872-4892$ & Sense & CCTGTGATGAGGAGTGTTGC & 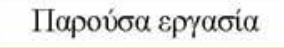 \\
\hline AR5275 & $5255-5275$ & Antisense & GAGACAAACGTGGTGAGTGC & 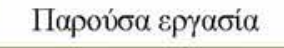 \\
\hline $\mathrm{CHR} 3$ & $5047-5065$ & Sense & CIACYCTWGARGCRCTVTT & 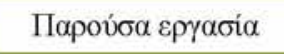 \\
\hline $\mathrm{CHR} 4$ & $5860-5841$ & Antisense & GACRTGAGIACHCCRCCRCA & 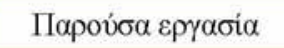 \\
\hline $5850 \mathrm{~F}$ & $5837-5859$ & Sense & CAGTGYGGIGGIGTICTCATGTC & Lukashev et al., 2003 \\
\hline $6500 \mathrm{R}$ & $6506-6531$ & Antisense & AGRTTGCCAAAYGTYTGYCTCATTGC & Lukashev et al., 2003 \\
\hline CHR5 & $6485-6507$ & Sense & ATCCAGYTTGAAYGAYTCIGIRG & 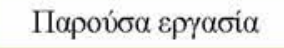 \\
\hline CHR6 & $7227-7250$ & Antisense & GAAYTCYTCRTAYTCKTGCTCYCC & 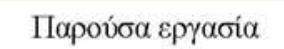 \\
\hline
\end{tabular}

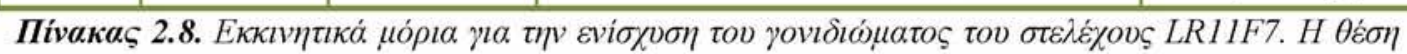

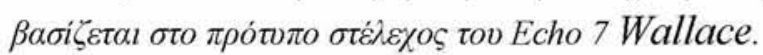




\begin{tabular}{|c|c|c|c|c|}
\hline Ovo $\mu \alpha$ & 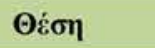 & 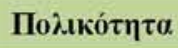 & 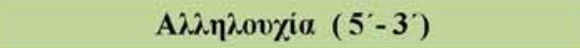 & Вıріноүрафік \\
\hline 72437 & $001-020$ & Sense & TTAAAACAGCTCTGGGGTTG & Mulders et al., 1999 \\
\hline 216616 & $545-565$ & Antisense & GAAACACGGACACCCAAAGTA & Blomqvist et al., 1999 \\
\hline 0340F & $310-333$ & Sense & TAGATCAGGCYGATGAGTCACCGC & Lukashev et al., 2005 \\
\hline $1200 \mathrm{R}$ & $1177-1196$ & Antisense & GGGAATTTCCACCACCACCC & Lukashev et al., 2005 \\
\hline BL870 & $870-890$ & Sense & CGACAGGATTTCACACAGGA & 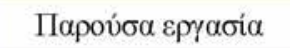 \\
\hline BR3231 & $3231-3211$ & Antisense & GCTTTTCACATACGGGCTAA & 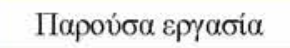 \\
\hline BL1369 & $1369-1389$ & Sense & GAGGTTGTCGCAGCTTCTCT & 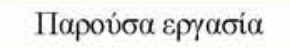 \\
\hline $\mathrm{BR} 2580$ & $2580-2560$ & Antisense & GGGAACCACTTGTGAGGTGT & П $\alpha \rho o v \sigma \alpha \alpha \varepsilon \gamma \alpha \sigma i \alpha$ \\
\hline 292 & $2612-2627$ & Sense & MIGCIGYIGARACNGG & Oberste et al., 2003 \\
\hline 222 & 2969-2951 & Antisense & CICCIGGIGGIAYRWACAT & Oberste et al., 2003 \\
\hline EUG3a & $2946-2965$ & Sense & TGGCAAACTTCCWCCAACCC & Caro et al., 2001 \\
\hline EUG3b & $2946-2965$ & Sense & TGGCAAACATCTTCMAATCC & Caro et al., 2001 \\
\hline EUG3c & $2946-2965$ & Sense & TGGCAGACTTCAACHAACCC & Caro et al., 2001 \\
\hline EUC2 & $4413-4433$ & Antisense & TTTGCACTTGAACTGTATGTA & Caro et al., 2001 \\
\hline EUC2a & $4428-4448$ & Antisense & GGTTCAATACGGCATTTG & Caro et al., 2001 \\
\hline EUC2b & $4428-4448$ & Antisense & GGTTCAATACGGTGTTTGCT & Caro et al., 2001 \\
\hline CHR1 & $4284-4308$ & Sense & CNTCHCARAGTGAYCARGARCARYT & Kottaridi et al., 2007 \\
\hline $\mathrm{CHR} 2$ & $5084-5081$ & Antisense & GTAYACYGGTGGWCCYTGRAAKA & Kottaridi et al., 2007 \\
\hline BL4709 & $4709-4729$ & Sense & TACATCCCCATTTGTGTTGG & 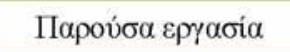 \\
\hline BR6063 & $6063-6043$ & Antisense & TGCTGGCTCCTTGTTACCTT & П $\alpha \rho \circ v ́ \sigma \alpha \varepsilon \rho \gamma \alpha \sigma i \alpha$ \\
\hline CHR3 & $5047-5065$ & Sense & CIACYCTWGARGCRCTVTT & 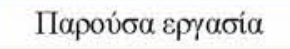 \\
\hline $\mathrm{CHR} 4$ & $5860-5841$ & Antisense & GACRTGAGIACHCCRCCRCA & 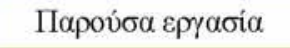 \\
\hline $5850 \mathrm{~F}$ & $5837-5859$ & Sense & CAGTGYGGIGGIGTICTCATGTC & Lukashev et al., 2003 \\
\hline $6500 \mathrm{R}$ & $6531-6506$ & Antisense & AGRTTGCCAAAYGTYTGYCTCATTGC & Lukashev et al., 2003 \\
\hline CHR5 & $6485-6507$ & Sense & ATCCAGYTTGAAYGAYTCIGIRG & 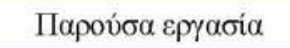 \\
\hline CHR6 & $7250-7227$ & Antisense & GAAYTCYTCRTAYTCKTGCTCYCC & 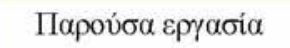 \\
\hline
\end{tabular}

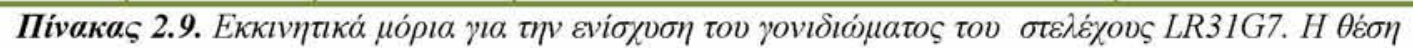

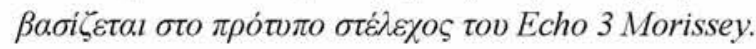




\begin{tabular}{|c|c|c|c|c|}
\hline Ovона & 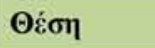 & 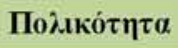 & 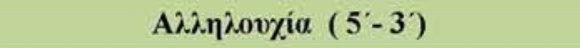 & 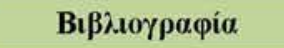 \\
\hline 72437 & $001-020$ & Sense & TTAAAACAGCTCTGGGGTTG & Mulders et al., 1999 \\
\hline 216616 & $545-565$ & Antisense & GAAACACGGACACCCAAAGTA & Blomqvist et al., 1999 \\
\hline $0340 \mathrm{~F}$ & $310-333$ & Sense & TAGATCAGGCYGATGAGTCACCGC & Lukashev et al., 2005 \\
\hline $1200 \mathrm{R}$ & $1177-1196$ & Antisense & GGGAATTTCCACCACCACCC & Lukashev et al., 2005 \\
\hline CL995 & $995-1015$ & Sense & CATCACTCTCGGCAACTCAA & 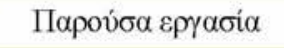 \\
\hline CR3152 & $3132-3152$ & Antisense & CTTGCTGGTAATCGGGCTAA & 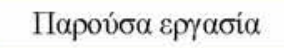 \\
\hline CL1653 & $1653-1676$ & Sense & CATCAACCTACATCCCAATAACC & 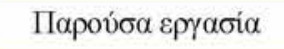 \\
\hline CR2648 & $2626-2648$ & Antisense & GAAGTTTTCCACTGACGATTCC & 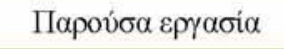 \\
\hline 292 & $2612-2627$ & Sense & MIGCIGYIGARACNGG & Oberste et al., 2003 \\
\hline 222 & $2969-2951$ & Antisense & CICCIGGIGGIAYRWACAT & Oberste et al., 2003 \\
\hline EUG3a & $2946-2965$ & Sense & TGGCAAACTTCCWCCAACCC & Caro et al., 2001 \\
\hline EUG3b & $2946-2965$ & Sense & TGGCAAACATCTTCMAATCC & Caro et al., 2001 \\
\hline EUG3c & $2946-2965$ & Sense & TGGCAGACTTCAACHAACCC & Caro et al., 2001 \\
\hline EUC2 & $4413-4433$ & Antisense & TTTGCACTTGAACTGTATGTA & Caro et al., 2001 \\
\hline EUC2a & $4428-4448$ & Antisense & GGTTCAATACGGCATTTG & Caro et al., 2001 \\
\hline EUC2b & $4428-4448$ & Antisense & GGTTCAATACGGTGTTTGCT & Caro et al., 2001 \\
\hline CL4058 & $4058-4078$ & Sense & CAAAACAACGGGTGGCTAAA & 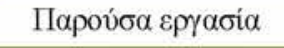 \\
\hline CR6052 & $6032-6952$ & Antisense & GCTGGTTCCTTATTGCCTTC & 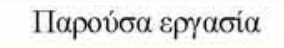 \\
\hline CL4703 & $4703-4723$ & Sense & TCGCCATTTGTTCTTGCTTC & 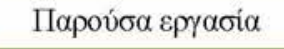 \\
\hline CR5487 & $5467-5487$ & Antisense & CCGCCCACCTGTCATAGATA & 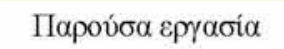 \\
\hline CHR3 & $5047-5065$ & Sense & CIACYCTWGARGCRCTVTT & 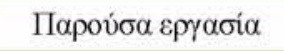 \\
\hline CHR4 & $5860-5841$ & Antisense & GACRTGAGIACHCCRCCRCA & 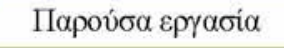 \\
\hline $5850 \mathrm{~F}$ & $5837-5859$ & Sense & CAGTGYGGIGGIGTICTCATGTC & Lukashev et al., 2003 \\
\hline $6500 \mathrm{R}$ & $6531-6506$ & Antisense & AGRTTGCCAAAYGTYTGYCTCATTGC & Lukashev et al., 2003 \\
\hline CL6333 & $6333-6364$ & Sense & CCAAAGACCTCACCAAACTGA & П $\alpha \rho o v \sigma \alpha \alpha \varepsilon \rho \gamma \alpha \sigma i \alpha$ \\
\hline CR6789 & $6768-6789$ & Antisense & TGCCACCTCTCACAAAGTAGT & 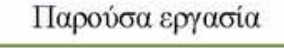 \\
\hline CHR5 & $6485-6507$ & Sense & ATCCAGYTTGAAYGAYTCIGIRG & 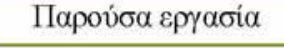 \\
\hline CHR6 & $7250-7227$ & Antisense & GAAYTCYTCRTAYTCKTGCTCYCC & 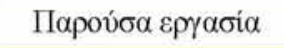 \\
\hline
\end{tabular}

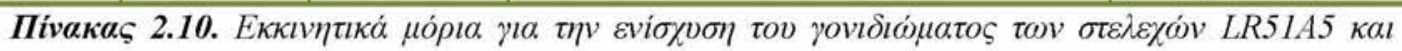

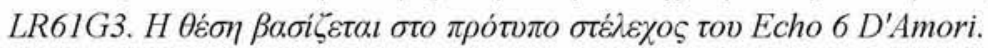




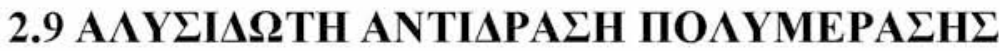

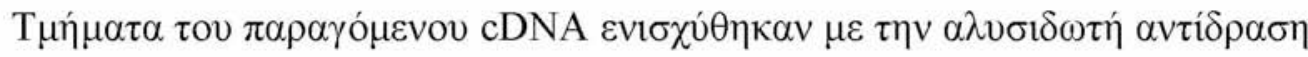

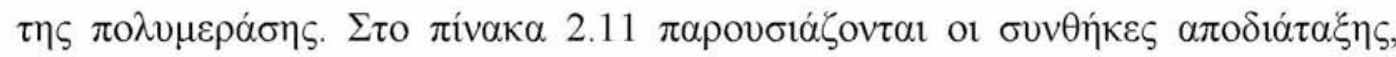

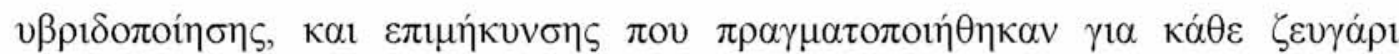

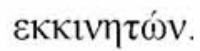

\subsubsection{PCR}

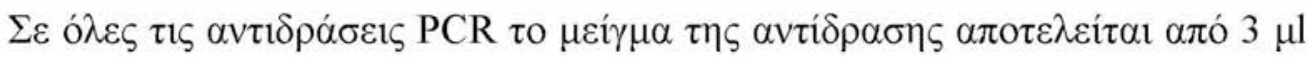

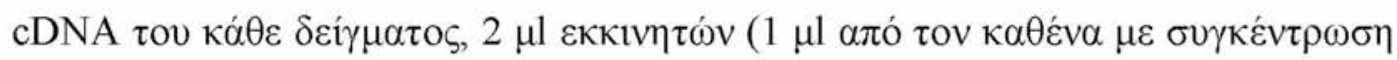

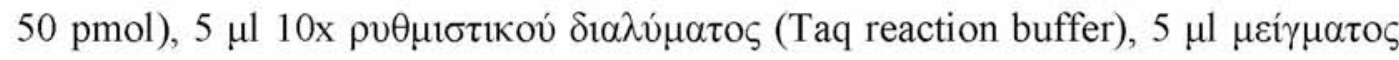

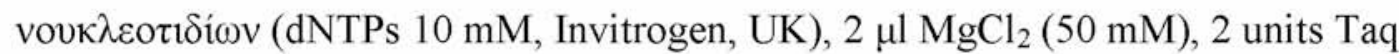

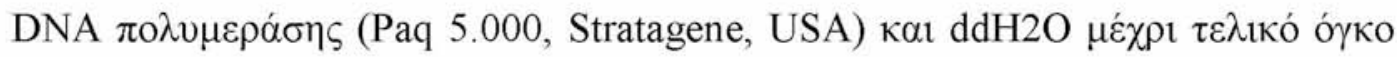

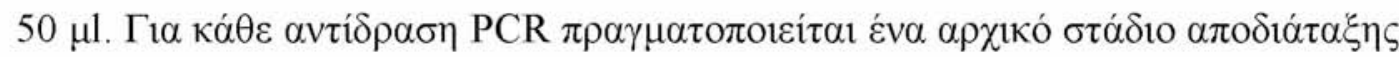

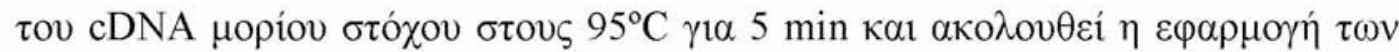

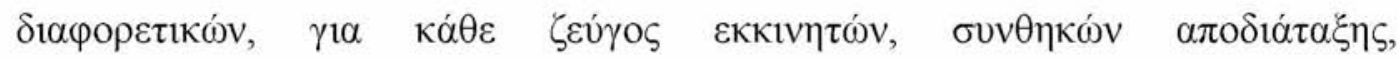

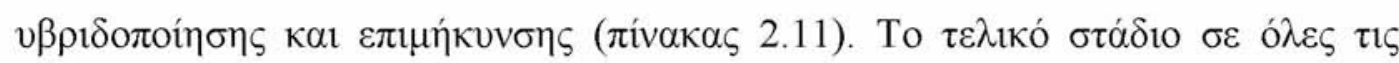

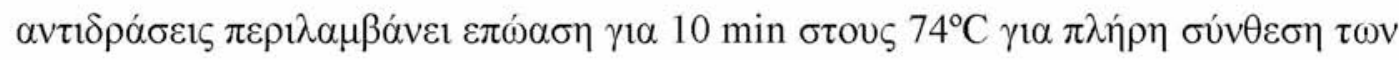

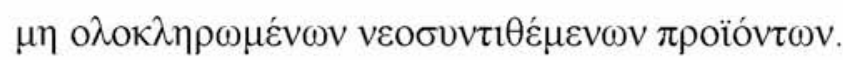

\subsection{2 semi-nested PCR}

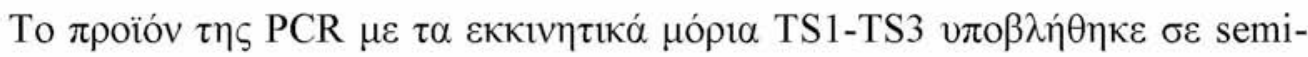

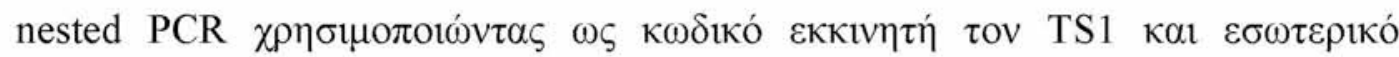

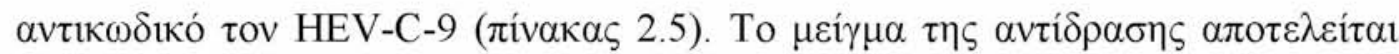

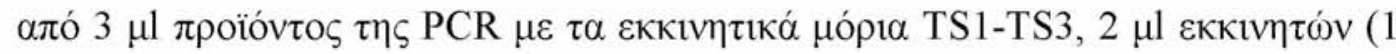

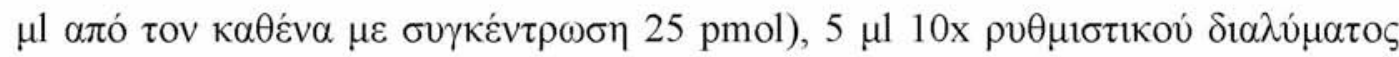

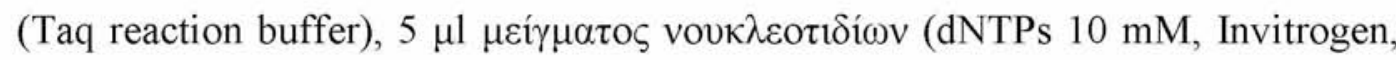
UK), $2 \mu \mathrm{MgCl}_{2}$ (50 mM), 2 units Taq DNA $\pi 0 \lambda v \mu \varepsilon \rho \alpha ́ \sigma \eta \varsigma$ (Paq 5.000,

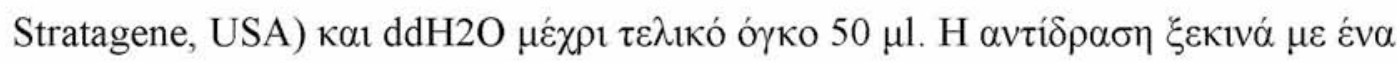

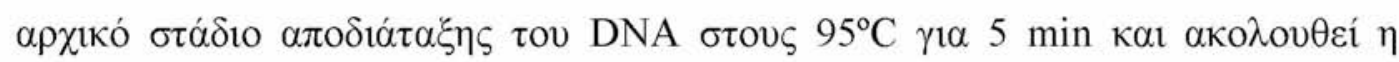

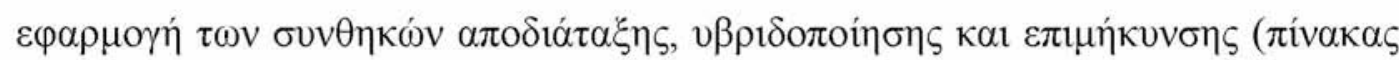
2.11). To $\tau \varepsilon \lambda 1$ кó $\sigma \tau \alpha ́ \delta 10 ~ \pi \varepsilon \rho ı \lambda \alpha \mu \beta a ́ v \varepsilon 1 ~ \varepsilon \pi \omega ́ \alpha \sigma \eta ~ \gamma 1 \alpha 10 \min \sigma \tau o v \varsigma 74^{\circ} \mathrm{C}$. 


\begin{tabular}{|c|c|c|}
\hline 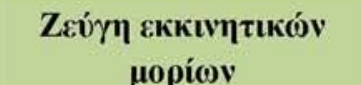 & \multicolumn{2}{|c|}{ 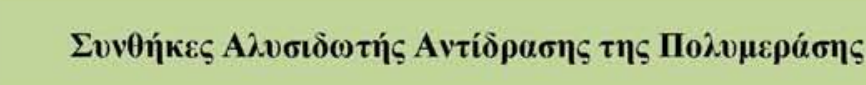 } \\
\hline $\begin{array}{l}72437-21661 \\
\text { UG16-UC12 }\end{array}$ & 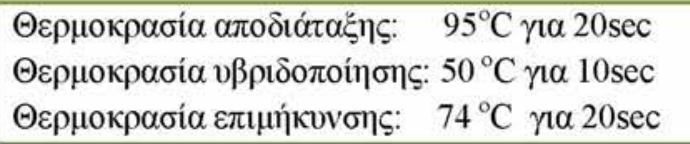 & 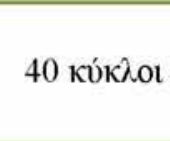 \\
\hline $\begin{array}{c}\text { Z752-Z1461 } \\
\text { Z1814-Z2478 } \\
\text { Z4202-Z4809 } \\
\text { Z6206-Z6643 } \\
\text { P11P-P13P }\end{array}$ & 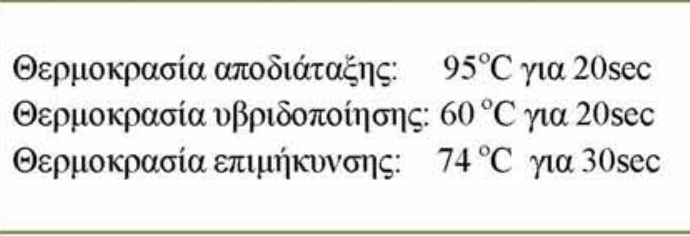 & 35 ки́кhot \\
\hline $\begin{array}{l}\text { Z1196-Z1941 } \\
Z 2378-Z 3021 \\
Z 4677-Z 5309 \\
Z 5228-Z 5890\end{array}$ & 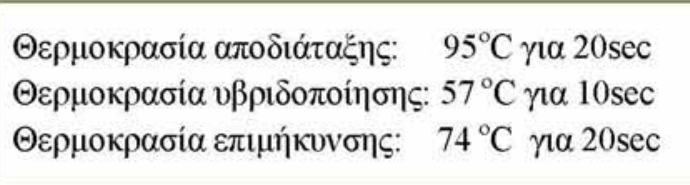 & 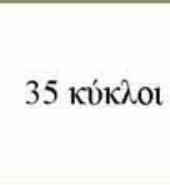 \\
\hline $\begin{array}{c}\text { EUC2- } \\
\text { EUG3a,EUG3b,EUG3c } \\
\text { CHR3-CHR4 } \\
\text { CHR5-CHR6 }\end{array}$ & 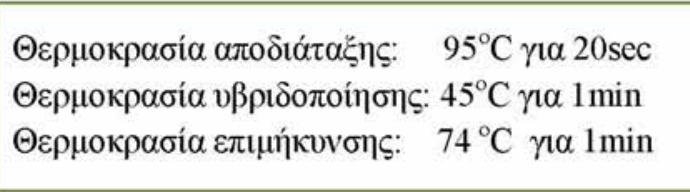 & 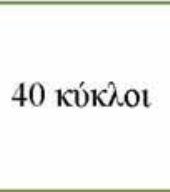 \\
\hline $\mathrm{S}_{2} 107-\mathrm{S}_{2} 872$ & 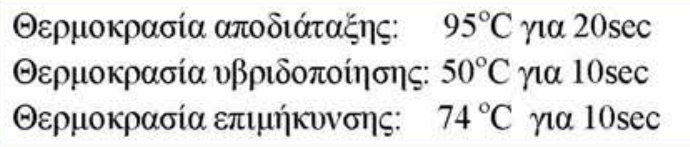 & 40 ки́кخ̀о \\
\hline $\begin{array}{l}\text { AL1632-AR2180 } \\
\text { ZL2550-ZR3040 }\end{array}$ & 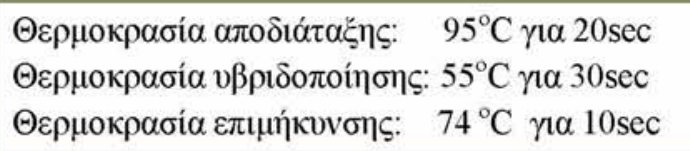 & 35 ки́кภо \\
\hline $\begin{array}{l}\text { AL939-AR2751 } \\
\text { BL1369-BR2580 } \\
\text { BL4709-BR6063 } \\
\text { CL4058-CR6052 }\end{array}$ & 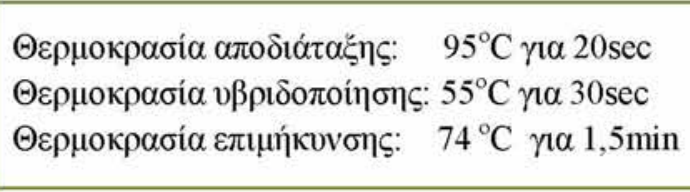 & 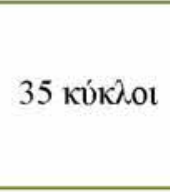 \\
\hline AL897-AR3091 & 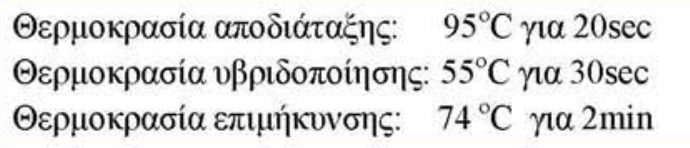 & 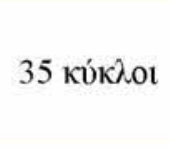 \\
\hline $\begin{array}{l}\text { AL4872-AR5275 } \\
\text { BL870-BR3231 } \\
\text { CL4703-CR5487 }\end{array}$ & 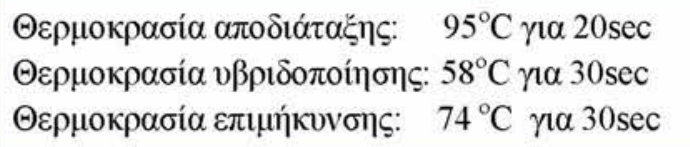 & 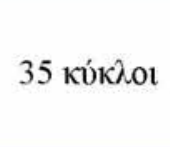 \\
\hline CL1653-CR2648 & 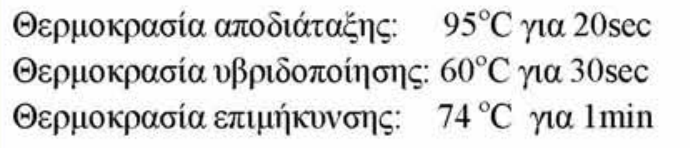 & 35 ки́кภо \\
\hline CL995-CR3152 & 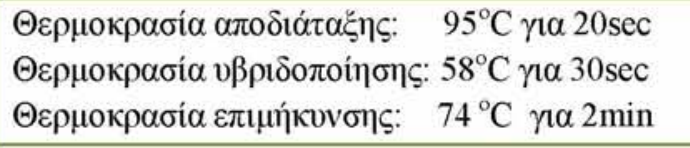 & 35 ки́кภо \\
\hline TS1-TS3 & 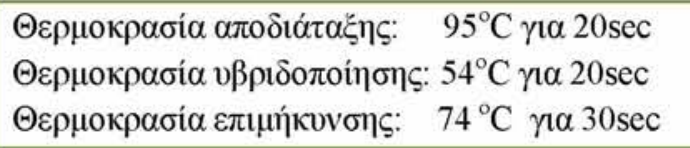 & 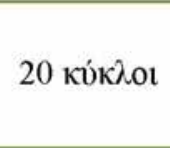 \\
\hline TS1-HEV-C-9 & 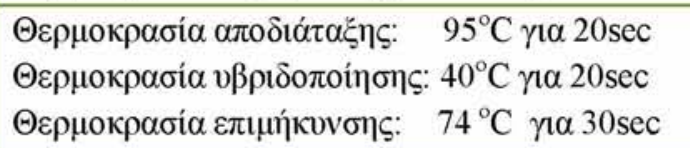 & 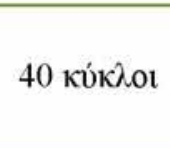 \\
\hline
\end{tabular}

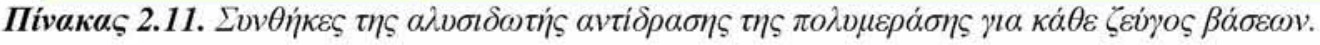




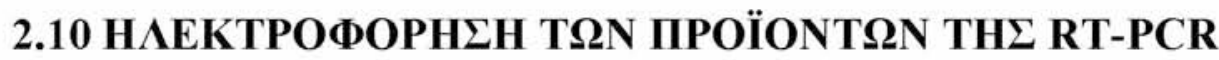

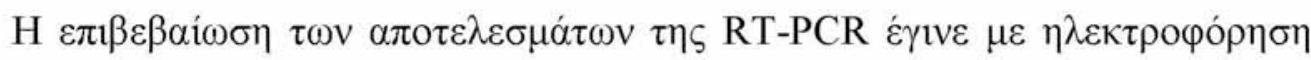

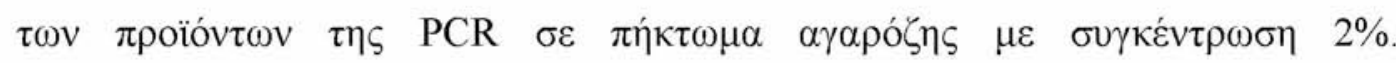

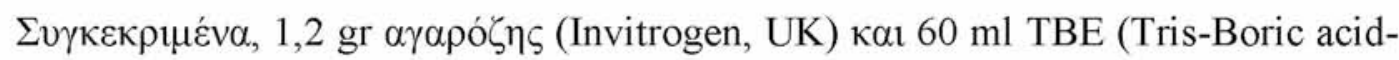

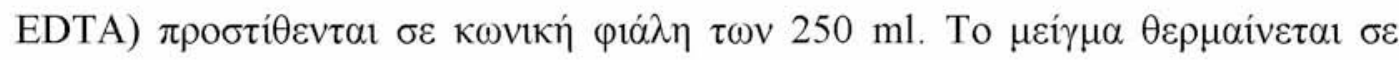

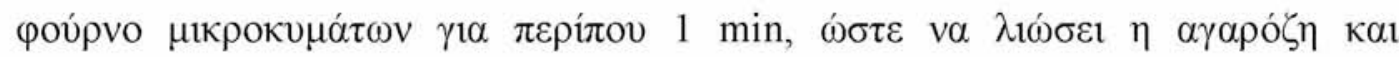

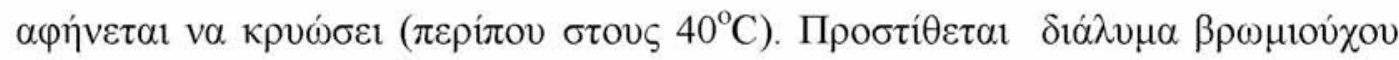

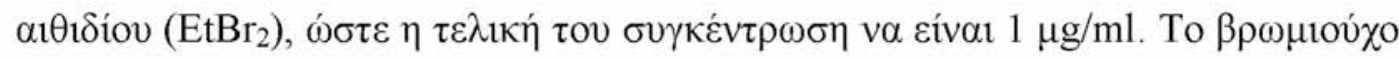

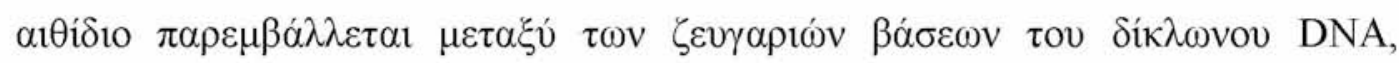

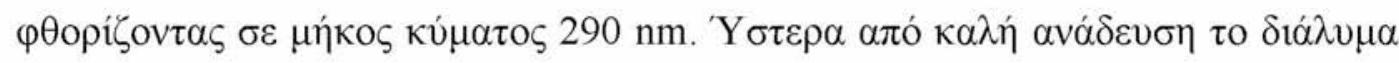

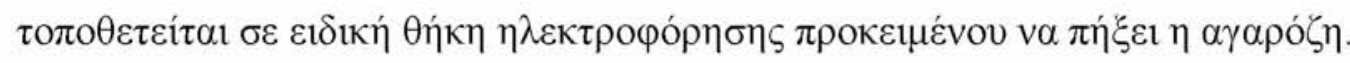

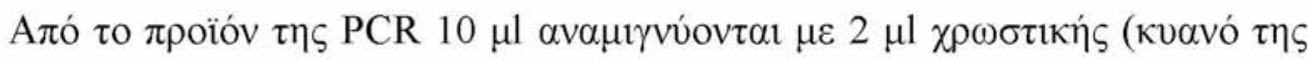

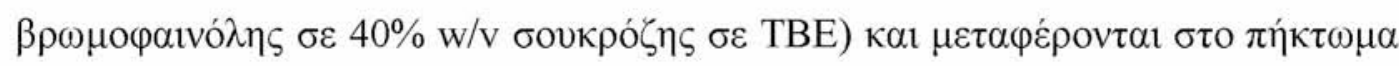

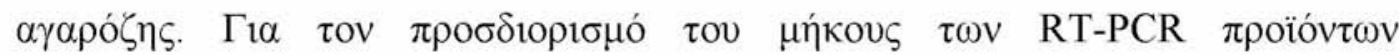

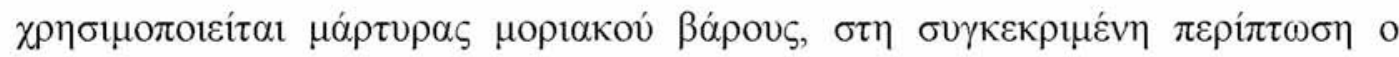

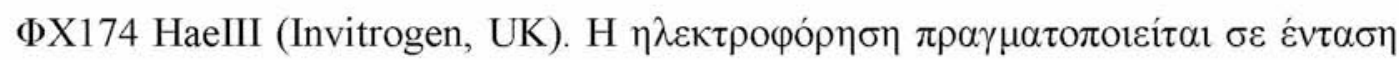

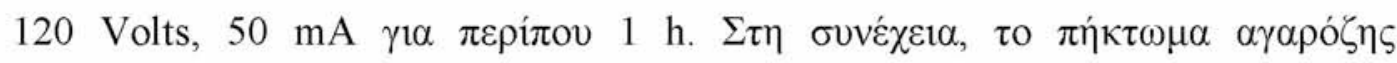

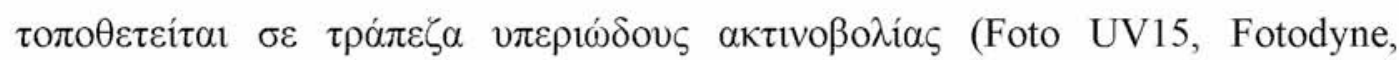
Hartland WI), ó

\section{$\Delta \mathbf{t} \lambda \hat{\nu} \mu \alpha \tau \alpha:$}

5x TBE: 5,4\% Tris Base, 2,75\% $\mathrm{H}_{3} \mathrm{BO}_{3}$ (Merck, Germany) $\kappa \alpha 110 \mu \mathrm{M}$

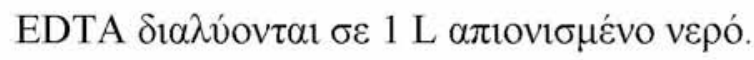

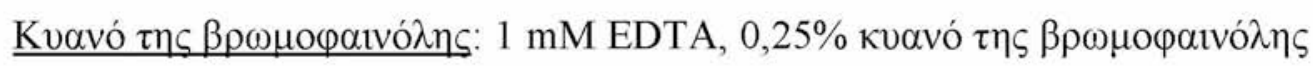

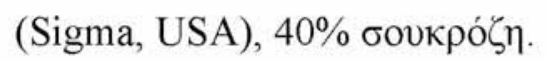

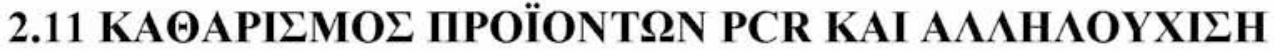

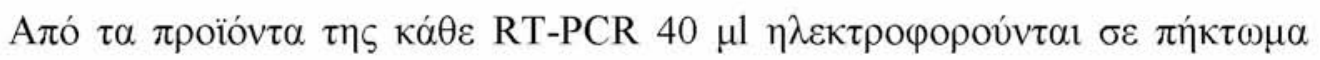

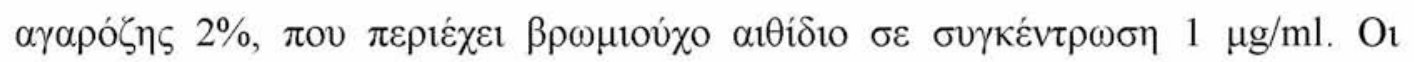

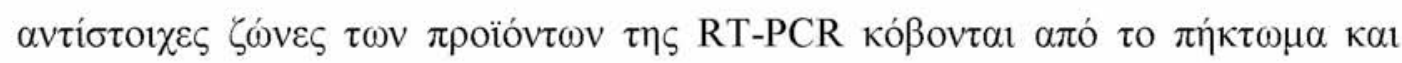

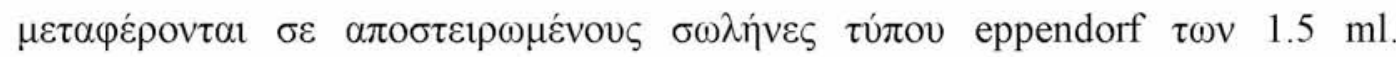

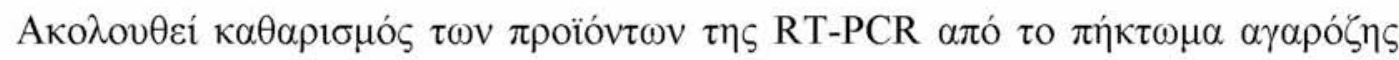

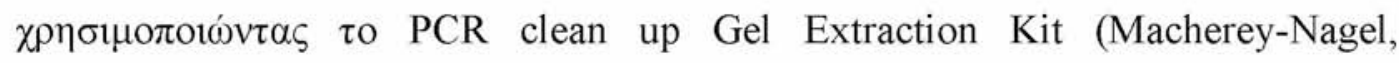

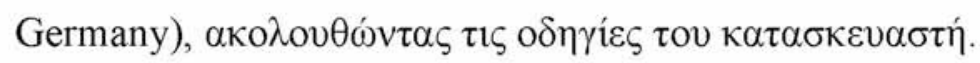




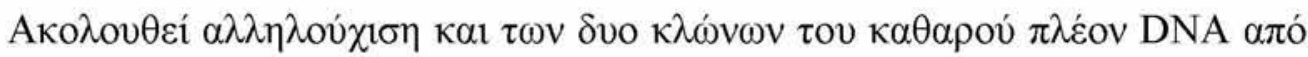

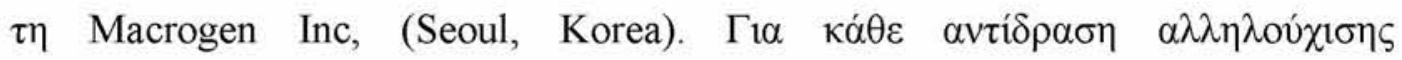

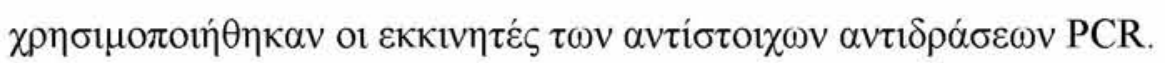

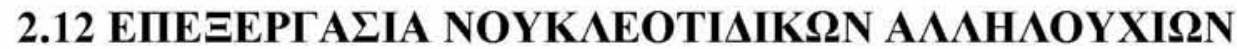

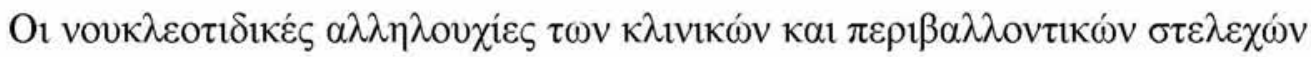

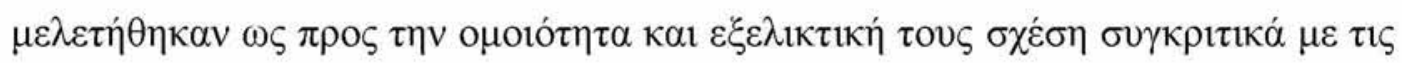

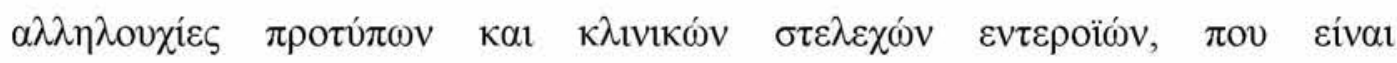

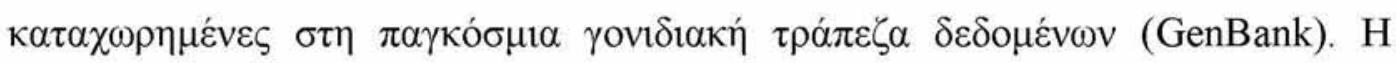

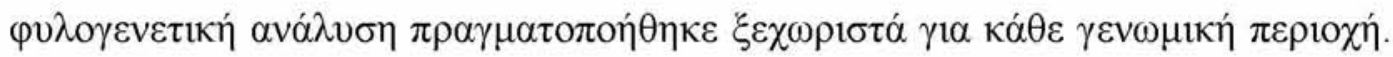

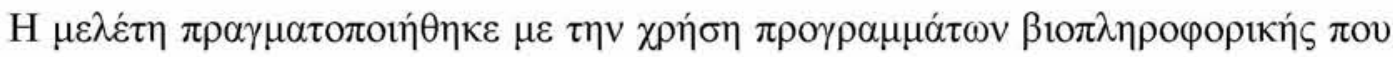

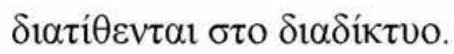

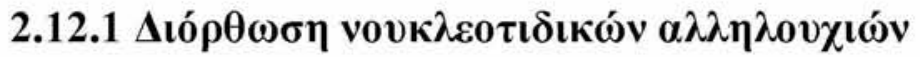

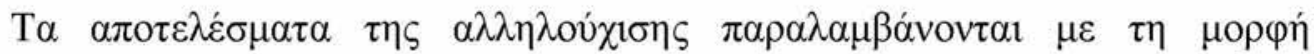

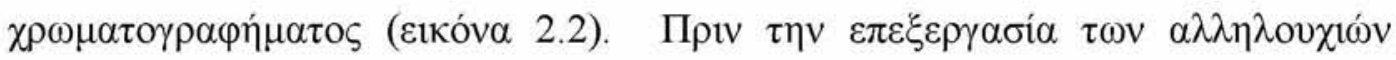

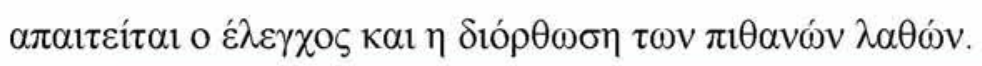

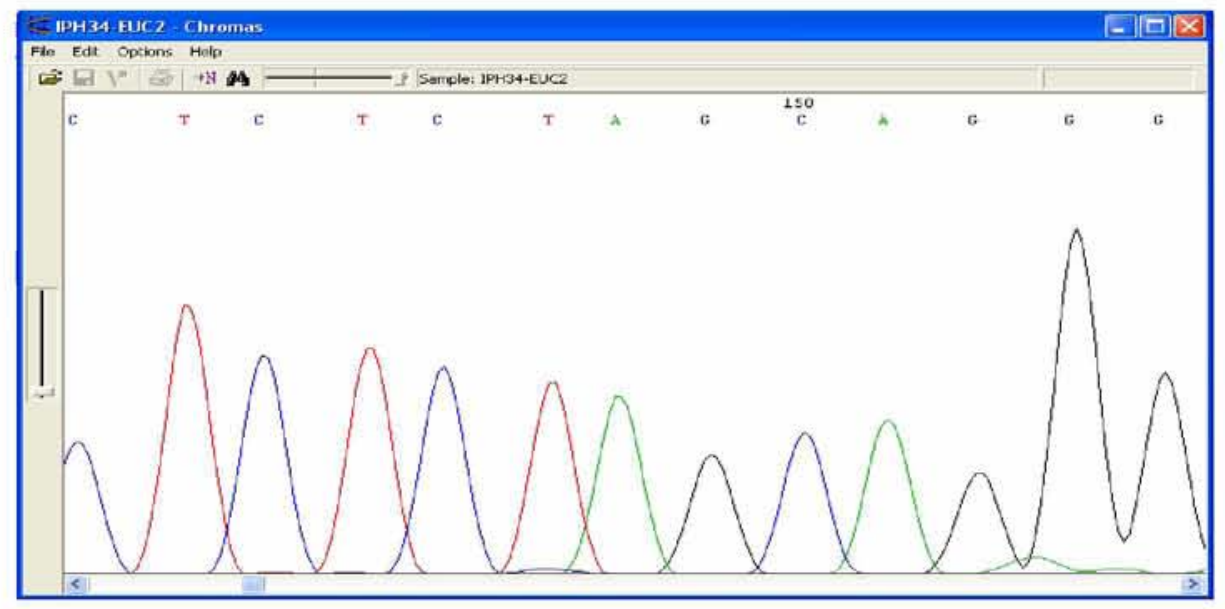

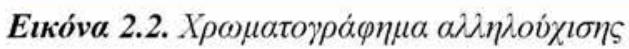

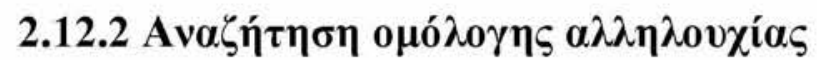

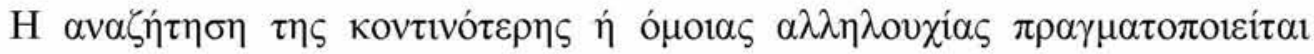

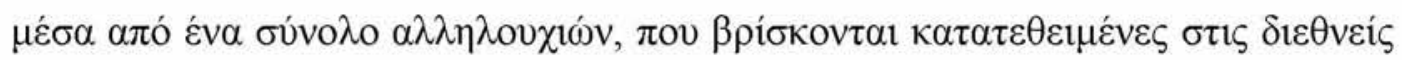

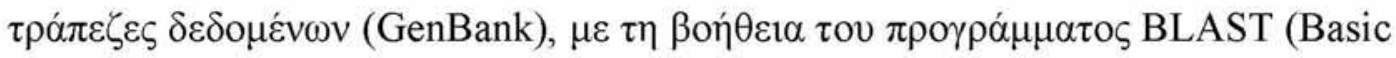

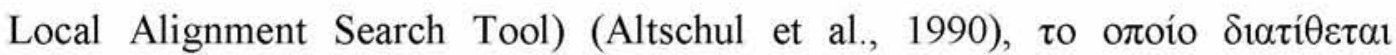

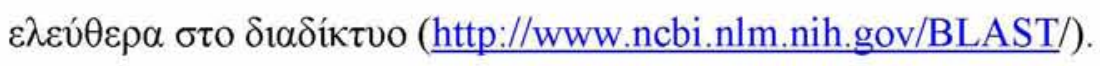




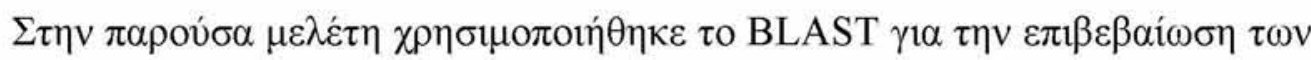

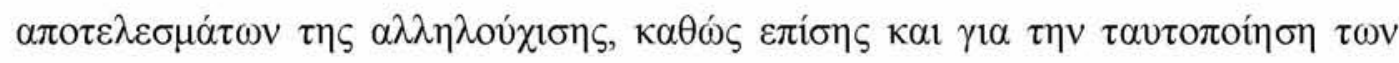

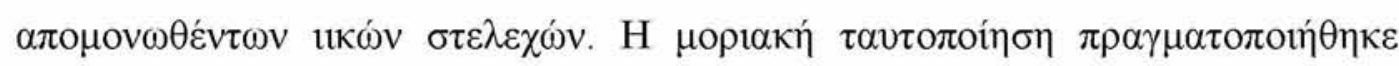

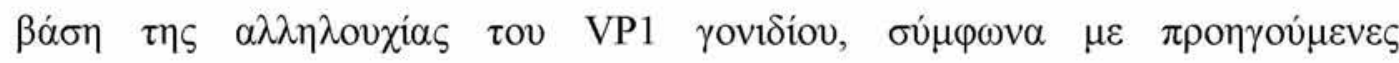

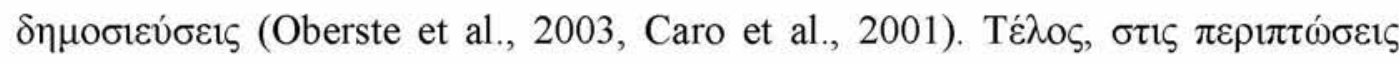

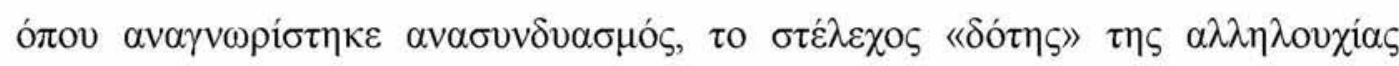

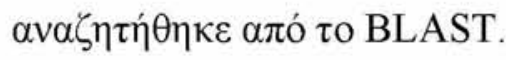

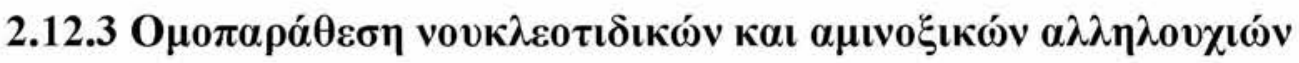

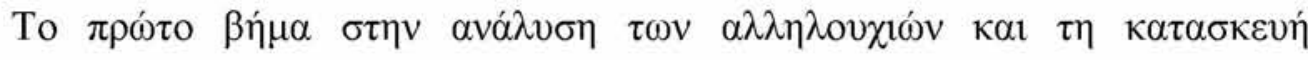

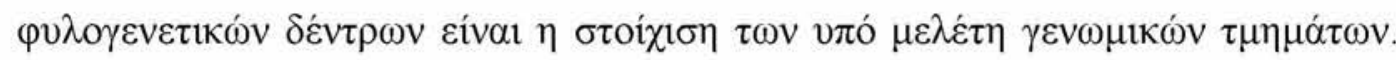

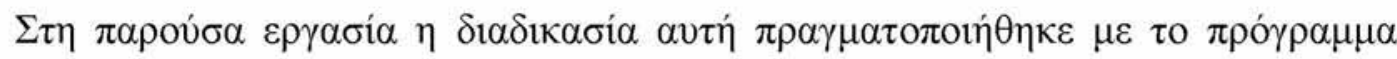

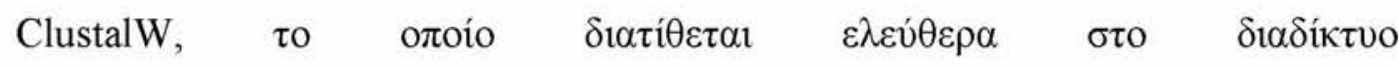

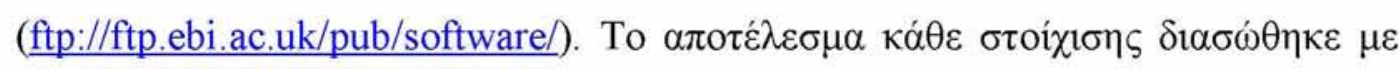

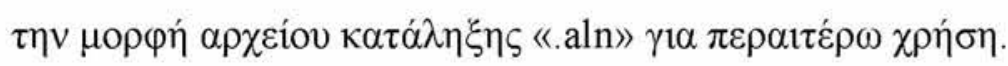

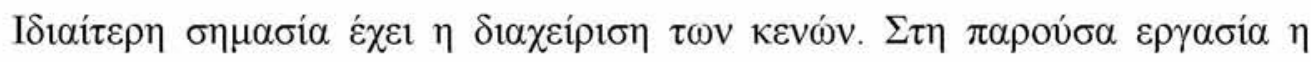

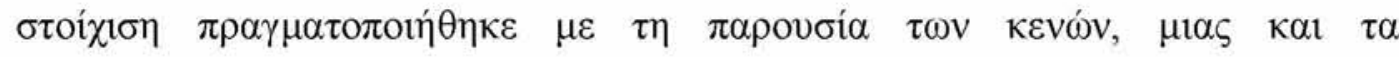

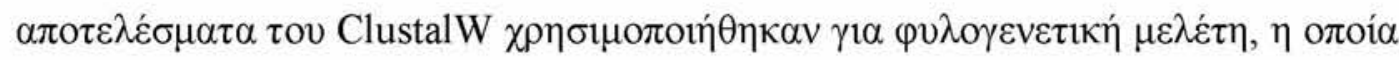

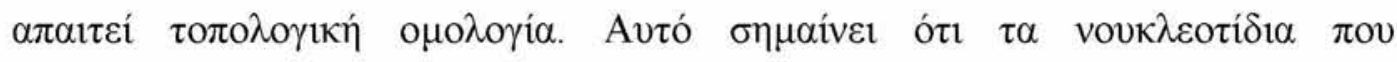

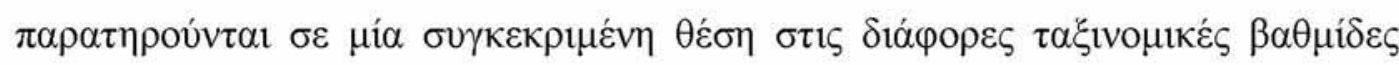

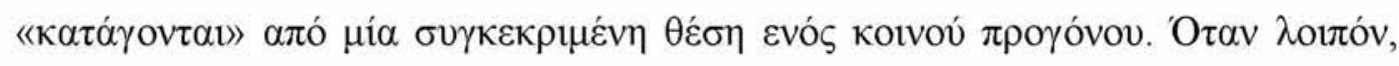

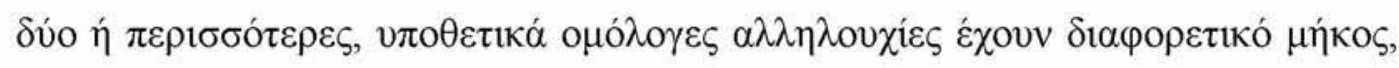

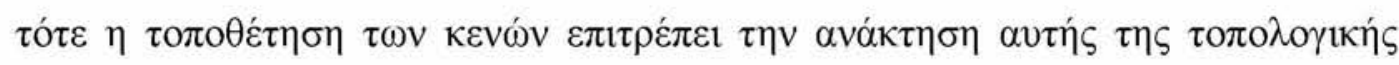
ouohorías.

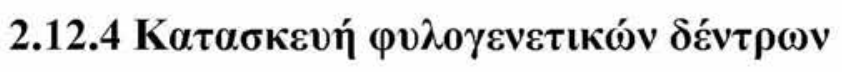

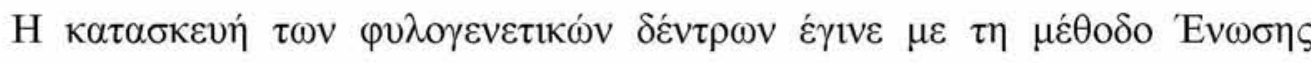

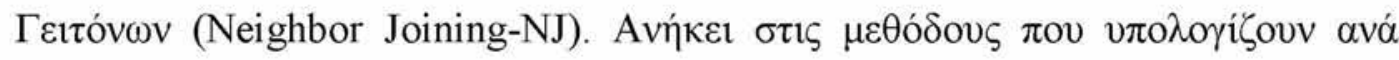

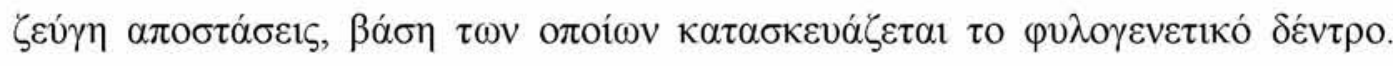

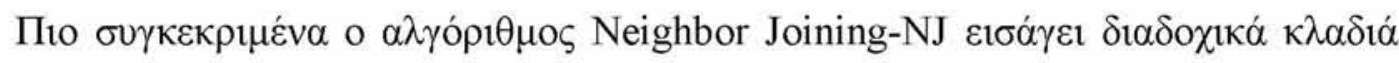

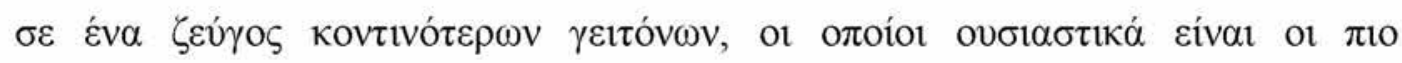

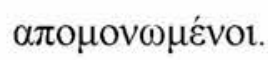




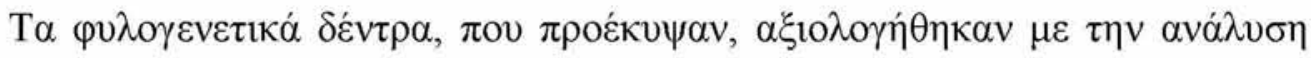

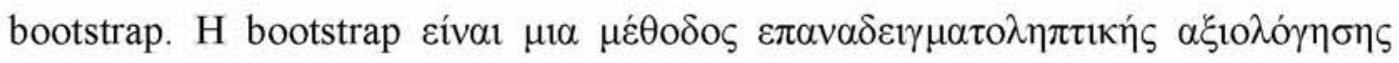

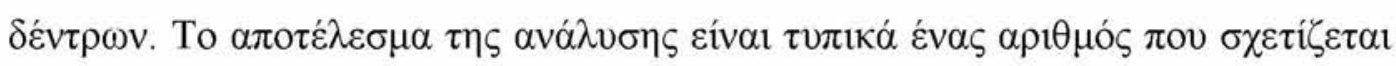

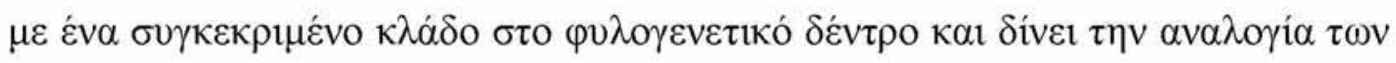

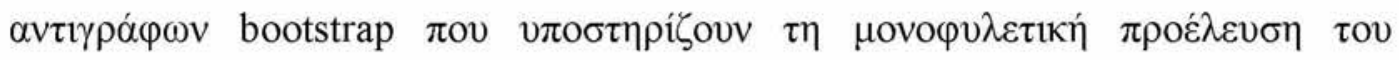

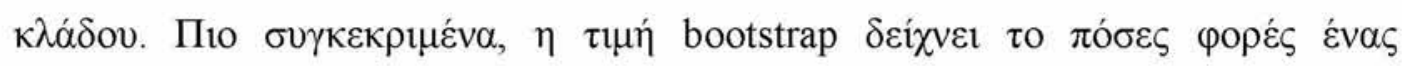

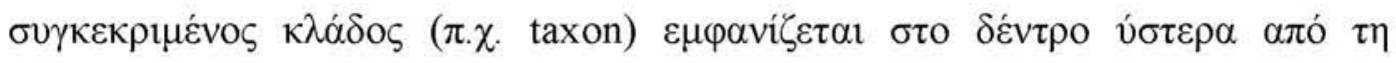

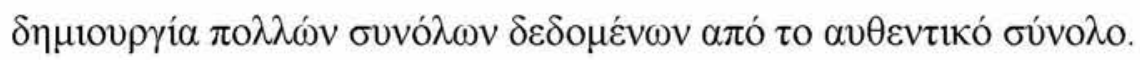

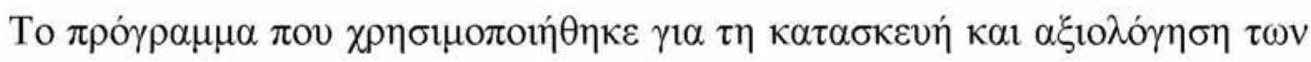

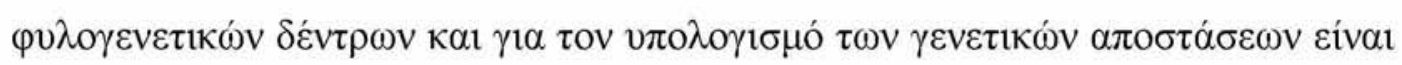

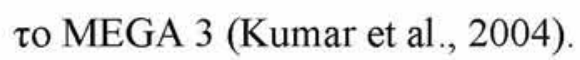

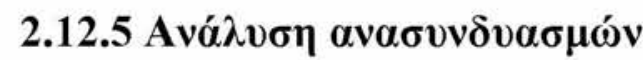

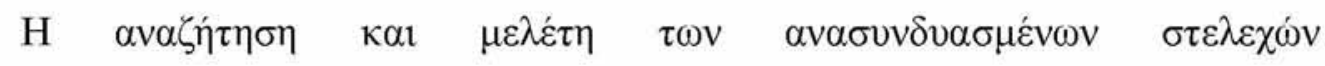

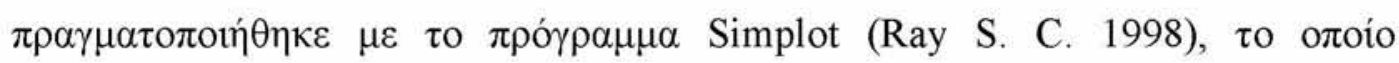
$\delta 1 \alpha \tau i \theta \varepsilon \tau \alpha \imath \quad \sigma \tau \eta v \quad 1 \sigma \tau 0 \sigma \varepsilon \lambda i \delta \alpha$ http://sray.med.som.jhmi.edu/RaySoft/SimPlot/. H

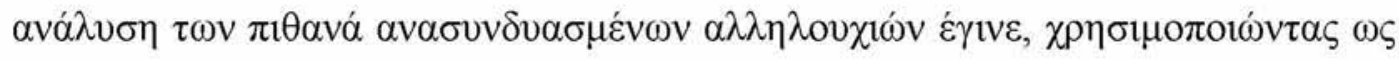

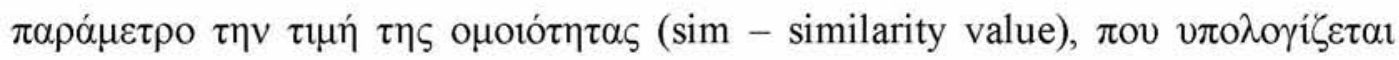

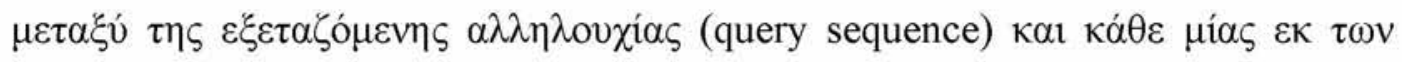

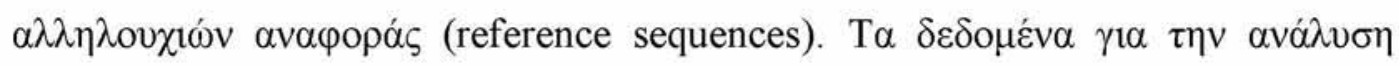

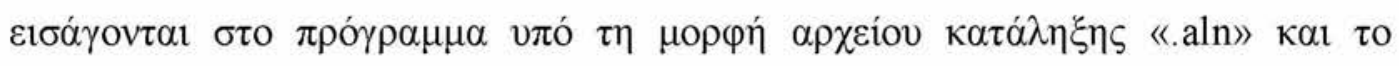

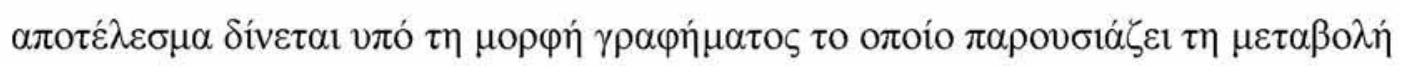

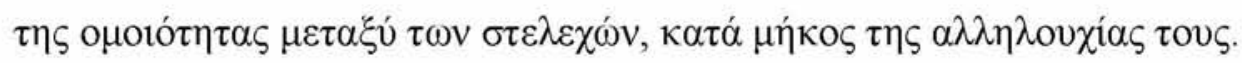




\section{КЕФАААIO 3}

\section{АПОТЕ $\Lambda$ ЕМАТА}

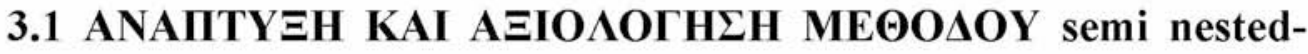

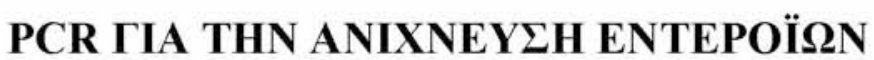

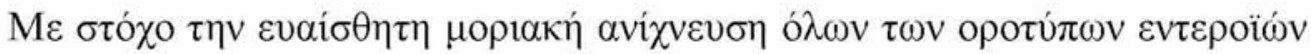

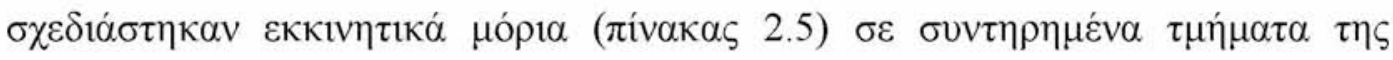

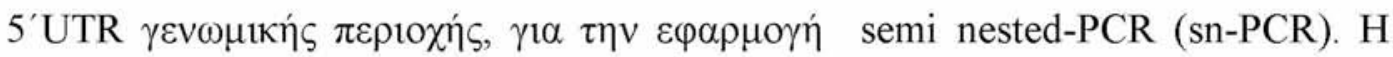

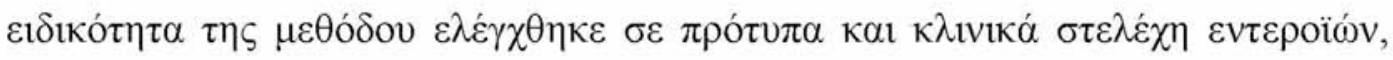

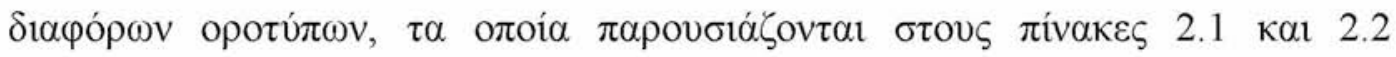

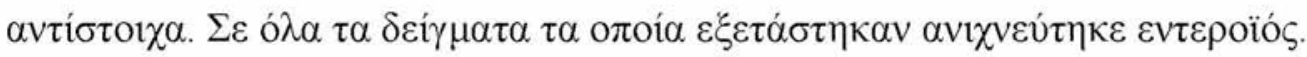

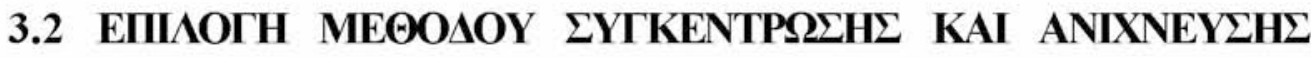

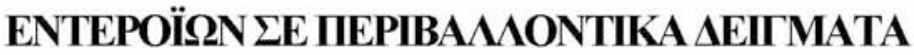

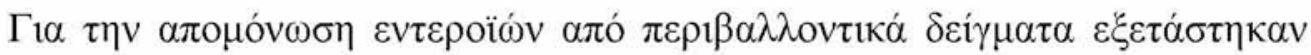

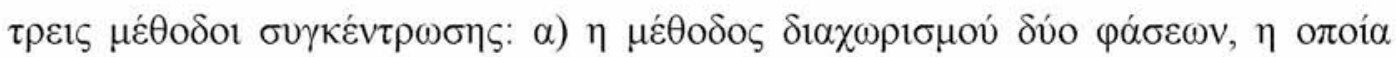

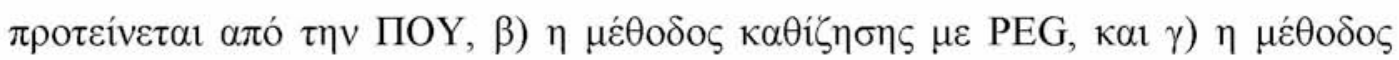

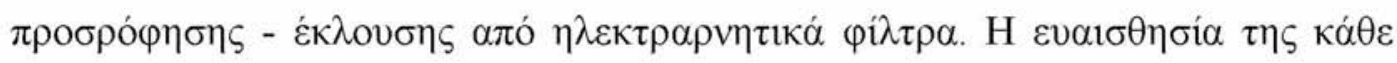

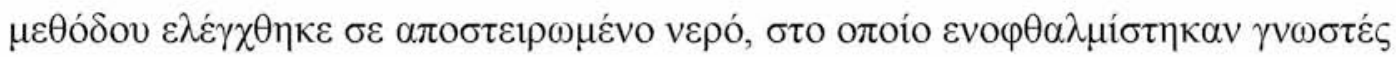

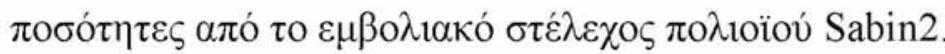

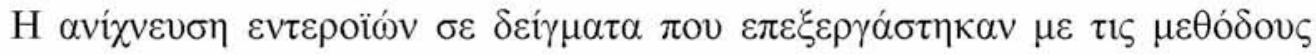

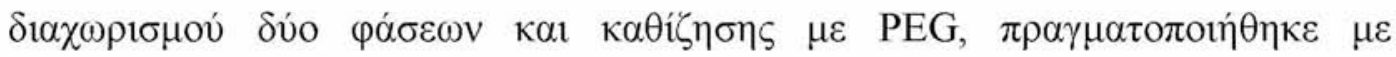

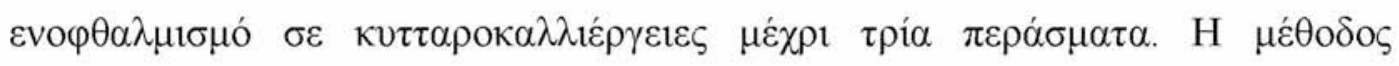

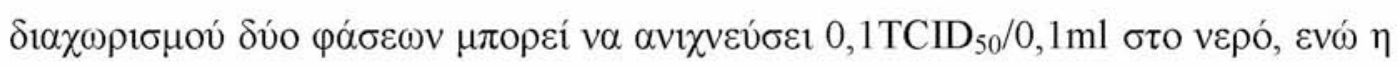

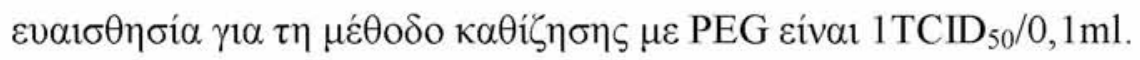

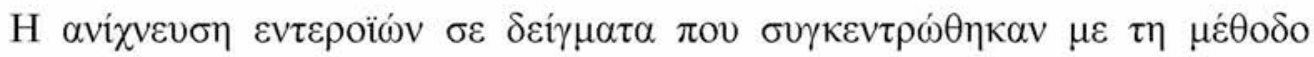

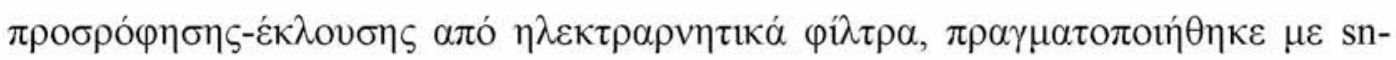

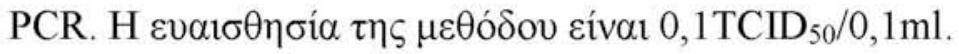

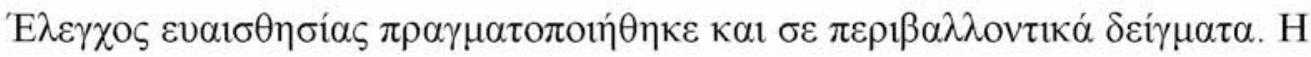

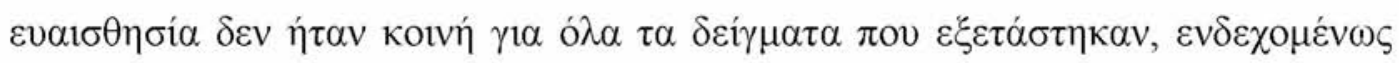




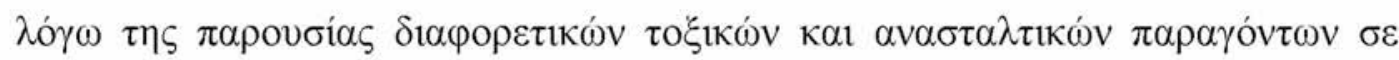

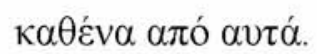

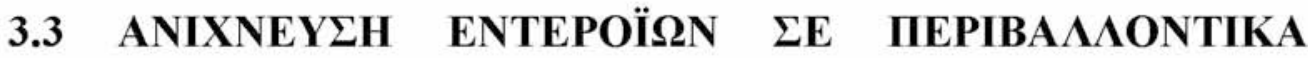

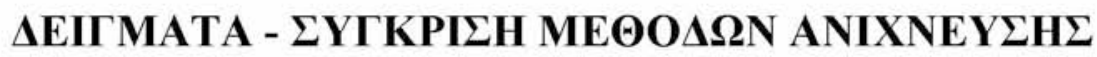

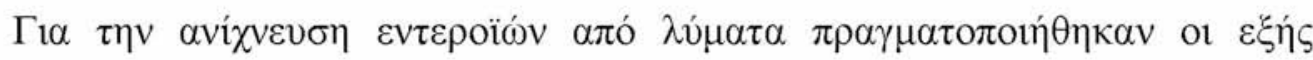

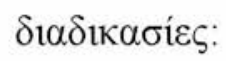

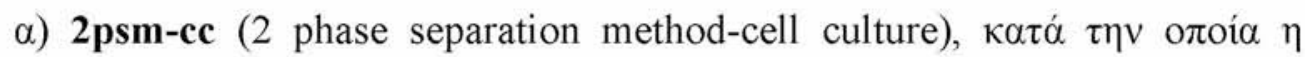

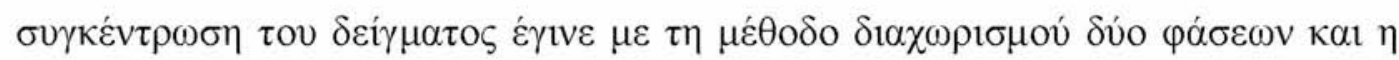

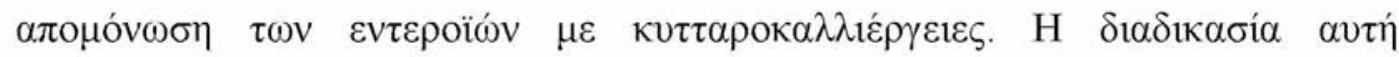

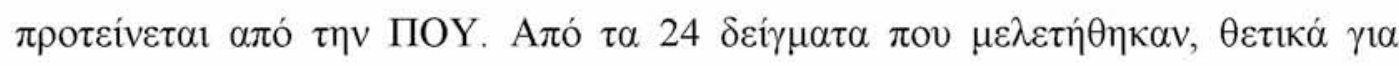

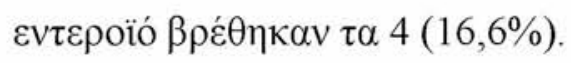

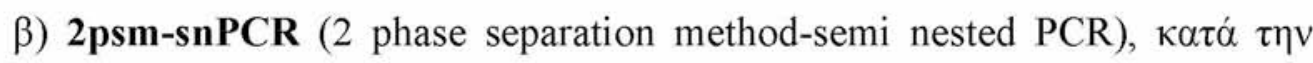

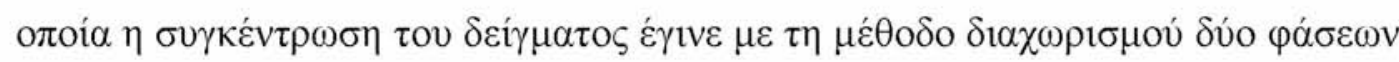

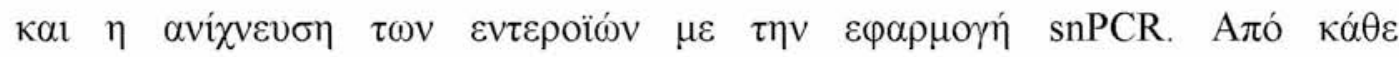

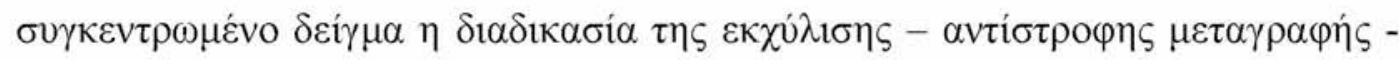

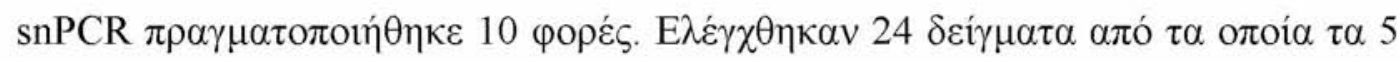

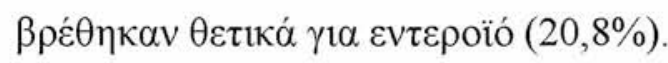

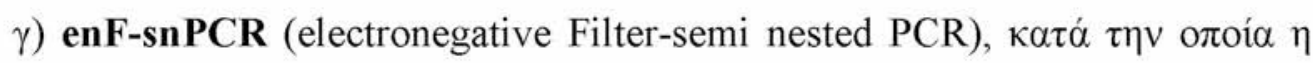

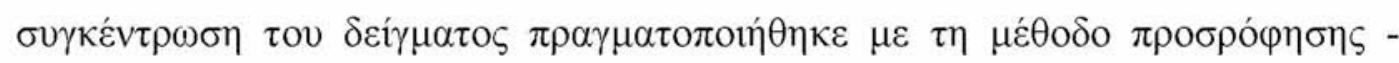

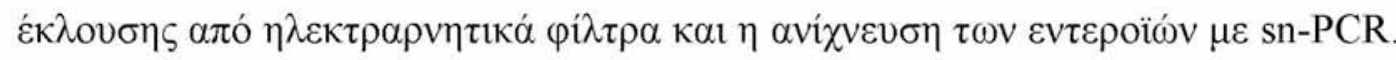

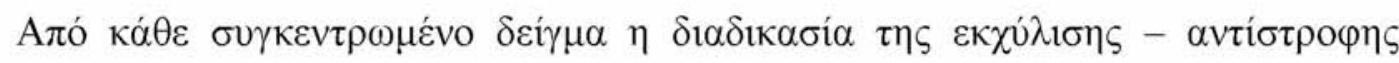

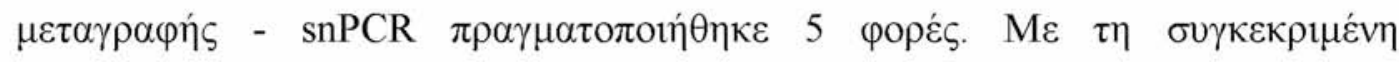

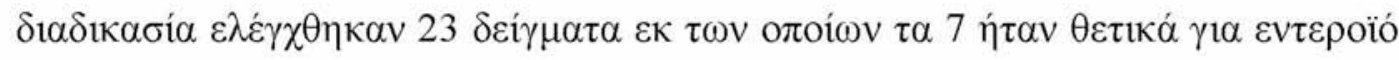
$(30,4 \%)$.

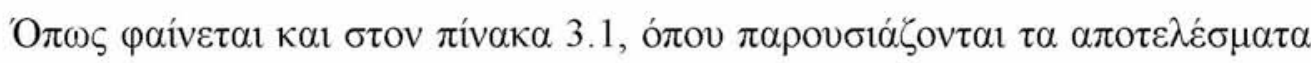

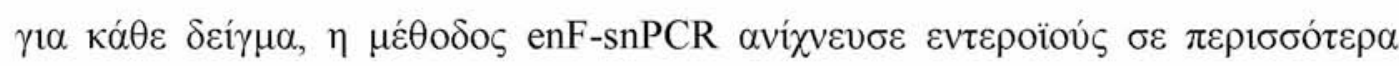

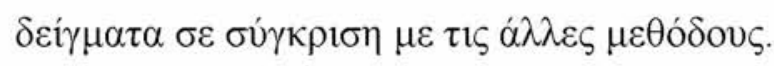

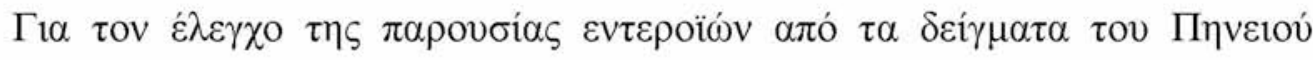

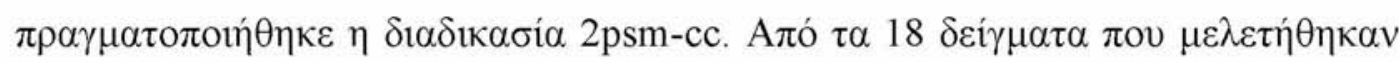

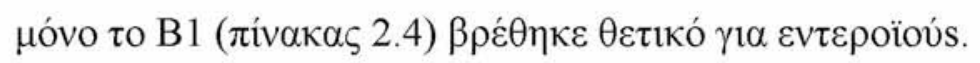




\begin{tabular}{|c|c|c|c|c|}
\hline$\Delta \varepsilon^{\prime} \gamma \mu \alpha$ & 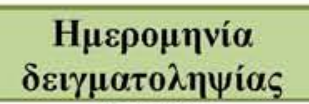 & 2psm-cc & 2psm-snPCR & enF-snPCR \\
\hline LR1 & $07 / 2005$ & + & + & * \\
\hline LR2 & $09 / 2005$ & - & - & + \\
\hline LR3 & $10 / 2005$ & + & + & + \\
\hline LR5 & $05 / 2006$ & + & - & + \\
\hline LR6 & $06 / 2006$ & + & + & + \\
\hline LR7 & $07 / 2006$ & - & + & - \\
\hline LR8 & $07 / 2006$ & - & - & - \\
\hline LR9 & $07 / 2006$ & - & - & - \\
\hline LR10 & $08 / 2006$ & - & - & - \\
\hline TR1 & $09 / 2006$ & - & - & + \\
\hline LR11 & $09 / 2006$ & - & - & - \\
\hline GN2 & $09 / 2006$ & - & - & - \\
\hline GN1 & $09 / 2006$ & - & - & + \\
\hline TR2 & $11 / 2006$ & - & - & - \\
\hline TR3 & $01 / 2007$ & - & - & - \\
\hline LR12 & $02 / 2007$ & - & - & - \\
\hline LR13 & $02 / 2007$ & - & + & - \\
\hline TR4 & $04 / 2007$ & - & - & - \\
\hline TR5 & $06 / 2007$ & - & - & - \\
\hline LR14 & $07 / 2007$ & - & - & - \\
\hline LR15 & $07 / 2007$ & - & - & - \\
\hline LRB1 & $07 / 2007$ & - & - & - \\
\hline LR16 & $08 / 2007$ & - & - & - \\
\hline LRB2 & $08 / 2007$ & - & - & + \\
\hline \multicolumn{2}{|c|}{ 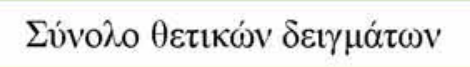 } & 4 & 5 & 7 \\
\hline \multicolumn{2}{|c|}{ 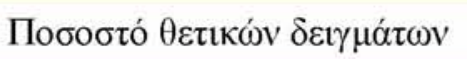 } & $16,6 \%$ & $20,8 \%$ & $30,4 \%$ \\
\hline
\end{tabular}

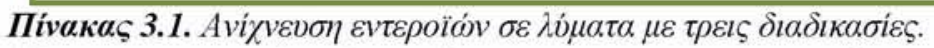

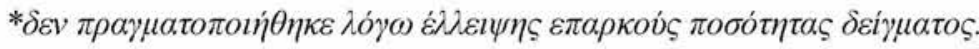

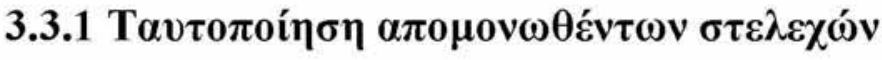

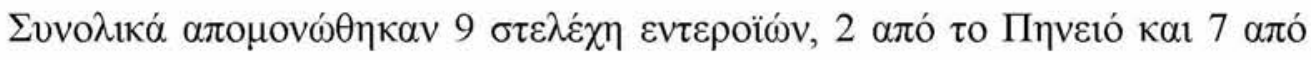

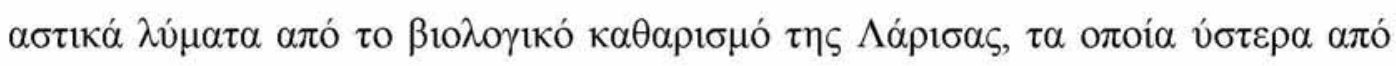

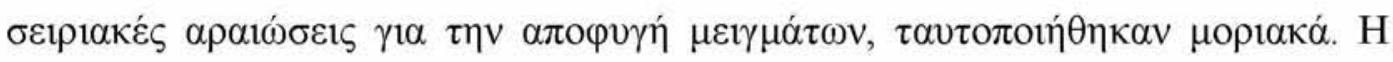

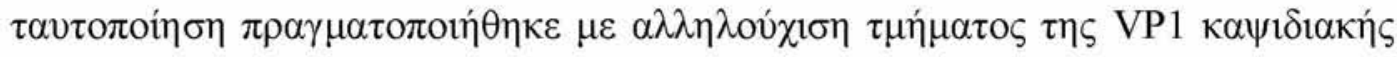

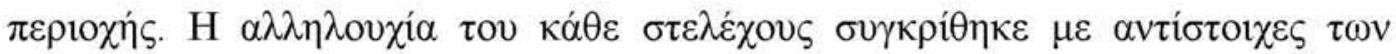

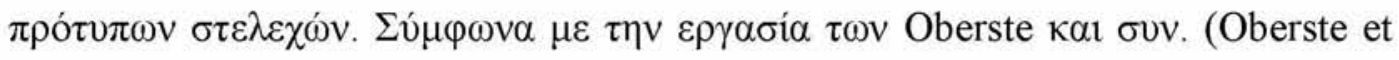

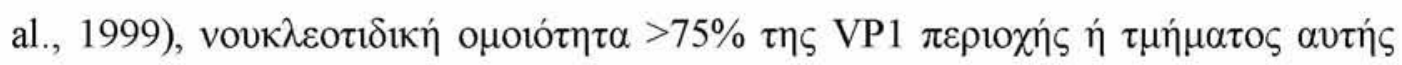

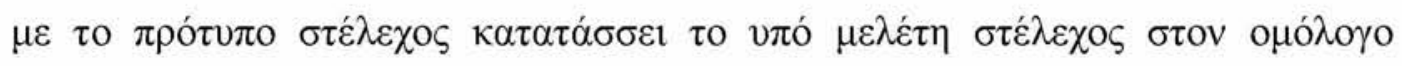

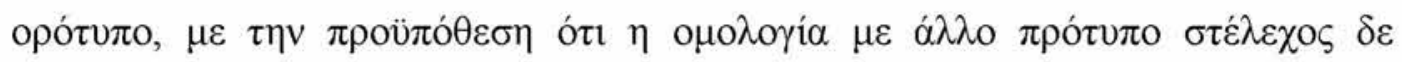

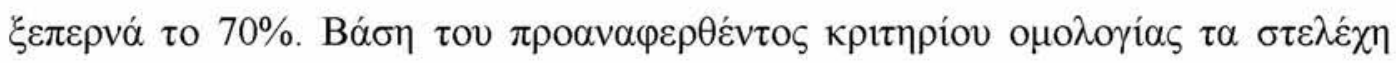




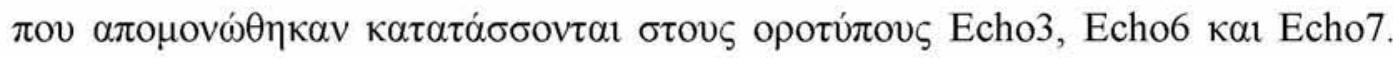

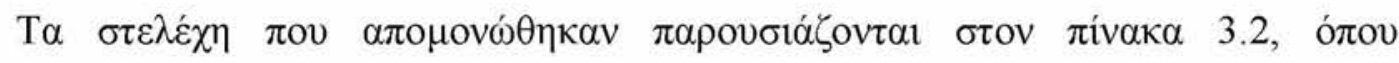

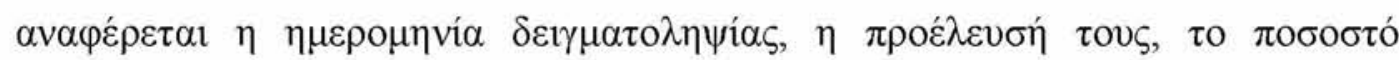

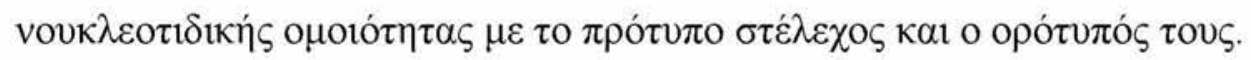

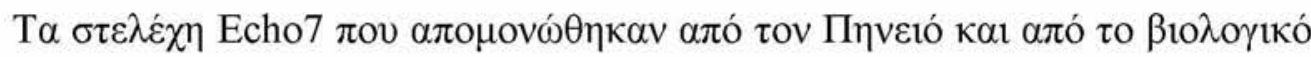

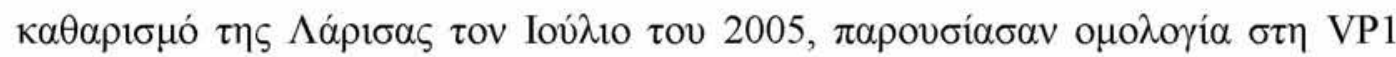

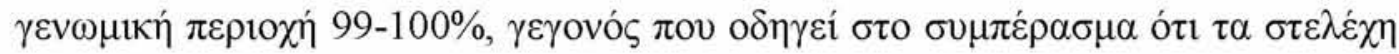

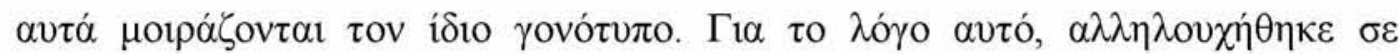

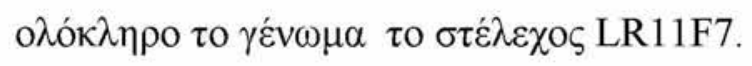

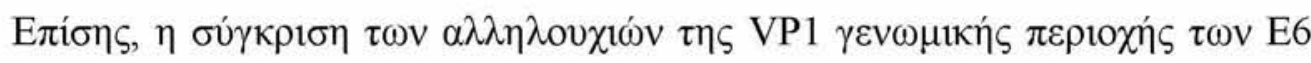

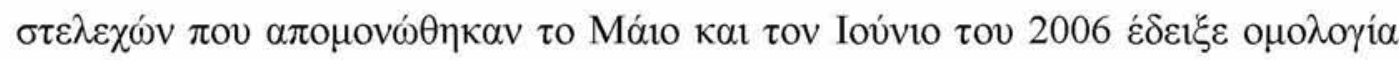

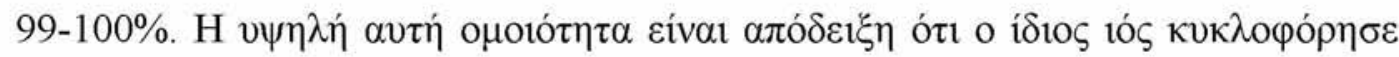

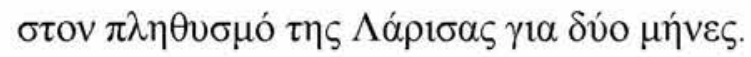

\begin{tabular}{|c|c|c|c|c|}
\hline$\Sigma \tau \varepsilon \dot{\lambda} \varepsilon \chi 0 \varsigma$ & 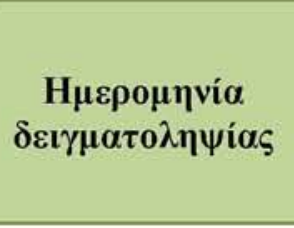 & 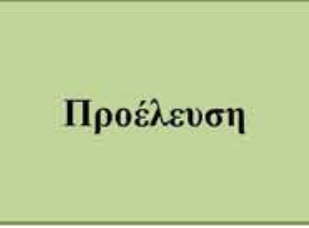 & 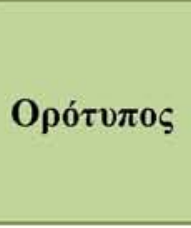 & 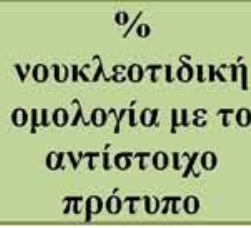 \\
\hline B11D2 & $13 / 07 / 2005$ & 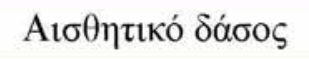 & Echo7 & $78 \%$ \\
\hline B11D6 & $13 / 07 / 2005$ & 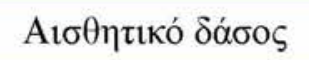 & Echo7 & $78 \%$ \\
\hline LR11D9 & $14 / 07 / 2005$ & 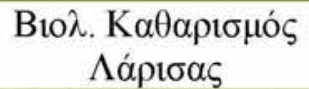 & Echo7 & $78 \%$ \\
\hline LR11E2 & $14 / 07 / 2005$ & 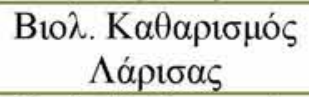 & Echo7 & $78 \%$ \\
\hline LR11E7 & $14 / 07 / 2005$ & 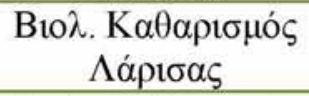 & Echo7 & $78 \%$ \\
\hline LR11F7 & $14 / 07 / 2005$ & 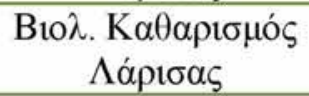 & Echo7 & $78 \%$ \\
\hline LR31G7 & $06 / 10 / 2005$ & 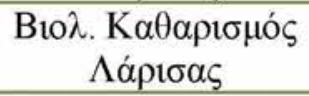 & Echo3 & $79 \%$ \\
\hline LR51A5 & $25 / 05 / 2006$ & 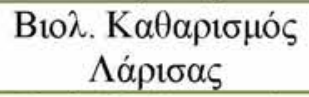 & Echo6 & $77 \%$ \\
\hline LR61G3 & $16 / 06 / 2006$ & 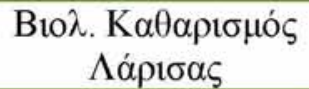 & Echo6 & $77 \%$ \\
\hline
\end{tabular}

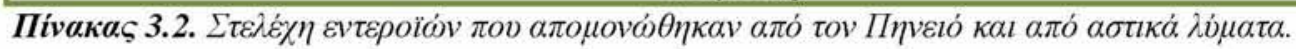




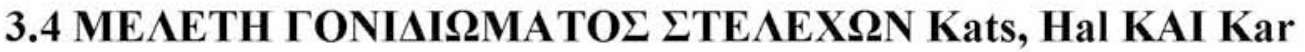

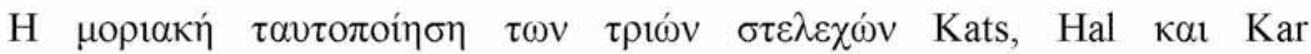

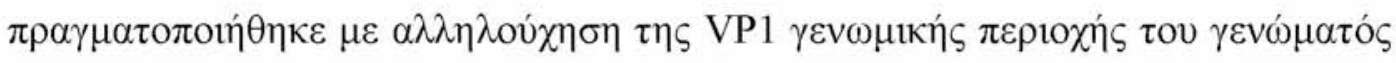

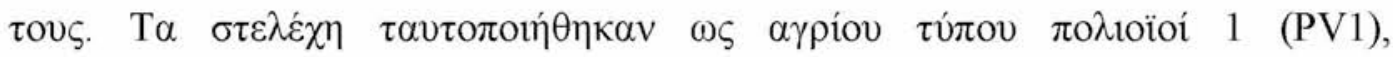

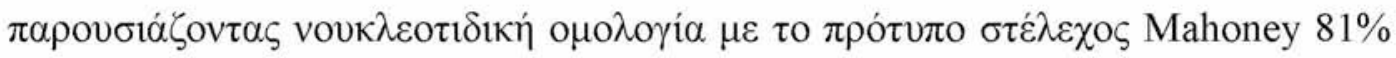

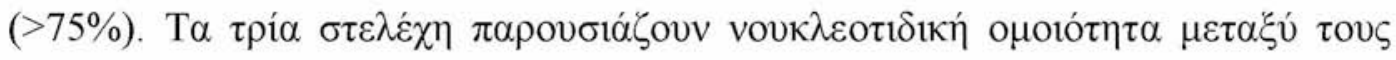

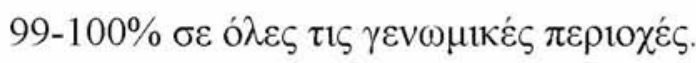

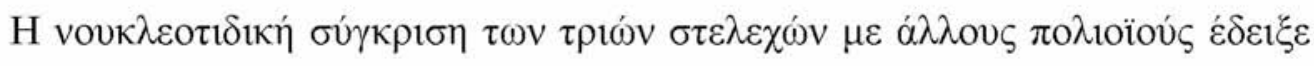

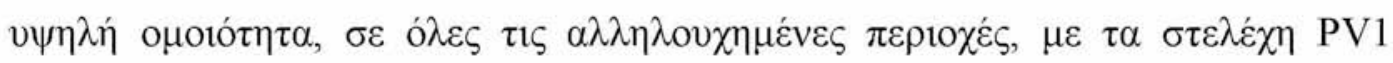

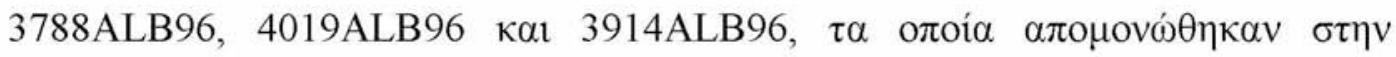

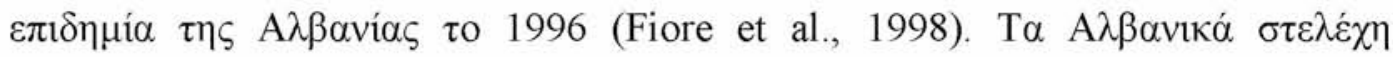

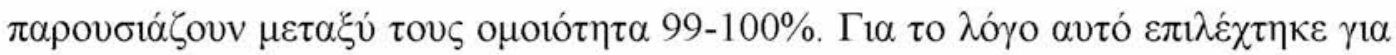

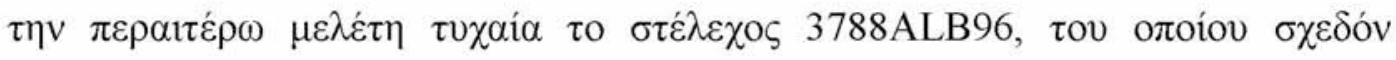
о

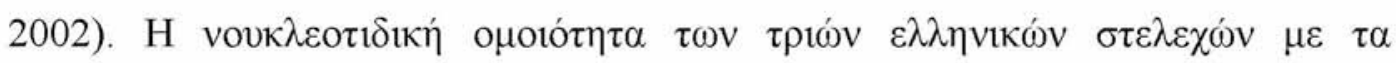

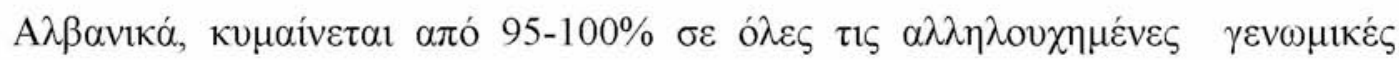

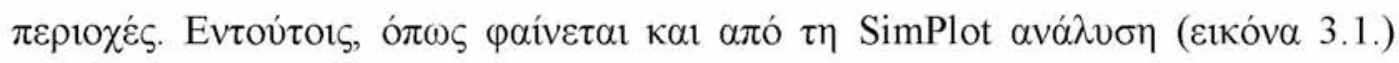

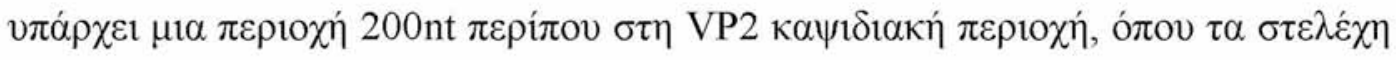

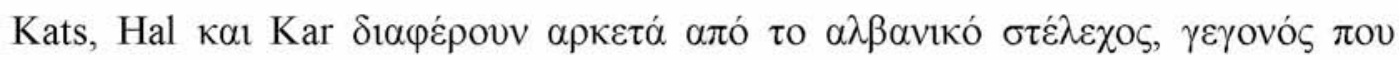

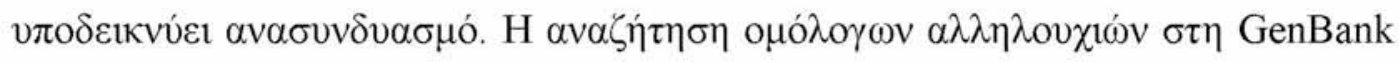

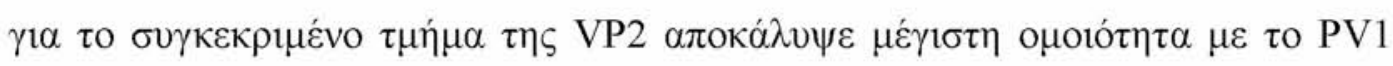

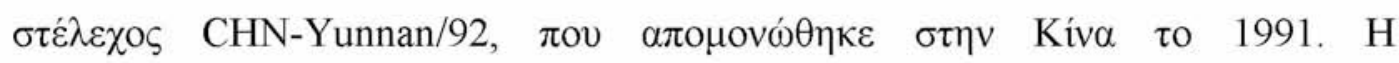

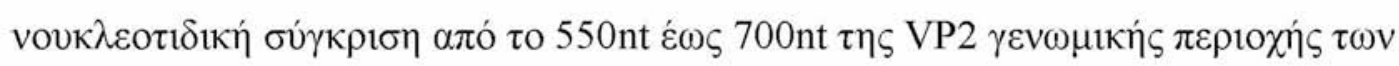

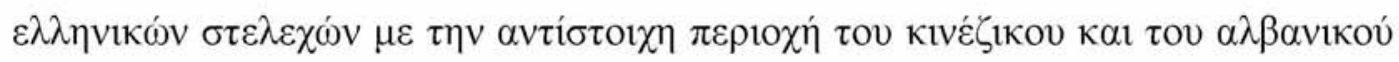

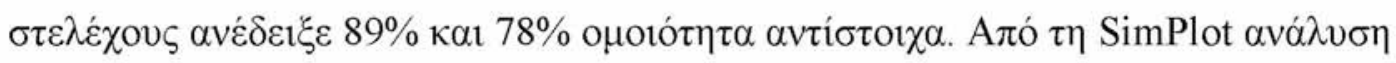

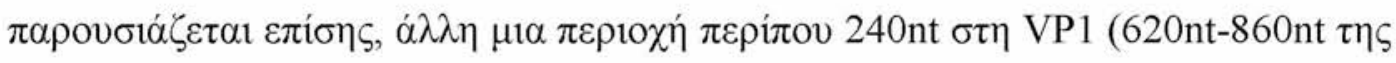

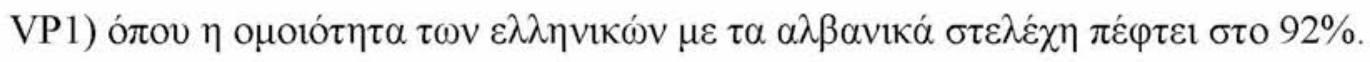

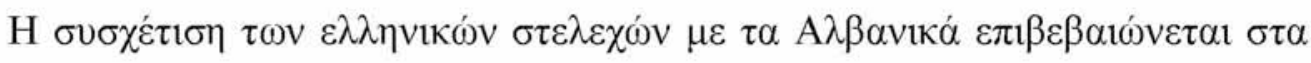

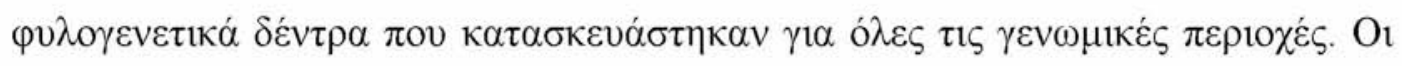


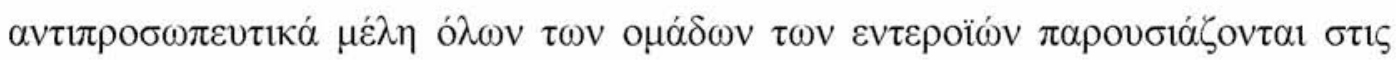




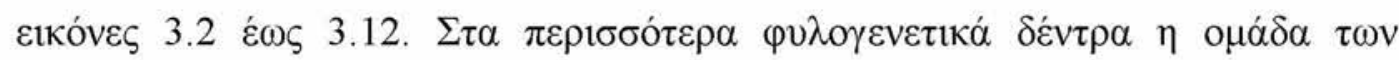

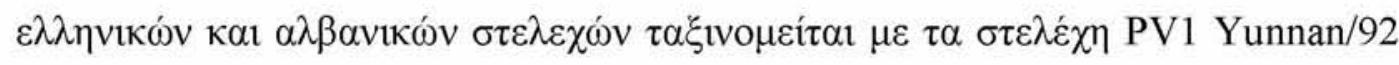

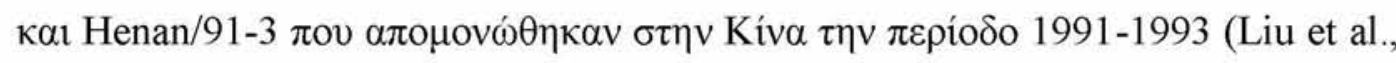

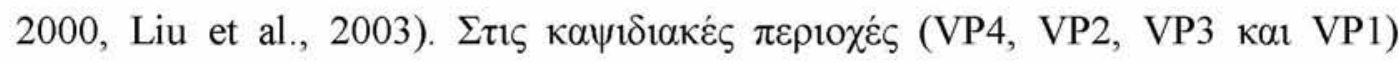

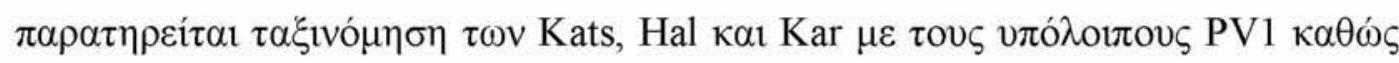

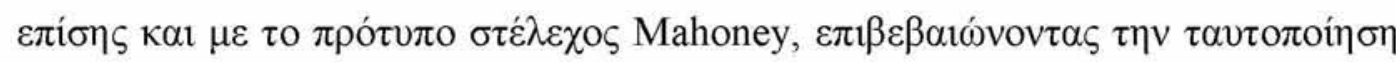

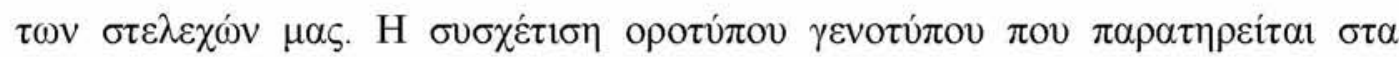

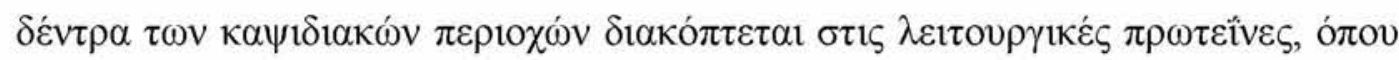

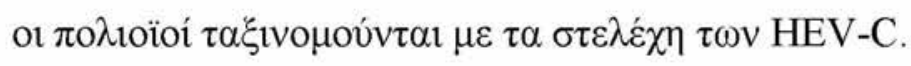

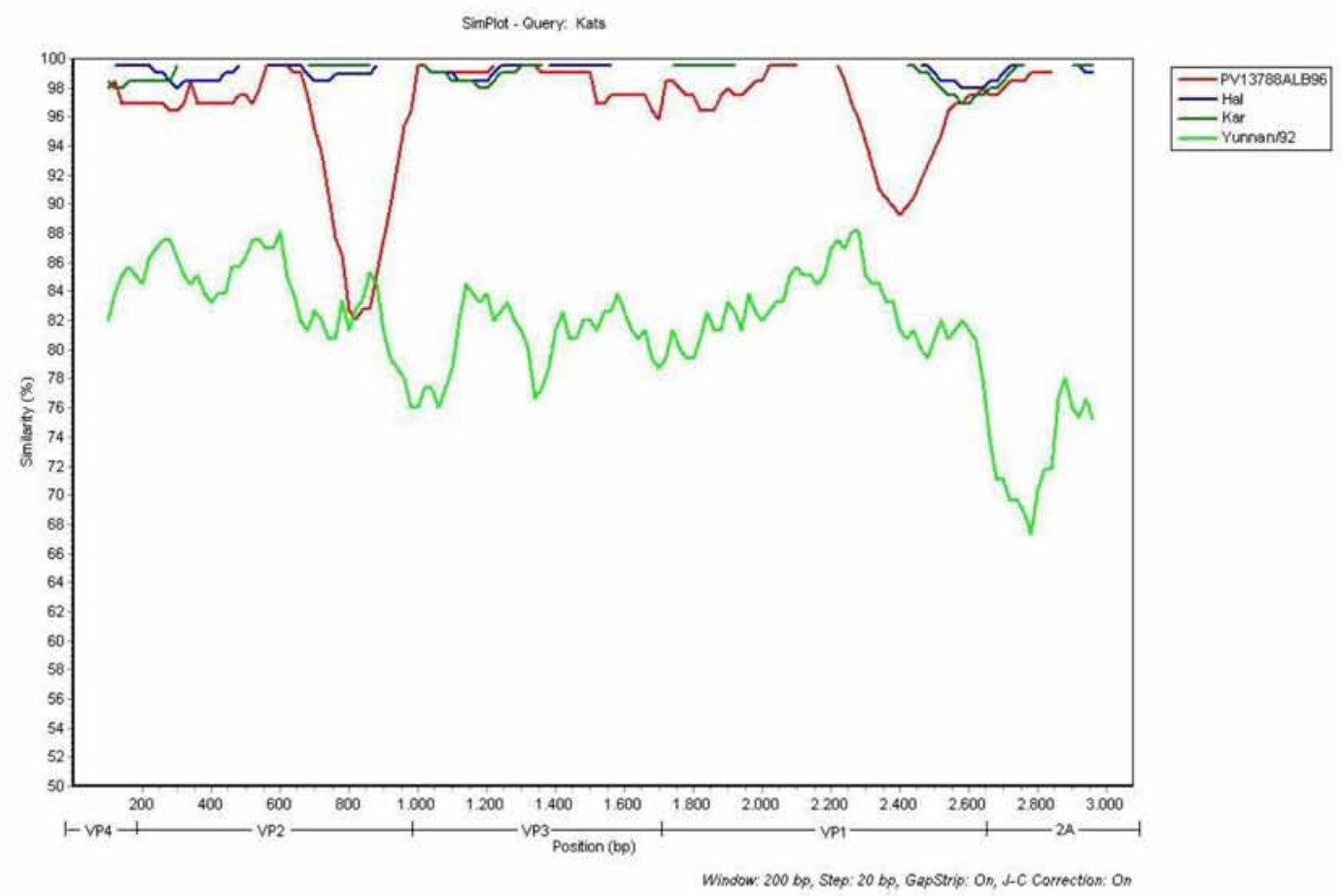

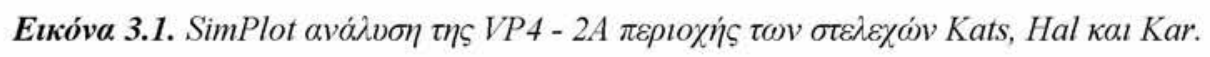




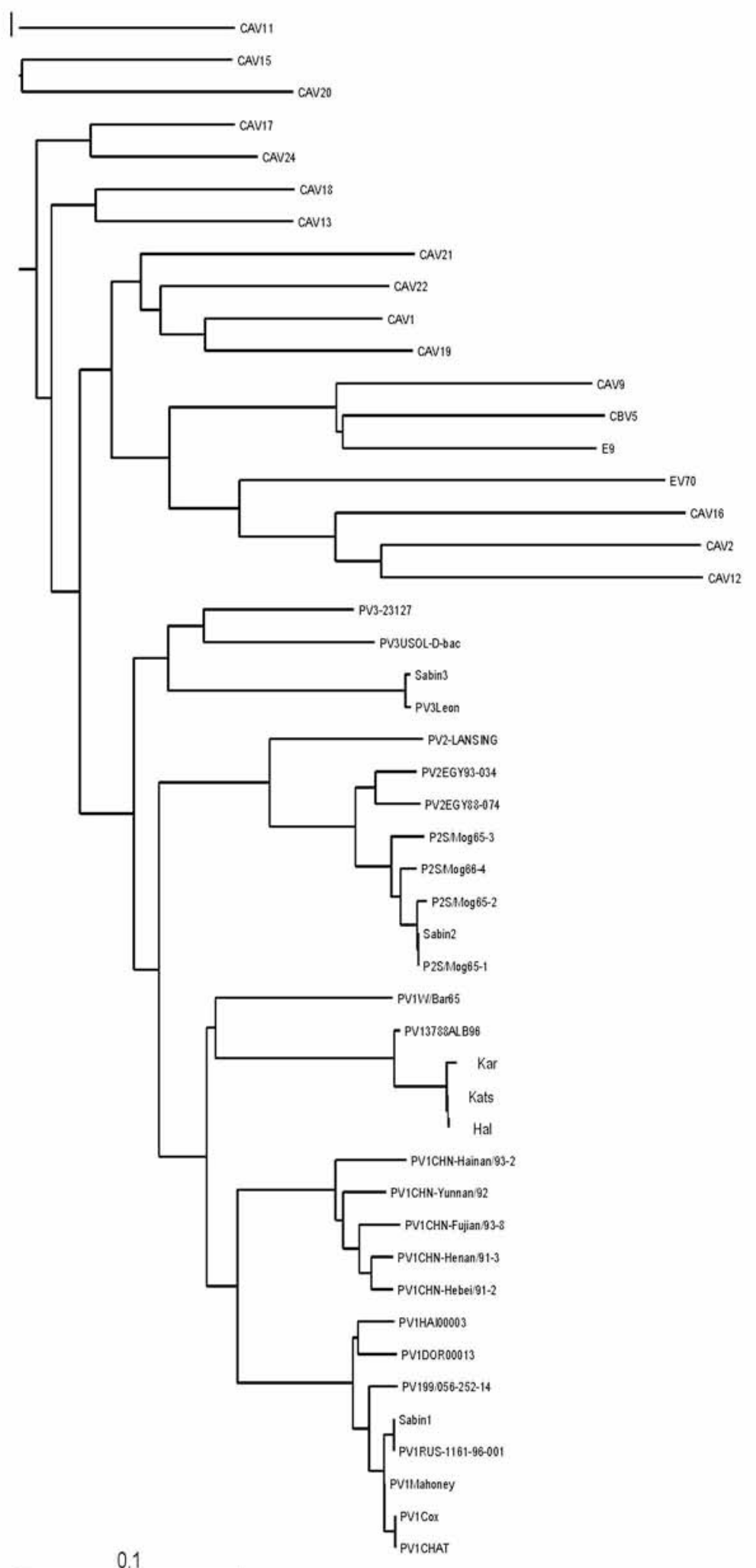

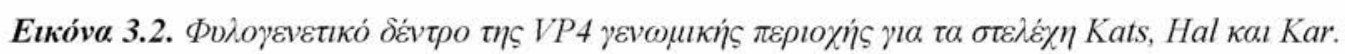




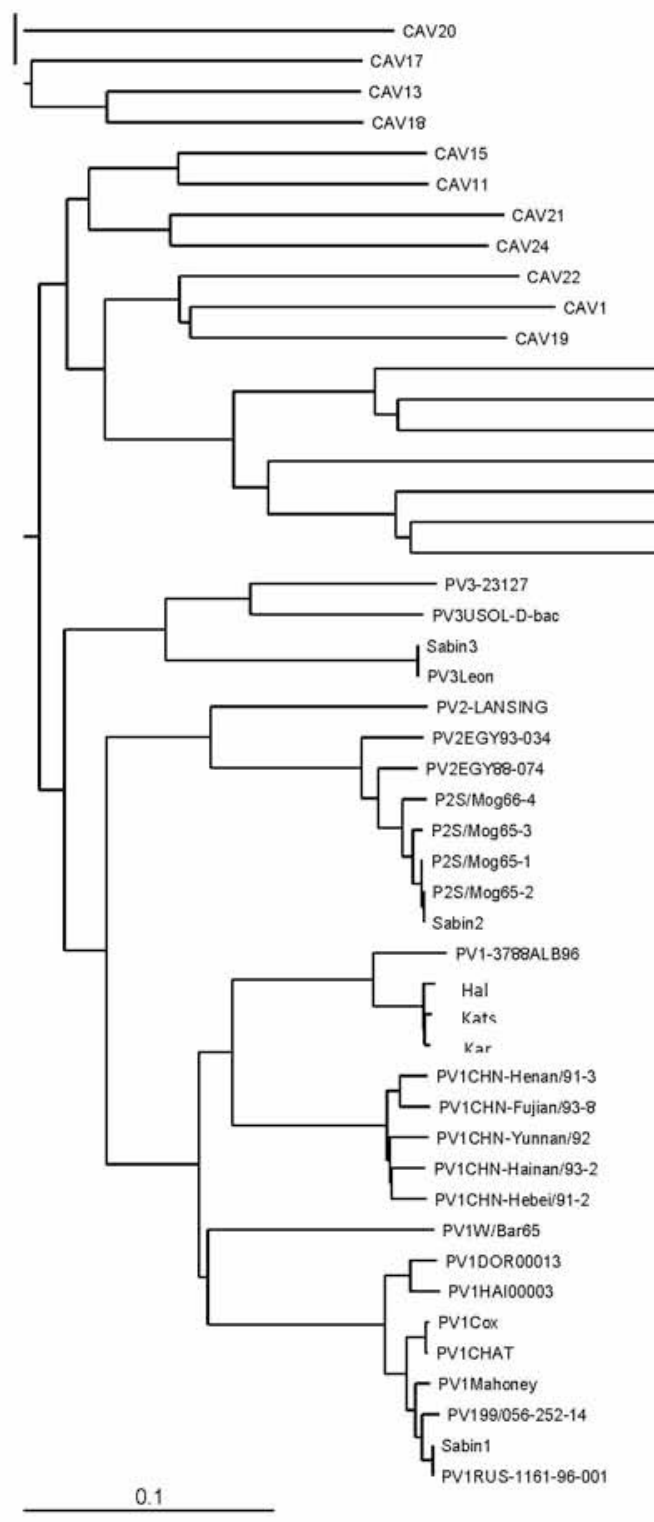

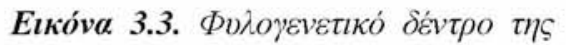

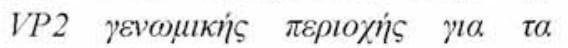
$\sigma \tau \dot{\alpha} \bar{\chi} \eta$ Kats, Hal kaı Kar.

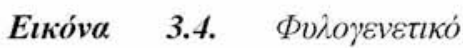

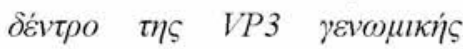

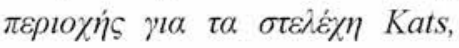
Hal kal Kar.

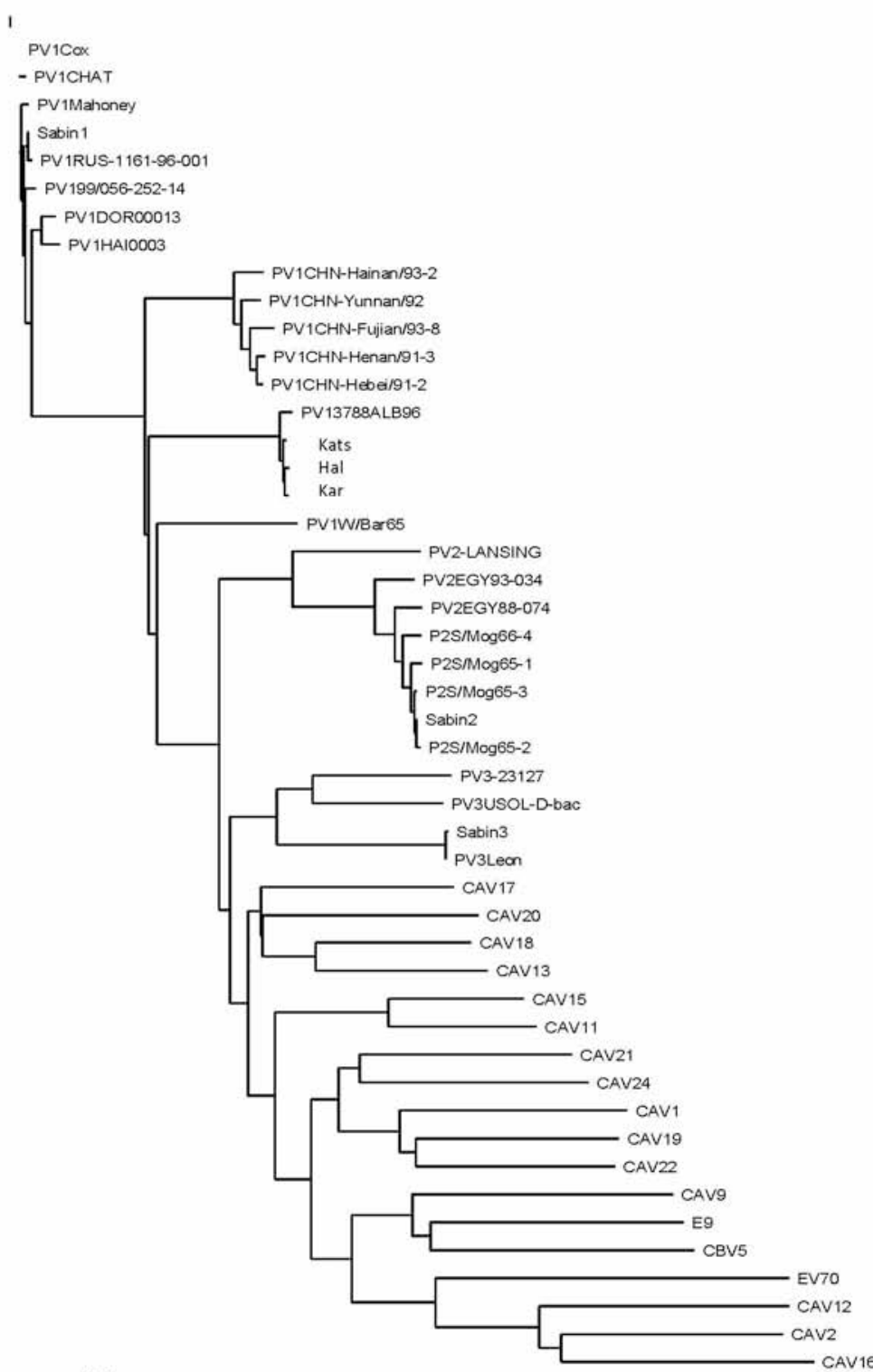




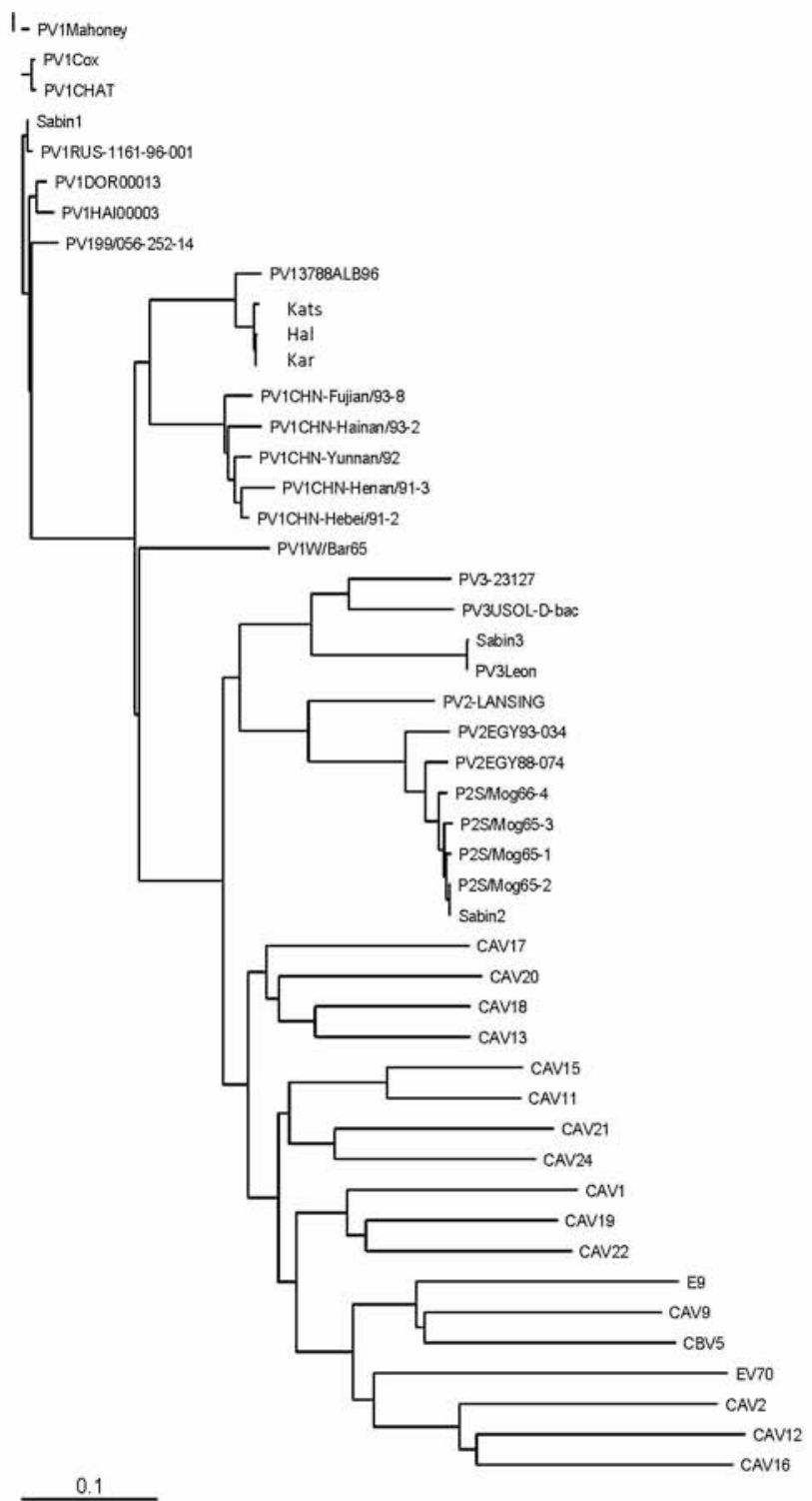

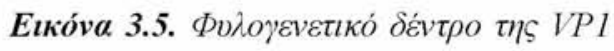

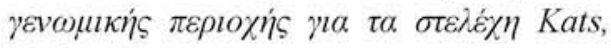
Hal kal Kar.

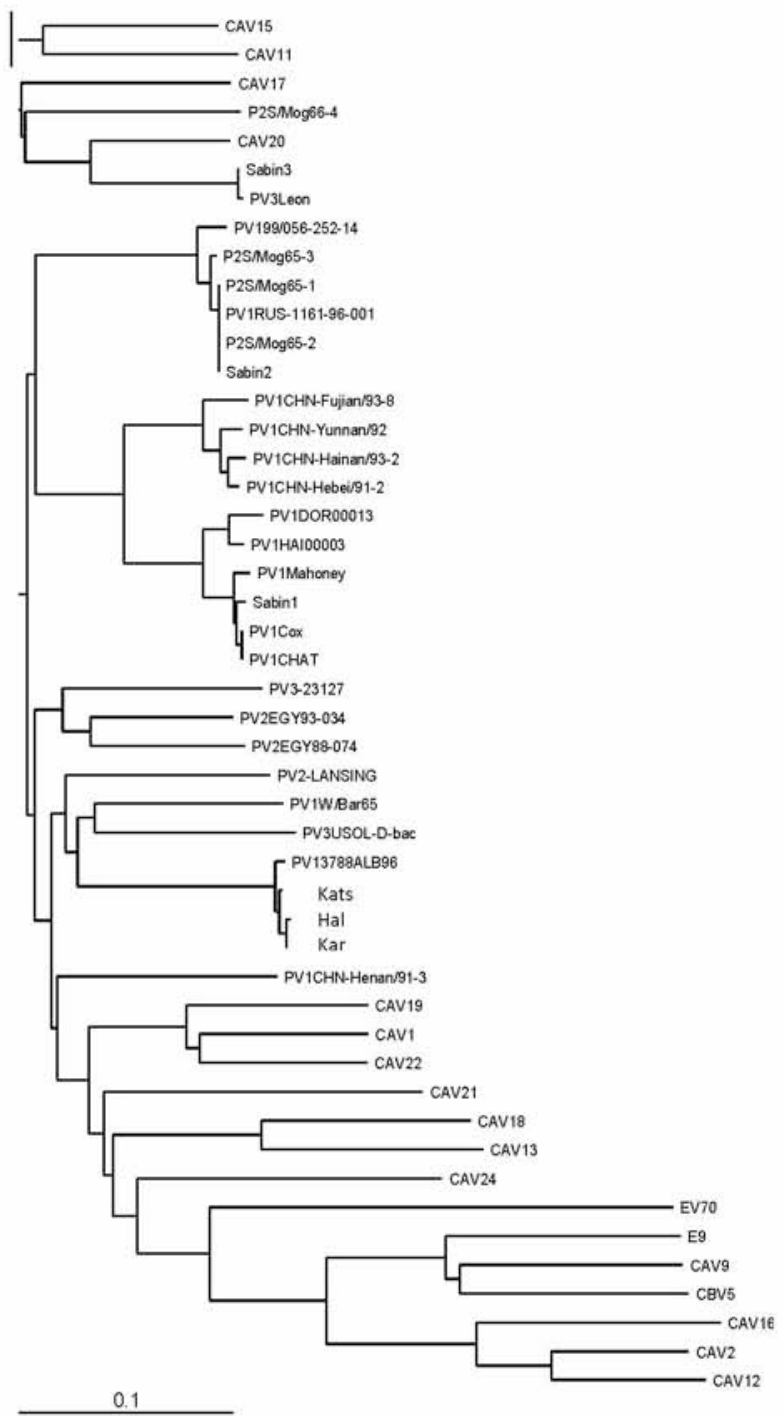




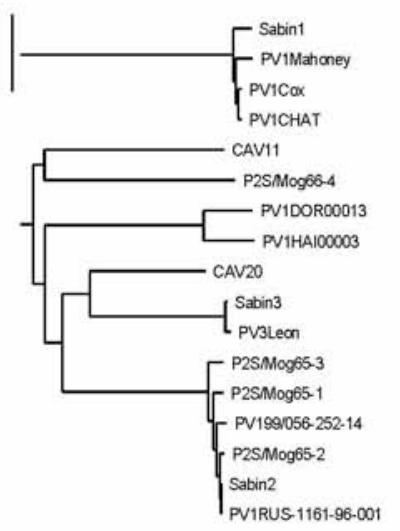

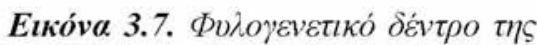

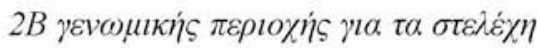
Kats, Hal kol Kar.

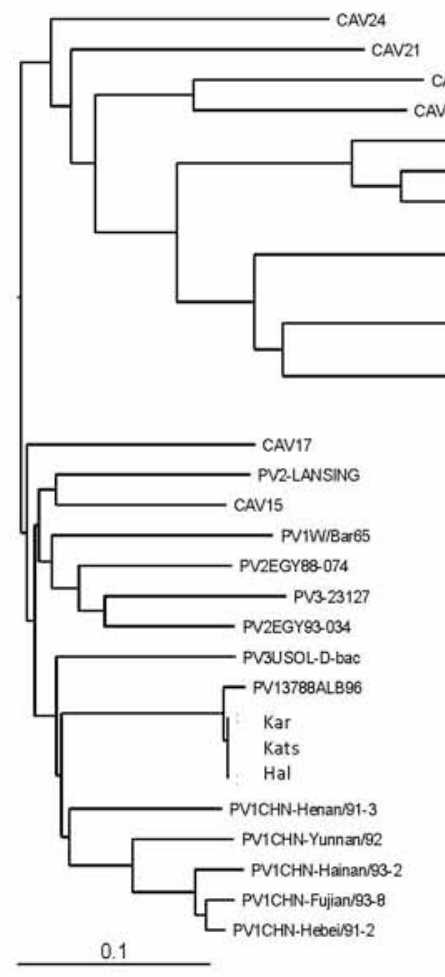

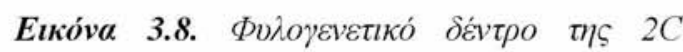

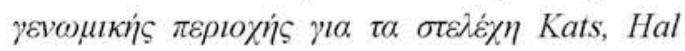
каl Kar. 


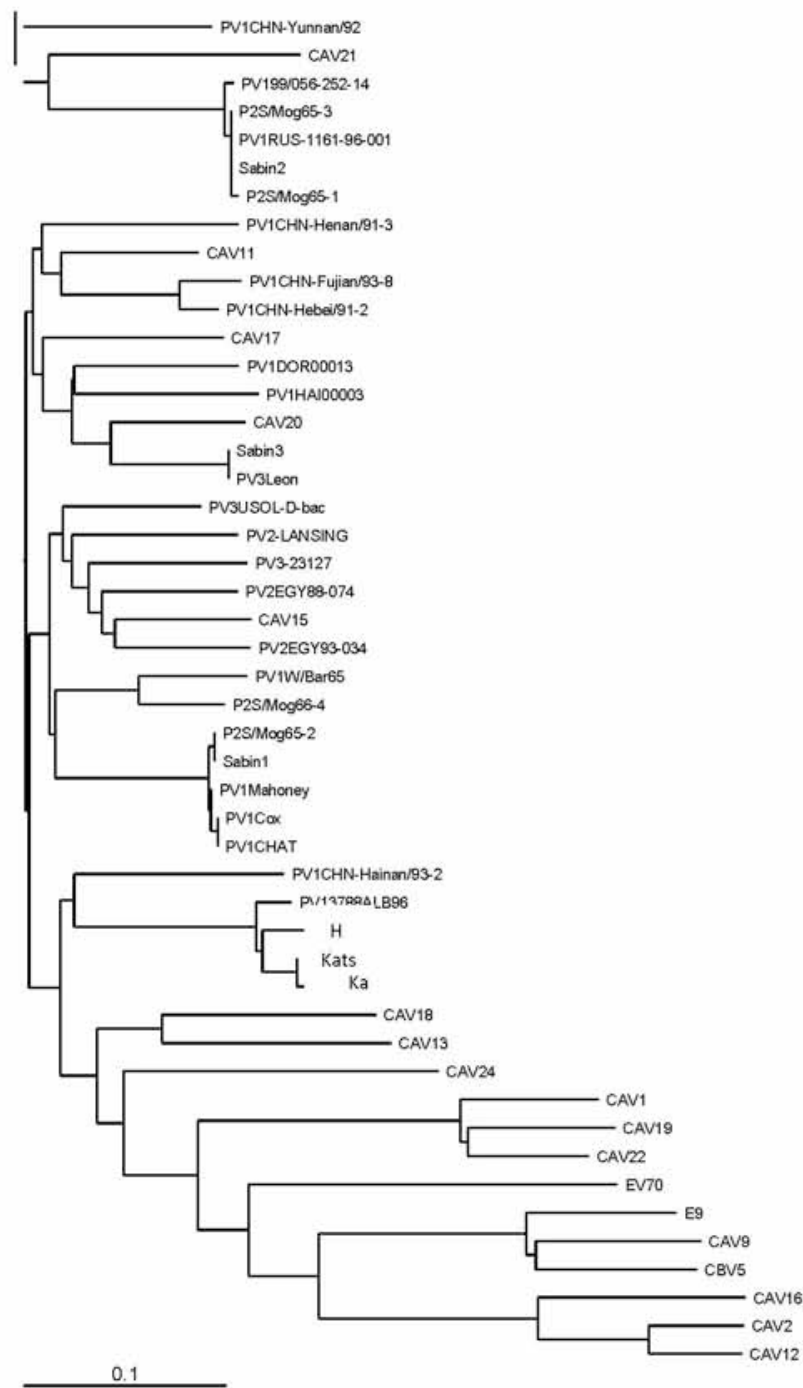

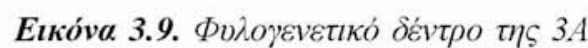

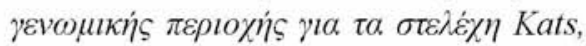
Hal kol Kar.

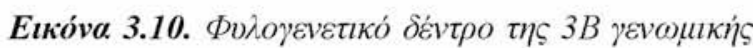

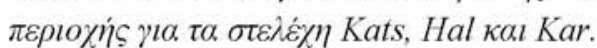

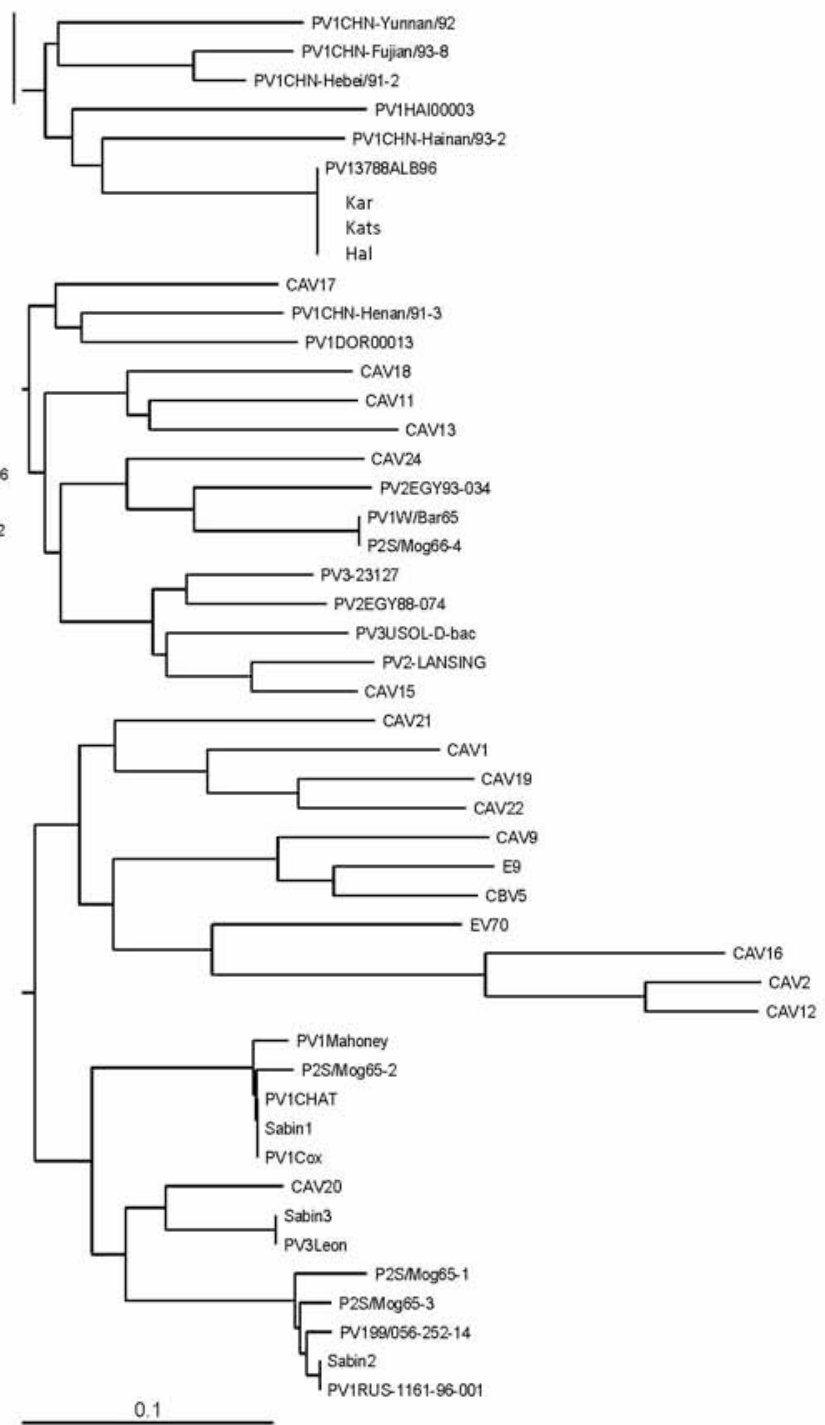




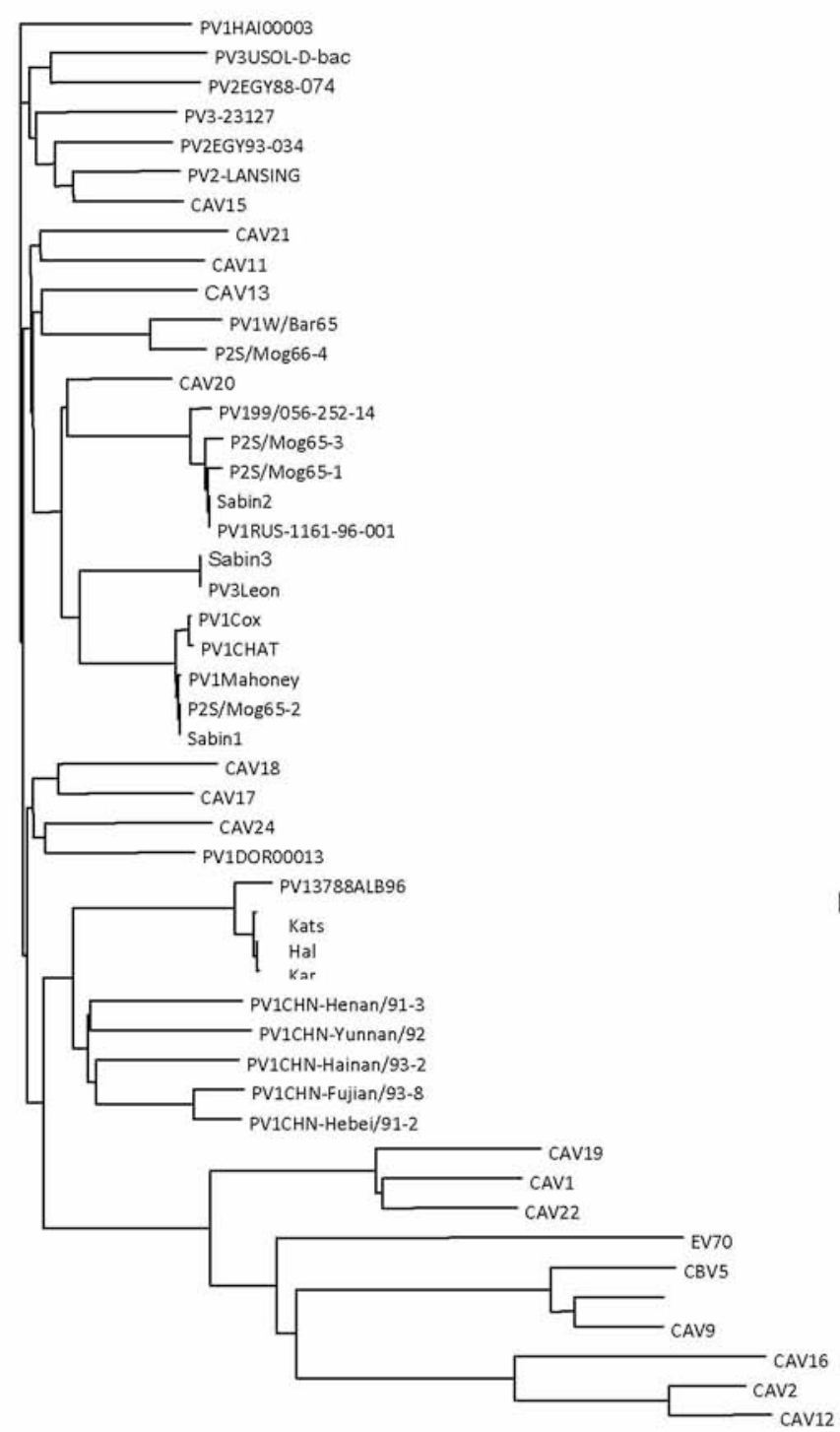

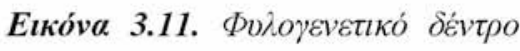

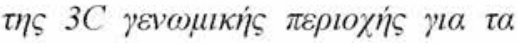

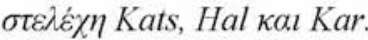

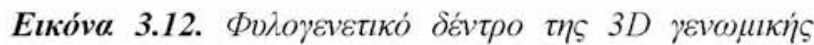

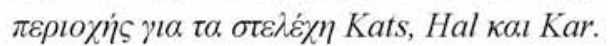

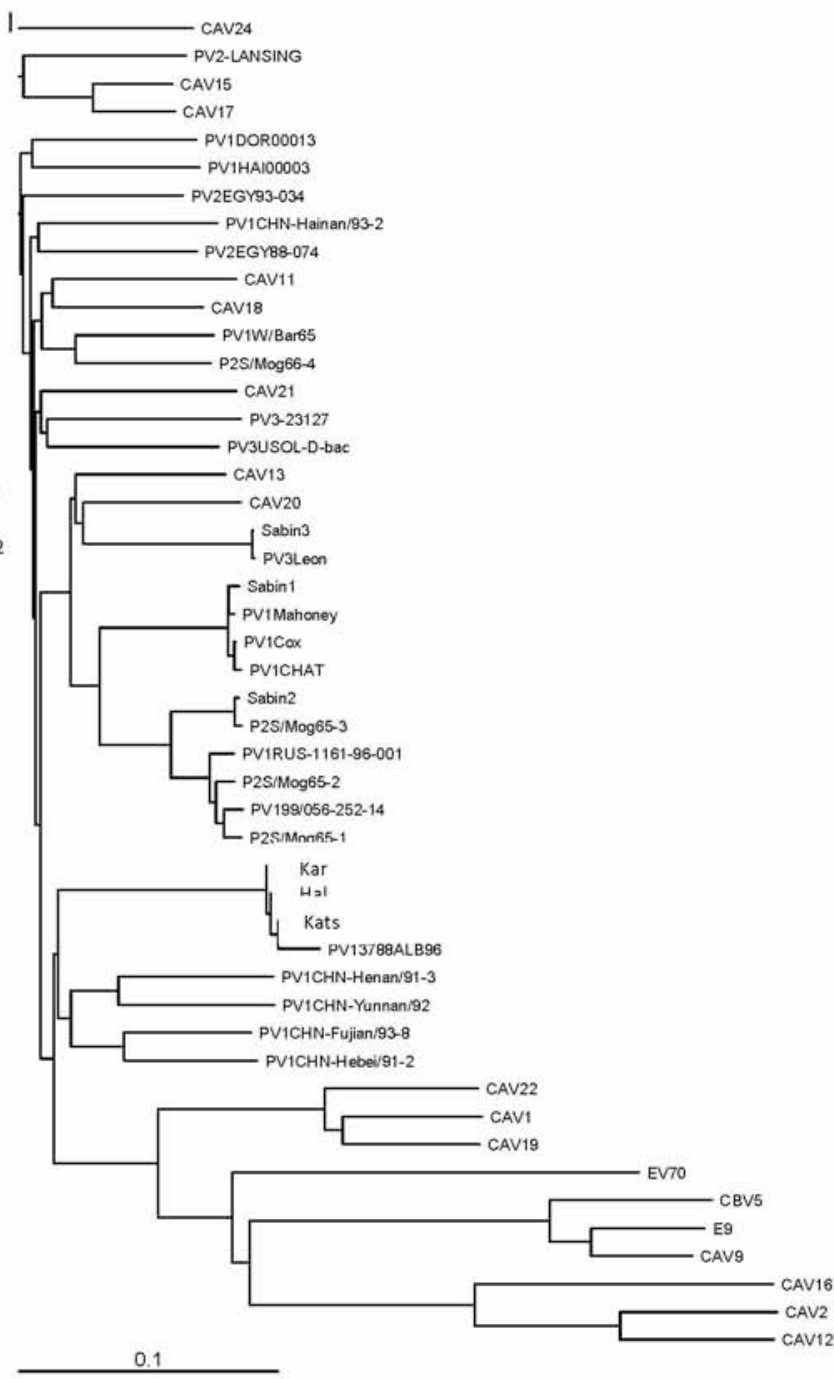




\subsection{MEAETH ГONIAISMATO $\Sigma$ TEAEXOY $\Sigma$ LR11F7}

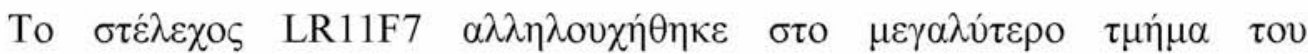

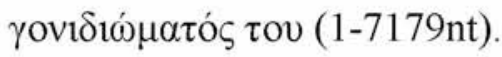

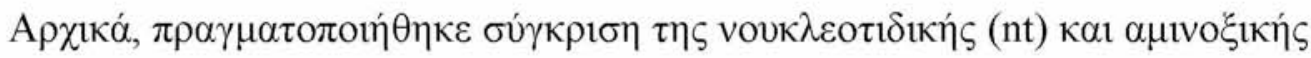

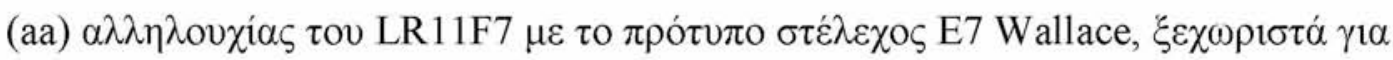

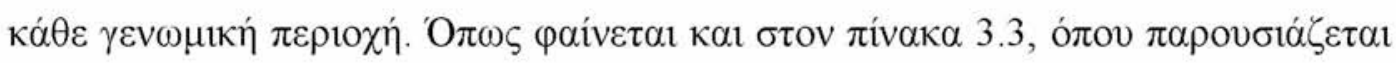

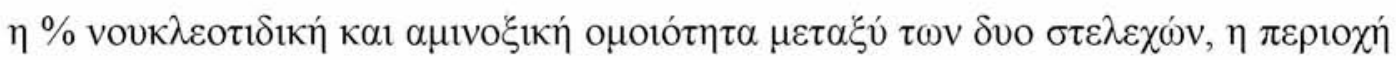

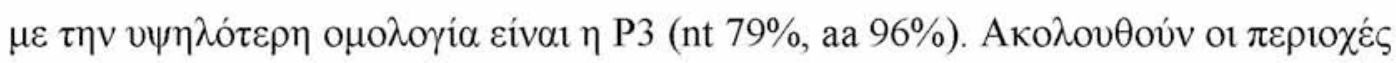

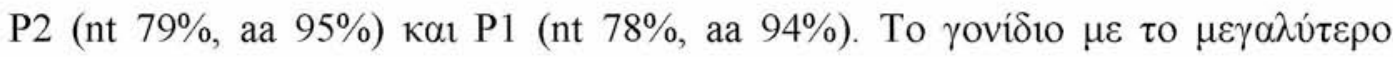

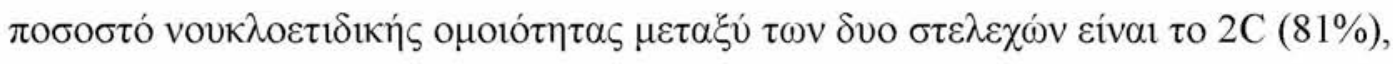

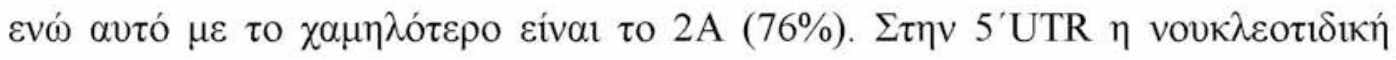

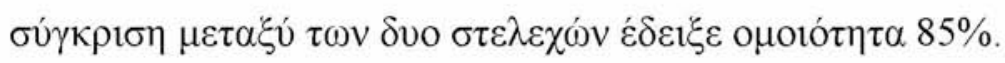

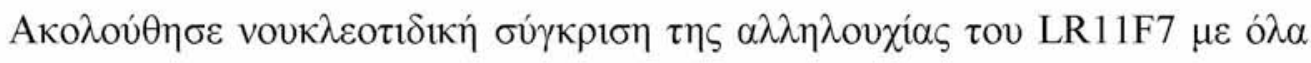

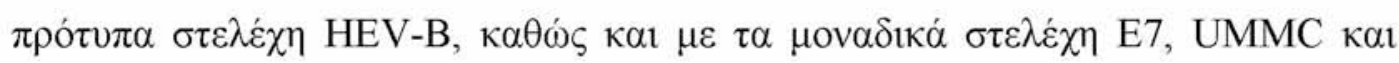

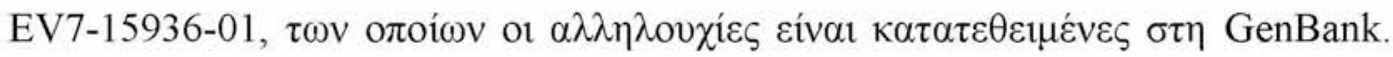

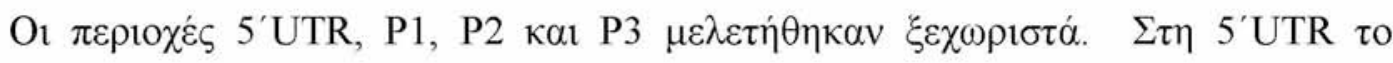

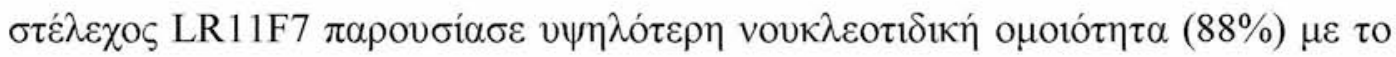

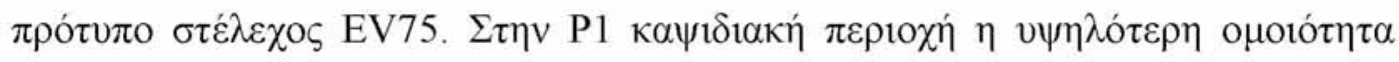

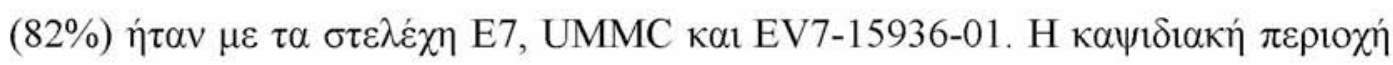

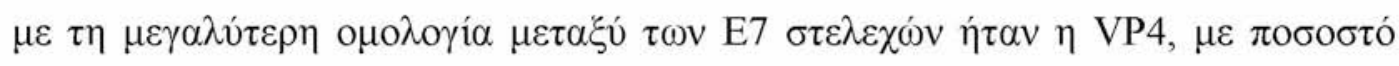

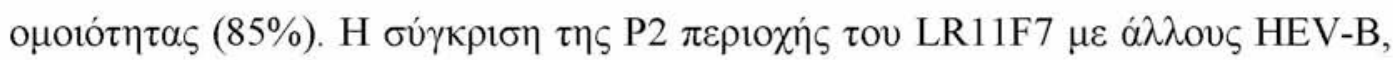

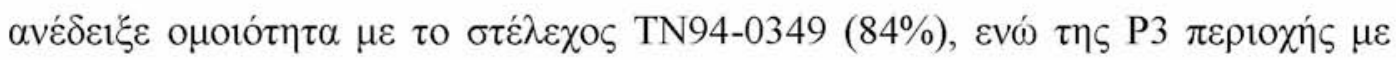

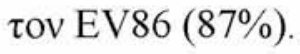

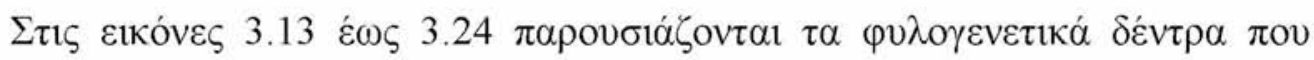

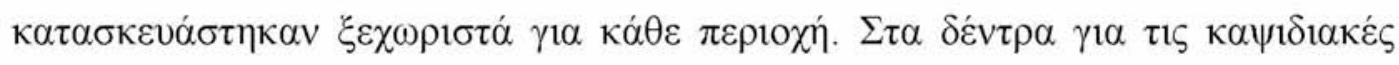

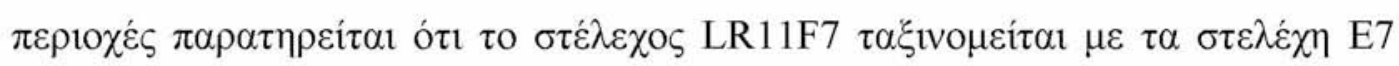

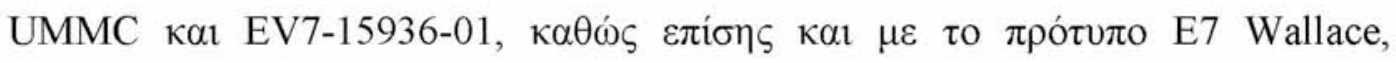

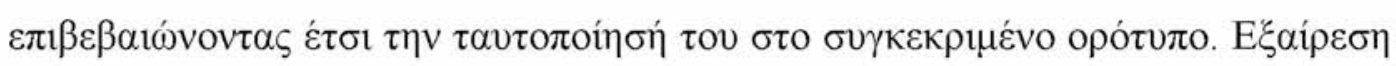

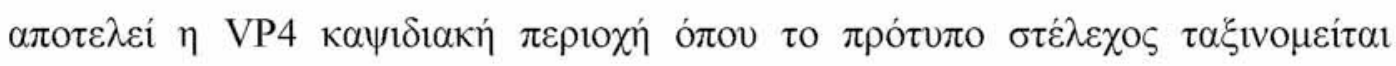

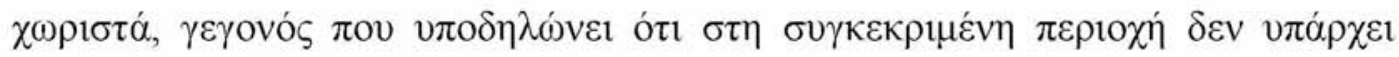

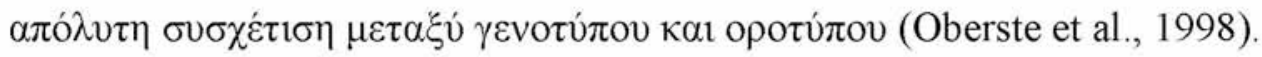




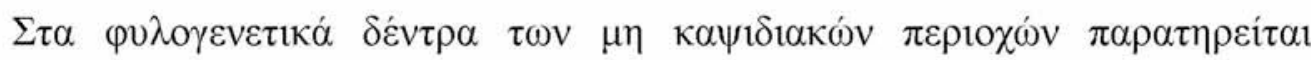

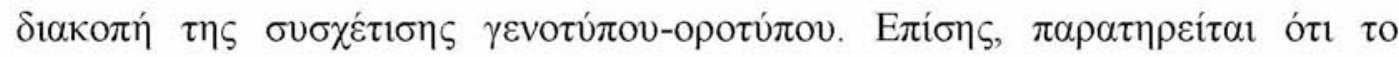

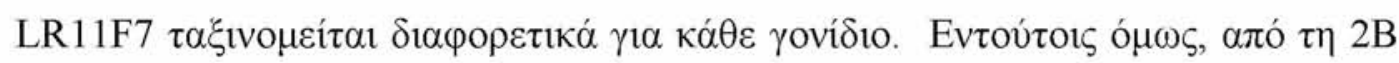

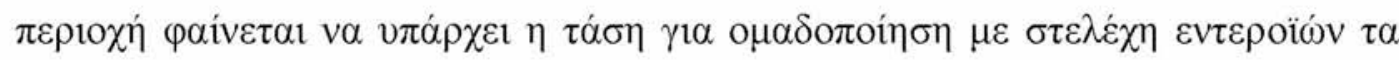

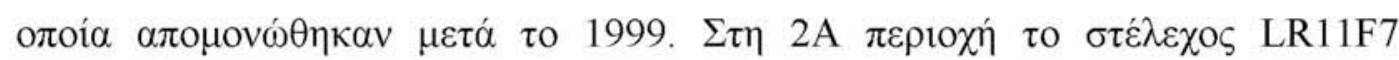

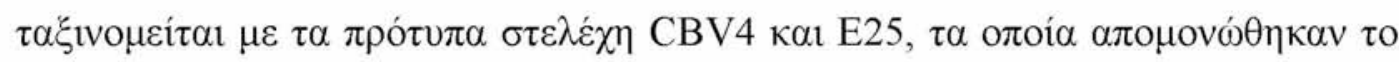

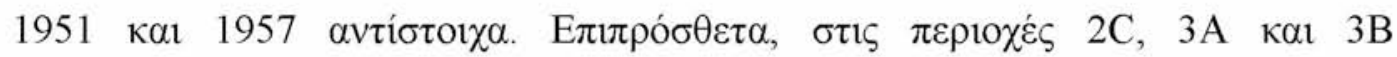

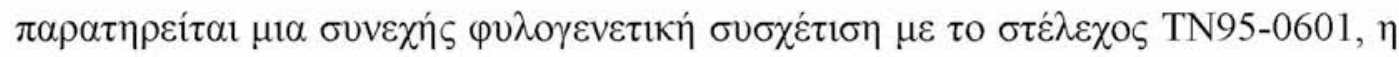

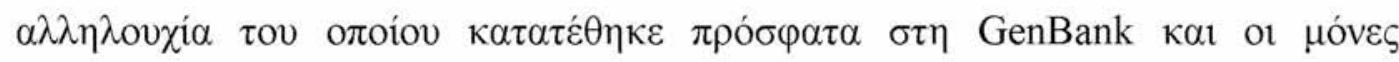

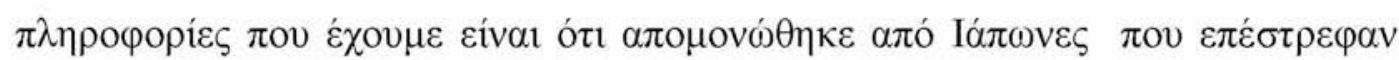
$\alpha \pi$ ó $\tau \eta$ Nó $\imath 1 \alpha$ Aбía.

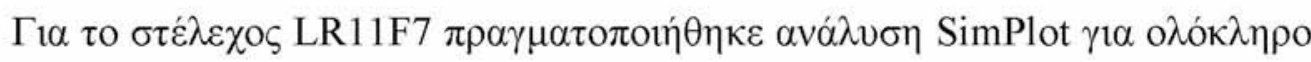

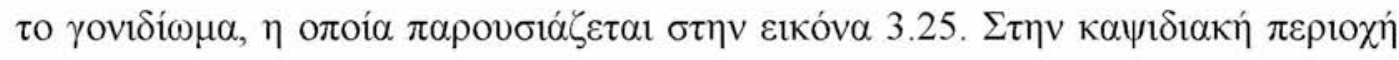

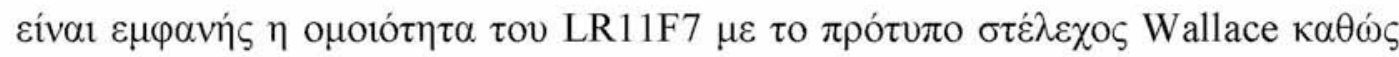

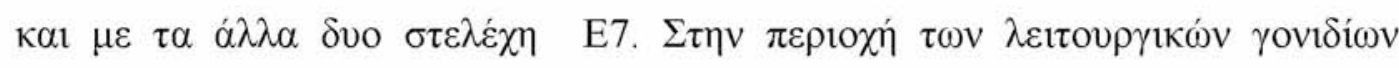

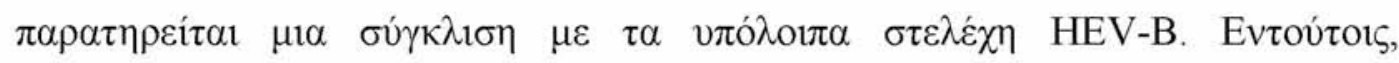

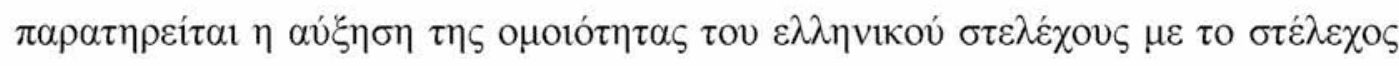

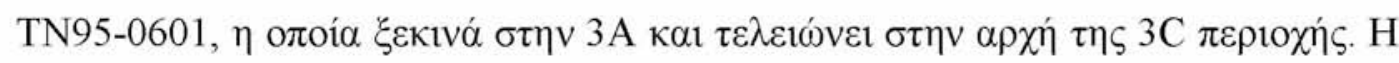

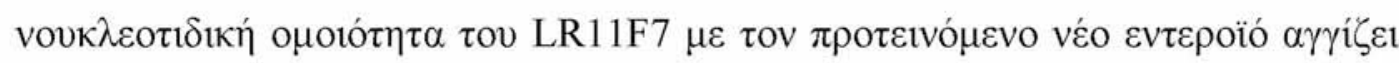

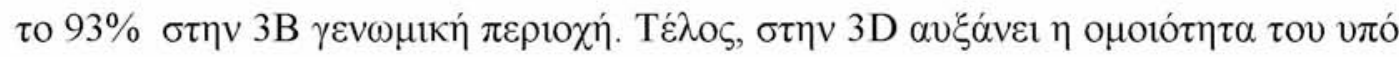

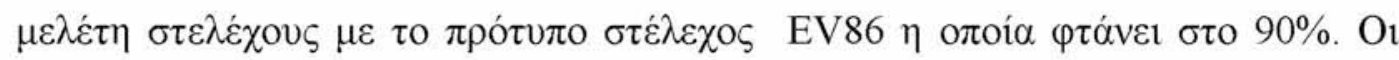

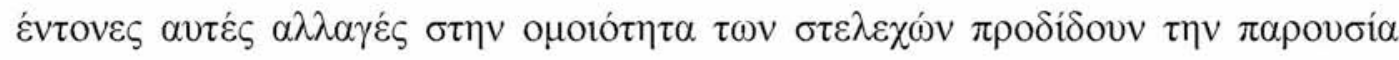

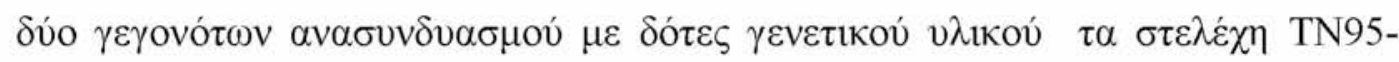

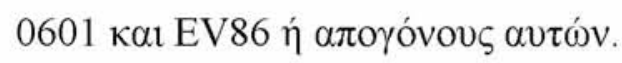

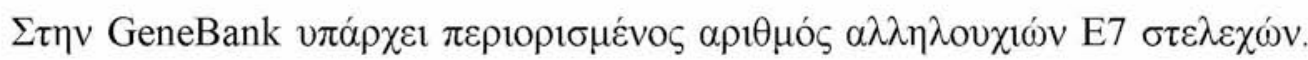

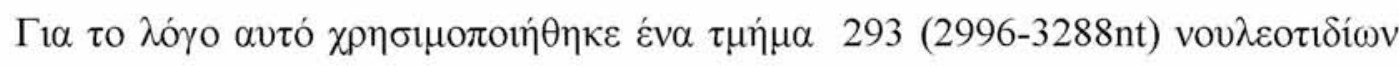

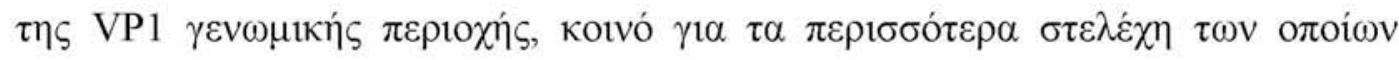

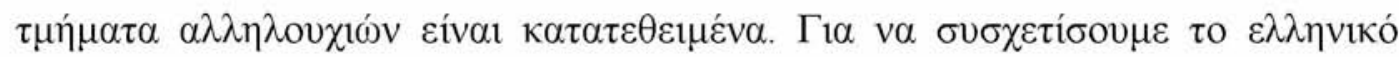

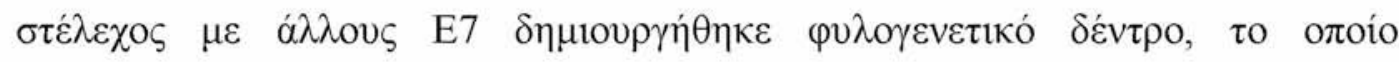

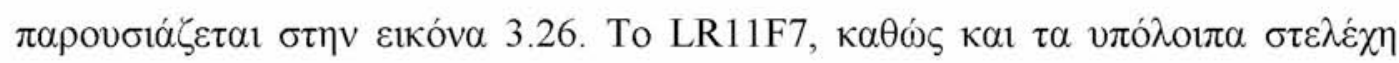

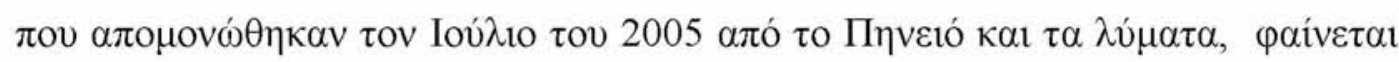

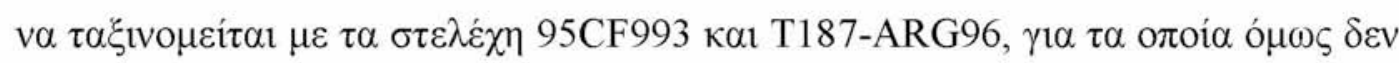

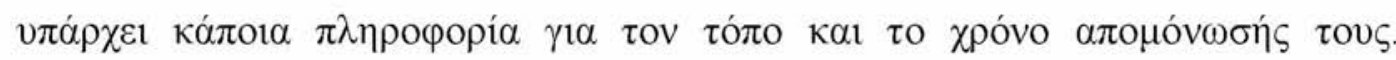




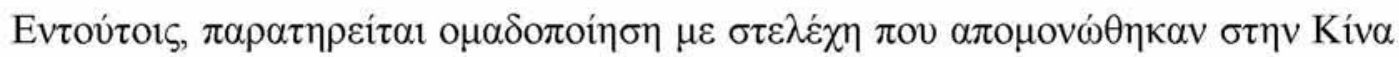

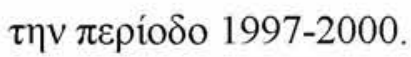

\begin{tabular}{|c|c|c|}
\hline 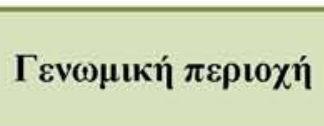 & 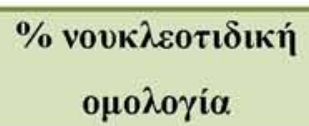 & 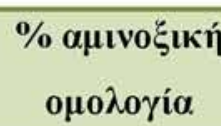 \\
\hline 5' UTR & 85 & - \\
\hline P1 & 78 & 94 \\
\hline VP4 & 79 & 100 \\
\hline VP2 & 78 & 93 \\
\hline VP3 & 79 & 94 \\
\hline VP1 & 78 & 93 \\
\hline P2 & 79 & 95 \\
\hline $2 \mathrm{~A}$ & 76 & 91 \\
\hline $2 \mathrm{~B}$ & 78 & 93 \\
\hline $2 \mathrm{C}$ & 81 & 98 \\
\hline P3 & 79 & 96 \\
\hline $3 \mathrm{~A}$ & 79 & 96 \\
\hline $3 \mathrm{~B}$ & 78 & 95 \\
\hline $3 \mathrm{C}$ & 79 & 96 \\
\hline $3 \mathrm{D}^{*}$ & 79 & 97 \\
\hline
\end{tabular}

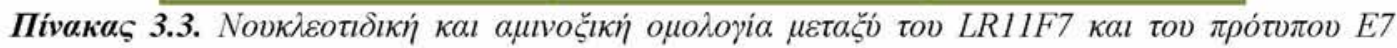

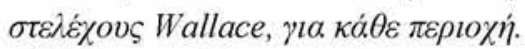

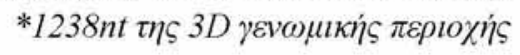




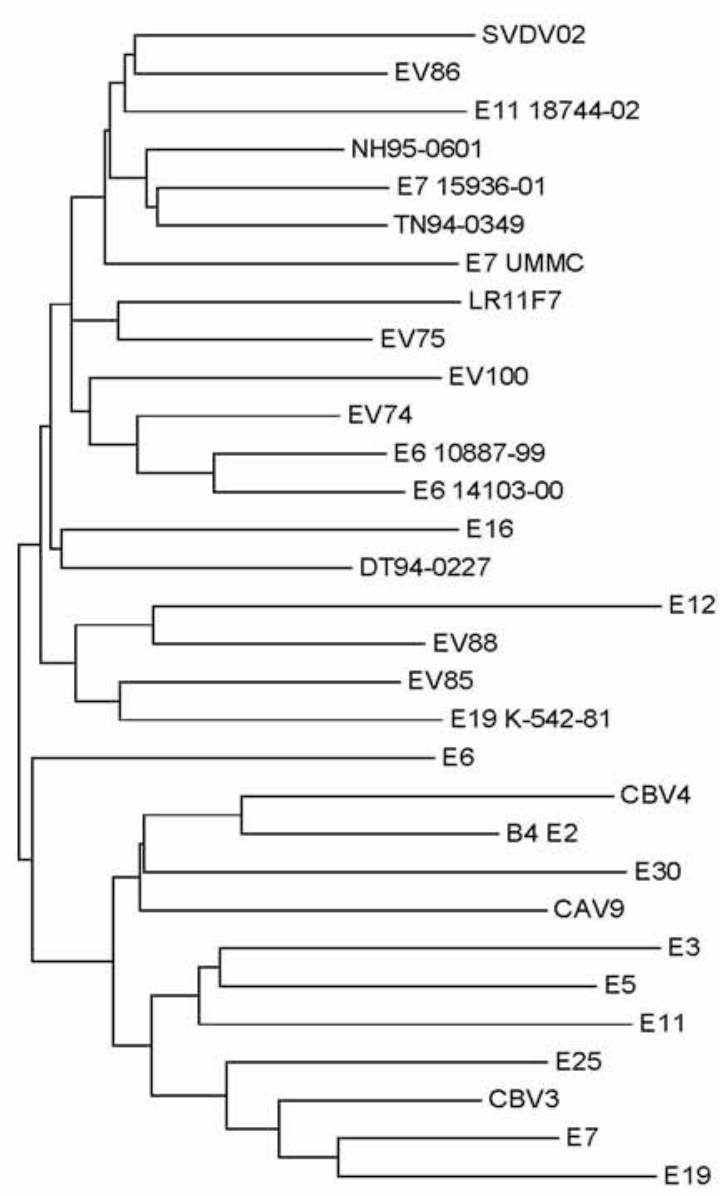

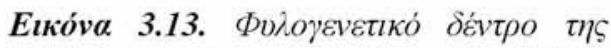

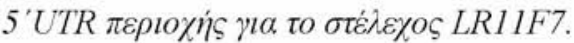

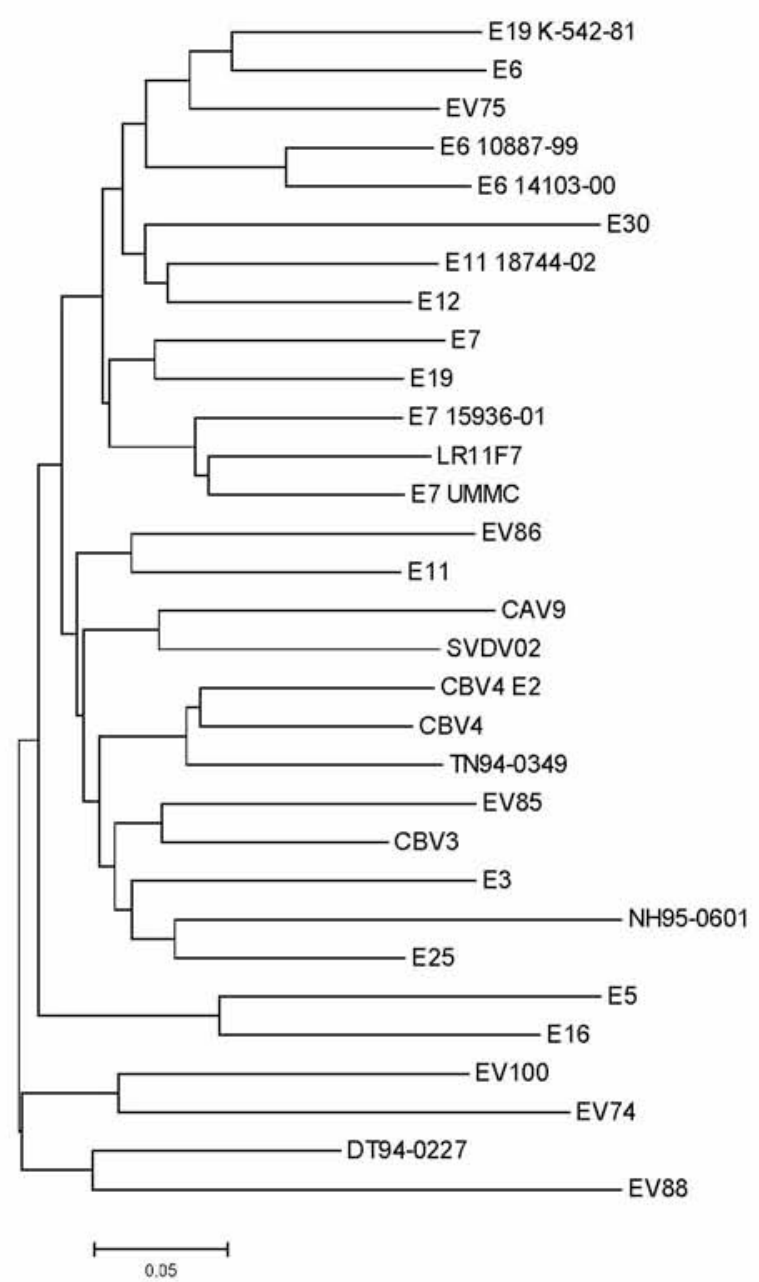




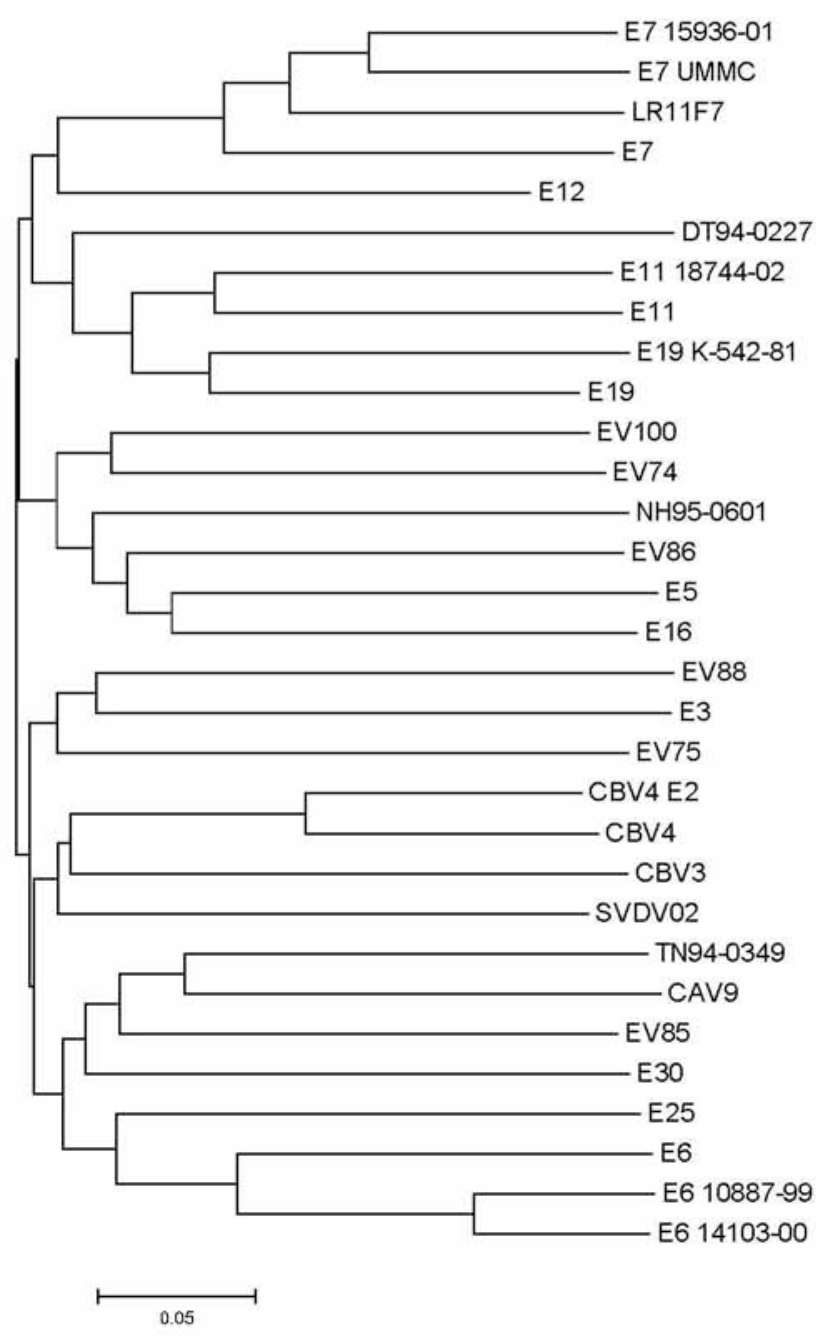

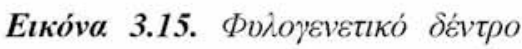

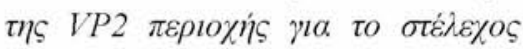
LRIIF7.

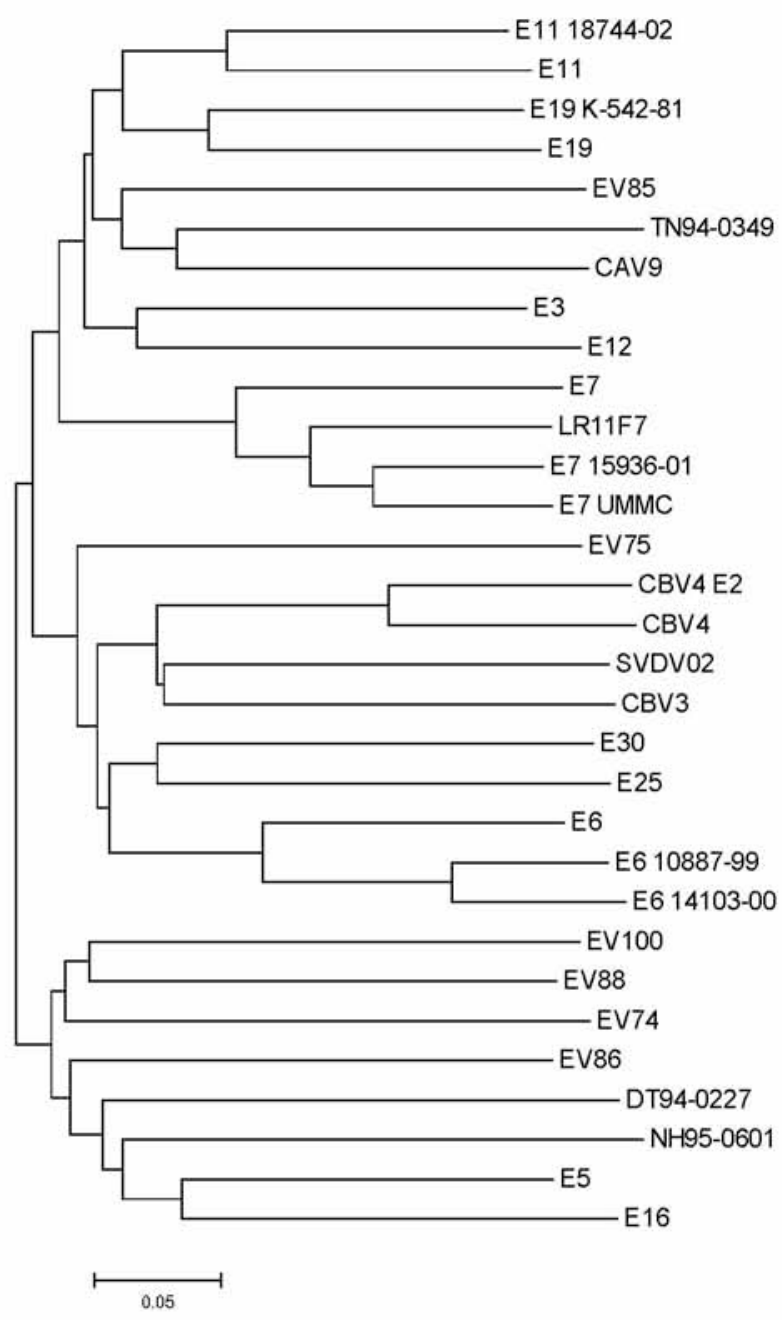

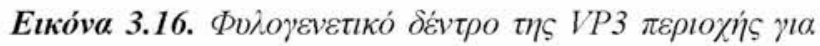

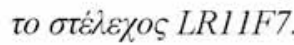




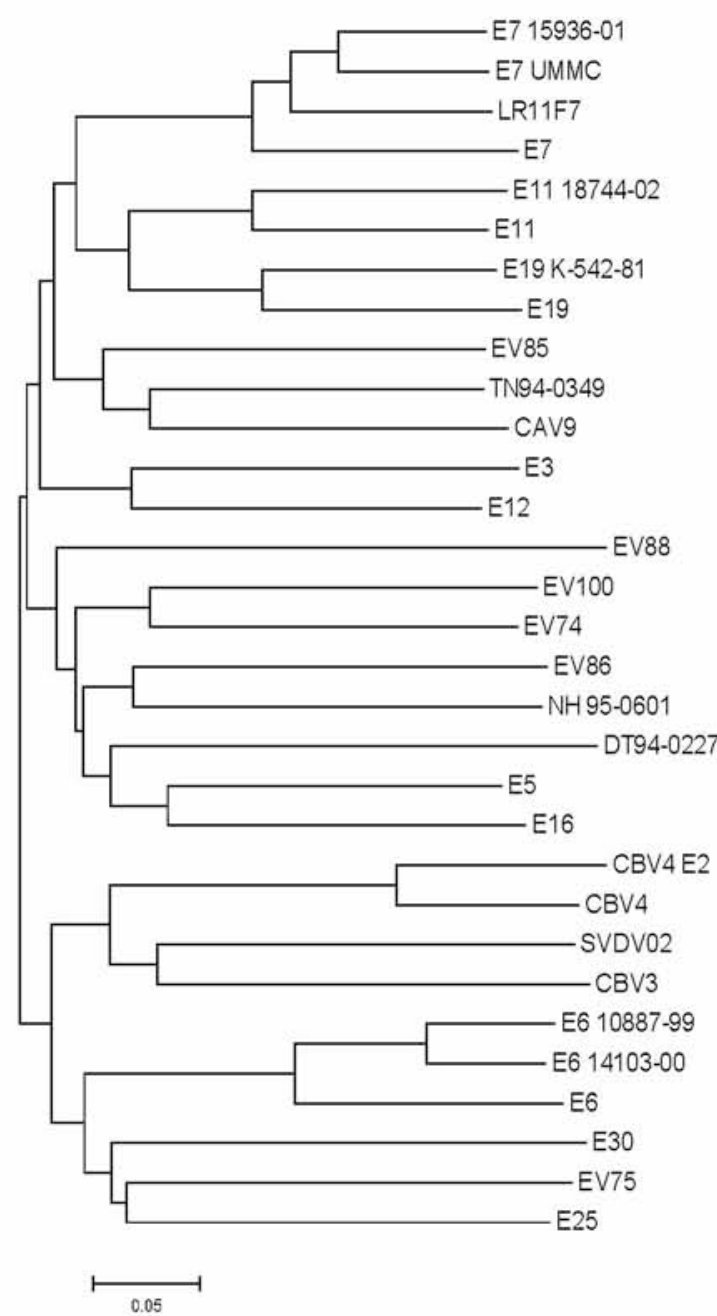

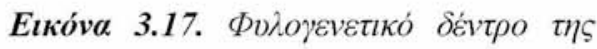

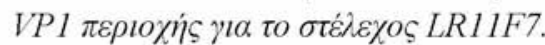

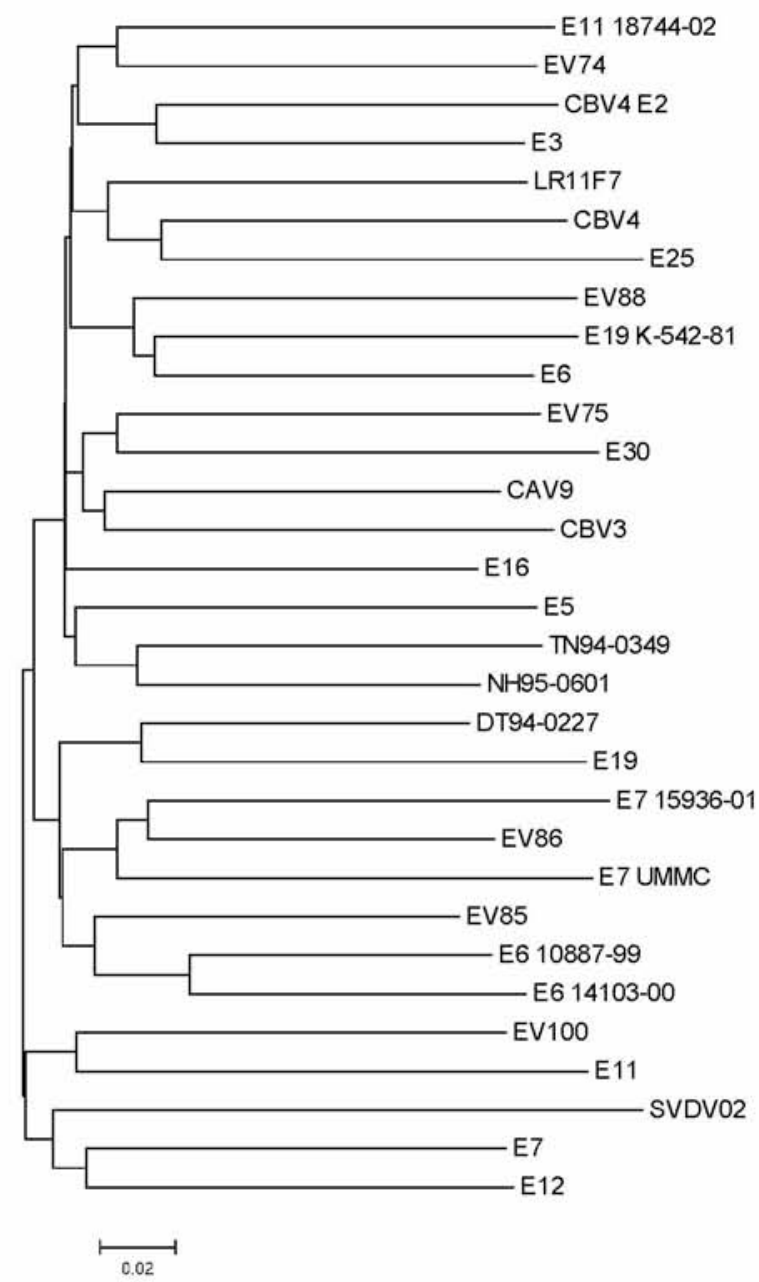




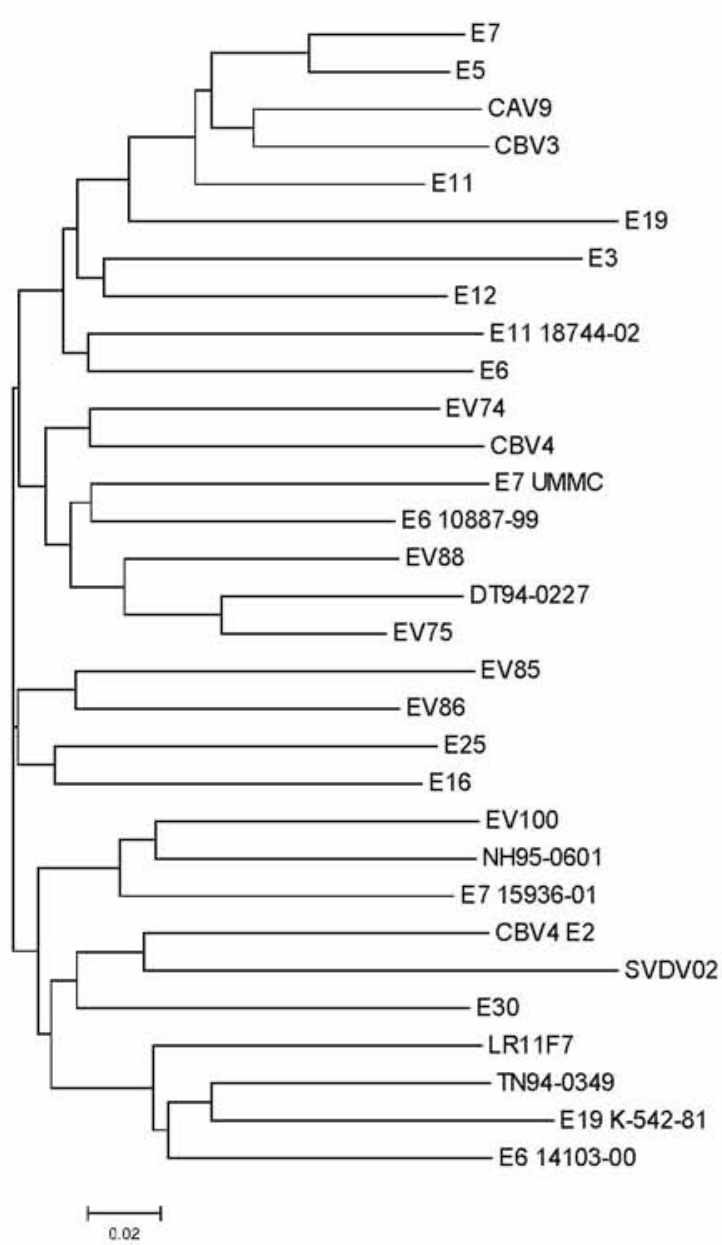

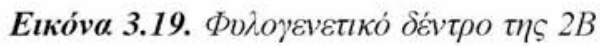

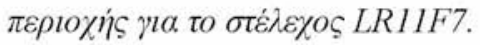

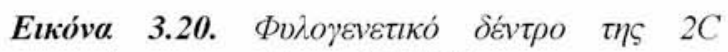

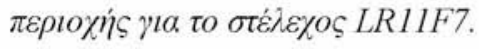

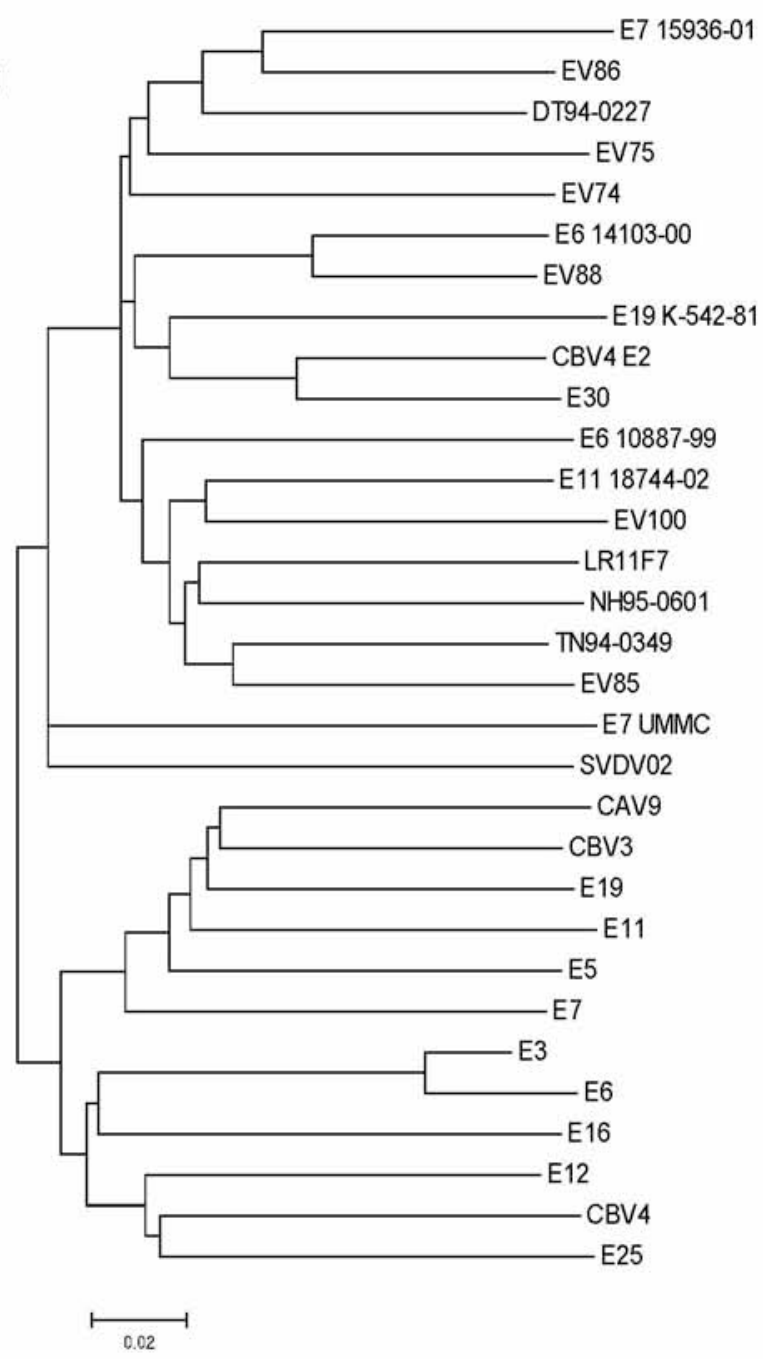




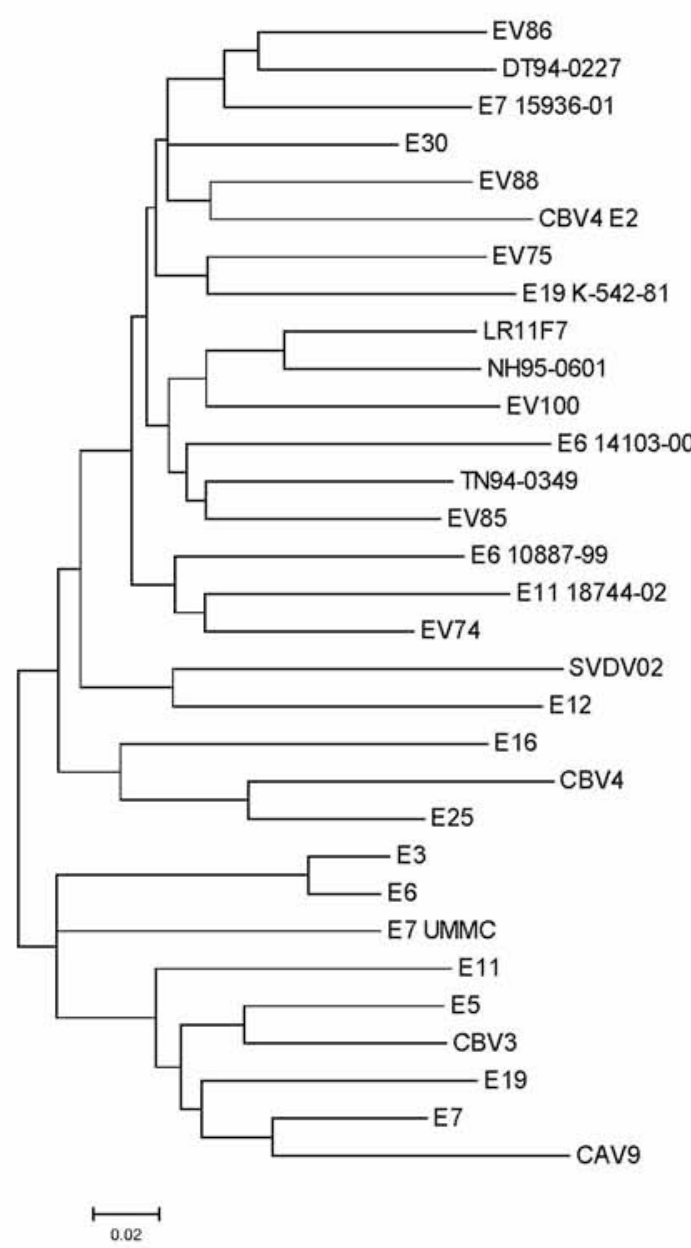

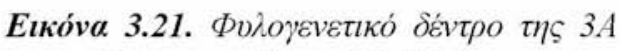

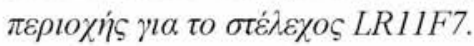

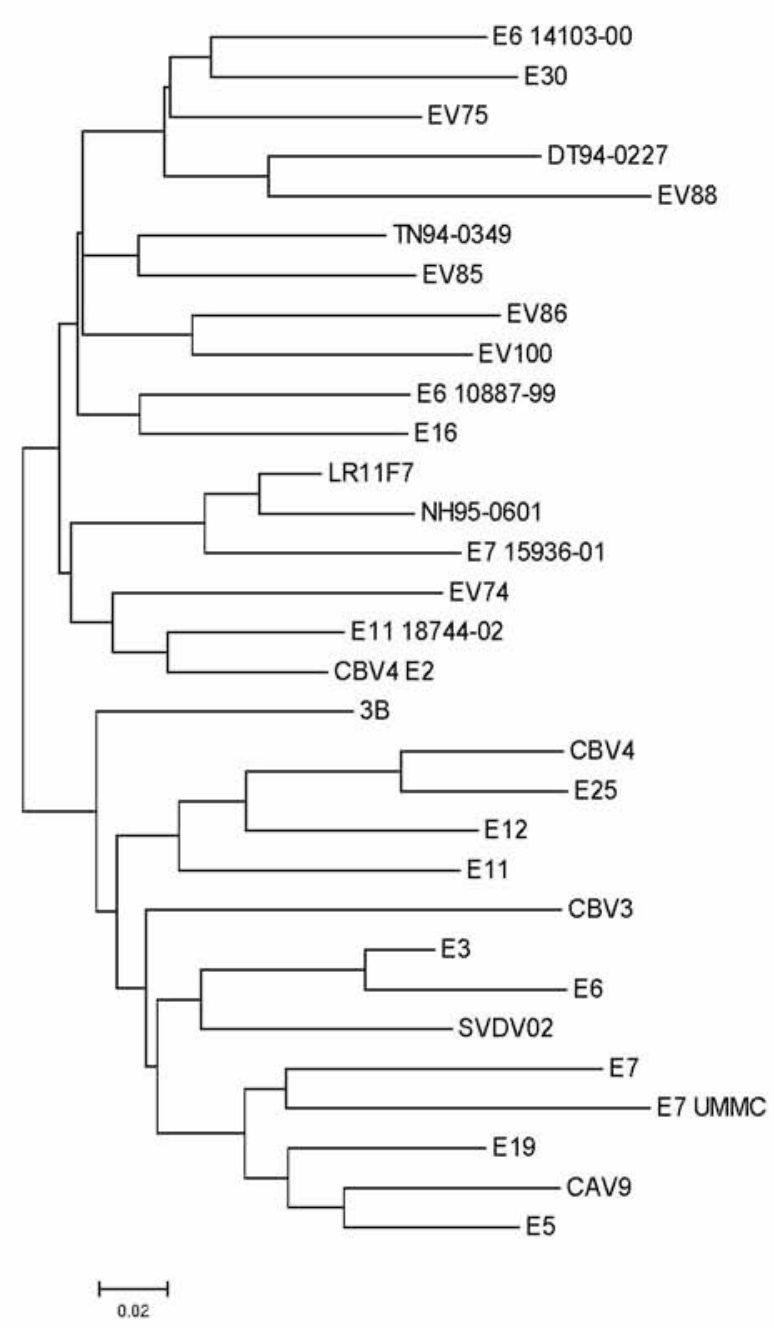




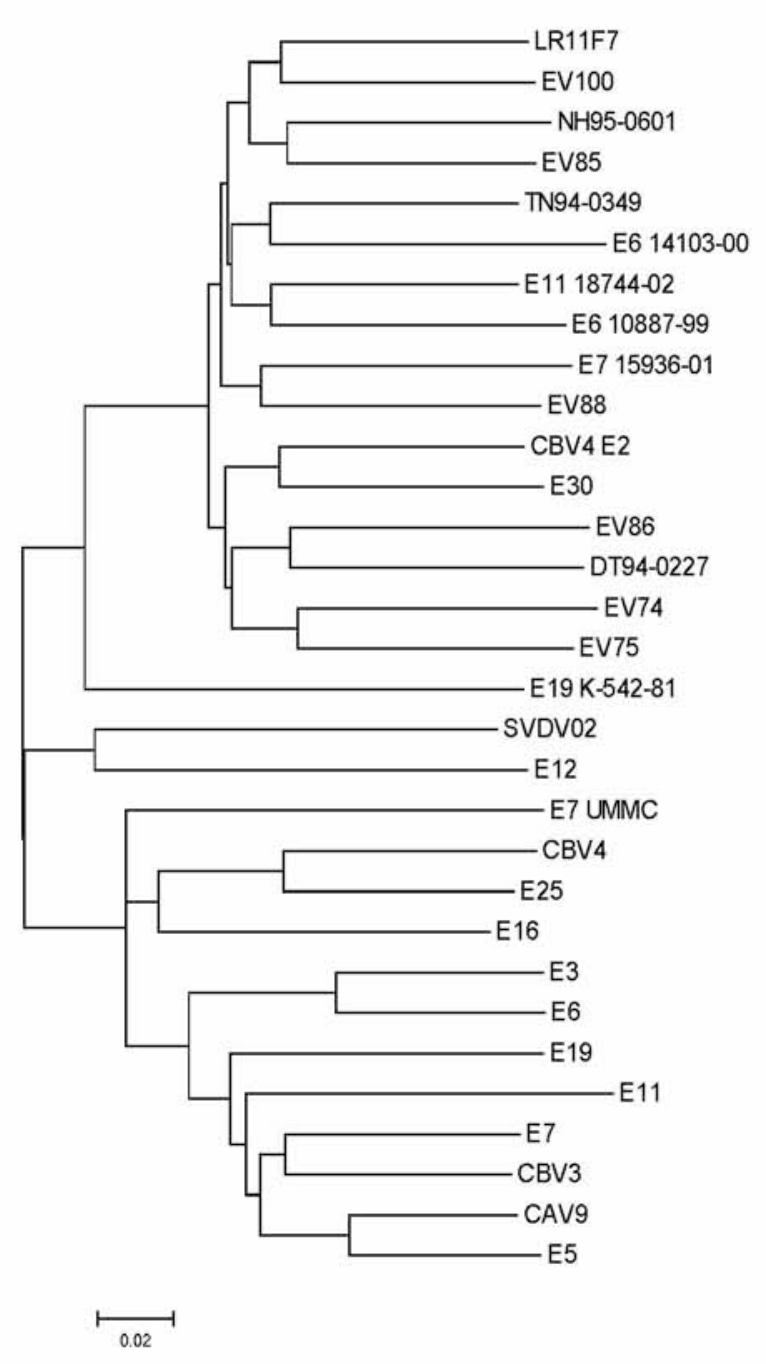

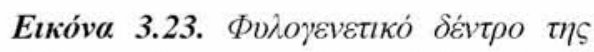

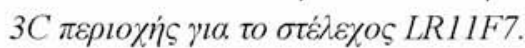

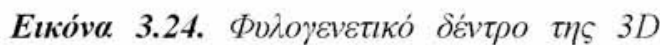

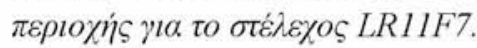

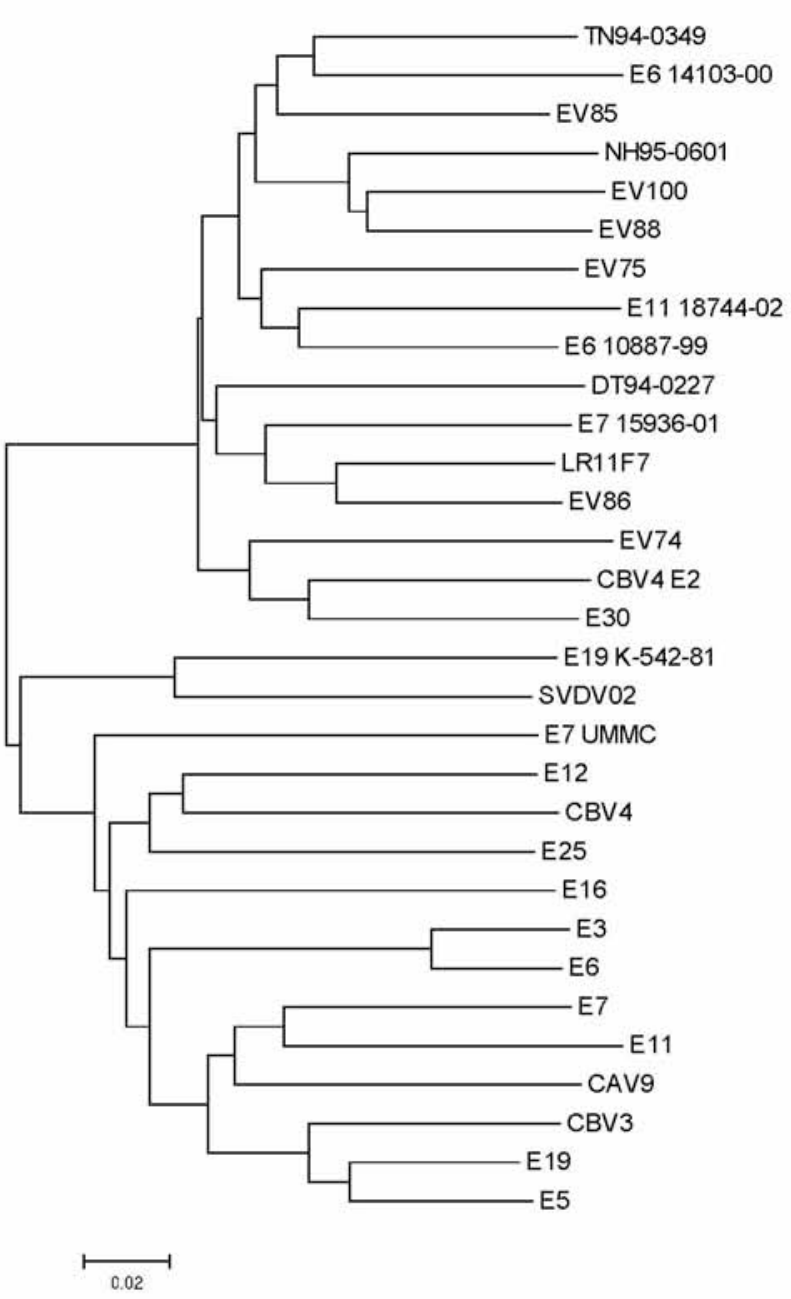




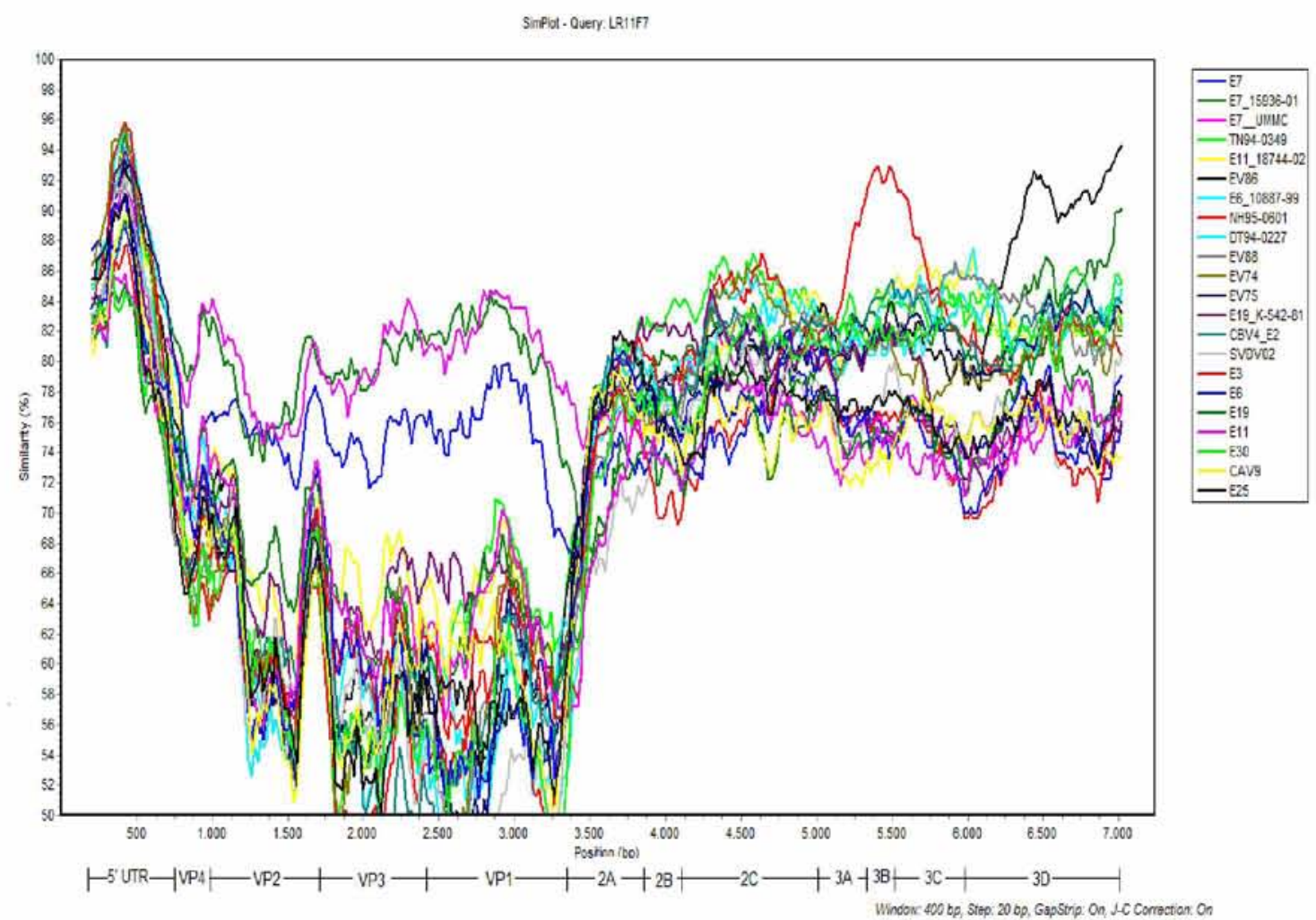

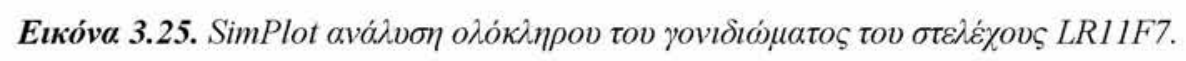

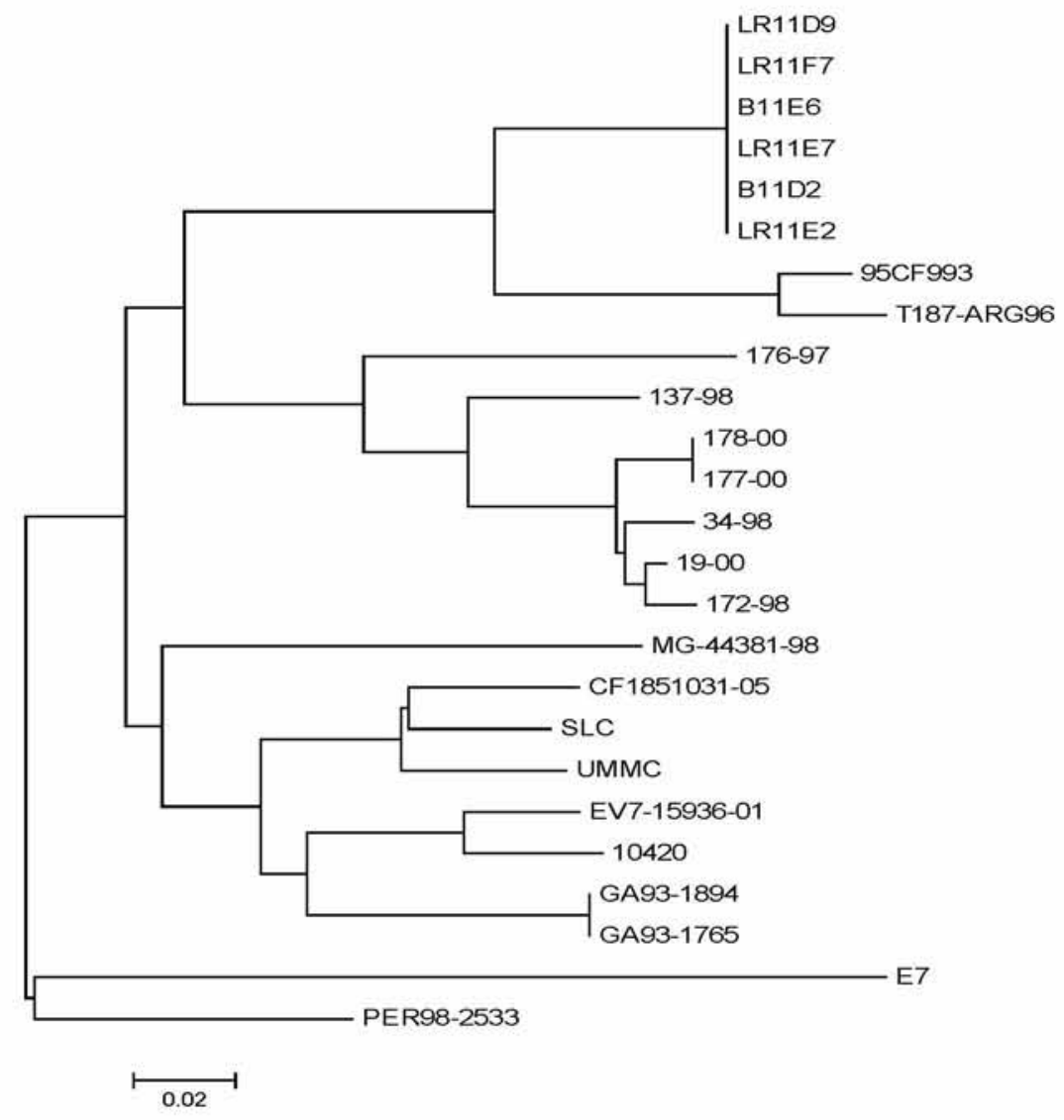

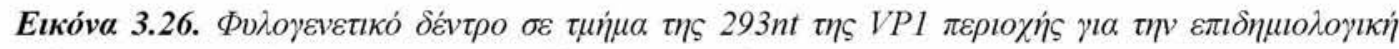

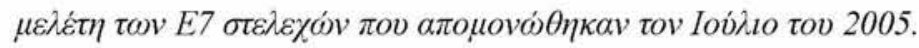




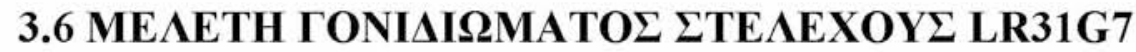

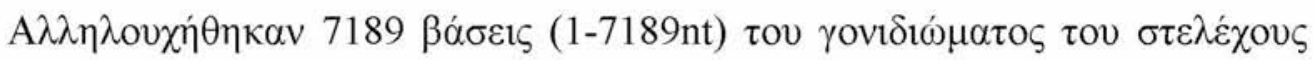
LR31G7.

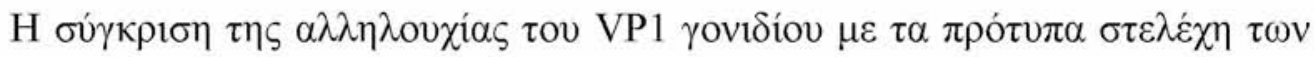

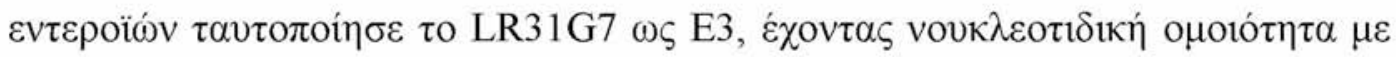

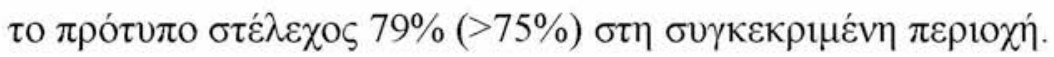

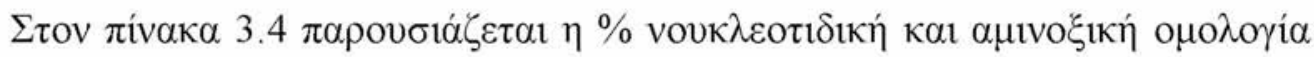

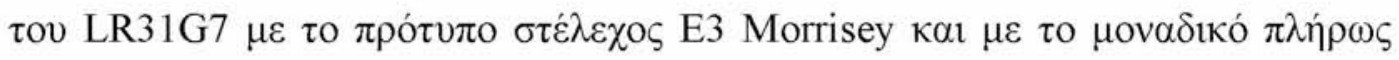

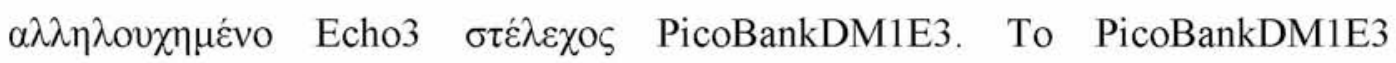

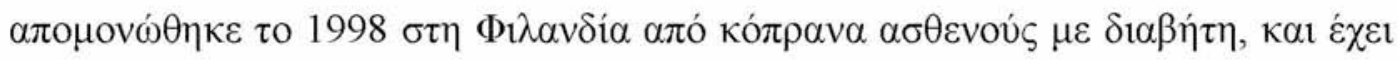

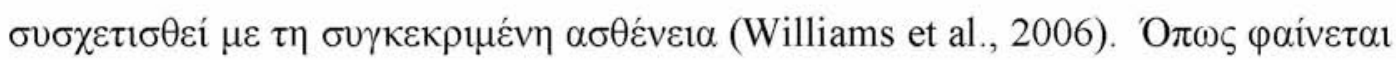

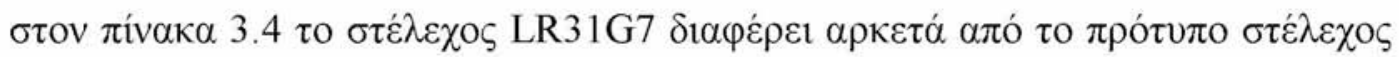

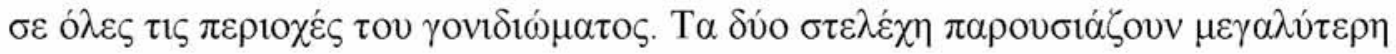

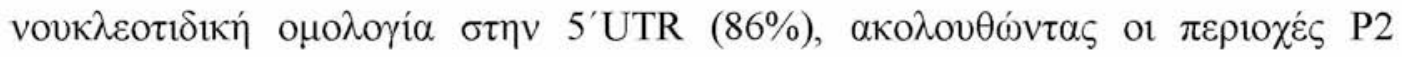

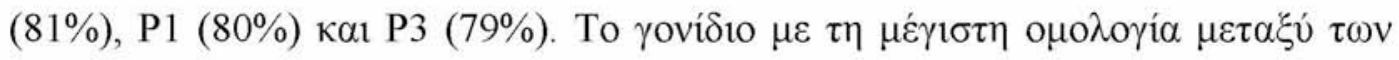

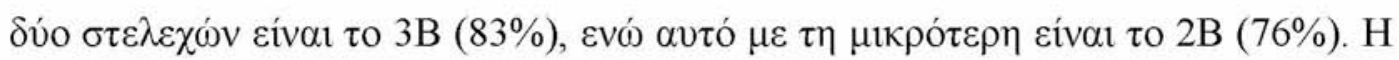

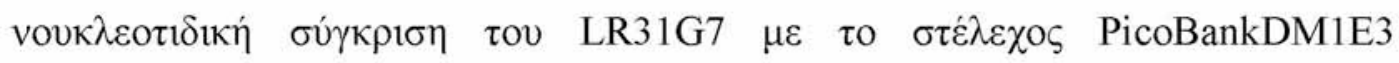

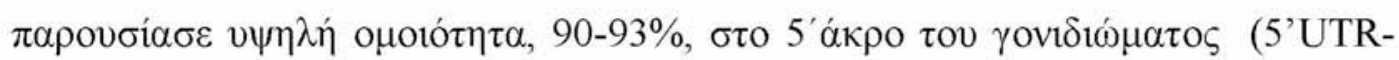

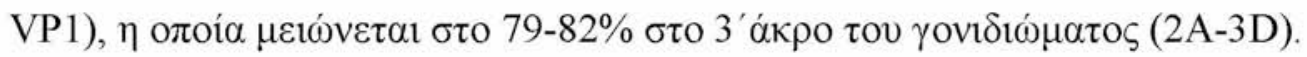

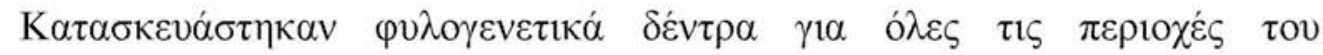

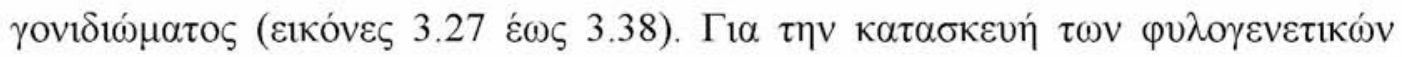

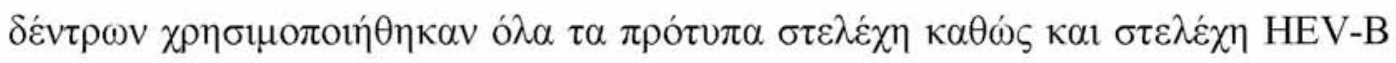

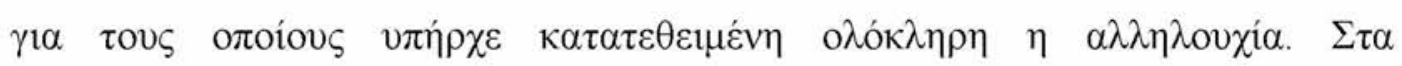

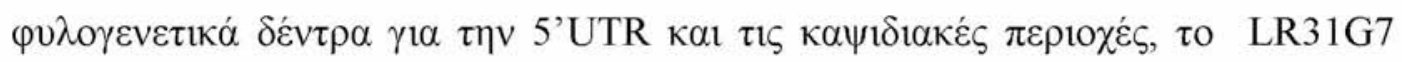

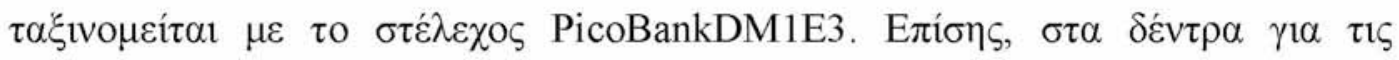

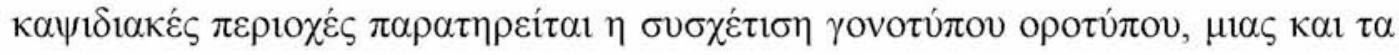

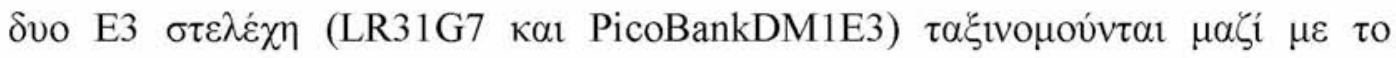

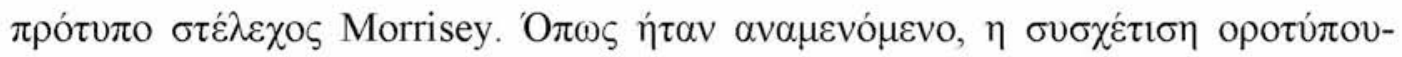

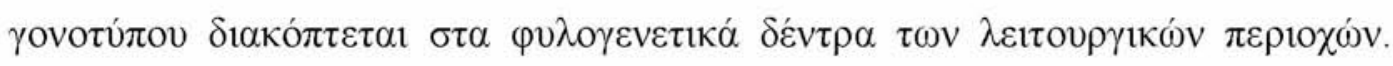

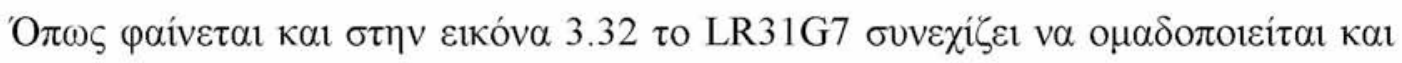

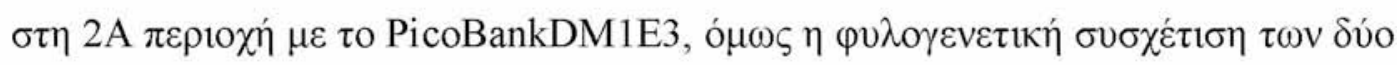

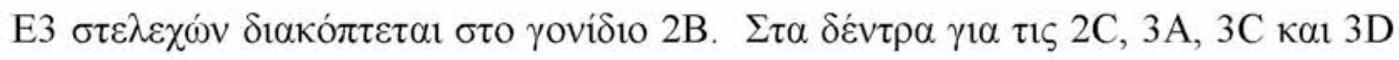

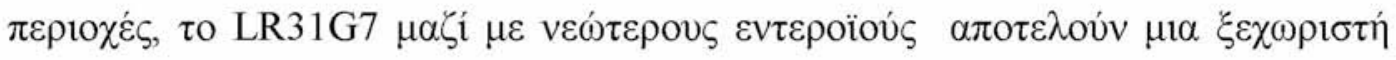




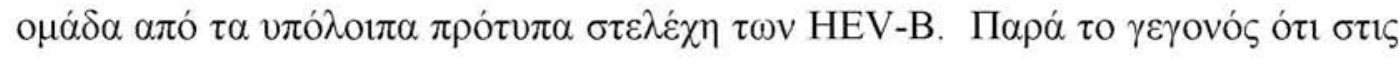

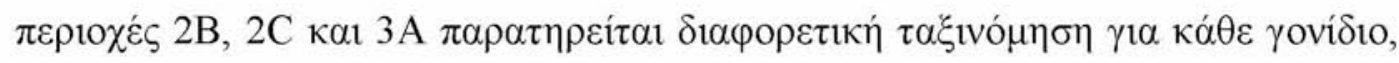

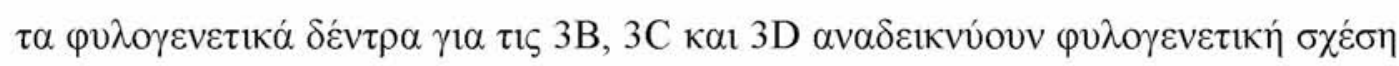

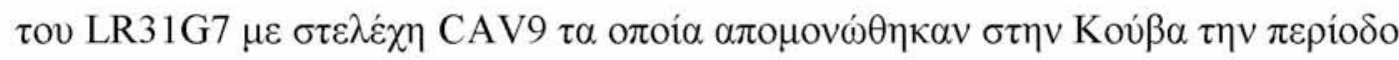

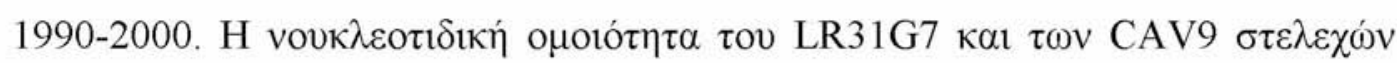

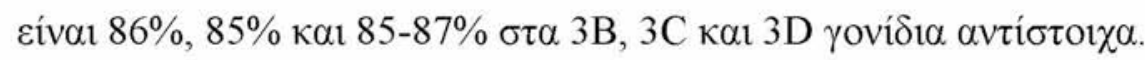

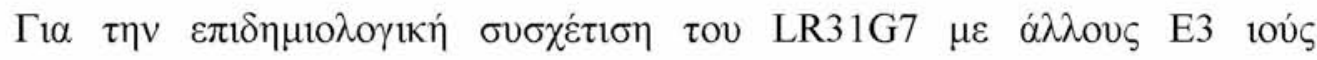

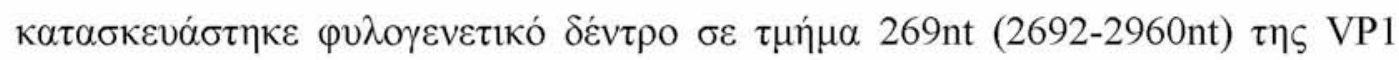

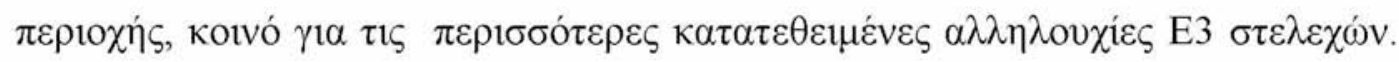

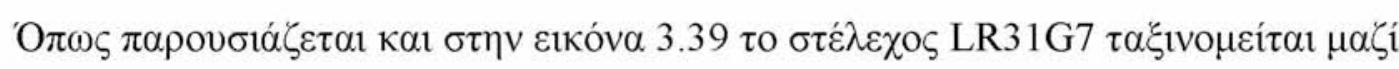

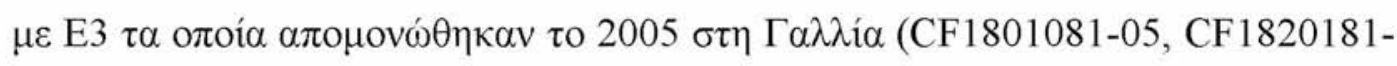
05) каı $\tau \eta v$ I $\alpha \pi \omega v i ́ \alpha$ (Fukuoka City2005-70, Fukuoka City2005-97). H

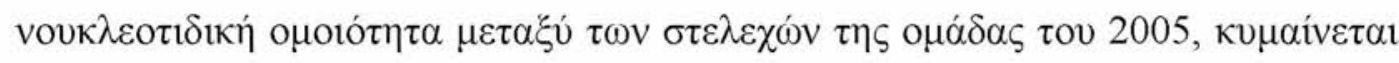

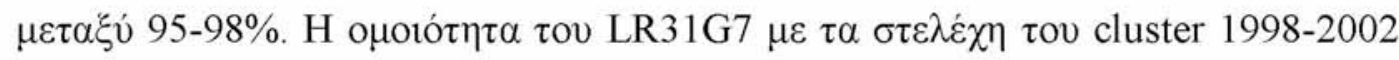

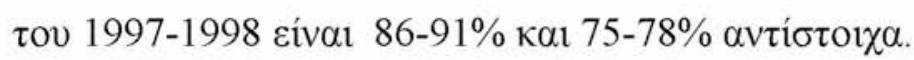

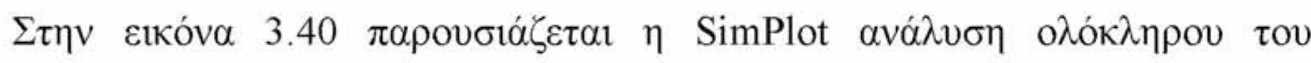

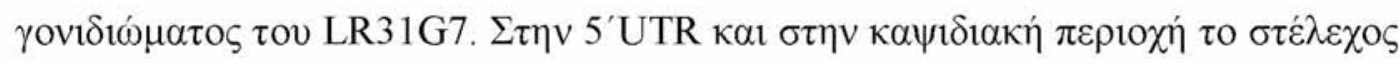

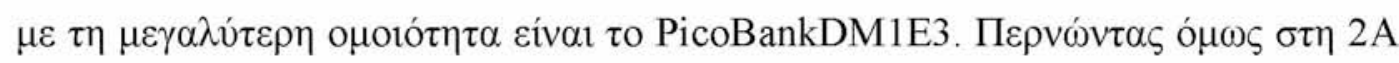

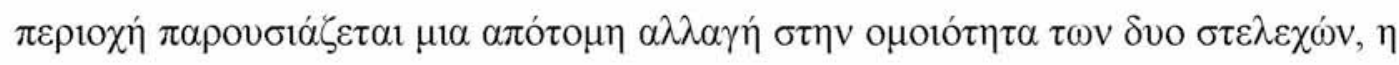

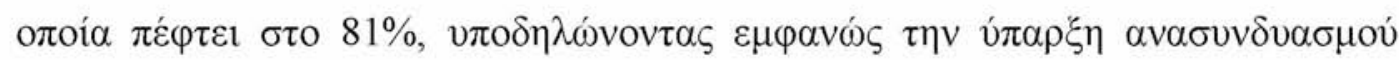

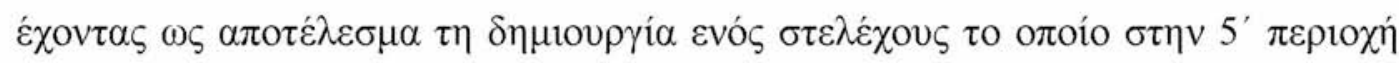

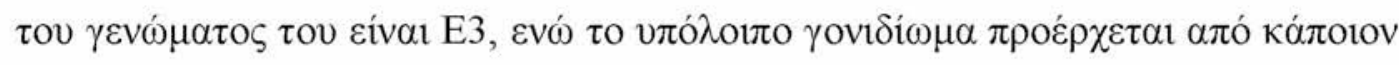

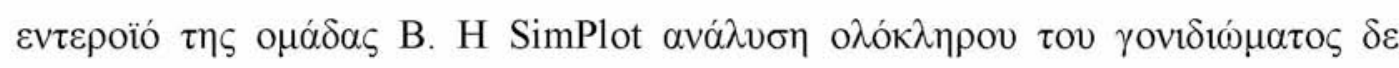

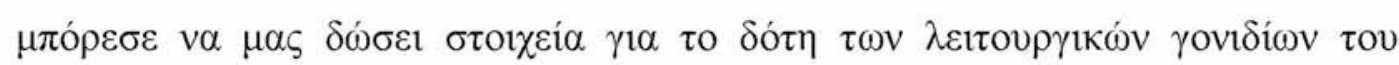

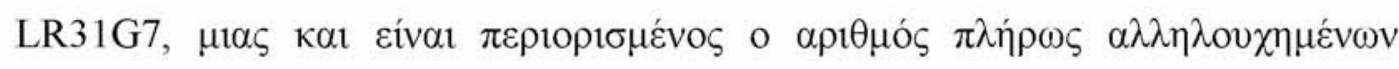

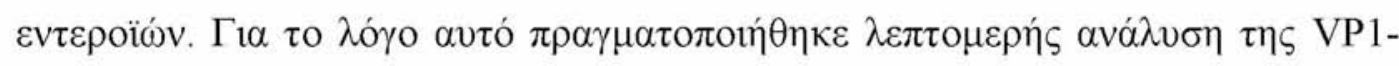

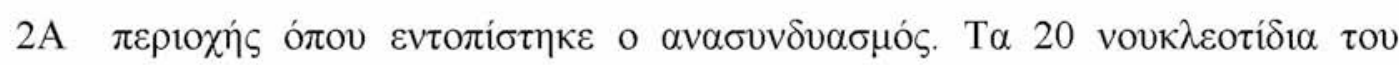

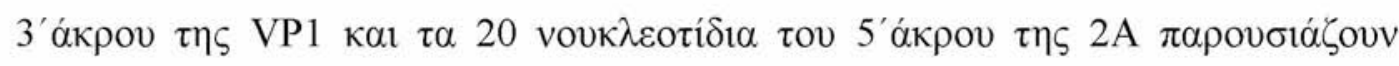

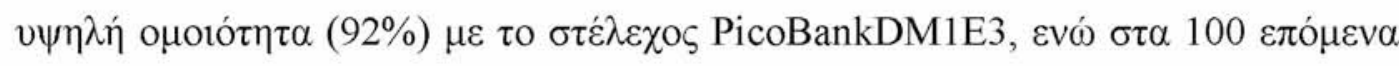

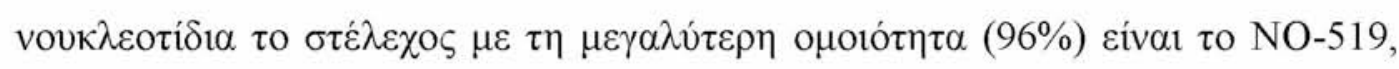

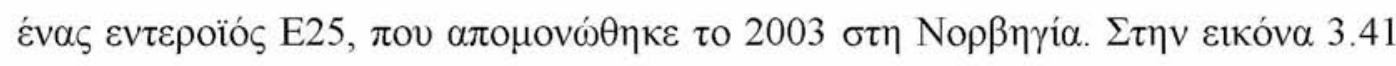

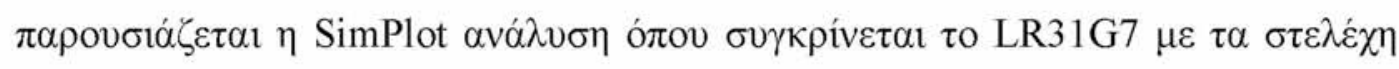

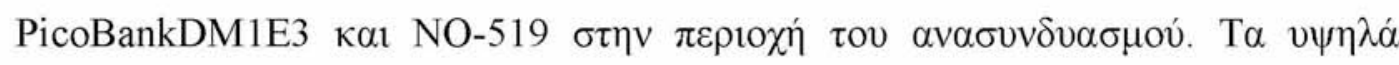

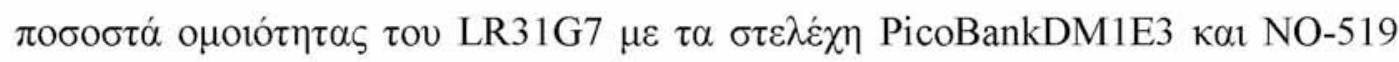




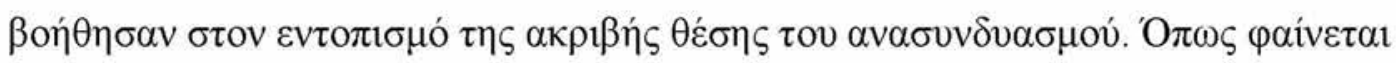

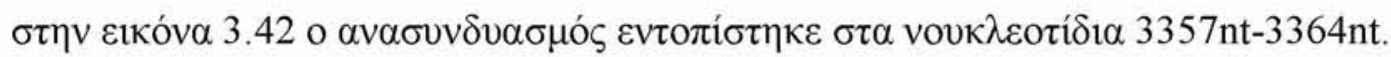

\begin{tabular}{|c|c|c|c|c|}
\hline \multirow{2}{*}{ 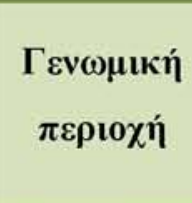 } & \multicolumn{2}{|c|}{ Morrisey } & \multicolumn{2}{|c|}{ PicoBankDM1E3 } \\
\hline & 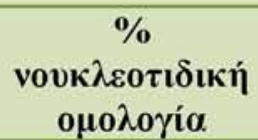 & 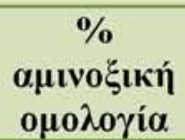 & 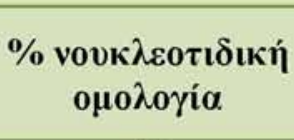 & 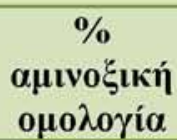 \\
\hline 5' UTR & 86 & - & 93 & - \\
\hline P1 & 80 & 97 & 90 & 99 \\
\hline VP4 & 80 & 98 & 92 & 100 \\
\hline VP2 & 79 & 97 & 90 & 99 \\
\hline VP3 & 82 & 97 & 91 & 99 \\
\hline VP1 & 79 & 96 & 90 & 98 \\
\hline P2 & 81 & 96 & 81 & 96 \\
\hline $2 \mathrm{~A}$ & 82 & 94 & 82 & 94 \\
\hline $2 B$ & 76 & 94 & 79 & 98 \\
\hline $2 \mathrm{C}$ & 81 & 98 & 82 & 97 \\
\hline P3 & 79 & 95 & 81 & 97 \\
\hline $3 \mathrm{~A}$ & 79 & 97 & 80 & 97 \\
\hline $3 \mathrm{~B}$ & 83 & 95 & 80 & 95 \\
\hline $3 \mathrm{C}$ & 78 & 93 & 81 & 97 \\
\hline $3 \mathrm{D}^{*}$ & 78 & 96 & 80 & 97 \\
\hline
\end{tabular}

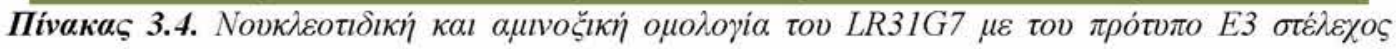

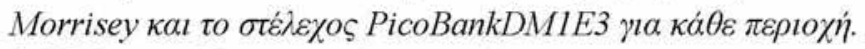

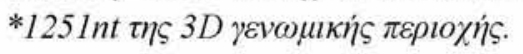




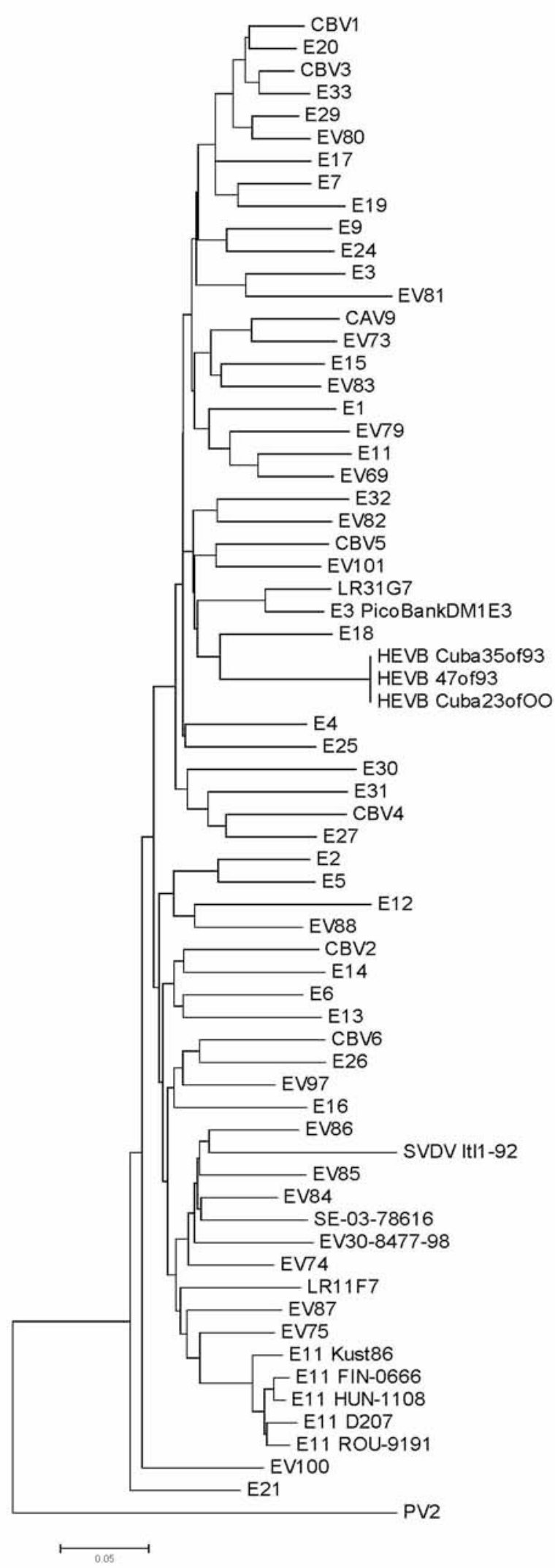

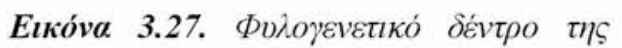

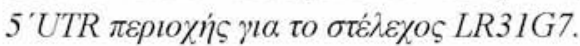

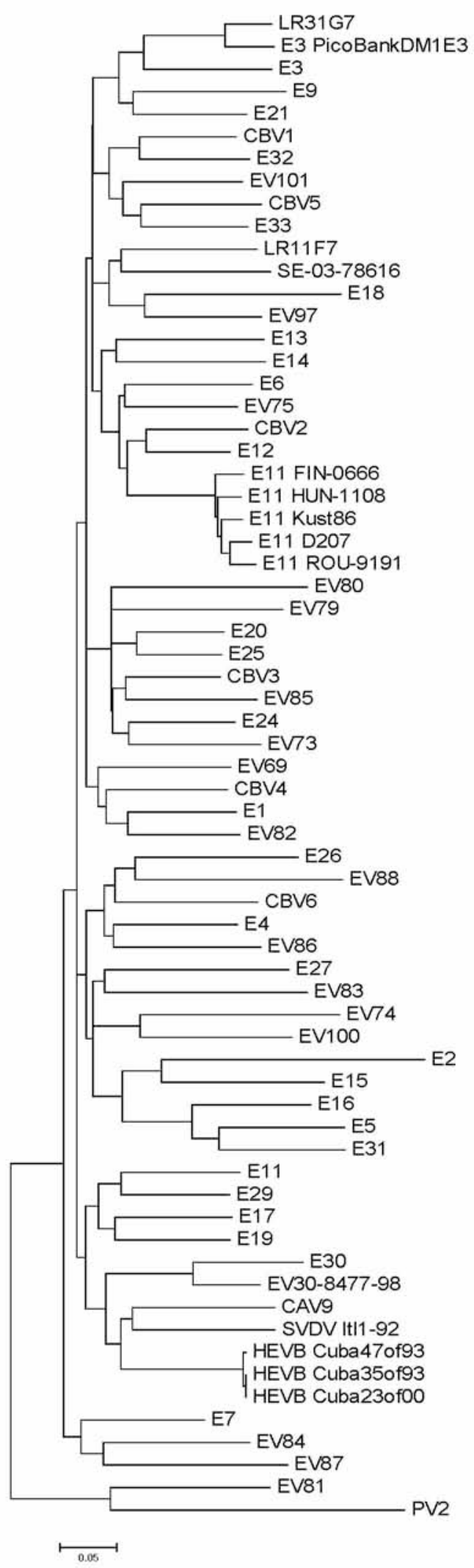

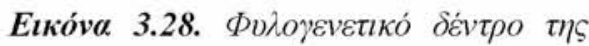

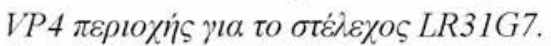




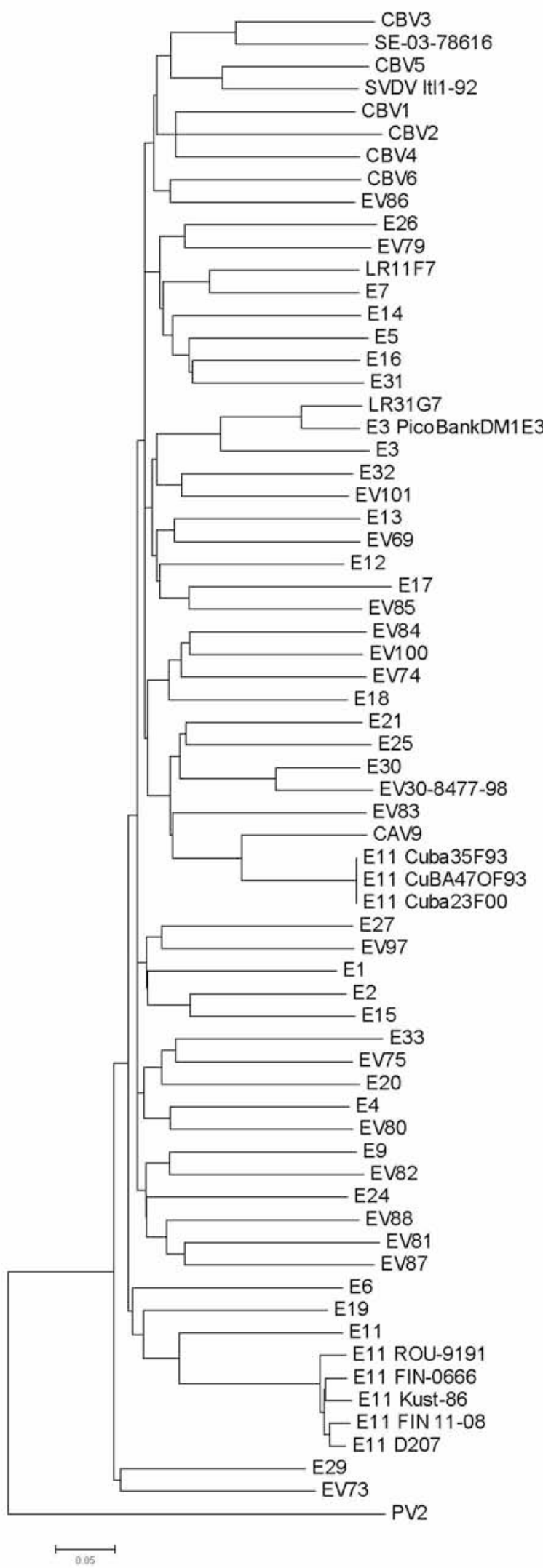

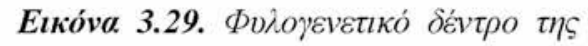

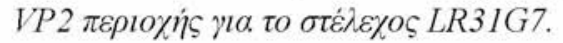

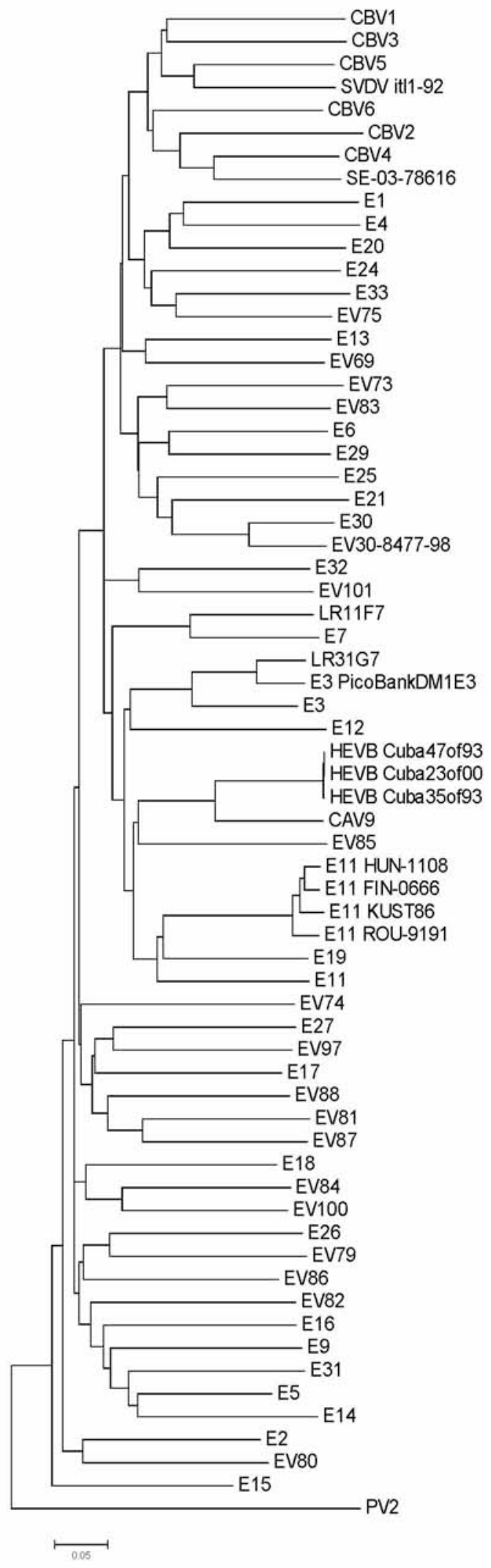

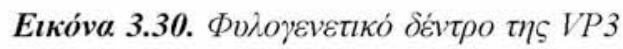

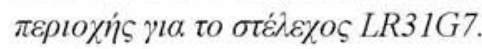




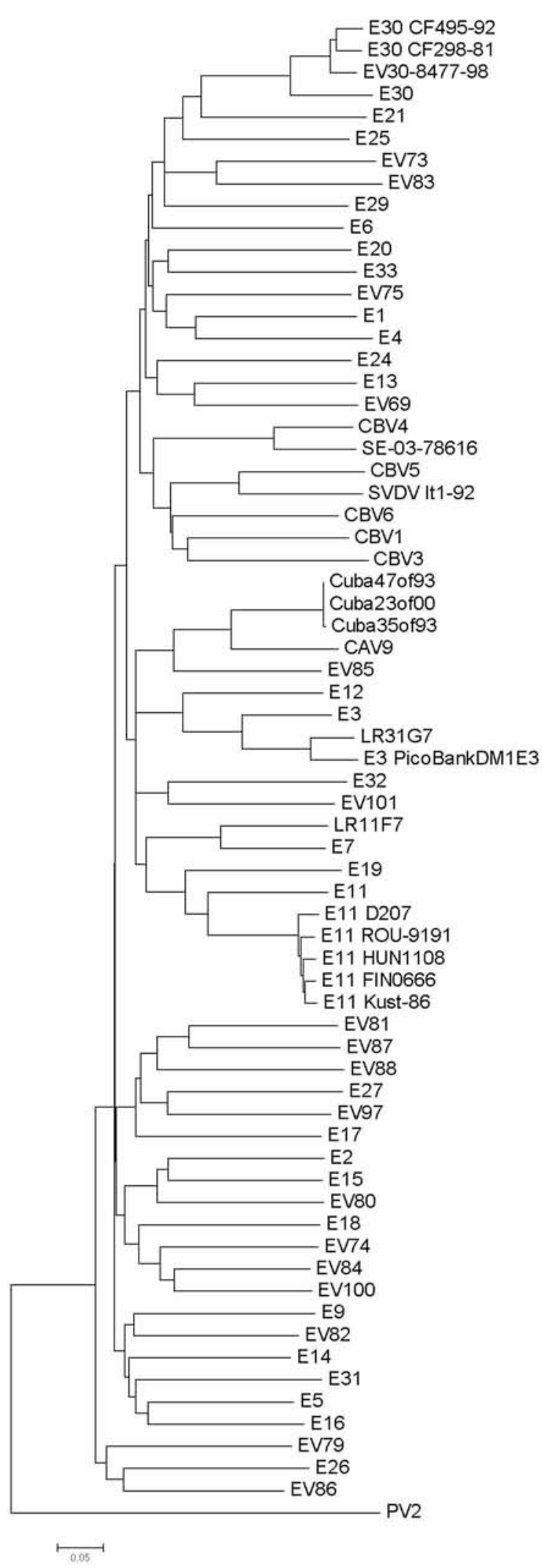

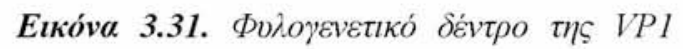

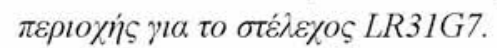

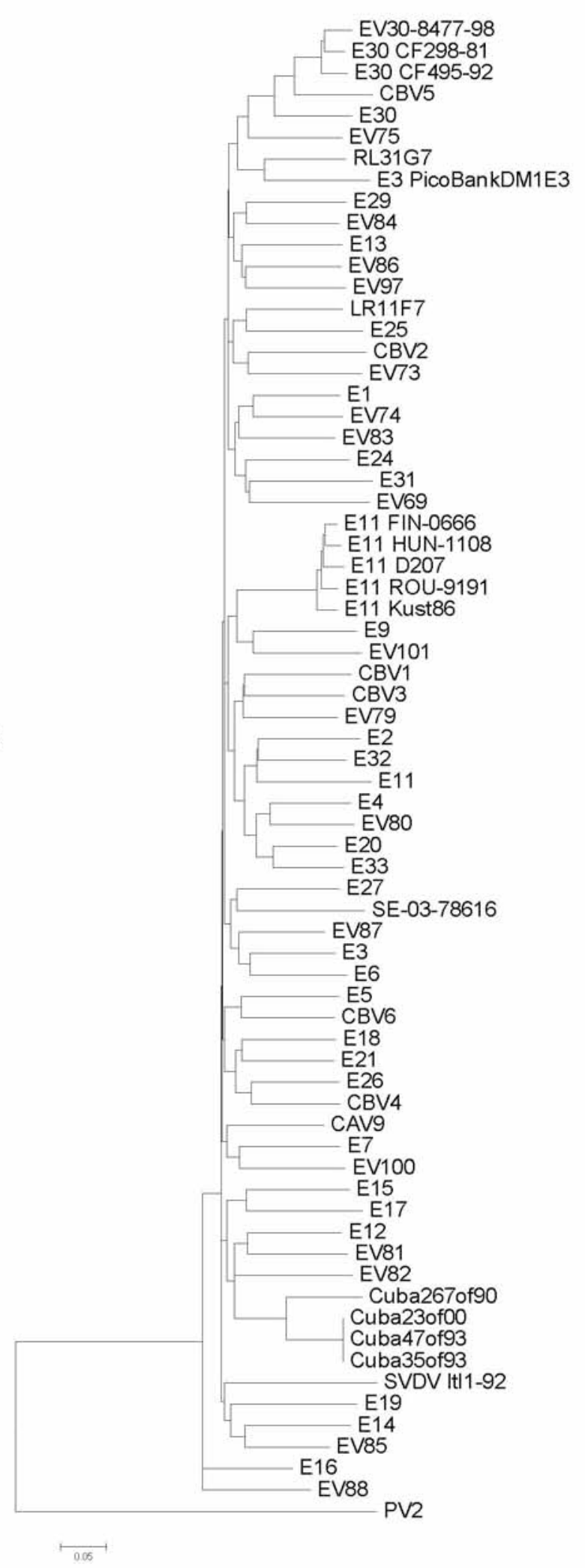

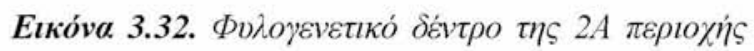

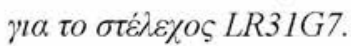




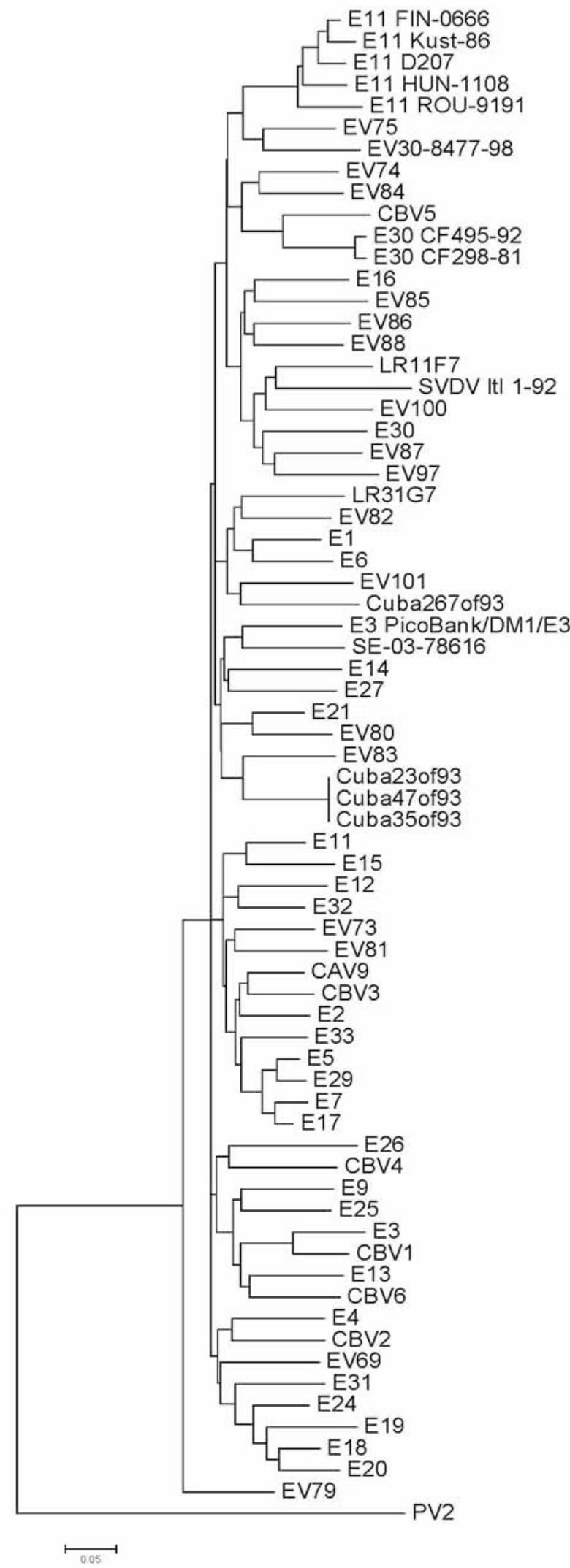

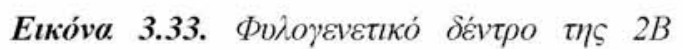

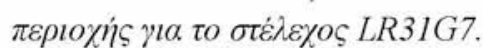

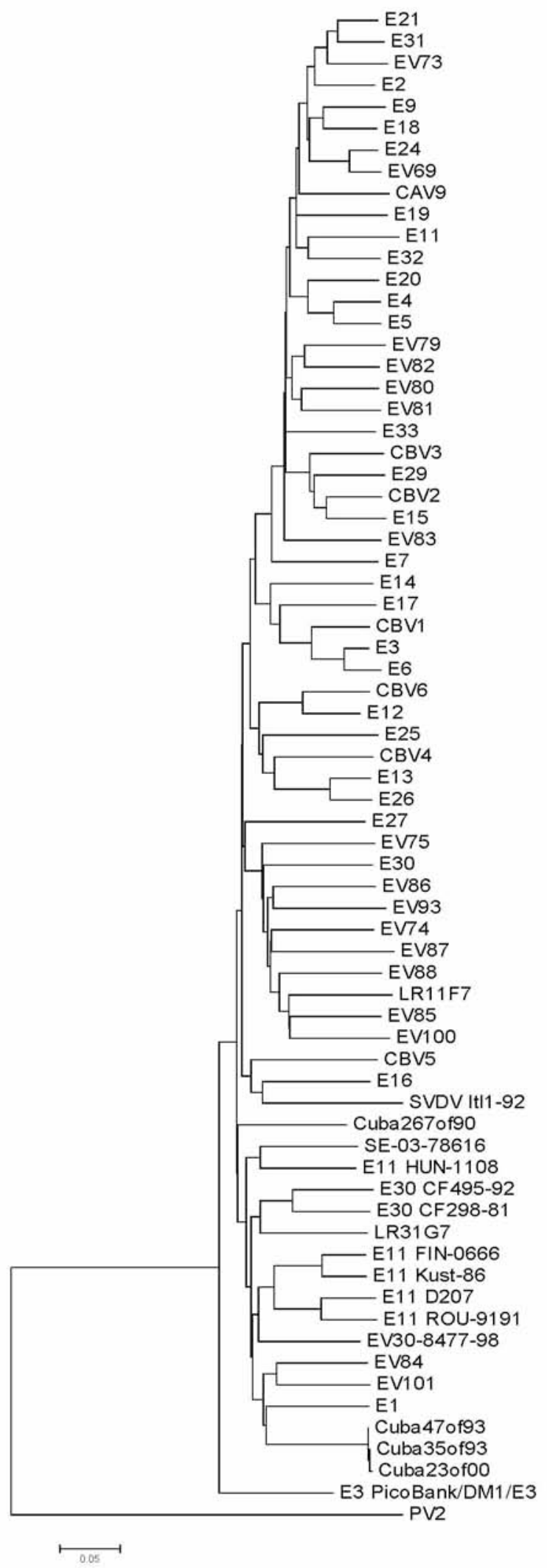

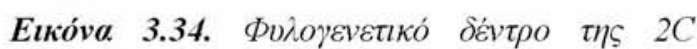

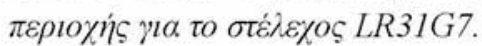




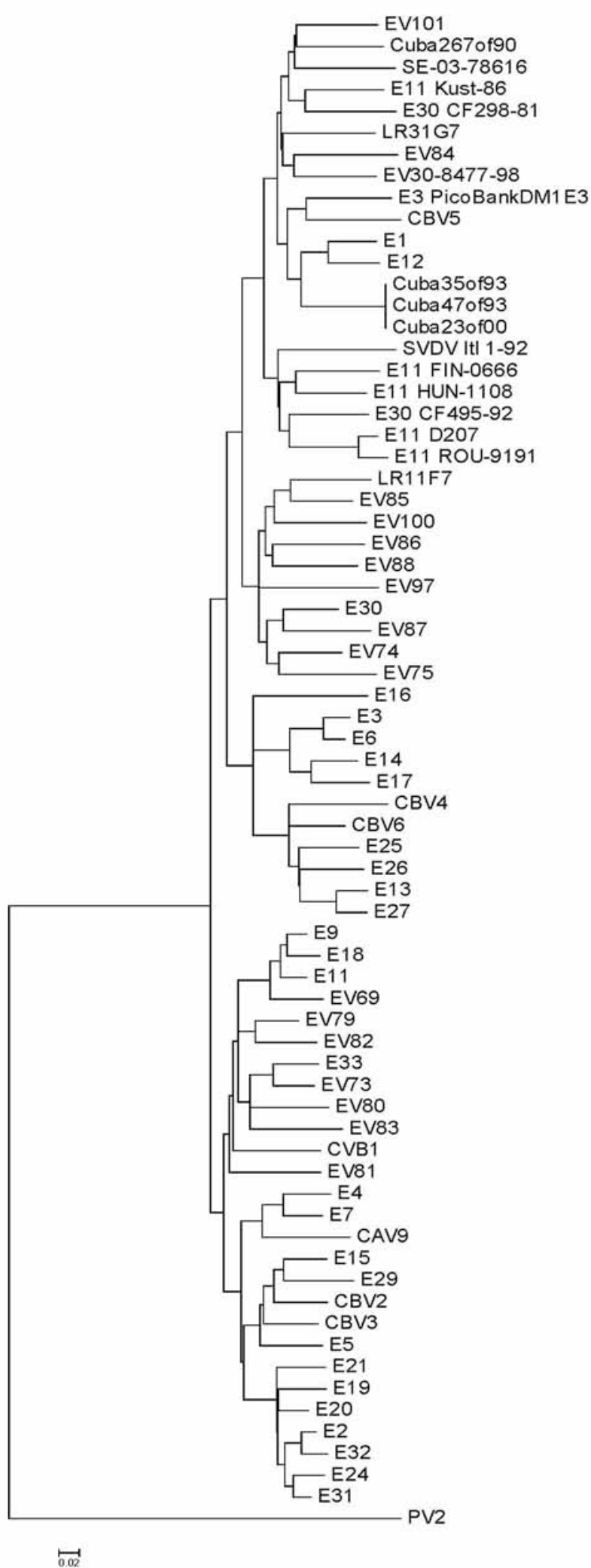

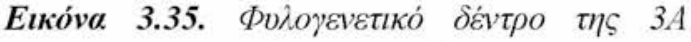

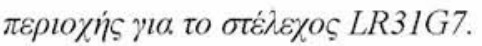

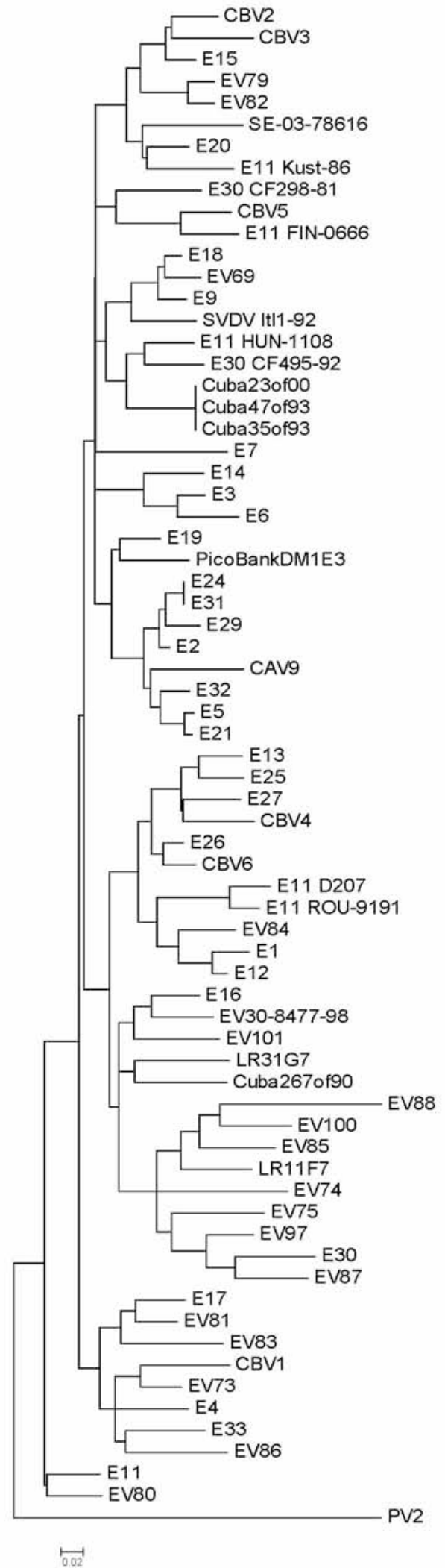

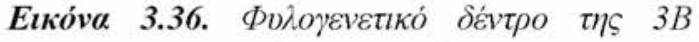

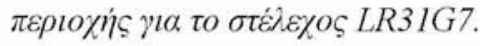




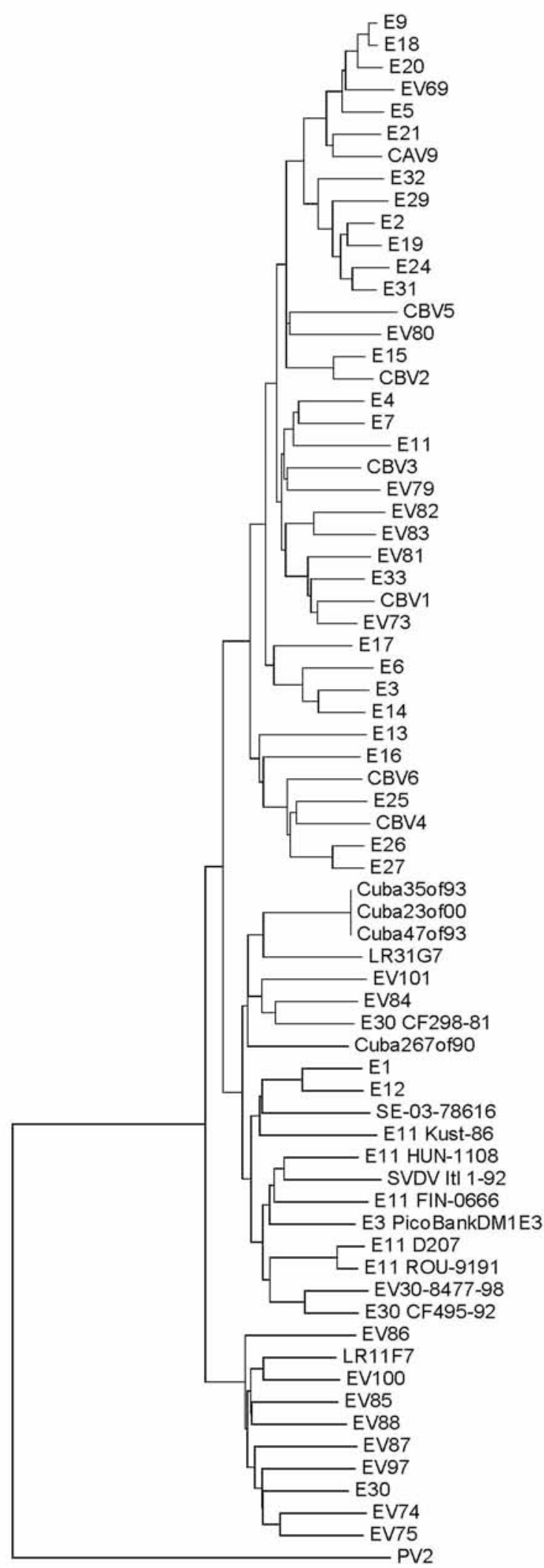

$\longmapsto$

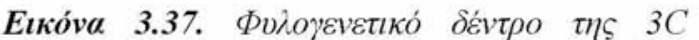

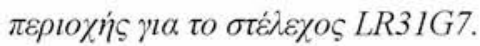

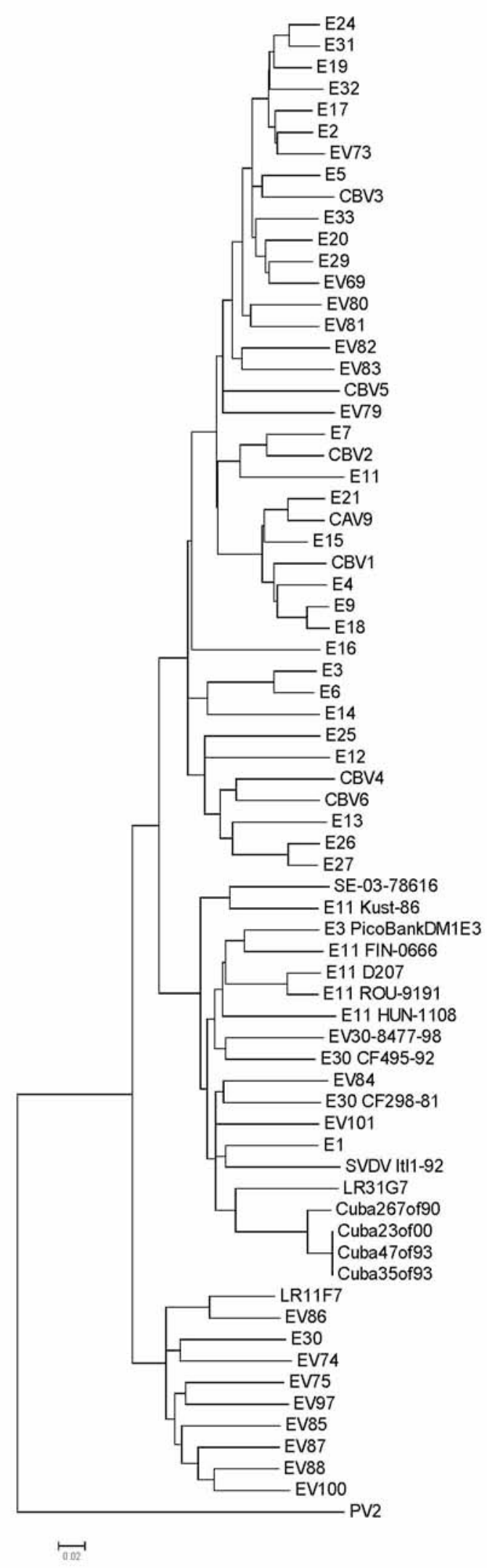

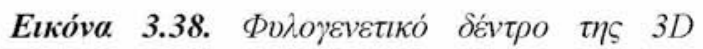

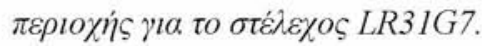




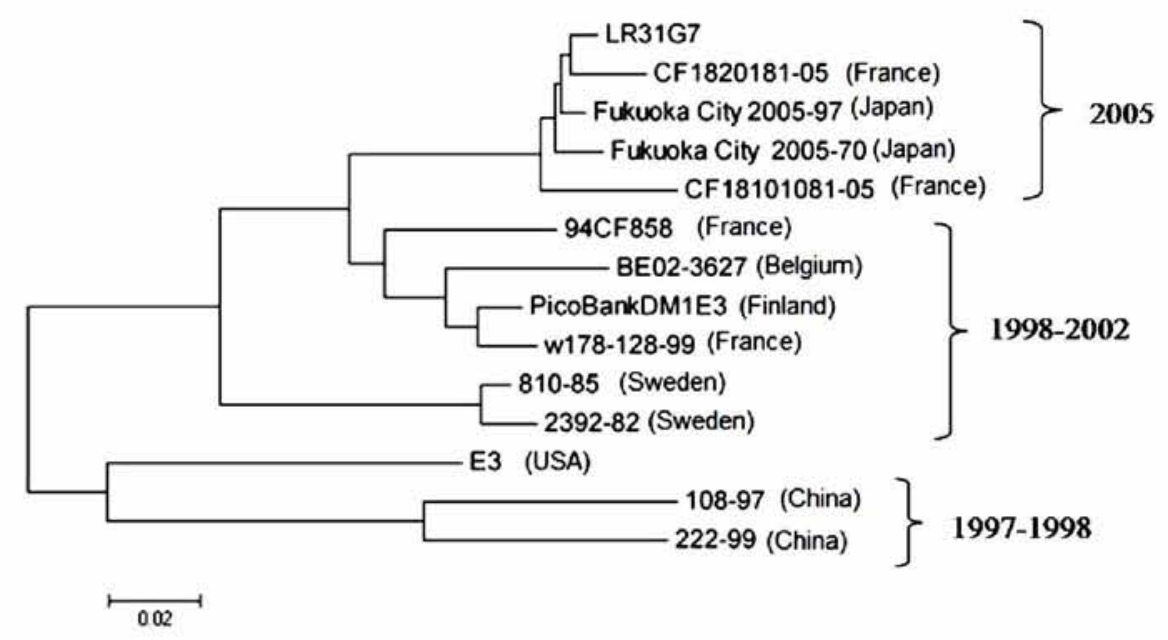

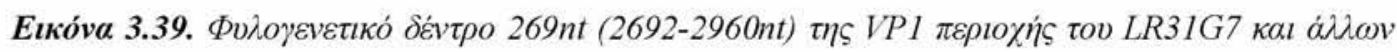
E3 $\sigma \varepsilon \lambda \varepsilon \chi \omega \dot{~}$
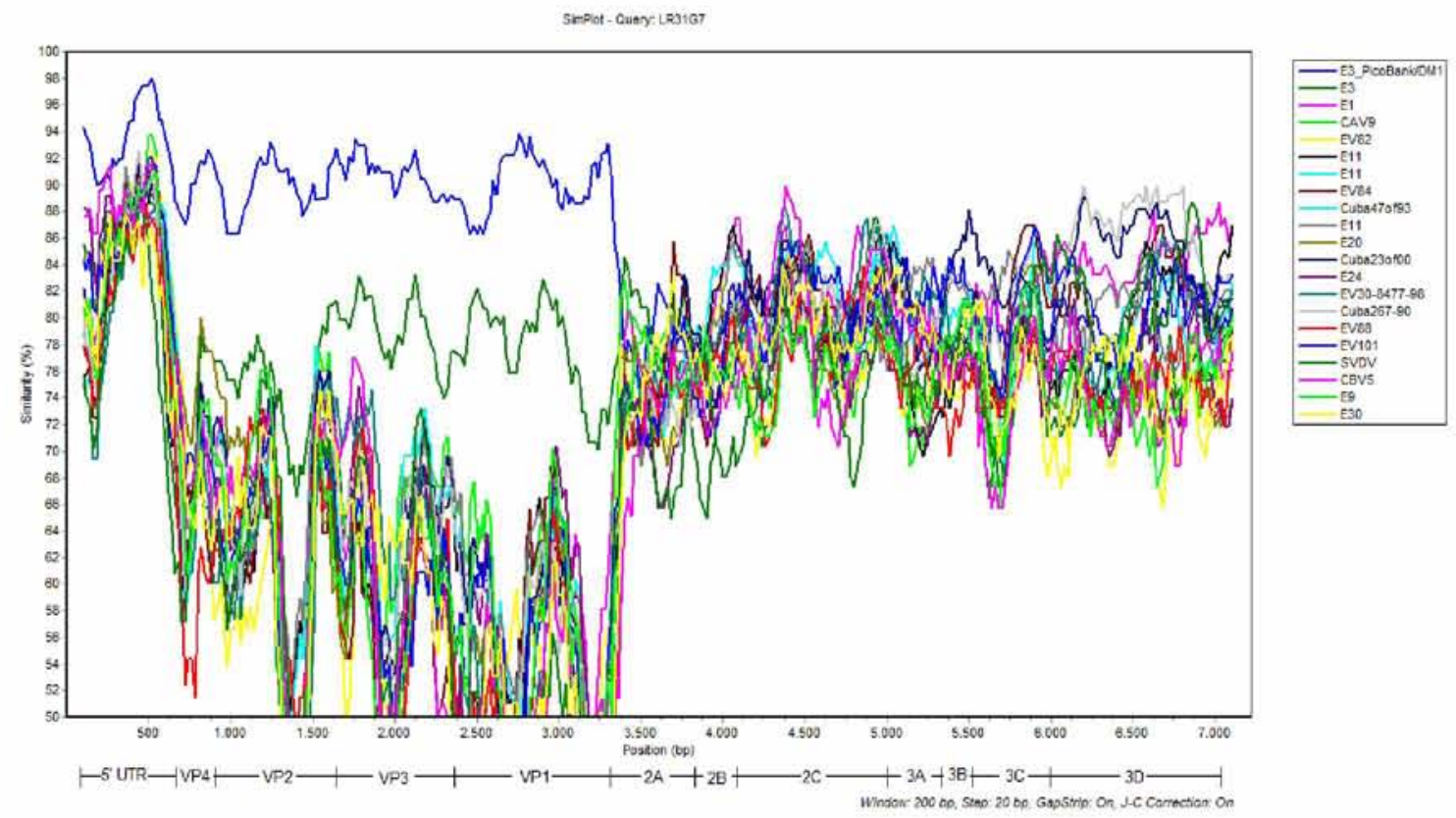

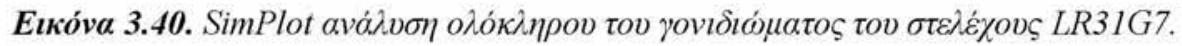




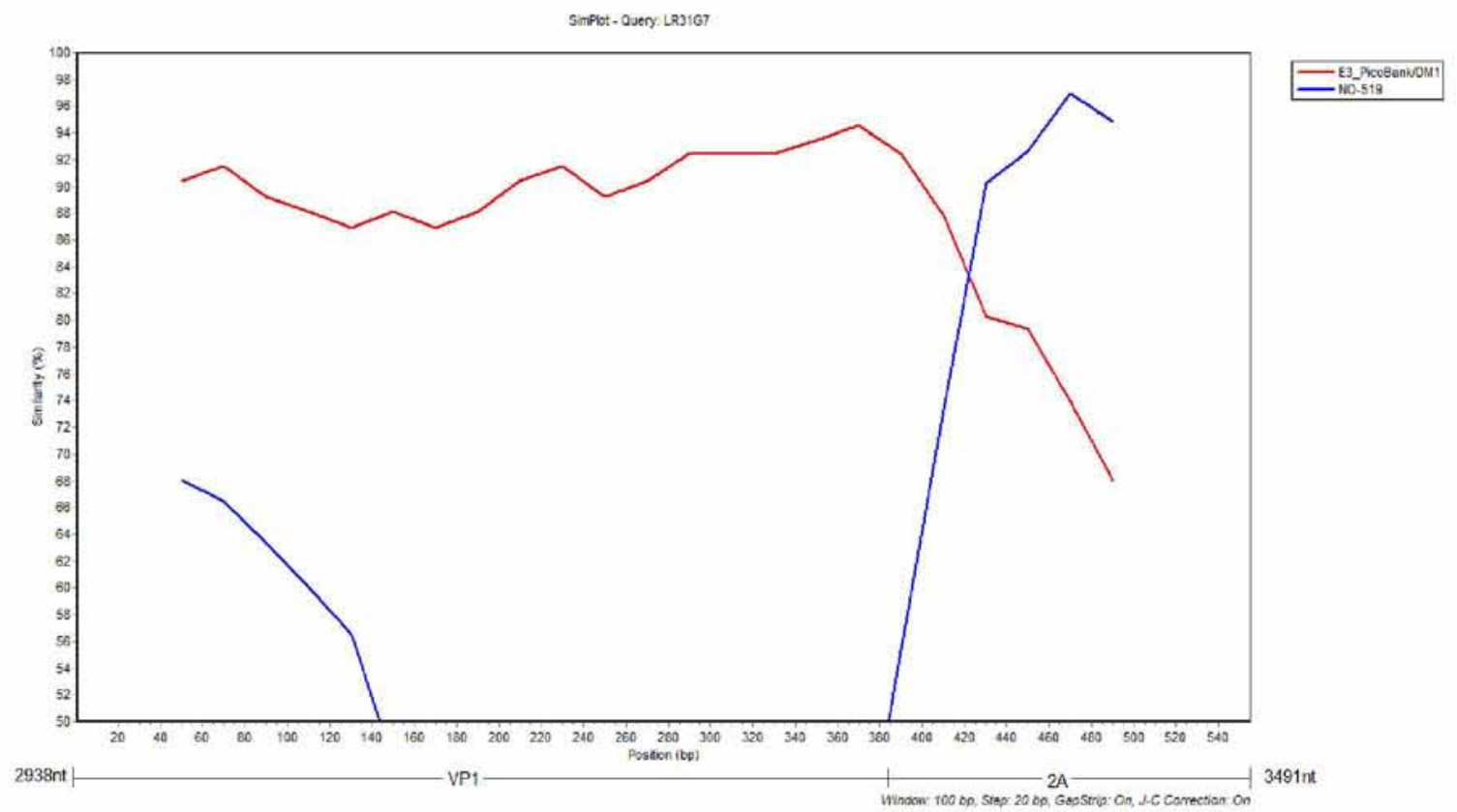

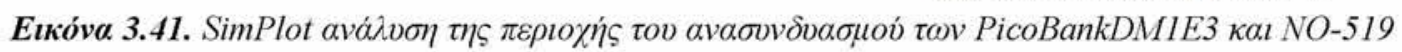
$\sigma \tau \varepsilon \lambda \varepsilon \chi \omega \dot{\omega}$.

PicoBank/DM1/E3 LR31G7

LR31G7

NO-519

$\mathrm{PicoBank/DM1/E3}$ LR31G7

NO-519
NO-519

PicoBank/DM1/E3

TATGGGGACGCTCCGCCCCATGGGTTATACCAGCCATGGGGCATTCGGGCAGCAGTCTGG TGTGGGGACGCTTCGCCCCATGGGTTATACTAACCATGGAGCATTCGGGCAGCAATCTGG AGAGAGATGACCATTTGCCCTCAATGGCA--AACCATGGGGCGTTCGGCCAGCAATCCGG

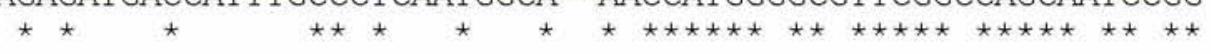
$3357 \quad 3364$

GGCTATCTATGTGGGTAACTACAGAGTAGTCAATAGGCACCTAGCCACGCATGTAGACTG GGCTATCIATGTGGG AAACTACAGAGT GTGAACCGGCACTTÄGCÄACÄCACACTGAC̈TG AGCTGTATATGTGGGCAACTACAGAGTGGTCAACCGGCACTTGGCAACACACACTGATTG

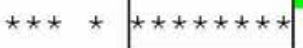

GAGCAATTGTGTCTGGGAGGACTATAACAGAGATTTACTAATCAGCACCACTACGGCCCT

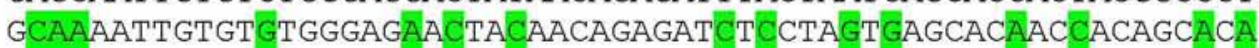
GCAAAATTGTGT GTGGGAGATTACAACAGAGATCTCCTAGTGAGCACAACCACTGCACA

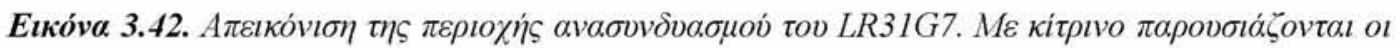

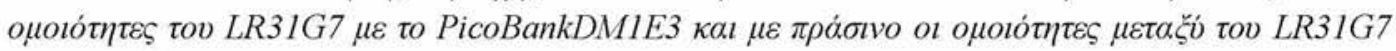
Ka. tov NO-519. 


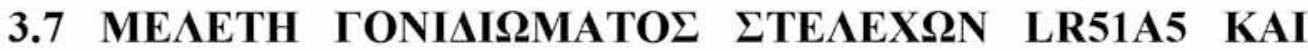

\section{LR61G3}

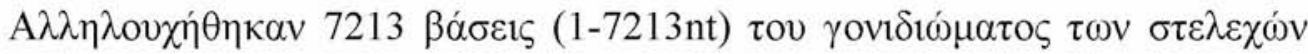

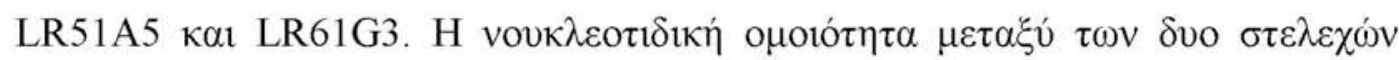

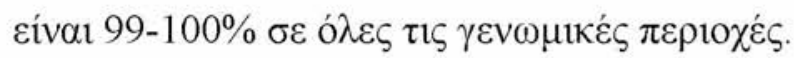

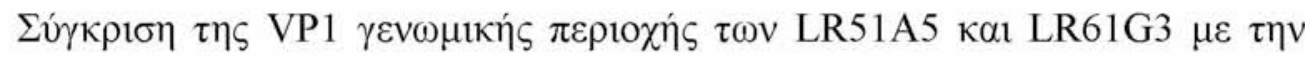

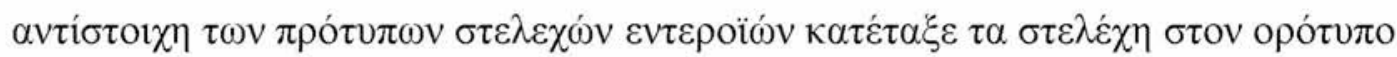

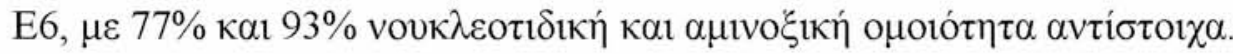

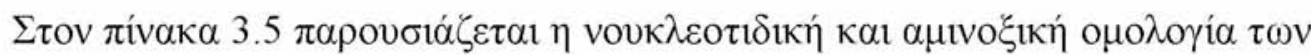

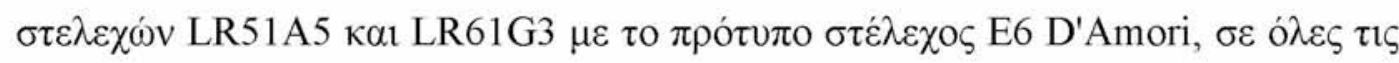

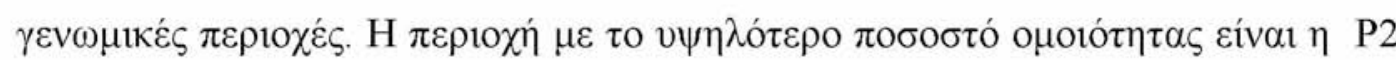

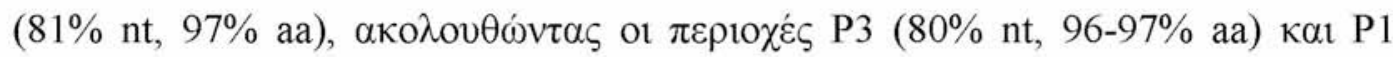

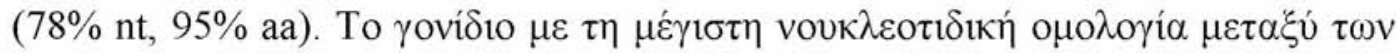

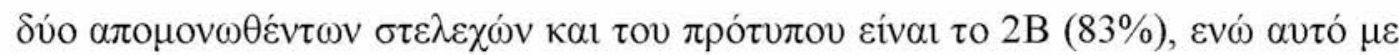

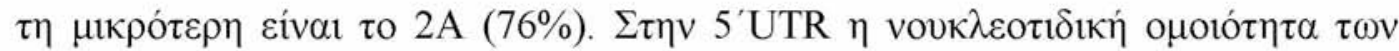

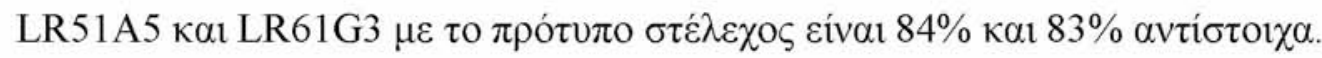

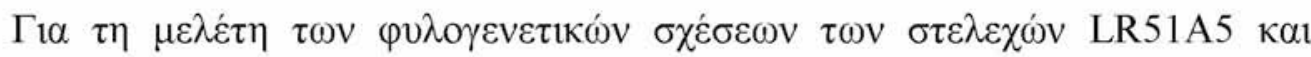

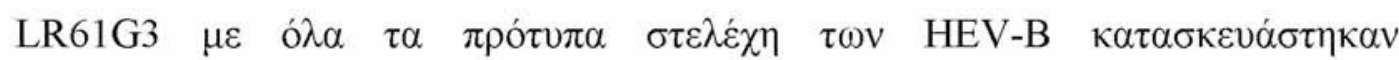

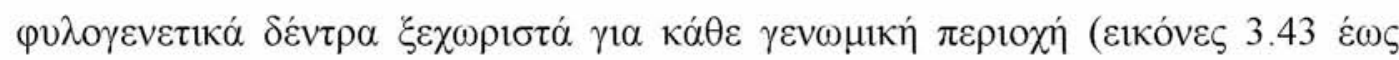
3.54). Хрпбцолоіп்

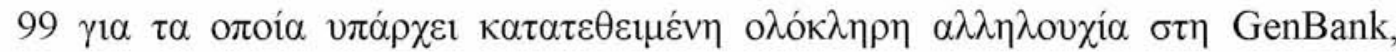

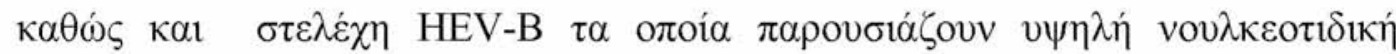

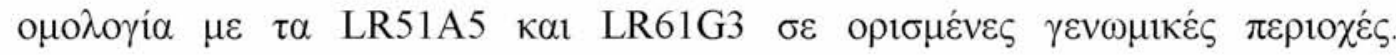

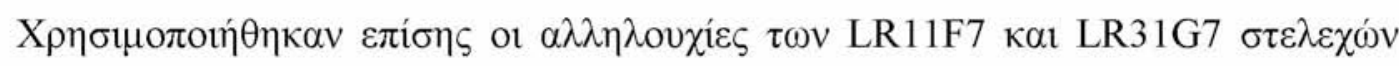

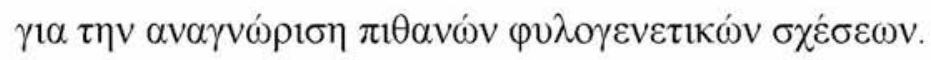

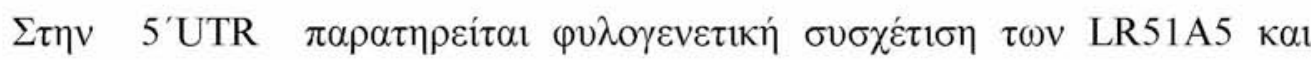

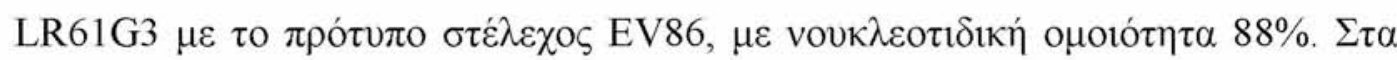

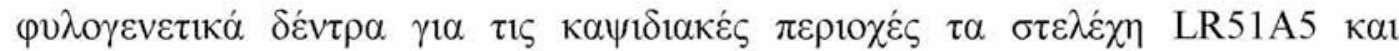

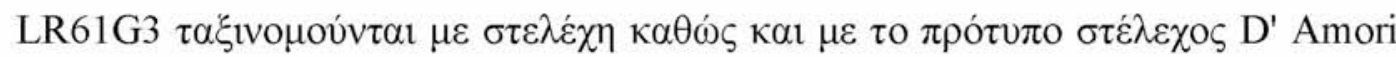

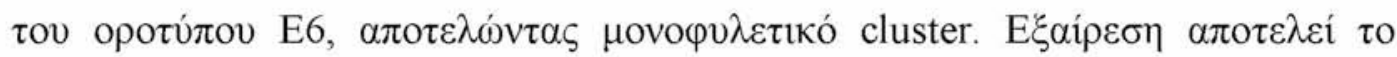

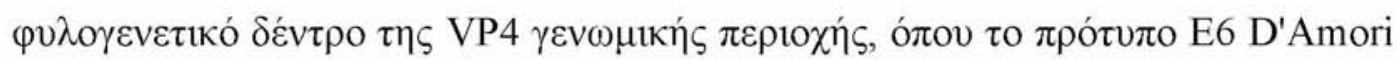

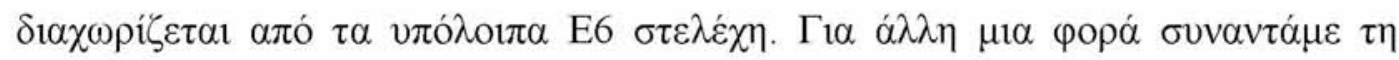




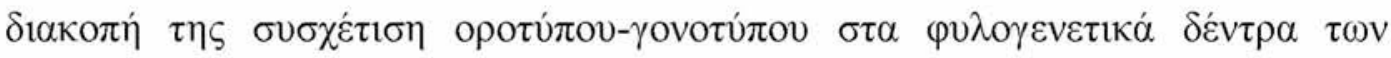

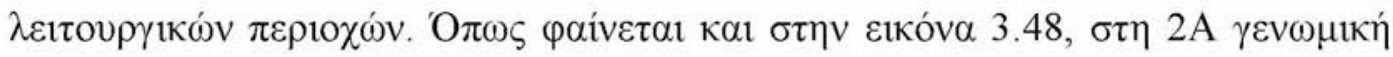

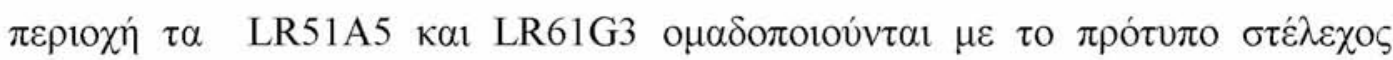

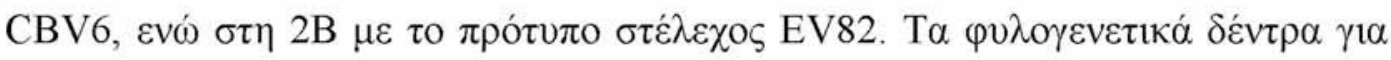

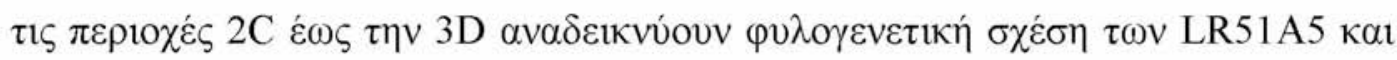

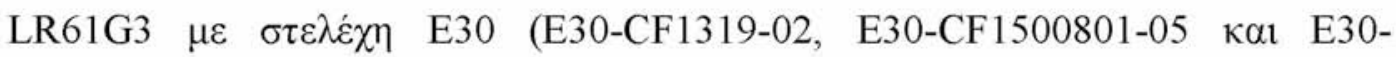

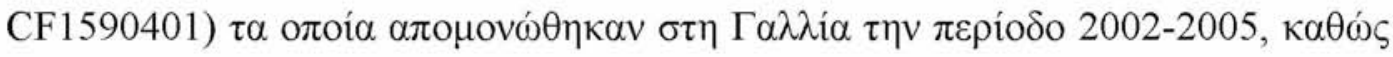

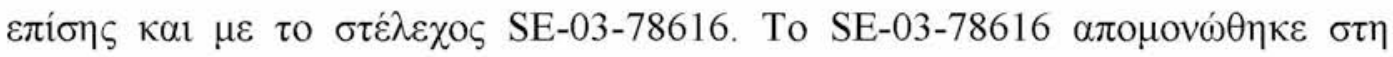

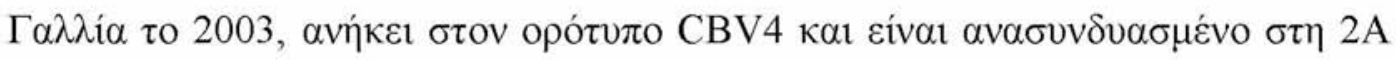

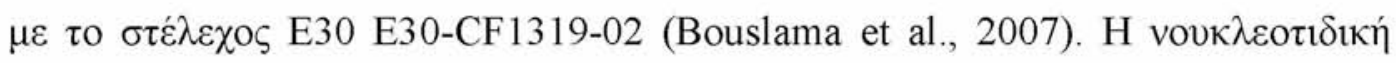

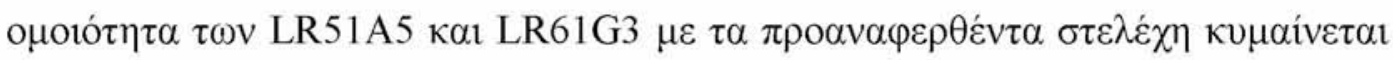

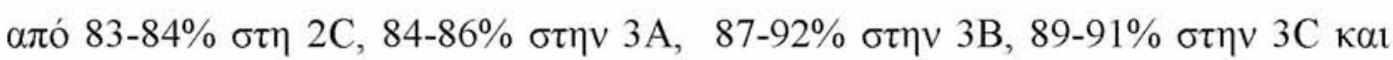

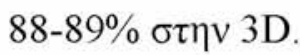

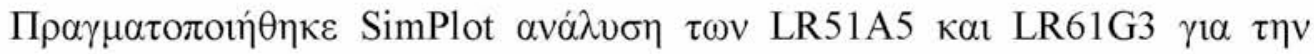

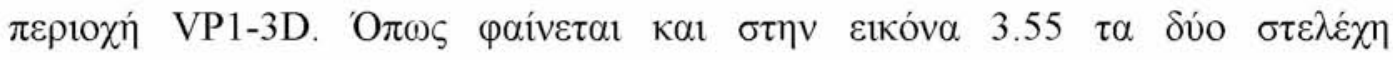

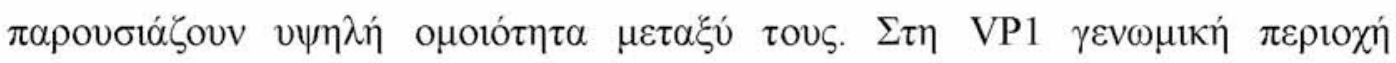

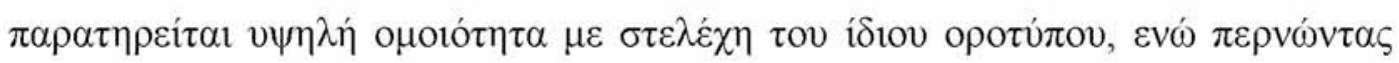

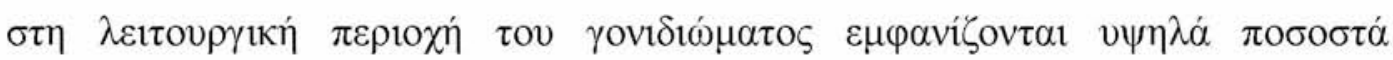

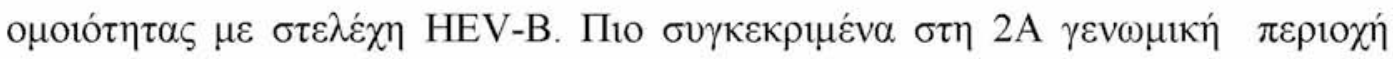

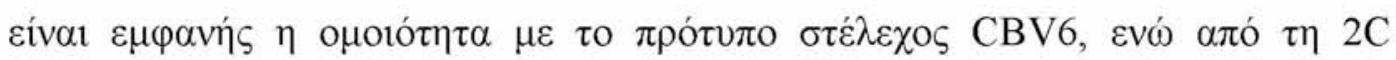

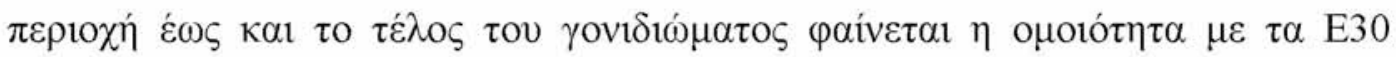

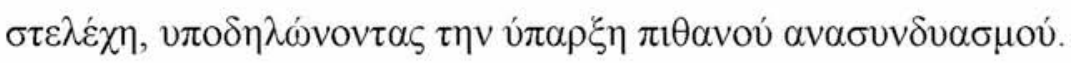

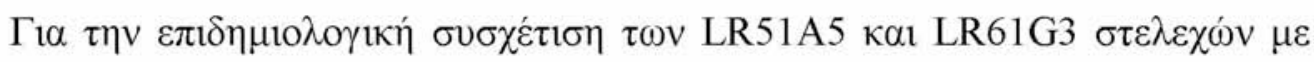

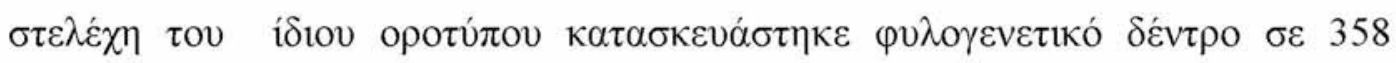

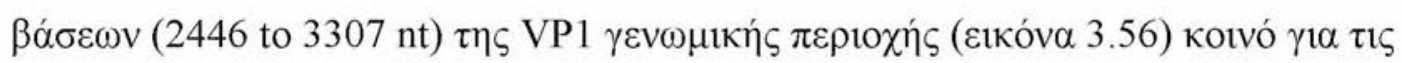

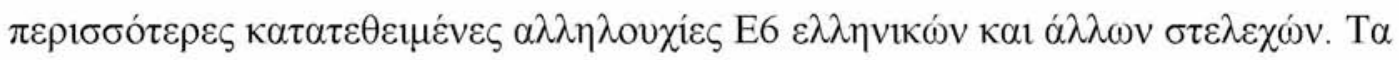

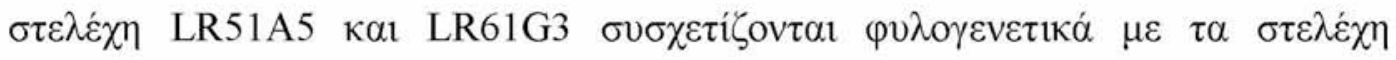

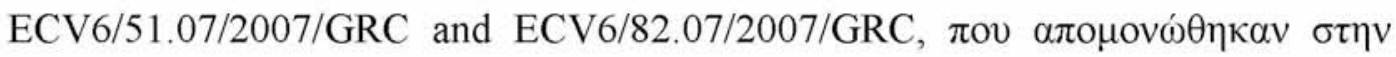

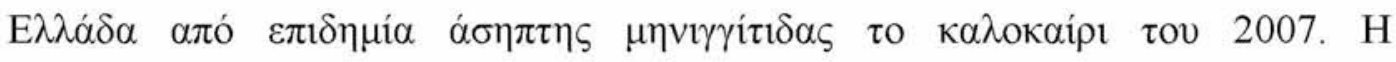

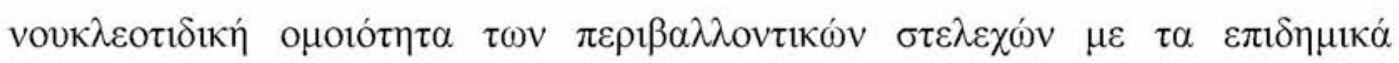

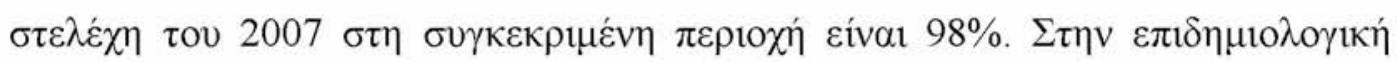

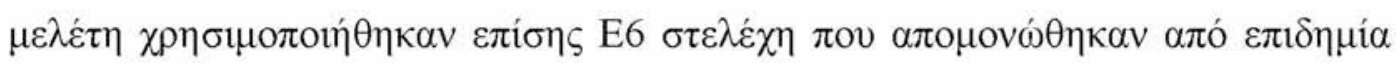

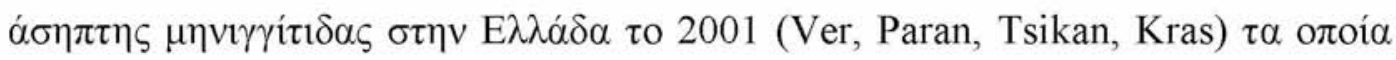

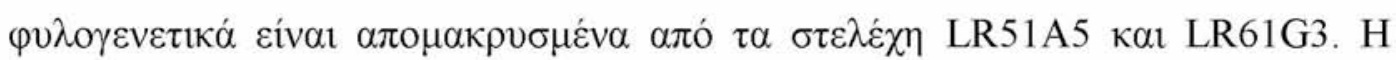




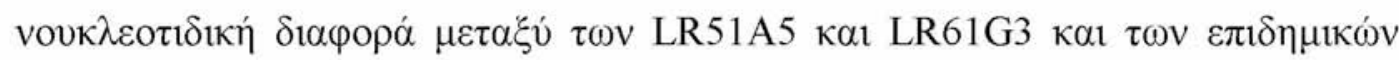

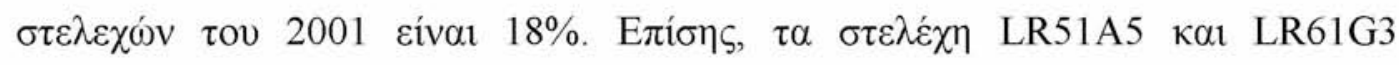

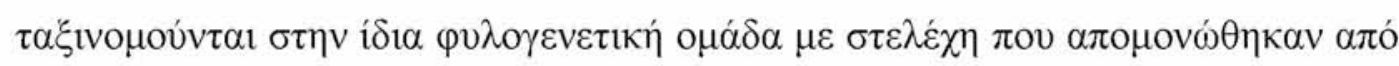

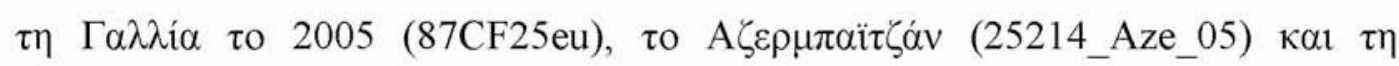

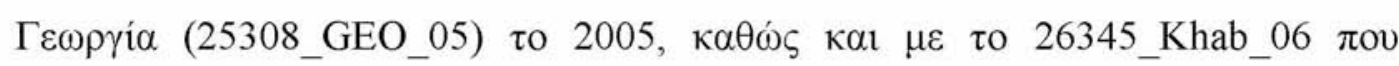

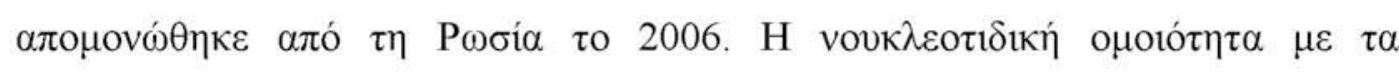

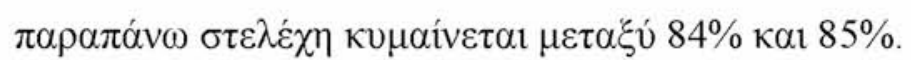

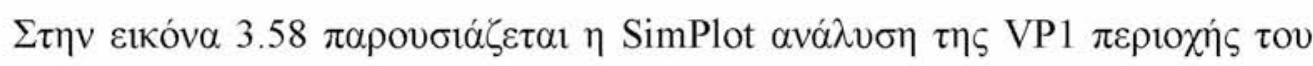

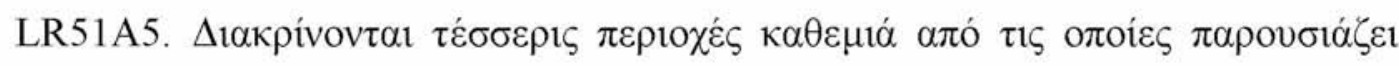

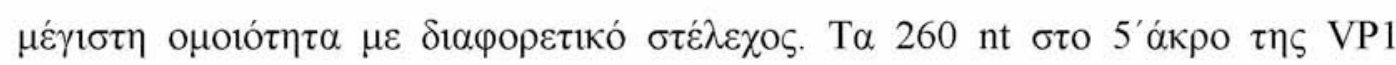

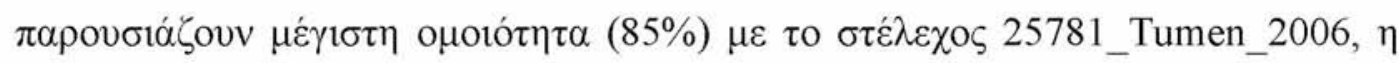

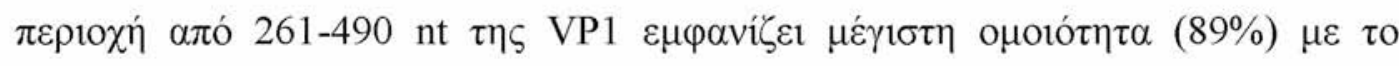

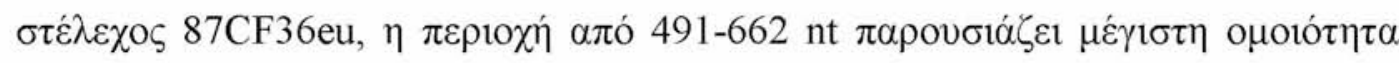

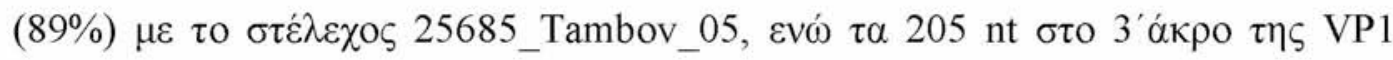

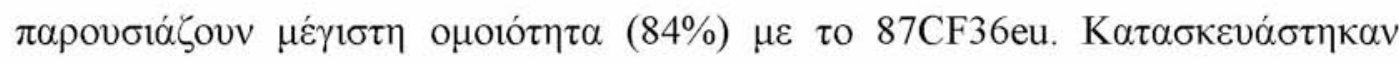

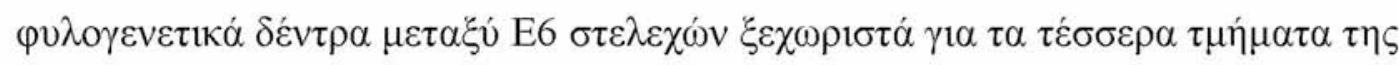

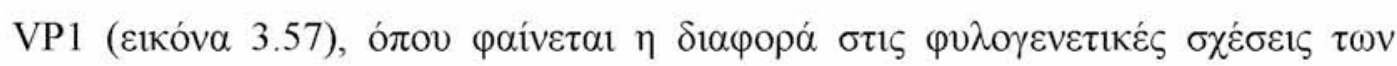

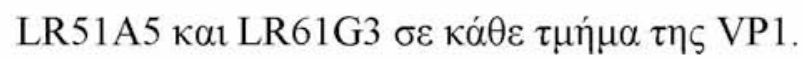




\begin{tabular}{|c|c|c|c|c|}
\hline \multirow{2}{*}{ 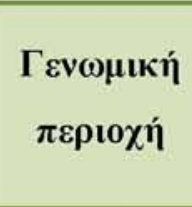 } & \multicolumn{2}{|c|}{ LR51A5 } & \multicolumn{2}{|c|}{ LR61G3 } \\
\hline & 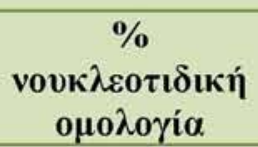 & 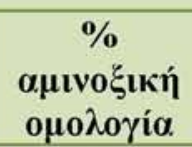 & 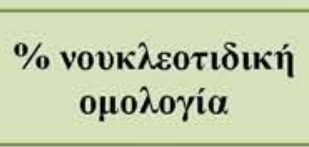 & 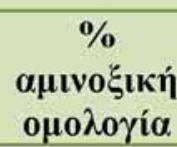 \\
\hline 5' UTR & 84 & - & 83 & - \\
\hline P1 & 78 & 95 & 78 & 95 \\
\hline VP4 & 80 & 95 & 79 & 95 \\
\hline VP2 & 79 & 96 & 78 & 97 \\
\hline VP3 & 78 & 99 & 78 & 100 \\
\hline VP1 & 77 & 93 & 77 & 93 \\
\hline P2 & 81 & 97 & 81 & 97 \\
\hline $2 \mathrm{~A}$ & 76 & 94 & 76 & 94 \\
\hline $2 \mathrm{~B}$ & 83 & 96 & 83 & 95 \\
\hline $2 \mathrm{C}$ & 82 & 99 & 82 & 99 \\
\hline P3 & 80 & 97 & 80 & 96 \\
\hline $3 \mathrm{~A}$ & 77 & 96 & 77 & 96 \\
\hline $3 \mathrm{~B}$ & 78 & 95 & 78 & 95 \\
\hline $3 \mathrm{C}$ & 80 & 97 & 79 & 97 \\
\hline $3 \mathrm{D}^{*}$ & 81 & 97 & 81 & 97 \\
\hline
\end{tabular}

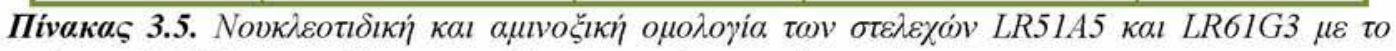

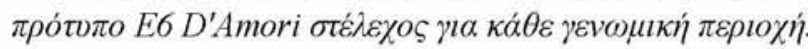

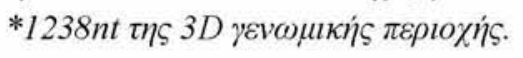




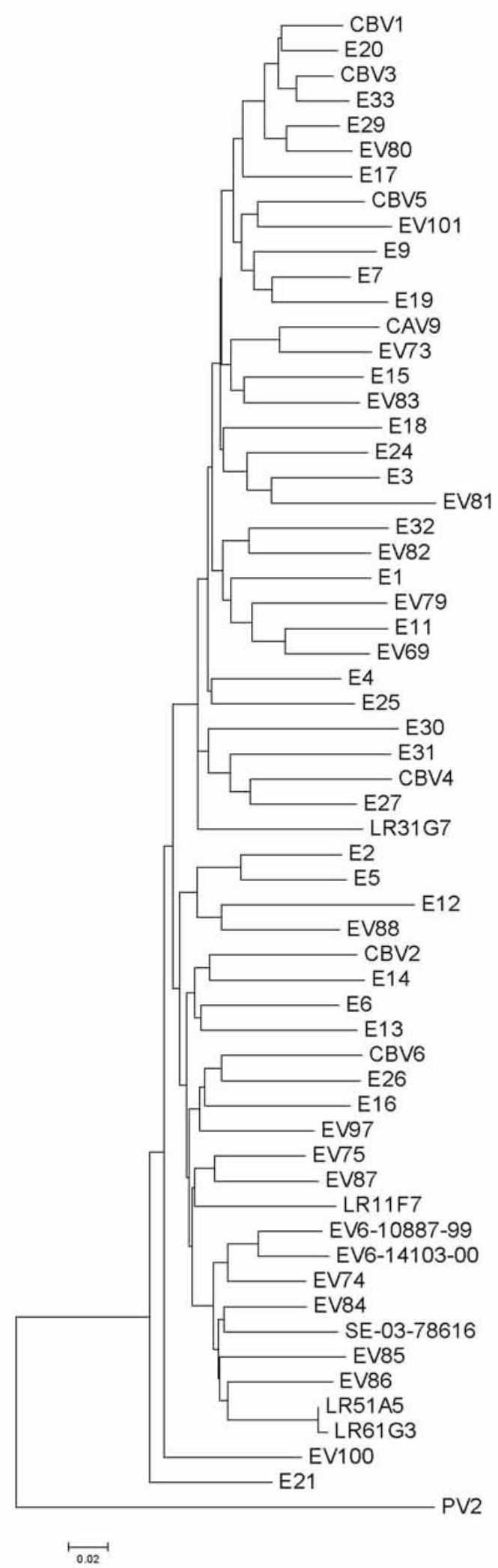

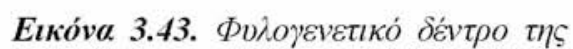

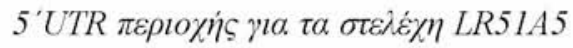
каı LR61G3.

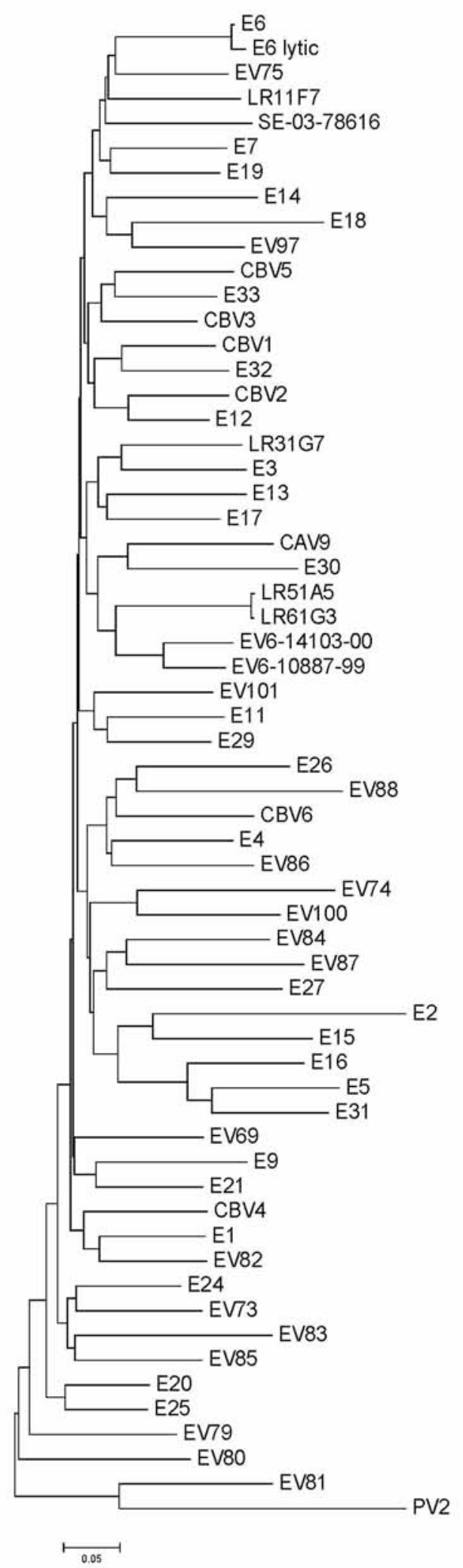

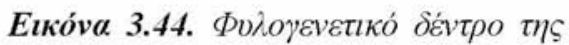

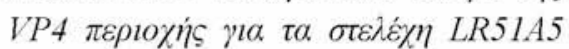
Kal LR61G3. 


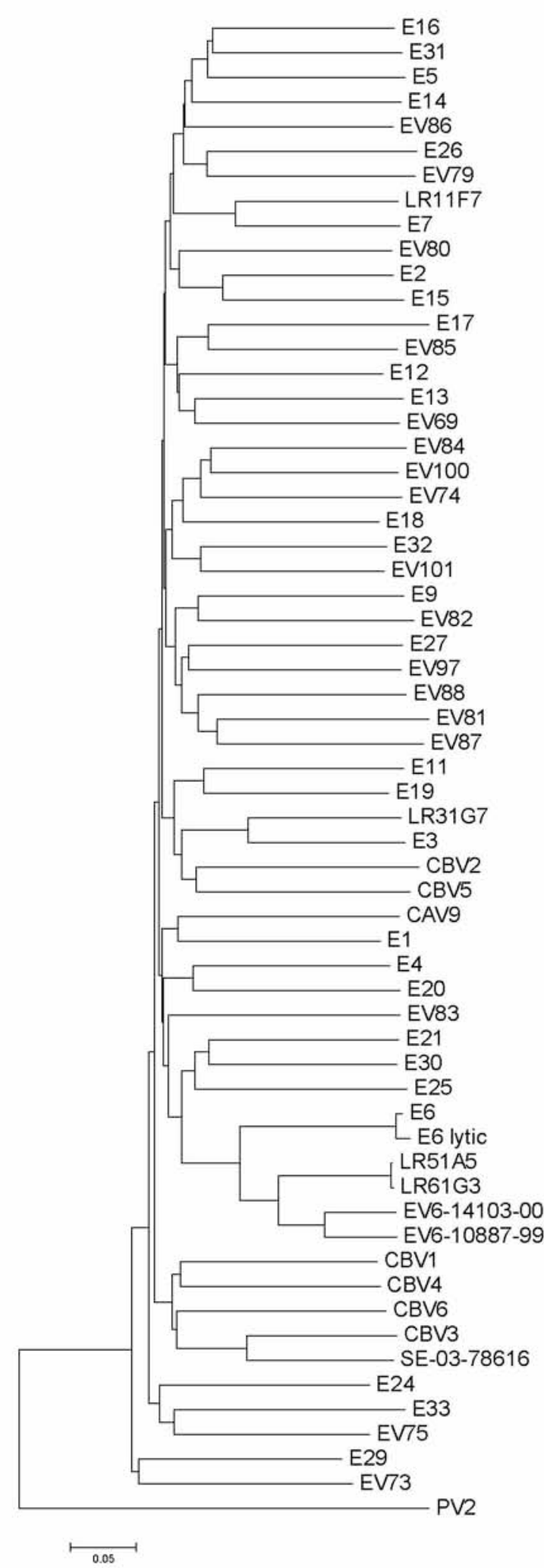

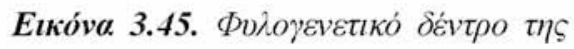

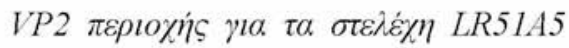
каı LR61G3.

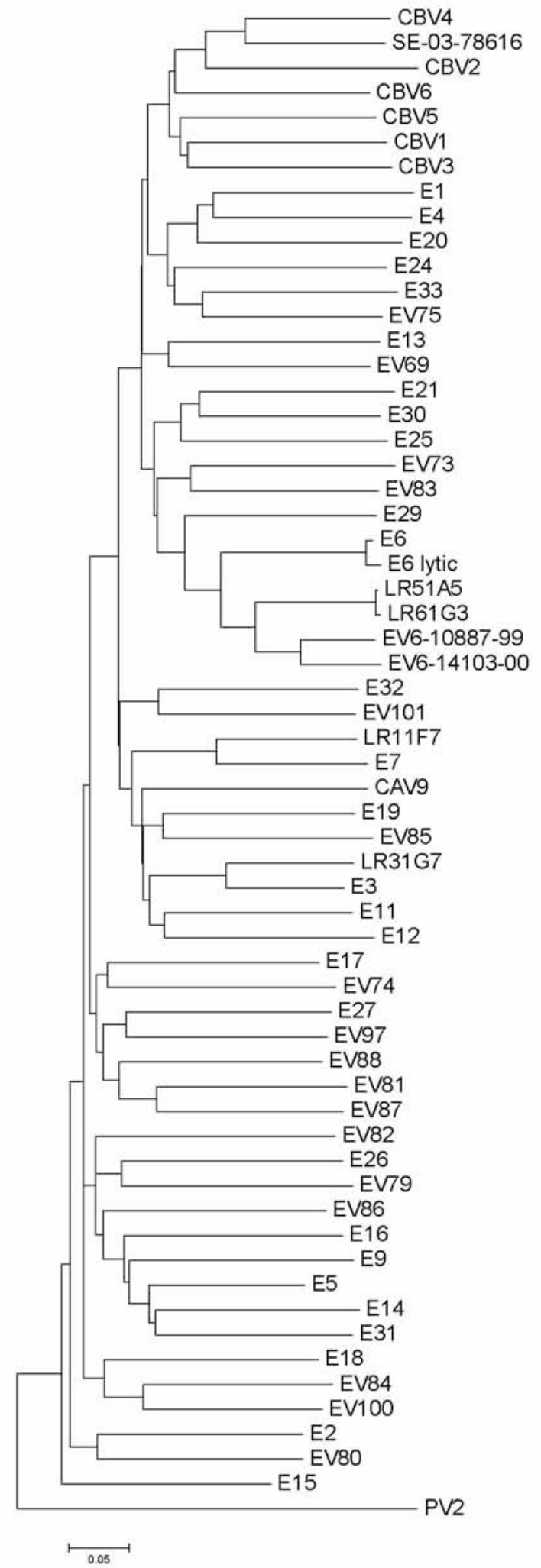

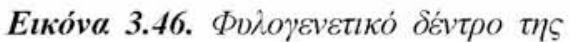

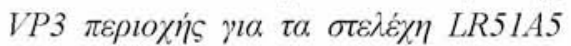
каı LR61G3. 


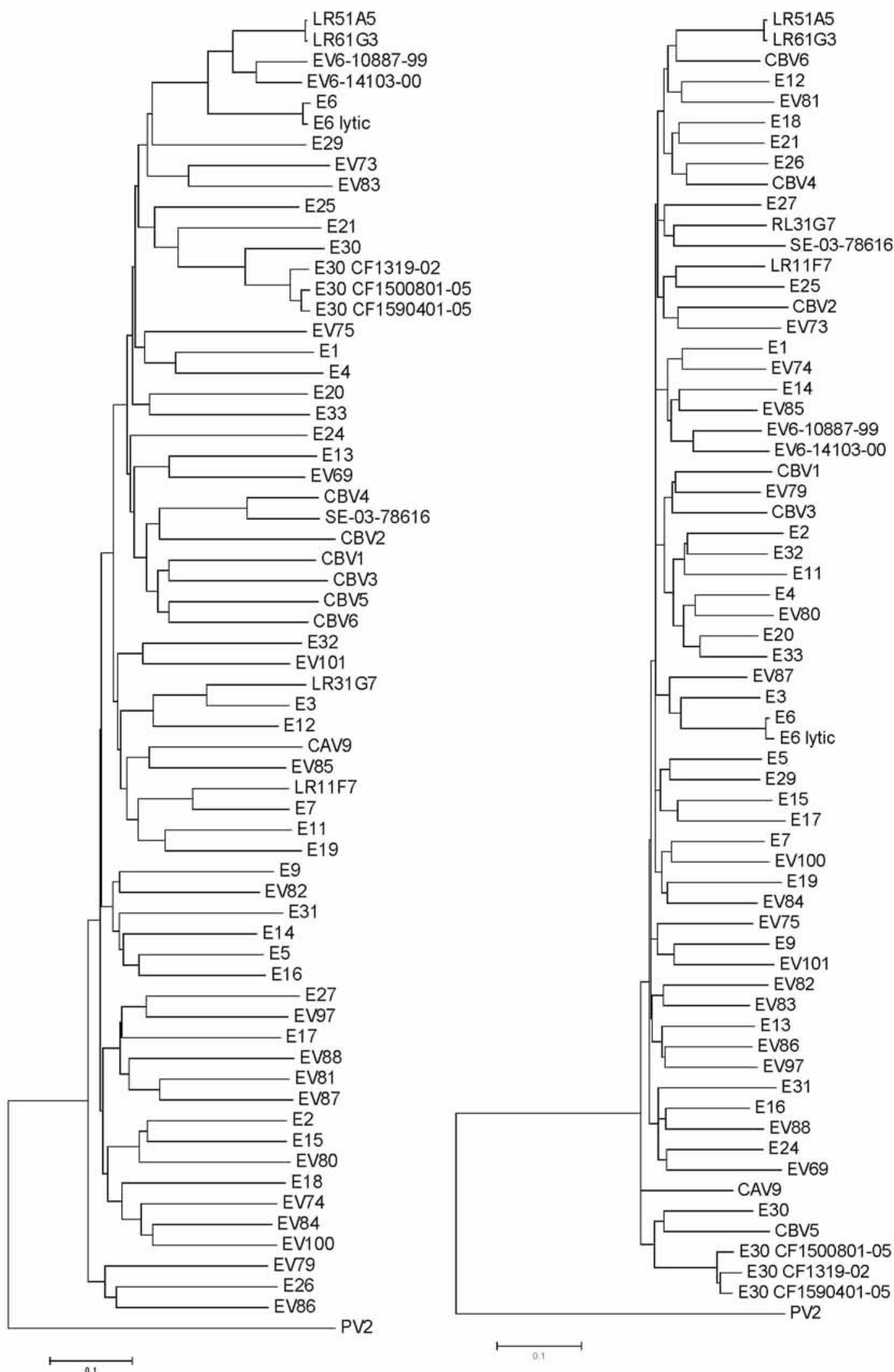

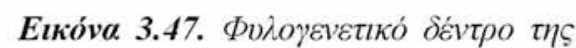

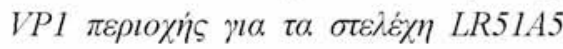
каı LR61G3.

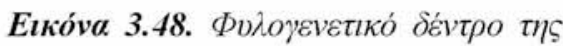

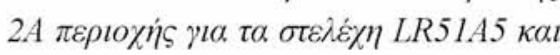
LR61G3. 


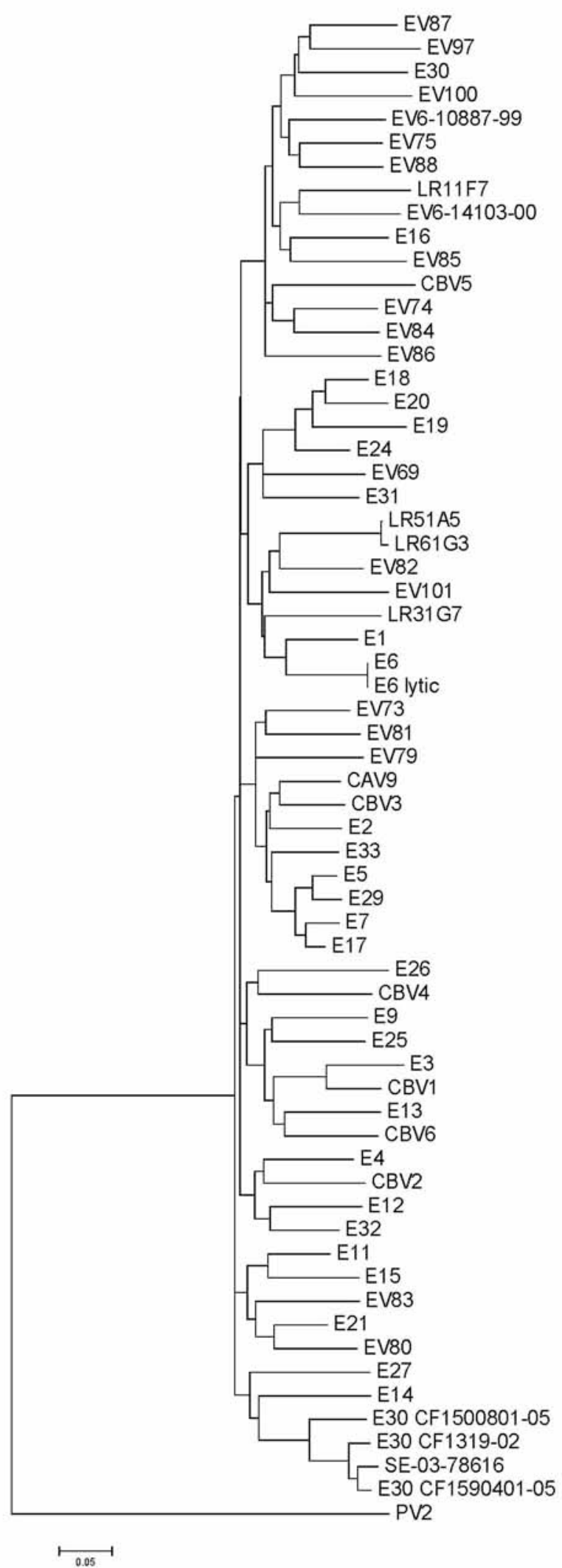

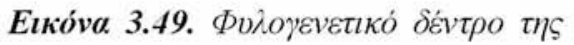

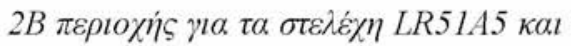
LR61G3.

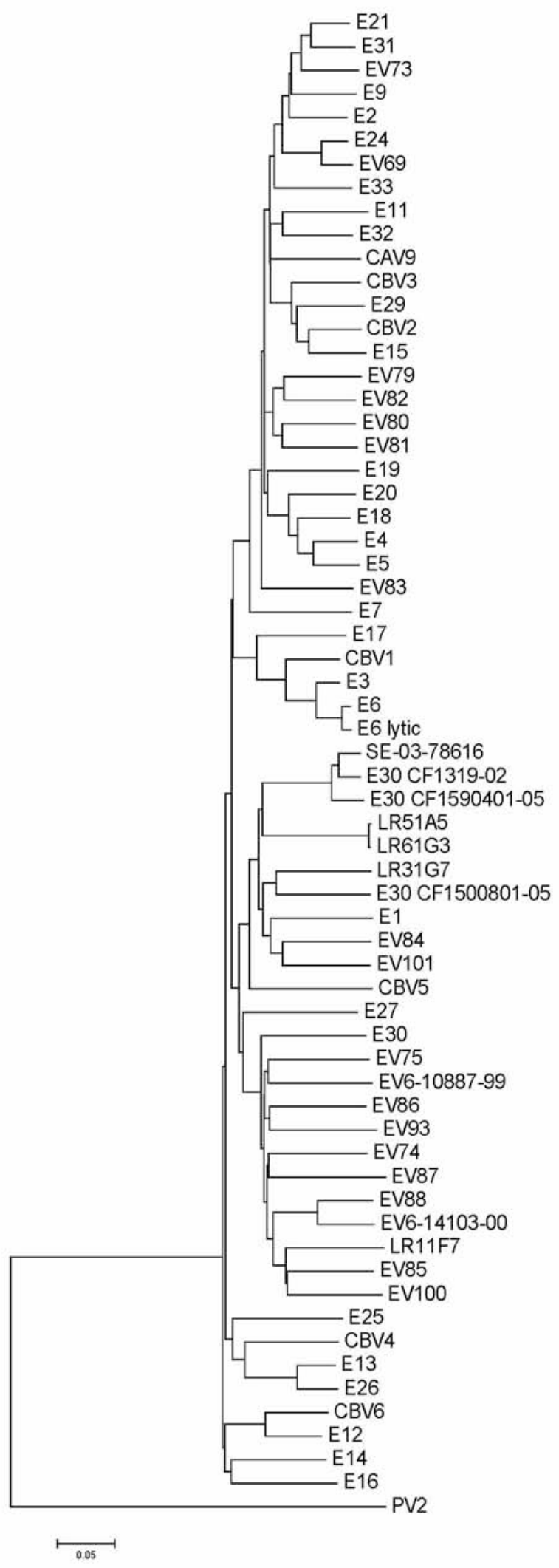

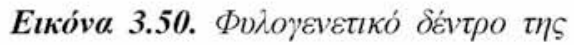

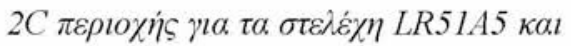
LR61G3. 


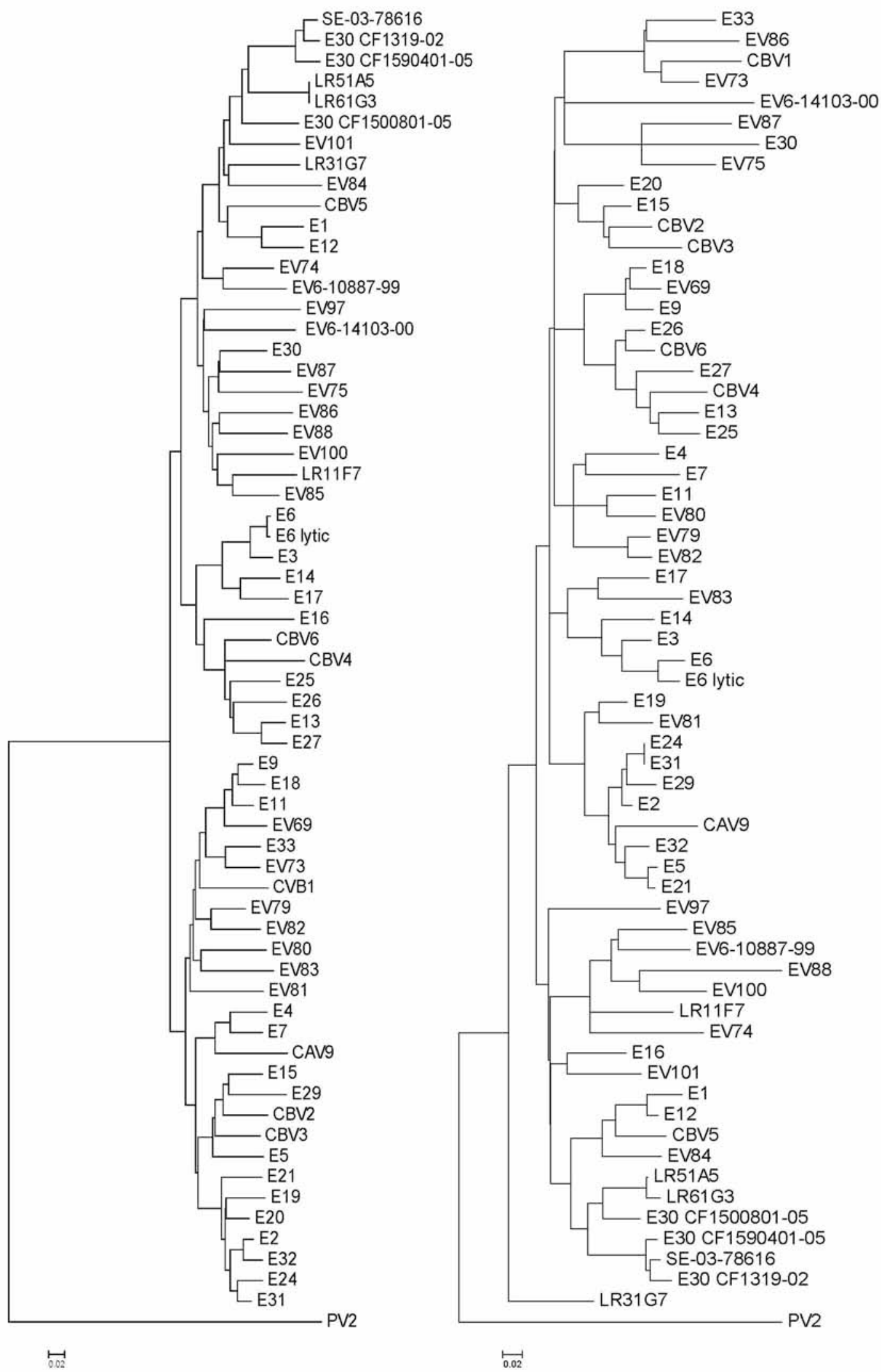

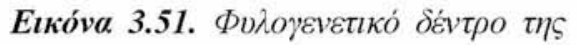

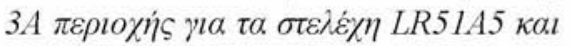
LR61G3.

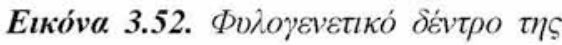

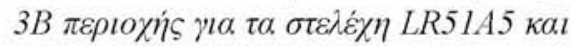
LR61G3. 


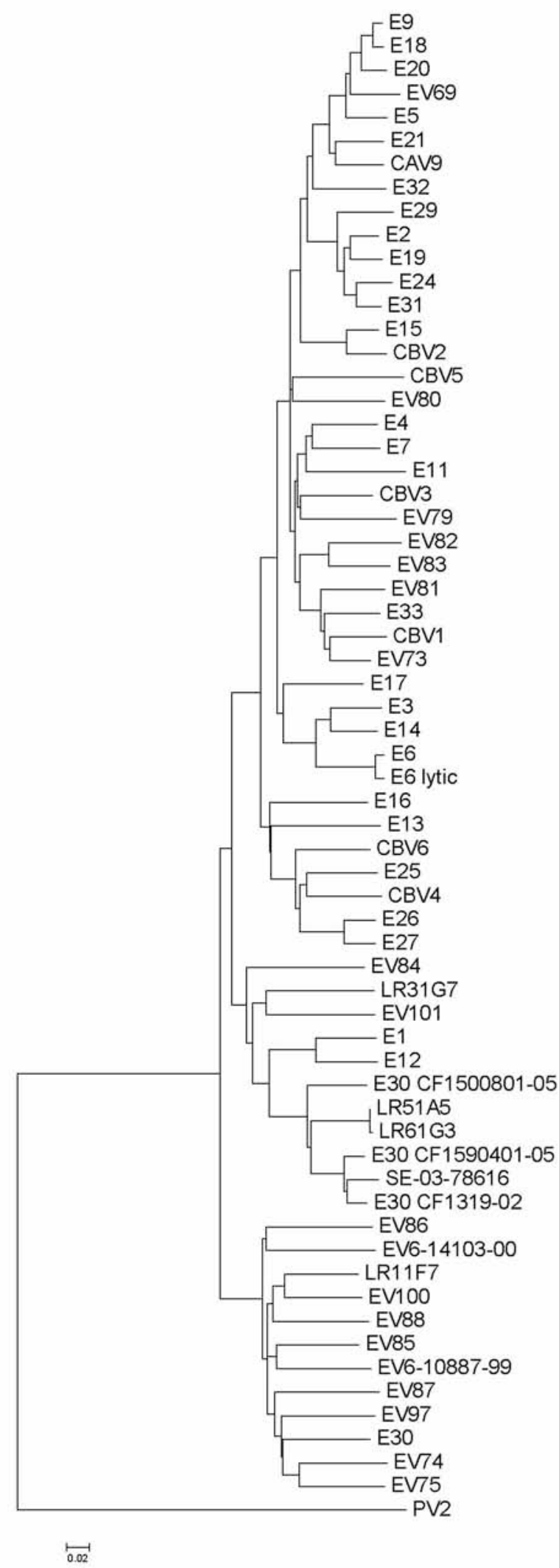

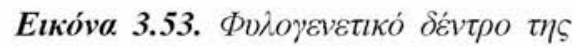

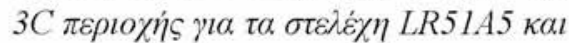
LR61G3.

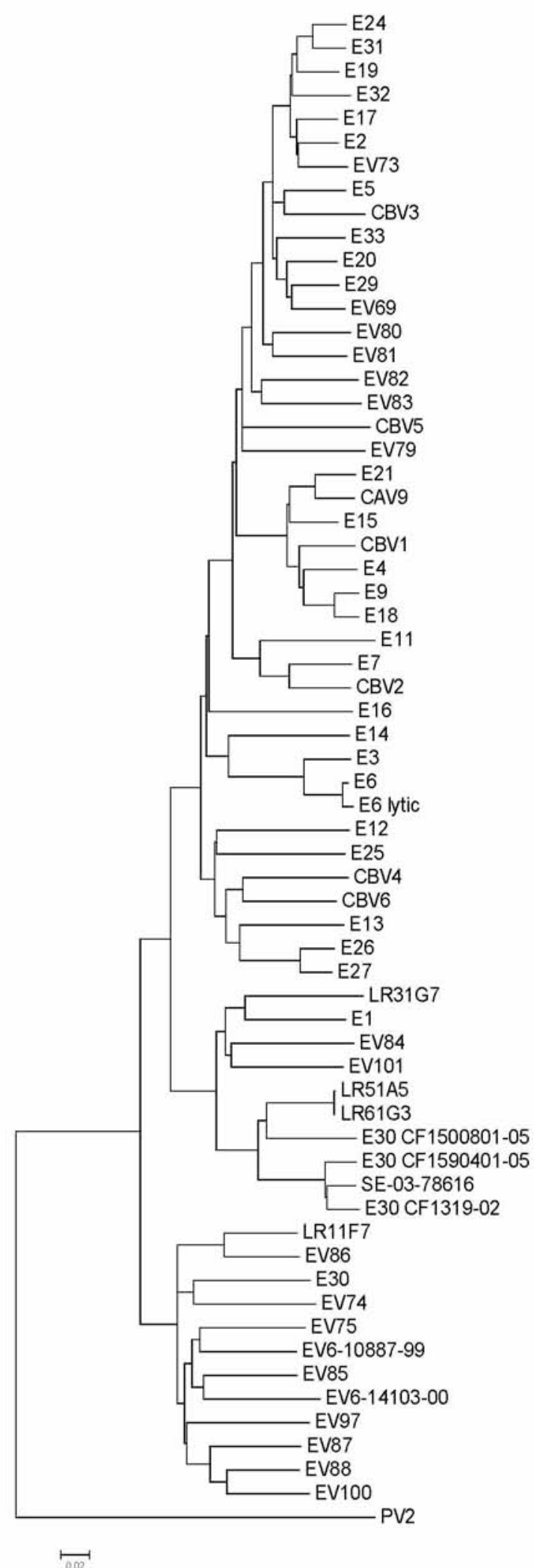

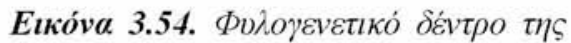

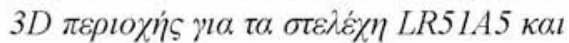
LR61G3. 

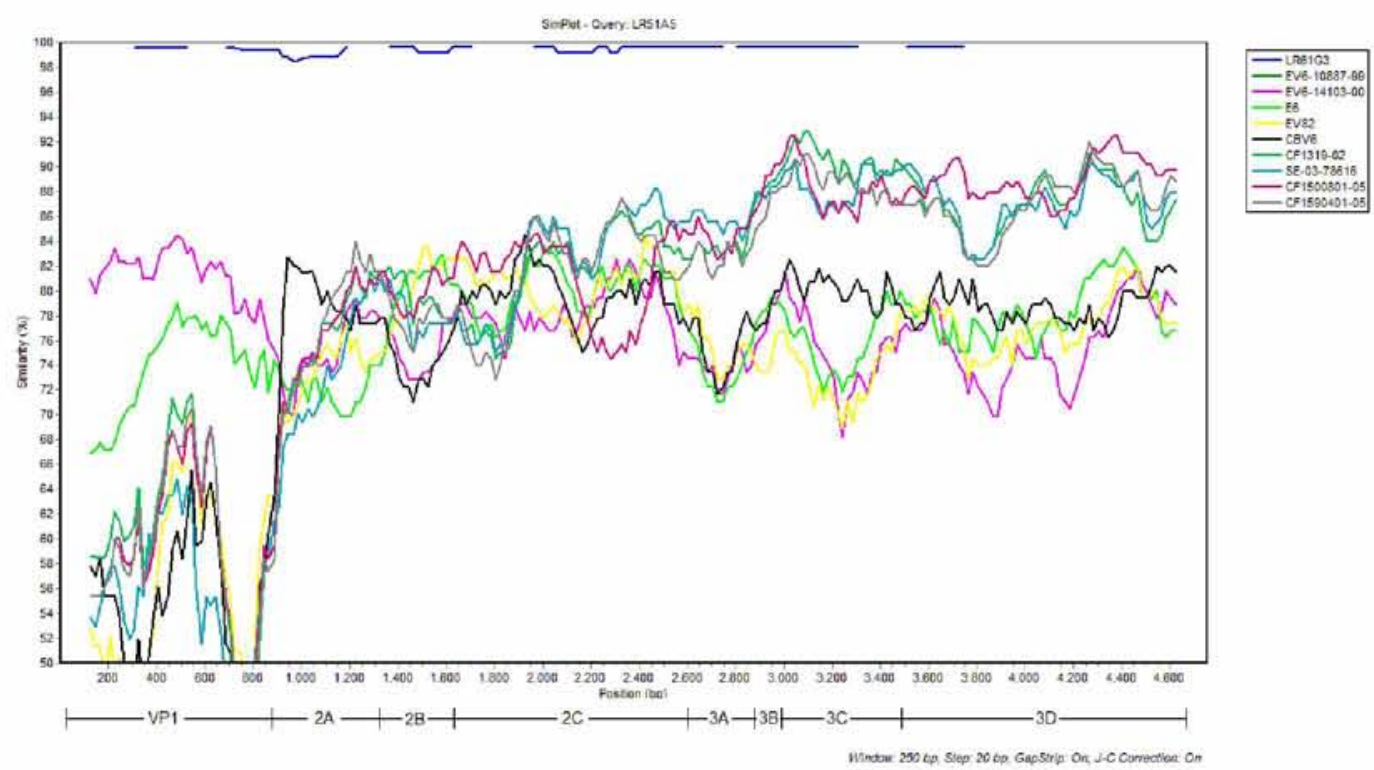

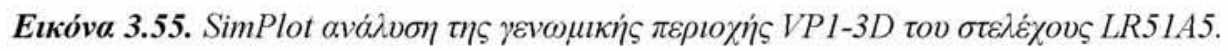

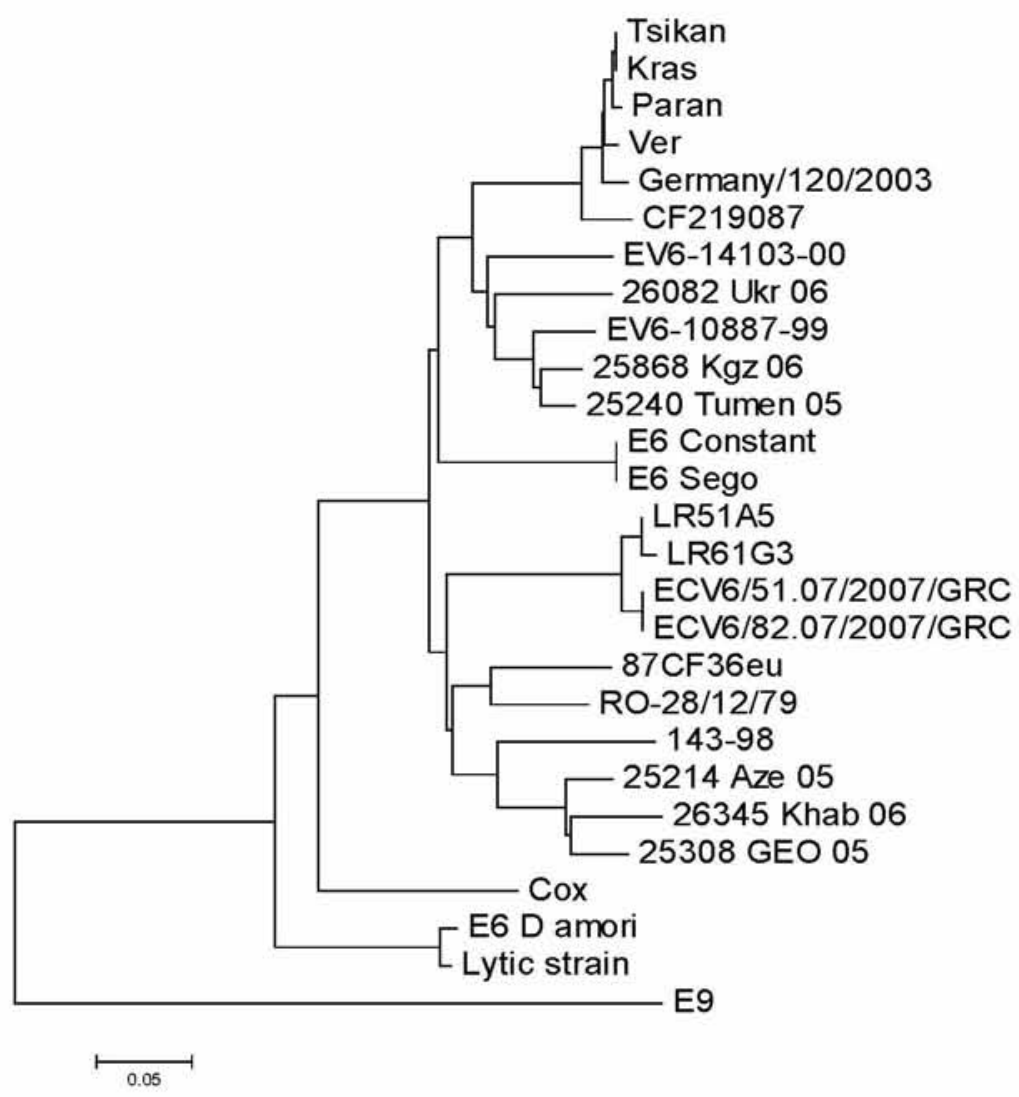

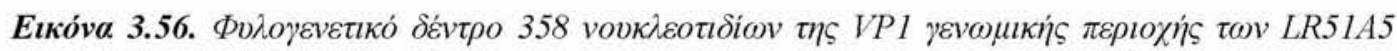

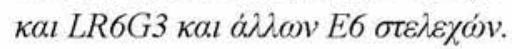




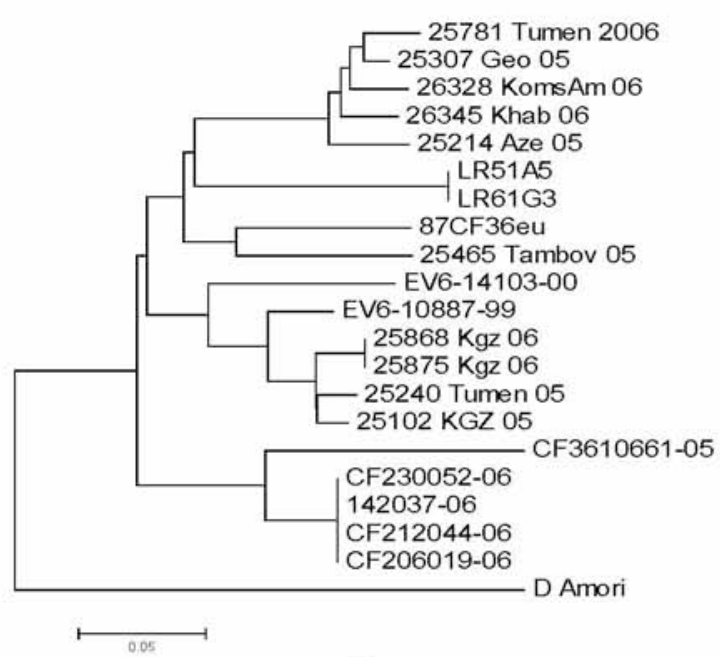

(a)

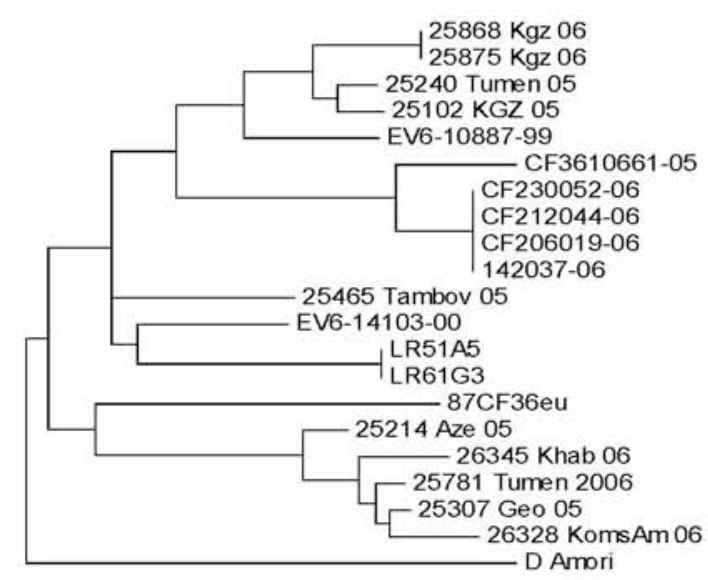

(c)

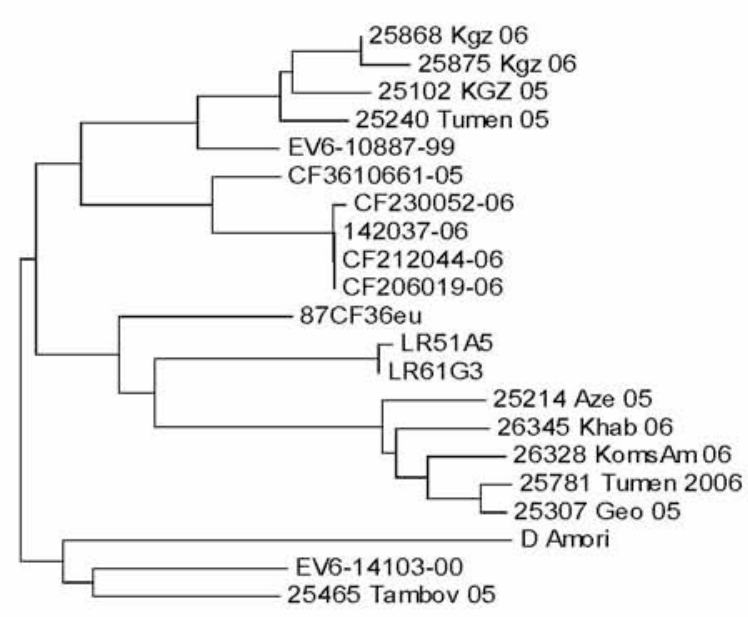

(b)

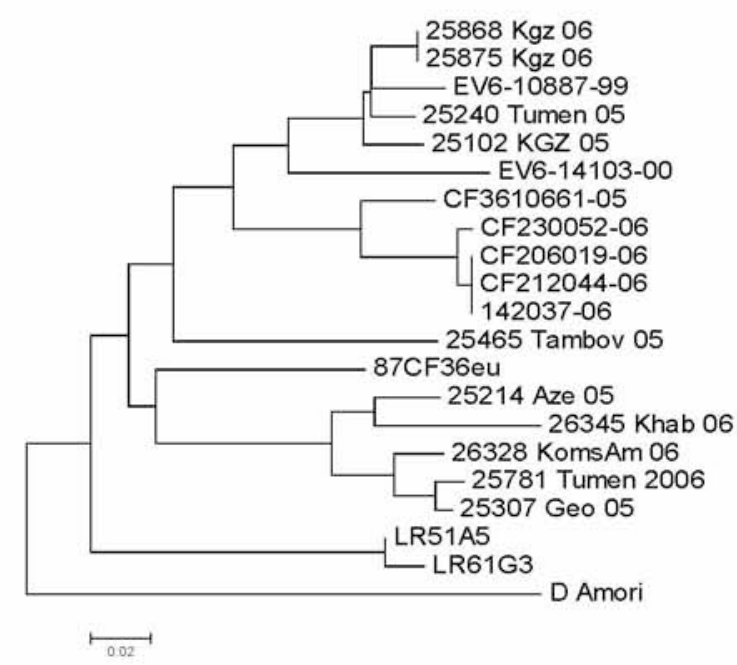

(d)

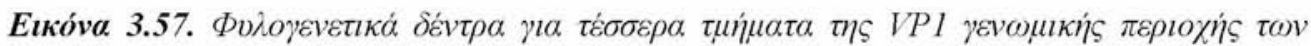

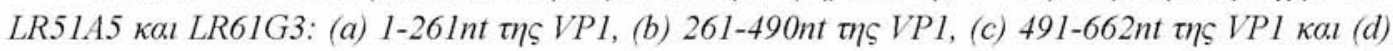
663-867 nt $\tau$ เ VPI. 

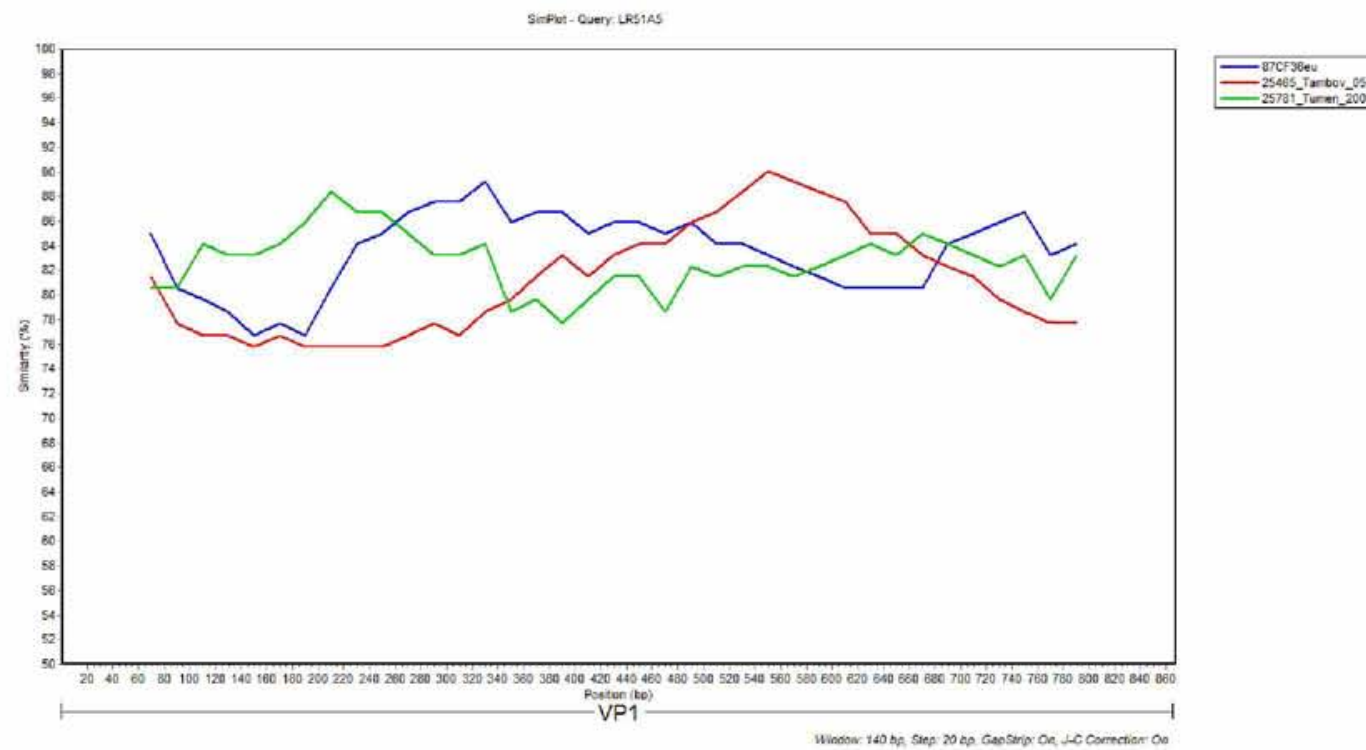

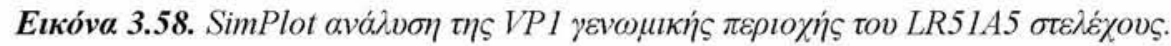




\section{КЕФАААIO 4}

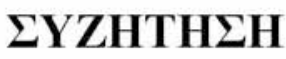

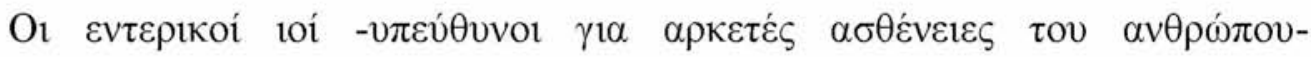

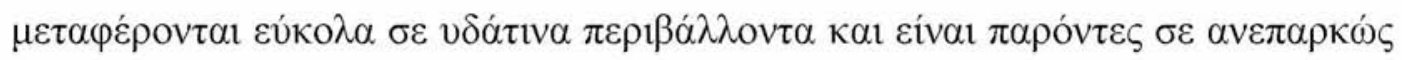

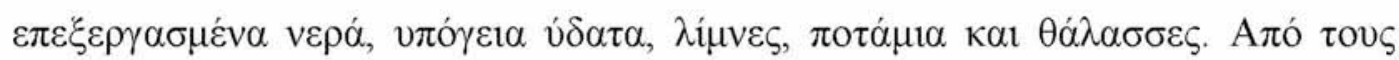

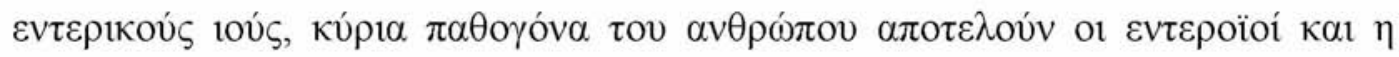

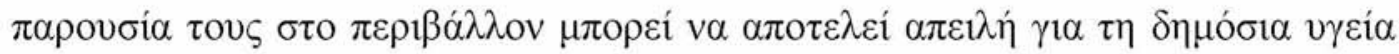

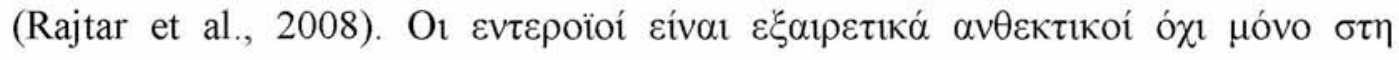

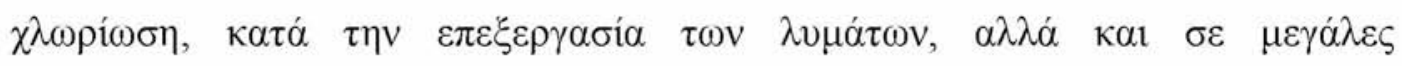

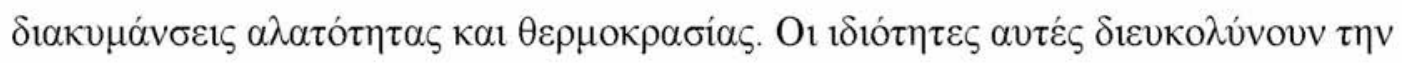

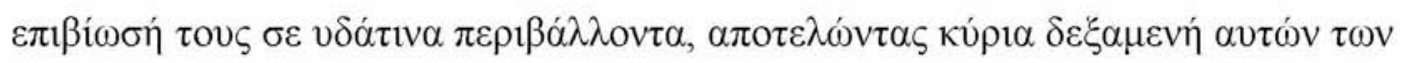

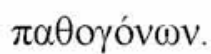

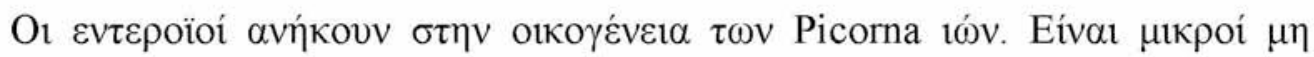

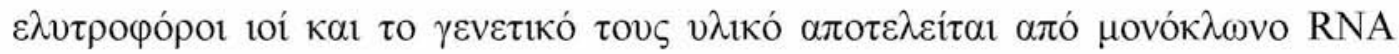

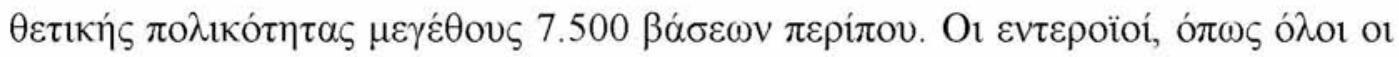

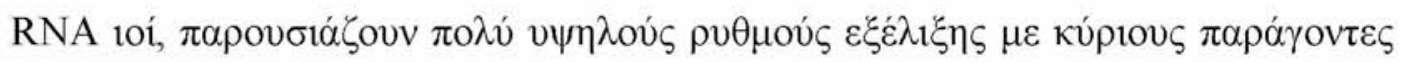

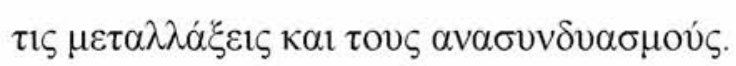

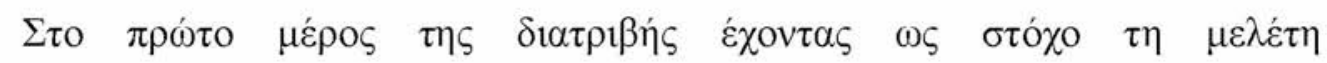

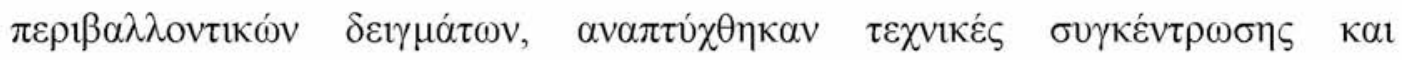

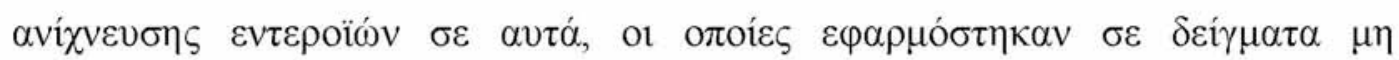

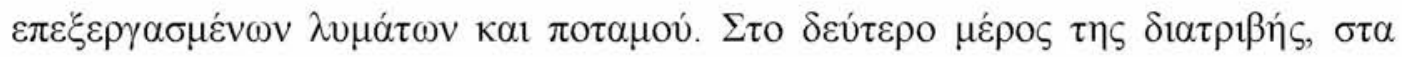

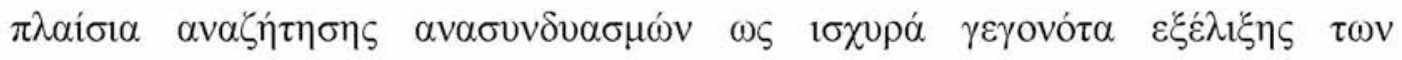

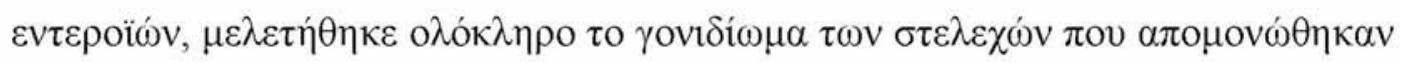

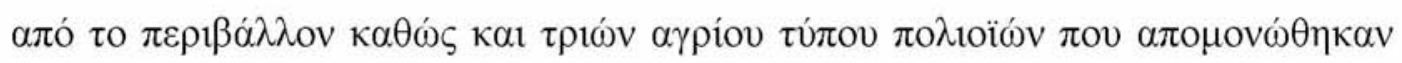

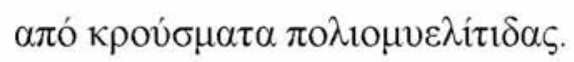

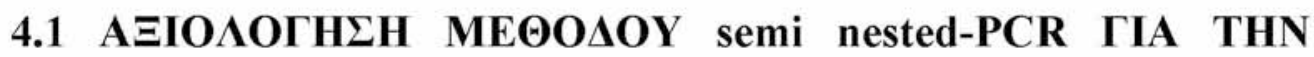

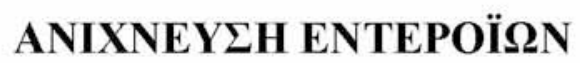

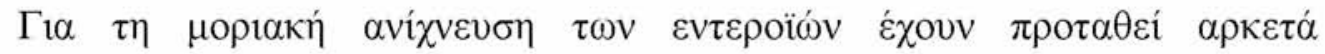

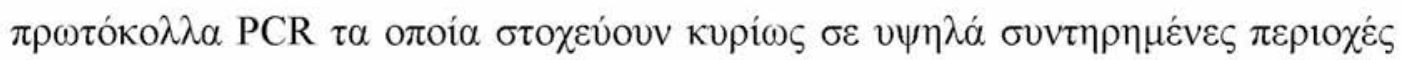




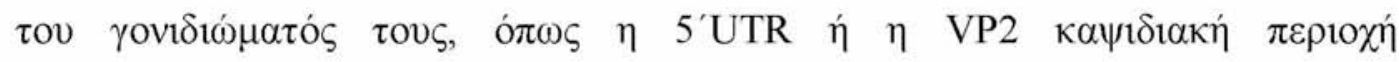
(Georgopoulou et al., 2000; Romero J.R. 1999; Poyry et al., 1996). H $\alpha \delta v v \alpha \mu i ́ \alpha$

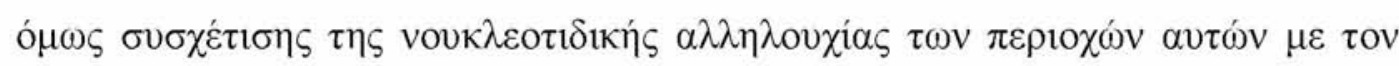

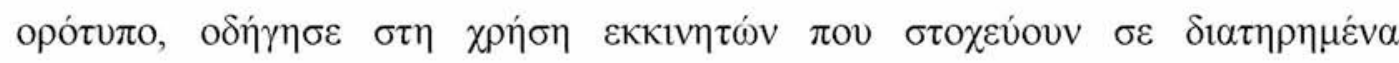

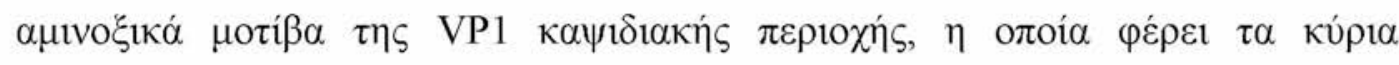

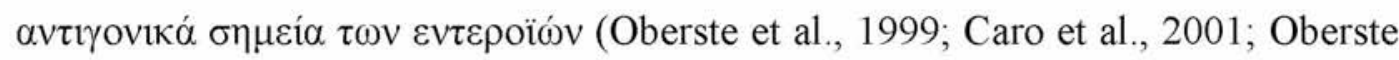

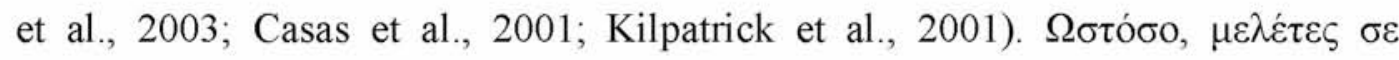

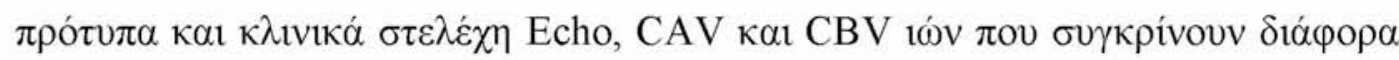

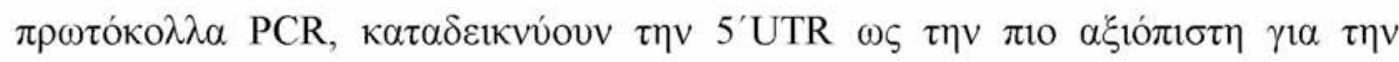

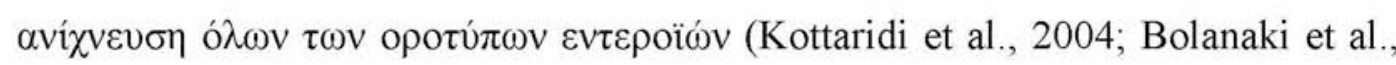
2005).

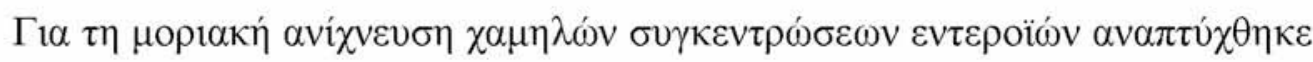

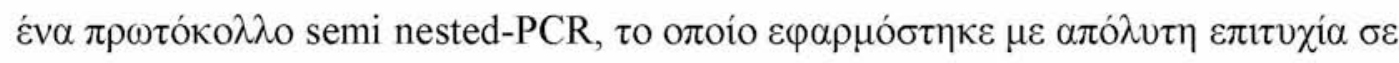

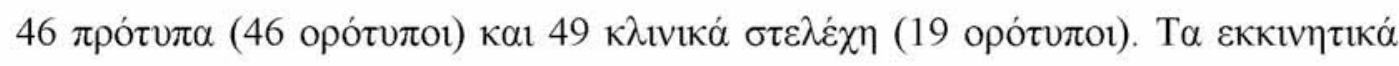

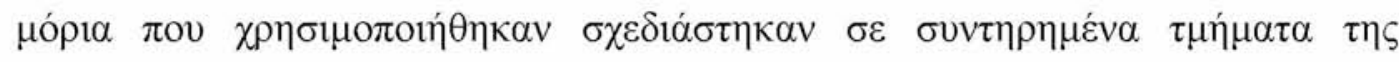

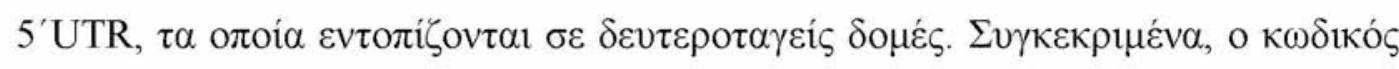

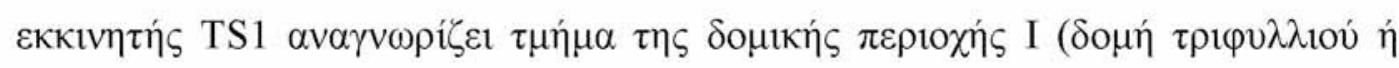

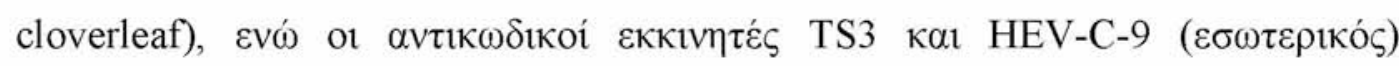

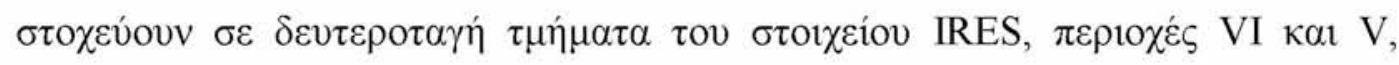

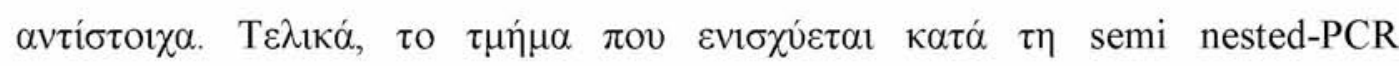

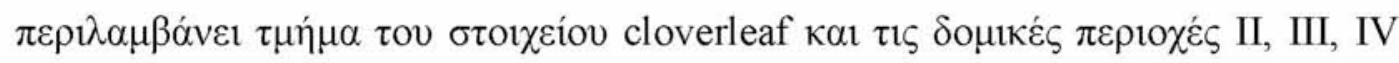

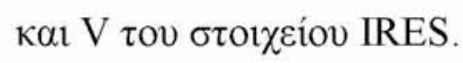

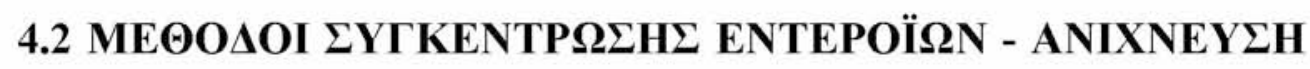

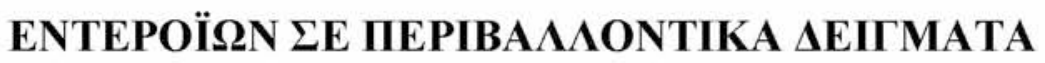

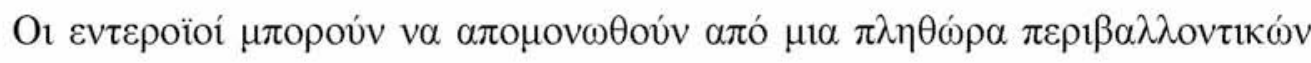

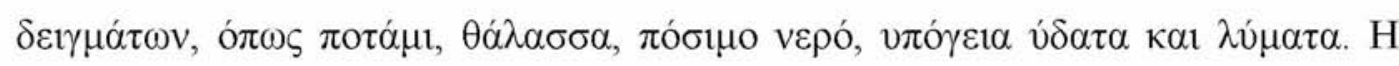

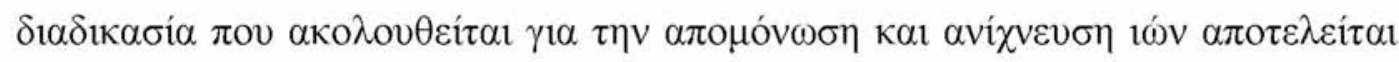

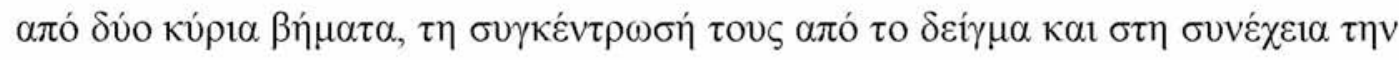

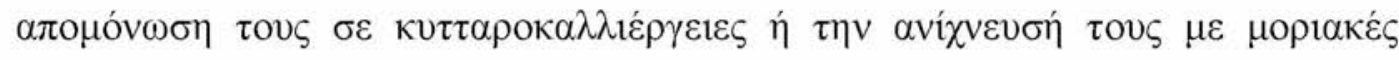
$\tau \varepsilon \chi \vee 1 \kappa \varepsilon ́ \varsigma$.

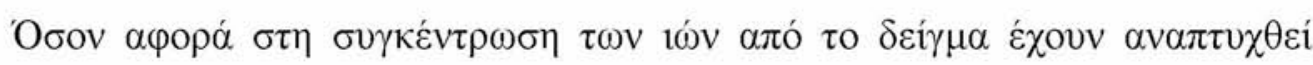

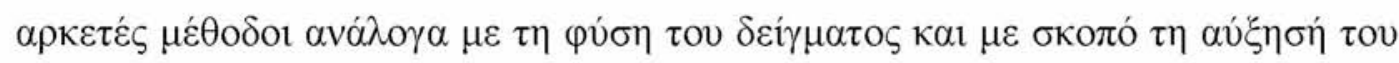




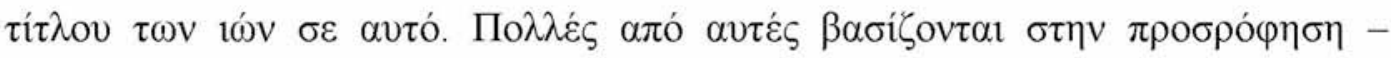

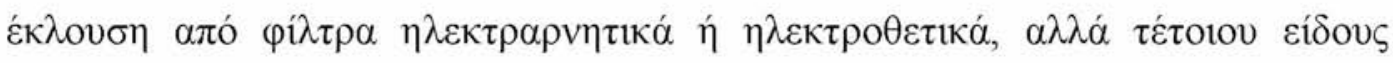

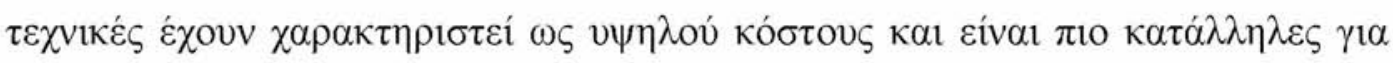

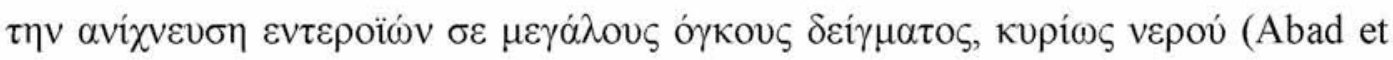
al., 1998; Crabtree et al., 1997; Katayama et al., 2002; Mocé-Llivina et al., 2002).

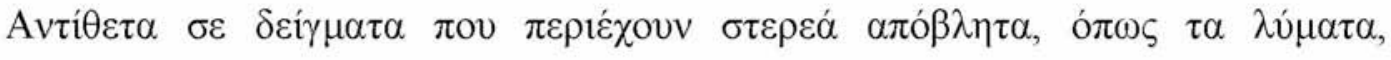

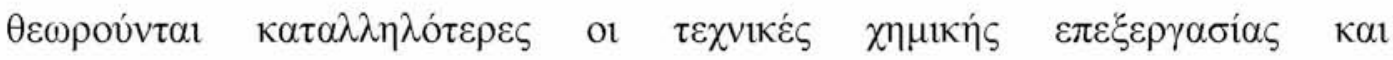

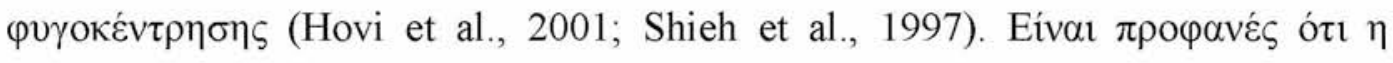

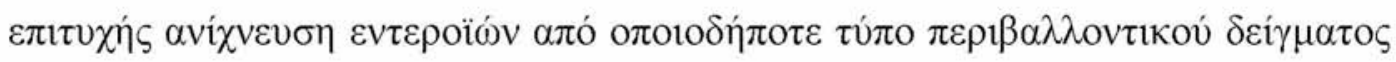

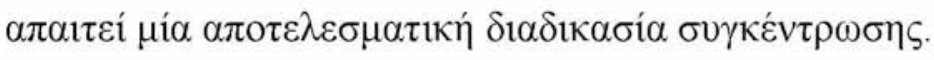

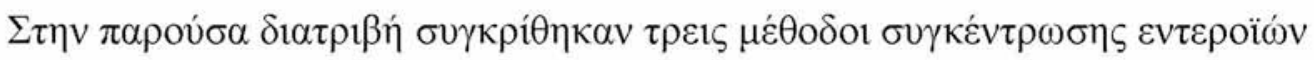

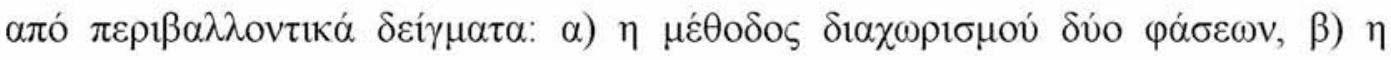

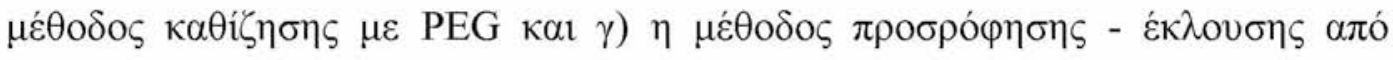
$\eta \lambda \varepsilon \kappa \tau \rho \alpha \rho v \eta \tau$ $\varepsilon v \alpha i \sigma \theta \eta \sigma i ́ \alpha$

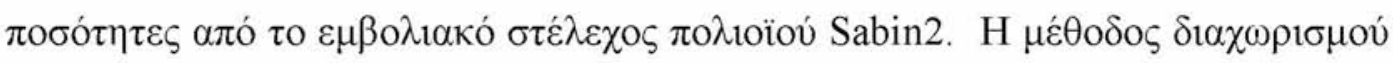

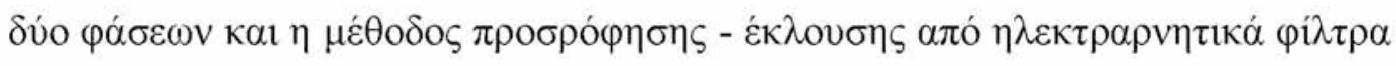

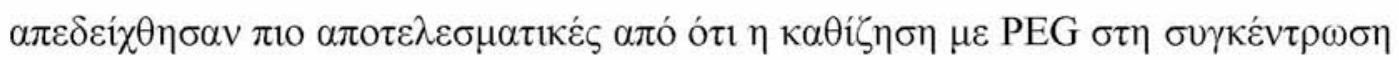

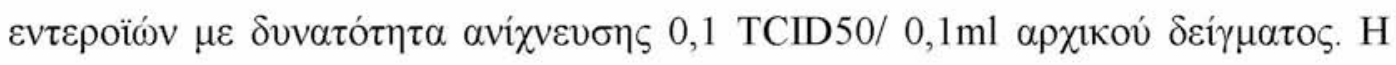

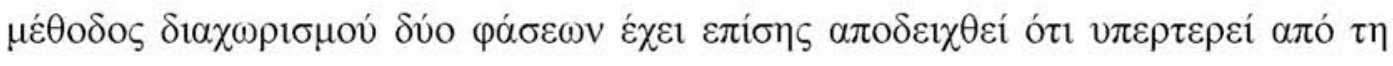

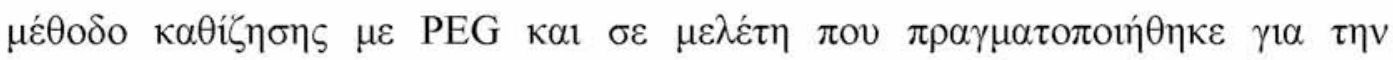

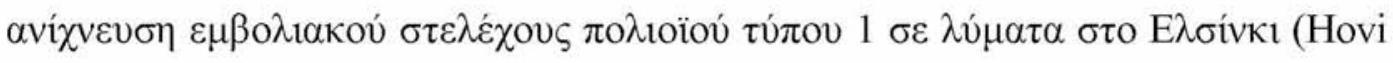

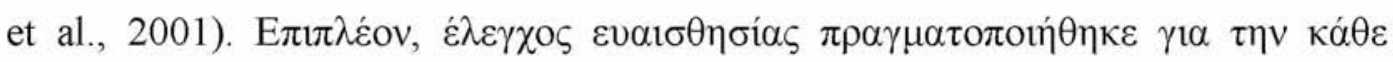

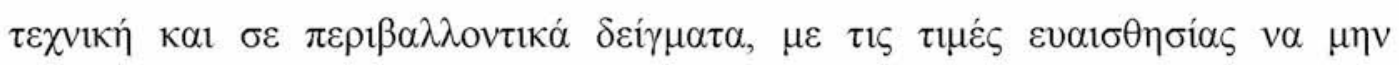

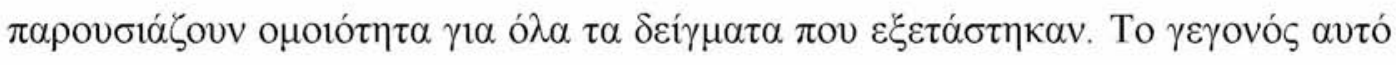

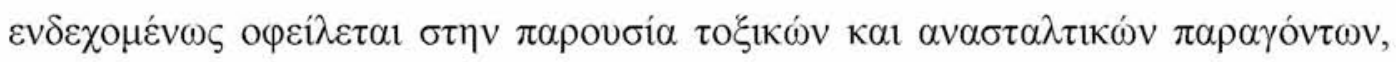

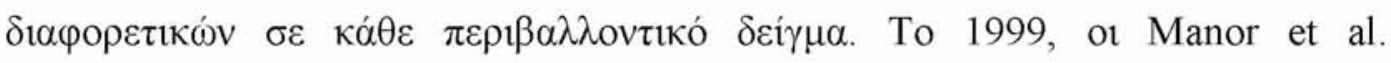

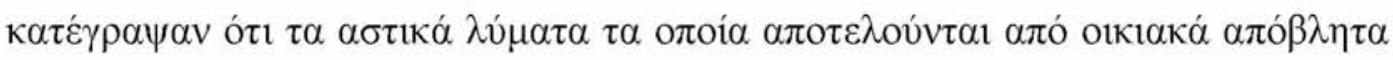

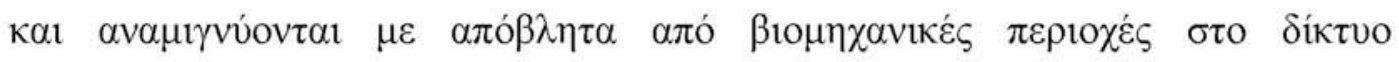

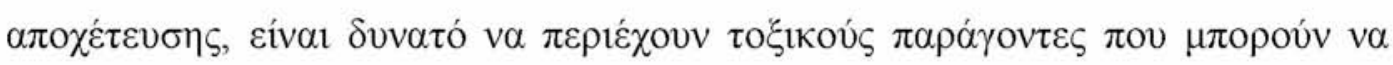

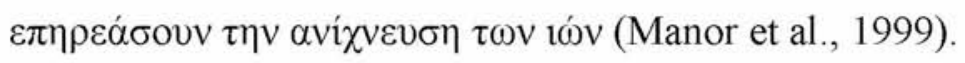

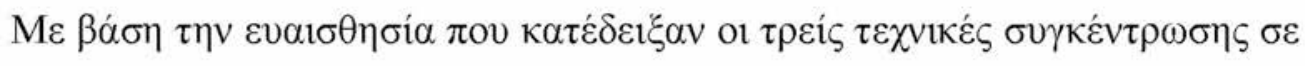

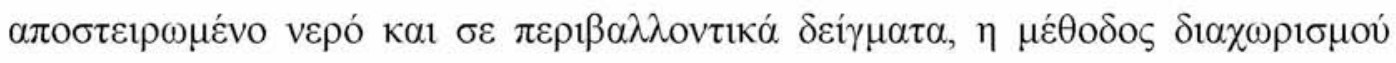

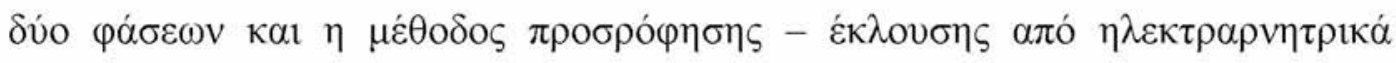




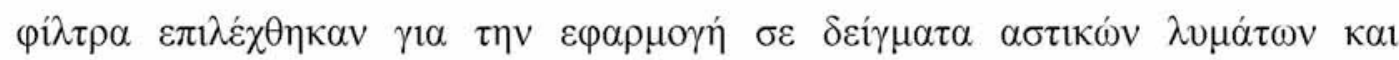

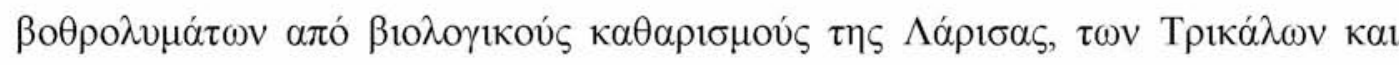

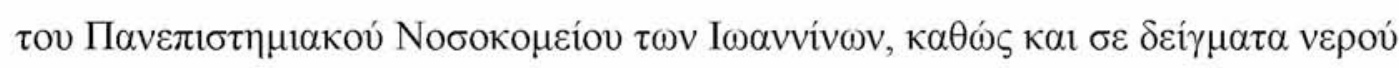

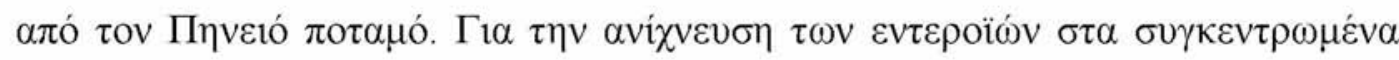

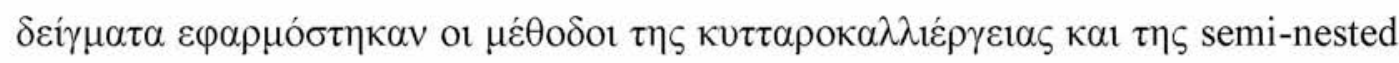

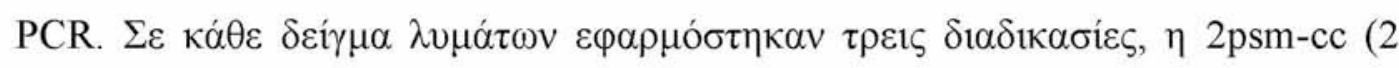
phase separation method-cell culture), $\eta 2$ psm-snPCR ( 2 phase separation methodsemi nested PCR) кaı $\eta$ enF-snPCR (electronegative Filter-semi nested PCR).

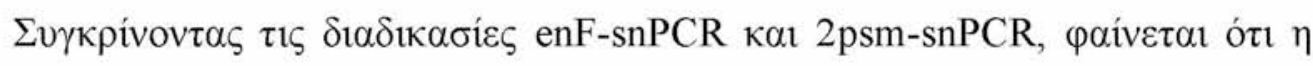

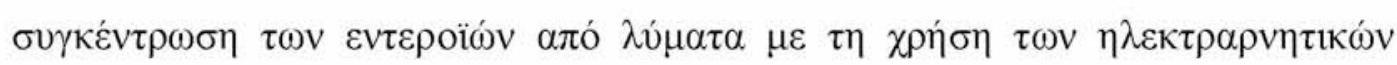

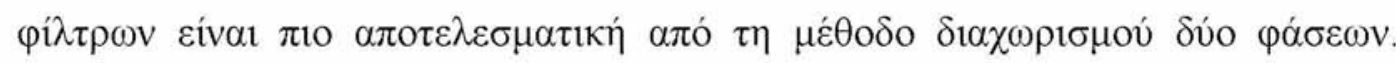

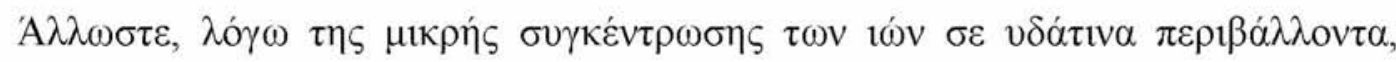

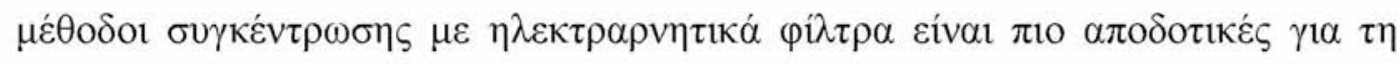

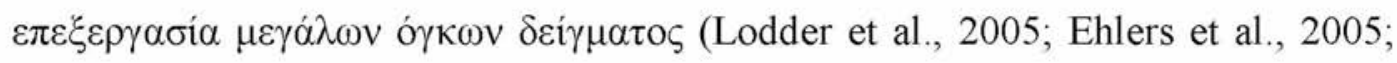

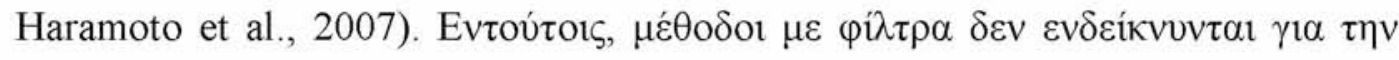

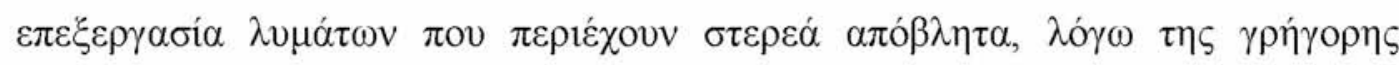

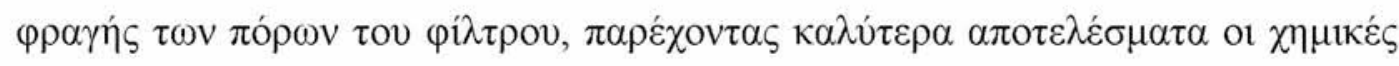
$\mu \varepsilon \dot{\theta}$ odor (Tsai et al., 1993; Shieh et al., 1997).

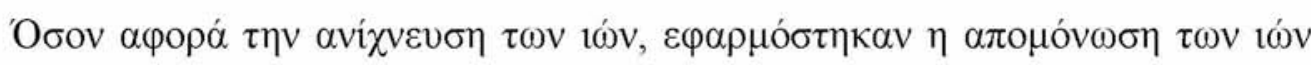

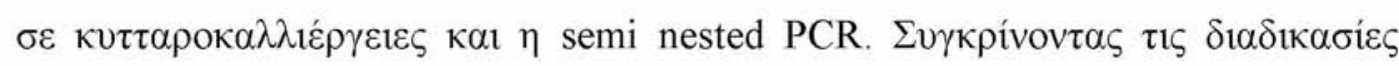

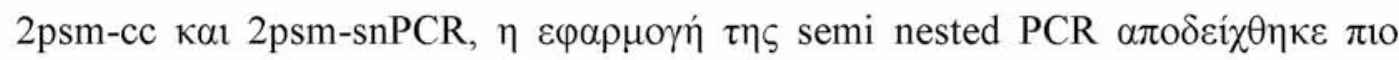

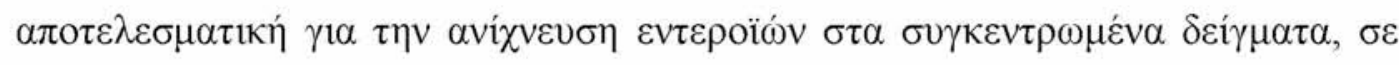

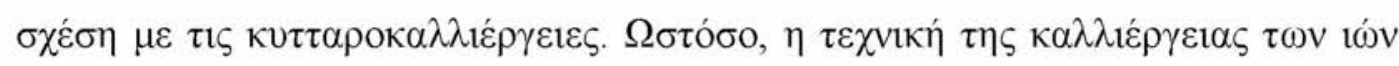

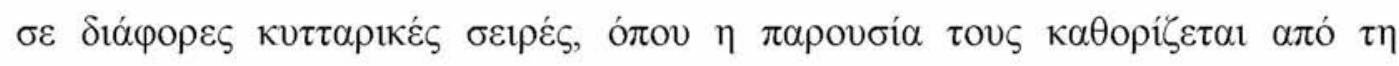

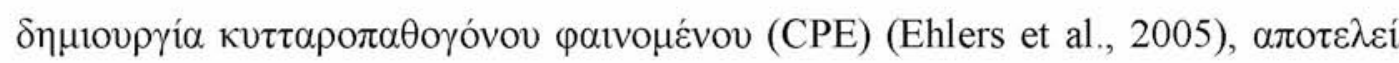

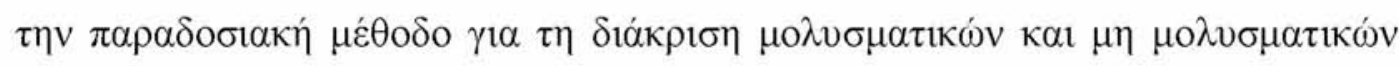

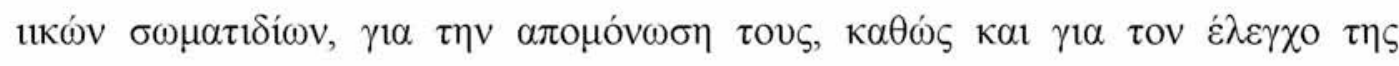

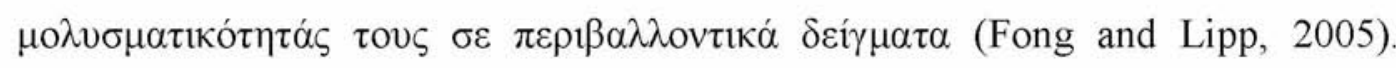

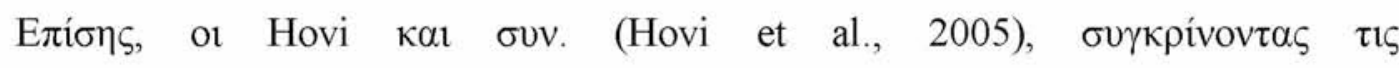

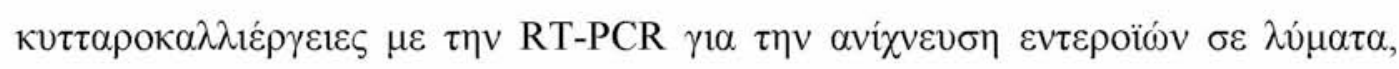

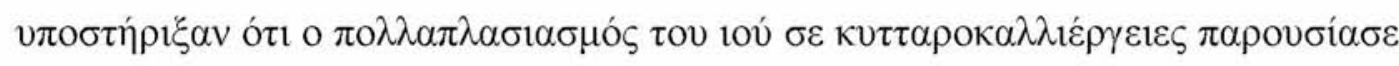

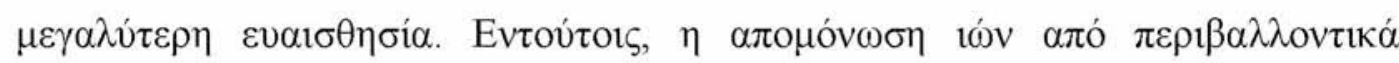

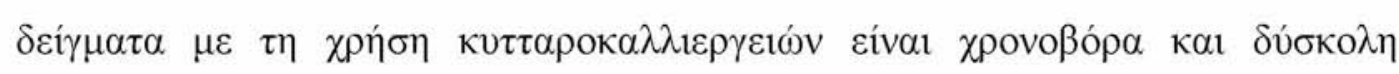

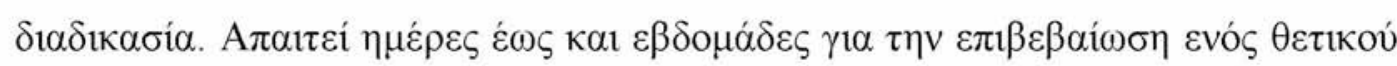




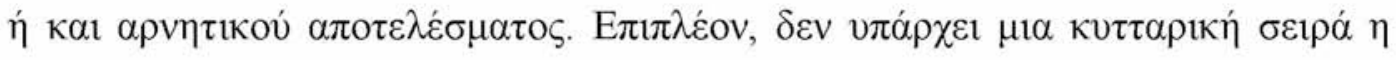

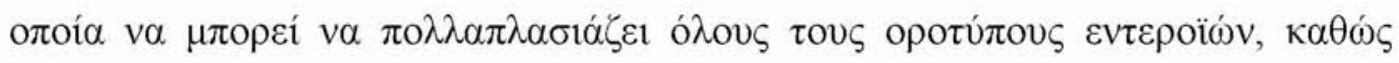

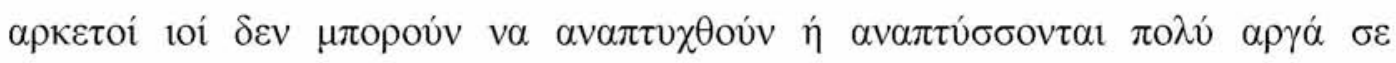

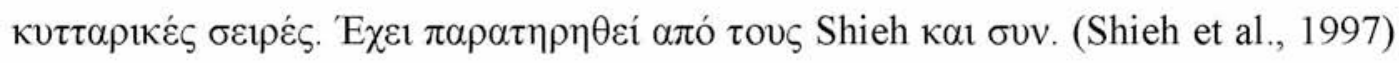

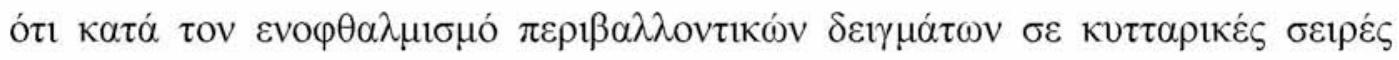

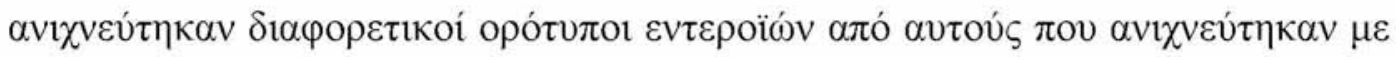

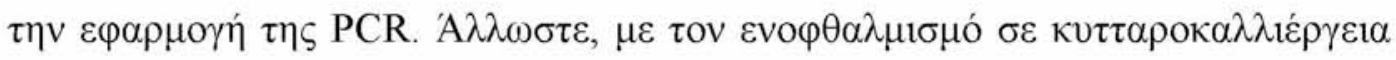

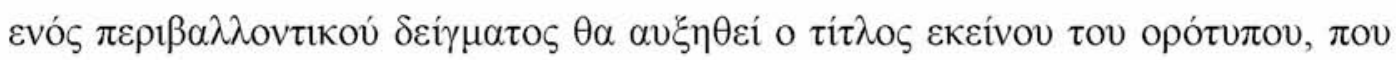

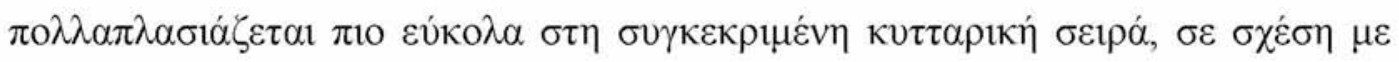

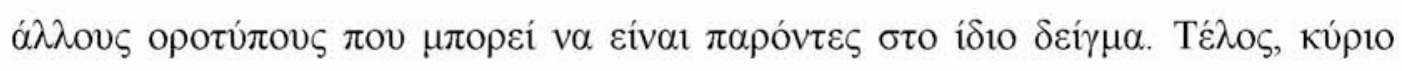

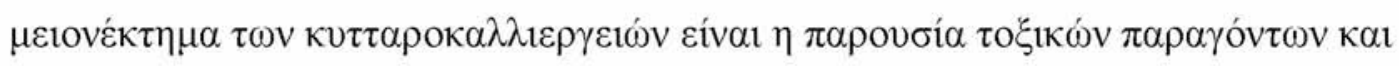

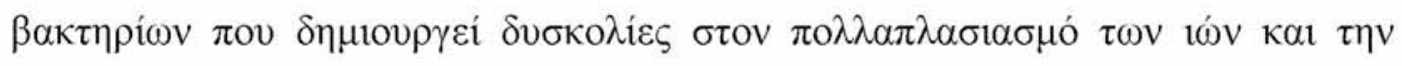

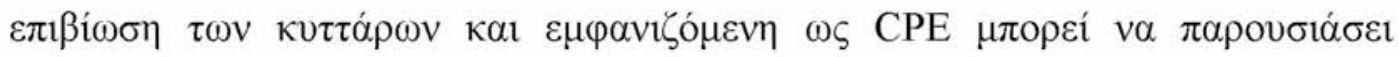

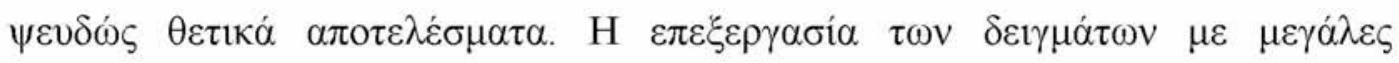

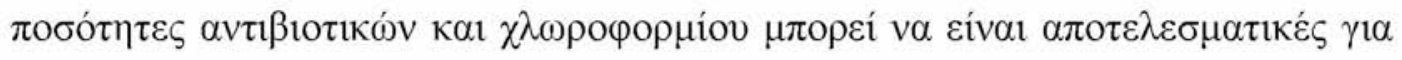

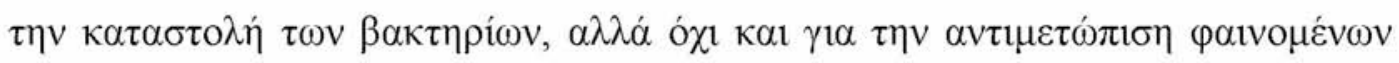

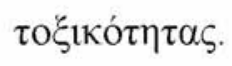

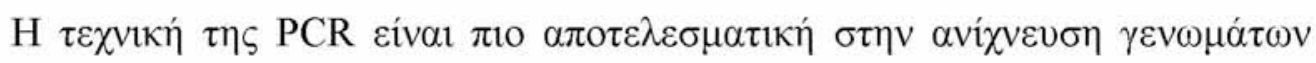

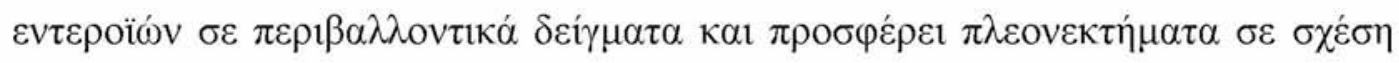

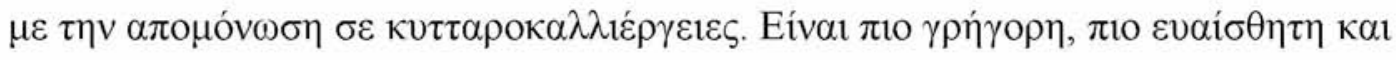

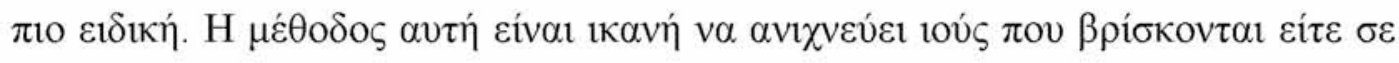

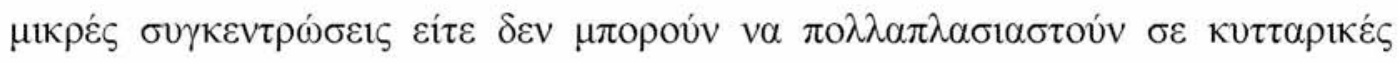

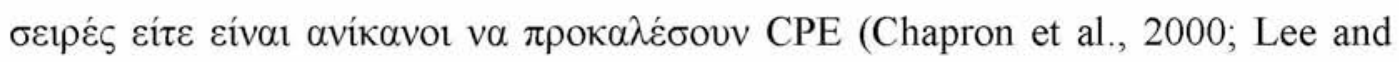

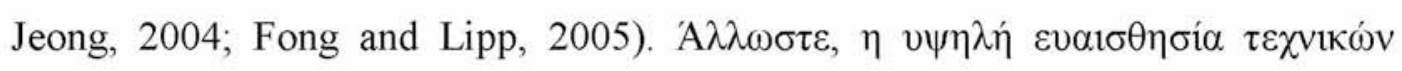

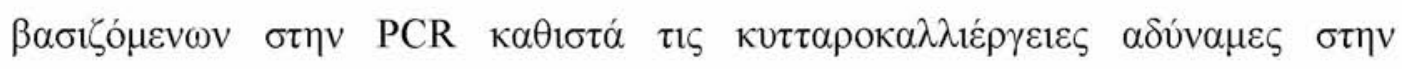

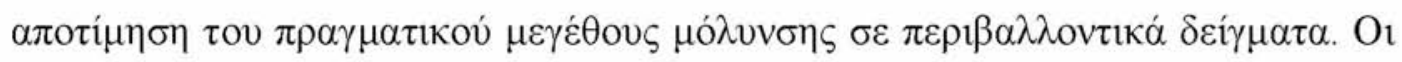

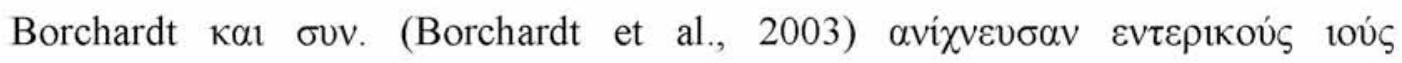

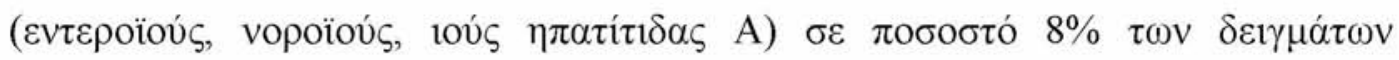

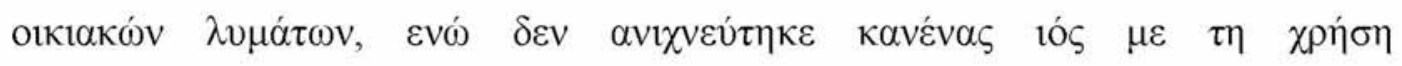

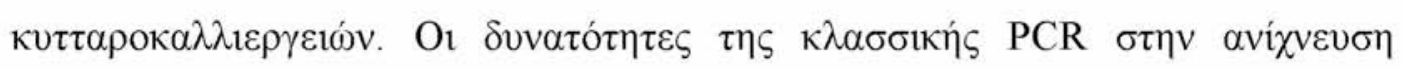

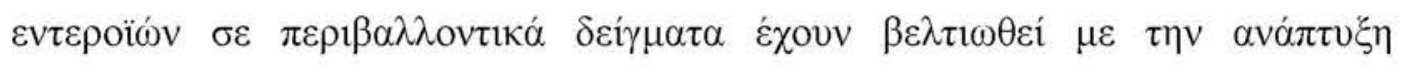
$\tau \varepsilon \chi v ı \kappa \omega ́ v ~ o ́ \pi \omega \varsigma \eta$ nested PCR, $\eta$ real time PCR $\kappa \alpha ı ~ \eta$ multiplex PCR (Fong and Lipp, 2005, Ehlers et al., 2005, Gregory et al., 2006, Fout et al., 2003). To kúpıo

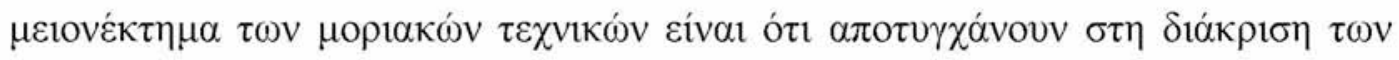




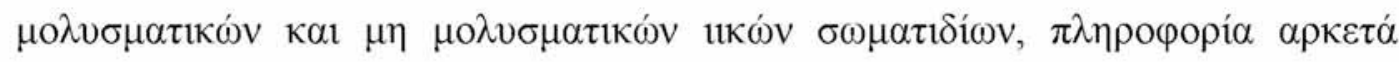

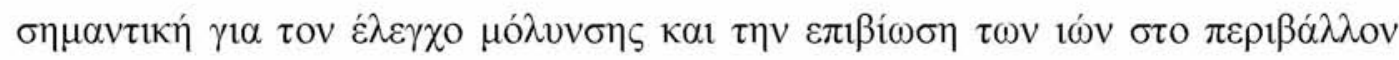
(Abad et al., 1997).

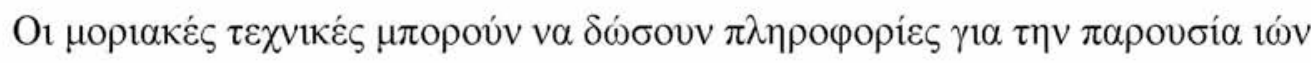

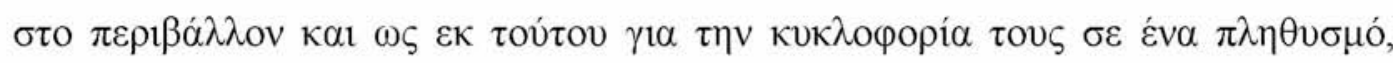

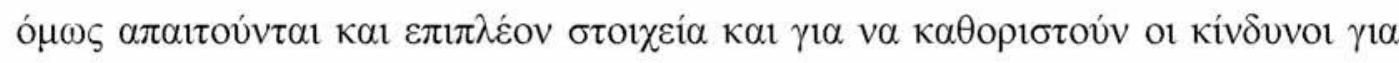

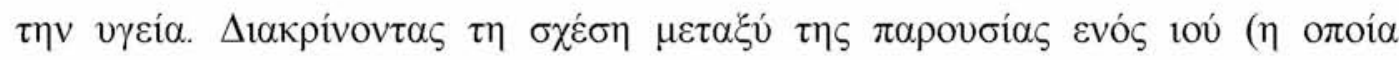

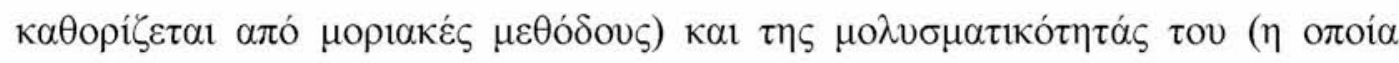

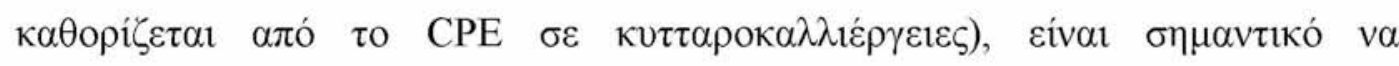

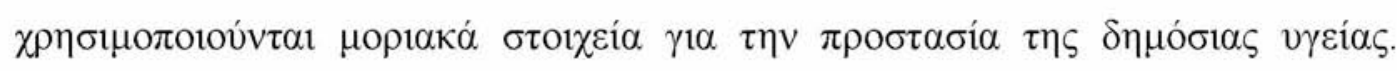

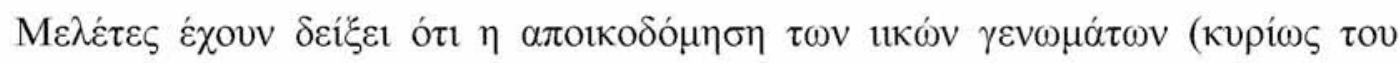

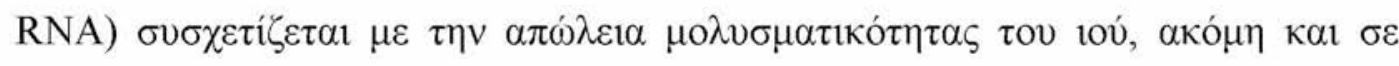

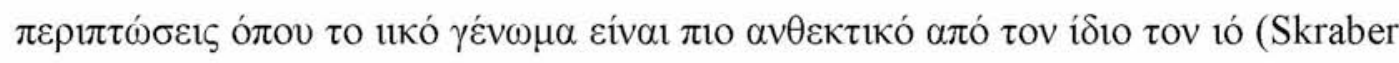

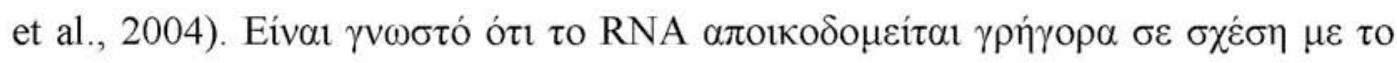

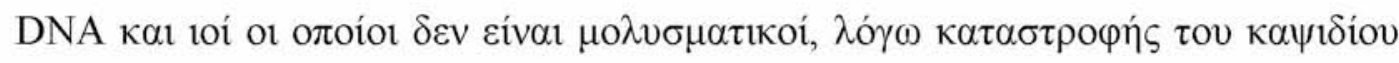

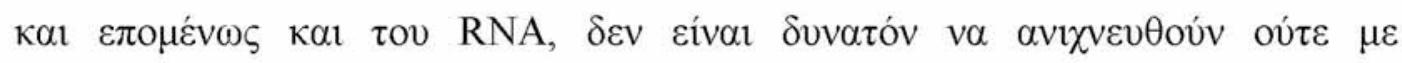

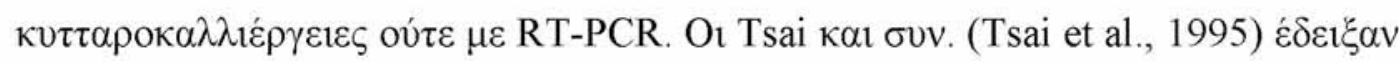

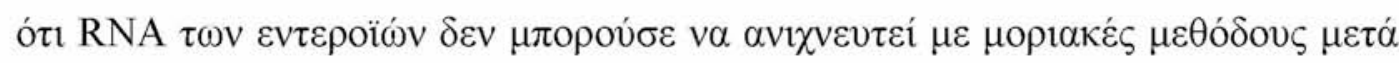

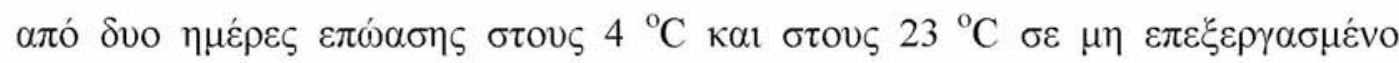

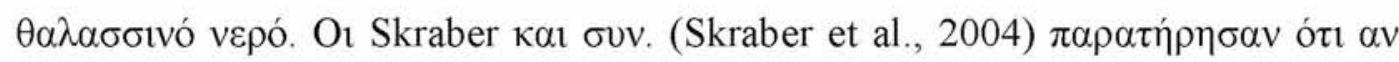

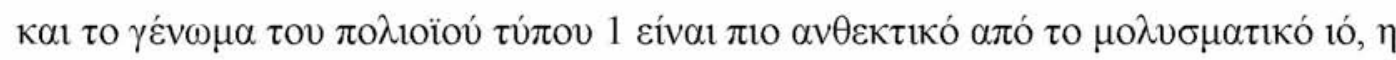

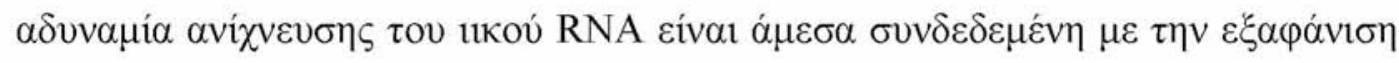

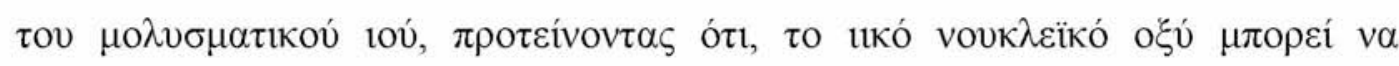

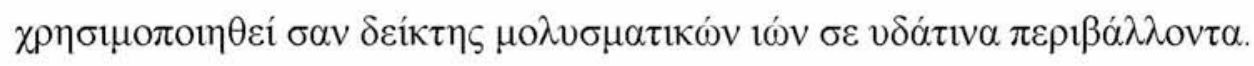

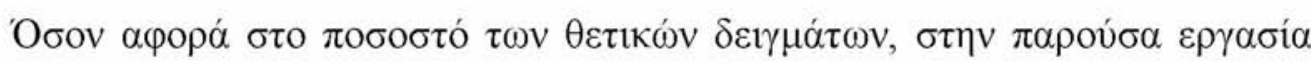

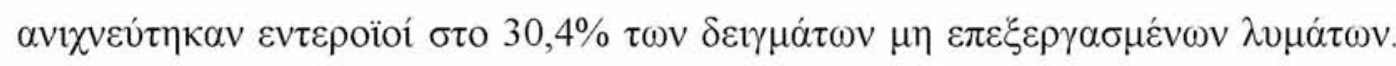

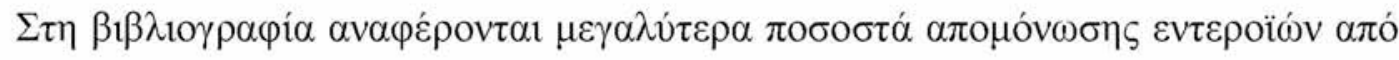

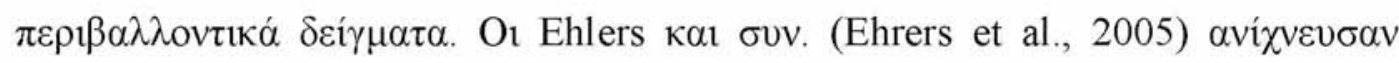

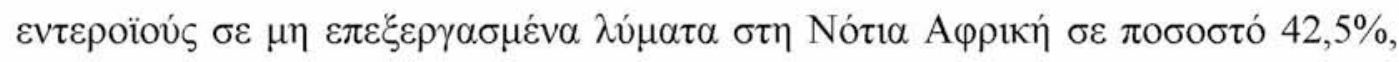

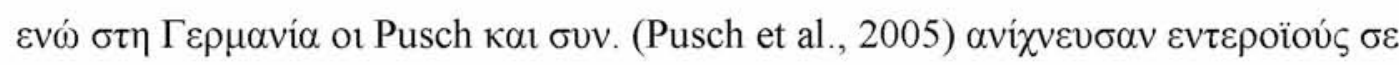

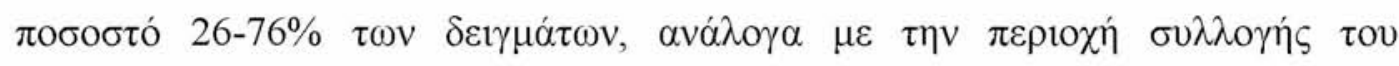

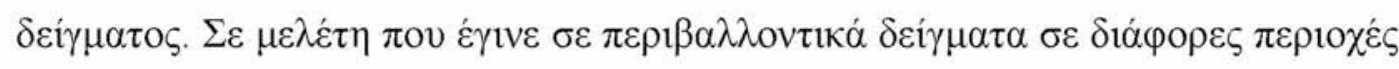

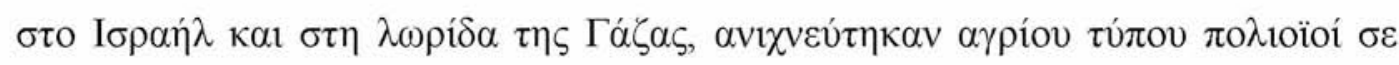

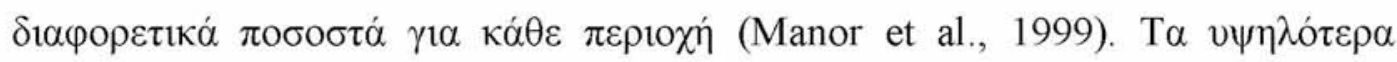




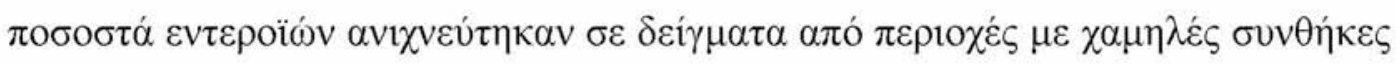

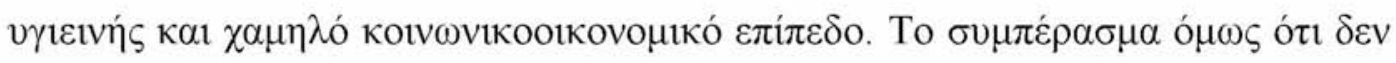

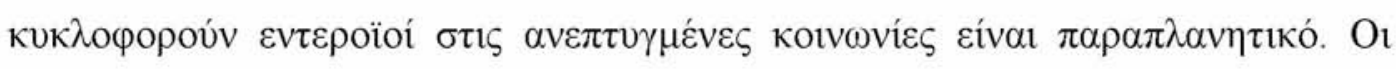

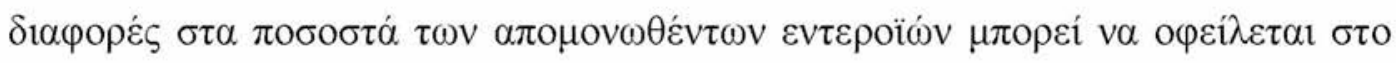

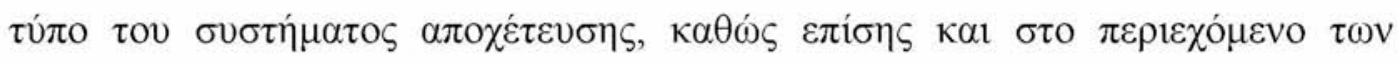

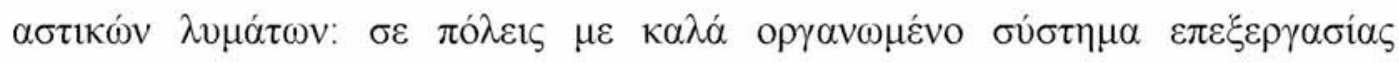

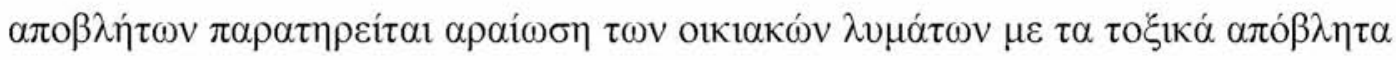
$\tau \omega \nu \beta 10 \mu \eta \chi \alpha \nu ı \omega ́ v$.

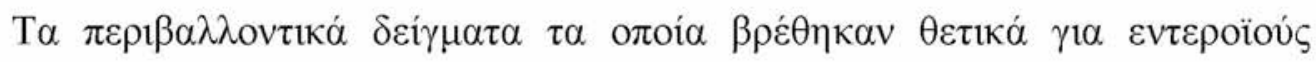

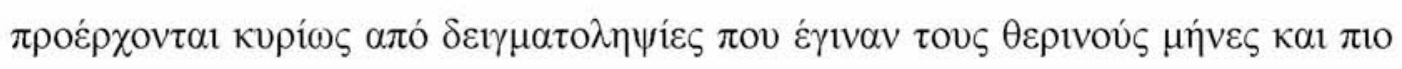

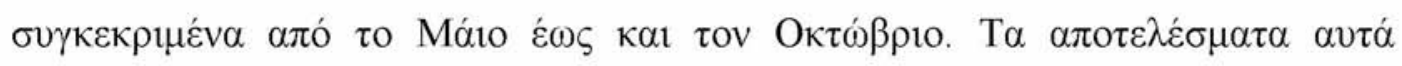

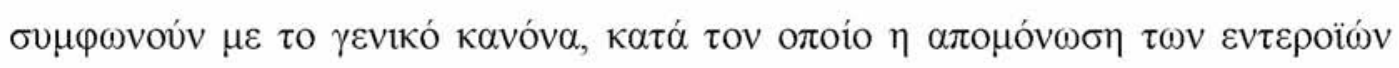

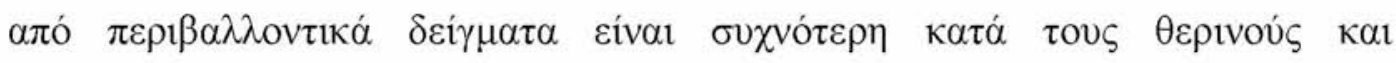

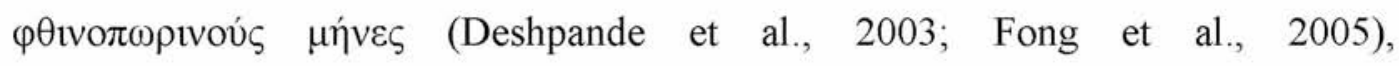

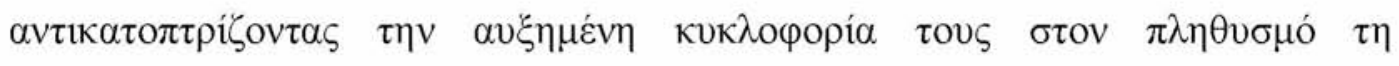

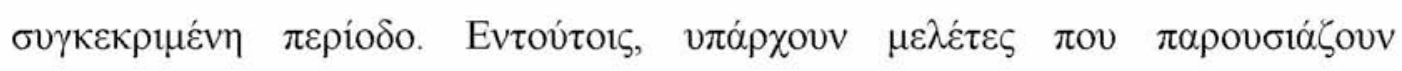

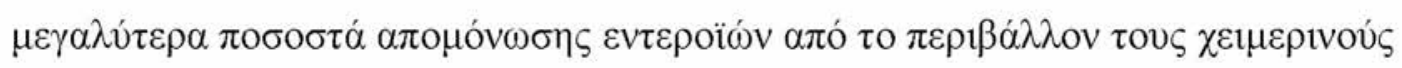

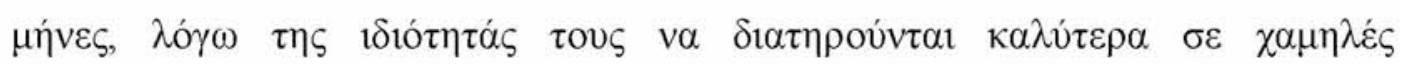

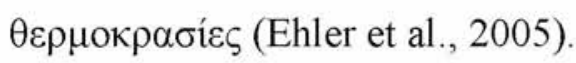

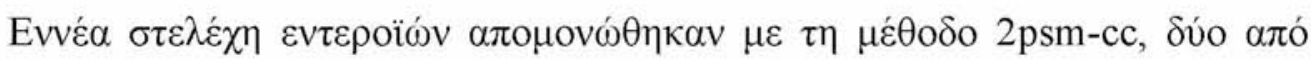

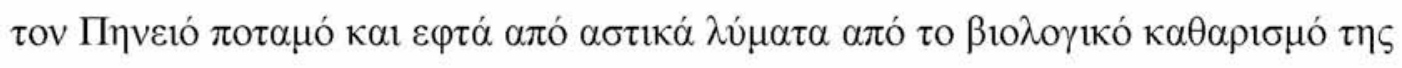

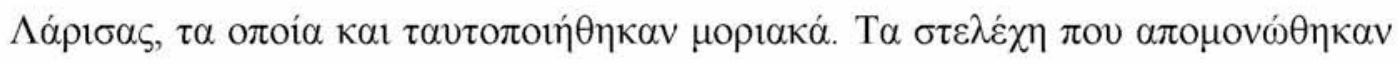

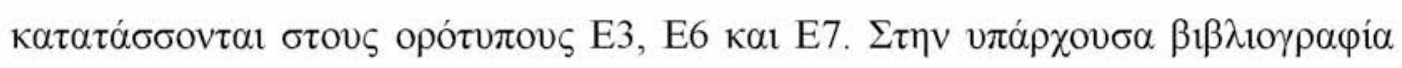

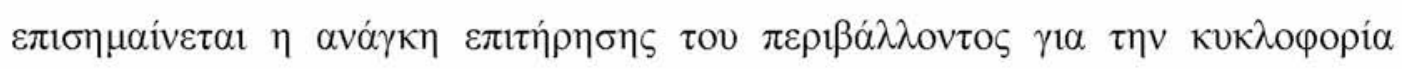

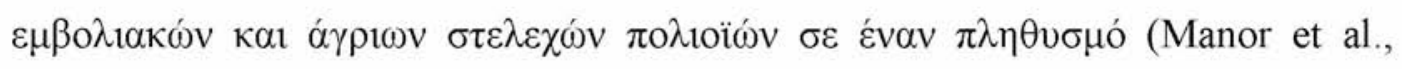

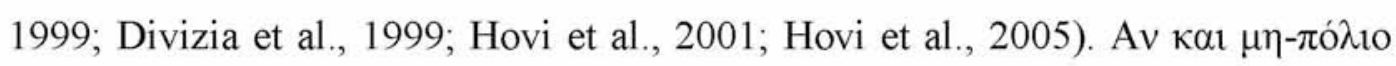

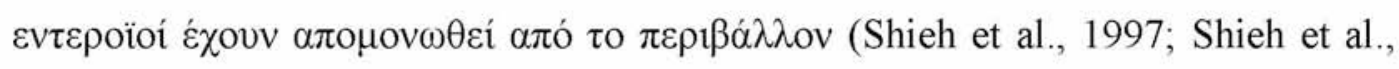

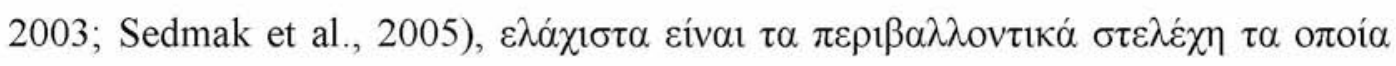

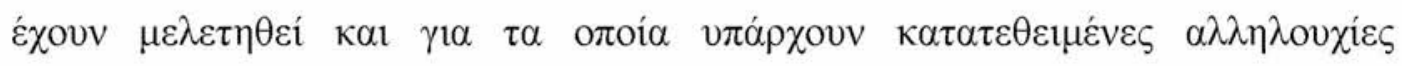
(Bolanaki et al., 2005b, Lukashev et al., 2005). 


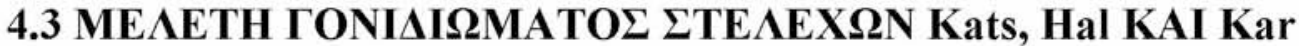

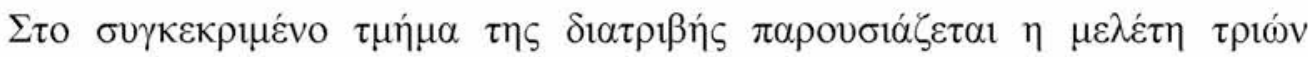

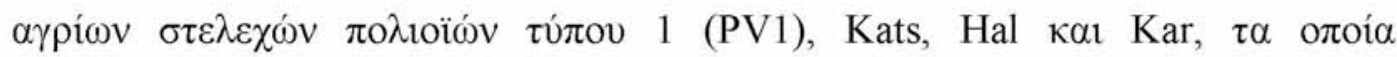

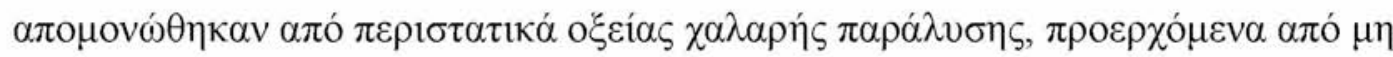

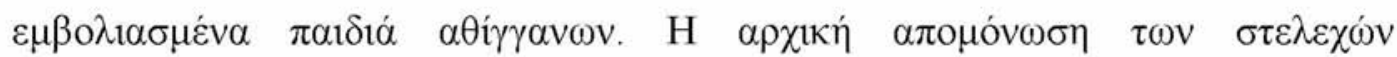

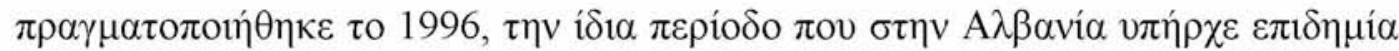

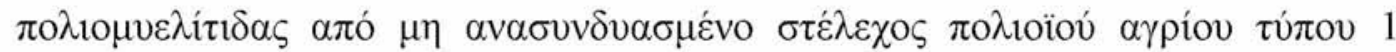

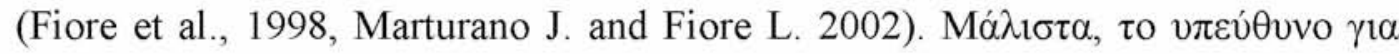

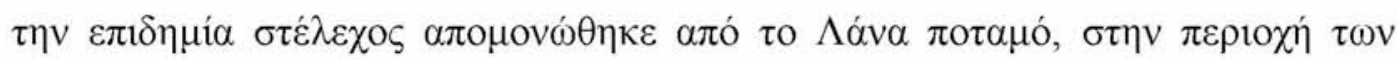

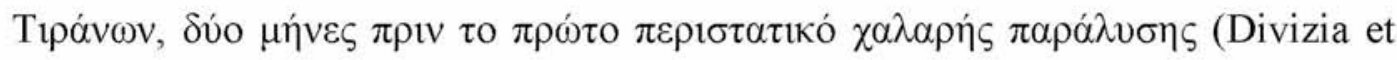

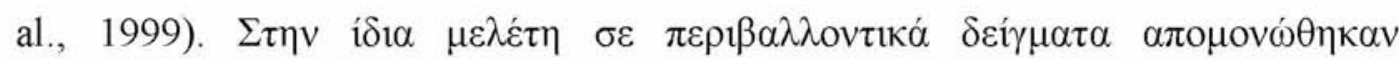

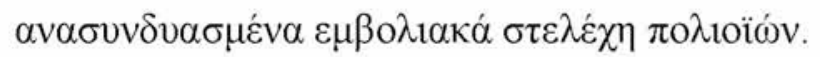

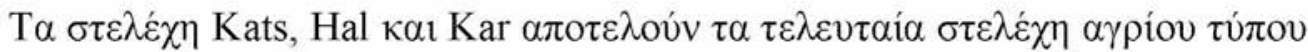

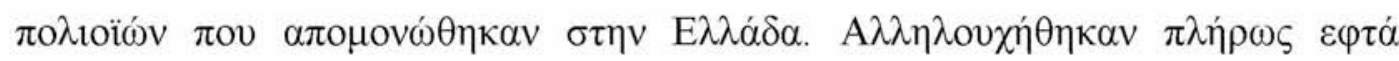

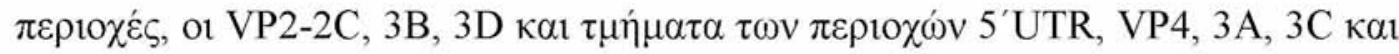

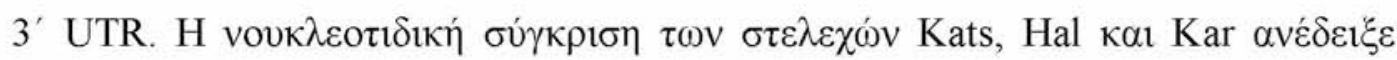

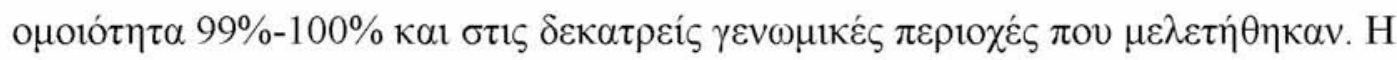

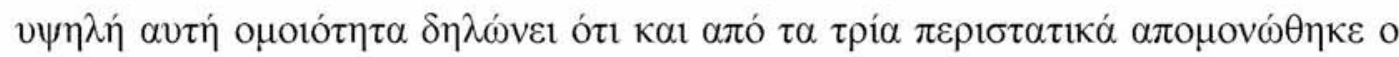

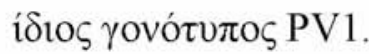

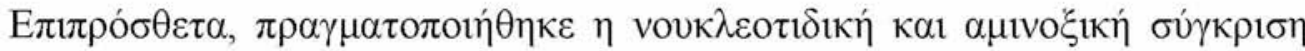

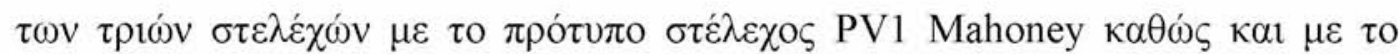

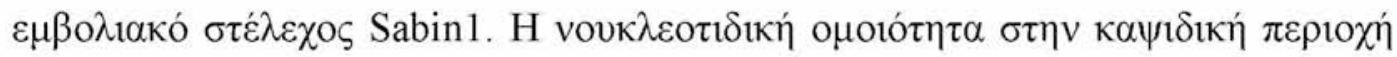

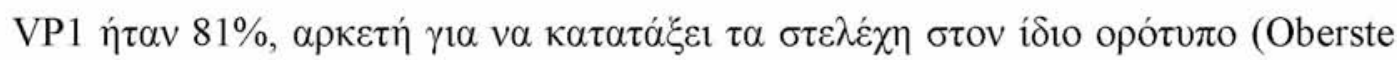

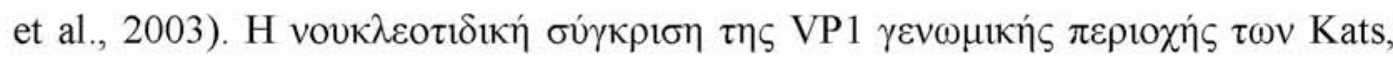

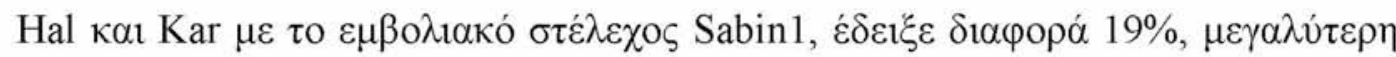

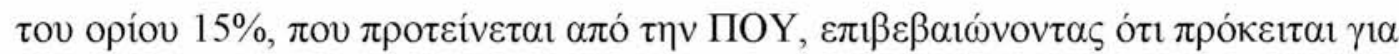

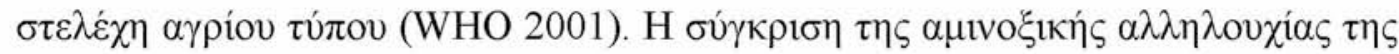

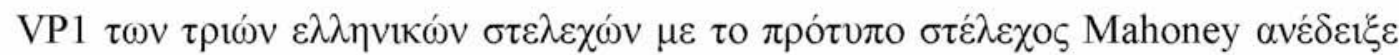

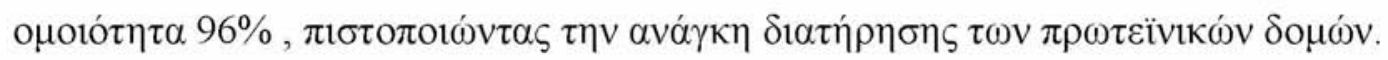

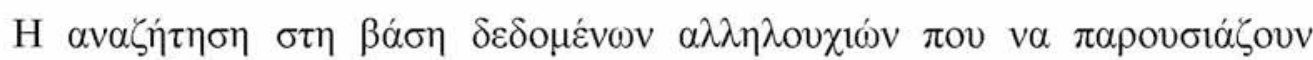

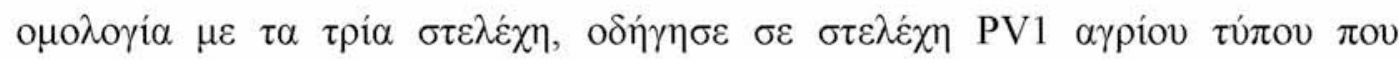
$\alpha \pi$ o

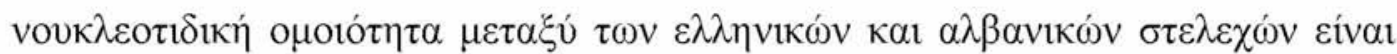




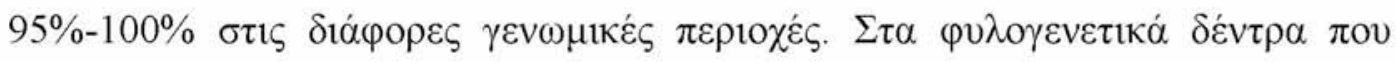

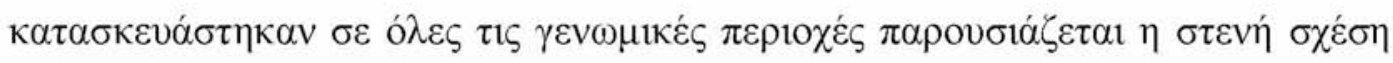

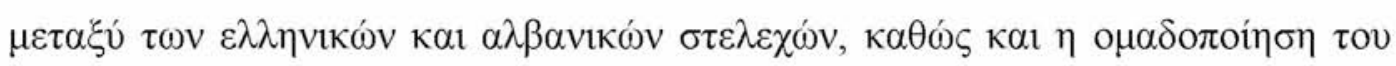

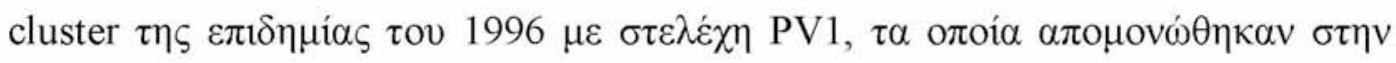

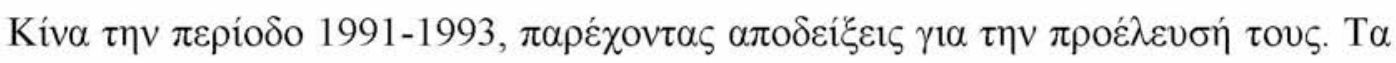

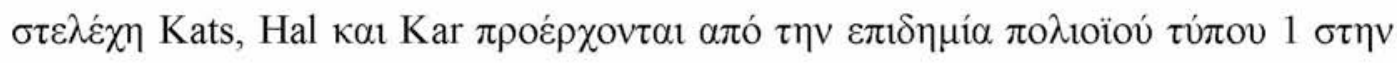

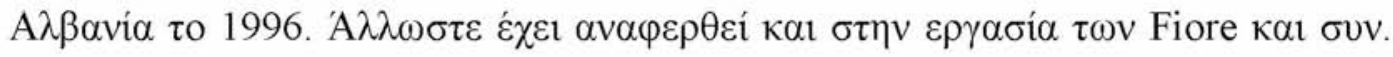

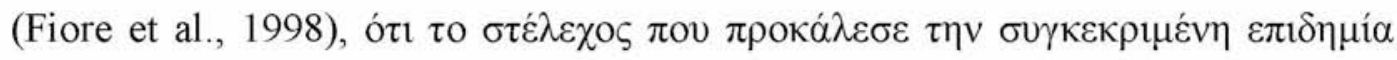

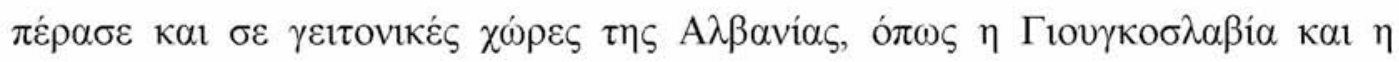
E $\lambda \lambda \alpha ́ \delta \alpha$.

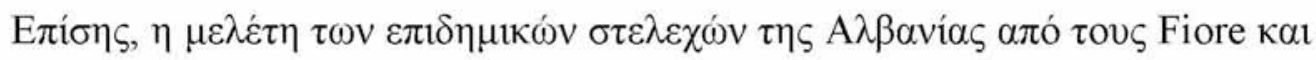

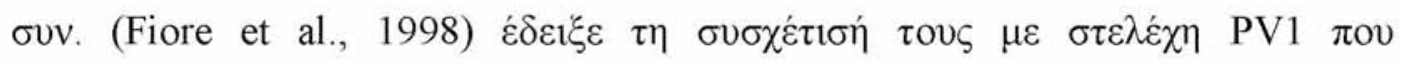

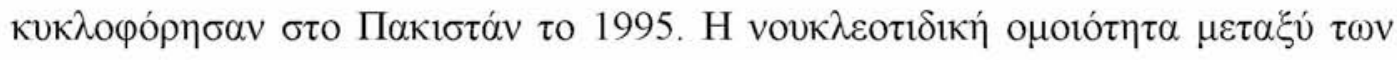

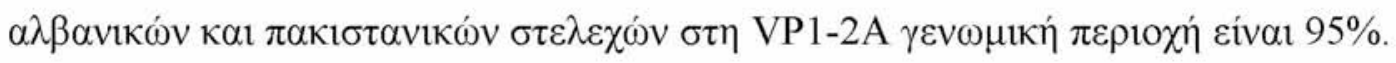

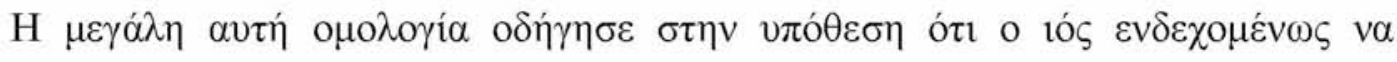

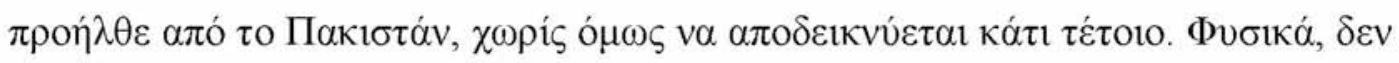

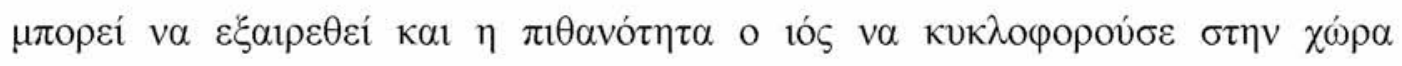

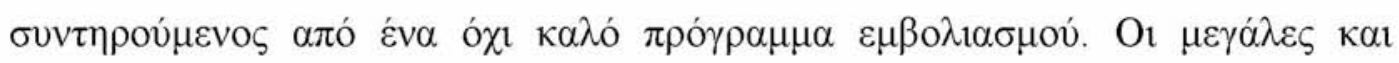

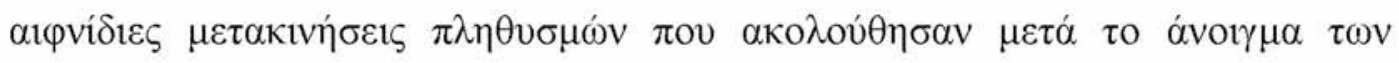

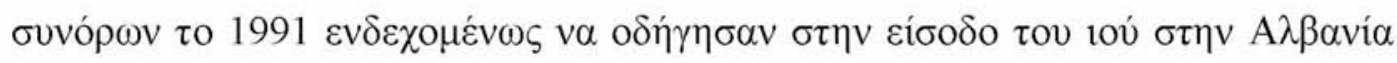

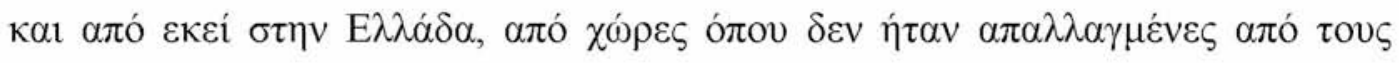

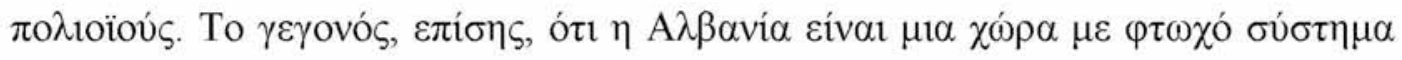

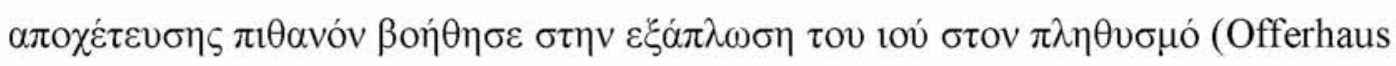
L., 1991).

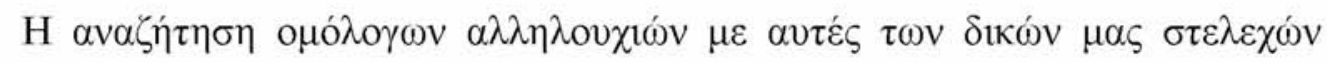

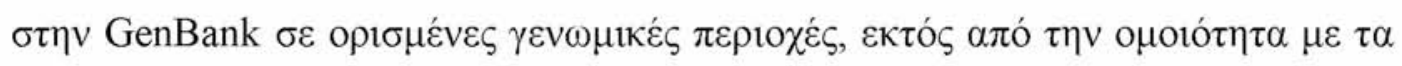

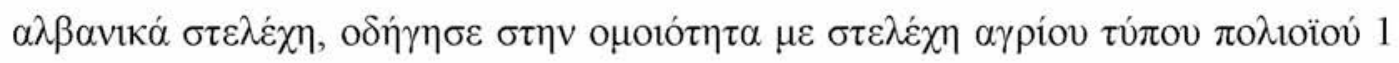

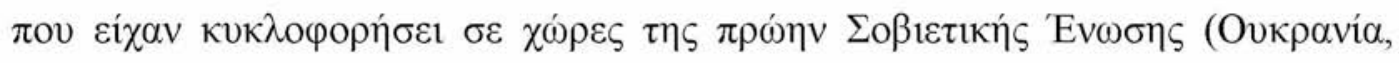

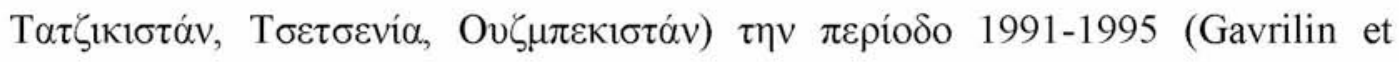

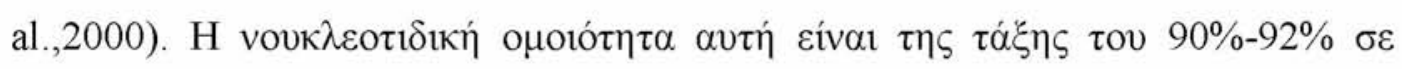

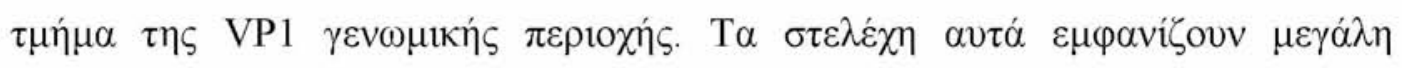

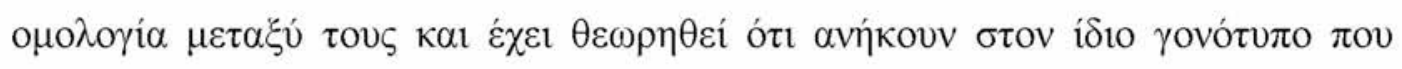

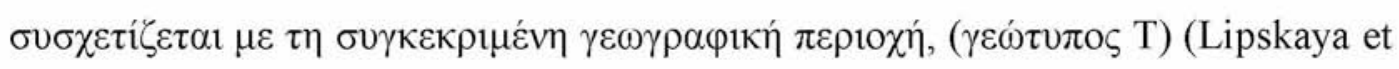

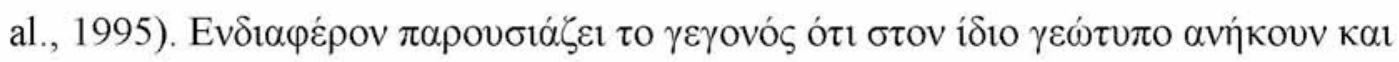




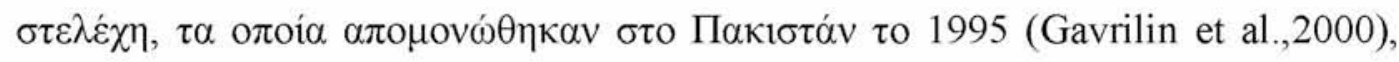

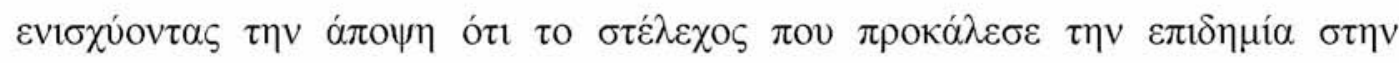

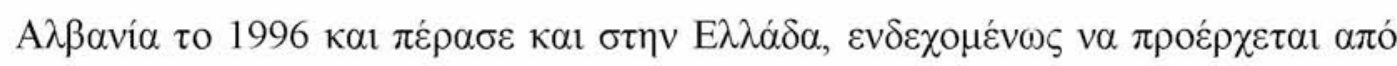

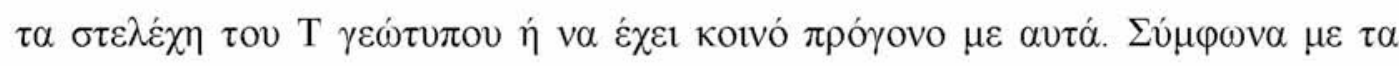
$\pi \alpha \rho \alpha \pi \alpha \dot{v} \omega, \delta \varepsilon v \alpha \pi \circ \kappa \lambda \varepsilon i \varepsilon \tau \alpha \iota \eta \eta$

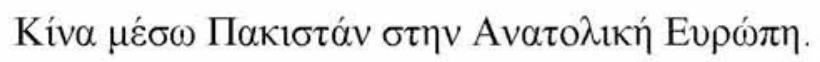

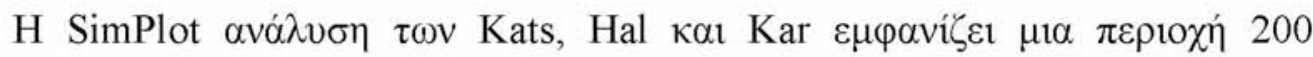

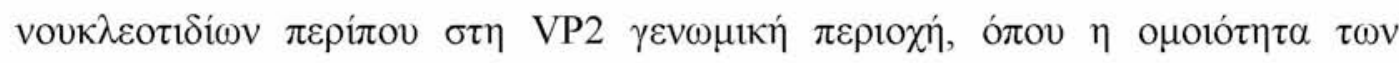

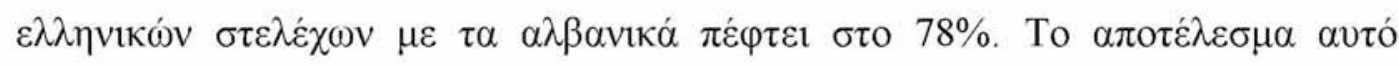

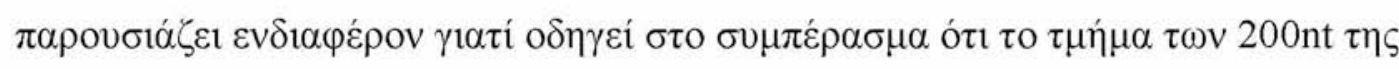

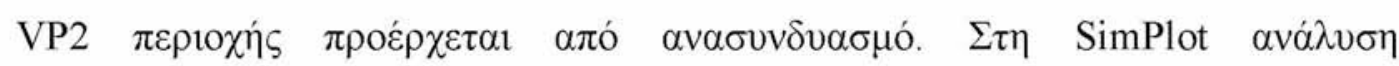

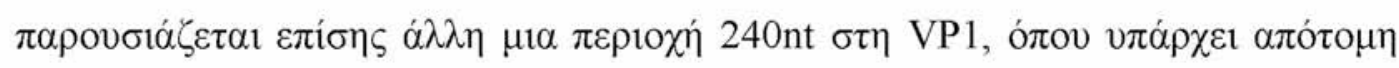

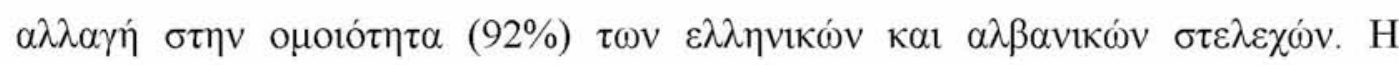

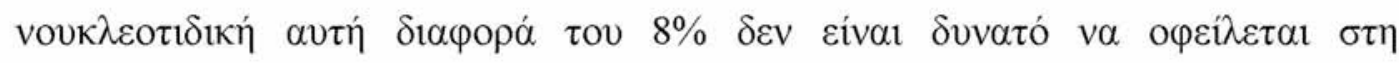

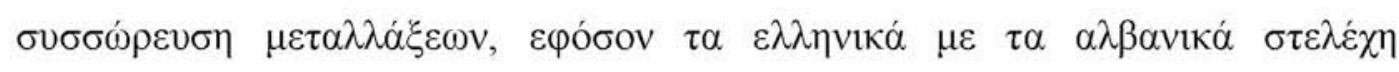

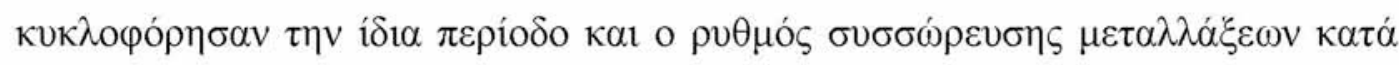

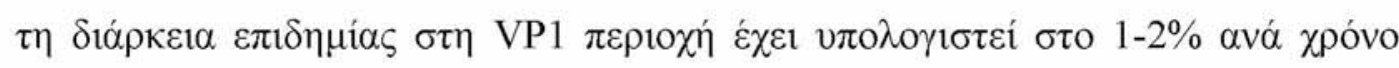

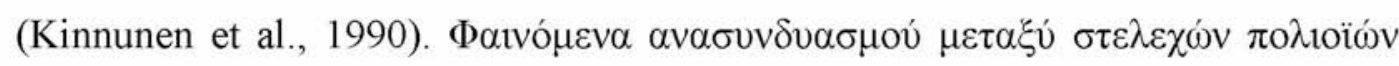

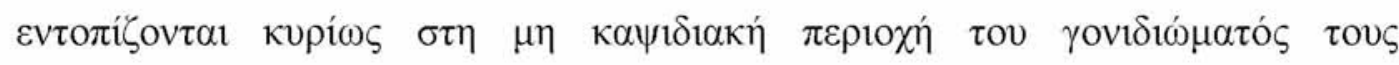
(Georgopoulou and Markoulatos , 2000; Cuervo et al., 2001; Karakasiliotis et al.,

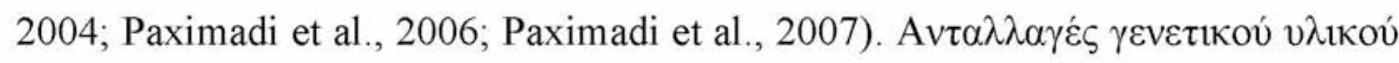

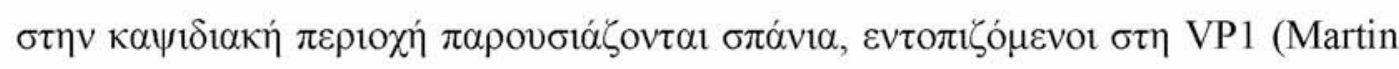

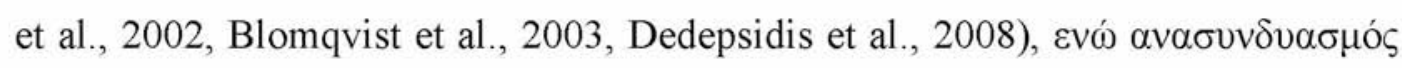

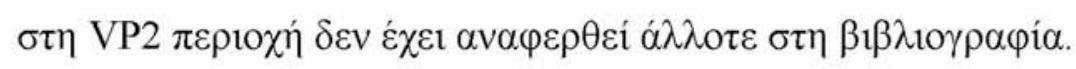

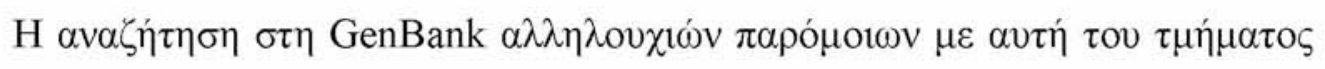

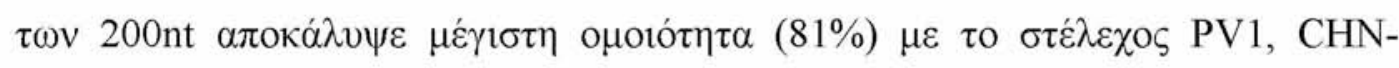

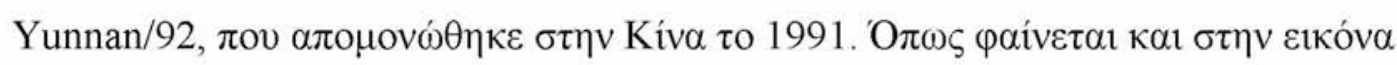

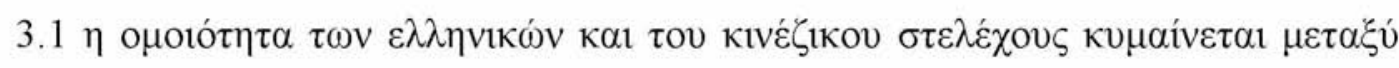

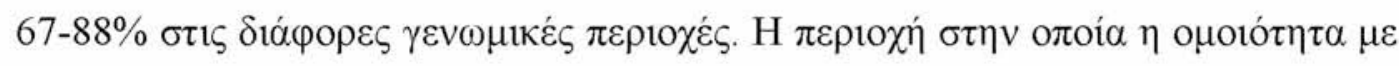

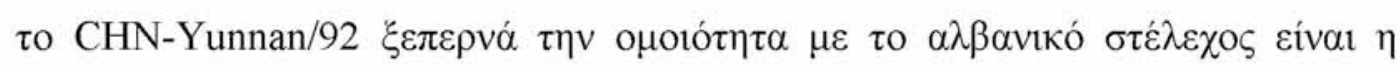

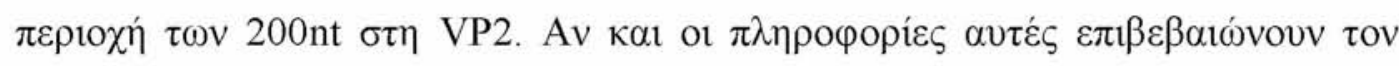

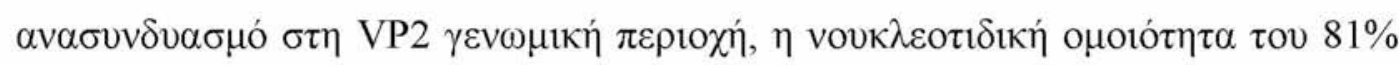

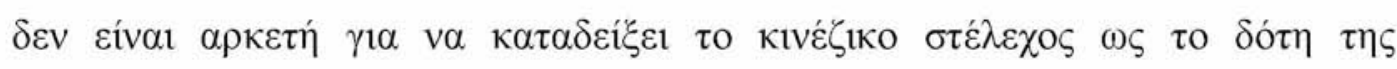




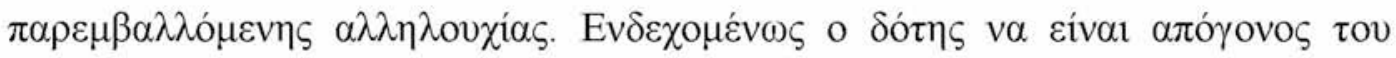

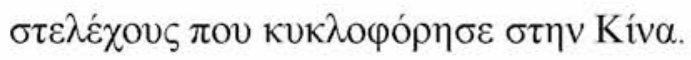

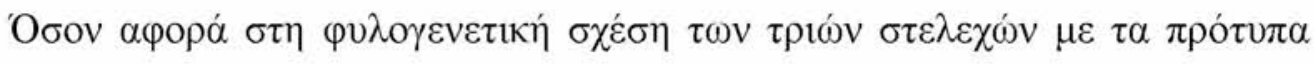

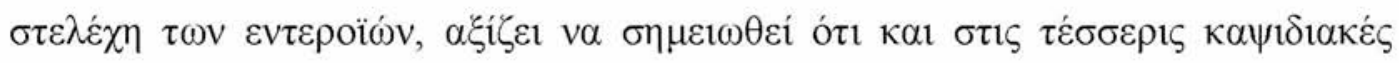

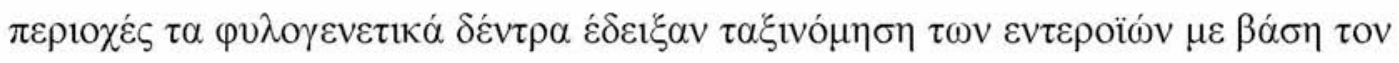

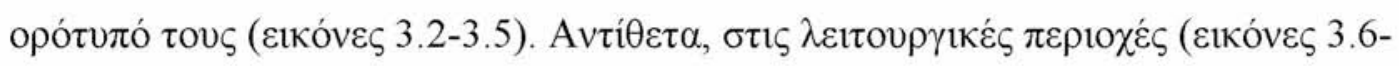

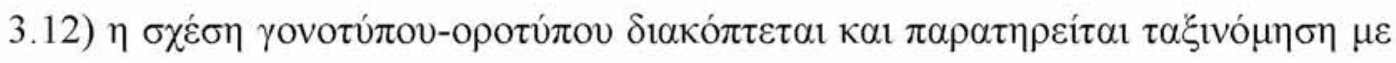

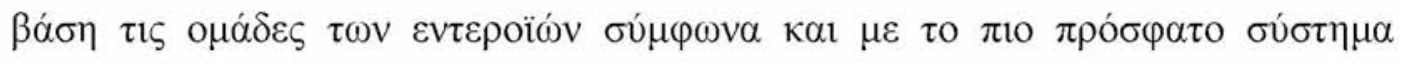

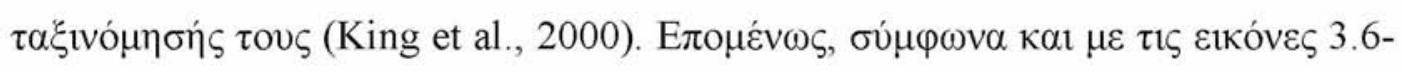

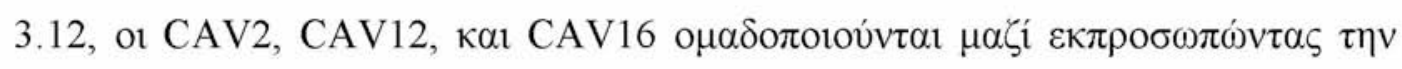

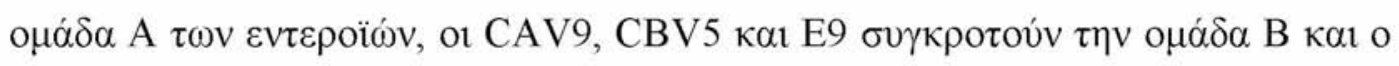

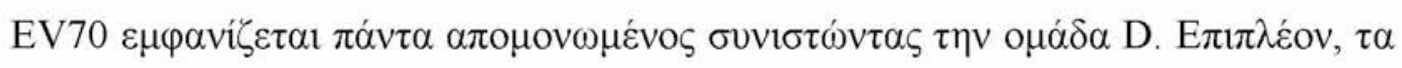

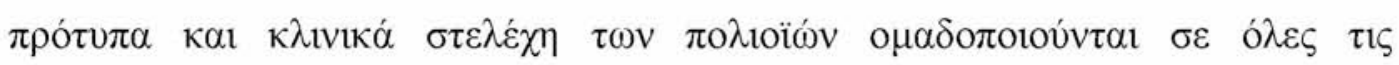

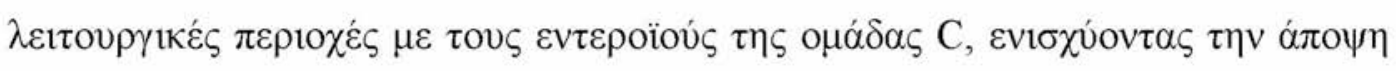

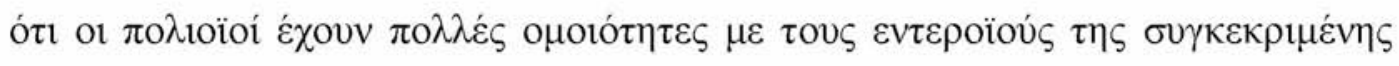

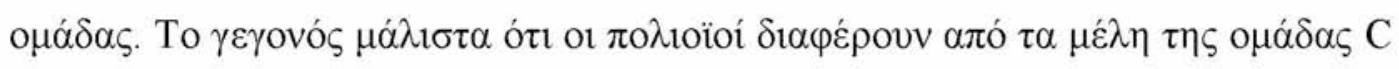

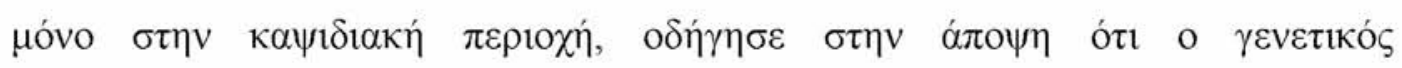

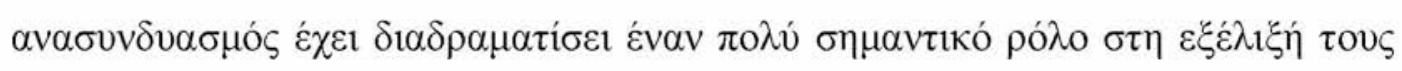

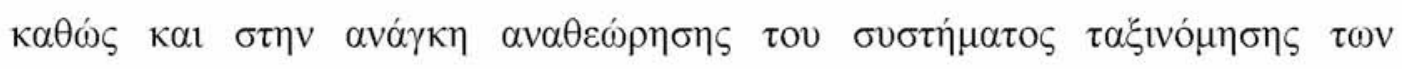

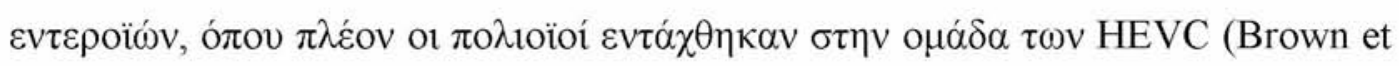
al., 2003).

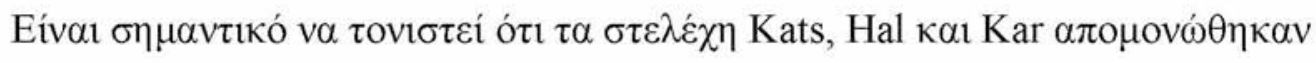

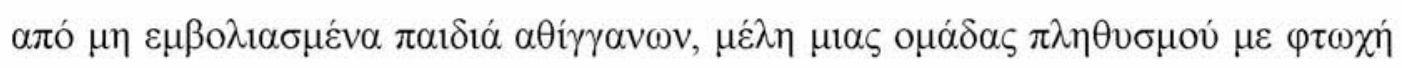

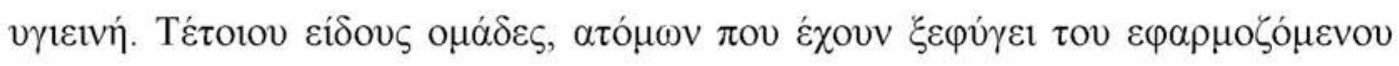

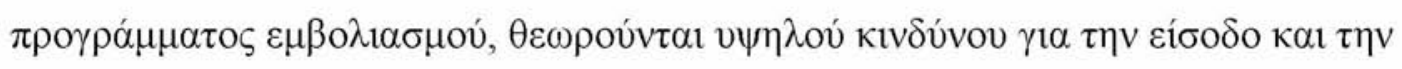

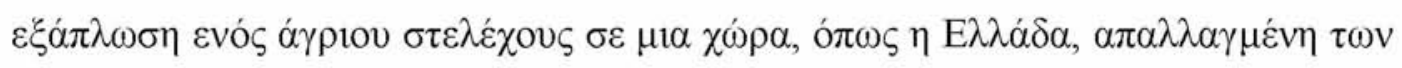

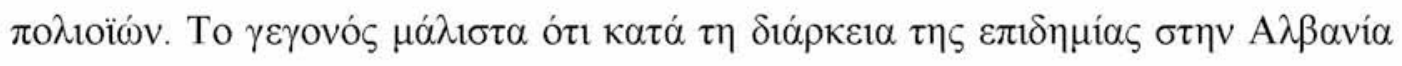

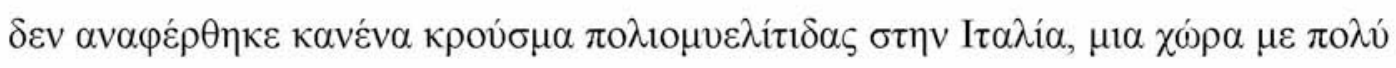

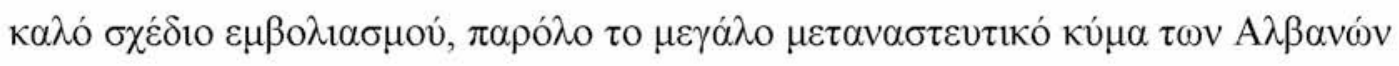

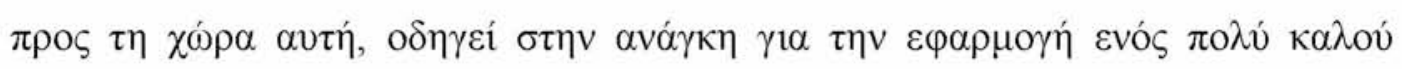

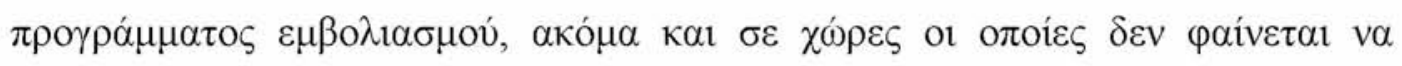

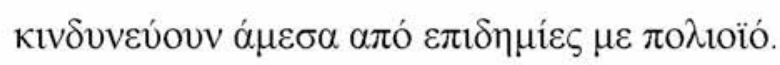

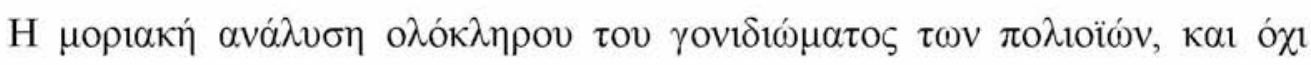

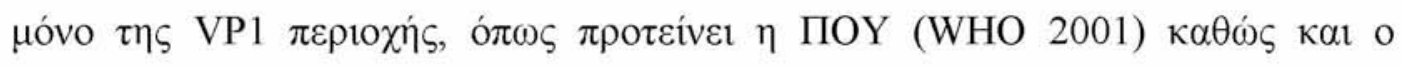




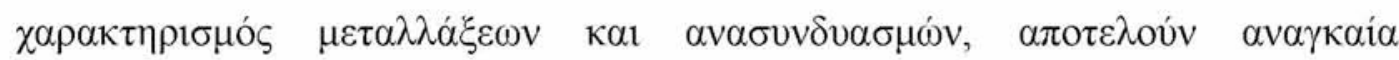

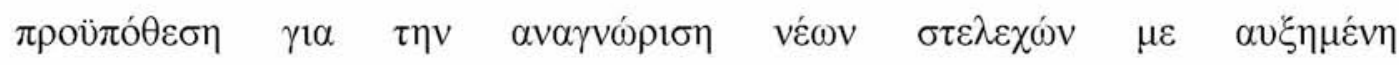

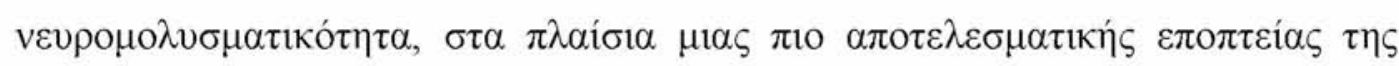

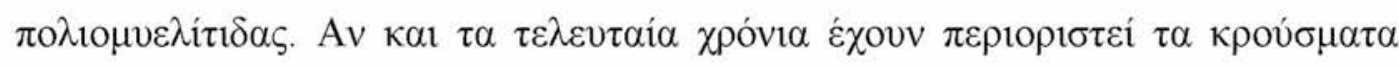

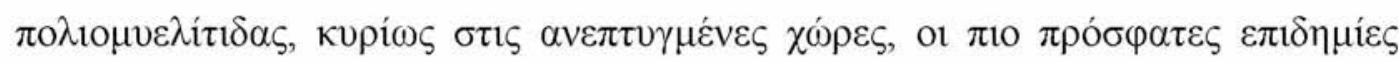

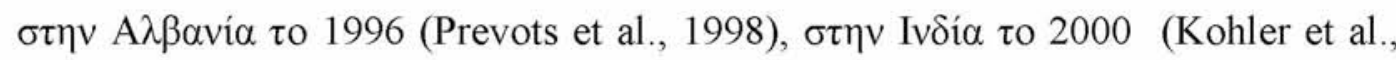

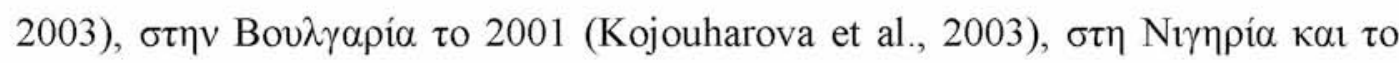

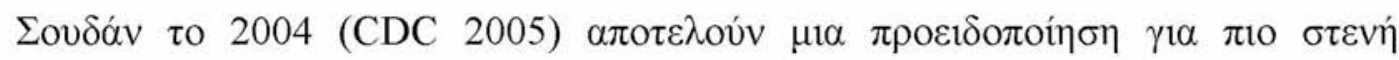

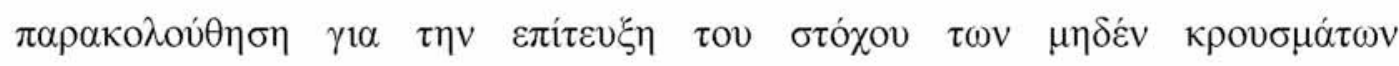

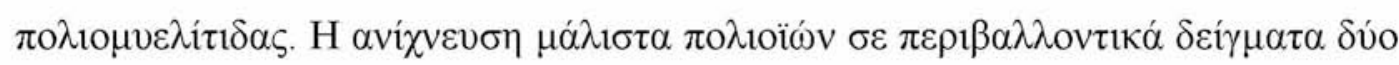

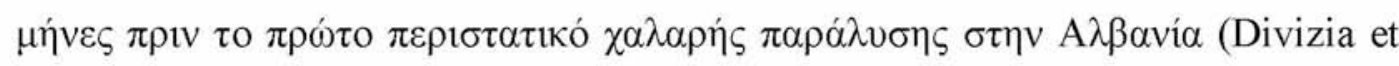

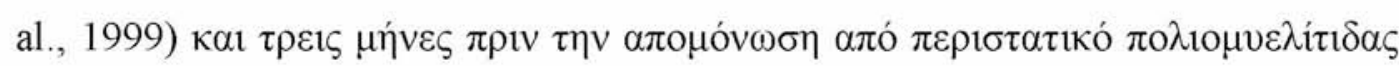

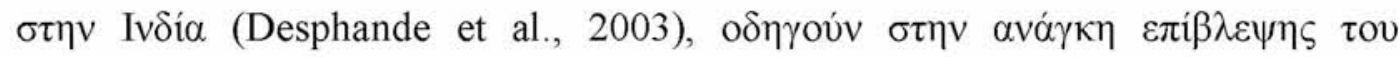

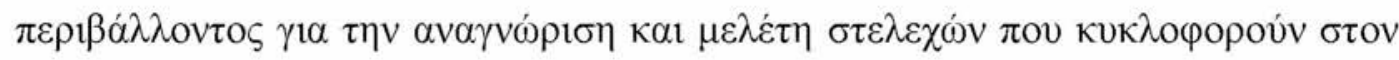
$\pi \lambda \eta \theta v \sigma \mu$ ó.

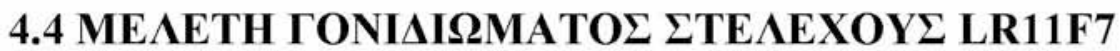

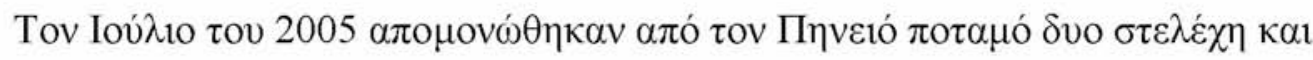

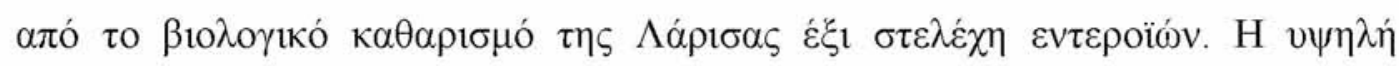

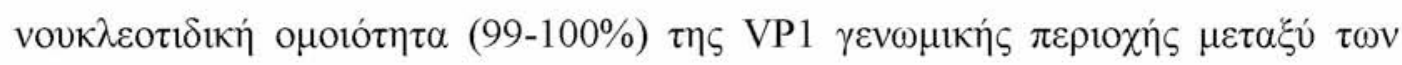

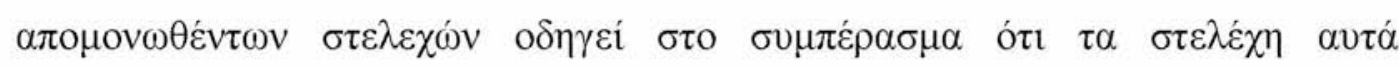

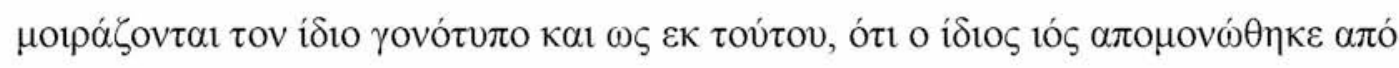

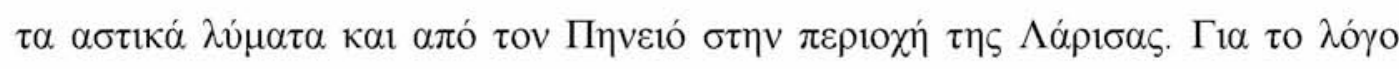

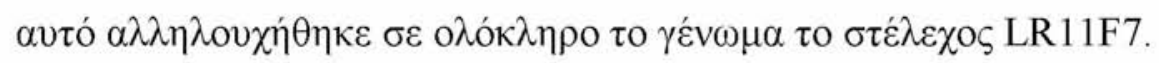

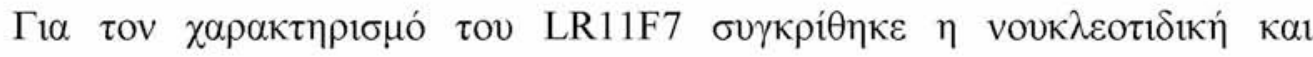

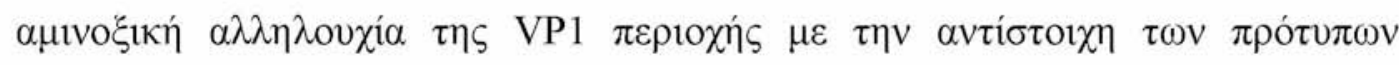

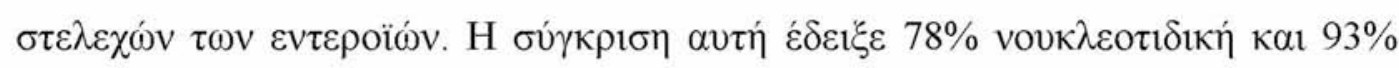

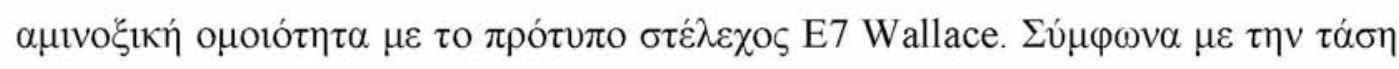

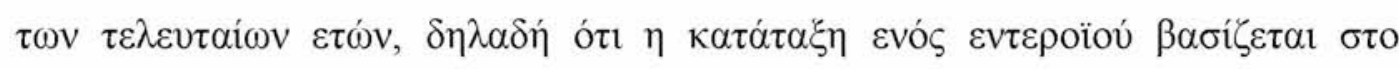

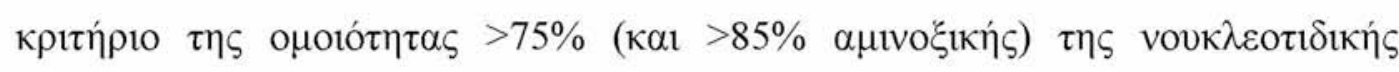

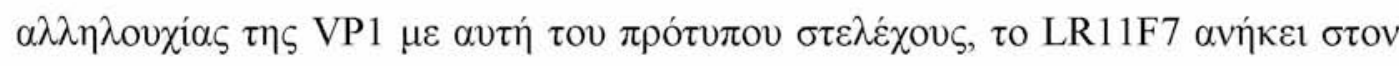

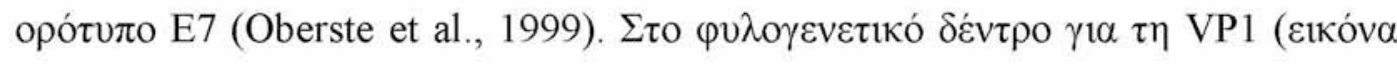

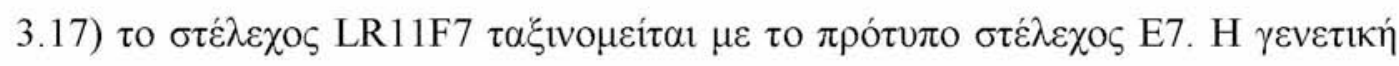




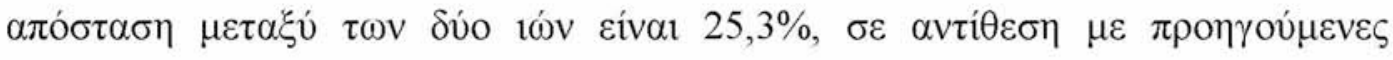

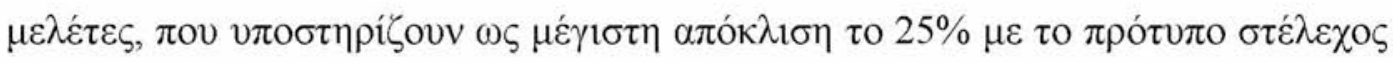

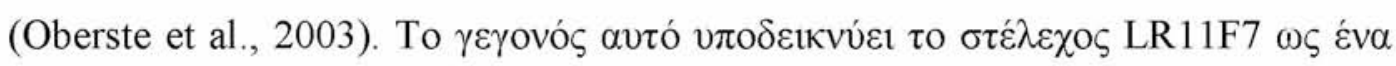

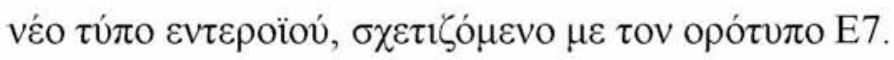

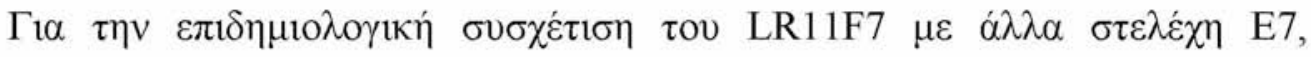

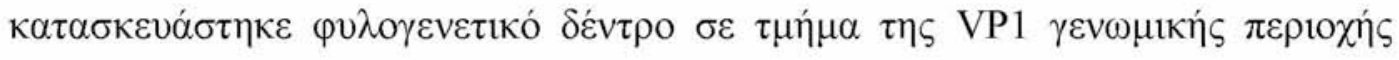

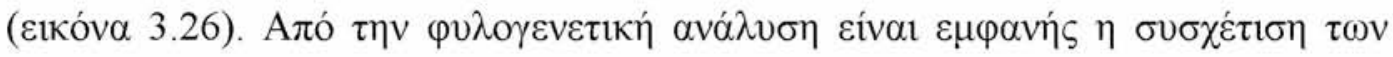

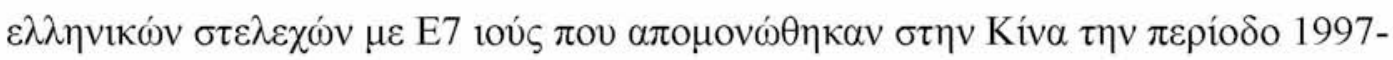

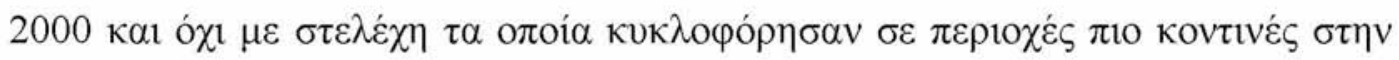

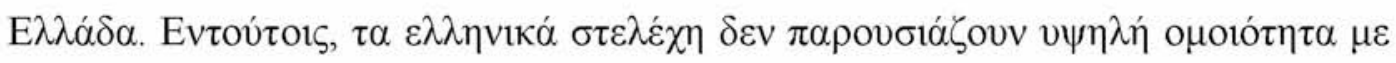

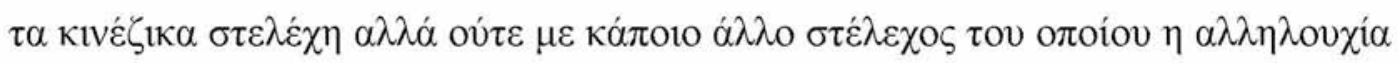

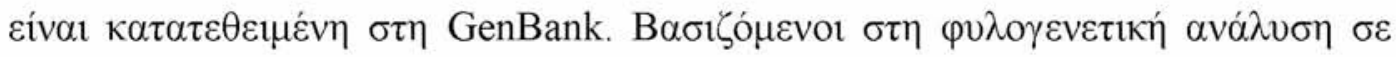

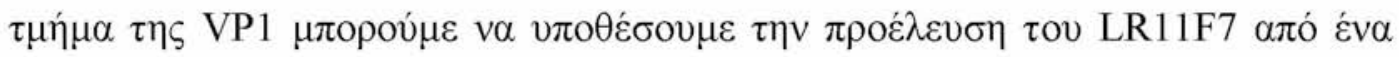

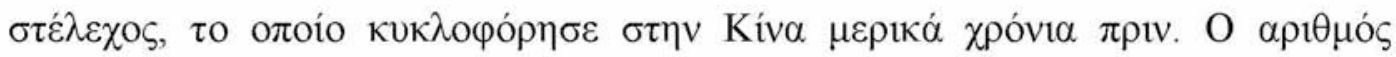

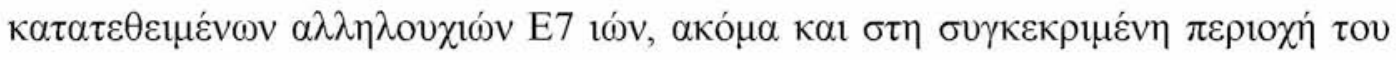

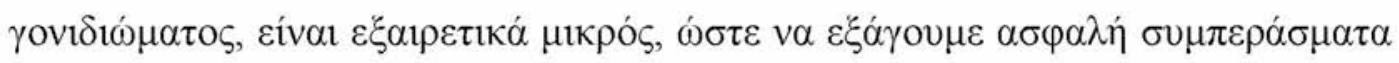

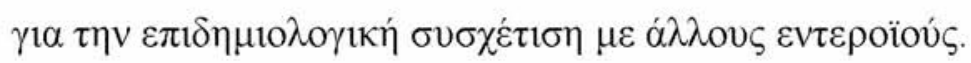

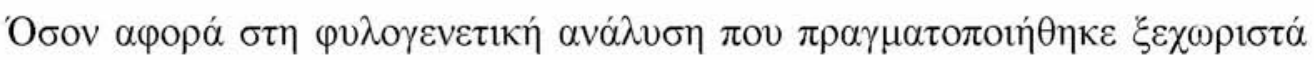

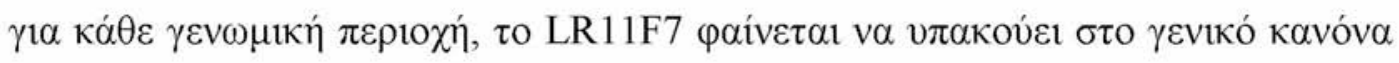

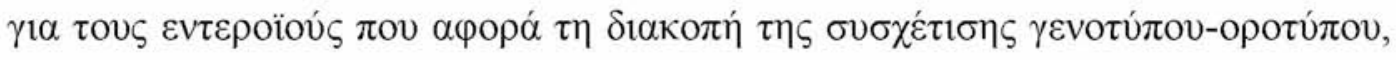

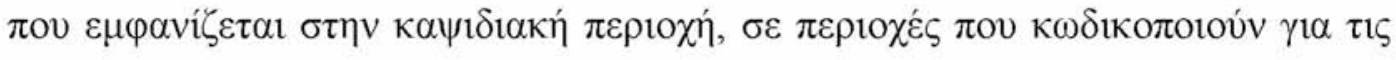

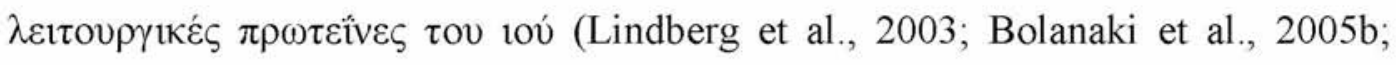

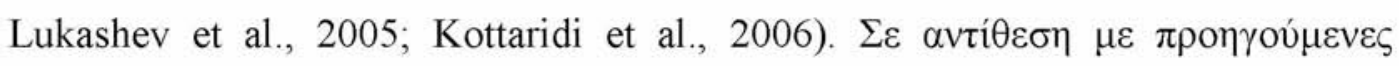

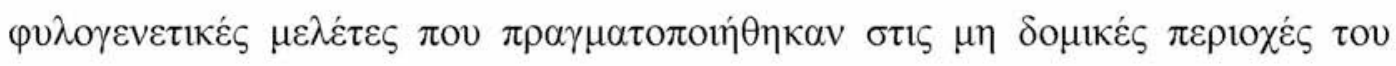

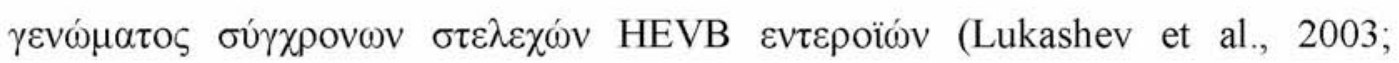
Lukashev et al., 2005; Bolanaki et al., 2007; Kottaridi et al., 2007), к

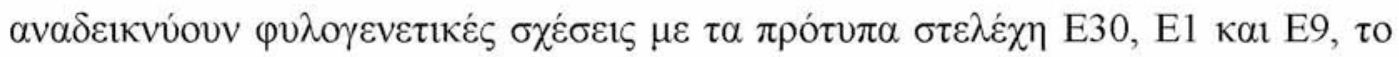

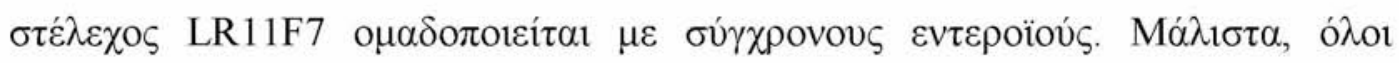

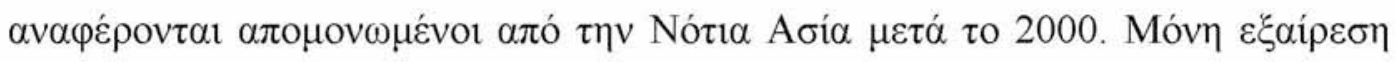

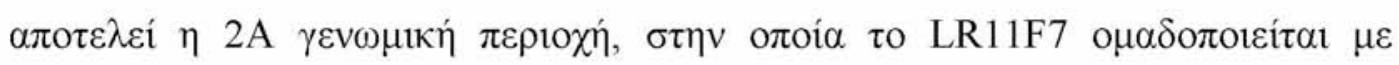

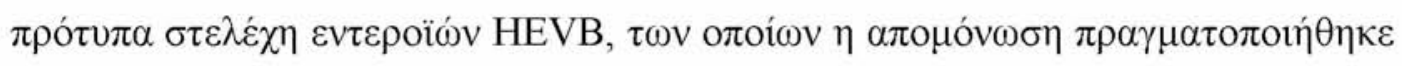

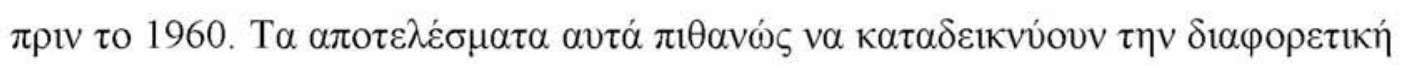

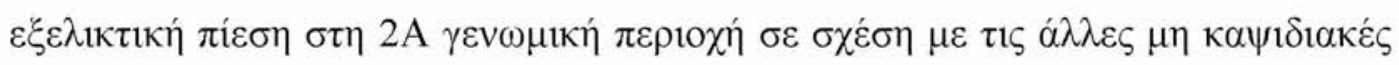

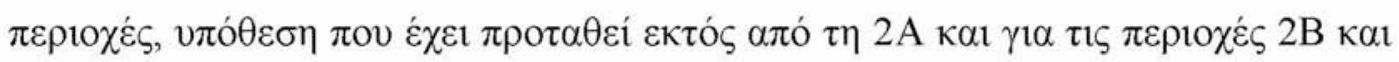




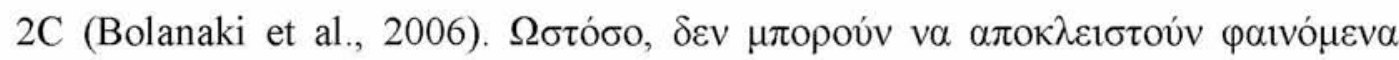

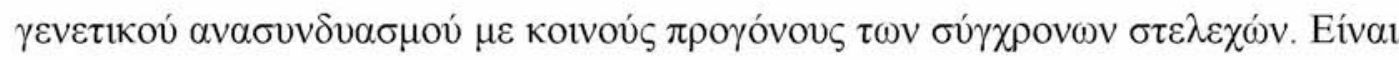

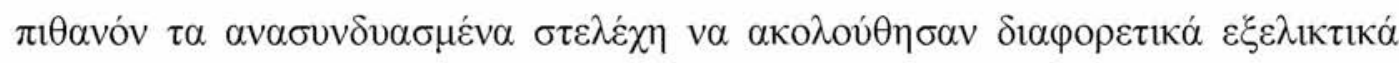

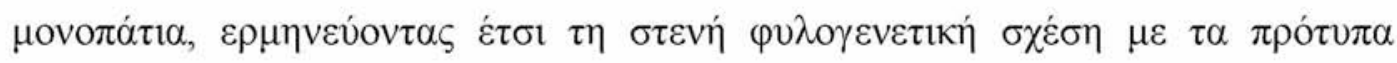

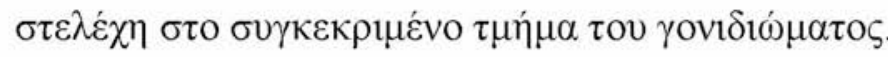

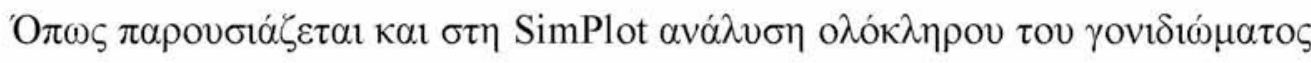

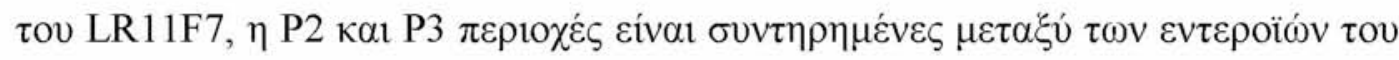

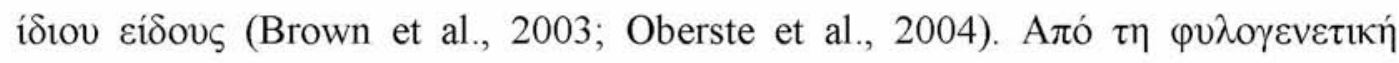

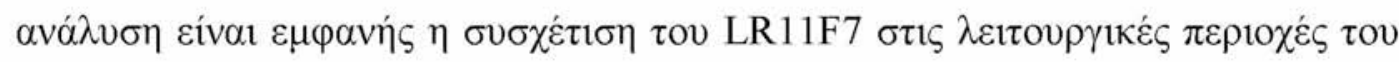

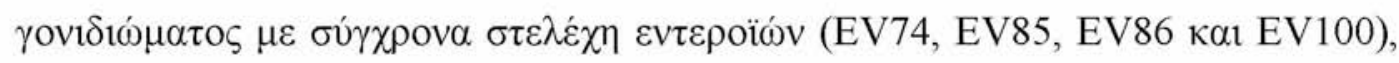

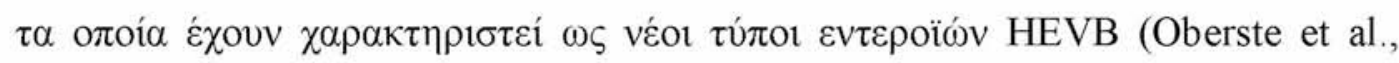

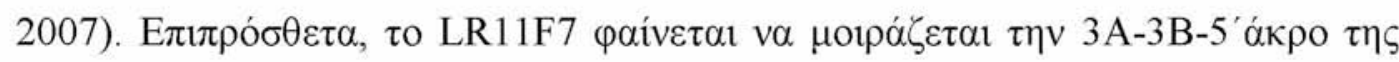

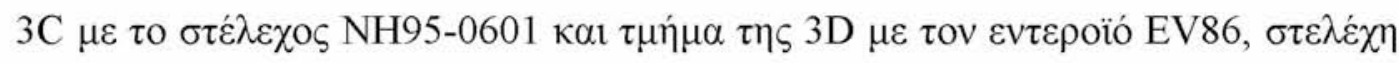

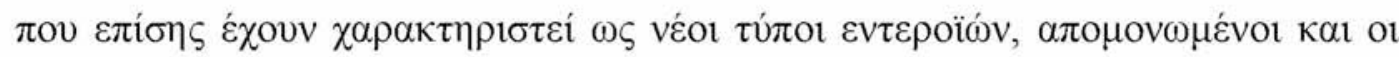

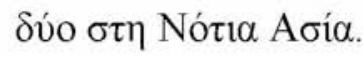

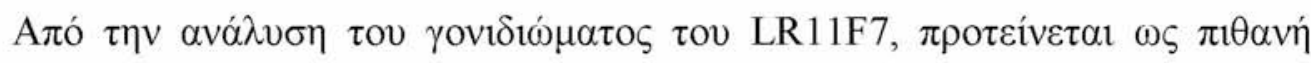

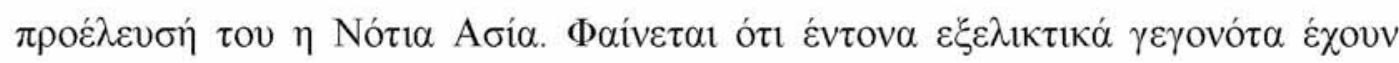

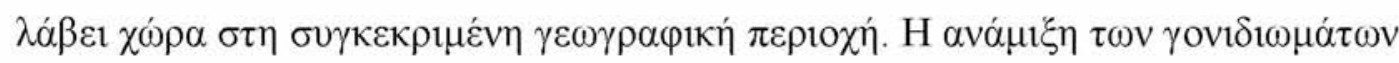

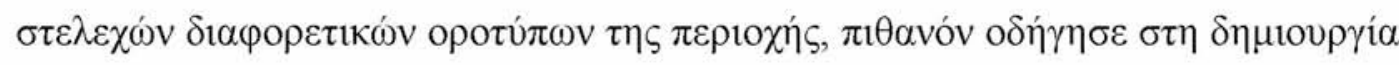

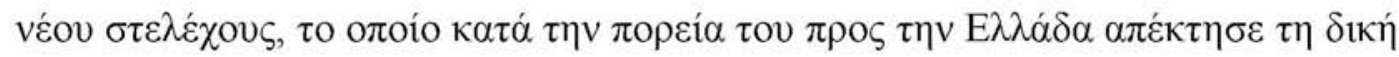

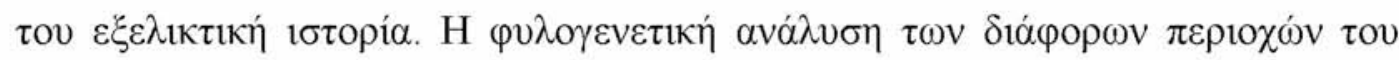

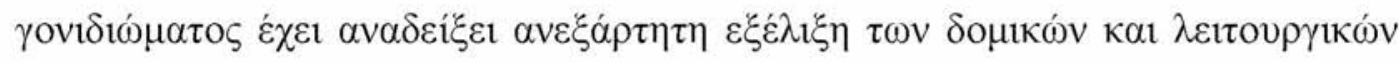

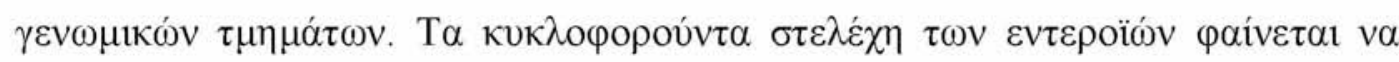

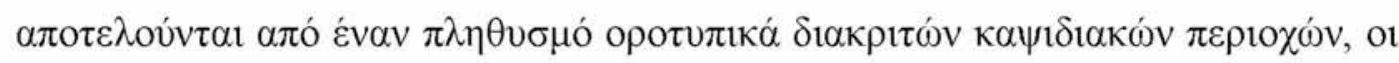

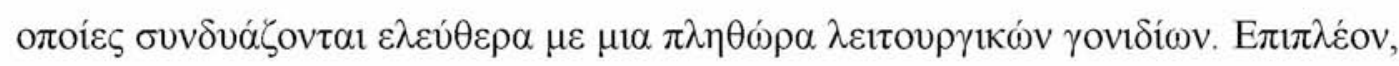

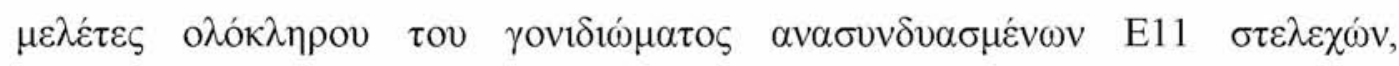

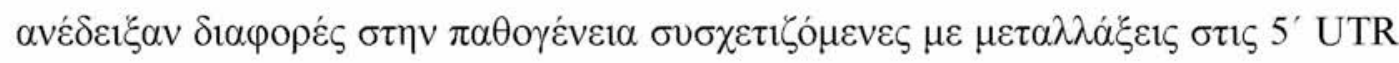

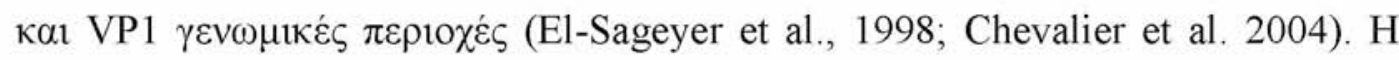

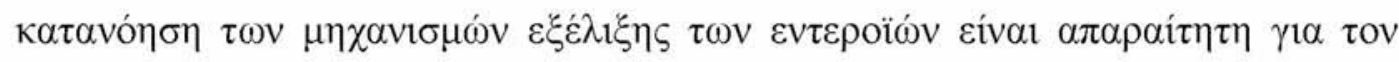

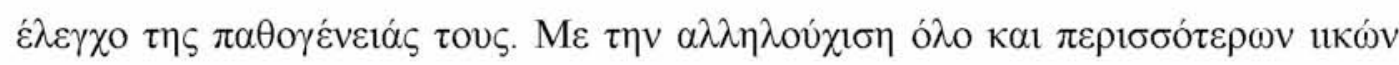

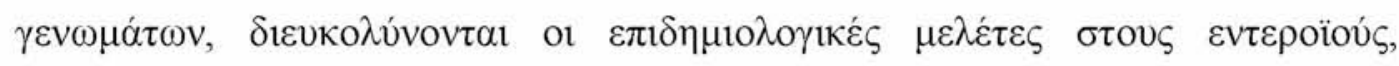

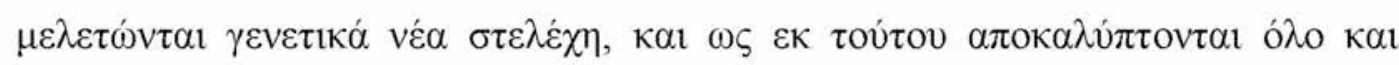

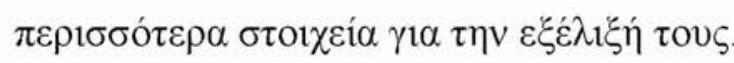




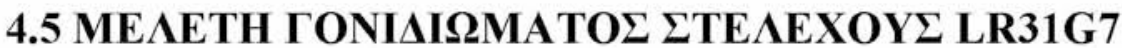

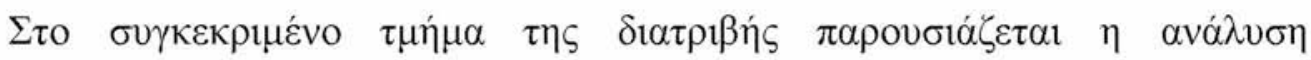

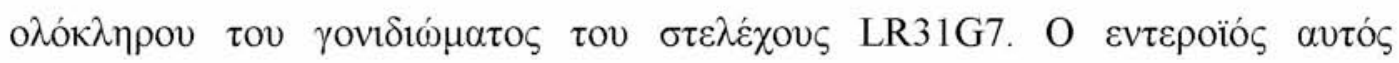
$\alpha \pi$ о

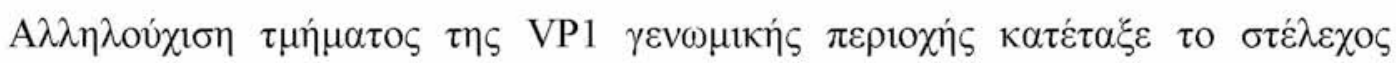

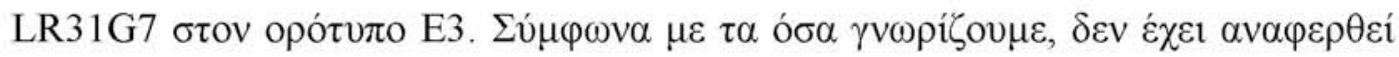

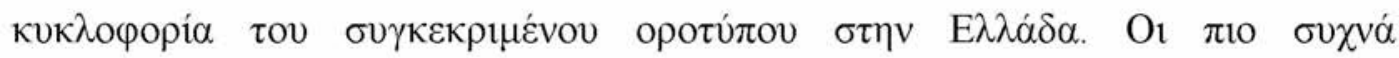

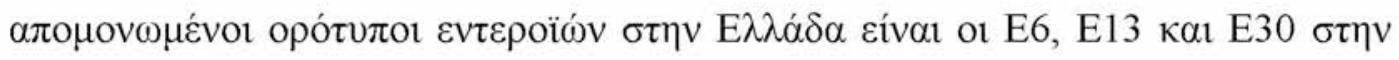

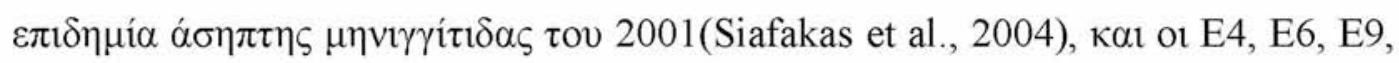

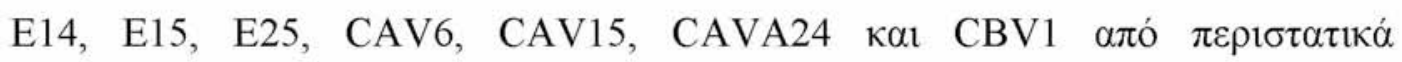

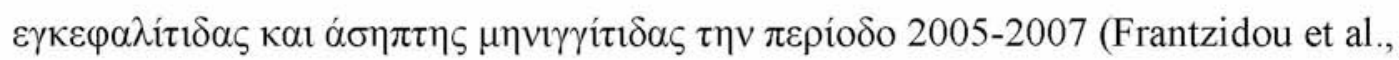
2007; Papa et al., 2009; Logotheti et al., 2009).

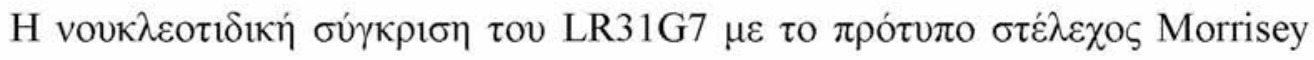

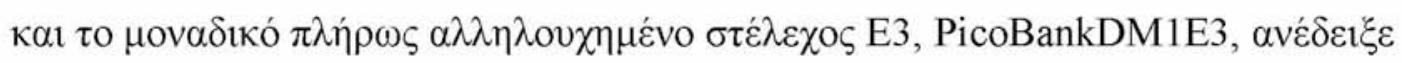

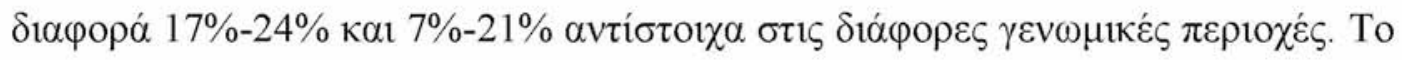

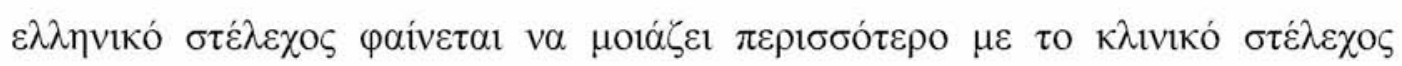

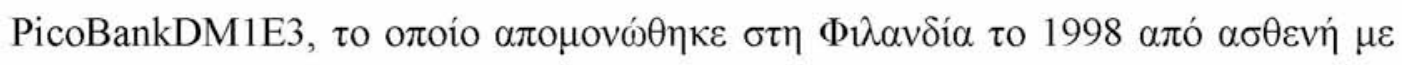

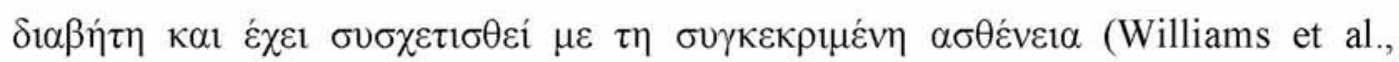

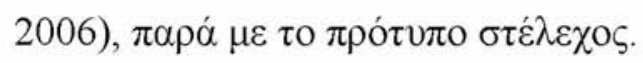

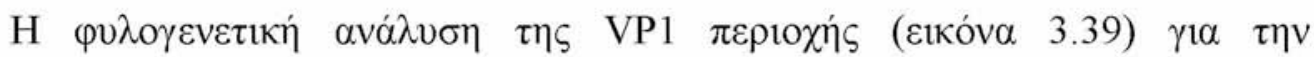

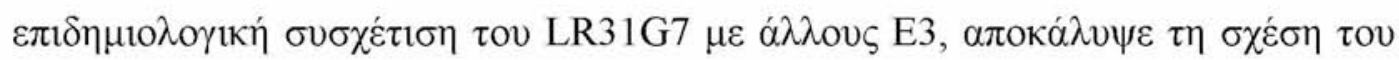

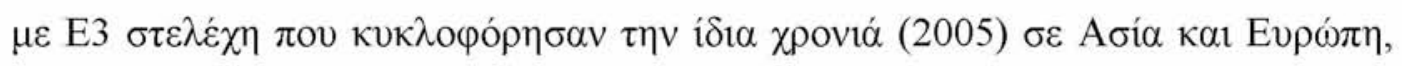

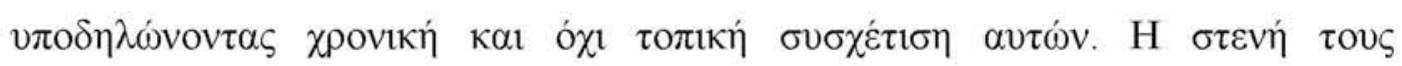

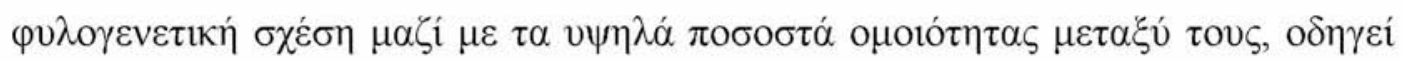

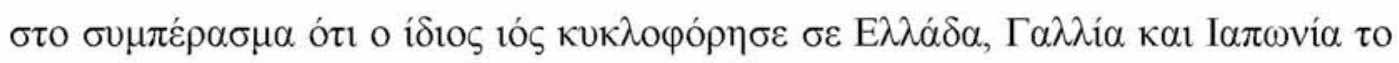

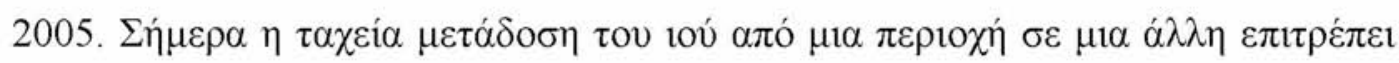

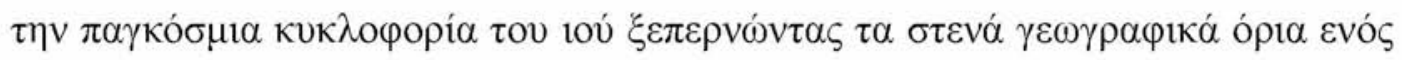

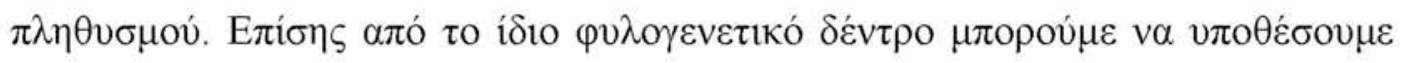

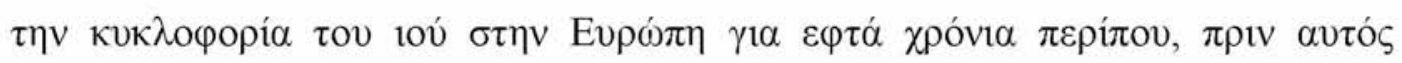

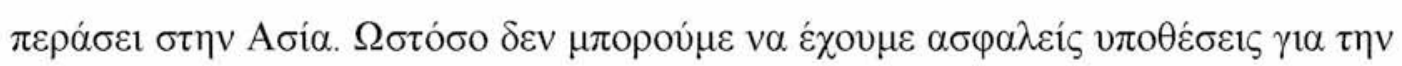

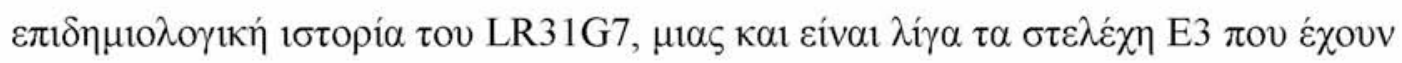

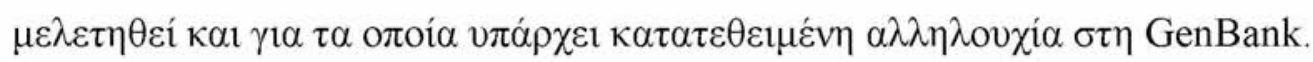




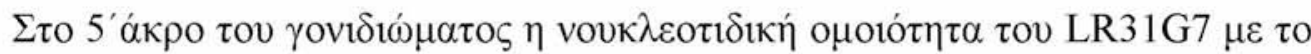

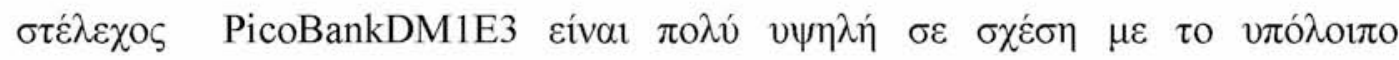

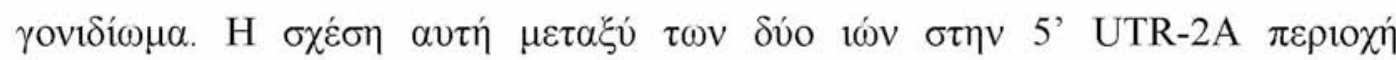

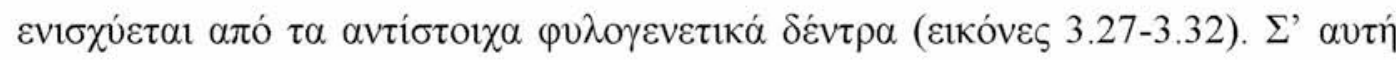

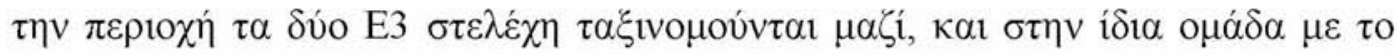

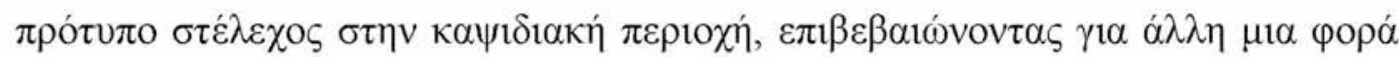

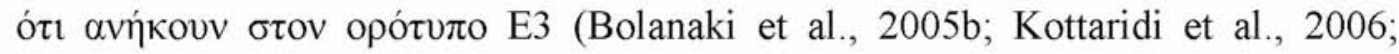

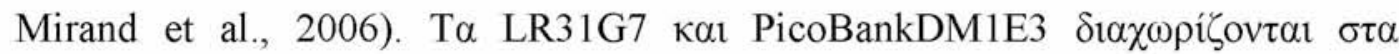

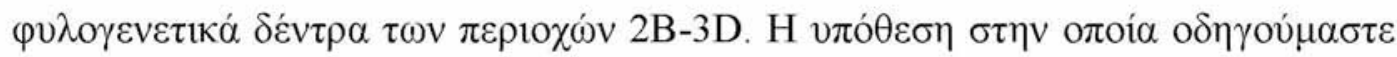

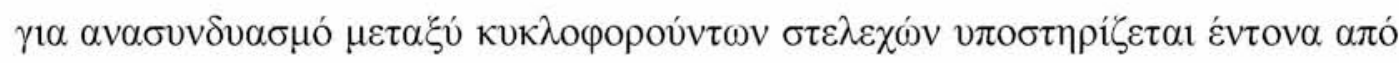

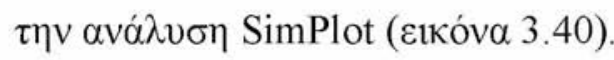

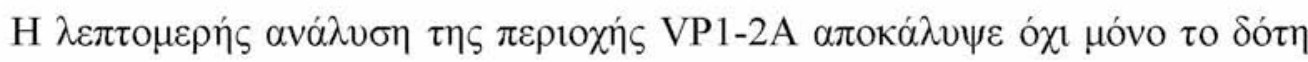

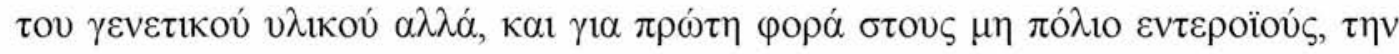

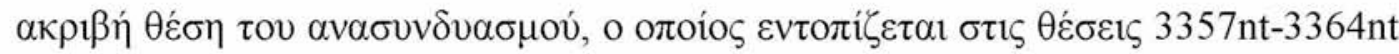

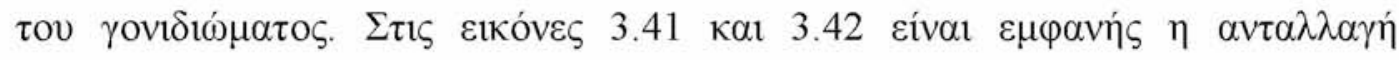

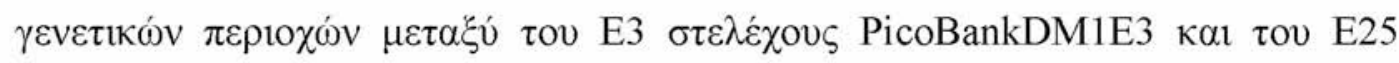

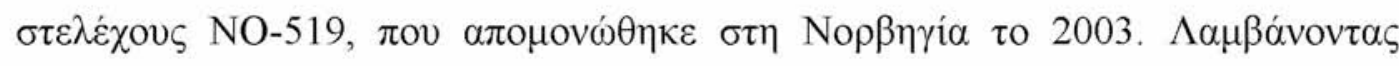

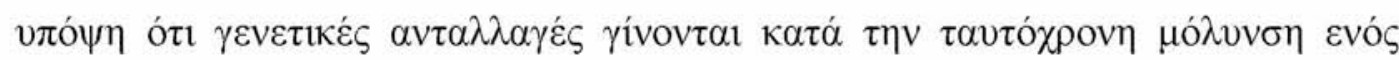

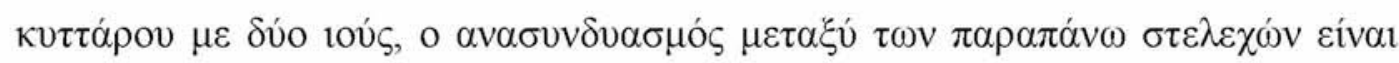

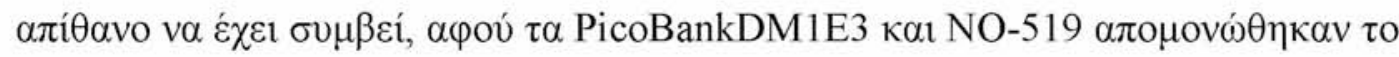

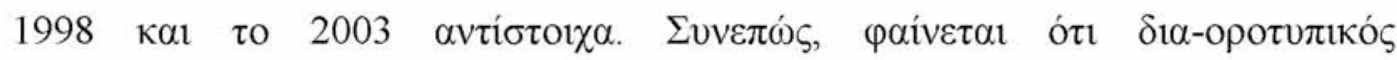

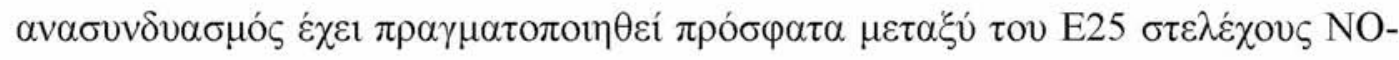

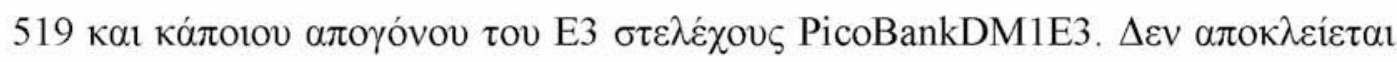

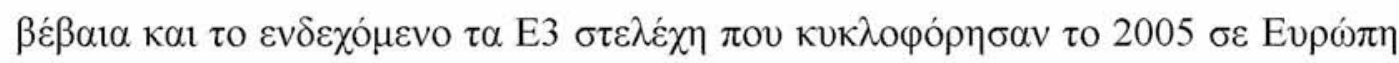

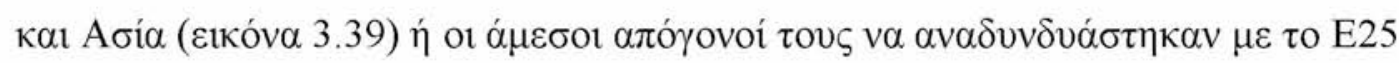

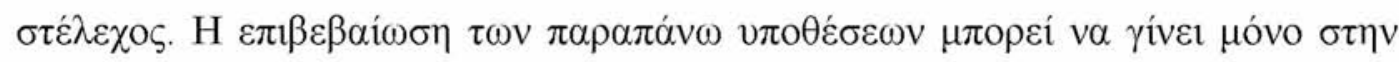

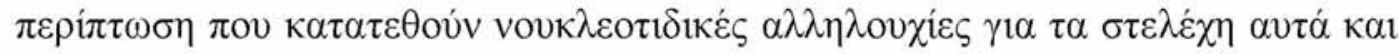
$\sigma \varepsilon \dot{\alpha} \lambda \lambda \varepsilon \zeta \gamma \varepsilon v \omega \mu 1 \kappa \varepsilon \dot{\varepsilon} \pi \varepsilon \rho 10 \chi \varepsilon \dot{\varepsilon}$.

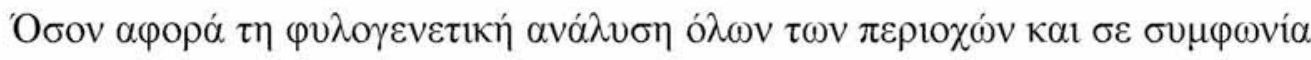
$\mu \varepsilon \pi \rho \circ \gamma$

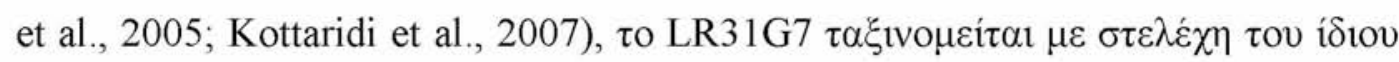

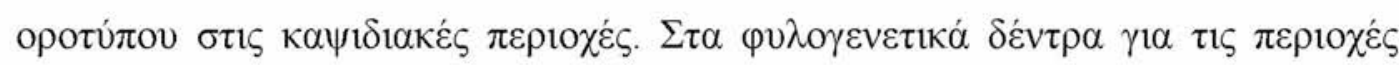

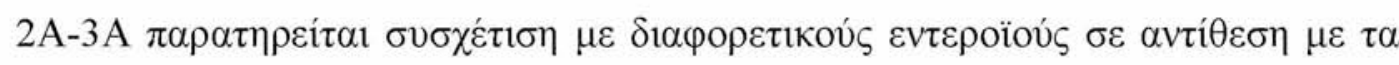

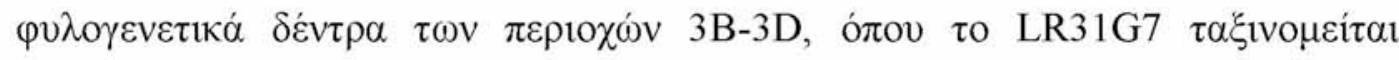




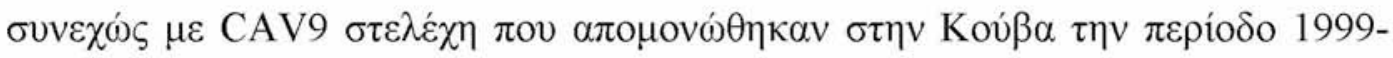

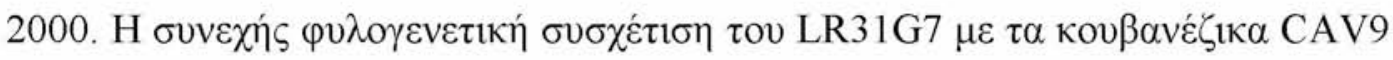

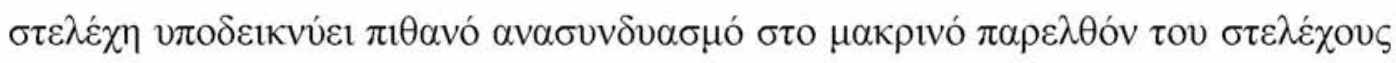
$\mu \alpha \varsigma$.

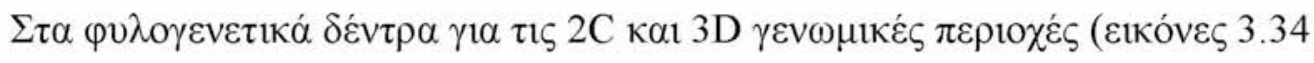

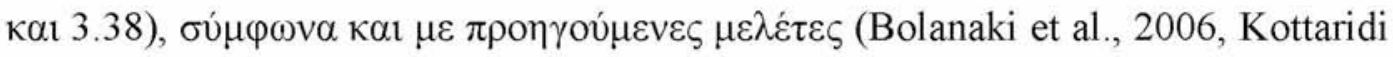

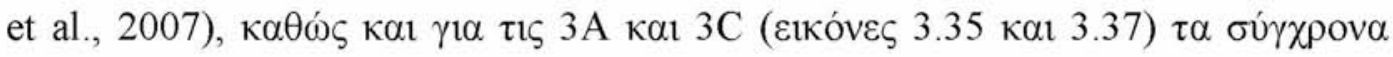

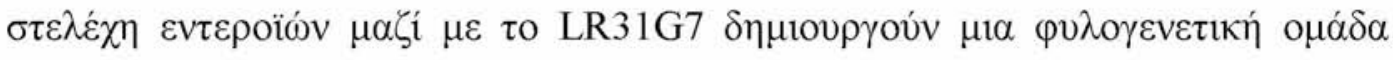

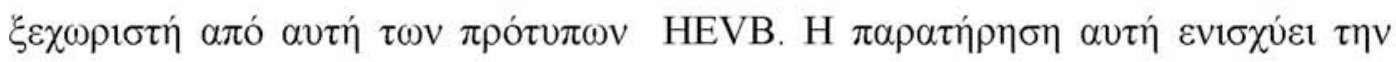

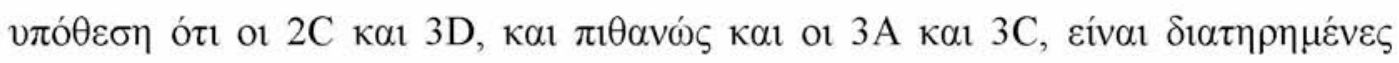

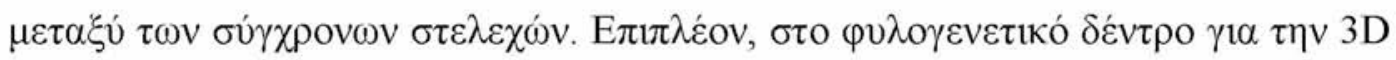

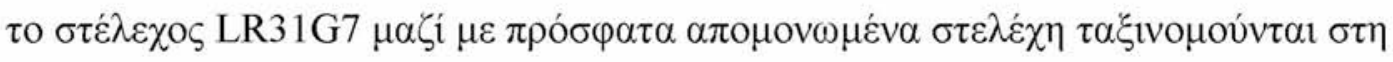

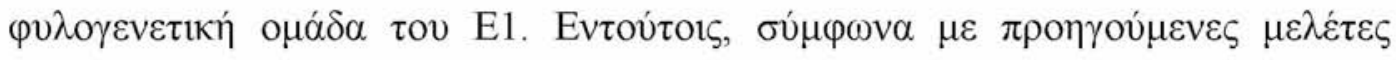

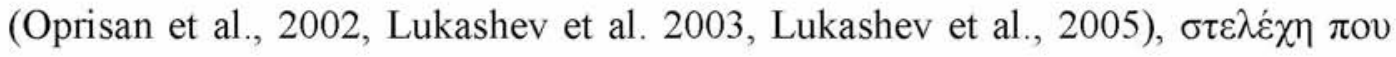

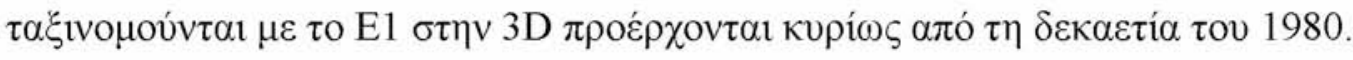

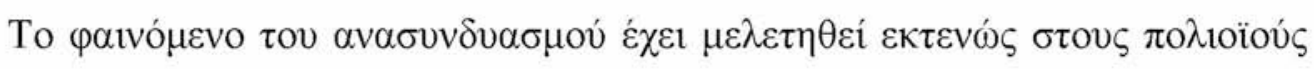
(Georgopoulou and Markoulatos, 2001; Dahourou et al., 2002; Guillot et al., 2000;

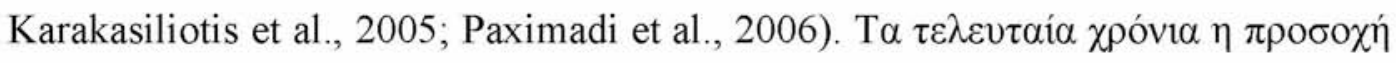

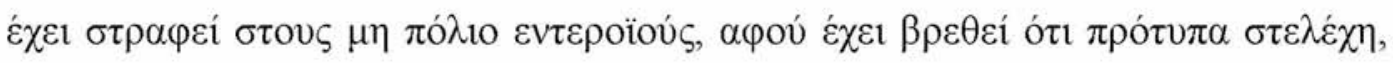

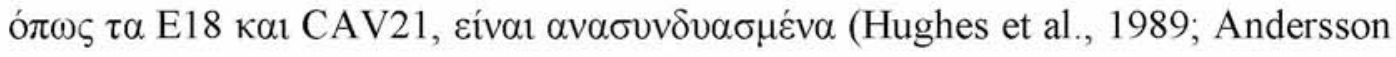

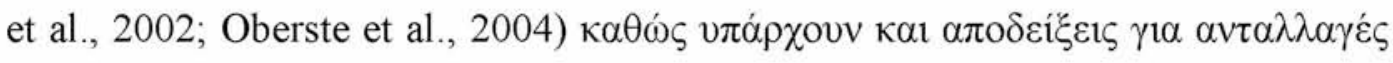

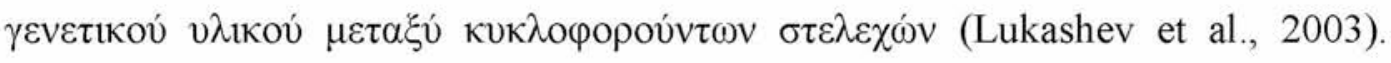

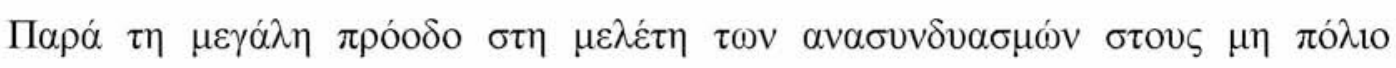

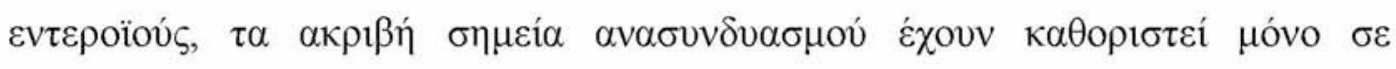

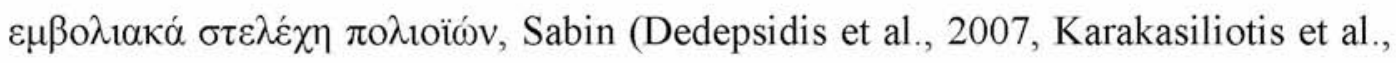
2004).

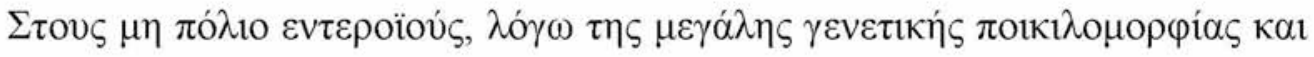

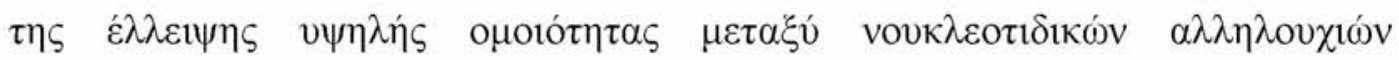

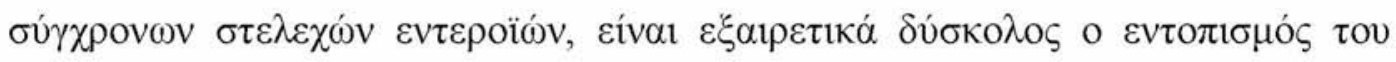

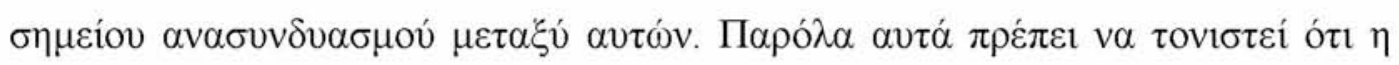

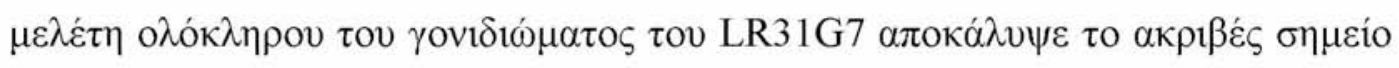

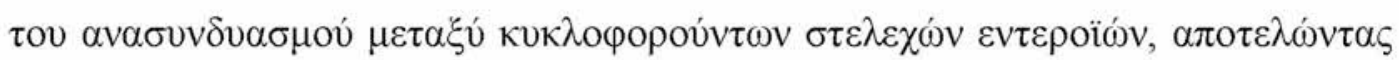

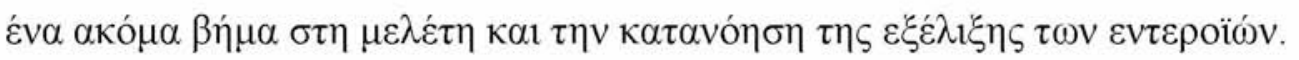




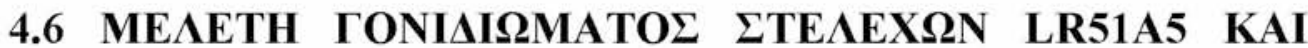 LR61G3}

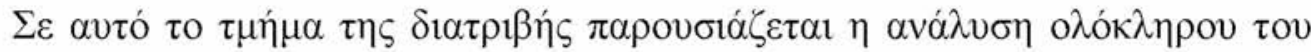

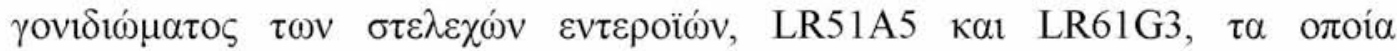

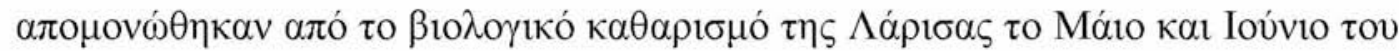

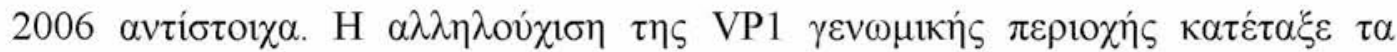

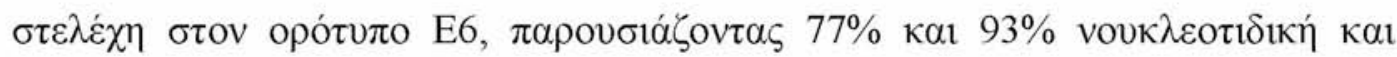

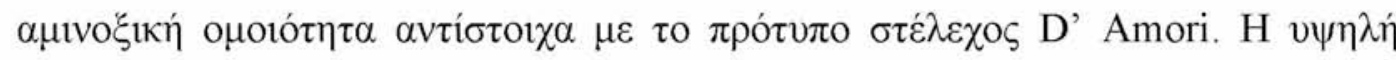

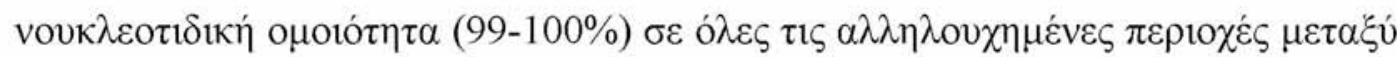

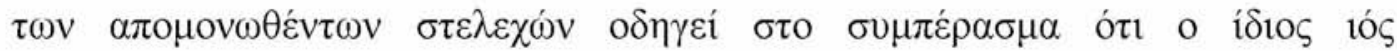

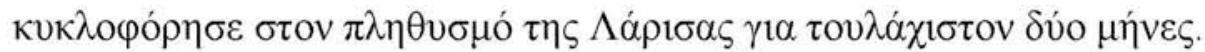

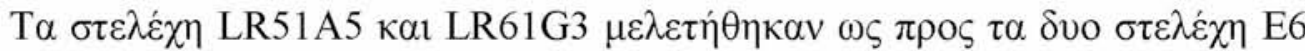

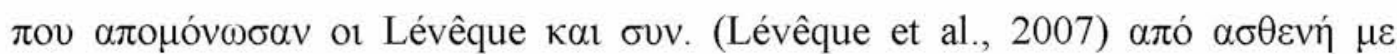

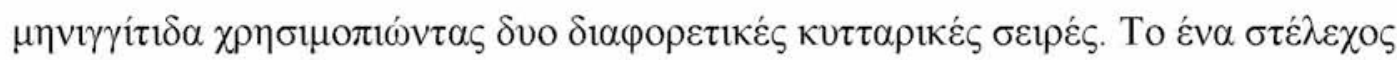

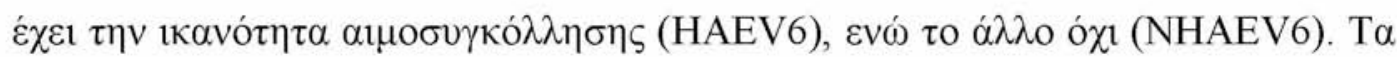

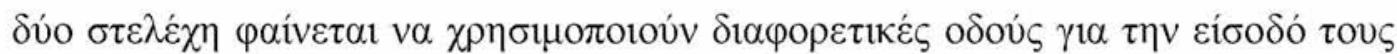

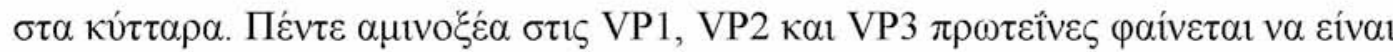

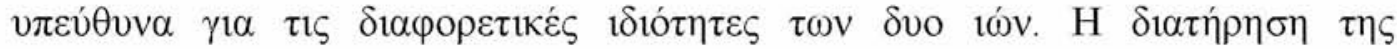

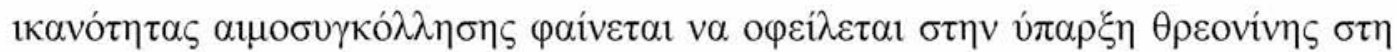

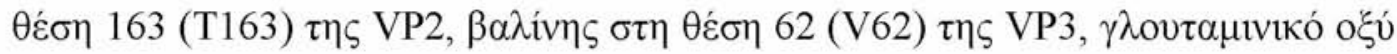

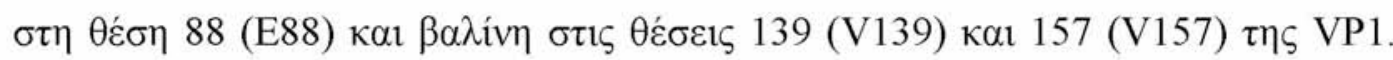

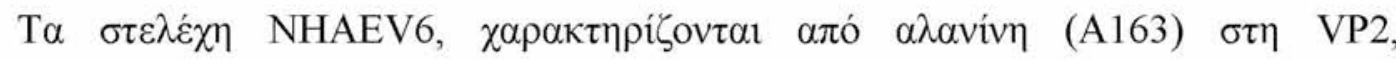

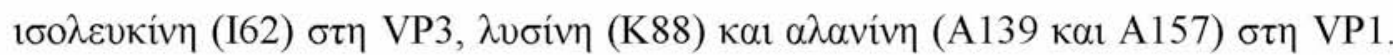

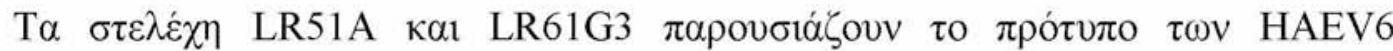

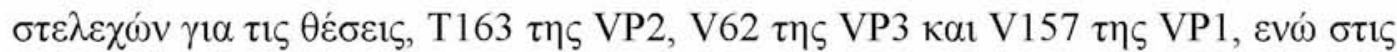

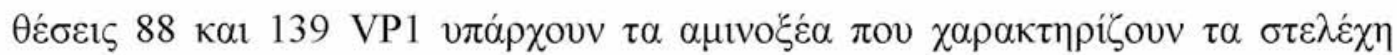

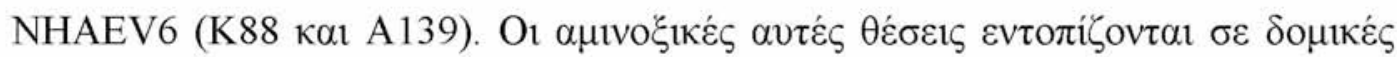

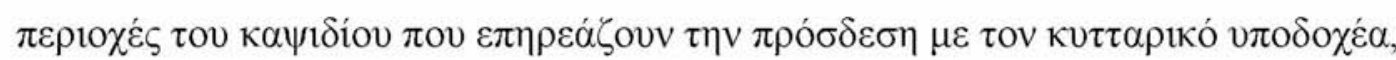

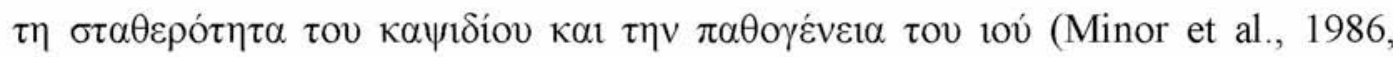

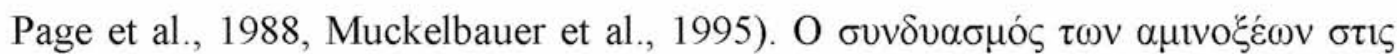

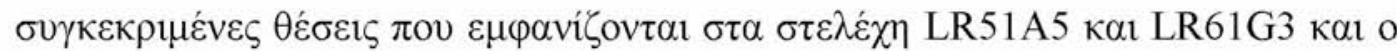

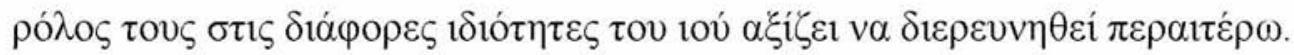




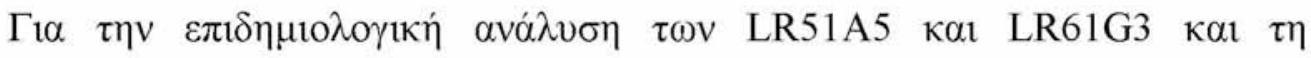

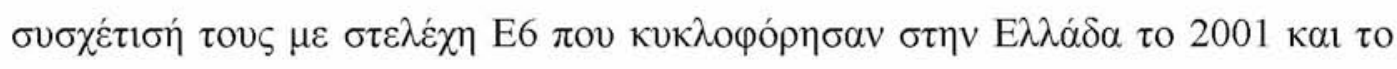

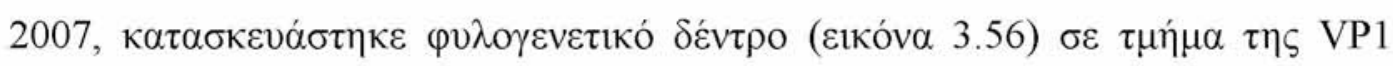

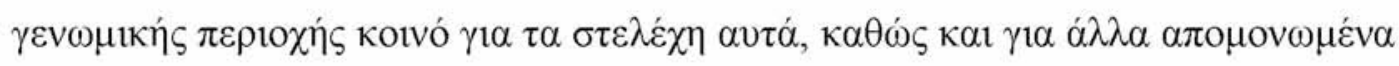

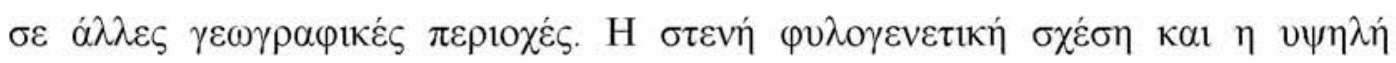

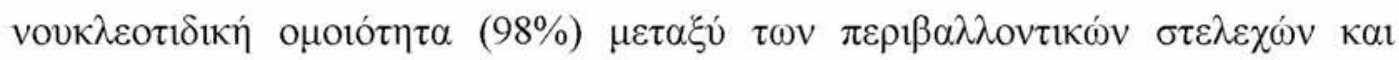

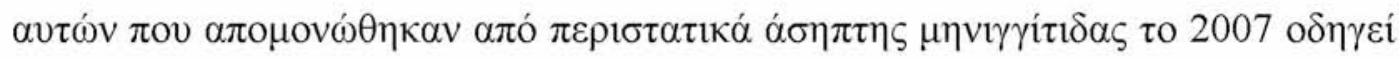

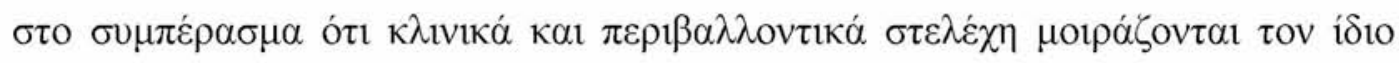

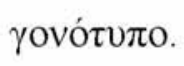

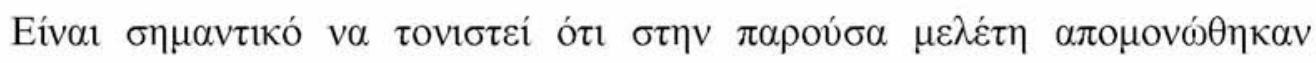

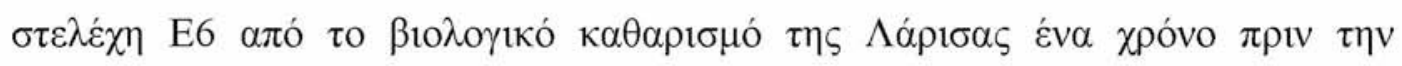

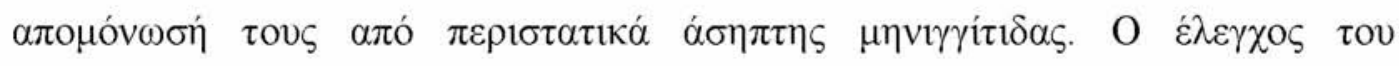

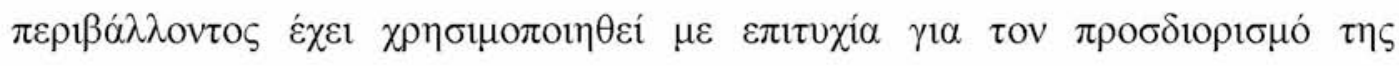

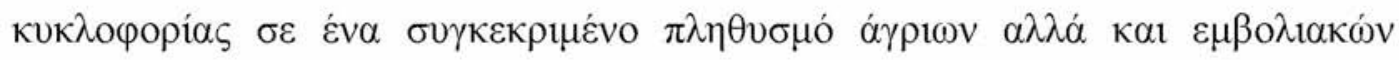

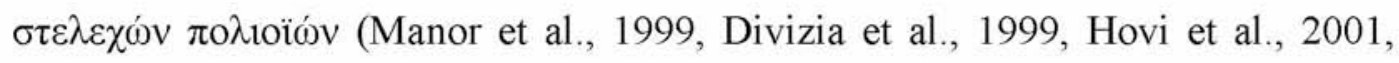

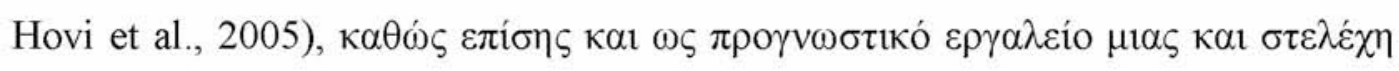

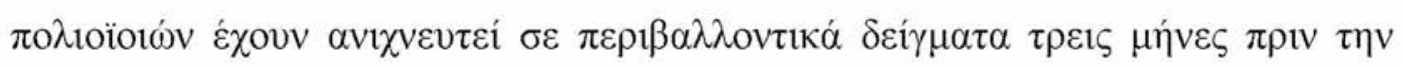

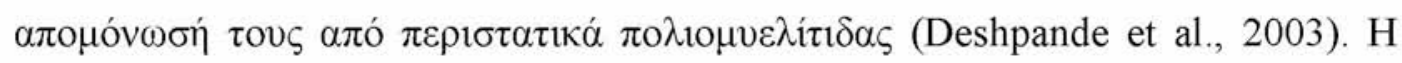

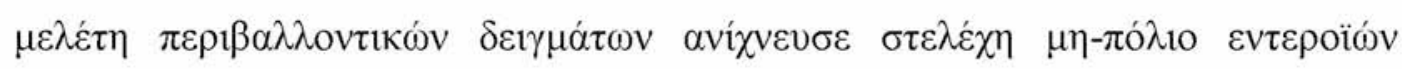
(Shieh et al., 1997; Shieh et al., 2003; Sedmak et al., 2005), $\alpha \lambda \lambda \alpha \dot{\alpha} \sigma \dot{\mu} \mu \varphi \omega v \alpha \mu \varepsilon \tau \eta v$

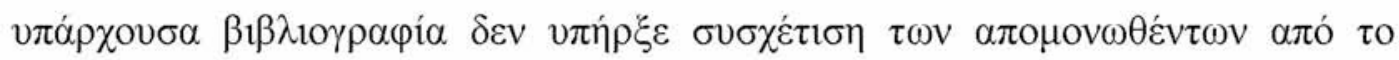

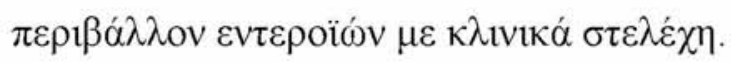

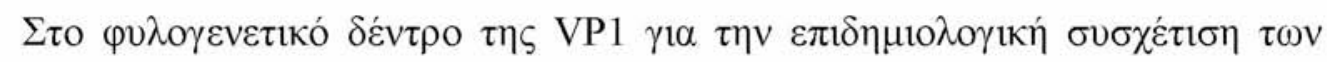

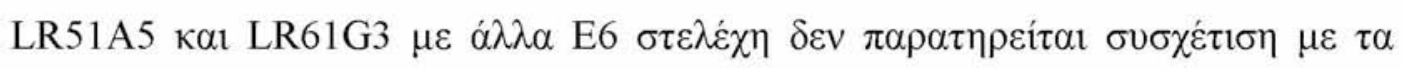

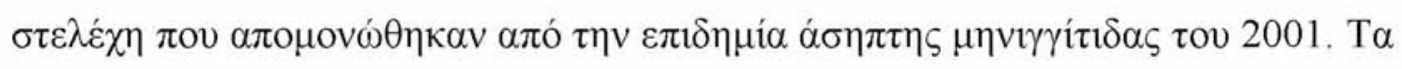

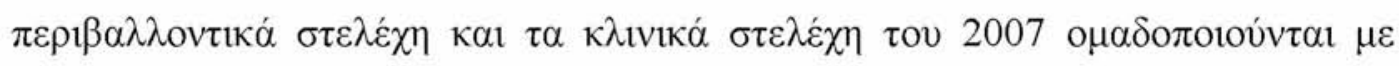

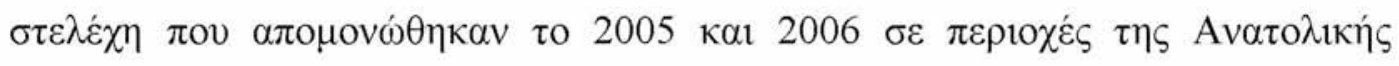

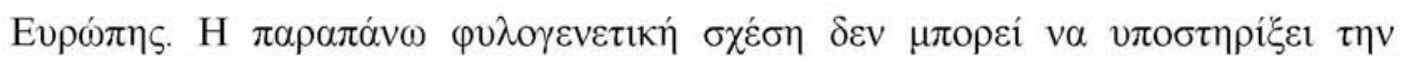

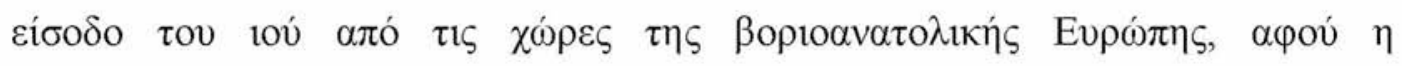

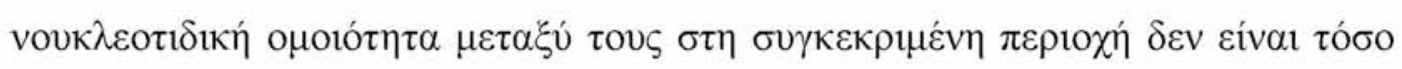

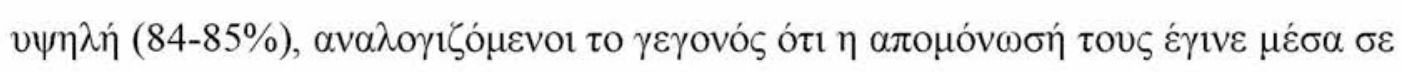

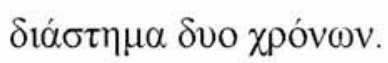

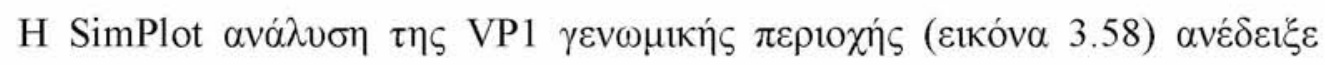

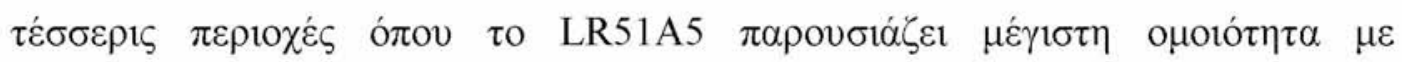




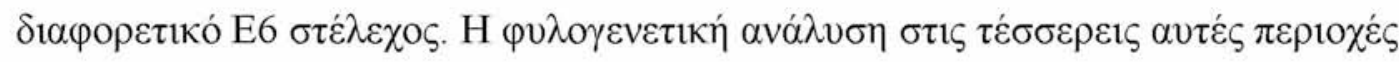

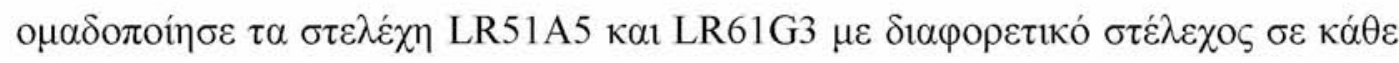

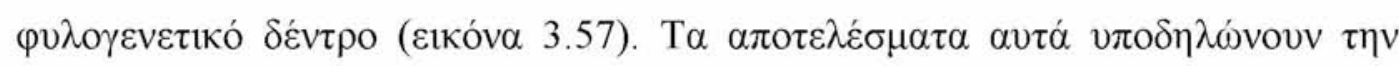

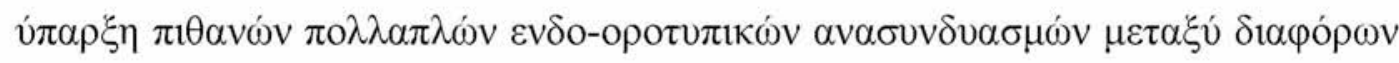

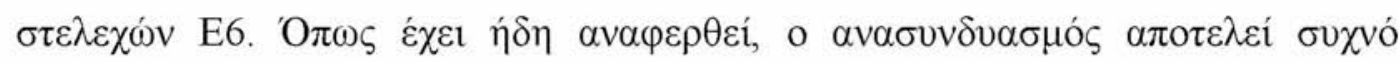

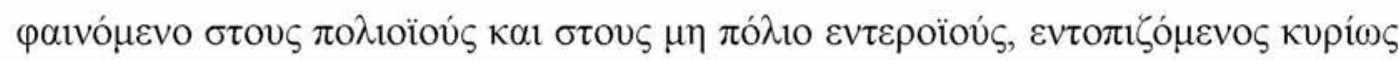

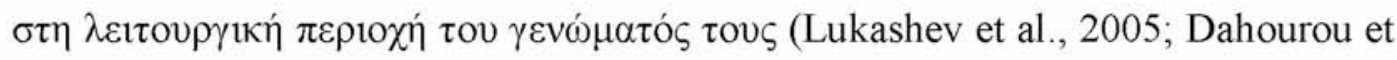
al 2002; Dedepsidis et al., 2008; Georgopoulou and Markoulatos, 2001; Kottaridi

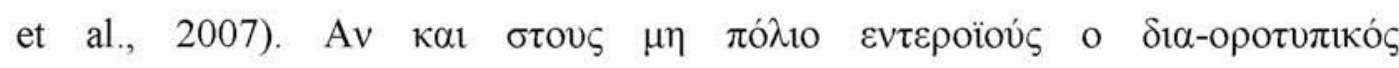

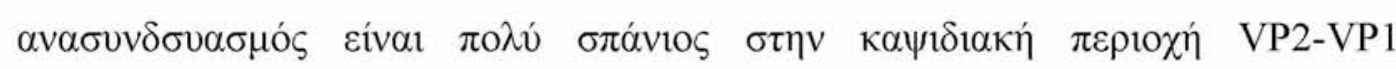

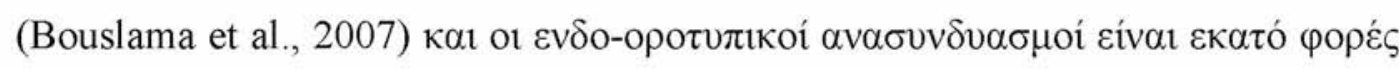

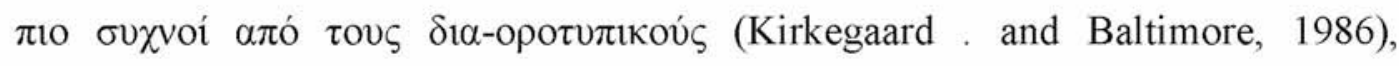

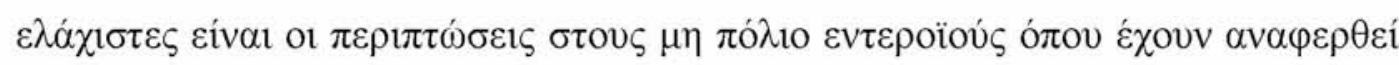

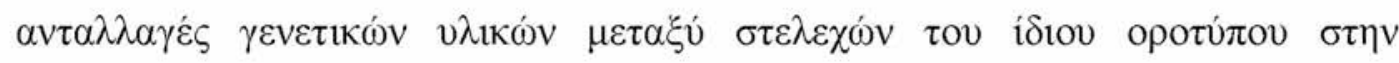

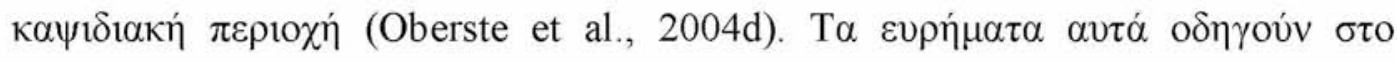

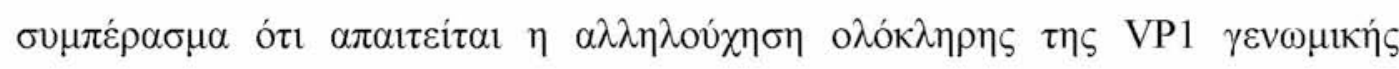

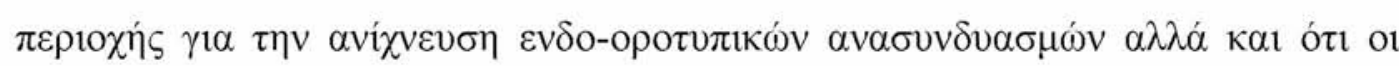

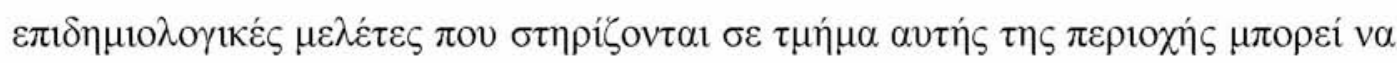

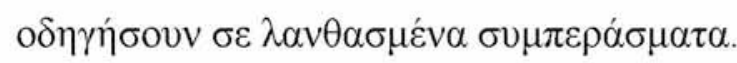

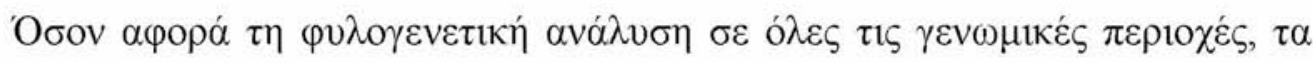

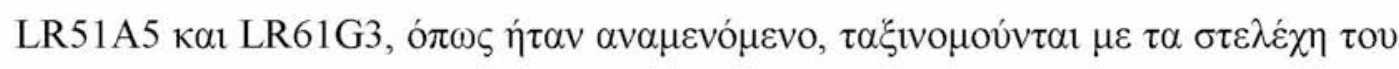

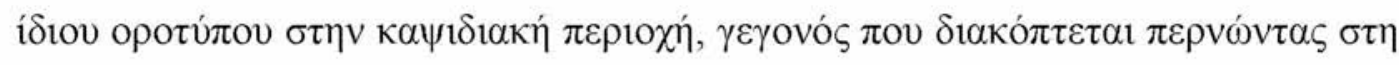

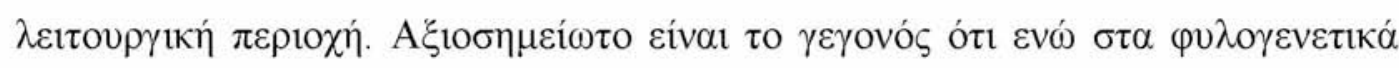

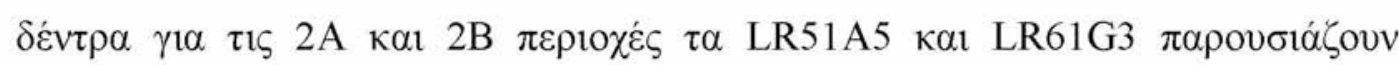

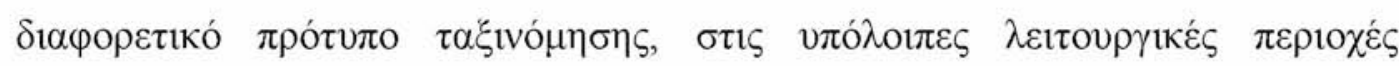

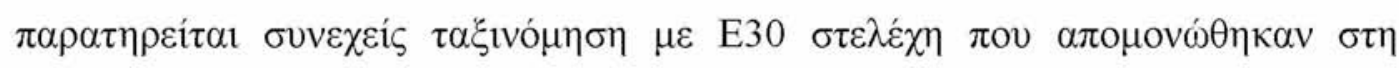

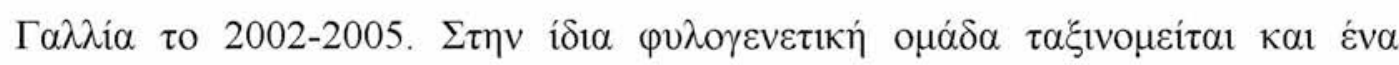

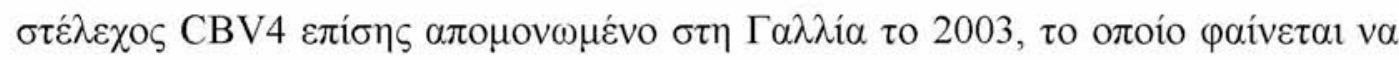

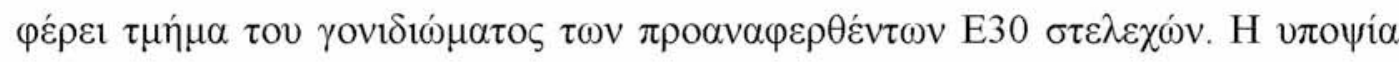

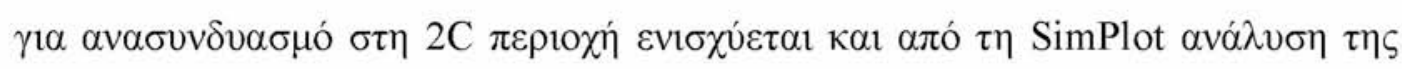
عıкóvas 3.55 .

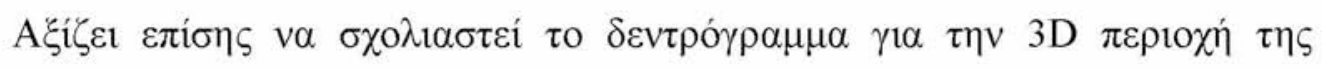

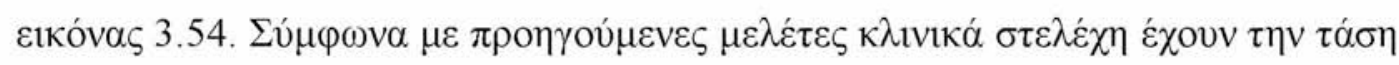

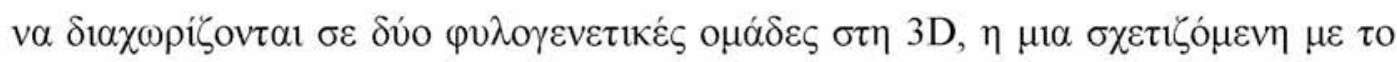




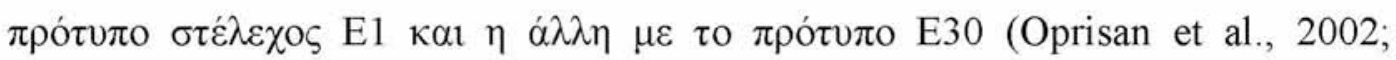

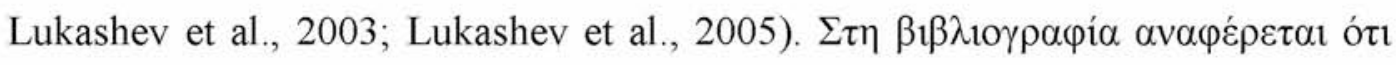

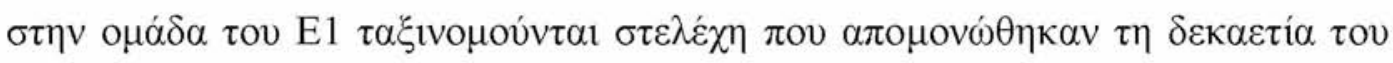

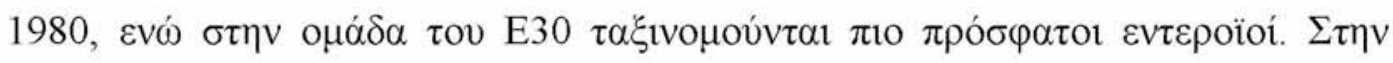

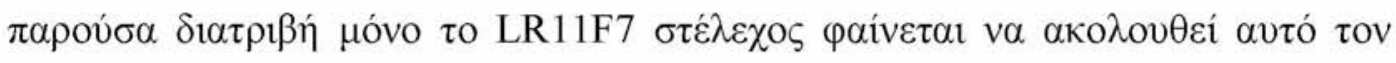

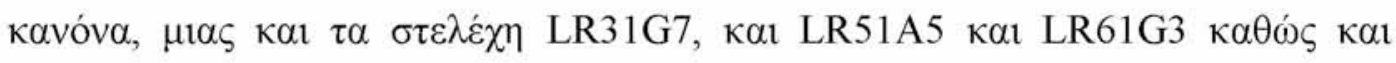

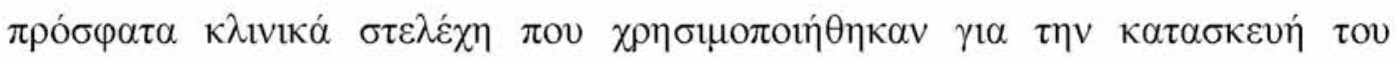

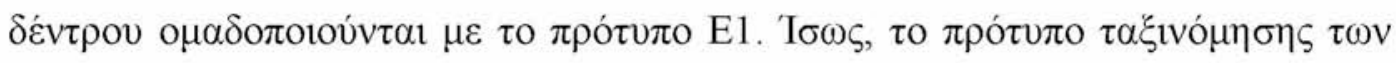

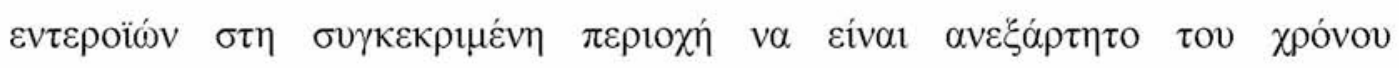

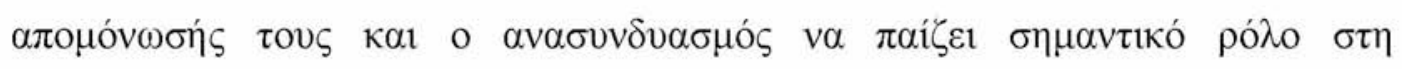

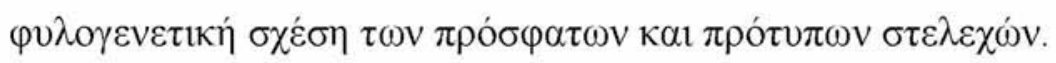

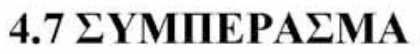

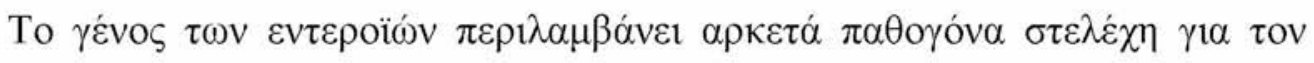

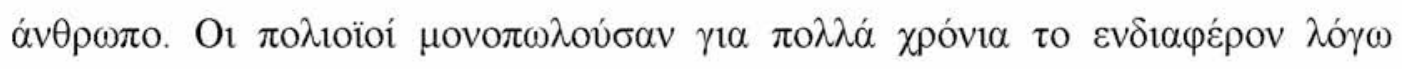

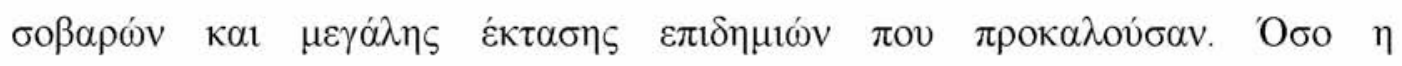

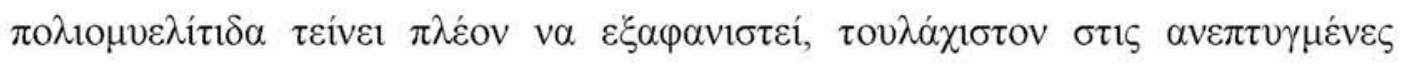

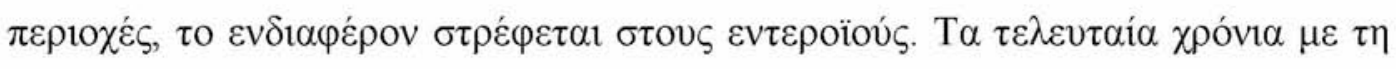

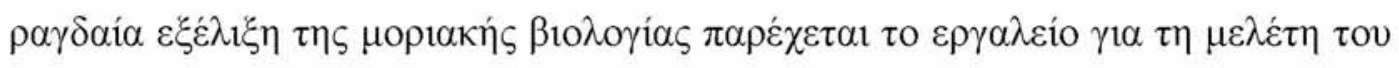

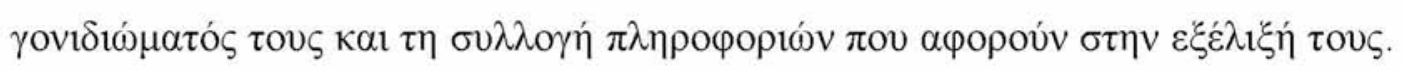

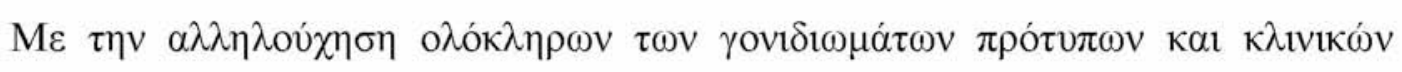

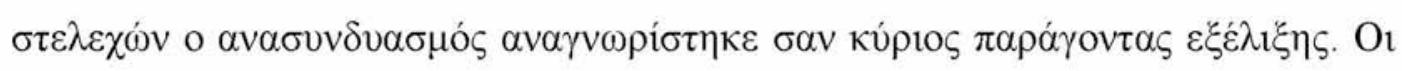

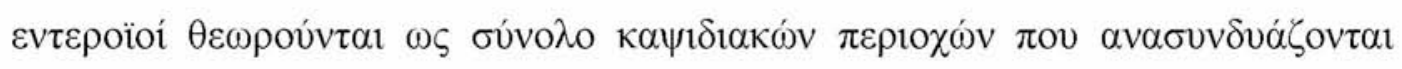

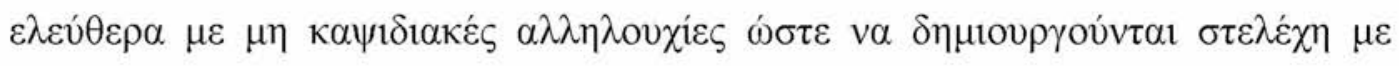

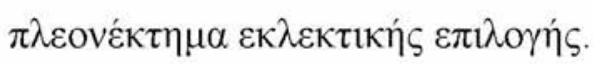

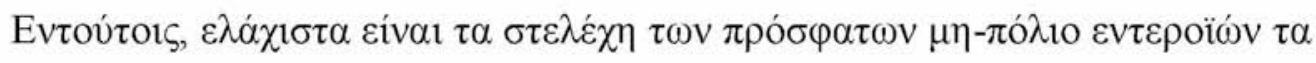

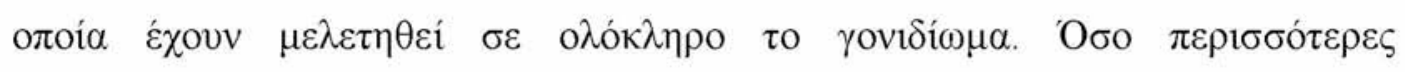

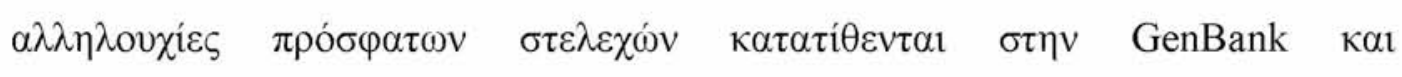
$\pi \rho \alpha \gamma \mu \alpha \tau$ o

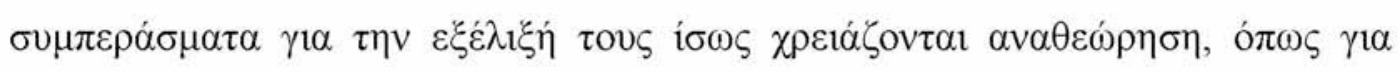

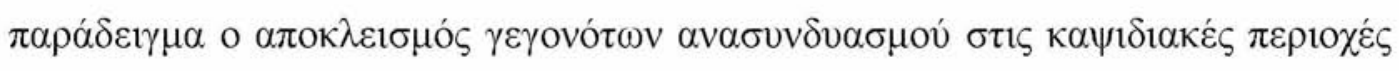

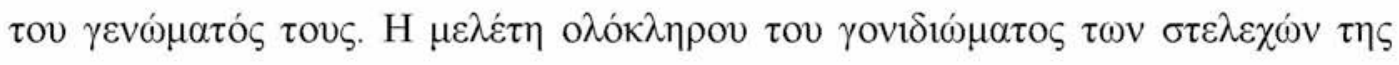

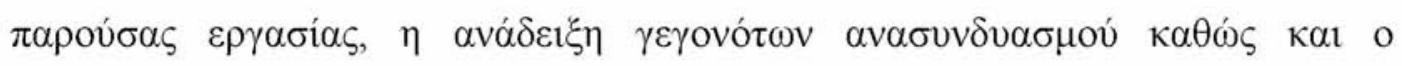




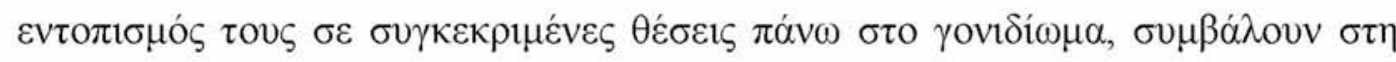

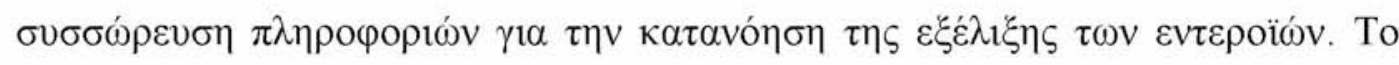

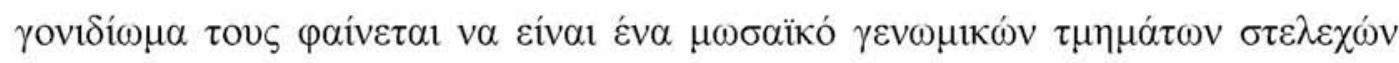

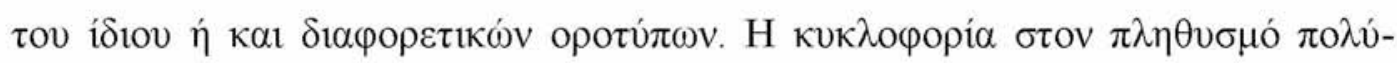

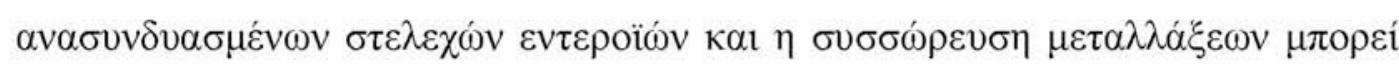

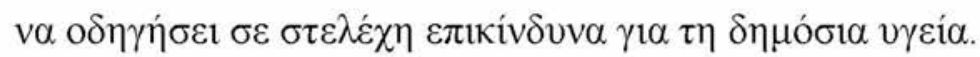




\section{ВIВАІОГРАФІА}

Abad F. X., Pintó R. M., and Bosch A. (1998) Flow cytometry detection of infectious rotaviruses in environmental and clinical samples. Appl. Environ. Microbiol. 64:2392-6.

Altschul S. F., Gish W., Miller W., Myers E. W., and Lipman D. J. (1990) Basic local alignment search tool. J. Mol. Biol. 215: 403-410.

Amvrosieva T. V., Paklonskaya N. V., Biazruchka A. A., Kazinetz O. N., Bohush Z. F., and Fisenko E. G. (2006) Enteroviral infection outbreak in the Republic of Belarus: principal characteristics and phylogenetic analysis of etiological agents. Cent. Eur. J. Public Health. 14:6773.

Andersson P., Edman K., and Lindberg A. M. (2002) Molecular analysis of the echovirus 18 prototype: evidence of interserotypic recombination with echovirus 9. Virus Res. 85:71-83.

Arnold E., Luo M., Vriend G., Rossmann M. G., Palmenberg A. C., Parks G. D., Nicklin M. J., and Wimmer E. (1987) Implications of the picornavirus capsid structure for polyprotein processing. Proc. Natl. Acad. Sci. USA 84: 21-25.

Baboonian C., Davies M. J., Booth J. C., and McKenna W. J. (1997) Coxsackie B viruses and human heart disease. Curr. Top. Microbiol. Immunol. 223:31-52.

Back S. H., Kim Y. K., Kim W. J., Cho S., Rang Oh H., Kim J.E., and Jang S. K. (2002) Translation of Polioviral mRNA Is Inhibited by Cleavage of Polypyrimidine Tract-Binding Proteins Executed by Polioviral 3Cpro. J. Virol. 76:2529-2542.

Barton D. J., and Flanegan J. B. (1997) Synchronous replication of poliovirus RNA: initiation of negative-strand RNA synthesis requires the guanidine-inhibited activity of protein 2C. J. Virol. 71:8482-8489.

Bazan J. F. and Fletterick R. J. (1988) Viral cysteine proteases are homologous to the trypsinlike family of serine proteases: structural and functional implications. Proc. Natl. Acad. Sci. USA. 85:7872-6.

Belnap D. M., McDermott B. M. Jr, Filman D. J., Cheng N., Trus B. L., Zuccola H. J., Racaniello V. R., Hogle J. M., and Steven A. C. (2000) Three-dimensional structure of poliovirus receptor bound to poliovirus. Proc. Natl. Acad. Sci. USA. 97:73-8.

Belov G. A., Bonnet N. A., Kovtunovych G., Jackson C. L., Lippincott-Schwartz J., and Ehrenfeld E. (2007) Hijacking Components of the Cellular Secretory Pathway for Replication of Poliovirus RNA. J. Virol. 81:558-567.

Bergelson J. M., Chan M., Solomon K. R., St John N. F., Lin H., and Finberg R. W. (1994) Decay-accelerating factor (CD55), a glycosylphosphatidylinositol-anchored complement regulatory protein, is a receptor for several echoviruses. Proc. Natl. Acad. Sci. USA. 91: 62456248.

Bienz K., Egger D., Troxler M., and Pasamontes L. (1990) Structural organization of poliovirus RNA replication is mediated by viral proteins of the P2 genomic region. J. Virol.. 64: 1156-63.

Blomqvist S., Bruu A. L., Stenvik M., and Hovi T. (2003) Characterization of a recombinant type 3 /type 2 poliovirus isolated from a healthy vaccinee and containing a chimeric capsid protein VP1. J. Gen. Virol. 84: 573-80.

Blomqvist S., Skytta A., Roivainen M., and Hovi T. (1999) Rapid detection of human rhinoviruses in nasopharyngeal aspirates by a microwell reverse transcription-PCR-hybridisation assay. J. Clin. Microbiol. 37: 2813-2816. 
Bolanaki E., Kottaridi C., Markoulatos P., Kyriakopoulou Z., Margaritis L., and Katsorchis T. (2007) Partial 3D gene sequences of Coxsackie viruses reveal interspecies exchanges. Virus Genes. 35:129-40.

Bolanaki E., Kottaridi C., Markoulatos P., Margaritis L., and Katsorchis T. A. (2005) Comparative amplification of five different genomic regions on Coxsackie A and B viruses. Implications in clinical diagnostics. Mol. Cell. Probes. 19:127-35.

Bolanaki E., Kottaridi C., Markoulatos P., Margaritis L., and Katsorchis T. (2005b) Nucleotide analysis and phylogenetic study of the homology boundaries of coxsackie A and B viruses. Virus Genes 31:307-20.

Bolanaki E., Kottaridi C., Markoulatos P., Margaritis L., and Katsorchis T. (2006) Evolution of $2 \mathrm{~B}$ and $2 \mathrm{C}$ genomic parts of species B Coxsackie viruses. Phylogenetic study and comparison with other regions. Virus Genes 32:249-59.

Borchardt M. A., Bertz P. D., Spencer S. K., and Battigelli D. A. (2003) Incidence of enteric viruses in groundwater from household wells in Wisconsin. Appl. Environ. Microbiol. 69:1172-80.

Bouslama L., Nasri D., Chollet L., Belguith K., Bourlet T., Aouni M., Pozzetto B., and Pillet S. (2007) Natural recombination event within the capsid genomic region leading to a chimeric strain of human enterovirus B. J. Virol. 81:8944-52.

Boussadia O., Niepmann M., Creancier L., Prats A. C., Dautry F., and Jacquemin-Sablon H. (2003) Unr is required in vivo for efficient initiation of translation from the internal ribosome entry sites of both rhinovirus and poliovirus. J. Virol. 77: 3353-3359.

Brown B., Oberste M. S., Maher K., and Pallansch M. A. (2003) Complete genomic sequencing shows that polioviruses and members of human enterovirus species $\mathrm{C}$ are closely related in the noncapsid coding region. J. Virol. 77:8973-8984.

Brown E. H. (1972) Enterovirus Infections. British Medical Journal. 2:169-171.

Caro V., Guillot S., Delpeyroux F., and Crainic R. (2001) Molecular strategy for 'serotyping' of human enteroviruses. J. Gen. Virol. 82: 79-91.

Casas I., Palacios G. F., Trallero G., Cisterna D., Freire M. C., and Tenorio A. (2001) Molecular characterization of human enteroviruses in clinical samples: comparison between VP2, VP1, and RNA polymerase regions using RT nested PCR assays and direct sequencing of products. J. Med. Virol. 65:138-48.

Casas I., Powell L., Klapper P. E., and Cleator G. M. (1995) New method for the extraction of viral RNA and DNA from cerebrospinal fluid for use in the polymerase chain reaction assay. J. Virol. Methods. 53:25-36.

Centers for Disease Control and Prevention. (2005) Progress toward interruption of wild poliovirus transmission-Worldwide, January 2004-March 2005. Morb. Mortal. Wkly. Rep. 54:408412 .

Chan K. P., Goh K. T., Chong C. Y., Teo E. S., Lau G., and Ling A. E. (2003) Epidemic Hand, Foot and Mouth Disease Caused by Human Enterovirus 71, Singapore. Emerging Infectious Diseases. 9:78-85.

Chapron C. D., Ballester N. A., Fontaine J. H., Frades C. N., and Margolin A. B. (2000) Detection of astroviruses, enteroviruses, and adenovirus types 40 and 41 in surface waters collected and evaluated by the information collection rule and an integrated cell culture-nested PCR procedure. Appl. Environ. Microbiol. 66:2520-5.

Chetverin A. B. (1999) The puzzle of RNA recombination. FEBS 460:1 - 5. 
Chevalier S., Szendrői A., Caro V., Balanant Jean, Guillot S., Berencsi Gy., and Delpeyroux, F. (2004) Molecular comparison of echovirus 11 starins circulating in Europe during an epidemic of multisystem haemorrhagic disease of infants indicates that evolution generally occurs by recombination. Virology 20:56-70.

Chua B. H., McMinn P. C., Lam S. K., and Chua K. B. (2001) Comparison of the complete nucleotide sequences of echovirus 7 strain UMMC and the prototype (Wallace) strain demonstrates significant genetic drift over time. J. Gen. Virol. 82:2629-39.

Clark M. E. and Dasgupta A. (1990) A transcriptionally active form of TFIIIC is modified in poliovirus-infected HeLa cells. Mol. Cell. Biol. 10:5106-5113.

Cochi S. L, Hull H. F, and Ward N. A. (1995) To conquer poliomyelitis forever. Lancet. 345(8965):1589-90.Costa-Mattioli M., Svitkin Y., Sonenberg N. (2004). La autoantigen is necessary for optimal function of the poliovirus and hepatitis $\mathrm{C}$ virus internal ribosome entry site in vivo and in vitro. Mol. Cell Biol. 24:6861-70.

Crabtree K. D., Gerba C. P., Rose J. B., and Haas C. N. (1997) Waterborne adenovirus: a risk assessment. Water Sci. Technol. 35:1-6.

Cuervo N. S., Guillot S., Romanenkova N., Combiescu M., Aubert-Combiescu A., Seghier M., Caro V., Crainic R., and Delpeyroux F. (2001) Genomic features of intertypic recombinant sabin poliovirus strains excreted by primary vaccinees. J. Virol. 75:5740-51.

Curry S., Fry E., Blakemore W., Abu-Ghazaleh R., Jackson T., King A., Lea S., Newman J., and Stuart D. (1997) Dissecting the roles of VP0 cleavage and RNA packaging in picornavirus capsid stabilization: the structure of empty capsids of foot-and-mouth disease virus. J. Virol. 12:9743-52.

Dahourou G., Guillot S., Le Gall O., and Crainic R. (2002) Genetic recombination in wild-type poliovirus. J. Gen. Virol. 83:3103-3110.

Dalldorf G., and Sickles G. M. (1948) An unidentified, filtrable agent isolated from the feces of children with paralysis. Science. 108:61-62.

Dedepsidis E., Kyriakopoulou Z., Pliaka V., Kottaridi C., Bolanaki E., Levidiotou-Stefanou S., Komiotis D., and Markoulatos P. (2007) A Retrospective characterization of a Vaccine derived Poliovirus type 1 isolated from sewages in Greece. Appl. Environ. Microbiol. 73:6697704 .

Dedepsidis E., Pliaka V., Kyriakopoulou Z., Brakoulias C., Levidiotou-Stefanou S., Pratti A., Mamuris Z., and Markoulatos P. (2008) Complete genomic characterization ofan intertypic Sabin3/Sabin2 capsid recombinant. FEMS. 52:342-351.

Deshpande J. M., Shetty S. J., and Siddiqui Z. A. (2003) Environmental surveillance system to track wild poliovirus transmission. Appl. Environ. Microbiol. 69:2919-27.

Divizia M., Palombi L., Buonomo E., Donia D., Ruscio V., Equestre M., Leno L., Panà A., and Degener A. M. (1999) Genomic characterization of human and environmental polioviruses isolated in Albania. Appl Environ Microbiol. 65:3534-9.

Doedens J. R., Giddings T. H. Jr, and Kirkegaard K. (1997) Inhibition of endoplasmic reticulum-to-Golgi traffic by poliovirus protein $3 \mathrm{~A}$ : genetic and ultrastructural analysis. J. Virol. 71:9054-64.

Domingo E., Martinez-Salas E., Sobrino F., de la Torre J. C., Portela A., Ortin J., LopezGalindez C., Perez-Brena P., Villanueva N., Najera R., et al. (1985) The quasispecies (extremely heterogeneous) nature of viral RNA genome populations: biological relevance--a review. Gene. 40:1-8. 
Dotta F., Censini S., van Halteren A. G., Marselli L., Masini M., Dionisi S., Mosca F., Boggi U., Muda A. O., Prato S. D., Elliott J. F., Covacci A., Rappuoli R., Roep B. O., and Marchetti P. (2007) Caxsackie B4 virus infection of $\beta$ cells and natural killer cell insulitis in recent-onset type 1 diabetic patients. PNAS. 104:115-5120.

Egger D., Teterina N., Ehrenfeld E. and Bienz K. (2000) Formation of the poliovirus replication complex requires coupled viral translation, vesicle production and viral RNA synthesis. J. Virol. 74:6570-6580.

Ehlers M. M., Grabow W. O., and Pavlov D. N. (2005) Detection of enteroviruses in untreated and treated drinking water supplies in South Africa. Water Res. 39:2253-8.

EI Bassioni L., Barakat I., Nasr E., de Gourville E. M., Hovi T., Blomqvist S., Burns C., Stenvik M., Gary H., Kew O. M., Pallansch M. A., and Wahdan M. H. (2003) Prolonged detection of indigenous wild polioviruses in sewage from communities in Egypt. Am. J. Epidemiol. 158:807-15.

EI-Sageyer M. M., Szendrői A., Hütter E., Új M., Szücs Gy., Mezey I., Tóth I., Kátai A., Kapiller Z., Páll G., Petrás Gy., Szalay E., Mihály I., Gourova S., and Berencsi Gy. (1998) Characterization of an echovirus type 11' (prime) epidemic strain causing haemorrhagic syndrome in newborn babies in Hungary. Acta Virol. 42:157-166.

Filman D. J., Syed R., Chow M., Macadam A. J. Minor P. D., and Hogle J. M. (1989) Structural factors that control conformational transitions and serotype specificity in type 3 poliovirus. EMBO J. 8: 1567-79.

Fiore L., Genovese D., Diamanti E., Catone S., Ridolfi B., Ibrahimi B., Konomi R., van der Avoort H. G., Hovi T., Crainic R., Simeoni P., and Amato C. (1998) Antigenic and molecular characterization of wild type 1 poliovirus causing outbreaks of poliomyelitis in Albania and neighboring countries in 1996. J .Clin. Microbiol, 36:1912-8.

Fong T. T., and Lipp E. K. (2005) Enteric viruses of humans and animals in aquatic environments: health risks, detection, and potential water quality assessment tools. Microbiol. Mol. Biol. Rev. 69:357-71.

Fong T. T., Griffin D. and W., and Lipp E. K. (2005) Molecular assays for targeting human and bovine enteric viruses in coastal waters and their application for library-independent source tracking. Appl. Environ. Microbiol. 71:2070-8.

Formiga-Cruz M., Hundesa A., Clemente-Casares P., Albiñana-Gimenez N., Allard A., and Girones R. (2005) Nested multiplex PCR assay for detection of human enteric viruses in shellfish and sewage. J. Virol. Methods. 125:111-8.

Fout G. S., Martinson B. C., Moyer M. W., and Dahling D. R. (2003) A multiplex reverse transcription-PCR method for detection of human enteric viruses in groundwater. Appl Environ Microbiol. 69:3158-64.

Frantzidou F., Dumaidi K., Spiliopoulou A., Antoniadis A., and Papa A. (2007) Echovirus 15 and autumn meningitis outbreak among children, Patras, Greece, J. Clin. Virol. 40:77-9.

Fricks C. E., and Hogle J. M. (1990) Cell-induced conformational change in poliovirus: externalization of the amino terminus of VP1 is responsible for liposome binding. J. Virol 64:1934-45.

Furione M., Guillot S., Otelea D., Balanant J., Candrea A., and Crainic R. (1993) Polioviruses with natural recombinant genomes isolated from vaccine-associated paralytic poliomyelitis. Virology. 196:199-208.

Gamarnik, A. V, and Andino R. (1998) Switch from translation to RNA replication in a positivestranded RNA virus. Genes Dev. 12:2293-2304. 
Gammack N. J., Phillips A., Dunn G., Patel V. and Minor P. D. (1989) Intertypic genomic rearrangements of poliovirus strains in vaccines. Virology. 167: 505-514.

Gavrilin G.V., Cherkasova E. A., Lipskaya G. Y., Kew O. M., and Agol V. I. (2000) Evolution of circulating wild poliovirus and of vaccine-derived poliovirus in an immunodeficient patient: a unifying model. J. Virol. 74:7381-90.

Georgopoulou A., and Markoulatos P. (2001) Sabin type 2 polioviruses with intertypic vaccine/vaccine recombinant genomes. Eur. J. Clin. Microbiol. Infect. Dis. 20:792-799.

Georgopoulou A., Markoulatos P., Spyrou N., and Vamvakopoulos N. C. (2000) Improved genotyping vaccine and wild-type poliovirus strains by restriction fragment length polymorphism analysis: clinical diagnostic implications.. J. Clin. Microbiol. 38:4337-42.

Gmyl A. P., Belousov E. V., Maslova S. V., Khitrina E. V., Chetverin A. B., and Agol A. I. (1999) Nonreplicative RNA Recombination in Poliovirus. J. Virol. 73: 8958 - 8965.

Grabow W. O., Botma K. L., de Villiers J. C., Clay C. G., and Erasmus B. (1999) Assessment of cell culture and polymerase chain reaction procedures for the detection of polioviruses in wastewater.Bull. World. Health Organ. 77:973-80.

Gregory J. B., Litaker R. W., Noble R. T. (2006) Rapid one-step quantitative reverse transcriptase PCR assay with competitive internal positive control for detection of enteroviruses in environmental samples. Appl Environ Microbiol. 72:3960-7.

Griffin D. W., Donaldson K. A., Paul J. H., Rose J. B. (2003) Pathogenic human viruses in coastal waters. Clin. Microbiol. Rev. 16:129-43.

Grimwood K., Huang S. Q., Sadleir G. L., Nix A. W., Kilpatrick D. R., Oberste M. S., and Pallansch M. A. (2003) Acute Flaccid Paralysis from Echovirus Type 33 Infection. J. Clin. Microb. 41:2230-2232.

Gromeier M., Alexander L., and Wimmer E. (1996) Internal ribosomal entry site substitution eliminates neurovirulence in intergeneric poliovirus recombinants. Proc. Natl. Acad. Sci. USA. 93: $2370-5$.

Guillot S., Caro V., Cuervo N., Korotkova E., Combiescu M., Persu A., Aubert-Combiescu A., Delpeyroux F., and Crainic R. (2000) Natural genetic exchanges between vaccine and wild poliovirus strains in humans. J. Virol. 74:8434-43.

Gulevich A.Y., Yusupova R. A., Drygin Y. F. (2001) A phosphodiesterase from ascites carcinoma Krebs II cells specifically cleaves the bond between VPg and RNA of encephalomyocarditis virus. Biochemistry. 66:345-9.

Haramoto E., Katayama H., Oguma K., and Ohgaki S. (2007) Recovery of naked viral genomes in water by virus concentration methods. J. Virol. Methods. 142:169-73.

Herold J. and Andino R. (2001) Poliovirus RNA replication requires genome circularization through a protein-protein bridge. Mol. Cell 7:581-591.

Hirst G., (1962) Genetic recombination with Newcastle disease virus, poliovirus, and influenza. Cold Spring Harbor Symp. Quant. Biol. 27:303 - 308.

Hogle J. M., Chow M., and Filman D. J. (1985) Three-dimensional structure of poliovirus at 2.9 A resolution. Science. 229:1358-1365.

Hovi T., Blomqvist S., Nasr E., Burns C. C., Sarjakoski T., Ahmed N., Savolainen C., Roivainen M., Stenvik M., Laine P., Barakat I., Wahdan M. H., Kamel F. A., Asghar H., Pallansch M. A., Kew O. M., Gary H. E. Jr, deGourville E. M., and El Bassioni L. (2005) Environmental surveillance of wild poliovirus circulation in Egypt--balancing between detection sensitivity and workload. J. Virol. Methods. 126:127-34. 
Hovi T., Stenvik M., Partanen H., and Kangas A. (2001) Poliovirus surveillance by examining sewage specimens. Quantitative recovery of virus after introduction into sewerage at remote upstream location. Epidemiol. Infect. 127:101-6.

Hughes P. J., North C., Minor P. D., and Stanway G. (1989) The complete nucleotide sequence of coxsackievirus A21. J. Gen. Virol. 70:2943-52.

Hunt S. L., and Jackson R. J. (1999) Polypyrimidine-tract binding protein (PTB) is necessary, but not sufficient, for efficient internal initiation of translation of human rhinovirus-2 RNA. RNA. 5: 344-359.

Jacobson S. J., Konings D. A., and Sarnow P. (1993) Biochemical and genetic evidence for a pseudoknot structure at the 3 ' terminus of the poliovirus RNA genome and its role in viral RNA amplification. J. Virol. 67:2961-2971.

Jurgens C. K., Barton D. J., Sharma N., Morasco B. J., Ogram S. A., and Flanegan J. B. (2006) 2Apro is a multifunctional protein that regulates the stability, translation and replication of poliovirus RNA. Virology. 345:346-357.

Kamer G. and Argos P. (1984) Primary structural comparison of RNA-dependent polymerases from plant, animal and bacterial viruses. Nucleic Acids Res. 12:7269-7282.

Karakasiliotis I., Markoulatos P., and Katsorchis T. (2004) Site analysis of recombinant and mutant poliovirus isolates of Sabin origin from patients and from vaccinees. Mol. Cell. Probes. 18: $103-9$.

Katayama H., Sh.imasaki A., and Ohgaki S. (2002) Development of a virus concentration method and its application to detection of enterovirus and norwalk virus from coastal seawater. Appl. Environ. Microbiol. 68:1033-9.

Kauder S. E. and Racaniello V. R. (2004) Poliovirus tropism and attenuation are determined after internal ribosome entry. J. Clin. Invest. 113: 1743-53.

Kilpatrick D. R., Quay J., Pallansch M., and Oberste M. S. (2001) Type-specific detection of echovirus 30 isolates using degenerate reverse transcriptase PCR primers. J. Clin. Microbiol. 39:1299-302.

King A. M. Q.,Brown F., Christian P., Hovi T., Hyypia T., Knowles N. J., Lemon S. M., Minor P. D., Palmenberg A. C., Skern T., and Stanway G. (2000) "Virus taxonomy. Seventh report of the International Committee on Taxonomy of Viruses". Academic Press. San Diego, New York.

Kinnunen L., Huovilainen A., Poyry T., and Hovi T. (1990) Rapid molecular evolution of wild type 3 poliovirus during infection in individual hosts. J. Gen. Virol. 71:317-24.

Kirkegaard K., and Baltimore D. (1986) The mechanism of RNA recombination in poliovirus. Cell 47: $433-443$.

Knipe D. M., and Howley P. M. (2007) Fields Virology, 5th Edition, Vol:1, Copyright: Lippincott Williams \& Wilkins.

Kohler K. A., Hlady W. G., Banerjee K., Gupta D., Francis P., Durrani S., Zuber P. L., and Sutter R.W. (2003) Compatible poliomyelitis cases in India during 2000. Bull World Health Organ. 81:2-9.

Kojouharova M., Zuber P. L., Gyurova S., Fiore L., Buttinelli G., Kunchev A., Vladimirova N., Korsun N., Filipova R., Boneva R., Gavrilin E., Deshpande J. M., Oblapenko G., and Wassilak S. G. (2003) Importation and circulation of poliovirus in Bulgaria in 2001. Bull World Health Organ 81:476-481. 
Kottaridi C., Bolanaki E., and Markoulatos P. (2004) Amplification of Echoviruses genomic regions by different RT-PCR protocols--a comparative study. Mol. Cell. Probes. 18:263-9.

Kottaridi C., Bolanaki E., Kyriakopoulou Z., Dedepsidis E., Pratti A., and Markoulatos P. (2007) Possible recombination and gene adaptation exchanges among clinical echovirus strains: crossing the temporal and topological barriers. Diagn. Microbiol. Infect. Dis. 58:407-12.

Kottaridi C., Bolanaki E., Mamuris Z., Stathopoulos C., Markoulatos P. (2006) Molecular phylogeny of VP1, 2A, and 2B genes of echovirus isolates: epidemiological linkage and observations on genetic variation. Arch. Virol. 151:1117-32.

Kumar S., Tamura K., and Nei M. (2004) MEGA3: integrated software for molecular evolutionary genetics analysis and sequence alignment. Brief. Bioinform. 5:150-163.

Lévêque N., Norder H., Zreik Y., Cartet G., Falcon D., Rivat N., Chomel J. J., Hong S. S., and Lina B. (2007) Echovirus 6 strains derived from a clinical isolate show differences in haemagglutination ability and cell entry pathway. Virus Res. 130:1-9.

Li J. P. and Baltimore D. (1990) An intragenic revertant of a poliovirus 2C mutant has an uncoating effect. J. Virol. 64:1102-1107.

Lindberg A. M., Andersson P., Savolainen C., Mulders M. N., and Hovi T. (2003) Evolution of the genome of Human enterovirus B: incongruence between phylogenies of the VP1 and 3CD regions indicates frequent recombination within the species. J. Gen. Virol. 84:1223-35.

Lipskaya G. Y., Chervonskaya E. A., Belova G. I., Maslova S. V., Kutateladze T. N., Drozdov S. G., Mulders M., Pallansch M. A., Kew O. M., and Agol V. I. (1995) Geographical genotypes (geotypes) of poliovirus case isolates from the former Soviet Union: relatedness to other known poliovirus genotypes. J. Gen. Virol. 76:1687-1699.

Liu H. M., Zheng D. P., Zhang L. B., Oberste M. S., Kew O. M., and Pallansch M. A. (2003) Serial recombination during circulation of type 1 wild-vaccine recombinant polioviruses in China. J. Virol. 77:10994-1005.

Liu H. M., Zheng D. P., Zhang L. B., Oberste M. S., Pallansch M. A., Kew O. M. (2000) Molecular evolution of a type 1 wild-vaccine poliovirus recombinant during widespread circulation in China. J. Virol. 74:11153-61.

Lodder W. J., and de Roda Husman A. M. (2005) Presence of noroviruses and other enteric viruses in sewage and surface waters in The Netherlands. Appl Environ Microbiol. 71:1453-61.

Logotheti M., Pogka V., Horefti E., Papadakos K., Giannaki M., Pangalis A., Sgouras D., and Mentis A. (2009) Laboratory investigation and phylogenetic analysis of enteroviruses involved in an aseptic meningitis outbreak in Greece during the summer of 2007. J. Clin. Virol. 46:270-4.

Lukashev A. N., Lashkevich V. A., Ivanova O. E., Koroleva G. A., Hinkkanen A. E., and Ilonen J. (2003) Recombination in circulating enteroviruses. J. Virol. 77: 10423-31.

Lukashev A. N., Lashkevich V. A., Ivanova O. E., Koroleva G. A., Hinkkanen A. E., and Ilonen J. (2005) Recombination in circulating Human enterovirus B: independent evolution of structural and non-structural genome regions. J. Gen. Virol. 86:3281-90.

Lukashev A. N., Lashkevich V. A., Koroleva G. A., Ilonen J., and Hinkkanen A. E. (2004) Recombination in uveitis-causing enterovirus strains. J. Gen. Virol. 85:463-70.

Macadam A. J., Arnold C., Howlett J., John A., Marsden S., Taffs F., Reeve P., Hamada N., Wareham K., and Almond J. (1989) Reversion of the attenuated and temperature-sensitive phenotypes of the Sabin type 3 strain of poliovirus in vaccinees. Virology. 172:408-14.

Madan V., Castelló A., and Carrasco L. (2008) Viroporins from RNA viruses induce caspasedependent apoptosis. Cellular Microbiology 10:437-451. 
Manor Y., Handsher R., Halmut T., Neuman M., Bobrov A., Rudich H., Vonsover A., Shulman L., Kew O., Mendelson E. (1999) Detection of poliovirus circulation by environmental surveillance in the absence of clinical cases in Israel and the Palestinian authority. J Clin. Microbiol. 37:1670-5.

Marcotte L. L., Wass A. B., Gohara D. W., Pathak H. B., Arnold J. J., Filman D. J., Cameron C. E., and Hogle J. M. (2007) Crystal Structure of Poliovirus 3CD Protein: Virally Encoded Protease and Precursor to the RNA-Dependent RNA Polymerase. J. Virol. 81:3583-3596.

Martin J., Samoilovich E., Dunn G., Lackenby A., Feldman E., Heath A., Svirchevskaya E., Cooper G., Yermalovich M., and Minor P. D. (2002) Isolation of an intertypic poliovirus capsid recombinant from a child with vaccine-associated paralytic poliomyelitis. J. Virol. 76:1092110928.

Marturano J., and Fiore L. (2002) Investigation of the presence of recombinant polioviruses in the hit population in Albania during the 1996 outbreak. J. Clin. Microbiol. 40:316-7.

McDermott B. M. Jr, Rux A. H., Eisenberg R. J., Cohen G. H., and Racaniello V. R. (2000) Two distinct binding affinities of poliovirus for its cellular receptor. J. Biol. Chem 275:2308923096.

Melchers W. J., Zoll J., Tessari M., Bakhmutov D. V., Gmyl A. P., Agol V. I., and Heus H. A. (2006) A GCUA tetranucleotide loop found in the poliovirus oriL by in vivo SELEX (un)expectedly forms a YNMG-like structure: Extending the YNMG family with GYYA. RNA. 12:1671-82.

Melnick J. L. (1996) "Fields Virology.". 3rd Ed., PA: Lippincott/Raven, Philadelphia

Melnick J. L., Shaw E. W., and Curnen E. C. (1949) A virus isolated from patients diagnosed as non-paralytic poliomyelitis or aseptic meningitis. Proc. Soc. Exp. Biol. Med .71:344-349.

Metcalf T. G, Melnick J. L., and Estes M. K. (1995) Environmental virology: from detection of virus in sewage and water by isolation to identification by molecular biology--a trip of over 50 years. Annu. Rev. Microbiol. 49:461-87.

Minor P. D., Ferguson M., Evans D. M., Almond J. W., and Icenogle J. P. (1986) Antigenic structure of polioviruses of serotypes 1, 2 and 3. J. Gen. Virol. 67:1283-1291.

Mirand A., Archimbaud C., Henquell C., Michel Y., Chambon M., Peigue-Lafeuille H., and Bailly J. L. (2006) Prospective identification of HEV-B enteroviruses during the 2005 outbreak. J. Med. Virol. 78:1624-34.

Mirand A., Henquell C., Archimbaud C., Peigue-Lafeuille H., and Bailly J. L. (2007) Emergence of recent echovirus 30 lineages is marked by serial genetic recombination events. J. Gen. Virol, 88:166-76.

Mocé-Llivina L., Jofre J., Méndez X., Akkelidou D, Lucena F., and Papageorgiou G. T. (2002) Counting cytopathogenic virus adsorbed to cellulose nitrate membrane filters as a simple method for counting viruses in raw sewage and sewage effluents. J. Virol. Methods. 102:83-92.

Molla A., Harris K. S., Paul A. V., Shin S. H., Mugavero J., and Wimmer E. (1994) Stimulation of poliovirus proteinase 3 Cpro-related proteolysis by the genome-linked protein VPg and its precursor 3AB. J. Biol. Chem. 269:27015-27020.

Muckelbauer J. K., Kremer M., Minor I., Diana G., Dutko F. J., Groarke J., Pevear D. C., and Rossmann M. G. (1995) The structure of coxsackievirus B3 at 3.5 A resolution. Structure. 153:653-67.

Muir P., Kammerer U., Korn K., Mulders M.N., Poyry T., Weissbrich B., Kandolf R., Cleator G.M, and van Loon A.M. (1998) Molecular typing of enteroviruses: current status and 
future requirements. The European Union Concerted Action on Virus Meningitis and Encephalitis. Clin. Microbiol. Rev. 11: 202-27.

Mulders M. N., Reimerink J. H., Stenvik M., Alaeddinoglu I., van der Avoort H. G., Hovi, T. and Koopmans M. P. (1999) A Sabin vaccine-derived field isolate of poliovirus type 1 displaying aberrant phenotypic and genetic features, including a deletion in antigenic site 1. J. Gen. Virol. 80:907-16.

Murray K. E., and Barton D. J. (2003) Poliovirus CRE-dependent VPg uridylylation is required for positive-strand RNA synthesis but not for negative-strand RNA synthesis. J. Virol. 77: 473950 .

Murray K. E., Roberts A. W. and Barton D. J. (2001) Poly(rC) binding proteins mediate poliovirus mRNA stability. RNA 7: 1126-1141.

Oberste M. S, Maher K., Nix W. A., Michele S. M., Uddin M., Schnurr D., al-Busaidy S., Akoua-Koffi C., and Pallansch M. A. (2007) Molecular identification of 13 new enterovirus types, EV79-88, EV97, and EV100-101, members of the species Human Enterovirus B. Virus Res. 128:34-42.

Oberste M. S., Maher K., and Pallansch M. A. (1998) Molecular phylogeny of all human enterovirus serotypes based on comparison of sequences at the 5' end of the region encoding VP2. Virus Res. 58:35-43.

Oberste M. S., Maher K., and Pallansch M. A. (2004) Evidence for frequent recombination within species human enterovirus B based on complete genomic sequences of all thirty-seven serotypes. J. Virol. 78:855-67.

Oberste M. S., Maher K., Kilpatrick D. R., Flemister M. R., Brown B. A., and Pallansch M. A. (1999) Typing of human enteroviruses by partial sequencing of VP1. J. Clin. Microbiol. 37:1288-93.

Oberste M. S., Maher K., Schnurr D., Flemister R. M., Lovchik C. J., Peters H., Sessions W., Kirk C., Chatterjee N., Fuller S., Hanauer J. M. and Pallansch A. M. (2004b). Evnterivirus 68 is associated with respiratory illness and shares biological features with both the enterovirus and the rhiniviruses. J. Gen. Virol. 85:2577-2584.

Oberste M. S., Michele S. M., Maher K., Schnurr D., Cisterna D., Junttila N., Uddin M., Chomel J. J., Lau C. S., Ridha W., al-Busaidy S., Norder H., Magnius L. O., and Pallansch M. A. (2004) Molecular identification and characterization of two proposed new enterovirus serotypes, EV74 and EV75. J. Gen. Virol. 85:3205-3212.

Oberste M. S., Peñaranda S., and Pallansch M. A. (2004d) RNA recombination plays a major role in genomic change during circulation of coxsackie B viruses. J. Virol. 78:2948-55.

Oberste M.S., Nix W. A., Maher K., and Pallansch M. A. (2003) Improved molecular identification of enteroviruses by RT-PCR and amplicon sequencing. J. Clin. Virol. 26:375-7.

Ochs K., Saleh L., Basssili, G., Sonntag V., Zeller A., and Niepmann M. (2002) Interaction of translation factor eIF4B with poliovirus internal ribosome entry site. J. Virol. $76: 2113-2122$.

Offerhaus L. (1991) Albania: a plundered country. Lancet. 337:44-45.

Oprisan G., Combiescu M., Guillot S., Caro V., Combiescu A., Delpeyroux F., and Crainic R. (2002) Natural genetic recombination between cocirculating heterotypic enteroviruses. J. Gen. Virol. 83:2193-2200.

Page G. S, Mosser A. G, Hogle J. M, Filman D. J, Rueckert R. R, and Chow M. (1988) Threedimensional structure of poliovirus serotype 1 neutralizing determinants. J. Virol. 62:1781-94. 
Pallin R., Wyn-Jones A. P., Place B. M., and Lightfoot N. F. (1997) The detection of enteroviruses in large volume concentrates of recreational waters by the polymerase chain reaction. J. Virol. Methods. 67:57-67.

Papa A., Skoura L., Dumaidi K., Spiliopoulou A., Antoniadis A., and Frantzidou F. (2009) Molecular epidemiology of Echovirus 6 in Greece. Eur. J. Clin. Microbiol.. Infect Dis. 28:683-7.

Parvin J. D., Smith F. I., and Palese P. (1986) Rapid RNA sequencing using double-stranded template DNA, SP6 polymerase, and 3'-deoxynucleotide triphosphates. DNA. 5:167-71.

Paul A. V., Mugavero J., Molla A., and Wimmer E. (1998) Internal ribosomal entry site scanning of the poliovirus polyprotein: implications for proteolytic processing. Virology. 250:241253.

Paul A. V., Rieder E., Kim D. W., van Boom J. H., and Wimmer E. (2000) .Identification of an RNA hairpin in poliovirus RNA that serves as the primary template in the in vitro uridylylation of VPg. J. Virol. 74:10359-10370.

Paximadi E., Karakasiliotis I., Bolanaki E., Krikelis A., and Markoulatos P. (2007) Vaccine derived bi- and multi-recombinant Sabin strains. Virus Genes 35:541-548.

Paximadi E., Karakasiliotis I., Mamuris Z., Stathopoulos C., Krikelis V., and Markoulatos P. (2006) Genomic analysis of recombinant sabin clinical isolates. Virus Genes 32: 203-210.

Perera R., Daijogo S., Walter B. L., Nguyen J. H. C., and Semler B. L. (2007) Cellular Protein Modification by Poliovirus: the Two Faces of Poly(rC)-Binding Protein. J. Virol. 81:8919-8932.

Pfister T., and Wimmer E. (1999) Characterization of nucleoside triphosphatase activity of poliovirus protein $2 \mathrm{C}$ reveals a mechanism by guanidine inhibits poliovirus replication. J. Biol. Chem. 274: 6992-7001.

Pfister T., Mirzayan C. and Wimmer E. (1999) Molecular biology. In "Encyclopedia of Virology", second edition (A. Granoff \& R.G. Webster, Eds), pp 1330-1348 Academic Press Ltd.

Pilipenco E.V., Gmyl A. P., and Agol V. I. (1995) A model for rearrangements in RNA genomes. Nucleic Acids Res. 23:1870-1875.

Pöyry T., Kinnunen L., Hyypia T., Brown B., Horsnell C., Hovi T., and Stanway G. (1996) Genetic and phylogenetic clustering of enteroviruses. J. Gen. Virol. 77:1699-1717.

Poyry T., Stenvik M., and Hovi T. (1988) Viruses in sewage waters during and after a poliomyelitis outbreak and subsequent nationwide oral poliovirus vaccination campaign in Finland. Appl. Environ. Microbiol. 54:371-4.

Prevot D., Decimo D., Herbreteau C. H., Roux F., Garin J., Luc Darlix J., and OhImann T. (2003) Characterization of a novel RNA - binding region of elF4GI critical for ribosomal scanning. The EMBO Jour. 22:1909-1921.

Prevots D. R., Ciofi degli Atti M. L., Sallabanda A., Diamante E., Aylward R. B., Kakariqqi E., Fiore L., Ylli A., van der Avoort H., Sutter R. W., Tozzi A. E., Panei P., Schinaia N., Genovese D., Oblapenko G., Greco D., and Wassilak S. G. (1998) Outbreak of paralytic poliomyelitis in Albania, 1996: high attack rate among adults and apparent interruption of transmission following nationwide mass vaccination. Clin. Infect. Dis. 26:419-25.

Pusch D., Oh D. Y, Wolf S., Dumke R., Schröter-Bobsin U., Höhne M., Röske.I, and Schreier E. (2005) Detection of enteric viruses and bacterial indicators in German environmental waters. Arch. Virol. 150:929-47.

Rajtar B., Majek M., Polański L., and Polz-Dacewicz M. (2008) Enteroviruses in water environment--a potential threat to public health. Ann. Agric. Environ. Med. 15:199-203. 
Ray S. C. (1998) SimPlot for Windows (version 1.6). Baltimore, Md. Distributedby author (http://www.welch.jhu.edu/;sray/download).

Richards O. C., and Ehrenfeld E. (1998) Effects of poliovirus 3AB protein on 3D polymerasecatalyzed reaction. J. Biol. Chem. 273:12832-12840.

Robbins F. C., Enders J. F., Weller T. H., and Florentino G. L. (1951) Studies on the cultivation of poliomyelitis viruses in tissue culture. V. The direct isolation and serologic identification of virus strains in tissue culture from patients with nonparalytic and paralytic poliomyelitis. Am. J. Hyg. 54:286-293.

Rohll J. B., Moon D. H., Evans D. J., and Almond J. W. (1995) The 3' untranslated region of picornavirus RNA: features required for efficient genome replication. J. Virol. 69: 7835-44.

Romanova L. I., ,Blinov V. M., Tolskaya E. A., Viktorova E. G., Kolesnikova M. S., Guseva E. A., and Agol V. I. (1986) The primary structure of crossover regions of intertypic poliovirus recombinants: A model of recombination between RNA genomes. Virology 155:202-213

Romero J. R. (1999) Reverse-transcription polymerase chain reaction detection of the enteroviruses. Arch. Pathol. Lab. Med. 123:1161-9.

Rossmann M. G. (1994) Viral cell recognition and entry. Protein Sci. 3:1712-1725.

Santti J., Harvala H., Kinnunen L., and Hyypiä T. (2000) Molecular epidemiology and evolution of coxsackievirus A9. J. Gen Virol. 81:1361-72.

Santti, J., T. Hyypiä, L. Kinnunen, and M. Salminen. 1999) Evidence of recombination among enteroviruses. J. Virol. 73:8741-9.

Schein C. H., Oezguen N., Volk D. E., Garimella R., Paul A., and Braun W. (2006) NMR structure of the viral peptide linked to the genome (VPg) of poliovirus. Peptides. 27:1676-1684.

Sedmak G., Bina D., and MacDonald J. (2003) Assessment of an enterovirus sewage surveillance system by comparison of clinical isolates with sewage isolates from milwaukee, wisconsin, collected august 1994 to december 2002. Appl. Environ. Microbiol. 69:7181-7.

Sedmak G., Bina D., Macdonald J., and Couillard L. (2005) Nine-year study of the occurrence of culturable viruses in source water for two drinking water treatment plants and the influent and effluent of a Wastewater Treatment Plant in Milwaukee, Wisconsin (August 1994 through July 2003). Appl. Environ. Microbiol. 71:1042-50.

Shafren D. R., Dorahy D. J., Ingham R. A., Burns G. F., and Barry R. D. (1997) Coxsackievirus A21 binds to decay-accelerating factor but requires intercellular adhesion molecule 1 for cell entry. J. Virol. 71:4736-4743.

Sharma R., Raychaudhuri S., and Dasgupta A. (2004) Nuclear entry of poliovirus proteasepolymerase precursor 3CD: implications for host cell transcription shut-off. Virology 320:195205 .

Shieh Y. C., Baric R. S., Woods J. W., and Calci K. R. (2003) Molecular surveillance of enterovirus and norwalk-like virus in oysters relocated to a municipal-sewage-impacted gulf estuary. Appl, Environ. Microbiol. 69:7130-6.

Shieh Y. S., Baric R. S., and Sobsey M. D. (1997) Detection of low levels of enteric viruses in metropolitan and airplane sewage. Appl. Environ. Microbiol. 63:4401-7.

Shulman L.M., Manor Y., Azar R., Handsher R., Vonsover A., Mendelson E., Rothman S., Hassin D., Halmut T., Abramovitz B., and Varsano N. (1997) Identification of a new strain of fastidious enterovirus 70 as the causative agent of an outbreak of hemorrhagic conjunctivitis. J. Clin. Microbiol. 35:2145-9. 
Siafakas N., Georgopoulou A., Markoulatos P., and Spyrou N. (2000) Isolation of polioviruses and other enteroviruses in south Greece between 1994 and 1998. J. Clin. Lab. Anal. 14:157-63.

Siafakas N., Markoulatos P., and Levidiotou-Stefanou S. (2004) Molecular identification of enteroviruses responsible for an outbreak of aseptic meningitis; implications in clinical practice and epidemiology. Mol. Cell. Probes. 18:389-98.

Skraber S., Gassilloud B., Schwartzbrod L., and Gantzer C. (2004) Survival of infectious Poliovirus-1 in river water compared to the persistence of somatic coliphages, thermotolerant coliforms and Poliovirus-1 genome. Water Res. 38:2927-33.

Stanway G. (1990) Structure, function and evolution of picornaviruses. J. Gen. Virol. 71:24832501.

Stanway G., Hughes P. J., Mountford R. C., Minor P. D. and Almond J. W. (1984) The complete nucleotide sequence of a common cold virus; human rhinovirus 14. Nucleic Acids Res. 12:7859-7875.

Strauss D. M., Glustrom L. W., and Wuttke D. S. (2003) Towards an understanding of the poliovirus replication complex: the solution structure of the soluble domain of the poliovirus $3 \mathrm{~A}$ protein. J. Mol. Biol. 330:225-234.

Sutter R. W., Caceres V. M., and Mas Lago P. (2004) The role of routine polio immunization in the post-certification era. Bull. World. Health Organ. 82:31-9.

Tambini G., Andrus J. K, Marques E., Boshell J., Pallansch M., de Quadros C. A., and Kew O. (1993) Direct detection of wild poliovirus circulation by stool surveys of healthy children and analysis of community wastewater. J. Infect.Dis. 168:1510-4.

Teterina N. L., Levenson E., Rinaudo M. S., Egger D., Bienz K., Gorbalenya A. E., and Ehrenfeld E. (2006) Evidence for Functional Protein Interactions Required for Poliovirus RNA Replication. J. Virol. 80:5327-5337.

Tobin G. J., Young D. C., and Flanegan J. B. (1989) Self-catalyzed linkage of poliovinus terminal protein VPg to poliovirus RNA. Cell. 59:511-9.

Todd S., Nguyen J. H., and Semler B. L. (1995) RNA-protein interactions directed by the 3' end of human rhinovirus genomic RNA. J. Virol. 69:3605-14.

Tosteson M. T., and Chow M. (1997) Characterization of the ion channels formed by poliovirus in planar lipid membranes. J Virol. 71:507-11.

Towner J. S., Brown D. M., Nguyen J. H. C., and Semler B. L. (2003) Functional conservation of the hydrophobic domain of polypeptide $3 \mathrm{AB}$ between human rhinovirus and poliovirus. Virology. 314:432-442

Toyoda H.., Nicklin M. J., Murray M. G., Anderson C. W., Dunn J. J., Studier F. W. and Wimmer E. (1986) A second virus-encoded proteinase involved in proteolytic processing of poliovirus polyprotein. Cell. 45:761-70.

Tsai Y. L., Tran B., and Palmer C. J. (1995) Analysis of viral RNA persistence in seawater by reverse transcriptase-PCR. Appl. Environ. Microbiol. 61:363-6.

Tsai Y. L., Sobsey M. D., Sangermano L. R., and Palmer C. J. (1993) Simple method of concentrating enteroviruses and hepatitis A virus from sewage and ocean water for rapid detection by reverse transcriptase-polymerase chain reaction. Appl. Environ. Microbiol. 59:3488-91.

van Kuppeveld F. J., Galama J. M., Zoll J., and Melchers W. J. (1995) Genetic analysis of a hydrophobic domain of coxsackie B3 virus protein $2 \mathrm{~B}$ : a moderate degree of hydrophobicity is required for a cis-acting function in viral RNA synthesis. J. Virol. 69;7782-7790. 
Verlinden Y., Cuconati A., Wimmer E., and Rombaut B. (2000) Cell-free synthesis of poliovirus: $14 \mathrm{~S}$ subunits are the key intermediates in the encapsidation of poliovirus RNA. J. Gen. Virol. 81:2751-4.

Wang J., Bakkers J., Galama J., Bruins Slot H., Pilipenko E. V., Agol V. I., and Melchers W. (1999) Structural requirements of the higher order RNA kissing element in the enteroviral 3 UTR. Nucleic Acids Res. 27:353-436.

Ward C. D., and Flanegan J. B. (1992) Determination of the poliovirus RNA polymerase error frequency at eight sites in the viral genome. J. Virol. 66:3784-93.

Weidman M. K., Sharma R., Raychaudhuri S., Kundu P., Tsai W., and Dasgupta A. (2003) The interaction of cytoplasmic RNA viruses with the nucleus. Virus Res. 95:75-85.

World Health Organization. (2003) posting date. Guidelines for environmental surveillance of poliovirus circulation. World Health Organisation, Geneva, Switzerland.

World Health Organization. (2001) Network strategy for detecting cVDPV is defined. Polio Lab Network. Quarterly update.Vol VII, Issue 4.

Williams C. H., Oikarinen S., Tauriainen S., Salminen K., Hyöty H., and Stanway G. J. (2006) Molecular analysis of an echovirus 3 strain isolated from an individual concurrently with appearance of islet cell and IA-2 autoantibodies. Clin. Microbiol. 44:441-8.

Wimmer E., Harber J. J., Bibb J. A., Gromeier M., Lu H. H. and Bernhardt G. (1994) in Cellular Receptors for Animal Viruses, ed. Wimmer E.(Cold Spring Harbor Lab. Press, Plainview, NY), pp 101-127.

Witwer C., Rauscher S., Hofacker I.L., and Stadler P. F. (2001) Conserved RNA secondary structures in Picornaviridae genomes. Nucleic. Acids Res. 15:5079-89.

Widjojoatmodjo M. N, Fluit A. C., and Verhoef J. (1995) Molecular identification of bacteria by fluorescence-based PCR-single-strand conformation polymorphism analysis of the 16S rRNA gene. J Clin. Microbiol. 33:2601-6.

Chuan X., Bator M. C., Bowman D. V., Rieder E., He Y., Hébert B., Bella J., Baker S. T., Wimmer E., Kuhn J. R., and Rossmann M. G. (2001) Interaction of Coxsackievirus A21 with Its Cellular Receptor, ICAM-1. J. Virol. 75:2444-2451.

Yang C. F., Naguib T., Yang S. .J, Nasr E., Jorba J., Ahmed N., Campagnoli R., van der Avoort H., Shimizu H., Yoneyama T., Miyamura T., Pallansch M., and Kew O. (2003) Circulation of endemic type 2 vaccine-derived poliovirus in Egypt from 1983 to 1993. J. Virol 77:8366-8377.

Yongning H., Chipman R. P., Howitt J., Bator M. C., Whitt A. M., Baker S. T., Kuhn J. R., Anderson W. C., Freimuth P., and Rossmann G. M. (2001) Interaction of coxsackievirus B3 with the full length coxsackievirus-adenovirus receptor. Nature Structural Biology 8:874-878. 


\section{ABSTRACT}

The genus of Enteroviruses belongs to Picornaviridae family. According to the degree of their genetic relatedness, they are divided into four species: HEVA, HEVB, HEVC and HEV-D Enteroviruses are non enveloped viruses with a 7.500 nt single stranded positive RNA genome protected by an icosahedral capsid. The enterovirus genome consists of three major regions: the 5 'untranslated region (5'UTR), the open reading frame (ORF) and the 3 'untranslated region ( $3^{\prime}$ UTR). $\mathrm{ORF}$ is translated into a single polyprotein and is then processed to generate four structural (VP1-VP4) and seven non-structural (2A-2C and 3A-3D) proteins.

Enteroviruses are transmitted by fecal-oral route and multiply in gastrointestinal tract. Infected people, symptomatic or asymptomatic, shed enteroviruses in large amounts in the environment by their faeces. Despite the reduction of viral amount following sewage treatment, enteric viruses can contaminate environmental waters causing a potential risk for public health by rejoining the food chain. The environmental surveillance constitutes a great tool for estimating the extent and the duration of poliovirus circulation in a population.

Enteroviruses, like other RNA viruses, have high mutation rate due to the lack of proofreading activity during genome replication. In addition, recombination plays a paramount role in the evolution of enteroviruses. Genetic exchanges between enteroviruses can give rise to new viral genotypes that may be extremely virulent and dangerous for public health. Environmental surveillance could be a useful tool for the study of enterovirus evolution, as long as a great number of enteroviruses can be isolated from environmental samples.

The aim of the present thesis was the isolation of enteroviruses from environmental samples and the molecular study of any putative recombinant strains. The first step was the selection of the appropriate method for the detection and the isolation of enteroviruses from environmental samples. The most appropriate methods that were the two phase separation method-cell culture and the electronegative filter-semi nested PCR used for the detection of enteroviruses in river water and sewage. Enteroviruses were detected in $30 \%$ of the sewage 
samples. Finally, two enteroviruses from river water and seven from sewage were isolated in cell cultures. The full genome analysis of the environmental strains and three poliovirus strains isolated from poliomyelitis cases, revealed that inta- and inter-serotypic recombination events have played a major role in their evolutionary history. The contribution of recombination to enterovirus evolution is substantial, giving rise to new multi recombinant genetic lineages with unknown properties. 


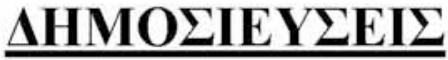




\title{
Molecular Characterization of Wild-Type Polioviruses Isolated in Greece during the 1996 Outbreak in Albania
}

\author{
Zaharoula Kyriakopoulou, ${ }^{1}$ Christine Kottaridi, ${ }^{1}$ Evaggelos Dedepsidis, ${ }^{1}$ Eugenia Bolanaki, ${ }^{2}$ \\ Stamatina Levidiotou-Stefanou, ${ }^{3}$ and Panayotis Markoulatos ${ }^{1 *}$ \\ Department of Biochemistry and Biotechnology, University of Thessaly, 26, Ploutonos and Aeolou St., Larissa 41 221, Greece ${ }^{1}$; \\ Virology Laboratory, Hellenic Pasteur Institute, 127, Vasilissis Sofias Ave., Athens 115 21, Greece ${ }^{2}$; and \\ Department of Microbiology, Medical School of Ioannina, Ioannina, Greece ${ }^{3}$
}

Received 2 December 2005/Accepted 8 December 2005

\begin{abstract}
During the present study three type 1 poliovirus strains isolated in Greece during the 1996 poliomyelitis outbreak in Albania were retrospectively investigated and determination of their relationship with other epidemic strains isolated in Albania or elsewhere during previous epidemics was attempted. SimPlot analysis revealed that the three Greek strains are the result of a recombination event in the VP2 coding region.
\end{abstract}

Three non-Sabin-like poliovirus type 1 (PV1) isolates similar to the serotypes that circulated in the epidemic in Albania in 1996 were isolated from members of a Gypsy community in Greece between the end of June 1996 and September 1996 (15). The outbreak in Albania was caused by a wild-type 1 poliovirus and lasted from May till November $1996(6,14)$. The three non-Sabin-like type 1 poliovirus strains were isolated in and recorded by the National Enterovirus Reference Laboratory for Greece at the Hellenic Pasteur Institute in Athens (15). The purpose of the present study was to further characterize in retrospect the specific poliovirus isolates throughout $5^{\prime}$ untranslated region (UTR) and coding regions VP4, VP2, VP3, VP1, and $2 \mathrm{~A}$, in an attempt to investigate their relationship with strains isolated in Albania at the same period or elsewhere in the world during previous or more recent epidemics. Since the outbreak in Albania started soon after the national immunization days in Albania (April to May 1996) there was also an attempt to define whether these isolates had a vaccine origin and, if they did have, to elucidate the mutation and recombination events that may have contributed to the reversion of the attenuated phenotype to a neurovirulent one.

The three non-Sabin-like type 1 poliovirus strains were isolated from stool samples at the Enterovirus Reference Laboratory for Greece from cases of acute flaccid paralysis diagnosed in nonvaccinated Gypsy children, aged 7 months old, $9[1 / 2]$ months old, and 2 [1/2] years old, in 1996. Initial isolation and identification of the strains by cell culture and seroneutralization with mixed, equine antiserum pools (supplied by the National Institute for Public Health and the Environment, Bilthoven, The Netherlands) took place in summer 1996, simultaneously with a serious poliomyelitis outbreak in Albania (15).

Enterovirus RNA was extracted from $200 \mu \mathrm{l}$ of the inoculated Rd cell cultures (4). Six different primer sets (72437 and 216616 [2, 12]; EUG3a, EUG3b, EUG3c, and EUC2 [3]; and Z752 and Z1461, Z1196 and Z1941, Z1814 and Z2478, and Z2378 and Z3021 [present study]) were used in reverse tran-

\footnotetext{
* Corresponding author. Mailing address: Department of Biochemistry \& Biotechnology, University of Thessaly, 26, Ploutonos \& Aeolou St., Larissa 41221, Greece. Phone: 00302410 565274. Fax: 00302410 565290. E-mail: markoulatos@bio.uth.gr.
}

scription-PCR, and sequence information from a part of the $5^{\prime}$ UTR and VP4 capsid region and complete VP2, VP3, VP1, and $2 \mathrm{~A}$ coding regions was obtained. Table 1 shows details of the primers designed in this study. The isolated RNA was reversed transcribed with all primer pairs (7). PCR was performed with primers EUC2, EUG3a, EUG3b, and EUG3c, while reverse transcription was performed with primers EUC2a and EUC2b, as previously described (3). The produced cDNA was amplified by PCR. Thirty-five cycles of denaturation $\left(95^{\circ} \mathrm{C}, 20 \mathrm{~s}\right)$, annealing $\left(57^{\circ} \mathrm{C}, 20 \mathrm{~s}\right.$, for primer pairs $\mathrm{Z} 752$ Z1461 and Z1814-Z2478 and $60^{\circ} \mathrm{C}, 10 \mathrm{~s}$, for primer pairs Z1196-Z1941 and Z2378-Z3021), and extension (74 ${ }^{\circ} \mathrm{C}, 20 \mathrm{~s}$ ), followed by incubation for $15 \mathrm{~min}$ at $78^{\circ} \mathrm{C}$ were performed in an Eppendorf master cycler.

Initial identification of the obtained genomic sequences was carried out by comparing them with all available sequences in the database using BLAST software. Pairwise comparison of the sequences of the three PV1 strains (accession numbers AY956405, AY956408, AY960848, AY956406, AY956409, AY960849, AY956407, AY956410, and AY960850) and the sequences of the respective genomic regions of reference and wild-type poliovirus strains, reference human enterovirus $\mathrm{C}$ strains (HEV-Cs), and other HEV representative species (CBV5, CAV9, CAV2, CAV12, and EV70) for which such data are available in GenBank was made with the aid of ClustalW software. Finally, plots of nucleotide similarity between poliovirus strains were created with the aid of SimPlot software (9), in an attempt to define genomic regions that display significant percentages of nucleotide sequence identity among the PV

TABLE 1. Designed primers

\begin{tabular}{lclll}
\hline Primer & Position & Polarity & \multicolumn{1}{c}{ Sequence $\left(5^{\prime}-3^{\prime}\right)$} & Gene \\
\hline Z752 & $752-774$ & Sense & CAGGTCTCATCCCAGAAAGTTG & VP4 \\
Z1461 & $1461-1483$ & Antisense & GGGTGTCGTTTGGTTATTGTCT & VP2 \\
Z1196 & $1196-1216$ & Sense & ACCGAACACACTAAGGGACA & VP2 \\
Z1941 & $1941-1963$ & Antisense & GGTAGCACTCAAATCAAAAGGA & VP3 \\
Z1814 & $1814-1836$ & Sense & GACAACTTTCAGTCTCCGTGTG & VP3 \\
Z2478 & $2478-2500$ & Antisense & GCCTTGTGCTATTGCTTTTTGT & VP3 \\
Z2378 & $2378-2400$ & Sense & GAAATGGATATTCTCGGGTTTG & VP3 \\
Z3021 & $3021-3043$ & Antisense & TTGATGGGTTTGATGAAGTCTG & VP1 \\
\hline
\end{tabular}



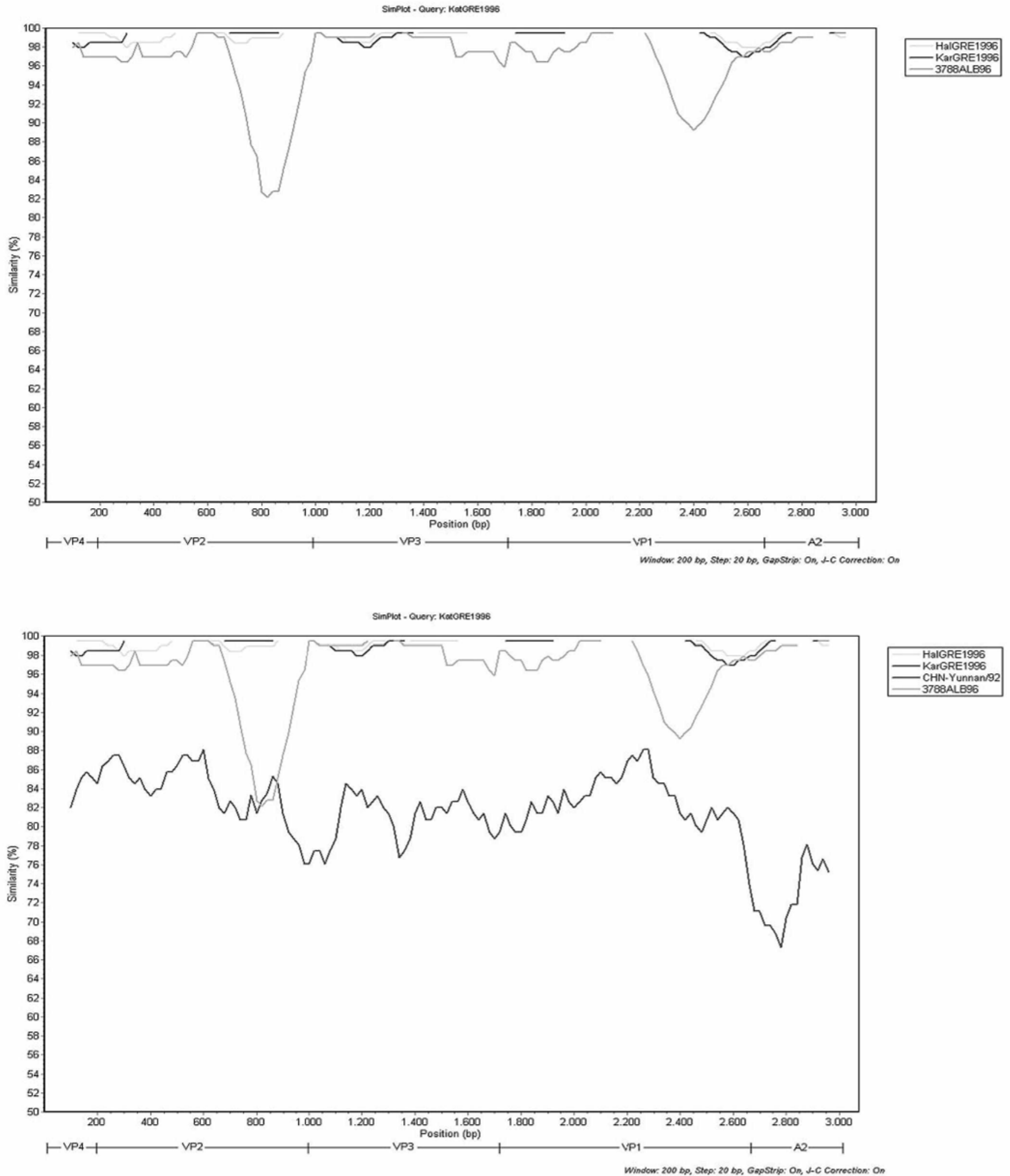

FIG. 1. SimPlot analysis of the 3' ends of VP4, VP2, VP3, VP1, and 2A genomic regions. KatGRE1996 is compared against (top) HalGRE1996, KarGRE1996, and the Albanian strain 3788ALB96 and (bottom) HalGRE1996, KarGRE1996, the Albanian strain 3788ALB96, and the Chinese isolate CHN-Yunnan/92. 
strains, providing indications of possible recombination events throughout the genome.

Sequencing alignment of the three PV1 strains referred to as KatGRE1996, HalGRE1996, and KarGRE1996 with ClustalW revealed the great degree of relatedness of these strains, with sequence identity of $99 \%$ in all sequenced genomic regions: nucleotides (nt) 43 to 565 of the $5^{\prime}$ UTR; the $3^{\prime}$ end of VP4; and complete VP2, VP3, VP1, and 2A. Sequencing of the VP1 coding region verified the serotype of the three poliovirus type 1 strains (13). The nucleotide comparison of VP1 regions of the isolates with the respective regions of the poliovirus Sabin 1 strain and reference strain Mahoney showed the same homology $(81 \%)$.

The nucleotide sequence comparison of the three PV1 isolates with other poliovirus strains using the BLAST software showed that the closest strains were those isolated from the epidemic in Albania in 1996, in all sequenced regions. Figure 1, top, shows the SimPlot comparison of the 3' ends of the VP4, VP2, VP3, VP1, and 2A coding regions of the three PV1 Greek isolates and the Albanian strain 3788ALB96, whose almostcomplete sequence is available in GenBank (11). The similarity between the three PV1 strains and the Albanian strains ranges between $95 \%$ and $100 \%$ in different genomic regions. Obviously, the epidemic PV1 strain that circulated in Albania in 1996 was transferred in Greece, as has also been suggested by Fiore et al. (6). Nevertheless, as represented in Fig. 1, top, there is a region containing part of the almost $200 \mathrm{nt}$ in the VP2 coding region that differs a lot from the respective region of the Albanian strain. Sequence analysis of this part of VP2 with BLAST software indicated a high degree of homology with PV1 strains isolated in China in 1991 (CHN-Yunnan/92). Multiple alignment of nt 550 to 700 of VP2 of the Greek isolates with the respective sequences of Chinese and Albanian strains revealed $89 \%$ and $78 \%$ homology, respectively. There is also another region of approximately $240 \mathrm{nt}$ in the VP1 coding region (nt 620 to 860 of VP1) where the similarity to the Albanian strains falls to $92 \%$.

The above result leads to the assumption that this small part of VP2 is the product of a recombination event. Natural genetic exchange within the capsid region appears to be rare (1, $10)$, but a recombination event in the VP2 gene was never mentioned before. Sequence analysis of this part of the genome with BLAST software indicated a high degree of homology $(81 \%)$ with PV1 strain isolated in China in 1991 (CHNYunnan/92) (5). As shown in Fig. 1, bottom, the homology of the Greek and the Chinese strains ranges between $67 \%$ and $88 \%$ in different genomic regions and the only part of the genome where this homology exceeds the homology with the Albanian strains is the region of the $200 \mathrm{nt}$ of VP2. According to these results we can assume that there was a recombination event in the VP2 gene, but the $81 \%$ similarity is not high enough to prove that the Chinese strains are the donors of the inserted sequence. Potentially, the donor is a descendant of the Chinese strains.

As shown by the Simplot analysis there is also another genomic region in the VP1 gene (nt 620 to 860 of VP1) where the similarity of the Greek strains with the Albanian strains falls to $92 \%$. Nucleotide substitutions accumulate at a rate of approximately 1 to $2 \%$ per year during an epidemic (8). For this reason, this $8 \%$ nucleotide difference couldn't be attributed to the accumulation of nucleotide substitutions, as the Greek and the Albanian isolates circulated at the same period.

In conclusion the nucleotide sequence analysis revealed that there has been a recombination event in the VP2 coding region. The Greek isolates seem to be the product of recombination of the Albanian strains with an unknown virus. The similarity of the inserted sequence to the respective sequence of the type 1 polioviruses isolated in China in 1991 to 1993 can lead to the hypothesis that an offspring of the Chinese isolates is the donor of the unknown sequence.

The present work was cofunded by the European Union (75\%) and the Greek Ministry of Education (25\%) under the framework of the Education and Initial Vocational Training Program "Pythagoras II."

This is research project "Molecular Detection of Enteroviruses in Clinical Samples and the Environment: Implications for the Public Health," code 52213.11.

\section{REFERENCES}

1. Blomqvist, S., A. L. Bruu, M. Stenvik, and T. Hovi. 2003. Characterization of a recombinant type $3 /$ type 2 poliovirus isolated from a healthy vaccinee and containing a chimeric capsid protein VP1. J. Gen. Virol. 84:573-580.

2. Blomqvist, S., A. Skyttä, M. Roivainen, and T. Hovi. 1999. Rapid detection of human rhinoviruses in nasopharyngeal aspirates by a microwell reverse transcription-PCR-hybridization assay. J. Clin. Microbiol. 37:2813-2816.

3. Caro, V., S. Guillot, F. Delpeyroux, and R. Crainic. 2001. Molecular strategy for "serotyping" of human enteroviruses. J. Gen. Virol. 82:79-91.

4. Casas, I., L. Powell, P. E. Klapper, and G. M. Cleator. 1995. New method for the extraction of viral RNA and DNA from cerebrospinal fluid for use in the polymerase chain reaction assay. J. Virol. Methods 53:25-36.

5. Centers for Disease Control and Prevention. 2005. Progress toward interruption of wild poliovirus transmission-worldwide, January 2004-March 2005. Morb. Mortal. Wkly. Rep. 54:408-412.

6. Fiore, L., D. Genovese, E. Diamanti, S. Catone, B. Ridolfi, B. Ibrahimi, R. Konomi, H. G. van der Avoort, T. Hovi, R. Crainic, P. Simeoni, and C. Amato. 1998. Antigenic and molecular characterization of wild type 1 poliovirus causing outbreaks of poliomyelitis in Albania and neighboring countries in 1996. J. Clin. Microbiol. 36:1912-1918.

7. Karakasiliotis, I., P. Markoulatos, and T. Katsorchis. 2004. Site analysis of recombinant and mutant poliovirus isolates of Sabin origin from patients and from vaccinees. Mol. Cell. Probes. 18:103-109.

8. Kinnunen, L., A. Huovilainen, T. Poyry, and T. Hovi. 1990. Rapid molecular evolution of wild type 3 poliovirus during infection in individual hosts. J. Gen. Virol. 71:317-324

9. Lole, K. S., R. C. Bollinger, R. S. Paranjape, D. Gadkari, S. S. Kulkarni, N. G. Novak, R. Ingersoll, H. W. Sheppard, and S. C. Ray. 1999. Full-length human immunodeficiency virus type 1 genomes from subtype C-infected seroconverters in India, with evidence of intersubtype recombination. J. Virol. 73:152-160.

10. Martin, J., E. Samoilovich, G. Dunn, A. Lackenby, E. Feldman, A. Heath, E. Svirchevskaya, G. Cooper, M. Yermalovich, and P. D. Minor. 2002. Isolation of an intertypic poliovirus capsid recombinant from a child with vaccineassociated paralytic poliomyelitis. J. Virol. 76:10921-10928.

11. Marturano, J., and L. Fiore. 2002. Investigation of the presence of recombinant polioviruses in the hit population in Albania during the 1996 outbreak. J. Clin. Microbiol. 40:316-317. (Letter.)

12. Mulders, M. N., J. H. Reimerink, M. Stenvik, I. Alaeddinoglu, H. G. van der Avoort, T. Hovi, and M. P. Koopmans. 1999. A Sabin vaccine-derived field isolate of poliovirus type 1 displaying aberrant phenotypic and genetic features, including a deletion in antigenic site 1. J. Gen. Virol. 80:907-916.

13. Oberste, M. S., W. A. Nix, K. Maher, and M. A. Pallansch. 2003. Improved molecular identification of enteroviruses by RT-PCR and amplicon sequencing. J. Clin. Virol. 26:375-377.

14. Prevots, D. R., M. L. Ciofi degli Atti, A. Sallabanda, E. Diamante, R. B. Aylward, E. Kakariqqi, L. Fiore, A. Ylli, H. van der Avoort, R. W. Sutter, A. E. Tozzi, P. Panei, N. Schinaia, D. Genovese, G. Oblapenko, D. Greco, and S. G. Wassilak. 1998. Outbreak of paralytic poliomyelitis in Albania, 1996: high attack rate among adults and apparent interruption of transmission following nationwide mass vaccination. Clin. Infect. Dis. 26:419-425.

15. Siafakas, N., A. Georgopoulou, P. Markoulatos, and N. Spyrou. 2000. Isolation of polioviruses and other enteroviruses in south Greece between 1994 and 1998. J. Clin. Lab. Anal. 14:157-163. 


\title{
Molecular identification and full genome analysis of an echovirus 7 strain isolated from the environment in Greece
}

\author{
Zaharoula Kyriakopoulou - Evaggelos Dedepsidis • Vaia Pliaka • \\ Panayotis Mastorakos - Anastassia Stamati - Anastassia Pratti · \\ Stamatina Levidiotou-Stefanou $\cdot$ Panayotis Markoulatos
}

Received: 10 September 2009/Accepted: 30 December 2009/Published online: 22 January 2010

(C) Springer Science+Business Media, LLC 2010

\begin{abstract}
Two enteroviruses from river water and four from sewage treatment plant were isolated in Larissa, Greece, that all shared the same sequence. A full genome analysis was conducted in an attempt to reveal the evolutionary pathways of one of the isolated strains (LR11F7). VP1 nucleotide and phylogenetic analysis revealed that the isolated strain had 78\% homology with the echovirus 7 prototype strain Wallace. Full genome analysis revealed that LR11F7 P1 region is related to echoviruses 7 and that $\mathrm{P} 2$ and P3 regions are originating from contemporary enteroviruses isolated in South Asia. Two recombination events were shown to be involved into the evolutionary history of LR11F7, the one event concerning 3A, 3B, and $2 \mathrm{C}$, and the other concerning $3 \mathrm{D}$ genomic region, both with new types of HEV-B. The contribution of recombination to enterovirus evolution is substantial, giving rise to new genetic lineages with unknown properties.
\end{abstract}

Keywords Enteroviruses - Environment $\cdot$ Recombination

\section{Introduction}

Human enteroviruses belong to the genus Enterovirus, family Picornaviridae. According to the degree of their genetic relatedness, they are divided into seven species:

Z. Kyriakopoulou - E. Dedepsidis - V. Pliaka - P. Mastorakos ·

A. Stamati - A. Pratti P. Markoulatos $(\bowtie)$

Department of Biochemistry \& Biotechnology, University of

Thessaly, 26, Ploutonos \& Aeolou Str., Larissa 41221, Greece

e-mail: markoulatos@bio.uth.gr

S. Levidiotou-Stefanou

Department of Microbiology, Medical School, University

of Ioannina, Ioannina, Greece
HEV-A (CAV-2, $-3,-5$ to $-8,-10,-12,-14,-16$, EV71, EV76, EV89-92, and SV19, SV43, SV46, and A13), HEV$B$ (CBV-1 to -6, CAV-9, all echoviruses, EV69, EV73-75, EV77-80, EV81-88, EV93, EV97-98, EV100-101, EV106107, and SA5), HEV-C (PV-1, -2, -3, CAV-1, -11, -13, -17, 19 -22, -24, EV95-96, EV99, EV102, EV104-105, EV109), HEV-D (EV68, EV70, and EV94), HRV-A (HRV-1, -2, -7 to $-13,-15,-16,-18$ to $-25,-28$ to $-34,-36$ to $-38,-39$ to -41 , -43 to $-47,-49$ to $-51,-53$ to $-68,-71,-73$ to $-78,-80$ to -82 , $-85,-88$ to $-90,-94$ to $-96,-98$, and -100 ), HRV-B (HRV -3 to $-6,-14,-17,-26,-27,-35,-37,-42,-48,-52,-69,-70$, $-72,-79,-83,-84,-86,-91$ to $-93,-97$ and -99 , and HRV-C.

Enteroviruses are non-enveloped viruses with a 7500-nt single-stranded positive RNA genome protected by an icosahedral capsid. The enterovirus genome consists of three major regions: the $5^{\prime}$ untranslated region ( $5^{\prime}$ UTR), the open reading frame (ORF), and the $3^{\prime}$ untranslated region ( $3^{\prime}$ UTR). ORF is translated into a single polyprotein and is then processed to generate four structural (VP1-VP4) and seven non-structural $(2 \mathrm{~A}-2 \mathrm{C}$ and $3 \mathrm{~A}-3 \mathrm{D})$ proteins.

Enteroviruses are transmitted by fecal-oral route and multiply in gastrointestinal and respiratory tract. Infected people, symptomatic or asymptomatic, shed enteroviruses in large amounts in the environment by their feces. Despite the reduction of viral amount following sewage treatment, enteric viruses can contaminate environmental waters [1]. They can spread to river, lake waters, and the sea causing a potential risk for public health by rejoining the food chain. The environmental surveillance constitutes an efficient tool for estimating the extent and the duration of enterovirus circulation in a population [2-4]. Wastewater screening can be used to reveal wild poliovirus transmission, as well as to evaluate the efficiency of poliomyelitis immunization campaigns [5]. Environmental surveillance constitutes a sensitive method for monitoring enteroviruses, as just one 
individual shedding poliovirus can be detected among 10,000 inhabitants [6].

Enteroviruses, like other RNA viruses, have high mutation rate due to the lack of proofreading activity during genome replication. It has been estimated that the rate of mutations is one mutation per genome per replication cycle [7]. In addition, recombination plays a paramount role in the evolution of enteroviruses. Natural genetic recombination has been reported among vaccine strains of polioviruses, vaccine, and wild-type polioviruses, or even vaccine and non-polio enterovirus strains [8-12]. Recombination events have been reported also in wild polioviruses [13, 14]. Although recombination has been recognized as a main event in poliovirus evolution, several recent publications have revealed that it is also frequent in non-polio enteroviruses [9, 15-17]. Genetic exchanges between enteroviruses can give rise to new viral genotypes that may be extremely virulent and dangerous for public health. Recombinant strains of polioviruses have been detected from environmental samples [18]. Environmental surveillance could be a useful tool for the study of enterovirus evolution, as long as a great number of enteroviruses can be isolated from environmental samples.

In this study, an echovirus 7 (E7) strain isolated from the environment, circulating in the city of Larissa, Greece, is described, as enteroviruses are among the most important emerging waterborne pathogens. A full genome analysis was conducted in an attempt to reveal the evolutionary pathways of the isolate, the detailed analysis of genetic differences between the environmental strain LR11F7 and that of the prototype strain of E7-Wallace and finally the phylogenetic relationships with other E7 isolates, as well as, with other Human Enteroviruses B was performed. Our study indicates that recombination events may play major role in enteroviruses evolution and that circulation of multi-recombinant strains with unknown properties could be potentially dangerous for public health.

\section{Materials and methods}

Isolation of enteroviruses from river water and sewage samples

Environmental samples from the city of Larissa, Thessaly, Greece-two samples from the sewage treatment plant and four samples from Pinios River, where the treated sewages are discarded-were analyzed during a 4-month period, from July to September 2005. The samples arrived in the laboratory within an hour and were treated the same day. Two enteroviruses from river water (B11D2 and B11E6) and four from sewage (LR11F7, LR11E2, LR11E7, and LR11E6) were isolated.
Sample processing

The samples were concentrated by the two-phase separation method proposed by WHO [19]. $50 \mu \mathrm{l}$ of each concentrate was inoculated onto confluent monolayers of Rhabdomyosarcoma (Rd), Hep-2, and $\mathrm{L}_{20} \mathrm{~B}$ cells, which were grown in 96-well plates in Eagle minimum essential medium (MEM) containing $1 \%$ fetal calf serum (FCS). After absorption of the viruses for $2 \mathrm{~h}$ at $37^{\circ} \mathrm{C}$, the medium in each well was replaced by $100 \mu \mathrm{l}$ of Eagle MEM supplemented with $1 \%$ FCS and the 96-well plates were incubated at $37^{\circ} \mathrm{C}$ for 5-6 days. Then the plates were frozen and thawed thrice, before a second passage was performed. The same procedure was repeated, for the third passage. The contents of the wells in which cytopathic effects (CPE) were observed were inoculated into a $25-\mathrm{cm}^{2}$ flask to confirm CPE. In order to avoid viral mixtures, tenfold serial dilution was prepared and inoculated into 96-well plates. The last dilution in which CPE was observed was inoculated into a $25-\mathrm{cm}^{2}$ flask containing cells. The presence of enterovirus was confirmed by PCR with primer pair UG52/UC53 [20], subsequent to RNA extraction and reverse transcription. Finally, two enterovirus strains from river water and four from sewage were detected (isolated on Rd cells).

VP1 amplification and characterization of the isolates

Enterovirus RNA was extracted from $100 \mu \mathrm{l}$ of the inoculated cell cultures according to the method described by Casas et al. [21] and stored at $-20^{\circ} \mathrm{C}$ until further analysis. The isolated RNA was reverse transcribed into cDNA as previously described [12]. For VP1 amplification of the isolates and characterization of the viruses, PCR with primer pair 292/222 was performed [22]. PCR products were sequenced at Macrogen Inc. (Seoul, Korea).

\section{Genome amplification}

During this study, there was an attempt to obtain sequence information from all genomic regions of the cell cultured viruses. Table 1 shows details of the primers that were used, including their sequences and the genomic area that they specifically amplify. Fourteen of these primers were used for the first time in this study. They were designed with the aid of Primer3 software, obtained online from the Whitehead Institute (http://www.genome.wi.mit.edu/genome software/other/). For the design of primer pairs CHR3/ CHR 4 and CHR5/CHR6, the sequences of all HEV-B prototype strains were used. All of the other primer pairs were designed based on the sequences of the virus isolate LR11F7, as all the isolates revealed $99 \%$ similarity in every sequenced genomic area. All primers were synthesized by Metabion (Martinsried, Germany). 
Table 1 Primers used in this study

\begin{tabular}{|c|c|c|c|c|}
\hline Primer & Position & Polarity & Sequence $\left(5^{\prime}-3^{\prime}\right)$ & References \\
\hline 72437 & $001-020$ & Sense & TTAAAACAGCTCTGGGGTTG & [42] \\
\hline 216616 & $545-565$ & Antisense & GAAACACGGACACCCAAAGTA & [43] \\
\hline 0340F & $310-333$ & Sense & TAGATCAGGCYGATGAGTCACCGC & [32] \\
\hline $1200 \mathrm{R}$ & $1177-1196$ & Antisense & GGGAATTTCCACCACCACCC & [32] \\
\hline AL897 & $897-1007$ & Sense & GGAAGTTTACCGAGCCAGTT & This study \\
\hline AR3091 & $3071-3091$ & Antisense & TTGTTCAGAGCGTTGTAGGC & This study \\
\hline AL939 & $939-959$ & Sense & TGCCTGCTCTAAACTCACCA & This study \\
\hline AR2751 & $2731-2751$ & Antisense & GCACCATCCTTCTTGCATTT & This study \\
\hline AL 1632 & $1632-1652$ & Sense & GCACCCTTGGATTACACTGC & This study \\
\hline AR2180 & $2160-2180$ & Antisense & CATTGCCTGTTTCCTGTTCG & This study \\
\hline 292 & $2612-2627$ & Sense & MIGCIGYIGARACNGG & {$[22]$} \\
\hline 222 & $2969-2951$ & Antisense & CICCIGGIGGIAYRWACAT & {$[22]$} \\
\hline ZL2550 & $2550-2570$ & Sense & AGACGGGGCATACATCACAG & This study \\
\hline ZR3040 & $3040-3060$ & Antisense & TACCCCATTTTGCGAGAAGT & This study \\
\hline EUG3a & $2946-2965$ & Sense & TGGCAAACTTCCWCCAACCC & [23] \\
\hline EUG3b & $2946-2965$ & Sense & TGGCAAACATCTTCMAATCC & [23] \\
\hline EUG3c & $2946-2965$ & Sense & TGGCAGACTTCAACHAACCC & [23] \\
\hline EUC2 & $4413-4433$ & Antisense & TTTGCACTTGAACTGTATGTA & [23] \\
\hline EUC2a & $4428-4448$ & Antisense & GGTTCAATACGGCATTTG & [23] \\
\hline EUC2b & $4428-4448$ & Antisense & GGTTCAATACGGTGTTTGCT & [23] \\
\hline CHR1 & $4284-4308$ & Sense & CNTCHCARAGTGAYCARGARCARYT & {$[32]$} \\
\hline CHR2 & $5084-5081$ & Antisense & GTAYACYGGTGGWCCYTGRAAKA & [32] \\
\hline AL4872 & $4872-4892$ & Sense & CCTGTGATGAGGAGTGTTGC & This study \\
\hline AR5275 & $5255-5275$ & Antisense & GAGACAAACGTGGTGAGTGC & This study \\
\hline CHR3 & $5047-5065$ & Sense & CIACYCTWGARGCRCTVTT & This study \\
\hline CHR4 & $5860-5841$ & Antisense & GACRTGAGIACHCCRCCRCA & This study \\
\hline $5850 \mathrm{~F}$ & $5837-5859$ & Sense & CAGTGYGGIGGIGTICTCATGTC & [44] \\
\hline $6500 \mathrm{R}$ & $6531-6506$ & Antisense & AGRTTGCCAAAYGTYTGYCTCATTGC & [44] \\
\hline CHR5 & $6485-6507$ & Sense & ATCCAGYTTGAAYGAYTCIGIRG & This study \\
\hline CHR6 & $7250-7227$ & Antisense & GAAYTCYTCRTAYTCKTGCTCYCC & This study \\
\hline
\end{tabular}

The reverse transcription was performed as has been previously described [12] for all primer pairs, with the exception of EUC2, EUG3a, EUG3b, and EUG3c, for which a RT with the anti-sense primers EUC2a and EUC2b was carried out [23].

The cDNA produced was amplified by PCR, using a reaction mixture of $50 \mu \mathrm{l} /$ tube containing $3 \mu \mathrm{l}$ from each cDNA, $1 \mu \mathrm{l}$ of each primer at a concentration of $25 \mathrm{pmol} / \mu \mathrm{l}$, $5 \mu \mathrm{l} 10 \times$ PCR reaction buffer, $5 \mu \mathrm{ldNTPs} 10 \mathrm{mM}, 2.5$ units Paq5000 DNA polymerase (Stratagene, La Jolla, USA), and double-distilled nuclease-free water up to a final volume of $50 \mu \mathrm{l} /$ tube. The reaction was carried out using an Eppendorf Mastercycler. An initial, hot-start step at $95^{\circ} \mathrm{C}$ for 2 min was applied. Specific details of PCR reaction with primers designed in this study regarding number of cycles, primer annealing temperatures, and duration of each step are shown in Table 2. For the other primer pairs, PCR conditions were the same as those described in the original papers (Table 1).
A final step of $5 \mathrm{~min}$ at $72^{\circ} \mathrm{C}$ completed the PCR reaction. $10 \mu \mathrm{l}$ of each amplified product was analyzed by agarose gel electrophoresis in $2 \%$ ultrapure electrophoresis grade agarose gel (Invitrogen, Life Technologies, Paisley, UK) containing $1 \mu \mathrm{g} / \mathrm{ml}$ ethidium bromide in Tris-BorateEDTA buffer.

Sequencing and sequence analysis

Purification of PCR products was carried out directly from $2 \%$ agarose gels using PCR gel extraction kit (QIAGEN $\mathrm{GmbH}$, Hilden, Germany). Both clones were sequenced by Macrogen Inc., using the primers described in Table 1.

Initial identification of the genomic sequences obtained was carried out by comparing them with all available sequences in the database using BLAST software, obtained online from the National Centre for Biotechnology Information (NCBI) (http://www.ncbi.nlm.nih.gov/BLAST/). 
Table 2 PCR conditions for each new primer pair used in this study

\begin{tabular}{llll}
\hline Primer pairs & \multicolumn{2}{l}{ PCR conditions } & \\
\hline CHR3-CHR4 & Denaturation & $95^{\circ} \mathrm{C}$ for $30 \mathrm{~s}$ & 40 cycles \\
CHR5-CHR6 & Annealing & $45^{\circ} \mathrm{C}$ for $1 \mathrm{~min}$ & \\
& Extension & $72^{\circ} \mathrm{C}$ for $1 \mathrm{~min}$ & \\
AL1632-AR2180 & Denaturation & $95^{\circ} \mathrm{C}$ for $30 \mathrm{~s}$ & 35 cycles \\
ZL2550-ZR3040 & Annealing & $55^{\circ} \mathrm{C}$ for $30 \mathrm{~s}$ & \\
& Extension & $72^{\circ} \mathrm{C}$ for $30 \mathrm{~s}$ & \\
AL939-AR2751 & Denaturation & $95^{\circ} \mathrm{C}$ for $30 \mathrm{~s}$ & 35 cycles \\
& Annealing & $55^{\circ} \mathrm{C}$ for $30 \mathrm{~s}$ & \\
& Extension & $72^{\circ} \mathrm{C}$ for $1.5 \mathrm{~min}$ & \\
AL897-AR3091 & Denaturation & $95^{\circ} \mathrm{C}$ for $30 \mathrm{~s}$ & 35 cycles \\
& Annealing & $55^{\circ} \mathrm{C}$ for $30 \mathrm{~s}$ & \\
& Extension & $72^{\circ} \mathrm{C}$ for $2 \mathrm{~min}$ & \\
AL4872-AR5275 & Denaturation & $95^{\circ} \mathrm{C}$ for $30 \mathrm{~s}$ & 35 cycles \\
& Annealing & $58^{\circ} \mathrm{C}$ for $30 \mathrm{~s}$ & \\
& Extension & $72^{\circ} \mathrm{C}$ for $30 \mathrm{~s}$ & \\
\hline
\end{tabular}

Multiple sequence alignments were generated using ClustalW software, obtained online from the web site of the European Bioinformatics Institute (http://www.ebi.ac.uk/ clustalw/). Phylogenetic trees and the confidence values (bootstrap values) were carried out by neighbor-joining method using MEGA version 4 software. For the full genome phylogenetic analysis, complete genome sequences of representative prototype HEV-B, as well as those of the two echoviruses 7 and other $H E V-B$ isolates, deposited in GenBank, that showed high similarity in BLAST were used. For the epidemiology study, a phylogenetic tree was inferred with a 293-nt segment of the VP1 genomic region (from positions 2996 to 3288 within the genome), as it spanned most of the available sequences of E7 isolates in GenBank. Finally, nucleotide similarity plots between the aligned sequences were created with the aid of SimPlot software (version 2.5), with a window size of $400 \mathrm{nt}$, in an attempt to observe genomic regions that display significant percentages of nucleotide sequence identity, providing indications of possible recombination events throughout the genome. The accession numbers of the reference and other enterovirus strains that were used for the full genome computational analysis and phylogenetic analysis of VP1 genomic region are presented in Table 3.

Nucleotide sequence accession number

The almost complete genomic sequence of isolate LR11F7 (from nucleotides 1 to 7179) has been deposited in the GenBank library under accession number FJ460595.
Table 3 Enterovirus strains that were used for the full genome analysis and the phylogenetic analysis of VP1 coding region and their corresponding accession number

\begin{tabular}{|c|c|c|}
\hline Virus & Strain & Accession number \\
\hline CAV9 & Griggs & D00627 \\
\hline CBV3 & Nancy/Connecticut/US/49 & M33854 \\
\hline CBV4 & JVB/New York/US/51 (Benschoten) & $\mathrm{X} 05690$ \\
\hline CBV4 & E2 & AF311939 \\
\hline E3 & Morrisey/Connecticut/US/51 & AY302553 \\
\hline E5 & Noyce/Maine/54 & AF083069 \\
\hline E6 & D'Amori (Rhode Island/55) & AY302558 \\
\hline E6 & EV6-10887-99 from Russia & AY896760 \\
\hline E6 & EV6-14103-00 from Russia & AY896761 \\
\hline E7 & UMMC & AY036578 \\
\hline E7 & EV7-15936-01 from Azerbaijan & AY896765 \\
\hline E7 & $95 \mathrm{CF} 993$ & $\mathrm{AJ} 241447$ \\
\hline E7 & T187-ARG96 & AF403783 \\
\hline E7 & PER98-2533 & $\mathrm{AF} 081630$ \\
\hline E7 & GA93-1765 & AF081608 \\
\hline E7 & GA93-1894 & $\mathrm{AF} 081610$ \\
\hline E7 & 10420 & AY919404 \\
\hline E7 & SLC & DQ227458 \\
\hline E7 & CF1851031-05 & AM236932 \\
\hline E7 & MG-44381-98 & AJ279195 \\
\hline E7 & $172-98$ & $\mathrm{AB} 268178$ \\
\hline E7 & $19-00$ & AB268182 \\
\hline E7 & $34-98$ & AB268183 \\
\hline E7 & $177-00$ & AB268180 \\
\hline E7 & $178-00$ & AB268181 \\
\hline E7 & $137-98$ & $\mathrm{AB} 268177$ \\
\hline E7 & $176-97$ & $\mathrm{AB} 268179$ \\
\hline E11 & EV11-18744-02 from Moldova & AY896764 \\
\hline E12 & Travis (Philippines/53) (wild-type) & X79047 \\
\hline E16 & Harrington (Massachusetts/51) & AY302542 \\
\hline E19 & Burke (Ohio) & AY302544 \\
\hline E19 & $\mathrm{K} / 542 / 81$ & AY167107 \\
\hline E25 & JV-4 (Washington DC/57) & AY302549 \\
\hline $\mathrm{E} 30$ & Bastianni (New York/58) & AF162711 \\
\hline EV74 & USA/CA75-10213 & AY556057 \\
\hline EV75 & USA/OK85-10362 & AY556070 \\
\hline EV85 & BAN00-10353 & AY843303 \\
\hline EV86 & BAN00-10354 & AY843304 \\
\hline EV88 & BAN01-10398 & AY843306 \\
\hline EV100 & BAN2000-10500 & NC009887 \\
\hline EV97 & DT94-0227 & AB426611 \\
\hline EV79 & NH95-0601 & AB426610 \\
\hline EV 107 & TN94-0349 & AB426609 \\
\hline SVDV02 & & EU887279 \\
\hline
\end{tabular}




\section{Results}

Primary characterization of enteroviruses

Partial sequencing of the VP1 genomic region identified the six enteroviruses as belonging to E7 serotype, considering that the nucleotide and the amino acid sequence identity of VP1 region of the isolates was $78 \%(>75 \%)$ and $93 \%$ ( $>88 \%$ ), respectively, compared to that of the prototype strain [24].

Full genome nucleotide analysis

The six isolates showed $99-100 \%$ similarity in all sequenced regions leading to the assumption that it is the same virus isolated from both, the river water and the sewage treatment plant. Based on this, LR11F7 isolate was selected for the full genome analysis. The almost full genome sequence of LR11F7 (from 1 to $7179 \mathrm{nt}$ of the viral genome) was obtained, missing the $3^{\prime}$ end of $3 \mathrm{D}$ genomic region and the $3^{\prime}$ UTR. For genome analysis of the LR11F7 isolate, available information from the complete genomes of all $H E V-B$ prototype strains as well as those information of the two E7 isolates deposited in GenBank were used.

As shown in Table 4, P3 region has the highest degree of identity (nt 79\%, aa 96\%) with the reference strain E7 Wallace, followed by regions P2 (nt 79\%, aa 95\%) and P1

Table 4 Nucleotide and aminoacid sequence identity between LR11F7 and E7 Wallace

\begin{tabular}{llc}
\hline Genetic region & $\begin{array}{l}\text { Nucleotide } \\
\text { identity (\%) }\end{array}$ & $\begin{array}{l}\text { Amino acid } \\
\text { identity (\%) }\end{array}$ \\
\hline $5^{\prime}$ UTR & 85 & - \\
P1 & 78 & 94 \\
VP4 & 79 & 100 \\
VP2 & 78 & 93 \\
VP3 & 79 & 94 \\
VP1 & 78 & 93 \\
P2 & 79 & 95 \\
2A & 76 & 91 \\
2B & 78 & 93 \\
2C & 81 & 98 \\
P3 & 79 & 96 \\
3A & 79 & 96 \\
3B & 78 & 95 \\
3C & 79 & 96 \\
3D & 79 & 97
\end{tabular}

a $1238 \mathrm{nt}$ of $3 \mathrm{D}$ genomic region (nt $78 \%$, aa $94 \%$ ). The gene with the highest similarity is $2 \mathrm{C}(81 \%)$ and the one with the lowest is $2 \mathrm{~A}(76 \%)$.

Comparison of almost the full genome of LR11F7 with the sequences of all prototype strains of $H E V-B$ group, as well as with the sequences of the E7 isolates UMMC and EV7-15936-01, was performed. The genomic regions $5^{\prime}$ UTR, P1, P2, and P3 were aligned separately. Nucleotide similarity of $5^{\prime}$ UTR region with the prototype strain was $85 \%$. This same region showed high similarity $(88 \%)$ with the prototype strain EV75. The phylogenetic relationships of the above strains are presented in the phylogenetic tree of $5^{\prime}$ UTR (Fig. 1). The highest nucleotide similarity of P1 region, $(82 \%)$, was with $\mathrm{E} 7$ isolates, UMMC and EV715936-01. VP4 was the capsid protein with the highest similarity $(85 \%)$ between LR11F7 and the two E7 isolates, UMMC and EV7-15936-01. Comparison of $\mathrm{P} 2$ region with the $H E V-B$ respective regions revealed the highest similarity with TN94-0349 strain (84\%), and of P3 region with those of EV86 (87\%).

\section{Phylogenetic analysis}

Figure 1 shows phylogenetic trees derived separately for each genomic region. The LR11F7 clustered with the other E7 isolates and the reference strain E7 Wallace in the phylogenetic trees determined with gene sequences encoding the capsid proteins. The only exception was observed with VP4 capsid region where the reference strain is clustering separately; fact that indicates that there is no absolute correlation between serotype and genotype in this region [25].

An interruption of the serotype-genotype correlation is observed in phylogenetic trees for non-capsid regions and there is a different classification for each gene. Nevertheless, LR11F7 shows a tendency for grouping with recent enteroviruses isolated later than 1999 in the non-capsid regions, starting from $2 \mathrm{~B}$ region downstream to the end. It is worth pointing out that phylogenetic trees for $2 \mathrm{C}, 3 \mathrm{~A}$, and $3 \mathrm{~B}$ regions revealed that $\mathrm{LR} 11 \mathrm{~F} 7$ is consistently clustering with strain NH95-0601. The strain NH95-0601 belongs to EV79 serotype, isolated from Japanese travelers returning from Southeast Asia (Yamashita et al., unpublished data).

\section{SimPlot analysis}

SimPlot analysis of full genome isolate LR11F7 is presented in Fig. 2. The similarity analysis indicated that, in the genomic region encoding the capsid proteins, the LR11F7 isolate displayed the highest similarity with the other E7 strains. A convergence with $H E V-B$ strains in the regions coding for functional proteins is observed. 


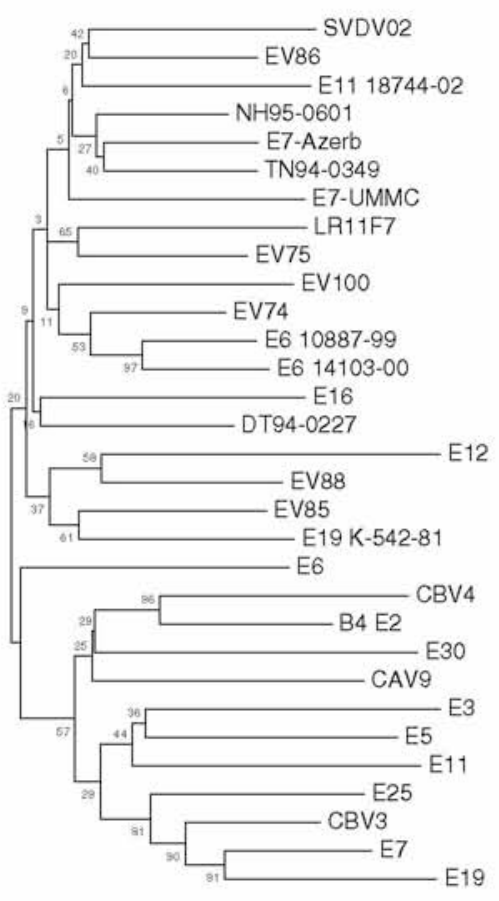

$\stackrel{\longmapsto}{0.02}$

5' UTR

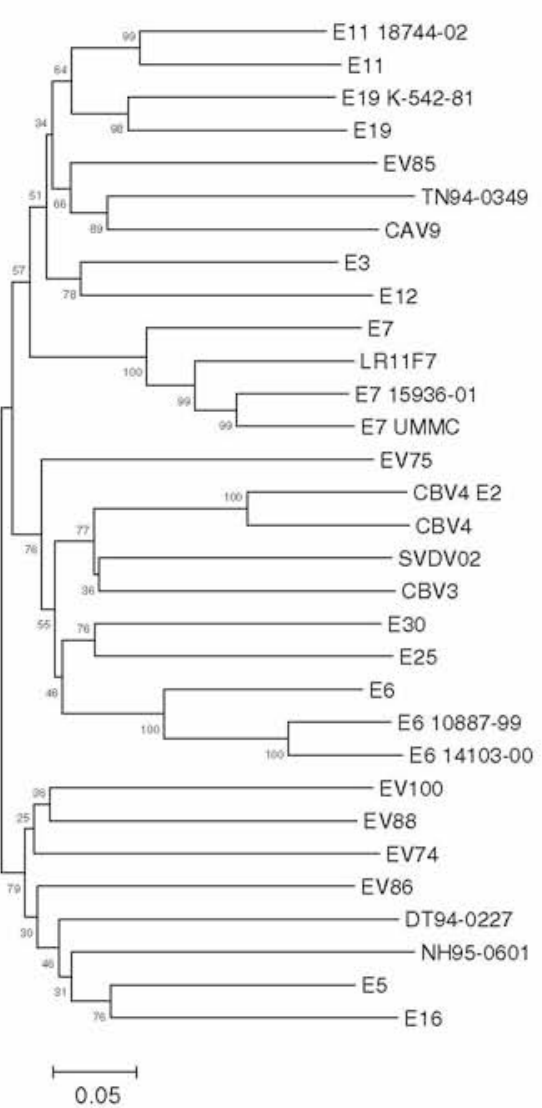

VP3

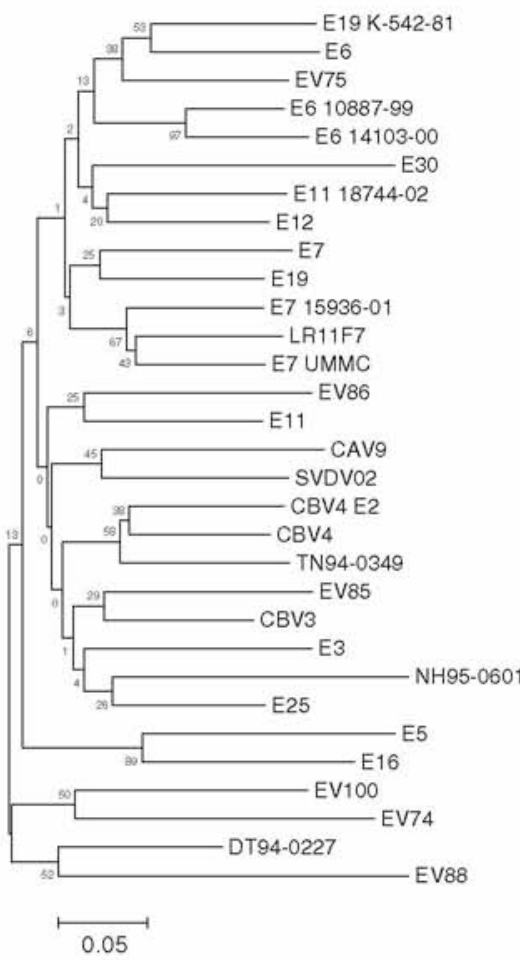

VP4

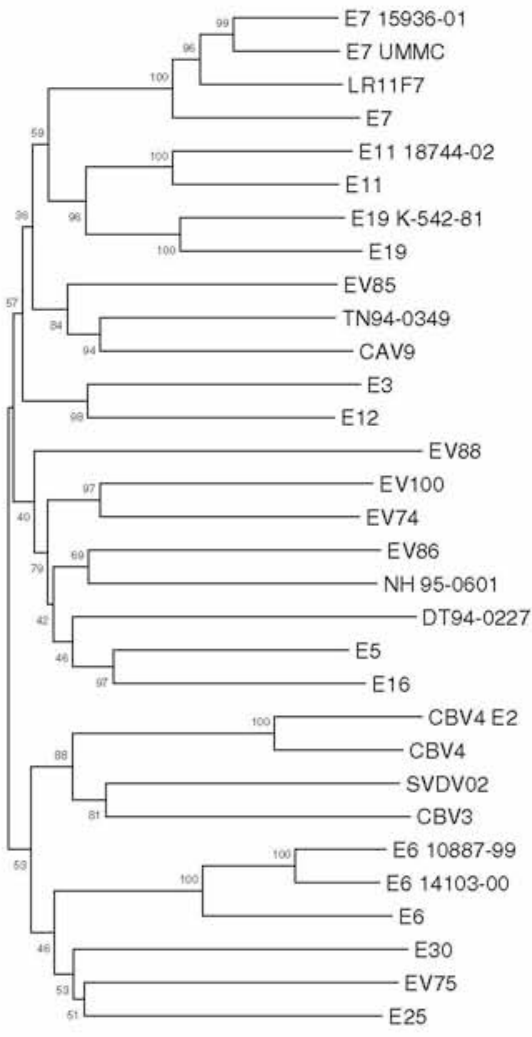

0.05

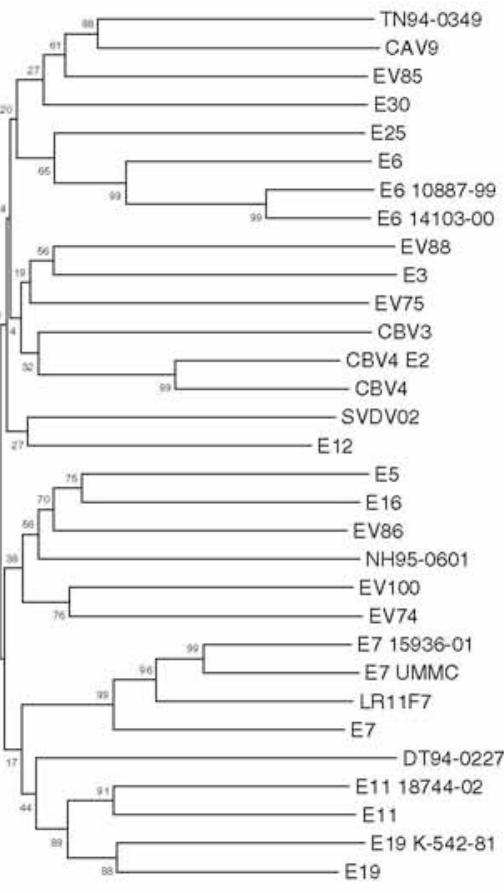

VP2

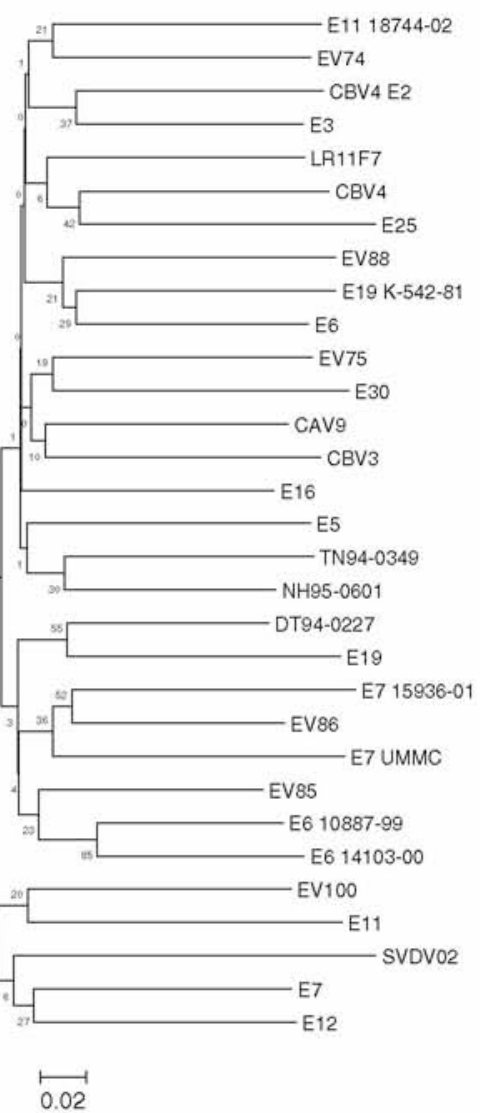

$2 \mathrm{~A}$

Fig. 1 Phylogenetic trees of all sequenced regions of LR11F7 with other $H E V-B$ strains 


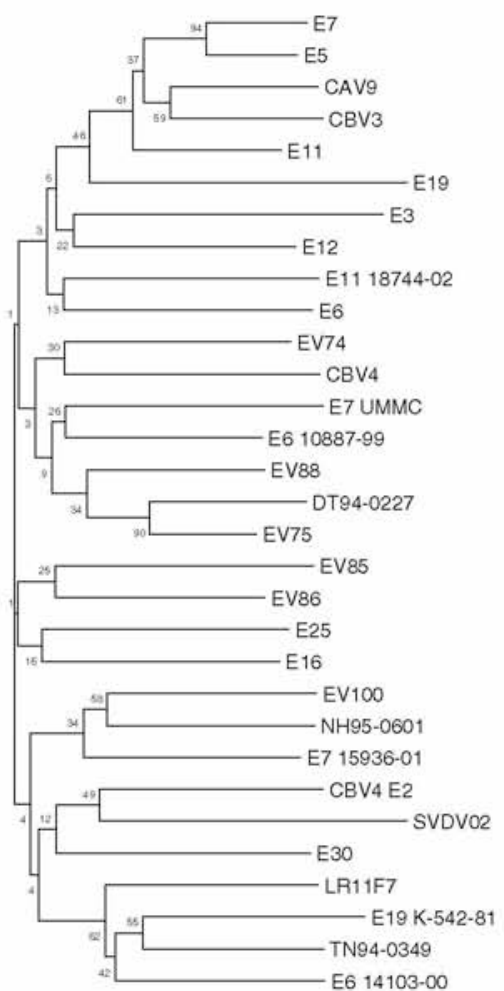

$\stackrel{\longmapsto}{0.02}$

2B

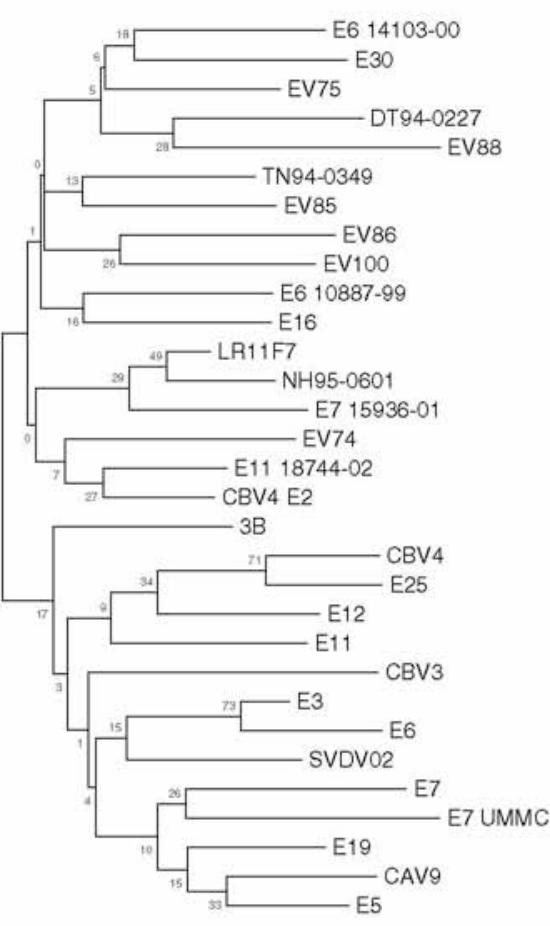

$\stackrel{\overrightarrow{0.02}}{ }$

3B

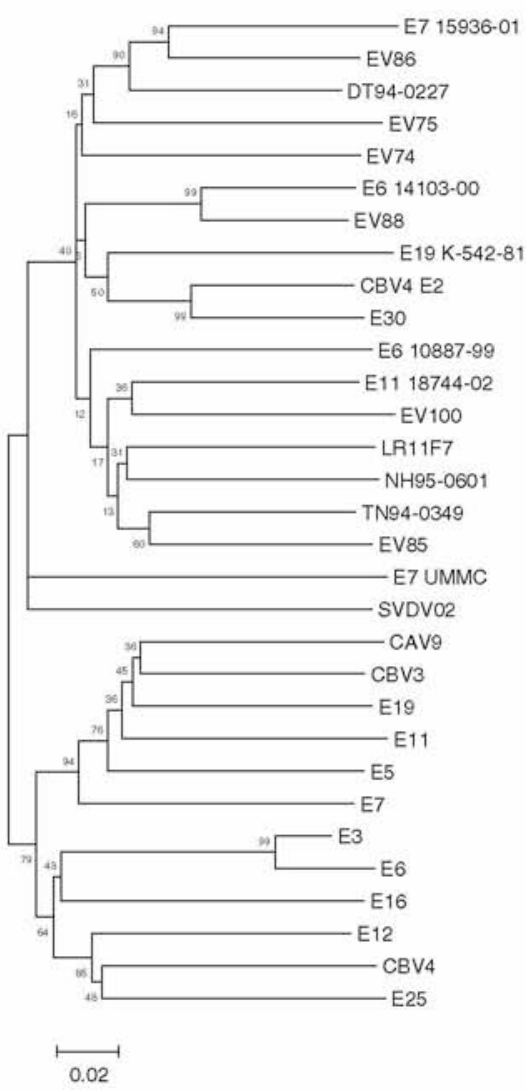

$2 \mathrm{C}$

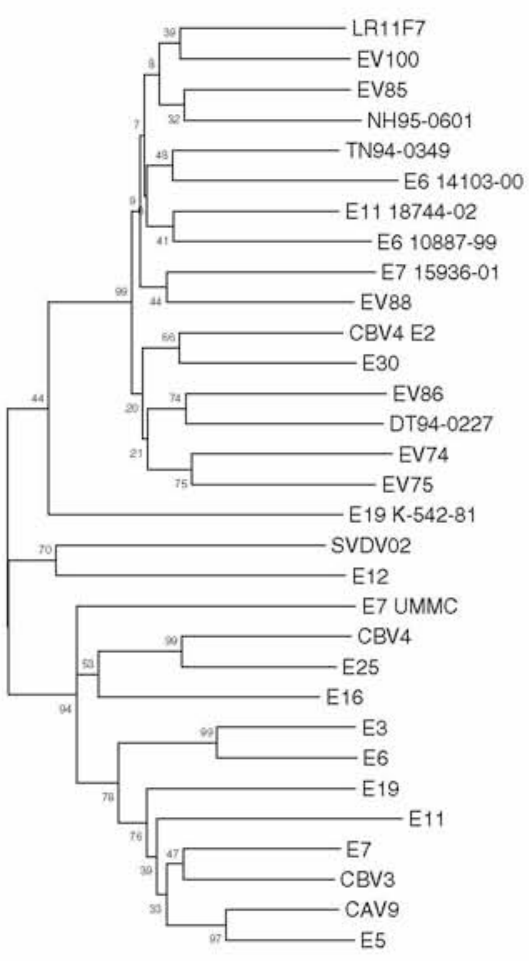

$\stackrel{\longmapsto}{0.02}$

$3 \mathrm{C}$

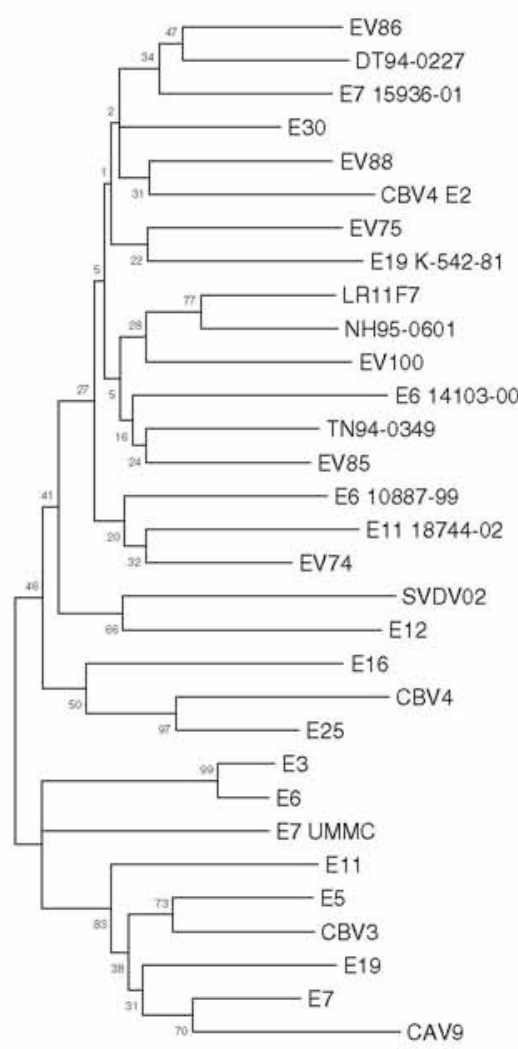

$3 A$

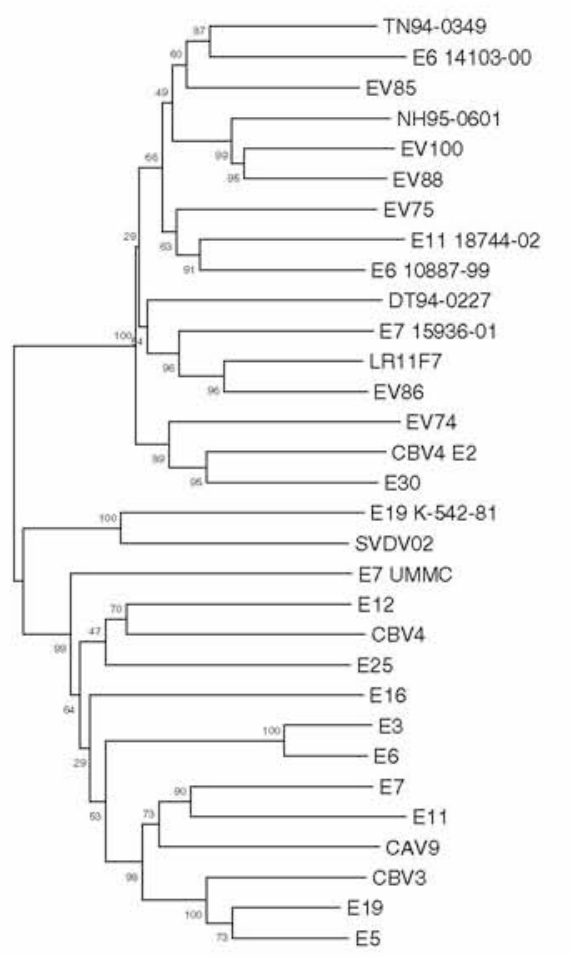

$\stackrel{\longmapsto}{0.02}$

$3 \mathrm{D}$

Fig. 1 continued 


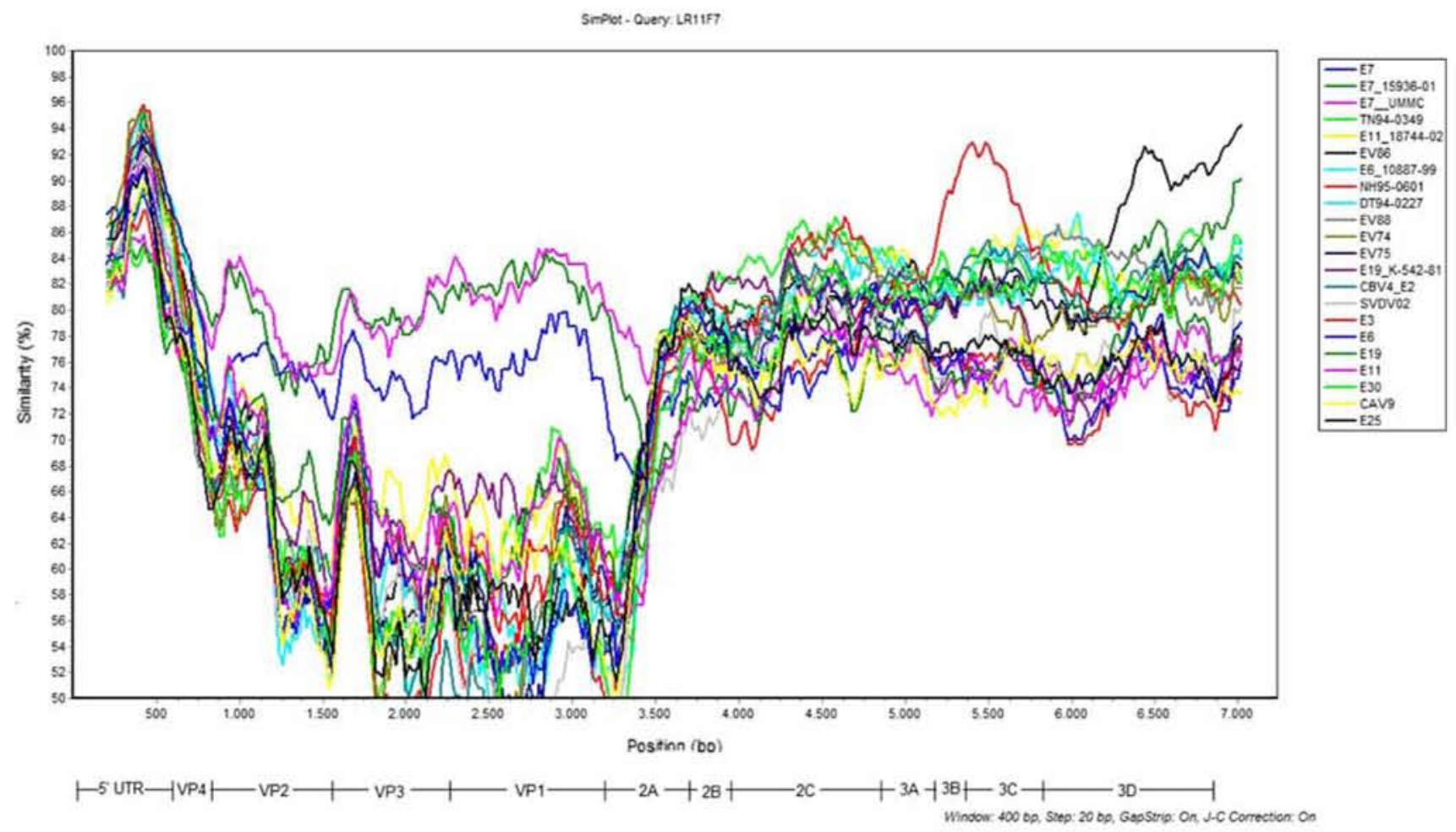

Fig. 2 Full genome SimPlot analysis of LR11F7

However, an increased homology of LR11F7 is observed with the enterovirus $\mathrm{NH} 95-0601$ beginning at the $3 \mathrm{~A}$ region and ending at the beginning of $3 \mathrm{C}$. The highest similarity of LR $11 \mathrm{~F} 7$ with the EV79 strain is in 3B region, with a value approaching 93\%. Following this region the homology drops, whereas in $3 \mathrm{D}$ region an increased homology with EV86 is observed reaching $90 \%$. These sharp changes in similarity of strains in the above-mentioned regions probably reveal two recombination events with donors NH95-0601 and EV86 or their descendants.

\section{Relationships with other E7 isolates}

Only few E7 isolates have been partially sequenced and their sequences are published in GenBank. For this reason, in order to correlate LR11F7 with other E7 isolates, a 293nt region (2996-3288 nt) of VP1 was chosen, as it lies within most of the available sequences. As shown in Fig. 3, the Greek isolates cluster together with enteroviruses 95CF993, isolated in France in 1995, and T187-ARG96 isolated in Argentina in 1996. On the other hand, there is a clustering of LR11F7 with E7 strains isolated in China during 1997-2000 (176-97, 137-98, 178-00, 177-00, 34-98, 19-00, and 172-98), supported by low bootstrap value, and not with the recent European isolates.

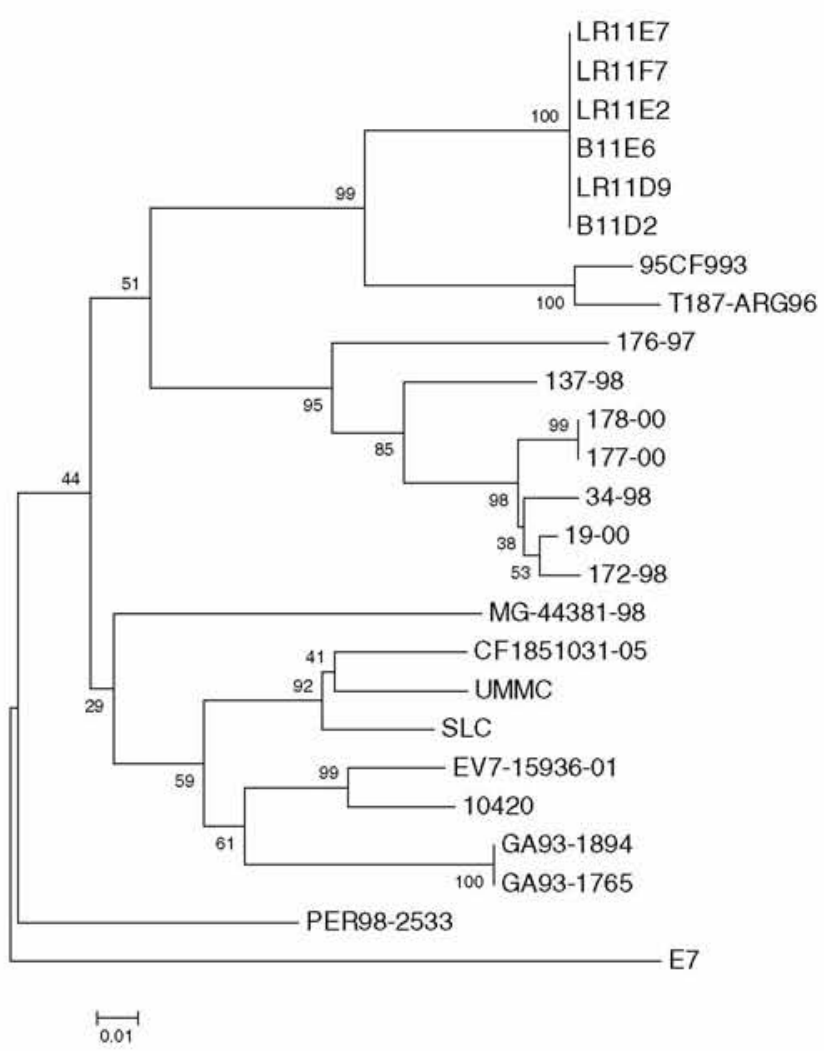

Fig. 3 Phylogenetic tree of part of VP1 of LR11F7 with other E7 strains 


\section{Discussion}

A lot of published research work denotes the need of surveying the environment for assessing the poliovirus circulation in a community. Environmental surveillance along with healthy children stool surveys was successfully used to demonstrate wild and vaccine-derived poliovirus transmission in a community [6, 26-28]. In addition, environmental surveillance has revealed the existence of polioviruses strains recombined with other poliovirus strains or with non-polio enteroviruses strains [12]. Existing research information on environmental samples has further revealed non-polio enterovirus strains [3, 29, 30], but there are only few cases of non-polio enteroviruses isolated from sewage or other environmental samples which have been partially or fully sequenced [31, 32].

In this study, there was an attempt to investigate the circulation of enteroviruses in sewage and river water in the city of Larissa. Two enteroviruses from river water and four from sewage treatment plant were isolated. Comparison of nucleotide sequences of these six isolates revealed that all of them share exactly the same genotype, indicating that the treated sewages discarded in the river have the potential of passing to the food chain.

The full genome sequence of the isolated virus was obtained. For the characterization of this enterovirus, a nucleotide and amino acid comparison of its VP1 genomic region with those of prototype strains of enteroviruses was conducted. This comparison showed $78 \%$ nucleotide similarity and $93 \%$ amino acid homology with E7 Wallace strain. In accordance with the tendency of latest years that the serotype identification of enteroviruses should be based on the criterion of sequence similarity of VP1 with a reference strain in GenBank higher than $75 \%$ (or amino acid sequence homology more than $88 \%$ ), LR $11 \mathrm{~F} 7$ is classified as an E7 strain [24]. Phylogenetic analysis of VP1 genomic region (Fig. 1) grouped LR11F7 with reference E7 strain, supporting the molecular classification of the Greek isolate.

From the phylogenetic analysis of part of VP1 region of LR11F7 and of other E7 isolates, it is obvious that there is a correlation of the Greek isolate with E7 viruses isolated in France in 1995, and in Argentina in 1996. At the same time, there is not high similarity with the Chinese isolates or with any other isolate deposited in GenBank. It is difficult to come to a sound conclusion about the origin of the Greek isolate, since enteroviral sequences deposited in GenBank are extremely few, not only for the whole genome but also for VP1 region and for epidemiological studies, a great number of available sequences will be necessary.

With respect to the phylogenetic analysis conducted for all the genomic regions, separately, LR11F7 was found to be complying with the general rule for enteroviruses concerning the interruption of serotype-genotype correlation in the region of non-structural proteins, a correlation which is present in the capsid protein region [31-34]. In contrast to previous phylogenetic studies $[9,17,32,35]$ in nonstructural proteins of modern $H E V-B$, where their evolutionary relationship with the prototype strains E30, E1, and E9 was revealed, LR11F7 is clustering together with recent enteroviruses that all were isolated in South Asia later than 2000. These data may lead to the hypothesis that there is a different evolutionary pressure in non-capsid protein regions, as was also shown recently for $2 \mathrm{~A}, 2 \mathrm{~B}$, and $2 \mathrm{C}$ genomic regions [36]. However, there is also the chance that phenomena of genetic exchanges may have taken place in common ancestors of these new enteroviruses and that the recombined viruses followed different evolutionary pathways, explaining by this way their close phylogenetic relationship in this part of the genome.

The high conservation of $\mathrm{P} 2$ and $\mathrm{P} 3$ regions within an enterovirus species [37, 38] was also supported in the case of LR11F7 by SimPlot analysis. The correlation of LR11F7 with recent viruses characterized as new types of enteroviruses [39], in functional genomic region, is obvious. LR11F7 seems to share the $3 \mathrm{~A}-3 \mathrm{~B}-5^{\prime}$ end of $3 \mathrm{C}$ region with NH95-0601 and a part of the 3D gene with EV86, both isolated in south Asia and characterized as new types of $H E V-B$.

Our findings indicate South Asia as a possible origin of LR11F7 virus. Great evolutionary events may have taken place in that specific geographical region in enteroviruses by mixing their genomes through recombination events giving rise to a strain that passing to Greece had its own evolutionary history. Analysis of sequence phylogenies between different parts of the genome has led to the concept of semi-independent evolution of structural and nonstructural genes in such a way that enteroviruses circulating worldwide are conceptualized as a population of serologically distinct capsid genes recombining with a range of non-structural genes with transient epidemiological linkage between genome regions associated with episodic spread of individual recombinants. In previous studies, full genome analysis of recombinant epidemic Echo 11 isolates revealed profound differences in pathogenicity, associated with nucleotide transitions in $5^{\prime}$ UTR and VP1 genomic regions [40, 41]. Understanding the tempo and mode of picornavirus evolution are of major importance in the control of pathogenesis of enteroviruses. As sequence data become more available, the evolutionary studies of enteroviruses will be facilitated, new sequence variants will be studied and new features of the evolution of $H E V-B$ genotypes will be revealed. This study indicates also that recombination events may play major role in enteroviruses evolution and that circulation of multi-recombinant strains 
with unknown properties could be potentially dangerous for public health.

Acknowledgments The study was supported by research grants of the Postgraduate Program "Biotechnology," code 3439, of the Department of Biochemistry \& Biotechnology, School of Health Sciences, University of Thessaly.

\section{References}

1. D.W. Griffin, K.A. Donaldson, J.H. Paul, J.B. Rose, Clin. Microbiol. Rev. 16, 129 (2003)

2. R. Pallin, A.P. Wyn-Jones, B.M. Place, N.F. Lightfoot, J. Virol. Methods 67, 57 (1997)

3. Y.S. Shieh, R.S. Baric, M.D. Sobsey, Appl. Environ. Microbiol. 63, 4401 (1997)

4. G. Sedmak, D. Bina, J. MacDonald, Appl. Environ. Microbiol. 69, $7181(2003)$

5. T. Poyry, M. Stenvik, T. Hovi, Appl. Environ. Microbiol. 54, 371 (1988)

6. T. Hovi, M. Stenvik, H. Partanen, A. Kangas, Epidemiol. Infect. 127, $101(2001)$

7. J.W. Drake, J.J. Holland, Proc. Natl Acad. Sci. USA 96, 13910 (1999)

8. N.S. Cuervo, S. Guillot, N. Romanenkova, M. Combiescu, A. Aubert-Combiescu, M. Seghier, V. Caro, R. Crainic, F. Delpeyroux, J. Virol. 75, 5740 (2001)

9. A.N. Lukashev, V.A. Lashkevich, O.E. Ivanova, G.A. Koroleva, A.E. Hinkkanen, J. Ilonen, J. Virol. 77, 10423 (2003)

10. S. Blomqvist, A.L. Bruu, M. Stenvik, T. Hovi, J. Gen. Virol. 84, 573 (2003)

11. I. Karakasiliotis, P. Markoulatos, T. Katsorchis, Mol. Cell. Probes 18, 103 (2004)

12. E. Dedepsidis, Z. Kyriakopoulou, V. Pliaka, C. Kottaridi, E. Bolanaki, S. Levidiotou-Stefanou, D. Komiotis, P. Markoulatos, Appl. Environ. Microbiol. 73, 6697 (2007)

13. H.M. Liu, D.P. Zheng, L.B. Zhang, M.S. Oberste, M.A. Pallansch, O.M. Kew, J. Virol. 74, $11153(2000)$

14. G. Dahourou, S. Guillot, O. Le Gall, R. Crainic, J. Gen. Virol. 83, 3103 (2002)

15. J. Santti, T. Hyypiä, L. Kinnunen, M. Salminen, J. Virol. 73, 8741 (1999)

16. G. Oprisan, M. Combiescu, S. Guillot, V. Caro, A. Combiescu, F. Delpeyroux, R. Crainic, J. Gen. Virol. 83, 2193 (2002)

17. E. Bolanaki, C. Kottaridi, P. Markoulatos, Z. Kyriakopoulou, L. Margaritis, T. Katsorchis, Virus Genes 35, 129 (2007)

18. S. Blomqvist, C. Savolainen, P. Laine, P. Hirttiö, E. Lamminsalo, E. Penttilä, S. Jöks, M. Roivainen, T. Hovi, J. Virol. 78, 4876 (2004)

19. World Health Organization: Guidelines for environmental surveillance of poliovirus circulation. http://www.who.int/vaccinesdocuments/DocsPDF03/www737pdf. Accessed 3 Mar 2003

20. A. Georgopoulou, P. Markoulatos, N. Spyrou, N.C. Vamvakopoulos, J. Clin. Microbiol. 38, 4337 (2000)
21. I. Casas, L. Powell, P.E. Klapper, G.M. Cleator, J. Virol. Methods 53, 25 (1995)

22. M.S. Oberste, W.A. Nix, K. Maher, M.A. Pallansch, J. Clin. Virol. 26, 375 (2003)

23. V. Caro, S. Guillot, F. Delpeyroux, R. Crainic, J. Gen. Virol. 82, $79(2001)$

24. M.S. Oberste, K. Maher, D.R. Kilpatrick, M.R. Flemister, B.A. Brown, M.A. Pallansch, J. Clin. Microbiol. 37, 1288 (1999)

25. M.S. Oberste, K. Maher, M.A. Pallansch, Virus Res. 58, 35 (1998)

26. Y. Manor, R. Handsher, T. Halmut, M. Neuman, A. Bobrov, H. Rudich, A. Vonsover, L. Shulman, O. Kew, E. Mendelson, J. Clin. Microbiol. 37, 1670 (1999)

27. M. Divizia, L. Palombi, E. Buonomo, D. Donia, V. Ruscio, M Equestre, L. Leno, A. Panà, A.M. Degener, Appl. Environ. Microbiol. 65, 3534 (1999)

28. T. Hovi, S. Blomqvist, E. Nasr, C.C. Burns, T. Sarjakoski, N. Ahmed, C. Savolainen, M. Roivainen, M. Stenvik, P. Laine, I. Barakat, M.H. Wahdan, F.A. Kamel, H. Asghar, M.A. Pallansch, O.M. Kew, H. E. Gary Jr., E.M. deGourville, L. El Bassioni, J. Virol. Methods 126, 127 (2005)

29. Y.C. Shieh, R.S. Baric, J.W. Woods, K.R. Calci, Appl. Environ. Microbiol. 69, 7130 (2003)

30. G. Sedmak, D. Bina, J. Macdonald, L. Couillard, Appl. Environ. Microbiol. 71, 1042 (2005)

31. E. Bolanaki, C. Kottaridi, P. Markoulatos, L. Margaritis, T. Katsorchis, Virus Genes 31, 307 (2005)

32. A.N. Lukashev, V.A. Lashkevich, O.E. Ivanova, G.A. Koroleva, A.E. Hinkkanen, J. Ilonen, J. Gen. Virol. 86, 3281 (2005)

33. A.M. Lindberg, P. Andersson, C. Savolainen, M.N. Mulders, T. Hovi, J. Gen. Virol. 84, 1223 (2003)

34. C. Kottaridi, E. Bolanaki, Z. Mamuris, C. Stathopoulos, P. Markoulatos, Arch. Virol. 151, 1117 (2006)

35. C. Kottaridi, E. Bolanaki, Z. Kyriakopoulou, E. Dedepsidis, A. Pratti, P. Markoulatos, Diagn. Microbiol. Infect. Dis. 58, 407 (2007)

36. E. Bolanaki, C. Kottaridi, P. Markoulatos, L. Margaritis, T. Katsorchis, Virus Genes 32, 249 (2006)

37. B. Brown, M.S. Oberste, K. Maher, M.A. Pallansch, J. Virol. 77, 8973 (2003)

38. M.S. Oberste, K. Maher, M.A. Pallansch, J. Virol. 78, 855 (2004)

39. M.S. Oberste, K. Maher, W.A. Nix, S.M. Michele, M. Uddin, D. Schnurr, S. al-Busaidy, C. Akoua-Koffi, M.A. Pallansch, Virus Res. 128, 34 (2007)

40. M.M. El-Sageyer, A. Szendrői, E. Hütter, Gy. Szücs, I. Mezey, I. Tóth, A. Kátai, Z. Kapiller, G. Páll, Gy. Petrás, E. Szalay, I. Mihály, S. Gourova, Gy. Berencsi, Acta Virol. 42, 157 (1998)

41. S. Chevalier, A. Szendrői, V. Caro, J. Balanant, S. Guillot, Gy. Berencsi, F. Delpeyroux, Virology 20, 56 (2004)

42. M.N. Mulders, J.H. Reimerink, M. Stenvik, I. Alaeddinoglu, H.G. van der Avoort, T. Hovi, M.P. Koopmans, J. Gen. Virol. 80, 907 (1999)

43. S. Blomqvist, A. Skytta, M. Roivainen, T. Hovi, J. Clin. Microbiol. 37, 2813 (1999)

44. A.N. Lukashev, V.A. Lashkevich, G.A. Koroleva, J. Ilonen, A.E. Hinkkanen, J. Gen. Virol. 85, 463 (2004) 
From: jcmfield@bellsouth.net>

To: <markoulatosabio.uth.gr>

Sent: Saturday, January 23, $20103: 46$ PM

Subject: Decision on manuscript JCM00475-09 Version 3

PROF PANAYOTIS MARKOULATOS

UNIVERSITY OF THESSALY

BIOCHEMISTRY \& BIOTECHNOLOGY

26 PLOUTONOS STREET

LARISSA 41221

Greece

Re: Full genome sequence analysis of a multi-recombinant echovirus 3 strain isolated from sewage in Greece (JCM00475-09 Version 3)

Dear Dr. MARKOULATOS:

Your manuscript has been accepted, and I am forwarding it to the ASM Journals Department for publication. For your reference, ASM Journals' address is given below. Before it can be scheduled for publication, your manuscript must be checked by the ASM production editor to make sure that

all elements meet the technical requirements for publication.

Charles Brown, the production editor for the Journal of clinical Microbiology (JCM), or his assistant will contact you if anything needs to be revised before copyediting and production begin.

Thank you for submitting your paper to JCM.

Sincerely,

Howard A. Fields

Editor, Journal of Clinical Microbiology (JCM)

Journals Department

American Society for Microbiology

$1752 \mathrm{~N}$ St., NW

Washington, DC 20036

E-mail: journalsrreasmusa.org

Phone: 202-942-9384

Fax: 202-942-9355 
1 Full genome sequence analysis of a multi-recombinant echovirus 3 strain

3 Kyriakopoulou Zaharoula ${ }^{1}$, Dedepsidis Evaggelos ${ }^{1}$, Pliaka Vaia ${ }^{1}$, Tsakogiannis

4 Dimitris $^{1}$, Pratti Anastassia ${ }^{1}$, Levidiotou-Stefanou Stamatina ${ }^{2}$, Markoulatos Panayotis $^{1}$

$5{ }^{1}$ Department of Biochemistry \& Biotechnology, University of Thessaly, 26, Ploutonos

$6 \quad \&$ Aeolou str., Larissa 41 221, Greece.

$7{ }^{2}$ Department of Microbiology, Medical School, University of Ioannina, Ioannina,

8 Greece

9 Running title: molecular analysis of an Echovirus 3 recombinant isolate

10

11

12

13

14

15
Corresponding Author: Professor Panayotis Markoulatos

Department of Biochemistry \& Biotechnology

University of Thessaly 26, Ploutonos \& Aeolou str.

Larissa 41221, Greece

Tel.: 00302410565274 , Fax: 00302410565290

E-mail: markoulatos@bio.uth.gr 
17 An echovirus 3 strain (LR31G7) was isolated from sewage treatment plant in Greece 18 in 2005. A full genome molecular, phylogenetic and SimPlot analysis was conducted 19 in order to reveal the evolutionary pathways of the isolate. Nucleotide and phylogenetic analysis of part of VP1 genomic region revealed that the isolated strain correlates with Echo3 strains isolated the same year in France and Japan, implying that the same virus circulated in Europe and Asia. LR31G7 was found to be recombinant sharing the 3'part of its genome with an Echo25 strain isolated from asymptomatic infants, in Norway, in 2003. Nucleotide and SimPlot analysis of the VP1-2A junction, where the recombination was located, revealed the exact recombination breakpoint (3357nt-3364nt). Moreover, there is evidence that recombination events had occurred in 3B-3D region in the past evolutionary history of the isolate. Our study indicates that recombination events play major role in enteroviruses evolution and that circulation of multi-recombinant strains with unknown properties could be potentially dangerous for public health. 


\section{Introduction}

Enteroviruses are among the most common viruses infecting humans. Although most infections are mild or asymptomatic, they can cause severe and potentially fatal diseases, such as acute hemorrhagic conjunctivitis, aseptic meningo-encephalitis and acute flaccid paralysis (39). They are small, non enveloped viruses with a positive strand RNA of approximately 7.500nt. The enterovirus genome consists of three major regions: the $5^{\prime}$ untranslated region ( $5^{\prime}$ UTR), the $3^{\prime}$ untranslated region ( $3^{\prime}$ UTR) and the open reading frame (ORF), which is translated to a long polyprotein, which contains the four structural (VP1-VP4) and the seven non-structural (2A-2C and 3A3D) viral proteins. Human enteroviruses include immunologically and genetically distinct types and are classified into five species HEV-A to $-\mathrm{D}$ and Polioviruses that show low molecular diversity with HEV-C (7).

An enterovirus population does not exist as a single genotype, but as a group of correlated sequences, named quasispecies. Quasispecies arise from high mutation rate, due to the lack of proofreading activity of the viral RNA polymerase and short generation times $(19,23)$. Despite the fact that high mutation rates result in nonviable viruses, it can lead to a swarm of potentially beneficial mutations to the population level, where under certain circumstances a dominant sequence may emerge after a long silent period, adapted to new environments and challenges during infection (44).

Recent publications state also that enteroviruses within species exist not as delimited lineages but as a pool of independently evolving genome fragments that recombine frequently to give rise to new virus variants $(30,33,45)$. There is a reservoir of a limited number of capsid sequences and a variety of functional regions that recombine giving rise to new viral genotypes exhibiting modified pathogenic properties (29). 
Recombination has been extensively described in polioviruses $(2,11,14,21,22,41$, 42 , 43), but much less attention has been devoted to recombination in non polio enteroviruses. Recent publications based on parts of enterovirus genome revealed that intertypic recombination is a frequent phenomenon in circulating non-polio enteroviruses $(6,24,28,45)$. Although sequences are available for all the prototype strains of enteroviruses, only a restricted number of full genome studies of modern enterovirus strains have been reported so far $(10,29)$.

In this study we present a full genome analysis of an Echo 3 (LR31G7) recombinant strain isolated in 2005 from the sewage treatment plant of Larissa city, Thessaly, Greece. A full genome sequence comparison of the isolate LR31G7 was conducted with prototype Echo 3-Morrisey as well as with the only available full sequenced E3 isolate PicoBankDM1E3. Finally a phylogenetic analysis of VP1 region comparing the LR31G7 isolate with E3 prototype strain and other E3 isolates is reported.

\section{Materials and methods}

Enterovirus isolation from sewages: An enterovirus strain (LR31G7) was isolated from the sewage treatment plant of the city of Larissa, Thessaly, Greece, in October 2005. The environmental sample was concentrated by the two-phase separation method recommended by WHO (48) and the virus was isolated from Rd cells. In order to avoid viral mixtures, 10 -fold serial dilutions were prepared and inoculated into 96-well plates. The last dilution in which CPE was observed was inoculated into $25-\mathrm{cm}^{2}$ flask containing Rd cells. The presence of the virus was confirmed by PCR with primer pair UG52/UC53 (17), following RNA extraction and reverse transcription. 
Primary characterization of the isolate: Enterovirus RNA was extracted from the inoculated Rd cells according to the method described by Casas et al, (9) and stored at $-20^{\circ} \mathrm{C}$. The isolated RNA was reverse transcribed into cDNA. Five $\mu$ l of extracted RNA were incubated with $1 \mu$ of random d(N6) primers (New England Biolabs, United States), $1 \mu \mathrm{l}$ of dNTPs $40 \mathrm{mM}$ and $5 \mu \mathrm{l}$ of RNase- DNase-free distilled water for five min at $65^{\circ} \mathrm{C}$. After cooling on ice, $8 \mu$ l of reaction mixture containing $4 \mu \mathrm{l} \mathrm{M}$ MLV reaction buffer (Invitrogen, Life Technologies, Paisley UK), 200 U M-MLV reverse transcriptase, $2 \mu \mathrm{l}$ of $0,1 \mathrm{mM}$ DTT, $20 \mathrm{U}$ RNase inhibitor (Promega Corporation, Madison, WI) and $0.5 \mu 1$ RNase DNase-free distilled water. The cDNA synthesis was completed by incubation at $25^{\circ} \mathrm{C}$ for $10 \mathrm{~min}, 37^{\circ} \mathrm{C}$ for $50 \mathrm{~min}$ and finally at $70^{\circ} \mathrm{C}$ for $15 \mathrm{~min}$ for $\mathrm{M}-\mathrm{MLV}$ reverse transcriptase inactivation. For the characterization of the isolate LR31G7, VP1 amplification by RT-PCR with primer pair 292/222 has been performed as previously described (37).

Genome amplification: Table 1 shows details of the primer pairs that were used in order to obtain sequence information from all genomic regions of the LR31G7 isolate. Ten of these primers were used for the first time in the present study. They were designed with the aid of Primer3 software, obtained on-line from the Whitehead Institute (http://www.genome.wi.mit.edu/genomesoftware/other/). For the design of primer pairs CHR3/CHR4 and CHR5/CHR6 the sequences of all HEV-B prototype strains were used. The rest primer pairs were designed based on the sequences of the virus isolate LR31G7. All primers were synthesized by Metabion (Martinsried, Germany).

The viral RNA was reverse transcribed into cDNA as described above for all primer pairs, with the exception of EUC2, EUG3a, EUG3b and EUG3c, for which a RT with 
102 the anti-sense primers EUC2a and EUC2b was carried out as has been previously 103 described (8).

104 The PCR mixture for each tube comprised of: $3 \mu 1$ cDNA from LR31G7 isolate, $1 \mu 1$ of 105 each primer at a concentration of $25 \mathrm{pmol} / \mu 1,5 \mu 1$ 10x PCR reaction buffer, $5 \mu 1 \mathrm{dNTPs}$ $10610 \mathrm{mM}, 2,5$ units Paq5000 DNA polymerase (Stratagene) and double-distilled 107 nuclease-free water up to a final volume of $50 \mu \mathrm{l} /$ tube. For the primer pairs CHR3108 CHR4 and CHR5-CHR6 40 cycles of denaturation $\left(95^{\circ} \mathrm{C}\right.$ for $30 \mathrm{sec}$ ), annealing (45 $109{ }^{\circ} \mathrm{C}$ for $\left.1 \mathrm{~min}\right)$ and extension $\left(72{ }^{\circ} \mathrm{C}\right.$ for $\left.1 \mathrm{~min}\right)$ were used. The reactions with primer 110 pairs BL870-BR3231, BL1369-BR2580 and BL4709-BR6063 were performed for 35 111 cycles. Each cycle consisted of $30 \mathrm{sec}$ of denaturation at $95^{\circ} \mathrm{C}, 30 \mathrm{sec}$ of annealing at $11258{ }^{\circ} \mathrm{C}$ (for BL870-BR3231) or at $55^{\circ} \mathrm{C}$ (for BL1369-BR2580 and BL4709-BR6063), 113 and 2min (for BL870-BR3231) or 1,5min (for BL1369-BR2580 and BL4709114 BR6063) of extension at $72{ }^{\circ} \mathrm{C}$. For the other primer pairs, PCR conditions were the 115 same as those described in the original papers (Table 1). PCR products were cleaned 116 up using a PCR gel extraction kit (Qiagen GmbH, Hilden, Germany). All PCR 117 products were sequenced at Macrogen Inc (Seoul, Korea) using the primers described 118 in Table 1.

119 Sequencing and sequence analysis: Initially the sequences were examined in terms 120 of closest homologous sequence using BLAST software, obtained on-line from the 121 National Centre for Biotechnology Information - NCBI 122 (http://www.ncbi.nlm.nih.gov/BLAST/). Multiple sequence alignments were 123 generated using ClustalW software, obtained on-line from the web site of the 124 European Bioinformatics Institute (htxtp://www.ebi.ac.uk/clustalw/). Phylogenetic 125 and molecular analysis was conducted using MEGA version 4 software. The 
126 127 data sets. Finally, nucleotide similarity plots between the aligned sequences were created with the aid of SimPlot software (version 3.1), with a window size of $200 \mathrm{nt}$ for full genome analysis and of 100nt in the case of analysis of recombination 130 junction (26). Partial sequences of the following Echo 3 strains were used for the 131 phylogenetic analysis of the LR31G7 isolate: CF1801081-05 (AM236930), 132 CF1820181-05 (AM236931), Fukuoka City2005-70 (AB234341), Fukuoka City200513397 (AB234342), 94CF858 (AJ241446), BE02-3627 (AY342794), W178-128/99

reliability of the trees was determined by bootstrap analysis with 100 pseudoreplicate (AY208114), 810/85 (AF295490), 2392-82 (AF295453), 108-97 (AB268162), 222-99 (AB268165). For the recombination junction analysis the partial sequence of the Echo25 strain NO-519 (DQ317208) was used.

Nucleotide sequence accession number: The almost complete genomic sequence of isolate LR31G7 (from nucleotide 1 to nucleotide 7189) has been deposited in the GenBank library under accession number FJ766334.

\section{Results}

An enterovirus strain was isolated from sewage treatment plant of the city of Larissa, Greece, in October 2005. Sequencing of the VP1 genomic region revealed that the RL31G7 belongs to the Echo3 serotype, as it shows $79 \%$ (>75\%) nucleotide and $96 \%$ $(>88 \%)$ amino acid identity with the respective region of the Echo3 reference strain (34). An almost full genome analysis (7189nt) of the isolate was conducted, as the only completed sequenced echo 3 strains are the reference strain Morrisey and the PicoBankDM1E3 isolate, associated with diabetes induction, isolated from stool sample in Finland, in 1998 (47). 
149 Table 2 shows the nucleotide and amino acid comparison of the LR31G7 strain with 150 the prototype strain Morrisey and the only complete sequenced Echo3 151 PicoBankDM1E3 isolate. As it is shown in table 2, LR31G7 isolate differs 152 significantly from the prototype strain in all sequenced regions. The region with the 153 highest identity of LR31G7 isolate with the prototype strain is 5' UTR (86\%), 154 followed by regions P2 (81\%), P1 (80\%) and P3 (79\%). The gene with the highest 155 identity is $3 \mathrm{~B}(83 \%)$ and the one with the lowest is $2 \mathrm{~B}(76 \%)$. The nucleotide and 156 amino acid comparison of LR31G7 with the isolate PicoBankDM1E3 revealed that, in 157 the 5' part of the genome (5'UTR-VP1) there is a high identity, 93-90\%, which falls 158 to $82-79 \%$ in the 3' part of the genome (2A-3D).

159 In order to investigate the relationship of the LR31G7 strain with the entire prototype 160 and other strains of enteroviruses species B, phylogenetic trees for each genomic 161 region were constructed (data not shown). In phylogenetic trees for the 5'UTR and 162 capsid regions LR31G7 clusters with the PicoBankDM1E3 isolate. In the 163 phylogenetic trees for the structural genes, there is absolute correlation between 164 serotype and genotype, as the two Echo3 isolates (LR31G7 and PicoBankDM1E3) are 165 clustering together with the Echo3 prototype strain Morrisey.

166 As it was expected the serotype genotype correlation that was observed in capsid 167 coding region, was interrupted in trees for non structural sequences. In $2 \mathrm{~A}$ region 168 LR31G7 is still clustering together with PicoBankDM1E3, but this phylogenetic 169 relationship between these two Echo3 isolates stops in $2 \mathrm{~B}$ coding region. It is also 170 observed that in $2 \mathrm{C}, 3 \mathrm{~A}, 3 \mathrm{C}$ and $3 \mathrm{D}$ phylogenetic trees $\mathrm{LR} 31 \mathrm{G}$ along with modern 171 enterovirus isolates belongs to a separate from the prototype stain cluster. Despite the 172 fact that in $2 \mathrm{~B}, 2 \mathrm{C}$ and $3 \mathrm{~A}$ there is a different classification for each genomic region, 
173 phylogenetic trees for 3B, 3C and 3D revealed that LR31G7 is consistently clustering

174 with CAV9 strains (Cuba35of93, Cuba47of93, Cuba23of00, Cuba267of90) isolated in

175 Cuba from 1990 to 2000 . Between the LR31G7 and the CAV9 isolates there is $86 \%$,

$17685 \%$ and $85-87 \%$ nucleotide identity in $3 \mathrm{~B}, 3 \mathrm{C}$ and $3 \mathrm{D}$ regions respectively.

177 In order to correlate LR31G7 with other short sequences available from Echo 3 178 isolates, a 269nt (2692-2960nt) region of VP1 was chosen for the construction of 179 phylogenetic tree, as it lies within most of the available sequences. As it is shown in 180 figure 1 the isolate LR31G7 clusters together with echoviruses 3 recently isolated 181 from France (CF1801081-05, CF1820181-05) and Japan (Fukuoka City2005-70, 182 Fukuoka City2005-97) in 2005. The nucleotide identity of this part of VP1 between 183 the LR31G7 and the Echo3 isolates of the 2005 cluster, is $95-98 \%$. The isolates of the $184 \quad 1998-2002$ and of the 1997-1998 cluster present between them 86-91\% and 75-78\% 185 nucleotide identity respectively.

186 SimPlot analysis of LR31G7 full genome analysis is presented in figure 2a. In 5'UTR 187 and capsid coding region the strain with the highest identity is PicoBankDM1E3. 188 Passing to the $2 \mathrm{~A}$ coding region there is an abrupt change of the identity that falls to $18981 \%$. It is obvious that a recombination event had occurred giving naissance to a 190 strain that in 5 ' half of the genome is echo 3 and the rest of the genome is derived 191 from another enterovirus B. Full genome SimPlot analysis couldn't help to the 192 detection of the donor of the functional genomic regions of the LR31G7 isolate, as 193 there is small number of complete sequenced enterovirus strains available in 194 GenBank. For this reason, we studied in detail the VP1-2A region, where the 195 recombination was located. The $20 \mathrm{nt}$ of the 3 ' end of VP1 and the 20 nt of the 5' end 196 of 2 A regions have the highest identity (92\%) with the PicoBankDM1E3 isolate but 
197

198

199

200

201

202

203

204

205

206

207

208

209

210

211

212

213

214

215

216

217

218

219

220

100nt downstream that point the closest strain to LR31G7 was an Echo25 strain, NO519, isolated in 2003 from Norway, with $96 \%$ nucleotide identity . SimPlot analysis for the VP1-2A region between LR31G7, PicoBankDM1E3 and NO-519 isolate, is presented in figure $2 b$, where the recombination between the last two strains is obvious. The high nucleotide identity between LR31G7 and the PicoBankDM1E3 and NO-519 isolates, allowed the identification of the exact location of the recombination. As it is shown in figure $2 \mathrm{c}$ the recombination junction was located in 3357nt-3364nt.

\section{Discussion}

In the present study the full genome sequence analysis of an enterovirus isolate (LR31G7) is reported. The strain was isolated from the sewage treatment plant of the city of Larissa, Thessaly, Greece, in October 2005. Primary characterization of the isolate by sequencing of part of VP1 capsid region revealed that LR31G7 belongs to the Echo3 serotype. To our knowledge none clinical Echo3 isolate identical to LR31G7 was reported in Greece. Previous studies in Greece revealed that the most frequently isolated serotypes were Echo6, Echo13, Echo30 in an outbreak of aseptic meningitis in 2001 (46) and Echo6, Echo15 implicated in aseptic meningitis and encephalitis cases in 2005-2007 (15,40).

Nucleotide comparison of LR31G7 with the prototype strain Morrisey and the only full sequenced Echo3 strain PicoBankDM1E3 showed that the Greek isolate differs $17 \%-24 \%$ and $7 \%-21 \%$ respectively, in different genomic regions. The Greek strain is more close to the PicoBankDM1E3 isolate than the prototype Echo3 strain.

Phylogenetic comparison of part of VP1 region of LR31G7 with other Echo3 isolates revealed that LR31G7 is related to Echo3 strains isolated in the same year (2005) in 
Asia and Europe, implying temporal and not topological correlation of these viruses.

The close phylogenetic relationship of these viruses along with the high nucleotide identity (95-98\%) between them, leads to the conclusion that the same virus circulated in Greece, France and Japan in 2005. At present, the rapid transmission of viruses from one region to other permit the viruses to circulate worldwide and not in the narrow topological boundaries of a specific population. From the phylogenetic tree of figure 1, we can also hypothesize that isolate LR31G7 circulated in Europe for almost seven years before pass to Asia. Nevertheless it is difficult to come to a sound conclusion about the epidemiology of LR31G7, as Echo3 viruses have been little studied and there are few isolates of which sequences are deposited in GenBank.

In the 5' part of the genome the nucleotide identity between LR31G7 and the PicoBankDM1E3 is extremely high in contrast with the rest of the genome. This correlation between LR31G7 and PicoBankDM1E3 in 5' UTR-2A region was also confirmed by phylogenetic analysis (data not shown). In this part of the genome the two Echo3 strains cluster together, while in the capsid coding region (data not shown) cluster together and in the same cluster with the prototype strain Morrisey, confirming once more that these strains belong to Echo3 serotype $(4,25,31)$. LR31G7 and PicoBankDM1E3 are separated in 2B-3D phylogenetic trees. This data along with the SimPlot analysis (figure 2), support strongly the hypothesis that a recombination event had occurred between circulating enteroviruses.

Analysis of VP1-2A junction (figure $2 \mathrm{~b}, \mathrm{c}$ ) revealed not only the donor but, for the first time, in non polio enteroviruses, the exact point of recombination (3357nt3364nt). In figures $2 \mathrm{~b}$ and $2 \mathrm{c}$, the exchange of genomic parts between the Echo3 strain PicoBankDM1E3 and the Echo25 strain NO-519 is obvious. Keeping in mind 
245 that genetic exchanges between enteroviruses occur when there is a co-infection of the 246 same cell, recombination between the above two viruses is unlikely to have occurred, 247 as PicoBankDM1E3 and NO-519 were isolated in 1998 and 2003 respectively. 248 Consequently, genetic exchanges have been recently occurred between a descendant 249 of PicoBankDM1E3 and NO-519. We can hypothesize that the Echo3 strains 250 circulated the same year worldwide (figure 1) or direct ancestors of them have 251 recombined with the Echo25 strain. Moreover, it cannot be excluded that the strains 252 of the 2005 cluster, in figure 1, are all recombined. The confirmation of our 253 assumption could be possible only in the case of which genome sequences of these 254 strains were available.

255 As far as phylogenetic analysis of all sequenced regions is concerned and in 256 agreement with previous studies $(4,25,28,29)$, LR31G7 clusters with strains of the 257 same serotype only in capsid coding genes. In phylogenetic trees for $2 \mathrm{~A}-3 \mathrm{~A}$ proteins, 258 correlation is observed with different enteroviruses, but phylogenetic trees for $3 \mathrm{~B}, 3 \mathrm{C}$ 259 and 3D regions revealed that LR31G7 is consistently clustering with CAV9 strains 260 isolated in Cuba from 1990 to 2000, demonstrating their close relationship. The 261 continuous clustering of LR31G7 with CAV9 strains in this part of the genome lead 262 us to the hypothesis that a recombination event might had occurred in the past 263 evolutionary history of LR31G7 strain.

264 In accordance with previous studies for $2 \mathrm{C}$ and $3 \mathrm{D}$ genomic regions $(5,24)$, as well as 265 in 3A and 3C modern enterovirus isolates along with LR31G7 form a cluster, separate 266 from the most HEV-B prototype strains. This observation reinforces the statement that 267 the $2 \mathrm{C}$ and $3 \mathrm{D}$ regions, probably the $3 \mathrm{~A}$ and $3 \mathrm{C}$, are conserved between modern 268 strains. Moreover, in 3D phylogenetic tree LR31G7 and the recently isolated viruses 
269 belong to the Echo1 cluster, whereas according to other studies $(38,30,29)$, isolates that grouped together with E1 prototype, mostly originated from the 1980s.

271 Recombination has been extensively studied in polioviruses $(12,16,18,21,27,43)$.

272 Last few years more attention has been shifted towards non-polio enteroviruses, since 273 it has been proven that a lot of the prototype strains, as Echo18 and CAV21 $(1,20$, $27435)$ are recombinant and there are more and more evidence for genetic exchanges 275 between circulating enteroviruses $(30,35)$. Even though a lot of progress has been 276 done in studies concerning genetic exchanges between enteroviruses, recombination 277 junctions have been determined only in recombinant Sabin poliovirus strains $(13,22)$.

278 In non polio enteroviruses, because of the genomic diversity and the paucity of high 279 nucleotide sequence identity between recent enteroviruses, it is very difficult to 280 allocate the exact point of genetic exchanges between circulating enteroviruses.

281 The present work, where, for the first time, the recombination breakpoint between 282 circulating enterovirus is presented, constitutes an additional step to the study and 283 understanding of enteroviruses evolution.

\section{Acknowledgements}

287 The work was supported by research grants of the Postgraduate Program 288 “Applications of Molecular Biology-Genetics. Diagnostic Biomarkers", code 3817 , 289 of the University of Thessaly, School of Health Sciences, Department of 290 Biochemistry 
292 1. Andersson, P., K. Edman, and A. M. Lindberg. 2002. Molecular analysis of 293 the echovirus 18 prototype: evidence of interserotypic recombination with 294 echovirus 9. Virus Res. 85:71-83.

2. Blomqvist, S., A. L. Bruu, M. Stenvik, and T. Hovi. 2003. Characterization of a recombinant type 3/type 2 poliovirus isolated from a healthy vaccinee and containing a chimeric capsid protein VP1. J. Gen. Virol. 84:573-580.

3. Blomqvist, S., A. Skytta, M. Roivainen, and T. Hovi. 1999. Rapid detection of human rhinoviruses in nasopharyngeal aspirates by a microwell reverse transcription-PCR-hybridisation assay. J. Clin. Microbiol. 37: 2813-2816.

4. Bolanaki, E., C. Kottaridi, P. Markoulatos, L. Margaritis, and T. Katsorchis. 2005. Nucleotide analysis and phylogenetic study of the homology

5. Bolanaki, E., C. Kottaridi, P. Markoulatos, L. Margaritis, and T. 305 Katsorchis. 2006. Evolution of 2B and 2C genomic parts of species B Coxsackie viruses. Phylogenetic study and comparison with other regions. Virus Genes. 32(3):249-59.

6. Bolanaki, E., C. Kottaridi, P. Markoulatos, Z. Kyriakopoulou, L. genomic sequencing shows that polioviruses and members of human enterovirus species $\mathrm{C}$ are closely related in the noncapsid coding region. J. Virol. 77:89738984. 
8. Caro, V., S. Guillot, F. Delpeyroux, and R. Crainic. 2001. Molecular strategy for "serotyping" of human enteroviruses. J. Gen. Virol. 82:79-91.

9. Casas, I., L. Powell, P. E. Klapper, and G. M Cleator. 1995. New method for the extraction of viral RNA and DNA from cerebrospinal fluid for use in the polymerase chain reaction assay. J. Virol. Methods. 53:25-36.

10. Chua, B. H., P. C. McMinn, S. K. Lam, and K. B. Chua. 2001. Comparison of the complete nucleotide sequences of echovirus 7 strain UMMC and the prototype (Wallace) strain demonstrates significant genetic drift over time. J. Gen. Virol. 82:2629-39.

11. Cuervo, N. S., S. Guillot, N. Romanenkova, M. Combiescu, A. AubertCombiescu, M. Seghier, V. Caro, R. Crainic, and F. Delpeyroux. 2001. Genomic features of intertypic recombinant sabin poliovirus strains excreted by primary vaccinees. J. Virol. 75:5740-5751.

12. Dahourou, G., S. Guillot, O. Le Gall, and R. Crainic. 2002. Genetic recombination in wild-type poliovirus. J. Gen. Virol. 83(12):3103-10.

13. Dedepsidis, E., V. Pliaka, Z. Kyriakopoulou, C. Brakoulias, S. LevidiotouStefanou, A. Pratti, Z. Mamuris, and P. Markoulatos. 2008. Complete genomic characterization of an intertypic Sabin 3/Sabin 2 capsid recombinant. FEMS Immunol. Med. Microbiol. 52(3):343-51.

14. Dedepsidis, E., Z. Kyriakopoulou, V Pliaka, C. Kottaridi, E. Bolanaki, S. Levidiotou-Stefanou, D. Komiotis, and P. Markoulatos. 2007. A Retrospective characterization of a Vaccine derived Poliovirus type 1 isolated from sewages in Greece. Appl. Environ. Microbiol. 73(21):6697-704. 
15. Frantzidou, F., K. Dumaidi, A. Spiliopoulou, A. Antoniadis, and A. Papa. 2007. Echovirus 15 and autumn meningitis outbreak among children, Patras, Greece, 2005. J. Clin. Virol. 40(1):77-9.

16. Georgopoulou, A., and P. Markoulatos. 2001. Sabin type 2 polioviruses with intertypic vaccine/vaccine recombinant genomes. Eur. J. Clin. Microbiol. Infect. Dis. 20(11):792-9.

17. Georgopoulou, A., P. Markoulatos, N. Spyrou, and N.C. Vamvakopoulos. 2000. Improved genotyping vaccine and wild-type poliovirus strains by restriction fragment length polymorphism analysis: clinical diagnostic implications. J. Clin. Microbiol. 38:4337-4342.

18. Guillot, S., V. Caro, N. Cuervo, E. Korotkova, M. Combiescu, A. Persu, A. Aubert-Combiescu, F. Delpeyroux, and R. Crainic. 2000. Natural genetic exchanges between vaccine and wild poliovirus strains in humans. J. Virol. 74(18):8434-43 .

19. Holland, J., and E. Domingo. 1998. Origin and evolution of viruses. Virus Genes. 16(1):13-21

20. Hughes, P. J., C. North, P. D. Minor, and G. Stanway. 1989. The complete nucleotide sequence of coxsackievirus A21. J. Gen. Virol. 70:2943-52.

21. Karakasiliotis, I., E. Paximadi, and P. Markoulatos. 2005. Evolution of a rare vaccine-derived multirecombinant poliovirus. J. Gen. Virol. 86:3137-42.

22. Karakasiliotis, I., P. Markoulatos, and T. Katsorchis. 2004. Site analysis of recombinant and mutant poliovirus isolates of Sabin origin from patients and from vaccinees. Mol. Cell. Probes. 18(2):103-9.

23. Kirkegaard, K., and D. Baltimore - 1986. The mechanism of RNA recombination in poliovirus. Cell. 47(3):433-43. 
24. Kottaridi, C., E. Bolanaki, Z. Kyriakopoulou, E. Dedepsidis, A. Pratti, and P. Markoulatos. 2007. Possible recombination and gene adaptation exchanges among clinical echovirus strains: crossing the temporal and topological barriers. Diagn. Microbiol. Infect. Dis. 58(4):407-12.

25. Kottaridi, C., E. Bolanaki, Z. Mamuris, C. Stathopoulos, and P. Markoulatos. 2006. Molecular phylogeny of VP1, 2A, and 2B genes of echovirus isolates: epidemiological linkage and observations on genetic variation. Arch. Virol. 151(6): 1117-32.

26. Kumar, S., K. Tamura, and M. Nei. 2004. MEGA3: integrated software for molecular evolutionary genetics analysis and sequence alignment. Brief. Bioinform. 5:150-163.

27. Kyriakopoulou, Z., C. Kottaridi, E. Dedepsidis, E. Bolanaki, S. LevidiotouStefanou, and P. Markoulatos. 2006. Molecular characterization of wild-type polioviruses isolated in Greece during the 1996 outbreak in Albania. J. Clin. Microbiol. 44(3):1150-2.

28. Lindberg, A. M., P. Andersson, C. Savolainen, M. N. Mulders, and T. Hovi. 2003. Evolution of the genome of Human enterovirus B: incongruence between phylogenies of the VP1 and 3CD regions indicates frequent recombination within the species. J. Gen. Virol. 84(5):1223-35.

29. Lukashev, A. N., V. A. Lashkevich, O. E. Ivanova, G. A. Koroleva, A. E. Hinkkanen, and J. Ilonen. 2005. Recombination in circulating Human enterovirus B: independent evolution of structural and non-structural genome regions. J. Gen. Virol. 86(12):3281-90. 
30. Lukashev, A. N., V. A. Lashkevich, O. E. Ivanova, G. A. Koroleva, A. E. Hinkkanen, and J. Ilonen. 2003. Recombination in circulating enteroviruses. J. Virol. 77:10423-10431.

31. Mirand, A., C. Archimbaud, C. Henquell, Y. Michel, M. Chambon, H. Peigue-Lafeuille, and J. L. Bailly. 2006. Prospective identification of HEV-B enteroviruses during the 2005 outbreak. J. Med. Virol. 78(12):1624-34.

32. Mulders, M. N., J. H. Reimerink, M. Stenvik, I. Alaeddinoglu, H. G. van der Avoort, T. Hovi, and M. P. Koopmans. 1999. A Sabin vaccine-derived field isolate of poliovirus type 1 displaying aberrant phenotypic and genetic features, including a deletion in antigenic site 1. J. Gen. Virol. 80:907-16.

33. Oberste, M. S., K. Maher, and M. A. Pallansch. 2004. Evidence for frequent recombination within species human enterovirus B based on complete genomic sequences of all thirty-seven serotypes. J. Virol. 78(2):855-67.

34. Oberste, M. S., K. Maher, D. R. Kilpatrick, M. R. Flemister, B. A. Brown, and M. A. Pallansch. 1999. Typing of human enteroviruses by partial sequencing of VP1. J. Clin. Microbiol. 37(5):1288-93.

35. Oberste, M. S., S. Peñaranda, and M. A. Pallansch. 2004. RNA recombination plays a major role in genomic change during circulation of coxsackie B viruses. J. Virol. 78(6):2948-55.

36. Oberste, M. S., S. Peñaranda, K. Maher, and M. A. Pallansch. 2004. Complete genome sequences of all members of the species Human enterovirus A. J. Gen. Virol. 85(6):1597-607.

37. Oberste, M. S., W. A. Nix, K. Maher, and M. A. Pallansch. 2003. Improved molecular identification of enteroviruses by RT-PCR and amplicon sequencing. J. Clin. Virol. 26(3):375-7. 
38. Oprisan, G., M. Combiescu, S. Guillot, V. Caro, A. Combiescu, F. Delpeyroux, and R. Crainic. 2002. Natural genetic recombination between cocirculating heterotypic enteroviruses. J. Gen. Virol. 83:2193-200.

39. Palacios, G., and M. S. Oberste. 2005. Enteroviruses as agents of emerging infectious diseases. J. Neurovirol. 11(5):424-33.

40. Papa A., L. Skoura, K. Dumaidi, A. Spiliopoulou, A. Antoniadis, and F. Frantzidou. 2009. Molecular epidemiology of Echovirus 6 in Greece. Eur. J. Clin. Microbiol. Infect. Dis. 28(6):683-7.

41. Paximadi, E., I. Karakasiliotis, D. Papaventsis, G. Papageorgiou, and P. Markoulatos. 2008. Recombinant Sabin environmental isolates in Greece and Cyprus. J. Appl. Microbiol. 104(4):1153-62.

42. Paximadi, E., I. Karakasiliotis, E. Bolanaki, A. Krikelis, and P. Markoulatos. 2007. Vaccine derived bi- and multi-recombinant Sabin strains. Virus Genes. 35(3):541-8.

43. Paximadi, E., I. Karakasiliotis, Z. Mamuris, C. Stathopoulos, V. Krikelis, and P. Markoulatos. 2006. Genomic analysis of recombinant sabin clinical isolates. Virus Genes. 32(2):203-10.

44. Ruiz-Jarabo, C. M., A. Arias, E. Baranowski, C. Escarmís, and E. Domingo. 2000. Memory in viral quasispecies. J. Virol. 74(8):3543-7.

45. Santti, J., T. Hyypiä, L. Kinnunen, and M. Salminen. 1999. .Evidence of recombination among enteroviruses. J. Virol. 73(10):8741-9.

46. Siafakas, N., P. Markoulatos, and S. Levidiotou-Stefanou. 2004. Molecular identification of enteroviruses responsible for an outbreak of aseptic meningitis; implications in clinical practice and epidemiology. Mol. Cell. Probes. 18(6):38998. 
444

47. Williams, C. H., S. Oikarinen, S. Tauriainen, K. Salminen, H. Hyöty, and G. J. Stanway. 2006. Molecular analysis of an echovirus 3 strain isolated from an individual concurrently with appearance of islet cell and IA-2 autoantibodies. Clin. Microbiol. 44(2):441-8.

48. World Health Organization. 3 March 2003, posting date. Guidelines for environmental surveillance of poliovirus circulation. World Health Organisation, Geneva, Switzerland. http://www.who.int/vaccinesdocuments/DocsPDF03/www737pdf. 


\section{Figure legends:}

446 Figure 1: Phylogenetic tree of 269nt (2692-2960nt) of a region of VP1 of LR31G7

447 and other Echo3 strains.

448 Figure 2: (a) Full genome SimPlot analysis of LR31G7, (b) SimPlot analysis of 449 recombination junction between PicoBankDM1E3 and NO-519 strain, (c) 450 recombination junction of LR31G7. Identities between LR31G7 and 451 PicoBankDM1E3 are highlighted in yellow, while identities between LR31G7 and 452 NO-519 are highlighted in green. 
Table 1: Primers used in the present study

\begin{tabular}{|c|c|c|c|c|}
\hline Primer & Position & Polarity & Sequence $\left(5^{\prime}-3^{\prime}\right)$ & Reference \\
\hline $\begin{array}{l}72437 \\
216616\end{array}$ & $\begin{array}{l}001-020 \\
545-565\end{array}$ & $\begin{array}{c}\text { Sense } \\
\text { Antisense }\end{array}$ & $\begin{array}{l}\text { TTAAAACAGCTCTGGGGTTG } \\
\text { GAAACACGGACACCCAAAGTA }\end{array}$ & $\begin{array}{l}\text { Mulders et al., } 1999 \\
\text { Blomqvist et al., } 1999\end{array}$ \\
\hline $\begin{array}{l}0340 \mathrm{~F} \\
1200 \mathrm{R}\end{array}$ & $\begin{array}{c}310-333 \\
1177-1196\end{array}$ & $\begin{array}{c}\text { Sense } \\
\text { Antisense }\end{array}$ & $\begin{array}{c}\text { TAGATCAGGCYGATGAGTCACCGC } \\
\text { GGGAATTTCCACCACCACCC }\end{array}$ & Lukashev et al., 2005 \\
\hline $\begin{array}{l}\text { BL870 } \\
\text { BR3231 }\end{array}$ & $\begin{array}{c}870-890 \\
3231-3211\end{array}$ & $\begin{array}{c}\text { Sense } \\
\text { Antisense }\end{array}$ & $\begin{array}{l}\text { CGACAGGATTTCACACAGGA } \\
\text { GCTTTTCACATACGGGCTAA }\end{array}$ & Present study \\
\hline $\begin{array}{l}\text { BL1369 } \\
\text { BR2580 }\end{array}$ & $\begin{array}{l}1369-1389 \\
2580-2560\end{array}$ & $\begin{array}{c}\text { Sense } \\
\text { Antisense }\end{array}$ & $\begin{array}{l}\text { GAGGTTGTCGCAGCTTCTCT } \\
\text { GGGAACCACTTGTGAGGTGT }\end{array}$ & Present study \\
\hline $\begin{array}{l}292 \\
222\end{array}$ & $\begin{array}{l}2612-2627 \\
2969-2951\end{array}$ & $\begin{array}{c}\text { Sense } \\
\text { Antisense }\end{array}$ & $\begin{array}{l}\text { MIGCIGYIGARACNGG } \\
\text { CICCIGGIGGIAYRWACAT }\end{array}$ & Oberste et al., 2003 \\
\hline $\begin{array}{l}\text { EUG3a } \\
\text { EUG3b } \\
\text { EUG3c } \\
\text { EUC2 } \\
\text { EUC2a } \\
\text { EUC2b }\end{array}$ & $\begin{array}{l}2946-2965 \\
2946-2965 \\
2946-2965 \\
4413-4433 \\
4428-4448 \\
4428-4448\end{array}$ & $\begin{array}{c}\text { Sense } \\
\text { Sense } \\
\text { Sense } \\
\text { Antisense } \\
\text { Antisense } \\
\text { Antisense }\end{array}$ & $\begin{array}{c}\text { TGGCAAACTTCCWCCAACCC } \\
\text { TGGCAAACATCTTCMAATCC } \\
\text { TGGCAGACTTCAACHAACCC } \\
\text { TTTGCACTTGAACTGTATGTA } \\
\text { GGTTCAATACGGCATTTG } \\
\text { GGTTCAATACGGTGTTTGCT }\end{array}$ & Caro et al. (2001) \\
\hline $\begin{array}{l}\text { CHR1 } \\
\text { CHR2 }\end{array}$ & $\begin{array}{l}4284-4308 \\
5084-5081\end{array}$ & $\begin{array}{c}\text { Sense } \\
\text { Antisense }\end{array}$ & $\begin{array}{l}\text { CNTCHCARAGTGAYCARGARCARYT } \\
\text { GTAYACYGGTGGWCCYTGRAAKA }\end{array}$ & Kottaridi et al 2007 \\
\hline $\begin{array}{l}\text { BLA709 } \\
\text { BR6063 }\end{array}$ & $\begin{array}{l}4709-4729 \\
6063-6043\end{array}$ & $\begin{array}{c}\text { Sense } \\
\text { Antisense }\end{array}$ & $\begin{array}{l}\text { TACATCCCCATTTGTGTTGG } \\
\text { TGCTGGCTCCTTGTTACCTT }\end{array}$ & Present study \\
\hline $\begin{array}{l}\text { CHR3 } \\
\text { CHR4 }\end{array}$ & $\begin{array}{l}5047-5065 \\
5860-5841\end{array}$ & $\begin{array}{c}\text { Sense } \\
\text { Antisense }\end{array}$ & $\begin{array}{l}\text { CIACYCTWGARGCRCTVTT } \\
\text { GACRTGAGIACHCCRCCRCA }\end{array}$ & Present study \\
\hline $\begin{array}{l}5850 \mathrm{~F} \\
6500 \mathrm{R}\end{array}$ & $\begin{array}{l}5837-5859 \\
6531-6506\end{array}$ & $\begin{array}{c}\text { Sense } \\
\text { Antisense }\end{array}$ & $\begin{array}{c}\text { CAGTGYGGIGGIGTICTCATGTC } \\
\text { AGRTTGCCAAAYGTYTGYCTCATTGC }\end{array}$ & Lukashev et al., 2003 \\
\hline $\begin{array}{l}\text { CHR5 } \\
\text { CHR6 }\end{array}$ & $\begin{array}{l}6485-6507 \\
7250-7227\end{array}$ & $\begin{array}{c}\text { Sense } \\
\text { Antisense }\end{array}$ & $\begin{array}{l}\text { ATCCAGYTTGAAYGAYTCIGIRG } \\
\text { GAAYTCYTCRTAYTCKTGCTCYCC }\end{array}$ & Present study \\
\hline
\end{tabular}

454 
455 Table 2: Nucleotide and amino acid sequence identity between LR31G7, Echo3 prototype

456 strain Morrisey and PicoBankDM1E3 strain.

$457 * 1251$ nt of 3D genomic region.

458

\begin{tabular}{|c|c|c|c|c|}
\hline $\begin{array}{c}\text { Genomic } \\
\text { Region }\end{array}$ & \multicolumn{2}{|c|}{ Morrisey } & \multicolumn{2}{c|}{ PicoBankDM1E3 } \\
\hline & $\begin{array}{c}\text { \% nucleotide } \\
\text { identity }\end{array}$ & $\begin{array}{c}\text { \%amino acid } \\
\text { identity }\end{array}$ & $\begin{array}{c}\text { \% nucleotide } \\
\text { identity }\end{array}$ & $\begin{array}{c}\text { \%amino acid } \\
\text { identity }\end{array}$ \\
\hline 5' UTR & 86 & & 93 & \\
\hline P1 & 80 & & 90 & \\
\hline VP4 & 80 & 98 & 92 & 100 \\
\hline VP2 & 79 & 97 & 90 & 99 \\
\hline VP3 & 82 & 97 & 91 & 99 \\
\hline VP1 & 79 & 96 & 90 & 98 \\
\hline P2 & 81 & & 81 & 94 \\
\hline & 82 & 94 & 82 & 98 \\
\hline 2A & 76 & 94 & 79 & 97 \\
\hline 2B & 81 & 98 & 82 & 97 \\
\hline 2C & 79 & & 81 & 95 \\
\hline P3 & 79 & 97 & 80 & 97 \\
\hline 3A & 83 & 95 & 80 & 97 \\
\hline 3B & 78 & 93 & 90 & \\
\hline 3C & 78 & 96 & & \\
\hline 3D* & & & & \\
\hline
\end{tabular}

459 


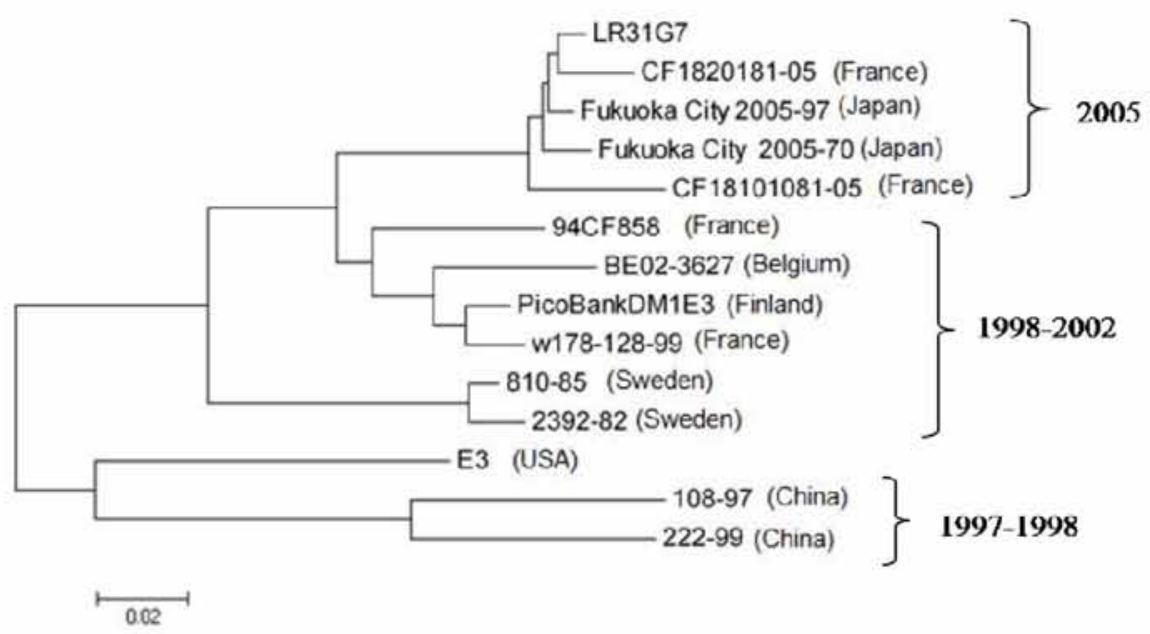


(a)

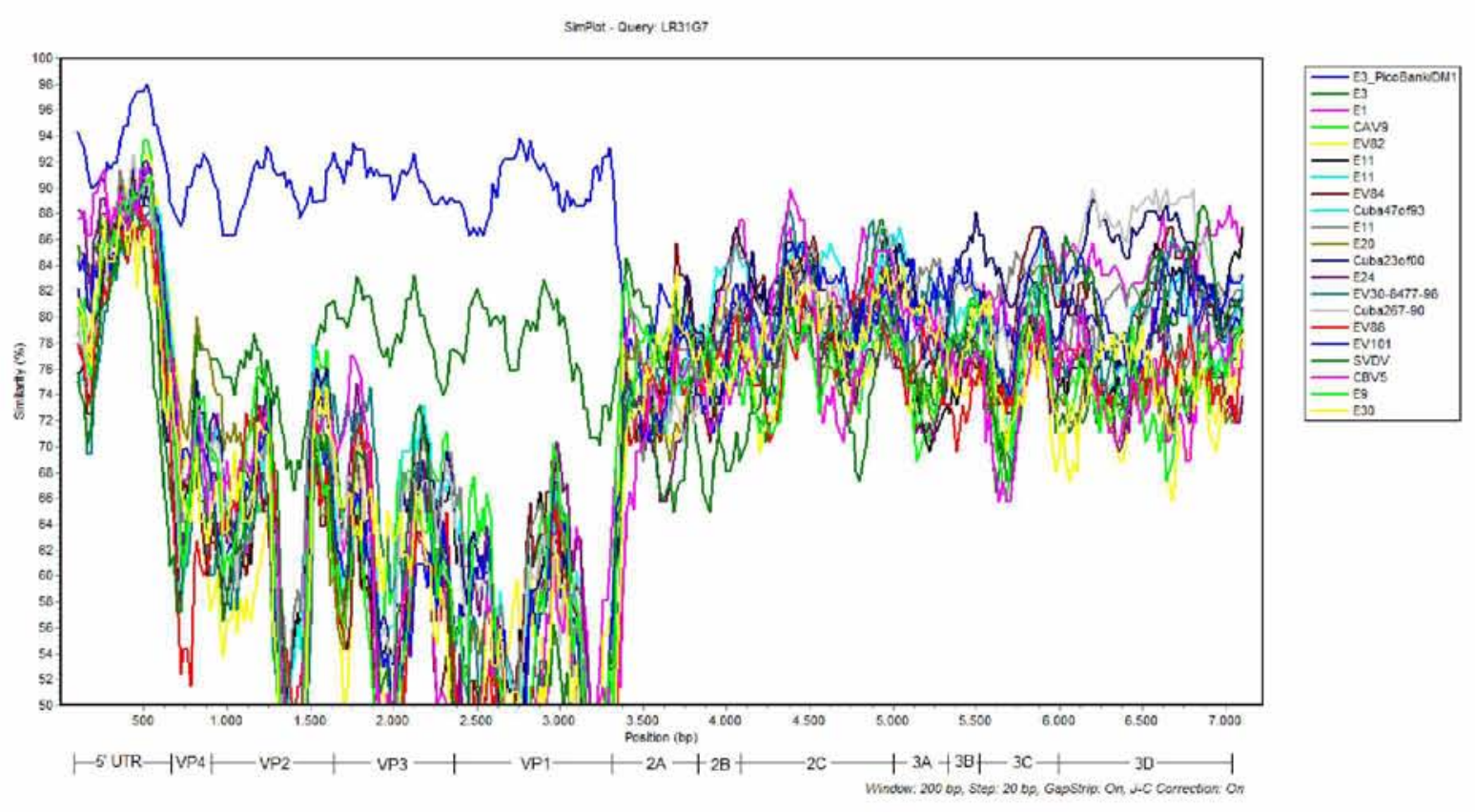

(b)

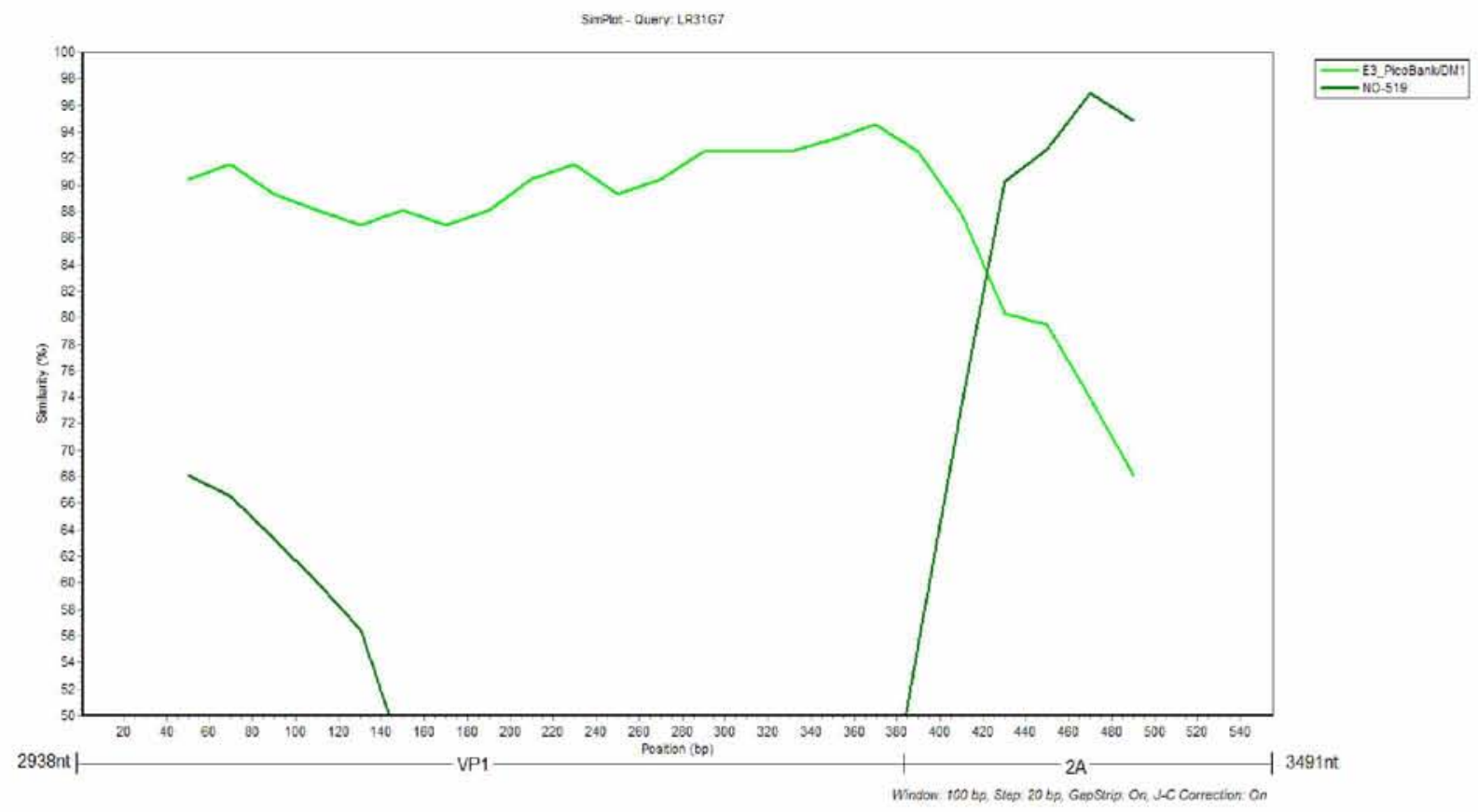


(c)

PicoBank/DM1/E3

LR31G7

NO-519

PicoBank/DM1/E3

LR31G7

NO-519

PicoBank/DM1/E3

LR31G7

NO-519
TATGGGGACGCTCCGCCCCATGGGTTATACCAGCCATGGGGCATTCGGGCAGCAGTCTGG TGTGGGGACGCTTCGCCCCATGGGTTATACTAACCATGGAGCATTCGGGCAGCAATCTGG AGAGAGATGACCATTTGCCCTCAATGGCA--AACCATGGGGCGTTCGGCCAGCAATCCGG

$3357 \quad 3364$

GGCTATdTATGTGGGAACTACAGAGTAGTCAATAGGCACCTAGCCACGCATGTAGACTG GGCTAT TATGTGGG AACTACAGAGTGGTCAACCGGCACTTAGCAACACACACTGACTG AGCTGTATATGTGGGCAACTACAGAGTGGTCAACCGGCACTTGGCAACACACACTGATTG

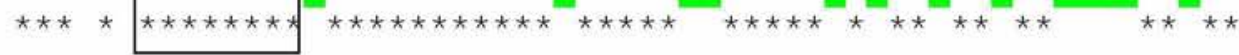

GAGCAATTGTGTCTGGGAGGACTATAACAGAGATTTACTAATCAGCACCACTACGGCCCT GCAAAATTGTGTGTGGGAGAACTACAACAGAGATCTCTAGT GAGCACAACCACAGCACA GCAAAATTGTGTGTGGGAGAATTACAACAGAGATCTCCTAGTGAGCACAACCACTGCACA 